LA-UR- $94-1510$

Title:

BEST AVAILABLE TECHNOLOGY FOR

THE LOS ALAMOS NATIONAL LABORATORY

RADIOACTIVE LIQUID WASTE TREATMENT FACILITY

\title{
RECEIVED
}

DEC 071994

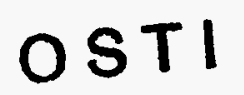

Submitted to:

U. S. Department of Energy

Defense Nuclear Facilities Safety Board

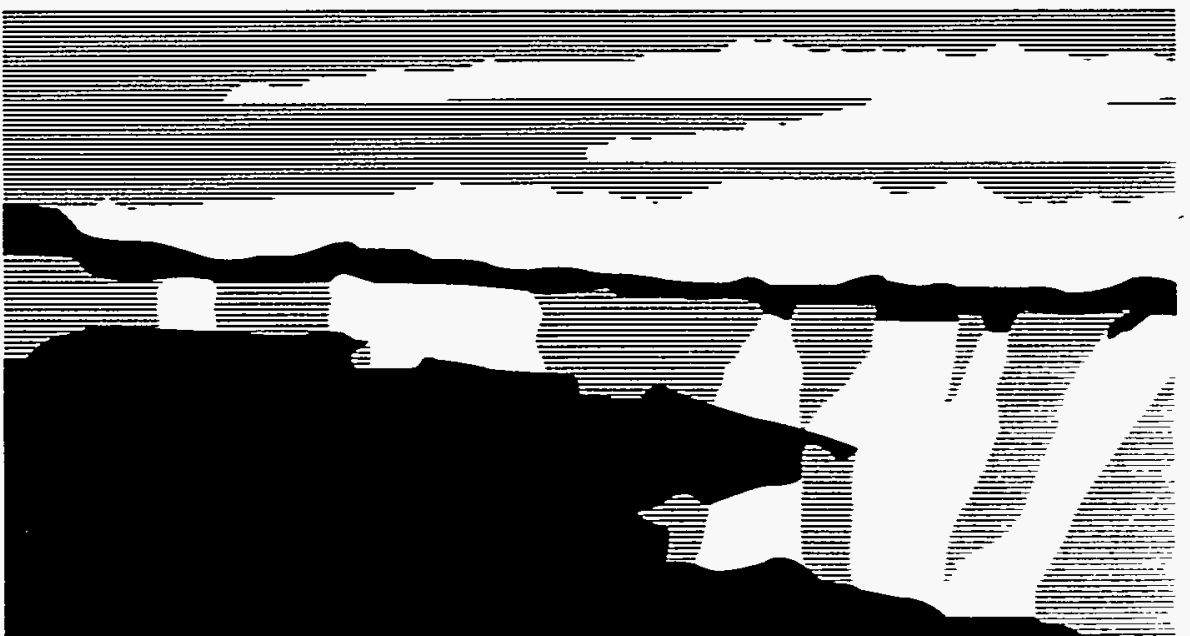

NATIONAL LABORATORY

Los Alamos National Laboratory, an affirmative action/equal opportunity employer, is operated by the University of California for the U.S. Department of Energy under contract W-7405-ENG-36. By acceptance of this article, the publisher recognizes that the U.S. Government retains a nonexclusive, royalty-free license to publish or reproduce the published form of this contribution, or to allow others to do so, Yor U.S. Government purposes. The Los Alamos National Laboratory requests that the publisher identify this article as work performed under the auspices of the U.S. Department of Energy. 


\title{
Best Available Technology Study
}

\author{
for the Los Alamos National Laboratory
}

\section{Radioactive Liquid Waste Treatment Facility}

\section{RALPH M. PARSONS CO.}

\begin{abstract}
DISCLAIMER
This report was prepared as an account of work sponsored by an agency of the United States Government. Neither the United States Government nor any agency thereof, nor any of their employees, makes any warranty, express or implied, or assumes any legal liability or responsibility for the accuracy, completeness, or usefulness of any information, apparatus, product, or process disclosed, or represents that its use would not infringe privately owned rights. Reference herein to any specific commercial product, process, or service by trade name, trademark, manufacturer, or otherwise does not necessarily constitute or imply its endorsement, recommendation, or favoring by the United States Government or any agency thereof. The views and opinions of authors expressed herein do not necessarily state or reflect those of the United States Government or any agency thereof.
\end{abstract}




\section{DISCLAIMER}

\section{Portions of this document may be illegible in electronic image products. Images are produced from the best available original document.}




\section{LOS ALAMOS NATIONAL LABORATORY RADIOACTIVE LIQUID WASTE TREATMENT FACILITY BEST AVAILABLE TECHNOLOGY STUDY SIGNATURES AND AUTHORIZATIONS}

W. Midkiff, Task Manager

I. L. Suazo, Deputy Project Manager

R. Garcia, Jr., Project Manager

A. Drypolcher, Program Manager 


\section{LOS ALAMOS NATIONAL LABORATORY \\ RADIOACTIVE LIQUID WASTE TREATMENT FACILITY \\ BEST AVAILABLE TECHNOLOGY STUDY}

TABLE OF CONTENTS

$\underline{\text { Page }}$

EXECUTIVE SUMMARY $\ldots \ldots \ldots \ldots \ldots \ldots \ldots \ldots \ldots$ i

1.0 INTRODUCTION $\ldots \ldots \ldots \ldots \ldots \ldots \ldots \ldots \ldots \ldots \ldots \ldots \ldots \ldots \ldots \ldots \ldots$

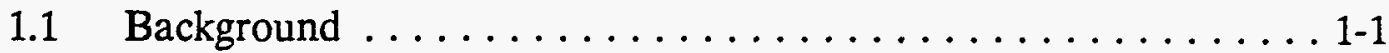

1.2 Analysis of Existing Liquid Effluent Treatment System . . . . . 1-1

1.3 Facility Description and Mission $\ldots \ldots \ldots \ldots \ldots \ldots \ldots \ldots \ldots \ldots .2$

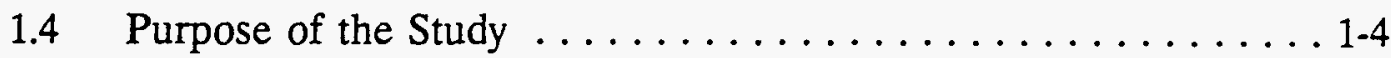

2.0 OPERATIONS AT EXISTING DEPARTMENT OF ENERGY

FACILITIES $\ldots \ldots \ldots \ldots \ldots \ldots \ldots \ldots \ldots \ldots \ldots \ldots \ldots \ldots \ldots \ldots \ldots \ldots, 1$

2.1 Los Alamos National Laboratory - Current TA-50

Waste Treatment Operations ................... 2-1

2.2 Savannah River Site Effluent Treatment Facility . . . . . . . 2-9

2.3 Hanford Site Effluent Treatment Plant . . . . . . . . . 2-19

2.4 Rocky Flats Plant Liquid Radwaste Treatment Plant . . . . . 2-28

2.5 Oak Ridge Central Pollution Control Facility ......... 2-31

2.6 Oak Ridge West End Treatment Facility $\ldots \ldots \ldots \ldots \ldots$ 2-37

3.0 SELECTION OF SUITABLE TREATMENT PROCESSES $\ldots \ldots \ldots$ 3-1

3.1 Bases for the Evaluation of Unit Processes . . . . . . . . . . 3-1

3.2 Analysis of Treatment Technologies and Processes ....... 3-6

3.3 Selection of Process Configurations $\ldots \ldots \ldots \ldots \ldots \ldots \ldots .3-26$ 
4.0 ANALYSIS OF ENVIRONMENTAL ISSUES $\ldots \ldots \ldots \ldots \ldots \ldots$ 4-1

4.1 Analysis of Environmental/Regulatory Issues $\ldots \ldots \ldots \ldots .4-1$

4.2 Design Basis Characterization of the Influent Waste Stream . . . 4-4

4.3 Environmental Bases for the Process Configurations .... . . 4-6

4.4 Environmental Issues for PC \#1 $\ldots \ldots \ldots \ldots \ldots \ldots \ldots .4 .8$

4.5 Environmental Issues for PC \#2 $\ldots \ldots \ldots \ldots \ldots \ldots \ldots$ 4.9

4.6 Environmental Issues for PC \#3 $\ldots \ldots \ldots \ldots \ldots \ldots \ldots$ 4-10

4.7 Environmental Issues for $\mathrm{PC} \# 4 \ldots \ldots \ldots \ldots \ldots \ldots$ 4-11

4.8 Environmental Issues for $\mathrm{PC} \# 5 \ldots \ldots \ldots \ldots \ldots \ldots \ldots$ 4-12

4.9 Environmental Issues for PC \#6 $\ldots \ldots \ldots \ldots \ldots \ldots \ldots$ 4-13

4.10 Environmental Issues for $\mathrm{PC} \# 7 \quad \ldots \ldots \ldots \ldots \ldots \ldots .4-14$

4.11 Environmental Issues for $\mathrm{PC} \# 8 \quad \ldots \ldots \ldots \ldots \ldots \ldots \ldots$ 4-14

4.12 Environmental Issues for PC \#9 $\ldots \ldots \ldots \ldots \ldots \ldots \ldots$ 4-15

4.13 Environmental Issues for $\mathrm{PC} \# 10 \ldots \ldots \ldots \ldots \ldots \ldots .4$ 4-16

4.14 Environmental Issues for $\mathrm{PC} \# 11 \ldots \ldots \ldots \ldots \ldots \ldots \ldots .4 .17$

4.15 Environmental Issues for $\mathrm{PC} \# 12 \ldots \ldots \ldots \ldots \ldots \ldots \ldots .4-17$

4.16 Evaluation and Elimination of PCs Based on Environmental Issues . . . . . . . . . . . . . . 4-18

5.0 SELECTION OF CANDIDATE TECHNOLOGIES $\ldots \ldots \ldots \ldots .5-1$

5.1 Development of Candidate Technologies $\ldots \ldots \ldots \ldots \ldots$ 5-1

5.2 Discussion of Candidate Technologies Selected $\ldots \ldots \ldots \ldots$. 5-2

5.3 Analysis of Environmental Issues for Candidate Technologies ... 5-6 
ANALYSIS OF OPERATIONAL ISSUES $\ldots \ldots \ldots \ldots \ldots \ldots \ldots-1$

6.1 Operational Evaluation of the Existing Facility $\ldots \ldots \ldots \ldots 6-1$

6.2 Definition of Functional Requirements . . . . . . . . 6-2

6.3 Operational Assumptions $\ldots \ldots \ldots \ldots \ldots \ldots \ldots \ldots .4$

6.4 Operational Issues and Criteria $\ldots \ldots \ldots \ldots \ldots \ldots \ldots 6-5$

6.5 Operational Issues for Candidate Technology \#1 $\ldots \ldots \ldots .6-8$

6.6 Operational Issues for Candidate Technology \#2 $\ldots \ldots \ldots .6$ 6-14

6.7 Operational Issues for Candidate Technology \#3 . . . . . . 6-19

6.8 Operational Issues for Candidate Technology \#4 . . . . . . . 6-24

6.9 Final Evaluation of Candidate Technologies . . . . . . 6 6-28

7.0 ANALYSIS OF ENERGY AND RESOURCE ISSUES $\ldots \ldots \ldots \ldots 7$-1

7.1 Evaluation of the Energy and Resources Use by the Existing

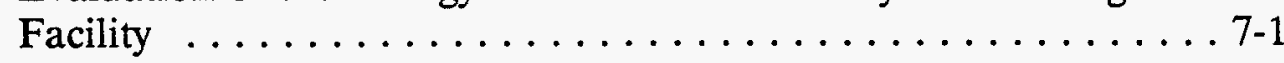

7.2 Technical Considerations for Energy Management and Resource Utilization ........................ 7-3

7.3 Energy and Resource Use Assumptions . . . . . . . . 7-4

7.4 Energy and Resource Use Issues and Criteria $\ldots \ldots \ldots \ldots \ldots 7-4$

7.5 Energy and Resource Use and Conservation Issues for Candidate Technology \#1 ................. 7-5

7.6 Energy and Resource Use and Conservation Issues for Candidate Technology \#2 ................. 7-7

7.7 Energy and Resource Use and Conservation Issues for Candidate Technology \#3

7.8 Energy and Resource Use and Conservation Issues for Candidate Technology \#4 
7.9 Evaluation and Elimination of Process Configurations

Based on Energy and Resource Use Issues . . . . . . . . . 7-14

8.0 ANALYSIS OF ECONOMIC/COST FEASIBILITY $\ldots \ldots \ldots \ldots 8-1$

8.1 Figure-Of-Merit Calculations $\ldots \ldots \ldots \ldots \ldots \ldots \ldots \ldots$. 1

8.2 Elements of Economic Analysis $\ldots \ldots \ldots \ldots \ldots \ldots \ldots$. 8 -1

8.3 Economic Considerations for Candidate Technologies $\ldots . .8$ 8-3

8.4 Cost Analysis of Candidate Technology \#1 ......... 8-6

8.5 Cost Analysis of Candidate Technology \#2 ........ 8-6

8.6 Cost Analysis of Candidate Technology \#3 . . . . . . . 8-7

8.7 Cost Analysis of Candidate Technology \#4 . . . . . . . . 8-8

8.8 Estimated Relative Project Cost Comparisons for Selected Candidate Technologies .................. 8 8.8

9.0 SELECTION OF THE BEST DEMONSTRATED AVAILABLE TECHNOLOGY $\ldots \ldots \ldots \ldots \ldots \ldots \ldots \ldots \ldots \ldots \ldots \ldots .9 .1$

9.1 Technology Issues $\ldots \ldots \ldots \ldots \ldots \ldots \ldots \ldots \ldots \ldots .1$

9.2 Economic Evaluation $\ldots \ldots \ldots \ldots \ldots \ldots \ldots \ldots \ldots \ldots .9 \ldots \ldots$

9.3 Final Cost-Effect Analysis and Technology Selection ...... 9-3

10.0 REFERENCES $\ldots \ldots \ldots \ldots \ldots \ldots \ldots \ldots \ldots \ldots \ldots \ldots \ldots \ldots \ldots \ldots \ldots \ldots \ldots$ APPENDICES 


\section{LOS ALAMOS NATIONAL LABORATORY \\ RADIOACTIVE LIQUID WASTE TREATMENT FACILITY \\ BEST AVAILABLE TECHNOLOGY STUDY}

\section{EXECUTIVE SUMMARY}

The existing Los Alamos National Laboratory TA-50 liquid radioactive waste treatment plant RLWP has been in service for over thirty years, during this period many technical, regulatory, and processing changes have occurred. The existing facility can no longer comply with the demands and requirements for continued operation, and would not be able to comply with anticipated stringent future contaminant discharge limitations. Either a major upgrading or replacement of the existing facility is required. In order to assess the most appropriate means of providing an adequate facility to comply with predicted requirements for TA-50, this Best Available Technology (BAT) Study was conducted to compare feasible technical and economic alternatives in order to define the most favorable technology configuration.

This report consists of eleven sections. Section 1 provides a general introduction and background of the TA-50 operations and the basis for this study. Section 2 provides a technical discussion of the unit processes at TA-50 and several other comparable operations at other DOE sites. Section 3 addresses the evaluation and selection of appropriate treatment processes. Section 4 provides an analysis of environmental issues and concerns. Section 5 presents the rationale for the selection of preferred process configurations. Section 6 is the evaluation of operational issues. Section 7 addresses energy and resource use topics. Section 8 provides an economic analysis, and Section 9 summarizes the evaluation and the identification of the BAT. These sections are augmented by appendices.

The report identifies the construction of a new radioactive liquid waste treatment facility as the BAT. Based on the information analyzed for this study, this option appears to provide the best combination of environmental compliance, operability, and economic value.

\section{PROCESS EVALUATION AND SELECTION}

A liquid rad-waste treatment process is selected and developed as part of this BAT study. There are many factors which impact the optimum choice recommended as the result of this study. The wastes to be treated are considered fixed, as are the requirements to meet release criteria, but plant costs, operational considerations, environmental issues, energy and resource usage, and even plant location are all strongly impacted by how the waste streams may be combined and the treatment process chosen. And a recommendation of a BAT encompasses all of these factors. In dealing with a radioactive waste, the volume and characteristics of any secondary waste produced is an additional important consideration.

The title of this report actually says a lot. This is a BAT report of a study for a RLWTF at Los Alamos National Laboratory. There is no GENERIC BAT to be looked up in an 
engineering handbook, each BAT developed is very site specific. This BAT has been developed to treat particular waste streams and comply with the environmental constraints particular to the Los Alamos site. The process chosen to do this, while providing an overall optimum combination of the other factors, is described in the following paragraphs.

Selection starts with a graph of existing, demonstrated technology. Preliminary choices can be made from a somewhat casual knowledge, but the "B" in BAT requires an in-depth understanding of the technology under consideration. Required pretreatments, supporting functions, operating problems, performance over time, response to impressed variables, and secondary wastes produced all can have a major impact on the validity of a particular choice. This requirement is not always obvious. Section 3.1.4 presents a shopping list of the more common unit operations and unit processes which can be combined into a treatment process (turned into hardware as a treatment plant), and then in the 14 pages of Section 3.2 attempts a brief presentation of each unit listed. The brevity of such a presentation is illustrated by the examination of a recent (1989) "AIChE TODAY SERIES" publication used as a text for a 4-day (short) course in "Advanced Waste Water Treatment". The looselead binder format used in this course contains over 500 page, $81 / 2 \times 11$, and does not address radioactivity, fixation, or the costs of secondary waste disposal. Even these 500 pages are a very condensed summary of the technology.

Next comes consideration of the waste stream(s) to be processed, the plant influent. Characterization is needed on not only the chemical species it contains, but how does it handle or respond to treatment. Is it filterable or will it, almost immediately, blind the filters? Can it be aged to improve filtration? Is it fouling to membranes or heat transfer surfaces, or so close to saturation that any concentrating will cause fouling? Foamy? Colloidal TSS that cannot be removed by filtration? Rapid settling, difficult to keep in suspension? Quantities? Variations in rates or composition? The list goes on. The answers to many of these questions determines the applicability of the various unit operations and unit processes, and the sequence of their application.

Finally there are the performance requirements to be met. This is a NPDES permit in the present case, with secondary requirements of a minimum impact on air pollution, and of secondary radwaste generation. With the influent identified and effluent requirements known, process selection, to get from influent to effluent, can begin.

It is often the case that experience with a similar situation provides an invaluable starting point for process selection. At the same capacity, and in the same temperature zone, most sanitary waste treatment plants are very similar. A new plant can be designed with only minor modifications required to take care of small differences. But a chemical based treatment plant for a cold climate can be very different from the bacteria based plant used in warm climates. But again, the process for treating say the waste stream from a refinery is not expected to be similar to a sanitary waste treatment plant even though located very near to each other. In the present case, a number of radioactive waste-water treatment facilities at other nuclear sites is available for comparison, and contrast. These have been documented in the other sections so that the similarities and differences can be pointed out, 
and used to justify the choices made in this report.

There is information from, and familiarity with the following nuclear sites: Savannah River (SRS), Hanford (HNF), Rocky Flats (RF), Oak Ridge (ORR), and Idaho Falls (INEL), in addition to Los Alamos (LANL). Paducah and Portsmouth are very similar in mission and in waste treatment to Oak Ridge. The liquid radwaste treatment facilities at these sites tend to be of one of two categories. This results from the fact that the liquid radwastes to be treated are different. At SRS and HNF, the liquid radwaste is a well characterized stream with small fluctuations in either composition or rate, fairly low TSS and TDS, and can be handled by such unit operation at R/O, Ion Exchange, or Electrodialysis without a lot of pretreatment. These waste streams are somewhat analogous to the waste streams from a chemical plant; the composition remains rather invariant, constant flow rates coming from a few identified sources. In contradistinction, the wastes at RF and LANL come from multi-sources, and each flow rate can be quite variable at any given time. Further differences are shown in the attached table. Waste treatment plants to treat these latter two wastes are more like sewerage treatment plants. They have to be capable of handling anything that may come down the pipeline. Compared to the one or two sources at SRS and HNF, there are over 40 identified sources for the LANL wastes, and there may be up to 25 changes in characterization of the waste streams over the near term.

Several of the differences in the composition of the liquid radwaste water at the different sites are crucial to the determination of the proper treatment required. Note the high TSS levels at RF. This is caused by the laundry wastes that have to be treated there. This is an average design value. When the instantaneous flow into the process is mostly laundry waste, this number could be much higher, and the process must be capable of handling it. If cross-flow filters (such as used at SRS) were to be used in the design, a very large excess capacity would have to be designed in if this flow could not be averaged out by use of surge tanks. SRS uses an R/O system to reduce the $1600 \mathrm{ppm}$ of TDS to about $160 \mathrm{ppm}$, and the feed suddenly surged to even 2000 ppm, and couldn't be dampened out, the plant would be out of compliance. 


\section{SOME DIFFERENCES IN LIQUID RADWASTE WATERS AT FOUR NUCLEAR SIETS}

$\begin{array}{lrrrr} & \text { SRS } & \text { HNF } & \text { LANL }^{*} & \text { RF } \\ \text { TSS - ppm } & 74 & 10 & 5 / 200 & 1153 \\ \text { TDS - ppm } & 1600 & 615 & 1000 / 6000 & 2430 \\ \mathrm{SiO}_{2}-\text { ppm } & & & 3140^{* *} & \\ \text { RADIOACTIVE } & \text { yes } & \text { low } & 80 / 87 & 400 \\ \text { TRU } & \text { no } & \text { yes } & \text { yes } & \text { yes } \\ \text { COMPOSITION } & \text { no } & \text { yes } & \text { yes } \\ \text { METAL HYDROXIDES } & \text { low } & \text { uniform } & \text { variable } & \text { variable } \\ \text { ORGANICS - ppm } & 54 & ? & \text { low } & 283 \text { ppm } \\ \end{array}$

* The range of variables for LANL based on operational sampling.

** 5-year average on TDS 
The values shown in the table are design values for the other sites, so the $1600 \mathrm{ppm}$ of TDS for SRS incorporates whatever safety factor was felt to be necessary from the characterization work done on this waste stream. The $160 \mathrm{ppm}$ TDS in the effluent meets the imposed criteria. Normally SRS does not use the Ion Exchange system that is installed. This was provided to remove any Iodides or Iodates and such cations as Cesium and Strontium on the rear occasions when these species were found in the waste waters. HNF must use the Ion Exchange system installed there at all times to meet their criteria.

Ion Exchange in a well operated system is a very effective normal unit operation, but does have the problems of leakage and upsets. With very tight release criteria, the effluent must be held up, $100 \%$, or analysis before release. This can entail large hold-up tanks and a fast turn-around on analytical capability. A little by-pass leakage of ions either during operation or regeneration, a sudden volumetric surge, a change in $\mathrm{pH}$ or redox potential, can cause a large volume of recycle.

The R/O systems at SRS and HNF are operated differently. SRS combines the permeate from all three stages to produce a final product. HNF uses only the permeate from the first stage, passing it through Ion Exchange, to produce a final product. The reject from the first stage goes to the second stage, but the permeate is recycled; the same is true for the third stage. This results in a much lower TDS going to the Ion Exchange system, but requires more $\mathrm{R} / \mathrm{O}$ equipment for the same production rates. The HNF arrangement reduces the load on the Ion Exchange equipment, and less secondary waste is produced from Ion Exchange regeneration. Both of these systems require extensive pre-treatment systems. The $\mathrm{R} / \mathrm{O}$ equipment itself is rather simple, but all $\mathrm{R} / \mathrm{O}$ units require prefiltration in the $5-25$ micron range to protect the high pressure pumps and membrane modules. Cellulose acetate membranes require $\mathrm{pH}$ control, and the waste water must be dechlorinated (if chlorine disinfectant is used) to protect polyamid membranes. Fouling of the membranes is always a consideration, and back-pulse, chemical feed, and cleaning systems are always required. The normal rejects, and the chemicals used in the protective systems add to the secondary waste streams which must be handled.

Calcium carbonate and calcium sulfate are the most common scaling species as they are found in most ground waters, and in the process of concentration can easily exceed the solubility limits. Silica is a problem if present in significant concentrations. Natural colloids or precipitated metal hydroxides are not removed by the normally used filters, and can lead to or worsen fouling build-ups, while some membranes are particularly susceptible to biological fouling and attack. All of these problems are enumerated to illustrate why there is no GENERIC BAT, it depends on the water being treated. A look at the table shows that silica is a particular problem if one wanted to use R/O at either LANL or RF. R/O was chosen as the particular method of removal for TDS at SRS because of the characterization of the waste water and the release criteria. But even here, after much preliminary characterization work, a number of changes were required before the system became fully operational. The initial design incorporated pre-coated candles as the filtration step. Much bench-scale testing showed that cross flow filtration would provide the needed function and reduce the amount of secondary waste production. In the actual installation, 
instead of being able to concentrate the TSS to $3 \%$ in the reject stream, a maximum of $1 \%$ had to be accepted. If the initial TSS was very much higher, this would have had a significant impact. The simulated wastes, after many cycles of separation and remixing, just did not behave like the fresh feed. The impact of this reduced concentration in the rejects has not been re-examined in terms of the filtration choices made.

The capacity of these filters was seriously impacted the first time some contaminated storm water accumulation was fed into the plant, not part of the normally expected waste stream. Biological material and metal hydroxides almost plugged the filters, and was very difficult to remove. This waste is now routinely rerouted directly to the evaporators, and the problem has been solved; but it points up the unexpected. The large equalization tanks provided for the influent are now agitated and chemical additions for $\mathrm{pH}$ control have been moved upstream of these tanks. This provides time for precipitation and aging of the precipitates which improves filtration. Despite all the preliminary characterization work, the presence of tributylphosphate (TBP) and its potential detrimental affects on the R/O membranes was overlooked. A carbon absorption system had to be added between the filters and $\mathrm{R} / \mathrm{O}$ units. Then to keep any mercury present from accumulating in the large carbon beds, a mercury removal unit was added.

With these changes, operation of the ETF at SRS has been very successful, a smooth, 24 hr-per-day, 7 days-per-week operation that meets its through-put and release requirements. This points to another consideration in selecting competing technologies: some unit operations require continuous operation to be successfully applied, this includes $\mathrm{R} / \mathrm{O}$, Ion Exchange and Electrodialysis. These units can be put on stand-by by continuously recycling the effluent and keeping the supporting functions in operation, but they cannot be simply turned off and then turned back on. Ion leakage and surface fouling is almost certain to result. If it is necessary to shut down these units, a full shutdown, place in storage, procedure must be performed, and then a normal start-up procedure must be followed. For this reason these unit operations are not the normal first choice for periodic operations. But for low TSS, low TDS, non-fouling waste waters, with continuous operation, the R/O - Ion Exchange combination can be justified and selected. Still at SRS $16 \%$ of the R/O feed rate must be recycled by evaporation (using a single-effect evaporator), and this will increase when Ion Exchange is also required to meet release criteria. In retrospect, it is not at all certain that the process scheme used for the ETF at SRS is the BAT. It may well be, but it would take a lot of alternate design work to determine this with certainty. It can be said, however, with a high degree of certainty that the Rocky Flats design would handle the STS liquid radwaste stream, but the SRS design could not handle the Rocky Flats waste, or the LANL waste water stream. One of the reasons for this is the obvious $\mathrm{R} / \mathrm{O}$ fouling problem from the high silica content of the LANL and RF wastes, but the other is a much less restrictive release criteria at the SRS site.

It is shown in the table of waste compositions that the RF silica content is far in excess of saturation, and the LANL concentration would exceed the solubility limit at a 1.5 to 1.0 concentration factor. Before these wastes could be fed into a R/O unit, this silica would have to be removed. This is usually accomplished by absorbing the silica on a 
formed-in-place magnesium hydroxide precipitate. If such a processing step was used, it would also precipitate all of the material presently being precipitated in the Room 60 and TA-50 operations at LANL and there is no savings of secondary waste generation by use of $\mathrm{R} / \mathrm{O}$. Then since R/O plus Ion Exchange will generate more secondary waste then will Evaporation, secondary waste generation would increase vs. what is presently proposed.

With precipitation being required before any $\mathrm{R} / \mathrm{O}$ system, generating a large volume of TSS, and Ion Exchange required downstream of the R/O system to meet release criteria (assuming the Ion Exchange effluent is the same high quality water as evaporator overheads), it is estimated that $30-35 \%$ of the plant capacity would have to be evaporated in any event. Considering the expected reduced efficiency of a small evaporation system, and the energy required to operate the R/O - I.X. combination, energy savings appear negligible.

Present operations of the Room- 60 precipitation steps and the flucculation/clarification steps of the main TA-50 operations are quite efficient, and not only efficient, but flexible. Precipitatable radioactive materials are selectively removed from the waste water, leaving only the TDS which is presently released. Since it is this TDS which must be reduced (or practically eliminated) to meet the new release criteria, Evaporation (vis-a-vis the other unit operations) appeared the logical choice to accomplish this. The resulting choice, precipitation plus evaporation, provides efficient removal of contaminants, flexibility, familiarity of operation, simple and rugged equipment, easy to start up and shut down, and relatively unaffected by any change in rate or composition. The combination of precipitation and evaporation was therefore chosen as a basis for design. With a preliminary precipitation step required to permit operation of a R/O system, and the increased energy costs of keeping a R/O - Ion Exchange combination on "hot" stand-by, there is no penalty for either increased secondary waste or energy usage inherent in this choice. Consideration of the secondary wastes that would be generated by regeneration of the Ion Exchange resins products that the chosen combination is the minimum secondary waste generator. With this choice made, it then remained to work out the details.

\section{PROCESS DEVELOPMENT}

The factors enumerated in the previous section, such as composition and variations in composition of the influent, operational considerations, and release criteria led to the choice of a combination of flocculation/sedimentation plus evaporation as the most likely candidate to provide a successful operation. But it remained to do a detail design to prove this choice, show applicability, and meet a number of rather severe constraints.

The new release criteria clearly indicated that there was going to be a significant increase in the amount of secondary waste generated. Most of the TDS presently released to the environment was going to have to be removed. The present average value of $3400 \mathrm{ppm}$, in $22,000,000$ liters per year equates to $165,000 \mathrm{lbs}$ per year. It therefore appeared necessary to selectively and effectively remove the alpha contamination before removing the TDS to minimize the amount of TRU waste produced. To meet release criteria it was necessary 
to reduce the residual plutonium and americium to the CDG levels spelled out in 5400.5. The nitrate levels had to be reduced to meet release criteria. Soluble radioactive species such as Cesium and Strontium had to be reduced before release, as did heavy metals, COD, chloride ions, TDS, and possibly aluminum and ammonia. The release criteria was, in fact, so "tight" that consideration was given to going to a "zero release" type system. This would have entailed taking the evaporator condensate to solar ponds or using it as feed to a cooling tower, recycling a concentrate from pond or tower to evaporation as the concentration build up over many cycles of operation. The input alpha levels, either in terms of $\mathrm{Ci} /$ year or $\mathrm{Ci} / \mathrm{L}$, was determined from the past annual reports, and even for the very efficient removal of alpha contamination demonstrated in current Room- 60 operations, it was apparent that sufficient removal to meet a $30 \mathrm{pCi} / \mathrm{L}$ release level could not be met by simple flocculation/sedimentation alone, or even combined with filtration.

The available operational data is difficult to interpret, and test data may be very specific to the waste being tested. An early SRS design assumed a D.F. of 2,000 across precoated candle filters, R/O and Ion Exchange, but the measured results was much less than this. The present pre-treatment, cross-flow filters and R/O is reported to give a D.F. of 2,000 for the alpha contamination. The D.F. achieved is very dependent upon the success of the precipitation step to precipitate soluble species, and upon initial concentrations. Ion Exchange is only effective on the non-precipitated ions, because any residual colloidal material would be too small to be removed by an filtering action. When reprocessing enriched uranium, material losses far in excess of solubility predictions were experienced due to retarded crystallization. The kinetics of crystallization were so slow, despite significant hold times and excess reagents, that a lot of uranium remained in solution and passed through a micro-filtration stage. The same thing can happen with plutonium and americium, so simple solubility data can often fail to indicate residual alphas. The main TA-50 operation reports a fairly consistent $99.7 \%$ removal of the alpha materials; for a D.F. of 370 . Based on a typical annual input of $3-4 \mathrm{Ci} / \mathrm{yr}$ in $22 \times 10^{6}$ liters of waste water, a D.F. equal to 5300 is required to reach a $30 \mathrm{pCi} / \mathrm{L}$ release level. The fact the series vs. parallel operation of the two clari-flocculators does not seem to change the results suggests that the residual alpha is either soluble or else colloidal material that does not get trapped and removed in the sedimentation step.

In comparison, the Room-60 operation reported annual averages (1990) of D.F. $=43,000$, with informal reports of up to 140,000 . The room- 60 operation consists of a pre-contact stage, precipitation/flocculation/clarification, followed by a long-term contact with precipitating chemicals and opportunity for sedimentation. The reported D.F.'s are comparable with Rocky Flats which reports a D.F. $=50 /$ stage with 3-stage contacting. Since $50^{3}=125,000$, the Room- 60 operation gives the results of $2+$ stages, which is about what should be expected. A very small amount of carry over of colloidal material during the three stage washing of the precipitated sludges could explain any reported divergence in measured D.F.'s.

The relatively high D.F.'s achieved in Room-60 vs. the main TA-50 plant may be explained by the differences in inlet concentrations, $1 \times 10^{-3} \mathrm{Ci} / \mathrm{L}$ for Room- 60 vs. $16 \times 10^{-6} \mathrm{Ci} / \mathrm{L}$ for 
TA-50, and perhaps a more easily removed particle size range, but the effluents are not that much different, $16 \mathrm{nCi} / \mathrm{L}$ from Room-60 and $0.4 \mathrm{nCi} / \mathrm{L}$ from TA-50. It must be inferred that the Room-60 operation is more efficient in removal of alph contamination, and since the objective is to maximize alpha removal from the waste stream, design follows the Room- 60 example of first contacting the influent with previously formed chemical sludge, then pass the waste stream through a precipitation/flocculation/sedimentation second stage.

Performance data consisted of averaged data from annual reports plus analysis of a few grap samples. This data was carefully evaluated and an acceptable basis of design established. Postulating a partial nitrate destruction, it could then be shown that flocculation/sedimentation plus evaporation could achieve the design objectives. This design is shown in the included sketches. Current treatments, in terms of chemicals used and quantities, had to be assumed because there was no data or possible alternates. The result is a maximum prediction of secondary waste generation for a proven performance. Many alternate chemical systems may be useful, and these should be tested as a means to minimizing secondary waste. One system that should definitely be tested is the commercial system used for "hot line softening". This is typically used for silica removal, and this could improve evaporator performance while enhancing alpha removal and reducing chemical usage of waste (TRU) minimization. Required contact times should also definitely be investigated. Most of the other unit operations shown are straight-forward applications of proven technology. The result should be an efficient, easy to operate plant, familiar to the present operators, flexible, and easy to start up and shut down. The only addition to secondary waste being produced is the chemicals used in the precipitation step, and this should be less than the chemical requirements for any conceivable alternate process. Use of a mechanical vapor recompression evaporator plus a finishing evaporator minimizes waste volumes resulting from removal of the TDS from the waste water, and the energy consumption should be comparable to, or less than, energy requirements to keep more exotic alternatives on long-term hot stand-by.

\section{ADDITIONAL RECOMMENDED STUDIES}

The following items reflect areas where additional studies should be performed to validate and reinforce the results of this BAT or to explore other promising areas which may yield improvements to the recommendations contain herein. Please note, Section 3.3.5 "Additional Considerations for Treatment Processes" also contains a listing of potential studies that could be performed.

1. This decontamination factors (DF) used in this study were established based on historical data from LANL and Rocky Flats operations. These along with optimization of the types and dosages of chemicals needed for the precipitation/flocculation/sedimentation operations should be thoroughly investigated in bench scale and later pilot plant operations. This will be an absolute necessity as input data to any detailed design effort that will have to preclude construction of this facility. 
2. Processes that prove viable in the in the clean up of the TA-55 and of this waste stream rather than disposal. Approximately 45,000 lbs/yr of secondary waste generation could be avoided if the recovery of the stream could be demonstrated as practical.

3. The unit process of nitrate destruction on the TA-55 acid stream should also be investigated in an effort to establish the overall requirements. The chemical usage, process configuration and anticipated destruction rate needs to be established prior to initiating detailed design.

4. The waste water characterization, currently in progress, should address the following items which were found to be deficient in our BAT review of data available.

a) The amount of information available on TA-55 effluent was minimal and needs to be reinforced.

b) Additional analysis of the composition of the Room 60 effluent needs to be provided. A single analysis was provided for this study.

c) Several of the NPDES regulated species had no compositional information available.

d) The constituents that compose the anticipated COD need to be defined.

5. The potential for silica scaling in the BAT proposed evaporator system needs to be researched and tested. This is a key element in the removal of the TDS and its ability to function without scaling on the LANL waste streams needs to be verified.

6. The potential for providing a silica removal unit process should be investigated if it is ascertained that scaling in the evaporator cannot be mitigated by other means. This would also be required to protect $\mathrm{R} / \mathrm{O}$ membranes if this unit process is under consideration. 


\subsection{INTRODUCTION}

\subsection{BACKGROUND}

At the Los Alamos National Laboratory (LANL) the management of radioactive, hazardous, and industrial liquid waste is an integral function of the mission programs and research and development activities, many of which involve nuclear materials. All radioactive waste streams that result from nuclear materials research must be treated to remove the radioactive components, and must be handled in an environmentally responsible manner that also provides the maximum health protection to employees and the public. Pursuant to these goals, the treatment process for these waste streams must produce a final effluent stream that meets the environmental discharge requirements of the State of New Mexico Environment Department (NMED), the U.S. Environmental Protection Agency Region 6 Office (EPA), and the U.S. Department of Energy (DOE). In addition, there is some potential that additional discharge requirements could be imposed on LANL should the area Indian Tribes be granted the status of statehood.

The existing LANL Technical Area 50 (TA-50) liquid radioactive waste (radwaste) treatment RLWP has been in service since 1963. Over the years, this facility has been successful in providing treatment of the liquid radioactive wastes for compliance with discharge limits, and has been modified a number of times to accommodate changing influent waste characteristics and effluent release requirements (including a major upgrade 10 years ago). However, due to the age of the facility and the associated equipment and systems, the requirements for maintenance to ensure continued operability and process efficiency have become elevated and continue to increase. It has become evident that either a major upgrading and modification of the existing facility or the construction of a new facility will be required to meet the continuing mission of the existing TA-50 Plant.

A new facility, the TA-63 Radioactive Liquid Waste Treatment Facilities (RLWTF) has been proposed to replace the existing facility under line item funding by the U.S. Department of Energy (DOE). During the week of April 6-10, 1992, an Interdisciplinary Value Engineering Study was conducted at LANL which identified several studies that would be required to meet the planning schedule for the RLWTF project. Among these studies was the requirement for the arrangement of a subcontractor to perform a Best Demonstrated Available Technology (BAT) study in accordance with the directives for the conduct of Best Available Technology (BAT) studies presented in DOE Order 5400.5, 'Radiation Protection for the Public and the Environment', for both the existing and proposed facilities. 


\subsection{ANALYSIS OF EXISTING LIQUID EFFLUENT TREATMENT SYSTEM}

The present radioactive liquid waste treatment operation at LANL integrates a process for the pretreatment of acid and caustic waste streams from the Plutonium Facility (TA-55) with a low-level radioactive wastewater treatment facility (TA-50). TA-55 pretreatment is a batch precipitation process utilizing lime addition to remove most of the alpha-emitting radionuclides consisting primarily of plutonium and americium. The supernatant/effluent from TA-55 pretreatment, containing a high concentration of chloride and nitrate ions, is mixed with incoming raw, low-level waste, to TA-50. There nitrates and chlorides historically have not been removed by the TA- 50 treatment process, and have passed through to the effluent. The TA-50 process removes most of the alpha-emitting contaminants, with the discharge concentrations of both plutonium and americium at or slightly higher than the Derived Concentration Guidelines (DCGs) as defined in DOE Order 5400.5. New discharge limits for the renewed permit may significantly reduce the concentration of allowable salts in the TA-50 effluent stream.

The existing plant does not meet the current criteria standards of DOE Order 6430.1A, 'General Design Criteria'. In particular, compliance cannot be demonstrated with Division 13, Section 23 for Radioactive Liquid Waste Facilities. Design features not incorporated in the existing plant include zoned ventilation for personnel protection, inspectable storage and treatment tanks, classification waste tanks for segregation and controlled treatment, special waste stream process equipment, and real time instrumentation for monitoring progress and the quality of treatment activities. Additional topics that are not in accordance with DOE 6430.1A include resistance to design basis earthquakes, safety class stack monitors, primary radiation control, and confinement, ventilation, and secondary containment for waste transfer lines.

This noncompliance exists primarily because the facility was constructed before these design and operational requirements were promulgated. For the same reason the facility does not provide a working environment that is designed to maintain personnel exposures to As Low As Reasonably Achievable (ALARA) criteria.

With the issuance of new and modified environmental regulations, the release criteria for discharges from water treatment facilities have become increasingly restrictive on certain contaminants of concern. As these release requirements have become more restrictive, the actual release levels of contaminants from the LANL facility are now approaching the modified limits. It is anticipated that the existing facility will not be able to provide waste processing and contamination reduction that will comply with the most recently proposed effluent standards (September 11, 1992) for chemical constituents regulated under the National Pollutant Discharge Elimination System (NPDES) requirements of the Clean Water Act, nor with the radioactive contaminant discharge limitations of DOE Order 5400.5 . 


\subsection{FACILITY DESCRIPTION AND MISSION}

The concept as presented herein for a new treatment facility includes the initial segregation of the TRU waste streams where they will receive separate, comprehensive pretreatment as required. The effluent from this pretreatment would then be combined with the main waste streams for further treatment before discharge. This concept of segregated treatment should also be considered as desirable for any upgrading of the existing facility.

\subsubsection{Process Streams to be Treated}

For the LANL BAT Study, it was necessary to consider three different influent scenarios: (1) the current Room 60 (TA-55) streams (2) the TA-50 stream without the Room 60 effluent, or the TA-50 'neat' waste stream and (3) the blended TA-50 waste stream including the Room 60 effluent.

The effluents produced by the TA-55 operations include a highly acidic waste stream and a very basic or high $\mathrm{pH}$ waste stream. The acidic waste stream comprises approximately $80 \%$ of the total flow to Room 60 , which is estimated at 180,000 liters per year.

The liquid waste volume routed to the TA-50 operations averages approximately 20,500,000 liters per year. The TA-50 influent is piped from various LANL sites and locations. A small fraction of the liquid wastes is transported in by trucks from some of the more remote and dispersed waste generation locations.

\subsubsection{Release/Discharge Limits}

At the time of generation of this BAT study the final release limits for the existing outfall in Mortandad Canyon had not yet been established. Therefore this report compares remedial technologies required to meet the two most probable discharge limit scenarios that may have been imposed. The discharge limit basis are use of the effluent as a (1) source drinking water for wildlife or watering livestock and (2) the more restrictive NPDES criteria that were proposed by NMED on September 11, 1992.

\subsubsection{Contaminants to be Controlled}

The BAT study was initiated to provide an evaluation of the technologies that are available to treat the primary and secondary waste streams currently managed by the existing facility. The primary concern is the treatment and disposal of the current TA-50 low-level radioactive liquid waste stream. 
The contaminants of concern include TRU waste contamination from the TA-55 influent, which also contains high nitrate and chloride levels. The TRU waste components contain relatively high concentrations of alpha-emitting radionuclides.

\subsection{PURPOSE OF THE STUDY}

\subsubsection{Study Objectives}

In support of LANL under Contract 9-XY2-Y9591-1, Work Release \#0015, the Ralph M. Parsons Company (Parsons) was subcontracted to prepare a BAT study in accordance with the directives for BAT studies in DOE Order 5400.5 for both the existing plant at TA-50-1 and the proposed new facility. The Scope of Work stated that the BAT should include an evaluation of the alternatives for treating the combined flows of raw influent to TA-50-1 and supernatant from the pretreatment of process wastes from TA-55, or for providing completely segregated treatment. The study was conducted to address the treatment and disposition of the primary low-level radioactive liquid waste stream that was to be routed to the TA-50-1 Plant. Included are the two TRU waste streams from TA-55 that are currently routed to Room 60 for pretreatment, which generates TRU solid waste for storage at TA-54.

DOE Order 5400.5 defines BAT as "the preferred technology for treating a particular process liquid waste, selected from among others after taking into account factors related to technology, economics, public policy, and other parameters. ... BAT is not a specific level of treatment, but the conclusion of a selection process that includes several treatment alternatives." This philosophy was used as the basis for the BAT evaluations and technology selections.

What will be entailed in the upgrade/modification of the existing facility or the construction of a new facility is not a simple determination. The choice of a BAT often is highly dependent upon all of the unique parameters that are involved with the existing and desired situations. In order to systematically evaluate and identify the BAT, the analysis requires (1) the process knowledge required to evaluate and weigh positive and negative aspects of candidate unit operations, (2) familiarity with the environmental regulations and discharge criteria that impact the decision process, (3) assessment of secondary processes (e.g., secondary waste generation) associated with the candidate technologies, (4) knowledge of the site and availability/lack of resources, and (5) economic considerations that will be factored into the evaluation process. 


\subsubsection{Background Evaluations}

In order to determine the current status of radioactive liquid waste treatment technology within the DOE complex, Parsons was tasked with the responsibility to investigate similar operations at other DOE sites. Other than the current operations at LANL, Parsons investigated facilities at:

- $\quad$ the Savannah River Site

- the Oak Ridge Reservation

- the Rocky Flats Plant

- the Hanford Site

Other possible sources of technology transfer cited by LANL included EPA projects and international radioactive waste treatment facilities.

Parsons was directed to conduct activities as necessary to support the preparation of the study, which included obtaining drawings and plans, visiting relevant DOE sites, and conducting interviews in person and by phone. Parsons was to collect by any or all of these means the necessary information to compile a BAT study that conformed to the requirements of LANL.

The specific topics to be presented and analyzed by the BAT were:

1. Extent of the characterization of the waste to be treated prior to design of the treatment Plant, the waste characterization that was used for design and the actual waste, if different

2. Process flow for each installation investigated or proposed

3. Function of each unit operation, and the portion of the total waste influent that this operation was provided to treat

4. Mass balance of each unit process on the anticipated waste stream for which the unit was designed, and on the actual waste stream to be treated (if characterization data is available); also, where potentially relevant, the mass balance through each unit operation based on theoretical waste streams defined by LANL and a related assessment of the relevance of treatment technologies at other sites to the LANL waste

5. Discussion of the final destination or ultimate disposition of each waste stream for each facility 
6. Project costs for construction and operation of facilities in service at other DOE sites. Also, estimated costs for LANL in the instances were a similar treatment scheme is proposed

7. Problems that have occurred at each operating facility that was investigated

8. Analysis of whether the best demonstrated available technology will accomplish the goals and requirements for LANL, and an evaluation on whether or not more advanced technologies are desirable and recommended

\subsubsection{Approach}

The initial approach for this project was to address the BAT with a level of detail and analysis similar to a BAT study. As stated in DOE Order 5400.5, selection of best available technology for a specific application is to be made from among candidate alternative treatment technologies which are identified by an evaluation process that includes factors related to technology, economics, and public policy considerations. Factors that are to be considered in selecting BAT, at a minimum, are to include:

- the age of the equipment and facilities involved

- the process employed

- the engineering aspects of the application of various types of control techniques

- process change

- the cost of achieving such effluent reduction;

- non-water quality environmental impacts (including energy requirements)

- $\quad$ safety considerations

- $\quad$ public policy considerations

The general techniques to be evaluated for the thermal, chemical, and/or physical treatment of the LANL wastes were to include (but not be limited to):

1. Coagulation/precipitation/flocculation

2. Filtration

3. Ion exchange

4. Sorption

5. Evaporation

6. Solidification 
BAT studies are required to identify the waste management processes that can be used as a basis to provide the required level of treatment. While generic treatment facilities may be used for simple operations (e.g., sanitary treatment plants), facilities that handle radioactive waste streams typically are unique designs engineered to treat specific wastes. Depending on the nature of the contaminants of concern, secondary contaminants, and waste matrix effects, any of a number of technologies must be analyzed for applicability and effectiveness. These technologies (and unit processes associated with the technologies) include not only those previously mentioned, but other state-ofthe-art treatment systems such as biological reaction and destruction, thermal oxidation, incineration, reverse osmosis, electrodialysis, solvent extraction, and sterilization.

The selection of a candidate technology from among the various unit processes is dependent on the waste characteristics, the method of operation, and the evaluation of trade-offs between capital and operating costs. Important parameters of the waste characteristics include flow volume/rates, chemical constituency, short-term and long-term variability and changes, effluent release or reuse criteria, and generation of secondary wastes. Methods of operation may include batch processing, batch/continuous operation, and continuous (or dynamic) processing, with subdivision into advanced technology or high technology operations (e.g., automated and computer controlled systems, complex technologies, sophisticated multi-step processes) and standard technology or low technology operations (e.g., manual operations, simple technology, processes with few steps). Also, the considerations of economical trade-off between capital and operating costs can be a significant factor. Capital costs for equipment, buildings, control systems, and large storage volumes must be analyzed together with operating costs such as labor, chemical usage, testing and analyses, utilities, replacement costs, maintenance, and disposal costs in order to evaluate the total expected costs of the candidate technology.

\subsubsection{Establishment of Alternatives}

The number of treatment alternatives to be considered was determined by a combination of the factors that drive the requirements to evaluate BAT: (1) the three waste streams to be considered, as discussed in Section 1.3.1; (2) the two sets of release criteria to be met, as discussed in Section 1.3.2; , and (3) the decision on whether to modify and upgrade the existing facility or construct a new facility, as discussed in Section 1.1. This combination of possible considerations yielded the total of twelve alternatives, or process configurations, as shown in the matrix provided as Table 1-1. 


\subsection{OPERATIONS AT EXISTING DEPARTMENT OF ENERGY FACILITIES}

\subsection{LOS ALAMOS NATIONAL LABORATORY - CURRENT TA-50 WASTE TREATMENT OPERATIONS}

In order to define the basis for further Best Available Technology development, it is important to understand the current status and situation that requires modification or replacement. Present wastewater operations at LANL involves the blending of effluent from pretreatment processes and other waste streams as influent to the TA50 facility.

The first process relevant to the LANL liquid radioactive waste treatment facility is the treatment of the TA-55 effluents in the Room 60 Operations, as depicted in the block flow diagram provided in Figure A-1 of Appendix A. The second process presented is the main TA-50 operation, where the effluent from Room 60 pretreatment is blended with the TA-50 influent as shown the second block flow diagram for the RLWP provided in Figure A-2 of Appendix A.

\subsubsection{Wastewater Characterization}

Most of the information available on LANL waste characterization is contained in a series of annual reports on operating data for the LANL Liquid Waste Management Group (HSE-7), which include information for both TA-50 and Room 60 operations (TA-55 waste streams). These reports contain information on influent flow rates, composition (averages and ranges), expected performance standards for current treatment, and information on the quantity and composition of secondary wastes that are generated in the treatment processes. Based on the information from these reports, along with other supporting analytical data, plant descriptions, and environmental documentation, it was possible to identify and define the controlling parameters of the relevant waste streams (e.g, volume, composition, TDS, TSS, COD, variations, etc.) and to determine in terms of release limits the approach to BAT evaluation that would satisfy the project requirements.

Two distinct effluents are produced by the TA-55 operations. One effluent is a very low $\mathrm{pH}$ (highly acidic) waste stream composed of approximately $3.5 \mathrm{~N}$ nitric acid $\left(\mathrm{HNO}_{3}\right)$ containing transuranics and other heavy metals. The second effluent is caustic comprised typically of a $0.5 \mathrm{~N}$ basic solution also containing transuranics and heavy metals. Both streams contain elevated levels of TDS. In addition the caustic stream contains elevated levels of TSS.

The acidic waste stream comprises approximately $80 \%$ of the total flow to Room 60 , which is estimated at 180,000 liters per year. The actual treatment rates for the past two years have been lower than this estimated annual 
average due to anomalies in operations, but it is expected that normal operations will reestablish the estimated annual processing rate.

The liquid waste volume routed to the TA-50 operations (not including the Room 60 effluent) has been relatively consistent over the past five years at $20,500,000$ liters per year. The annual averages of the particular species in the influent also are consistent over the same five-year period; occasional variations are normalized by the use of composite sampling and in the process of averaging data.

The Room 60 effluent comprises approximately $1.2 \%$ of the total flow of TA-50 and is intermittent. The small Room 60 effluent stream is a major contributor of sodium nitrate to the TDS in the TA-50 effluent. But without additional data, it is not possible to evaluate the peak contaminant loading on a point-in-time basis. A considerable degree of waste characterization is needed for design basis evaluation.

\subsubsection{Process Description}

The relationship between the processes conducted in Room 60 and in TA-50 represents neither a fully separate process for the treatment of the TA-55 waste streams, nor a process where two waste streams are completely blended. The TA-55 effluent first is treated separately in Room 60 to remove most of the contamination containing alpha-emitting radionuclides. After this step has been completed, the liquid phase wastes from Room 60 are blended into the main TA-50 waste stream for further treatment. This arrangement has the advantage of segregating the high alpha activity sludge solids from the solids produced in the TA-50 process so that only the Room 60 solid wastes must be handled as TRU. Given the specific restrictions for the management of TRU wastes (see DOE Order 5820.2A), the segregation and minimization of TRU wastes is an important technological and economical consideration.

These waste streams have not been characterized completely regarding the definitive waste constituents and the variability of waste stream composition. In the past, such a detailed characterization was not required for effective processing and compliance with release limits. A more detailed characterization study is currently in progress.

The TA-50 operations have a capacity slightly larger than the influent rate. Therefore, typical current operations involve collection in the Waste Holding Tanks, processing the accumulated waste volume on a batch/continuous flow basis, and then placing the facility in standby awaiting the accumulation of the next batch. The 1991 LANL Annual Report indicates a usage of 1054 operating hours, or about half-time for a 40-hour work week. At this operating level, the average flow rate for TA-50 would be approximately 
$86 \mathrm{gpm}$ (325 liters/min.). This flow rate represents the utilization of two Clarifiers in series, which has been the normal operating mode for the past several years (prior to this mode, the two clarifiers were operated in parallel). According to the Annual Report, some improvement in decontamination factors for several contaminants of concern could be attributed to this change in operations.

\subsubsection{TA-50-66 Acid and Caustic Waste Receiving and Storage $\underline{\text { Tanks }}$}

The two process waste streams from TA-55 are received and stored in separate 10,000 liter inspectable tanks located in an underground vault designated as TA-50-66. This storage volume is sufficient, under normal historical operations at TA-55, to store these wastes in TA-50-66 for at least 2 weeks without having to sample and operate the Room-60 treatment plant. Historically, 1986 through 1989, the Room-60 volumes have averaged: process acid -- 146,185 Liters, process caustic -- 32,930 Liters, cold caustic -- 47,875 Liters, and backwash water -- 25,000 Liters. The average total flow of 251,990 Liters per year corresponds to a volume of about 9,690 Liters per 2-week operational cycle as presented below.

Volume (Liters) of Process Waste from TA-55 and Room-60 Operations

\begin{tabular}{||l||c|c|c|c|c||}
\hline \multicolumn{1}{|c||}{} & \multicolumn{5}{|c||}{ CALENDAR YEAR } \\
\cline { 2 - 7 } & 1986 & 1987 & 1988 & 1989 & AVERAGE \\
\hline \hline PROCESS ACID & 158,000 & 148,167 & 138,910 & 139,661 & 146,185 \\
\hline PROCESS CAUSTIC & 36,000 & 37,073 & 31,455 & 27,196 & 32,930 \\
\hline COLD CAUSTIC & 51,700 & 48,100 & 45,500 & 46,200 & 47,875 \\
\hline WATER & 25,000 & 25,000 & 25,000 & 25,000 & 25,000 \\
\hline \hline TOTAL & 270,700 & 258,340 & 240,865 & 238,057 & 251,990 \\
\hline \hline
\end{tabular}

\subsubsection{2}

TA-50-1-60 (Room 60) Neutralization Process

Since about $80 \%$ of the waste is acidic, $3.5 \mathrm{~N}$ nitric acid, there is an excess of acid after neutralizing the caustic waste. The excess acidic waste is neutralized using $10 \mathrm{~N}$ "cold" caustic. All neutralizations are 
performed in a 2,600 Liter stainless steel tank with a water jacket for removing the heat of neutralization. The solution is thoroughly agitated during neutralization using mechanical mixing and recirculation. Each batch of neutralized waste is sampled and analyzed for gross alpha and americium-241. If the neutralized solution contains process caustic, the batch is usually transferred directly to the sludge storage, contacting, and decanting vessel (TK-7); otherwise, the batch is transferred to the feed tank (TK-2) for treatment using continuous treatment methods.

\subsubsection{Room 60 Sludge Storage, Contacting, and Decanting Vessel (TK-7)}

This vessel is used for receiving and for initially treating the neutralized process caustic solution. Initially, the seed solids in TK-7 consist of sludge that is drawn from the Room- 60 clarifier and stored in the small sludge storage and decanting tank (TK-4). The seed solids consist primarily of excess calcium hydroxide $\left(\mathrm{Ca}(\mathrm{OH})_{2}\right)$ that was formed from the initial treatment of the excess process acid waste stream. The only additional solids that are added to the tank are those from the solution being treated.

The neutralized process caustic solution is pumped directly from TK-1 to TK-7 in batches of about 1,500 Liters. Contact with the accumulated sludge is provided by mixing the tank contents for about one hour with a mechanical mixer. The solution is then allowed to settle for up to one week prior to decanting. The decant is pumped to the Room-60 feed tank (TK-2) and treated using the same process used to treat the excess process acid. Administrative controls limit the quantity of $\mathrm{Pu}^{239}$ in this tank to 250 grams which is $1 / 4$ of critical mass. After reaching the 250 gram limit, the sludge is rinsed to remove the soluble salts and the remaining settled solids are solidified for storage. The decanted rinse water containing the soluble salts is then treated using the process used to treat the excess process acid. Normally, three rinses are performed to remove the soluble salts.

\subsubsection{Small Room-60 Sludge Storage and Decant Vessel}

This 1,200 Liter mild steel tank provides temporary storage for sludge that is drawn from the Room-60 clarifier. After solidification of the TK-7 sludge, the sludge accumulated in TK-4 is pumped directly to TK-7 and provides the initial seed solids for treating the process caustic waste in TK-7. Recirculation and mechanical mixing is used to obtain a representative sample to baseline the seed solids. 


\subsubsection{Room 60 Clarifier}

Currently, the primary purpose of the Room-60 clarifier is to treat neutralized process acid waste from TA-55. Additionally, the decant from the sludge storage tanks and backwash effluents are treated through the clarifier. After ten years of corrosion and decay, the clarifier presently consists of 1) a flash mixer section where ferric sulfate, calcium hydroxide, and polymeric flocculating agents are added and mixed with the influent stream and 2) an 8,000 Liter rectangular settling tank. The fiberglass slant plate flocculation section of the unit has deteriorated and was removed. The clarification section of the unit was never installed because experience had showed that sludge would buildup in the tubes, causing unacceptable maintenance requirements. The current operations use only calcium hydroxide and a polymeric flocculating agent to form an adequate floc.

The settled sludge from the clarifier bottoms is transferred to the sludge storage tank (TK-4) for subsequent transfer to TK-7 as the initial seed solids for treating the caustic waste.

The original multi-media filter system on the outlet of the clarifier unit failed and was replaced with an external pressure filter. Liquid effluent is now pumped through this filter to remove any remaining suspended solids.

It is the RLWP operator's opinion that it is not feasible to use disposable cartridge filters to further decrease the residual actinide constituents after the liquid passes through the multi-media filter because an experiment showed that the cartridge filters deteriorated rapidly from the high salt concentrations and alpha activities. The activities removed during the early stages of the experiment were actually released back into the effluent stream during the late stages. The disposal problems associated with the filters (new TRU waste product) and their rapid deterioration led to abandoning the use of cartridge filters.

\subsubsection{6 $\quad$ Room 60 Overflow Filter}

The purpose of the overflow filter is to remove any suspended solids that might be carried over with the clarifier effluent. The original filter was an integral part of the clarifier unit. This filtration process was subsequently replaced with an external filter unit when the original unit succumbed to excess corrosion. The filter is backwashed with industrial water and the backwash effluent is collected in the effluent 
holding tank. It is then transferred to the feed tank and treated through the clarifier (Room 60).

\subsubsection{Room 60 Effluent Hold Tank}

The filtered effluent from the Overflow Filter is sampled and collected in the Effluent Holding Tank (TK-3). It is then transferred to the Large Sludge Contacting and Decant Tank (TK-8). TK-8 provides temporary storage in case the effluent does not meet criteria for acceptance by the main treatment plant for additional treatment. If treatment through the Room- 60 plant and subsequent treatment obtained from the additional sludge contact and settling in TK- 8 is inadequate, the decant from TK- 8 is recycled through the Room- 60 plant for additional treatment.

\subsubsection{Room 61 Large Sludge Contacting and Decant Tank}

This large tank (10,000 liters) provides storage for the entire effluent volume from a single operational campaign in Room 60 . This temporary storage was necessary because on-line instrumentation is not available to determine if the treatment was successful. Normal analysis of the effluent samples takes about 24 hours to complete. This tank has a mechanical mixer and decant nozzles to remove the clarified liquid after mixing and settling. The clarified liquid is transferred to the main treatment plant influent holding tank if it meets its acceptance criteria; otherwise it is recycled to Room 60 for additional treatment. The sludge from this tank can be pumped to the Room- 60 solidification operation if analyses indicates that it is TRU or to the sludge holding tank in the main treatment plan.

\subsubsection{9}

\section{Room 60 Sludge Washing Process}

The sludge in TK-7 settles between Room-60 operations. The minimum settling time is normally one week. The clarified liquid is - transferred to TK-3 and is then treated through the Room-60 clarifier. The $\mathrm{Pu}^{239}$ inventory in TK-7 is limited by administrative controls to less than 250 grams. After approaching this limit, the sludge is rinsed to remove most of the dissolved solids. The tank is filled with industrial water and is mechanically mixed for about one hour. The mixture is then allowed to settle. The clarified liquid is then removed for treatment and the rinse repeated. Three rinses are normally performed to ensure adequate removal of the salts from the sludge. After the final decant, the sludge is completely homogenized using mechanical mixing and recirculation. A sample is obtained from the 
recirculation pipe for analyses. A summary of the Room-60 sludge solidification operations for the years 1986 through 1989 is presented below.

\begin{tabular}{||r||r|r|r|r|r||}
\hline \multicolumn{1}{||}{} & \multicolumn{6}{|c||}{ CALENDAR YEAR } \\
\cline { 2 - 6 } & \multicolumn{1}{|c|}{1986} & \multicolumn{1}{|c||}{1987} & \multicolumn{1}{c|}{1988} & 1989 & AVERAGE \\
\hline \hline Sludge Vol (Liters) & 4,589 & 3,217 & 4,368 & 2,668 & 3,710 \\
\hline Barrels & 57 & 47 & 21 & 32 & 39 \\
\hline $\mathrm{Pu}^{238}(\mathrm{Ci})$ & 3.0 & 3.0 & 4.7 & 5.7 & 4.1 \\
\hline $\mathrm{Pu}^{239}(\mathrm{Ci})$ & 56.9 & 72.6 & 24.5 & 52.3 & 51.6 \\
\hline $\mathrm{Am}^{241}(\mathrm{Ci})$ & 42.0 & 50.2 & 33.2 & 70.8 & 49.0 \\
\hline
\end{tabular}

\subsubsection{0 $\quad$ Room 60 Sludge Metering Tank}

After rinsing, homogenizing, and sampling the sludge in TK-7; it is transferred in approximately 22 gallon batches to the sludge metering tank. This tank is precisely calibrated to measure 22 gallons of sludge for transfer to each specially prepared 55 gallon drum.

\subsubsection{Room 60 Cement Paste Operation}

Based on the results of the analysis, a metered amount of sludge (typically 22 gallons per drum) is added to a waste drum preloaded with the correct amount of cement, sodium silicate and vermiculite. The drum lid is put in place and secured, then the drum is rotated slowly to check for leaks. If no leaks are observed the drum along with a second companion drum is tumbled for 20 mins. The drums are removed from the tumbler box, vented and fitted with a carbon filter. The drums are then checked for external contamination and moved to Room 59 for temporary storage. The drums are cured with one edge elevated to allow the surface of the cement to harden at an angle and force any free water to collect in the subsequent low area. This allows the free water to be detected by $\mathrm{x}$-rays should it be present. After the curing process is complete the drum is ready for transfer to final storage.

\subsubsection{TA-50 Influent Holding Tanks}

The TA-50 influent (not including the Room 60 waste stream) is piped from various LANL sites and locations to large Influent Holding 
Tanks, where it is stored prior to processing. A small fraction of the liquid wastes is transported by trucks from some of the more remote and dispersed waste generation locations.

\subsubsection{TA-50 Clarifiers}

The blended TA-50 influent (from the TA-50 Waste Holding Tanks and from the Room 60 Hold Tanks) is neutralized with a caustic $(\mathrm{NaOH})$ solution and routed to the first of the precipitation, flocculation, and clarification stages of the serial Clarifiers. Ferric sulfate, calcium hydroxide, and polymeric flocculants are added to the waste stream in a flash mixer to induce precipitation. The mixed stream flows to a zone of gentle agitation where flocculation occurs. Sludges from previous settling operations are recirculated to increase contact efficiency.

In the clarification zone, the floc settles to the Clarifier sludge bed. This precipitated floc contains most of the alpha-emitting radionuclides, but only a portion of the beta-emitters. The remainder of the waste stream becomes part of the clarified volume. The clarified liquid overflows to the second-stage (serial) Clarifier. The same precipitation, flocculation, and clarification processes are repeated in the second-stage Clarifier, with the clarified overflow routed to a multi-media rapid filter sand.

\subsubsection{TA-50 Multi-Media Rapid Sand Filter}

The Multi-Media Filter removes particles from the overflow from the final (second) stage of the serial Clarifiers.

The saturation concentration of aqueous calcium hydroxide is approximately $700 \mathrm{ppm}$ (or $\mathrm{mg} / \mathrm{L}$ ) as $\mathrm{Ca}(\mathrm{OH})_{2}\left(\mathrm{pk}_{\mathrm{st}}=5.3\right.$ ), or 378 $\mathrm{ppm}$ as $\mathrm{Ca}^{+2}$. The value for calcium sulfate, $\mathrm{CaSO}_{4}\left(\mathrm{pk}_{\mathrm{st}}=4.6\right)$, is similar. It is expected that the overflow from the second-stage Clarifier is near saturation for both of these species due to the excess of chemicals used in the treatment process. Since the reported annual average effluent $\mathrm{Ca}^{+2}$ concentration value is $221 \mathrm{ppm}$, it is assumed that some of the excess is being removed by the filters as precipitate.

The filtrate is routed to the Effluent/Release Tank and the solids are backwashed into two large Influent Holding Tanks. 


\subsubsection{Carbon Dioxide Injection System}

Carbon dioxide $\left(\mathrm{CO}_{2}\right)$ is injected into the Multi-Media Rapid Sand Filter to prevent scaling of the filter media and to adjust the $\mathrm{pH}$ of the final effluent to comply with the NPDES permit requirements (pH 6-9).

\subsubsection{TA-50 Effluent/Release Tank}

The filtrate from the Multi-Media Filter is collected and held for release pending analysis in the Effluent/Release Tank. If the analysis indicates that the gross alpha radioactivity and $\mathrm{pH}$ are within the prescribed discharge limits, the contents of the tank are routed to a permitted outfall. However, if gross alpha radioactivity and/or $\mathrm{pH}$ are out of specification, the contents of the Effluent/Release Tank can be recycled back into the treatment operations for reprocessing.

\subsubsection{TA-50 Sludge Tank}

The sludge from the Clarifiers/Flocculator is collected in the Sludge Tank. The slurries are allowed to separate and settle (decant). The supernate (clarified decant liquid) is recycled back to the front end of the TA-50 treatment process, while the thickened sludge is transferred to a Rotary Vacuum Precoat Filter for further dewatering.

\subsubsection{TA-50 Rotary Vacuum Precoat Filter}

The thickened sludge from the bottoms of the Sludge Tank are routed to the Rotary Vacuum Precoat Filter for the final stage of dewatering. The vacuum filter substrate is coated with a perlite that increases solids retention. The sludge is drawn through the precoat and substrate and solids are deposited on the filter media. The filtrate from this process is recycled into the front end of the TA-50 treatment process, while the dewatered sludge is transferred for drumming, staging, and transport to storage and/or ultimate disposal.

Historically, most of the final solids from this process have exhibited alpha activity content below the onsite disposal restriction limits. The drums have been disposed of by onsite burial.

\subsubsection{Unused Equipment/Systems at TA-50}

Equipment systems exist in the TA-50 process line that are not in use, either for reasons of difficulty in operations and/or maintenance, or pending approval for operations via conduct and approval of a safety 
analysis. The equipment and systems that have experienced operating difficulties (or are not needed) include the Primary and Polishing Filters (downstream of the Waste Holding Tanks). Also, Cation Exchangers (immediately upstream of the Clarifiers) are not used, since the high TSS content of the waste stream has precluded filtration upstream of the Clarifier, and filtration is required prior to ion exchange. In addition, a Grit Chamber was installed upstream of the Waste Holding Tanks, but is no longer in service. A Wiped Film Evaporator (also downstream of the Rotary Vacuum Filter) also was installed but now is not in use.

\subsubsection{Problems and Concerns}

1. Alpha Activity in the TA-55 Waste Stream Sludge

The sludge is classified as a TRU waste and must be handled appropriately.

2. Total Dissolved Solids in the TA-55 Waste Stream

Since the TA-55 effluent waste stream from Room 60 has a very high TDS content, the question has been raised as to whether it is appropriate to blend this waste stream into the bulk flow of the TA-50 process where the TDS must be removed from a more dilute liquid phase. For past operations this was not a concern because it was not required that the TDS in the dilute TA-50 waste stream be removed. If future restrictions require the removal of TDS from the TA-50 effluent, this current approach of blending of TA-55 and TA-50 waste streams may not be desirable.

3. TA-50 Multi-Media Rapid Sand Filter Carbon Dioxide Injection System

Although the carbon dioxide injection above the filter media effectively prevents scale and depresses the $\mathrm{pH}$ to bring it within the 6 to 9 discharge limits, it also probably increases the radioactivity in the effluent by resolublizing some of the particles. This allows these particles to elude capture on the filter media and pass through to the Effluent/Release Tank.

4. Existing Facility Age

The existing LANL TA-50 liquid radioactive waste treatment plant has been in continuous service since 1963. Therefore, even with modifications and upgrades instituted over this period, the age of the 
basic facility and much of its equipment and services has become a source of concern. The requirements for maintenance to ensure sustained operation will only continue to increase and there will be a time in the not so distant future that it will no longer be feasible to maintain this facility.

\subsection{SAVANNAH RIVER SITE EFFLUENT TREATMENT FACILITY}

The Effluent Treatment Facility (ETF) at the Savannah River Site was constructed to manage the radioactive liquid waste streams originating in the site $\mathrm{F}$ - and $\mathrm{H}$ Areas. The primary facilities in these areas are the two 'canyon' buildings where reactor fuel is reprocessed. The areas also include a number of analytical and testing laboratories.

The block flow diagrams for the complete ETF process are provided in Figure A-3 of Appendix A. Additional diagrams are used in this section to elucidate particular activities or functions within the overall treatment process.

\subsubsection{Wastewater Characterization}

The volume reduction of high-level radioactive liquid wastes from the areas serviced by the ETF produces a low-level condensate. In addition to the process wastes, there is the potential that radioactively contaminated cooling water could be routed into the ETF influent system. Also, periodic storm water discharges that have become contaminated and are collected in diked areas around the high level waste storage facilities that may contain residual radioactivity could be routed to the ETF.

The wastewater characterization efforts prior to the design of Savannah River Site's ETF were particularly difficult in that the influent to the then proposed facility was to consist of literally hundreds of point sources throughout $F$ and $\mathrm{H}$ Areas. These point sources were not all operating and therefore many were not producing streams that could be sampled to ascertain their constituents. The historical data of discharges to the seepage basins was sketchy and of minimal value because most instances it was not accompanied by useable flow data.

The design team which consisted of DuPont-Louviers, Parsons Main and SRS personnel decided on a series of parallel efforts to attempt to ascertain a reasonable basis of design for the SRS ETF. The SRS personnel were to institute a program of obtaining grab samples at the operating point sources, to be accompanied by interviews with the building authorities for all sources. The goal here was to establish a data base of constituents and flows from each point source, whether operating or not, and gather information on future 
changes in their discharges. This information gathering effort was spread over an approximate 18 month period.

This information was integrated into a common data base to attempt to define the probable influent to the proposed SRS ETF.

In a parallel path, Parsons Main was to install composite sampling and flow measurement stations at the influents to the existing seepage basins for two non-consecutive thirty day periods. These samples were to be analyzed and the data used to confirm that influents predicted in the point source program.

In addition, grab samples were taken from the seepage basins water and sediments and these analyzed to further confirm the point source predicted influents.

It should be noted that a faction within the design team contended that this approach was not adequate and that it could potentially miss constituents from the non-operating as well as operating facilities. This faction proposed that flow monitoring and composite sampling should be initiated throughout the both $\mathrm{F}$ and $\mathrm{H}$ Areas for an extended period. (1 to 2 yrs) Then the data should be put through probability analyses to establish accurate design data. This was determined to be too costly and time consuming approach to be employed at SRS ETF.

The above described program in general was successful in providing enough data to design the SRS ETF, but it was not without its glitches. The largest of which was that the sample analysis associated with the monitoring of the seepage basin influents turned up an organic solvent, tri-butyl phosphate (TBP), in the stream that was not uncovered in the point source program. The treatment facility design which had been initiated by this point had to be retrofitted with a carbon absorption system to prevent organics from fouling the $\mathrm{R} / \mathrm{O}$ membranes.

This program while not strictly conforming to normally accepted practices in the wastewater treatment industry did yield a design basis that was usable in the conceptualizing of the SRS ETF. It should be noted that the lack of firm design data in some areas caused the involved parties to fill in the holes with data derived from their collective experience which in turn increased the risk potential. Its success here can be attributed to a combination of the quality of the personnel involved and to the widely varied experience of those parties.

The ETF design was based on more than two years of waste characterization, bench-scale testing on actual waste streams, and pilot plant testing on simulated waste. The result of these design efforts is a dedicated radioactive liquid waste treatment plant that has been engineered to treat a well- 
characterized wastewater stream that does not vary significantly in composition. The ETF does not function in a typical sewage treatment plant mode, that must be able to treat any type of waste stream that could be generated, but rather as a sophisticated operation that has been reasonably successful in providing specialized treatment for the waste forms for which it was designed.

A characterization of ETF wastes through the various processes is provided as Table 2-1. This table is keyed to the Block Flow Diagram, Figure A-3, provided in Appendix A.

\subsubsection{Process Description}

The design basis flow rate for routine operations is $165 \mathrm{gpm}$, with a maximum influent flow rate of $235 \mathrm{gpm}$. This flow volume is accommodated by the use of three parallel process lines, which can be considered as 3-50\% processing trains. Normal flow requires one or two processing lines in operation with the third processing train is in a standby mode to provide processing reliability and surge capacity. In the event of abnormal conditions (e.g., a large dump of cooling water or large volumes of stormwater runoff flow), the third processing line can be placed into service in order to provide additional treatment capacity. (See Section 2.2.3, Item 4)

\subsubsection{Waste Collection Tanks}

In order to maintain a constant flow and to regulate possible variations in waste composition, which is desirable when employing these type processing units, the ETF design incorporates the retention of a relatively large volume at the front end of the process. This influent volume in accumulated by alternating sequence in one of the two 500,000 gallon Waste Collection Tanks. These tanks serve as equalization units, each providing 50 hours of holdup retention at the ETF design influent rate. During the accumulation period, preliminary neutralization and concurrent precipitation reactions are conducted in the Waste Collection Tanks. Agitation systems were designed for retrofitting to the tanks to support this treatment process. A recirculation system, originally intended for tank to tank transfers, is currently serving as a $\mathrm{pH}$ adjustment and mixing system.

When the one of the Waste Collection Tanks that is on line as influent feed to the ETF drains to the level of a maintained heel, this tank is switched to receive and accumulate incoming wastewater while the second tank (which had been serving as the waste receiving vessel) is switched simultaneously to the feed position. In the normal operating mode the maximum volume accumulated in either tank will depend on 
the waste influent/generation rate, and will rarely reach capacity before switching occurs. In the event that both tanks are approaching capacity due to an unusually high influent rate, the third waste processing train will be brought into service before the tanks can be completely filled.

\subsubsection{2}

\section{pH Adjustment Tank}

After primary neutralization and precipitant aging in the Waste Collection Tanks, the waste stream is routed to the two stage $\mathrm{pH}$ Adjustment Tanks for final $\mathrm{pH}$ adjustment. This on-line adjustment is accomplished by the addition of solutions of nitric acid $\left(\mathrm{HNO}_{3}\right)$ or sodium hydroxide $(\mathrm{NaOH})$ depending on the $\mathrm{pH}$ of the influent. The $\mathrm{pH}$ is adjusted in process as a dynamic reaction just prior to the waste stream being routed to the Cross Flow Filters (CFF).

\subsubsection{Cross Flow Filters}

The early design stages of the ETF at SRS were focused on selection of the appropriate equipment to accomplish TSS and TDS removal within the proposed facility. The design conglomerate of Parsons Main, DuPont Wilmington and DuPont SRS provided "experts" in each of the areas of treatment anticipated at ETF. These individuals formed teams tasked with determining the best approach to each step in the ETF process. As one would expect the individuals assigned the task of determining the best method to remove precipitated solids from the ETF stream had different ideas on how to approach this objective. The Parsons Main representative favored ultrafilters, while DuPont Wilmington favored tubular precoat filters and SRS personnel were divided between ceramic cross flow filters and hollow fiber filters. Testing programs involving a simulated waste stream were initiated on each of these alternatives which eliminated the hollow fiber filters because of their inability to tolerate organics (TBP). The remainder were all found to be suitable to meet the ETF process needs.

The final selection of cross flow filters was based on the elimination of tubular precoat filters because of an undesirable increase in secondary waste generated due to the introduction of precoat. The ultrafilter was eliminated on the basis that it would be difficult to justify the increase in costs associated with ultrafilters, with only a minor improvement in performance over cross flow filters.

This is an overview of a complicated process that took 12 to 18 months to accomplish and only describes it in very general terms. 
Each of these three parallel filtration trains has three stages in series, as shown in Figure 2-1. Cross-flow filtration involves the recirculation of a high velocity stream of fluid down the axis of a number of porous wall tubes arranged in a parallel flow format, similar to the arrangement of tubes in a heat exchanger bundle. The permeate or filtrate passes through the porous walls and is collected in what would correspond to the shell side of the heat exchanger. The solids filtered from the stream are carried to the wall by the fluid flux, but are resuspended by the shear force at the wall that results from the high velocity axial flow. An equilibrium is established that is dependent on the physical and chemical parameters of fluid flow and the percent composition of solids in the flow stream. The fluid flux through the wall, the filtration rate, is determined by this equilibrium.

Some materials in the laminar sublayer adjacent to the wall surface are difficult to resuspend by shear forces alone, so a periodic backpulse of fluid, created by a pulsing backflow system, is used to force this layer back into suspension, and to prevent blinding of the filtration surface of the tube assemblies. This backpulse is an important part of the CFF, since this resuspension technique is used typically for applications where 'into the wall' filtration would blind the filter medium.

In each stage in series, part of the feed flows through the wall and is collected as filtrate, while the thickened fluid containing the contaminants is either recirculated or routed to the next processing stage. As the percent solids in each stage increases, there is a corresponding decrease in filtration rate and at some predetermined point, the thickened fluid is routed to waste for further concentration.

\subsubsection{Filter Concentrate Tank}

The rejected waste stream from the CFF is the thickened fluid containing the contaminants retained on the influent side of the CFF tube sheets. From the CFF, this waste stream is accumulated in the Filter Concentrate Tank. When sufficient quantities of waste are collected in the Filter Concentrate Tank, the waste is routed to the Evaporator Feed Tanks to be processed for further concentration by evaporation.

\subsubsection{Filter Cleaning Wastes Tank}

As a function of maintenance of the CFF units, periodic cleaning of the filters is conducted by the addition of heated cleaning chemicals. The secondary waste stream generated during the cleaning of the CFF units is collected in the Filter Cleaning Wastes Tank. When sufficient 
quantities of cleaning wastes are accumulated, the contents of the Filter Cleaning Waste Tanks are routed subsequently to the Waste Concentration Tanks for further processing.

\subsubsection{Mercury Adsorption Column}

While the reject stream is routed for concentration, the largest portion (approx. 99.2\%) of the CFF influent is the filtrate. This waste stream is routed to the Mercury Adsorption Columns for the next phase of treatment.

The Mercury Adsorption Columns are three parallel adsorption units ( $4 \mathrm{ft}$ diameter with a $4 \mathrm{ft}$ bed depth), used two at a time. The columns are filled with Rohm \& Haas Dualiter Resin (GT-73). These columns are disposable units that are not regenerated because the entire expended column is designed to be discarded. The expected mercury loading rate is very low, since the anticipated mercury concentration in the ETF influent stream is approximately $63 \mathrm{ppb}$. It was originally anticipated that the columns would require changeout once every 1-2 years, based primarily on radioactivity levels resulting from coincidental adsorption of radionuclides. Current operating data indicates that none of the columns have yet to be discarded and that the plant operator does not anticipate having to change-out any in the foreseeable future. The primary function of the Mercury Adsorption Columns is to provide a form of prior treatment that will prevent the downstream Carbon Adsorption Beds from becoming contaminated with mercury and therefore being classified as a mixed (radioactive and toxic) waste, which in turn would prevent them from being disposed of in the on site low level waste burial ground.

\subsubsection{Carbon Adsorption Beds}

The Carbon Adsorption Beds, along with the Mercury Adsorption Columns discussed in Section 2.2.2.6, were retrofitted into the overall ETF process when it was determined that the influent wastewater contained tributylphosphate (TBP) at concentrations high enough to present concerns regarding potential damage to the membranes in the downstream Reverse Osmosis R/O Units. The activated carbon in the Carbon Adsorption Beds removes the soluble TBP, along with any other soluble organics and residual oils and grease, thereby preventing damage to the $\mathrm{R} / \mathrm{O}$ membranes and ensuring that the discharges can comply with chemical oxygen demand (COD) release limits.

The activated carbon beds are contained in large (10 ft. diameter, $10 \mathrm{ft}$. height) disposable units. The units were originally 
conceptualized to be changed out and disposed of at a rate of approximately three per year, but current operating data has the change out at three units in the first five years of plant operation. Cartridge filters are utilized to remove any carbon fines in order to protect the reverse osmosis membranes from undue abrasion and mechanical fouling.

The activated carbon beds were originally designed to be a "temporary fix" initiated because of the late identification of organics in the influent and time constraints prior to start-up. A permanent organic removal system to replace this temporary system was envisioned as soon as another capital improvement project could be authorized. The conceptual design for the permanent system was completed using on a Ozone, Ultraviolet Organic Destruction system. This effort has been subsequently abandoned because it cannot be justified on a costs basis. The current carbon beds cost $\$ 250,000$ each including disposal and the Organic Destruction System operating costs alone were substantially higher without inclusion of the initial capital cost investment.

\subsubsection{Reverse Osmosis pH Adjustment and Feed Tanks}

After the removal of the organics in the Carbon Adsorption Beds, the wastewater stream is routed through a cooling unit and into the Reverse Osmosis treatment process. The first step in this process is collection of the wastewater in the $\mathrm{R} / \mathrm{O} \mathrm{pH}$ Adjustment and Feed Tanks. During this portion of the process, chemicals are added for $\mathrm{pH}$ adjustment $\left(\mathrm{HNO}_{3}\right)$, dechlorination (sodium bisulfite, $\mathrm{NaHSO}_{3}$, to remove residual chlorine that could attack and degrade the $\mathrm{R} / \mathrm{O}$ membrane), and anti-scaling chemicals (to prevent scaling of the R/O membrane surfaces by precipitation resulting from concentration of the contaminants). Once treated, the waste stream is fed into the $R / O$ Units.

\subsubsection{9 $\quad$ Reverse Osmosis Units}

The design basis for the R/O Units is a 90\% rejection of the TDS in $10 \%$ of the feed volume. As with the CFF systems, there are three $\mathrm{R} / \mathrm{O}$ treatment trains in parallel, each rated for a $100 \mathrm{gpm}$ flow rate. Each of these three parallel trains includes three R/O modules in series. With any two of the three trains in service, the normal treatment capacity of the ETF is satisfied, with the off-line unit held for cleaning or standby operation. A cleaning solution is injected into the units for maintenance of the membranes. 
Other than the treated waste stream (permeate), two other waste streams are released from the $\mathrm{R} / \mathrm{O}$ Units. The first is the reject waste from the R/O process, which is routed to the Evaporator Feed Tanks for further volume reduction and processing. The second waste stream from the R/O Unit is from cleaning operations and is routed to the Waste Concentration Tanks for further processing.

The arrangement of the ETF R/O Units is shown in Figure 2-2. This figure includes design flow rates and anticipated TDS concentrations to illustrate the reduction in permeate flux and increased TDS leakage as the TDS concentration increases.

Depending on particular requirements, other $\mathrm{R} / \mathrm{O}$ process and system arrangements can be designed (see Figure 2-3). If the permeate is routed through a second stage, a much lower TDS can be achieved at the cost of additional equipment and an increased evaporative load. The diagrams in Figure A-3 indicate that the optimization of the R/O process requires complex, integrated considerations, and is best performed by computer modeling of the parameters.

\subsubsection{Second Mercury Adsorption Columns}

The permeate from the $\mathrm{R} / \mathrm{O}$ Units is routed to a regeneratable Mercury Adsorption Column similar to those discussed in Sec. 2.2.2.6. Although the permeate is not expected to contain mercury it is combined with recycled condensate from the evaporators which may contain mercury in the Ion Exchange pH Adjustment and Feed Tanks from the downstream processes.

If the ETF influent contains mercury in excess of the solubility level, the excess is removed in the CFF process step and is routed with the waste stream to the evaporators. At this point the mercury may be volatilized and carried with the water vapors to the condensers, and subsequently to the Ion Exchange $\mathrm{pH}$ Adjustment and Feed Tanks. This stream is combined with the $\mathrm{R} / \mathrm{O}$ Units permeate and passed through the Second Adsorption Removal Column for final mercury removal. Unlike the upstream Mercury Adsorption Columns, this is not a disposable unit but the resin can be removed from the column and the column refilled. The decision to remove the unit from service is also based on radioactivity levels rather than bed exhaustion.

\subsubsection{Ion Exchange $\mathrm{pH}$ Adjustment and Feed Tanks}

The permeate waste stream from the R/O Units (via the Second Mercury Adsorption Column), along with the recycled condensate from 
the Condensate Hold Tank, are collected in the Ion Exchange pH Adjustment and Feed Tanks. These tanks are used to adjust the $\mathrm{pH}$ of the waste (using $\mathrm{NaOH}$ ) prior to routing to the Ion Exchange Columns.

\subsubsection{Ion Exchange Columns}

Following $\mathrm{pH}$ adjustment, the waste stream flows through the Ion Exchange (IX) Columns for removal of anionic and cationic contaminants. The IX Columns consist of an anion (cationic resin) and a cation (anionic resin) in series. These columns contain selected ion exchange resins, and are regenerable. The concentration of common ions in the waste stream is expected to be low enough at this point in the process for release considerations because these columns serve the primary function of removal of specific radioactive chemical species.

Under routine conditions, the Anion Column is bypassed. This unit is placed into service only when it is known (or reasonably suspected) that the waste stream contains anionic radioactivity constituents (e.g., iodides, iodates, ruthenium complexes, etc.).

The Cation Column is provided primarily for the removal of radioactive cesium and strontium by ionic exchange with the sodiumform resin. The resin loading is very selective, so it is anticipated that approximately one column per week will require regeneration.

The IX Columns are regenerated by the addition of strong ionic solutions that replace the ionically-bound contaminants. Once the active ionic sites have been replenished and the regeneration is complete, a rinse step is initiated in the process to remove excess chemicals. The regeneration discharge containing the removed contaminants is routed to the Evaporator Feed Tanks for volume reduction and processing. The IX Column regeneration rinse discharge is routed back to the Waste Collection Tanks at the front end of the ETF process.

\subsubsection{Treated Water Tanks}

The treated discharge from the IX Columns is the final treated product of the ETF process. This discharge is collected in the Treated Water Tanks awaiting discharge. If sampling and analysis of the treated water determines that contamination exists that is out of specification for discharge, the treated water may be recycled to the Waste Collection Tanks to repeat the treatment process. 


\subsubsection{NPDES Station}

The final effluent from the ETF is routed to an NPDES permitted outfall for discharge. An NPDES Sampling Station monitors the discharge, and provides on-line analysis of release parameters to determine the compliance of the discharged effluent with permit requirements.

\subsubsection{Evaporator Feed Tanks}

The Evaporator Feed Tanks collect waste streams to be fed into the Evaporator including the reject stream from the CFF Filter Concentrate Tank, the reject stream from the R/O Units, and the IX Columns regeneration process discharge streams. A pH adjustment by acid addition is performed as required in the Evaporator Feed Tanks.

\subsubsection{Evaporators}

Two $25 \mathrm{gpm}$ evaporators are provided for volume reduction. The average design value for the feed stream to the evaporators is $23 \mathrm{gpm}$, or $14 \%$ of the feed volume to the ETF.

The evaporation system consists of a steam heating unit, the Heater, that performs the primary process. The heated vapor is routed to the process Vapor Body. The vapor phase of the stream is routed to the Separator, where moisture separation is conducted. The separated vapor and gases are routed to the Air Cooled Condenser, where the vapor is cooled, condensed, and collected in the Condensate Hold Tank.

The bottom condensate from the Separator and the Vapor Body are routed back into the Heater for recycling. When the TDS in the bottoms of the Vapor Body become sufficiently concentrated, the waste stream is routed to the Waste Concentrate Tanks for further treatment. The gases separated in the Air Cooled Condenser are released to the vent.

The Evaporators are designed for very low carryover in the condensate. This condensate is recycled back upstream in the process, to be used as a feed source for the IX pH Adjustment and Feed Tanks. A volume reduction factor of 130 is claimed for the process, but with the volume increase due to fixation (Saltstone) process, the estimated volume reduction factor is closer to 100 . 


\subsubsection{Waste Concentrate Tanks}

The Waste Concentrate Tanks receive concentrated waste streams from several sources including the concentrated bottoms of the Vapor Body from the Evaporator System, the secondary wastes collected in the CFF Filter Cleaning Wastes Tank, and the R/O Units cleaning solutions waste. These waste streams are routed to the Waste Concentrate Tanks for further treatment (neutralization) and holdup for ultimate disposal. The concentrated wastes (at approximately $30 \%$ TDS) are transferred via underground pipe lines for final disposal in the Saltstone (Z-Area) process.

\subsubsection{Problems and Concerns}

The items in this section represent an analysis of the primary concerns and problems that have been addressed at the ETF. The overall assessment of the facility is that it has functioned well as a major wastewater treatment facility, utilizing the most current technology available. The problems and concerns listed in this system represent process improvements and operational controls implemented at the level that might be anticipated for any high-technology installation, and do not indicate design inadequacies or process shortcomings.

1. Retrofit of Agitation Systems for the Waste Collection Tanks

The original design of the Waste Collection Tanks did not accommodate a neutralization process. Retrofit agitation systems were design for addition to provide for preliminary neutralization and concurrent precipitation reactions at the front end of the process. The need to ensure proper mixing for the development of the precipitation and subsequent precipitant aging required the retrofitting of agitation systems for the tanks to support this treatment process. Currently the tank to tank transfer system is employed to recirculate within each tank and provide a minimal amount of mixing for $\mathrm{pH}$ adjustment and precipitant aging.

Complete continuous neutralization facilities are included downstream in the process. However, as part of the facility design, consideration was given to the possibility that under certain circumstances there might be insufficient holdup time between the neutralization and filtration process steps to allow adequate aging of the precipitate prior to filtration.

Aging of precipitates is a common chemical treatment practice. When chemicals are added during the precipitation process step, submicron particulates can form in the reaction phase, trapping a high water 
content in the precipitate. When the precipitate contains elevated water-to-waste ratios, recovery and dewatering of the precipitate by filtration becomes difficult, often involving the handling of a gelatinous mass. However, if the precipitate is allowed sufficient time to stand, or is gently agitated, a larger amount of the water entrained in the precipitate is released, resulting in the growth of larger flocculent particles at the expense of fine particles reclaimed back into the solution.

2. Total Suspended Solids in the Cross Flow Filters

Actual operation of the Cross Flow Filters under normal plant operating conditions has shown that if the concentration of TSS exceeds approximately $1 \%$ there is very rapid decrease in total flux rates. However, since the ETF CFF feed stream composition typically is approximately $63 \mathrm{ppm}$ (including recycle), the reject waste stream represents only $1.6 \mathrm{gpm}$ out of a feed of $197 \mathrm{gpm}$.

Plant pilot tests at the equipment vendor, using a simulated waste stream that was consistently reconstituted using both reject and filtrate, resulted in good performance of operation in the 3-5\% TSS range. It is surmised that the difference in the pilot and actual tests is based on the physical characteristics of the TSS after continuous recirculation, which emphasizes the importance of giving adequate consideration to all performance factors in waste characterization.

3. Bacteria Fouling of the Cross Flow Filters

Redundant capacity via two Evaporators was provided in the design. When the first processing run of stormwater runoff was routed to the ETF, the CFF system indicated a marked loss of capacity on the CFF Units. This problem was ultimately traced to unexpected bacteria in the waste stream which 'blinded' the filter medium, and were very difficult to remove. The problem was eliminated by routing the particular batch of waste directly to the Evaporators, utilizing the capacity that had been designed as redundant/backup capability. This example illustrates that problems may occur even when processing a well-characterized waste (supported by extensive tests and measurements) through a state-of-the-art treatment facility.

4. Retrofit of Carbon Adsorption Beds

The Carbon Adsorption Beds were installed as a retrofit project when it was determined that the wastewater could contain TBP at concentrations high enough to present concerns regarding potential 
damage to the membranes in the downstream R/O Units. It was anticipated that most of the insoluble oils and grease in the waste stream will be removed by the CFF, but it was discovered that soluble TBP (soluble at the concentrations present in the ETF influent) would not be extracted during this process. The activated carbon in the Carbon Adsorption Beds removes the soluble TBP, along with any other soluble organics and residual oils and grease.

5. Use of Disposable Carbon Adsorption Beds Versus Organic Destruction Processes

The activated carbon beds are large disposable units. This technology was originally selected as a temporary solution that would allow the ETF to meet its start-up date and thereby DOE's consent agreement with South Carolina DHEC. A conceptual design using a Ultraviolet/Ozone Organic Destruction system to replace the Carbon Adsorption Beds was completed, but subsequently abandoned because it could not be justified on a cost basis.

6. Design Considerations for Reverse Osmosis Units

There are many critical parameters to be considered in optimizing the design of an $R / O$ system. The parameters include influent TDS concentration, the desired TDS concentration of the permeate, the required recovery as permeate, downstream processing of the R/O permeate, and the acceptable amount of evaporative capacity. The SRS ETF system treats only the reject stream in successive stages. The mixed permeates contain (after treatment) TDS levels of $160 \mathrm{ppm}$, and the evaporative load is $20 \mathrm{gpm}$ (10\% of the feed). The acceptability of the design basis concentration of TDS at $160 \mathrm{ppm}$ is dependent upon what is contained in the dissolved solids, since anions are not routinely removed by downstream processes. It is assumed that the $160 \mathrm{ppm}$ TDS criterion is a maximum value, since the high-rejection Filmtec membranes were measured at a better performance level than that used for the design basis.

\subsection{HANFORD SITE EFFLUENT TREATMENT PLANT}

The new 242-A Evaporator/Purex Plant Process Condensate Treatment Facility (PCTF) similar to the SRS Effluent Treatment Facility is currently under construction to manage the radioactive liquid waste streams at the Hanford Site. Since this facility has not been completed, there is a certain amount of ongoing process development that is being conducted and must be considered in future process descriptions. For the purpose of this study, the most recent design and operational information for the Hanford PCTF will be utilized. 
The block flow diagrams for the complete Hanford PCTF process as currently designed for construction are provided in Figure A-4 of Appendix A. Additional diagrams are used in this section to elucidate particular activities or functions within the overall treatment process.

\subsubsection{Wastewater Characterization}

The PCTF at Hanford will be very similar in many aspects to the completed Savannah River Site ETF. This facility will employ state-of-the-art technology for the treatment, processing, and management of liquid radioactive wastes. The facility is dedicated to the processing of four well-characterized waste streams

The amount of information available on Hanford's wastewater characterization for the Process Condensate Treatment Facility (PCTF) is somewhat limited and therefore any conclusions drawn must be fairly general in nature.

The waste characterization tables provided in the request for quotes provides design numbers that are labelled as "90\% CI" and in the accompanying text defined as $90 \%$ Confidence Index. This would lead us to believe that a probability analysis was performed on most of the species associated with the three main waste streams into PCTF. This type of analysis requires a minimum of five to ten data points over a period of time, the longer the better, to provide any meaningful results and would seem to indicate that the approach used was based on normally accepted practices.

A cursory review of the data indicates some large variances in the maximum concentrations of selected species versus the $90 \% \mathrm{CI}$ value. For example, the TOC value for the 242-A Evaporator effluent is defined as $218 \mathrm{ppm}$, while the maximum value is $4,920 \mathrm{ppm}$ and Silicon has a $90 \%$ CI value of $24 \mathrm{ppm}$ with a maximum of $986 \mathrm{ppm}$. This provides a range of 20 to 40 times the $90 \% \mathrm{CI}$ values and ranges such as this would not normally be expected if an in depth analysis was performed. We can only suppose that this was some sort of sampling or antilical error that probably should have been excluded from the data base, but was not because of the limited data available. Design to variances this large would require some definition of the duration of the peaks to allow them to be dampened in head end storage rather than attempt to size process units to treat them. Duration data was not provided and therefore is assumed not to be available.

In addition the $90 \% \mathrm{CI}$ value for certain species exceeds the maximum value given which is not practical in this type of analysis. 
These examples exhibit flaws in at least the analysis of the wastewater characterization data, if not the actual raw data. The worth of a wastewater characterization is not best judged at this juncture of the Hanford PCTF project, but will become readily apparent during start-up and the early operations of the actual constructed facility. Conversely, at this point to determine the wastewater characterization was incomplete can be very costly proposition, which points out the need for flexibility in the design of any such facility.

A summary of the characterization of liquid radioactive wastes through various processes is provided in Table 2-2. This table is keyed to the Block Flow diagram shown in Figure A-4 of Appendix A.

\subsubsection{Process Description}

The design basis flow for routine operations is $150 \mathrm{gpm}$. This flow volume is the result of four influent waste streams that are the source of all liquid wastes routed to the PCTF. There are two waste streams from the Purex plants, a waste stream from the 242-A Evaporator, and the waste stream from the reactor recycle facility basins.

\subsubsection{1 $\quad$ Surge Tanks}

The four waste streams to be routed to the PCTF will be received, blended, and adjusted for $\mathrm{pH}$ in large volume Surge Tanks. These tanks will serve the purpose of reducing any short-term variations or fluctuations in the concentration of contaminants, and provide a more uniform blend of PCTF influent, which will assist in maintaining consistent process parameters.

If testing indicates that the influent to the Surge Tanks is not homogenous or the $\mathrm{pH}$ has not been adequately adjusted, the contents of the tanks can be recirculated for a more thorough mixing.

\subsubsection{Two-Stage Filters}

The blended and adjusted feed from the Surge Tanks will be passed through two stages of filtration to remove the influent TSS and heavy metals that will precipitate due to the $\mathrm{pH}$ adjustment process. These filters consist of multiple banks of stainless steel filter elements to be precoated with a selected filter material which is held in place on the filter substrate by the force of the waste stream flow.

Maintenance of the filter system will involve a continual process of removing a section of each bank from service. The filter material from 
this off-line unit will be dislodged from the substrate when the water pressure is released by a backblowing of compressed air. The waste precoat filter material will be collected in the Evaporator Feed Tank. The cleaned filters then may be precoated with new filter material and placed in service as needed.

The use of precoat type filters should give the facility the ability to process biological solids in the influent stream without the blinding of the filter substrate that SRS ETF experienced. The precoat can be disposed of relatively easily should it blind and therefore rigorous cleaning cycles can be avoided.

\subsubsection{Ultraviolet Oxidation Units}

The filtrate from the Two-Stage Filters will be routed through the Ultraviolet (UV) Oxidation stage of the process. This stage utilizes a combination of ultraviolet light and ozone $\left(\mathrm{O}_{3}\right)$ units to oxidize organic constituents of the waste stream to carbon dioxide and water.

\subsubsection{4 pH Adjustment Tank}

After ultraviolet/ozone treatment, the $\mathrm{pH}$ of the waste stream will be adjusted in the $\mathrm{pH}$ Adjustment Tank. The $\mathrm{pH}$ Adjustment Tank will utilize acid (sulfuric acid, $\mathrm{H}_{2} \mathrm{SO}_{4}$ ) and caustic (sodium hydroxide, $\mathrm{NaOH}$ ) to correct the process waste $\mathrm{pH}$. This tank can be recycled as necessary to ensure that the $\mathrm{pH}$ adjustment process has been completed. Chemicals are to be added to the waste stream to destroy (by chemical reduction) residual ozone that, if left in the waste stream, could attack the downstream R/O membranes. From this point, the chemically-adjusted waste stream will be fed to the Degasification Unit.

\subsubsection{Degasification Unit}

The Degasification Unit consists of an air stripper that will function to remove dissolved $\mathrm{CO}_{2}$ and other dissolved/entrained gasses which may be present in the waste stream. The waste gases are to be drawn from the waste stream and passed through a chilling unit. Condensate from this unit will be recycled to the Surge Tank at the front end of the PCTF process. The gas-stripped waste stream then will be routed to the Recycle Tank as feed to the $\mathrm{R} / \mathrm{O}$ process. 


\subsubsection{Reverse Osmosis Recycle Tank}

The R/O Recycle Tank will collect the process waste stream from the Degasification Unit as feed to the R/O Units. This tank also will collect the reject stream from the second stage $\mathrm{R} / \mathrm{O}$ unit.

\subsubsection{Reverse Osmosis System - First Stage}

The R/O System for the Hanford PCTF utilizes a two-stage recycle system as depicted in Figure 2-4. The process waste stream will pass through two stages of $\mathrm{R} / \mathrm{O}$ units in series. The feed for the first $\mathrm{R} / \mathrm{O}$ Unit will be taken from the R/O Recycle Tank. The permeate from this waste stream is to be collected in the R/O Surge Tank. The reject stream from the Stage One R/O Unit will be routed to the Evaporator Feed Tank for volume reduction and further processing.

When off-line for maintenance, a cleaning solution will be injected into the units for maintenance of the $\mathrm{R} / \mathrm{O}$ membranes. This secondary waste stream is to be routed to the Evaporator Tank for processing.

\subsubsection{Reverse Osmosis Surge Tank}

The R/O Surge Tank receives the process stream from the first $R / O$ stage and serves as the feed tank to the second $\mathrm{R} / \mathrm{O}$ stage. The tank ensures a consistent flow for second stage processing.

\subsubsection{Reverse Osmosis System - Second Stage}

The final $\mathrm{R} / \mathrm{O}$ cleanup of the waste stream is completed by the Stage Two R/O Unit. The feed for this unit is taken from the R/O Surge Tank. The processed waste stream from this unit is routed to the downstream Ion Exchange Polishers. The reject stream from the Stage Two R/O unit is recycled to the R/O Recycle Tank as part of the feed stream to the stage one $\mathrm{R} / \mathrm{O}$ unit.

As with the Stage One R/O Unit, off-line maintenance involves the use of a cleaning solution for the R/O membranes. This secondary waste stream also is to be routed to the Evaporator Tank for processing.

The two-stage recycling arrangement of the PCTF R/O Units is shown in Figure 2-4. This particular staging arrangement for $\mathrm{R} / \mathrm{O}$ operations provides a reduced (lower) concentration of TDS in the permeate than some of the other arrangements. The tradeoff is a considerable 
reduction in the volume throughput for the same amount of equipment.

\subsubsection{Mixed Bed Ion Exchange Polishers}

The final permeate from the Stage Two R/O Unit will be processed through two Mixed Bed Ion Exchange (IX) Polishers arranged in series. This final cleanup, or 'polishing', of the waste stream will provide a very high quality of water that is expected to contain negligible, if any, residual TDS. The first ion exchange column in series removes most ionic TDS species in the waste stream. The second ion exchange column provides a final removal for any ionic contaminants that might have passed through the first stage. (Refer to Figure 2-5.) If any TDS content is found in the final stage effluent, it is expected to be at most a few ppm due to ion leakage through the columns.

The IX Polishers utilized in the Hanford PCTF system consist of mixed anion and cation resins selected for the ion exchange requirements at Hanford. These resins are designed to provide removal of almost all anionic and cationic contaminants. Once water has been processed through this serial deionization system, it is expected that the effluent stream should be able to comply with the most stringent discharge limitations and release criteria.

The resins selected are regenerable by chemical replacement of the active ion exchange sites on the resin substrate. This is accomplished by the addition of strong ionic solutions that replace the ionicallybound contaminants. Once the active ionic sites have been replenished and the regeneration is complete, a rinse step is initiated in the process to remove excess chemicals. The regeneration discharge containing the removed contaminants and the regeneration rinsate are to be routed to the Evaporator Feed Tanks for volume reduction and processing.

Regenerable resin will eventually require replacement for a number of reasons, including strong bonding of contaminants to active exchange sites, loss of effective exchange site surface area due to chemical or physical stress, and damage to the resin substrate. When the resin must be replaced, the depleted (spent) resin is to be routed to a Resin Dryer for further treatment.

\subsubsection{Ion Exchange Spent Resin Dryer}

The IX Spent Resin Dryer will receive the depleted resin for processing prior to disposal. The spent resin will be dewatered by 
drying, which will reduce the volume by evaporation of water and also by dehydration and shrinkage of the resin substrate material. The dewatered resin will be shipped for disposal as a waste product.

\subsubsection{Final pH Adjustment Tank}

After passing through the IX Polishers, the highly purified water will be routed to the Final pH Adjustment Tank for any adjustment that may be required, although it is anticipated that little adjustment will be necessary during routine operations. The $\mathrm{pH}$ will be adjusted to comply with the discharge requirements established by the permit release requirements.

\subsubsection{Verification Tanks}

The Verification Tanks will receive the flow from the Final $\mathrm{pH}$ Adjustment Tank after the $\mathrm{pH}$ of the water has been adjusted to accommodate the discharge criteria. The quality of the water in these Tanks will be verified by analysis. If the water quality meets the prescribed discharge limits for contaminant levels, the water can be released via the permitted outfall. If the contaminant levels exceed the permit limits, the batch in the Verification Tanks will be rerouted back through the treatment process.

\subsubsection{Evaporator Feed Tank}

The IX Polisher acid and caustic regeneration chemical discharge, the IX Polisher regeneration rinse, the IX Polisher backwash, the R/O System reject stream, and the Two-Stage Filter blowback and backwash waste streams will be collected in the Evaporator Feed Tank. This tank serves as the feed to the Evaporator System, and provides a constant, regulated waste stream supply.

\subsubsection{Evaporator}

The Evaporator will provide volume reduction for the collected wastes supplied by the Evaporator Feed Tank. The average design value for the feed stream (based on a process feed rate of $150 \mathrm{gpm}$ ) to the Evaporator is $41 \mathrm{gpm}$, or $27 \%$ of the feed volume to the ETF. This waste stream is concentrated to over 50\% TDS, which is comprised primarily of sodium compounds and ammonium sulfates.

The evaporation system consists of a forced-circulation single effect heat-and-flash evaporator. The feed from the Evaporator Feed Tank is forced into the body of the Evaporator, where the water is 
evaporated (for volume reduction)and a vapor phase with entrained gases is extracted. The vapor phase from the Evaporator is passed through a Separator, a Steam Jet Compressor (for recompression and to establish the temperature differential), and through a Condenser. The condensate stream is routed to the Condensate Tank, while the Condenser bottoms are transferred to the Dryer Feed Tank. Any gases separated in the Condenser are released to the vent.

\subsubsection{Dryer Feed Tank}

The Dryer Feed Tank will collect the Condenser bottoms for feed to the Dryer, where additional volume reduction occurs. The contents of this tank can be recycled as necessary.

\subsubsection{7 $\quad$ Dryer}

A steam-heated scraped surface dryer will be utilized to evaporate and dry the feed from the Dryer Feed Tank to a powder or cake. The specification indicated for the dried TDS is approximately $0.3 \mathrm{gpm}$, which would represent a 500:1 volume reduction. A more realistic estimation might indicate that the dried powder TDS probably would result in approximately $1 \mathrm{gpm}$ of fixed waste, for a 150:1 volume reduction factor.

The dried residue from the Dryer is collected in a Bottoms Hopper, which utilizes a Conveyor to transfer the material for drumming and subsequent disposal.

\subsubsection{Condensate Tank}

The Condensate Tank will collect the condenser carryover from the Evaporator. This stream is to be fed back into the treatment system upstream of the UV Oxidation Units.

\subsubsection{Problems and Concerns}

The PCTF is currently in design, and therefore has some flexibility in the resolution of problems and concerns that have been postulated at this point. The following items are postulated, but may be considered during design development.

1. Perturbations in PCTF Influent Water Characterization

Due to the varying source of input to the PCTF (e.g., 242

Evaporator versus the Reactor Recycle Facility Basins, 
and the intermittent and variable flow rates from the sources (e.g., 0-150 gpm for the Reactor Recycle Facilities Basins), it is difficult to plan operations for a specific waste form. This problem has been mitigated by thorough characterization of the wastes and the use of Surge Tanks to homogenize each waste batch. However, it is anticipated that there will be times that specific treatment requirements will require alterations in treatment parameters and operations.

The Two-Stage Filter system utilizes a precoat filter material that, while increasing the flexibility of the filters to handle various types of solids, when expended constitutes a secondary waste stream. Although it is not anticipated that this secondary waste stream will be particularly excessive, actual facility operation could yield some unexpected increases in the quantities of this material requiring disposal. The use of precoat type filters offers a trade off of flexibility to process varied waste streams for an increase in secondary waste production.

3. Ozone Degradation of the Reverse Osmosis Membranes.

The UV Oxidation stage of the process, which is upstream from the $\mathrm{R} / \mathrm{O}$ system, utilizes a combination of ultraviolet light and ozone $\left(\mathrm{O}_{3}\right)$ units to oxidize organic constituents. After oxidation, chemicals are to be added to the waste stream to destroy residual ozone that could attack and affect the operability of the $\mathrm{R} / \mathrm{O}$ membranes. This step will require careful, conscientious operations to avoid the damaging of a very expensive treatment component.

4. Silica Fouling of the Reverse Osmosis Membrane

In order to avoid the fouling of the semipermeable $\mathrm{R} / \mathrm{O}$ membrane, the concentration increase across the first stage of the $\mathrm{R} / \mathrm{O}$ operation is limited to an approximate factor of 5 , so that the anticipated $14.8 \mathrm{ppm}$ of silica in the $\mathrm{R} / \mathrm{O}$ influent stream, will not exceed the solubility limit for silica in the second stage of $R / O$. With this limitation, no silica pretreatment is required. However, 
elevated silica levels, $986 \mathrm{ppm}$ the maximum indicated in the wastewater characterization, could create scaling problems within this system.

The two-stage recycling arrangement selected for the PCTF R/O Units is very effective for producing a process waste stream with a lower concentration of TDS in the permeate than will be provided by some of the other equipment configurations. The recycling of the second stage reject stream is the primary factor in the reduced TDS levels. However, the nature of design for a system that recycles the waste stream (as opposed to a once-through system) creates a considerable reduction in the volume of waste that can be treated per unit time by the same amount of equipment.

6. Disposal of Spent Resin from the Ion Exchange Polishers

The resins selected for use in the Ion Exchange Polishers can be regenerated, which should minimize the overall bulk of resin requiring disposal versus the use of a nonregenerable resin. However, regenerable resin also will require periodic replacement, so a secondary solid waste stream will be created from the PCTF. This dewatered spent resin must be shipped from the PCTF for disposal as a waste product, either on site or shipped offsite. In either case, the cost of transportation and disposal could be significant. This would be especially true if the resins require frequent changeout, or if the concentrated radioactivity levels are high enough to warrant special consideration.

\section{Cost Effectiveness of Multiple Effect Evaporators}

Evaporation rates are a significant fraction of the total feed waste stream. It bears consideration of at what point conducting the entire duty in multiple-effect evaporators (for energy cost considerations) becomes cost effective. The Hanford system varies from the requirements of LANL in that there are no high alpha solids which must be separately treated. 
8. Evaporator Arrangement for Fluid Flow Mechanics

The high solids (TDS) content in the Evaporator bottoms indicates that the 'working fluid' for fluid flow and heat transfer has a TDS of 50\% (or greater), which has rather undesirable fluid properties. An alternative would be to extract a fluid from the Evaporator with a lower TDS concentration, and require additional loading for the Dryer system. This is especially important if the system engineering (as currently designed) uses a vapor recompression operation. There is a limited temperature rise across the steam jet recompressor, and most of this temperature elevation would be required to overcome the boiling point elevation due to high salts content. This could provide a small (and possibly inadequate) margin in the temperature differential for heat transfer.

9.

\section{Steam Economy of the Evaporator}

In contrast to the design parameters provided for the Hanford PCTF evaporation system, typically one pound of fresh steam is required to recompress one pound of recycle steam, which is the typical steam economy of a two-effect evaporator. This would indicate that the equivalent of approximately $20 \mathrm{gpm}$ of steam condensate that must be extracted ahead of the steam jet compressor, which must be subsequently condensed and routed for disposal. This would require that all of the equipment downstream of the point of recycle should be slightly larger than in the current design.

\subsubsection{APPLICABILITY TO WASTE STREAMS}

During the last stages of construction of SRS-ETF the local DOE at Hanford issued funding for SRS to provide duplicate copies of all construction specifications and drawings for the SRS ETF. In addition it is our understanding that Hanford personnel toured the SRS facility and were in contact with the operations people at SRS during start-up of ETF. Knowing this and reviewing the Hanford flow diagram leads us to believe that the Hanford group was attempting to improve on the design of SRS-ETF by incorporating changes in areas where SRS had experienced difficulties.

The most notable examples of this occurs in the areas of the filtration and organic removal processes. The filtration step at Hanford was 
changed to a precoated sintered metal type filter rather than the cross flow ceramic filters that the SRS-ETF was having difficulties with at that time. This type of precoat filter was considered in the conceptual design stages at SRS and rejected because of the increased production of secondary waste due to the introduction of the precoat. It was realized during conceptual design at SRS that the precoat filter could meet the performance requirements for the ETF.

The other example is the use of an Ozone/Ultraviolet Organics Destruction System at Hanford where as SRS had installed a carbon absorption system for organics removal. At the time of Hanford's visit to SRS, the SRS-ETF design group had already investigated many options for organic removal/destruction to replace the "temporary" carbon system and established that the Ozone/Ultraviolet System showed the most promise. SRS may even have initiated the Conceptual Design on this system to replace the once through carbon system, at this time.

These along with other examples leads one to believe that the Hanford group had already established that their influent waste streams were similar enough to SRS's that the SRS facility could be duplicated, with selected improvements, and successfully applied at Hanford. While the success or failure of this approach remains to be seen, as Hanford is not yet an operating facility, it does have some merit in not expending funds by going paths already followed by the SRS ETF design group. Contrarily, it may have allowed Hanford to gloss over what were considered minor differences in the influent characteristics without giving them due consideration, which may manifest itself in costly modifications to the process after start-up of the Hanford Facility.

\subsection{ROCKY FLATS PLANT LIQUID RADWASTE TREATMENT PLANT}

The Rocky Flats Plant (RFP) Liquid Radwaste Treatment Plant (LRTP) is similar in many ways to the current treatment operations at LANL, and also is akin to the proposed upgrades or the new treatment facility that. have been considered for the LANL RIWTT line item project. Both sites use a precipitation-flocculation-sedimentation process step, followed by filtration, to remove TRU waste constituents. It is after this step has been completed that the removal of TDS from the filtrate is conducted by evaporation.

\subsubsection{Wastewater Characterization}

At the RFP LRTP the liquid radiological waste (radwaste) influent may comprise effluent waste streams from any number of sources, facilities, and/or processes. These waste streams are collected and stored in large volumes, 
which helps normalize the fluctuation in waste character and composition. In order to determine the requirements for waste processing, each batch of waste to be fed to the LRTP is analyzed and characterized prior to treatment.

A summary of the characterization of LRTP wastes through the various processes is provided as Table 2-3. This table is keyed to the Block Flow Diagram provided in Figure A-5 of Appendix A.

\subsubsection{Process Description}

The design basis flow of the LRTP for routine operations is $1500 \mathrm{gpm}$. This flow volume is the result of the requirements from a number of influent waste streams that are collected and stored as feed to the LRTP.

\subsubsection{Waste Collection and Storage Tanks}

The Waste Collection and Storage Tank system receives the waste streams routed from various RFP liquid radwaste sources and stores these wastes in large-volume tanks. The large volume within each tank provides the function of equalization of the waste character of each batch, and also aids in maintaining a relatively constant, regulated influent to the LRTP.

The tank system has a large total capacity in order to accommodate the numerous waste streams and waste volumes to be treated by the LRTP. The tanks accept waste streams contaminated with both transuranic and nontransuranic activity. The large collection and storage capacity provides a uniform supply feed to the waste treatment process, which must accommodate an average processing rate of 1300 gpm (with a design basis of $1500 \mathrm{gpm}$ ).

When a tank is filled with sufficient volume to be fed to the treatment process, the contents of the tank are sampled and analyzed to determine the contaminant concentrations. The chemical analysis of the tank contents is used as a basis for waste characterization, which determines the downstream processes to be utilized for the cleanup and decontamination of the waste stream. After characterization, the wastes can be routed to various points within the treatment process, dependent upon the required level of treatment. Since the volumes that must be treated represent very large flow rates, this design is very flexible and responsive. 


\subsubsection{Clarifier Feed Tanks}

The feed stream from the Waste Collection and Storage Tanks can be routed into the treatment system for one to three stages of precipitation, flocculation, and sedimentation in series. The first unit of any of these three precipitation and clarification systems is the Clarifier Feed Tank. This tank is used to mix the flocculating agents into the waste stream to be fed to the Clarifiers.

\subsubsection{Clarifiers}

Each of the three stages of precipitation and clarification is a two-step process. The first step is precipitation, which involves the addition of chemicals to induce flocculation and subsequent precipitation of the suspended solids. In the second step the flocculate separates from the waste solution in a precipitation and sedimentation step. The largest portion of the alpha activity-bearing constituents of the waste stream is precipitate into the sediment sludge.

Once the flocculating agent has been added and mixed with the waste stream in the Clarifier Feed Tanks as the first step of any of the three precipitation and clarification stages, the waste is transferred to the corresponding second stage process Clarifier. This unit allows the flocculate to form, precipitate and settle into a sludge bed. The clarified supernate is either routed to the next serial step of precipitation and clarification, or is routed directly downstream to the Evaporator.

\subsubsection{Filtration Units}

The sludges from the bottoms of the Clarification Units are extracted and routed to the Filtration Units for dewatering. These units are rotary vacuum precoat filters. The filtrate liquid from this process is routed back into the Clarifier Feed Tank in the first stage of the precipitation and clarification process. The filter cake from this process is routed to the Sludge Dryer.

\subsubsection{5 $\quad$ Sludge Dryer}

The dewatered sludge from the filtration units is processed in the Sludge Dryer, which is a unit that eliminates the remaining moisture. The desiccated sludge is then transferred for solids fixation, packaging, storage, disposal, or recycle. 
The offgasses from the Sludge Dryer are routed to a Scrubber System. The moisture vapor removed in the Dryer is condensed into the scrubber fluid, and recycled back into the treatment system.

\subsubsection{Evaporator Storage Tank}

The waste stream from the clarified supernatant from the last utilized stage of the precipitation and clarification process is routed to the Evaporator Storage Tank. This tank collects, stores, and maintains the feed supply to the Evaporator System.

\subsubsection{Organics Destruction Unit}

Prior to transfer to the Evaporator System, the waste stream from the Evaporator Storage Tank is routed through the Organics Destruction Unit. This treatment stage utilizes a combination of ultraviolet light and ozone units to oxidize organic constituents of the waste stream to carbon dioxide and water. The $\mathrm{CO}_{2}$ is released as a harmless byproduct gas, while the waste stream is routed to the Evaporator System.

\subsubsection{8 $\quad$ Evaporator System}

The LRTP evaporation process utilizes a typical four-effect forced circulation unit with interstage flash drums. The steam economy is approximately 3.4 pounds of evaporation per pound of fresh steam used. Large vapor bodies and internal demisters limit the carryover to the degree that the combined condensates are within the contamination limitations for release to the environment.

\subsubsection{9 $\quad$ Hold-Up Tank}

The condensate from the Evaporator System is collected in the HoldUp Tank. Chemical analyses are conducted on samples of the condensate to ensure that permit restrictions for release have been met. If is determined that the contaminant levels are within discharge requirements, the liquid stream is routed for release to a permitted discharge point. If the analysis indicates out-of-specification contaminant levels, the batch is recycled into the treatment process.

\subsubsection{Thin-Film Evaporator}

The Evaporator System bottoms are extracted and transferred to the Thin-Film Evaporator for further volume reduction. The bottoms from this process are transferred for cementation and storage, awaiting final 
disposal. The moisture vapor from this process is routed to a Condenser, where it is returned to a liquid phase and rerouted to the four-effect Evaporator System.

\subsubsection{Problems and Concerns}

1.

Solidification of the Thin-Film Evaporator Bottoms

In the initial design of the LRTF, the bottoms from the last effect of the Evaporator System were spray-dried and transferred for disposal. When the decision was made to solidify these bottoms in cement, it was necessary to add water to the dried cake/powder in order to meet the requirements for the cement mix. This process proved to be uneconomical, so a new design was developed. The current design routes the bottoms from the fourth Evaporator effect to a finishing evaporator, the ThinFilm Evaporator, which produces a concentrated bottoms with adequate residual moisture for incorporation into a cement matrix.

2. The addition of chemicals to induce flocculation in the Clarifiers and precoat on the rotary vacuum filters increases the secondary waste production, which in turn increases the facility waste disposal costs. In addition, it has the desirable effect of increasing the facilities flexibility to handle varied waste streams through manipulation of the concentration and type of flocculants and precoats used.

3. The routing of the entire waste stream to the evaporator is an energy intensive unit operation, which has been minimized through the use of a multiple effect evaporator.

\subsection{OAK RIDGE CENTRAL POLLUTION CONTROL FACILITY}

Unlike the 'second generation' wastewater treatment Plants at the Savannah River and Hanford Sites which were planned and/or built to meet the more stringent requirements of contemporary environmental legislation, the Oak Ridge Reservation currently utilizes smaller, distributed treatment plants that were built in the past to accommodate the effluents of a limited area. This is typical of the facilities in Oak Ridge at Y-12, K-25, and X-10, as well as the satellite sites at Portsmouth and Paducah. The facilities at these sites have been in compliance with discharge limits 
that are much less stringent than those anticipated for the new LANL discharge permit.

Prominent Oak Ridge facilities include the West End Treatment Facility (WETF) and the Central Pollution Control Facility (CPCF) at Y-12. These facilities have been storing the sludges produced in the treatment processes, and are faced with the regulatory issues associated with ultimate disposal of these accumulated wastes.

The CPCF is a batch treatment facility that uses process reactors, settlers, filters, a mop water treatment system, a chrome reduction unit, a hydrated lime system, sludge dewatering, and effluent polishing to treat non-nitrate bearing waste streams. Waste streams with nitrate loading are processed at the WEFT.

\subsubsection{CPCF Wastewater Characterization}

The primary waste streams treated at the CPCF include dilute wastewater, including mop water and coolant wastes, caustic and acidic wastes, caustic scrub waste, plating shop floor waste and other spent acids and bases; and effluent and sludges from the Plating Rinsewater Treatment Facility (co-with the CPCF). (Refer to Figure A-6 of Appendix A.) The dilute wastewater stream comprises approximately $90 \%$ of the total volume of wastes treated within this facility.

\subsubsection{CPCF Process Description}

The CPCF is the primary facility for the routine treatment of wastewaters that do not contain substantial nitrate-bearing waste components. However, some nitrate-bearing wastes are pretreated at the CPCF for metal (nickel or chromium) removal. After pretreatment, the nitrate-bearing wastes are transported to the West End Treatment Facility for final treatment. All batches of wastes not containing nitrate components are treated and discharged subsequently to the NPDES Outfall 501 at the East Fork of Poplar Creek.

The CPCF operates in batch and semi-batch modes which involve physical and chemical processing steps. The process includes oil/water separation, neutralization, precipitation, flocculation, coagulation, settling and decanting, carbon adsorption, and cartridge filtration. The CPCF is designed to process 2.7 million gallons of wastewater per year. A block flow diagram for the CPCF is provided in Appendix A.

\subsubsection{Plating Rinsewater Treatment Facility}

The Plating Rinsewater Treatment Facility (PRTF) is itself a treatment process that is co-located with the CPCF. The function of this 
front-end treatment facility is to receive dilute rinsewater from the Plating Shop and provide treatment of this waste stream prior to introduction into the CPCF process. The rinsewater received from the Plating Shop is routed through a process which includes a Neutralization Tank for $\mathrm{pH}$ adjustment, an Equalization Tank for waste stream collection and flow management, a Heavy Metal Removal System for elimination of the heavy metals from the plating process (primarily chromium, eliminated by ferric hydroxide $\left(\mathrm{Fe}(\mathrm{OH})_{3}\right.$ addition), a Degasification Tank for the removal of entrained gases and vapors, a Clarifier/Filter for further removal of contaminants by precipitation, and a final $\mathrm{pH}$ adjustment step prior to routing the treated waste stream into the downstream portion CPCF process. The liquid waste stream either may be collected in the CPCF Sump Tank, or (if determined not to require treatment in the CPCF Carbon Adsorption Columns) may be routed for final treatment to the CPCF Final Polishing Filter.

The bottoms from the Clarifier/Filter processes are routed to Settling Tanks for subsequent storage. These solids once were routed to the CPCF Filter Press for dewatering in previous operations. However, under current operations, the solids are not processed by the Filter Press and are stored as received from the Clarifier/Filter.

Two additional processes are included with the PRTF that have not been utilized (i.e., the PRTF has not received shipments of wastes to be treated by these processes). The first additional process involves the capability of treating cyanide-bearing wastewaters. The liquid wastes would be collected in the Cyanide Holding Tank, then routed to the Cyanide Destruction Unit for treatment prior to collection in the PRTF Equalization Tank. The second additional process provides the capability to pretreat acidic wastes by utilizing a Neutralization Tank prior to routing to the Equalization Tank.

\subsubsection{Primary Influent Waste Stream}

The primary (i.e., largest volume) waste stream treated at the CPCF is the dilute wastewater or rinsewater stream, which includes cleaning rinsate and mop water, along with coolant wastes. This waste stream represents approximately $90 \%$ of the annual volume of liquid wastes treated at the CPCF, with the approximate volume calculated at one million gallons per year. This waste stream is treated as a batch operation, with each batch comprised of approximately 14,000 gallons. The waste stream is routed to the Oil/Water Separator as the first element of the treatment process. 
The Oil/Water Separator provides the first stage of treatment by assisting in the removal of insoluble organic waste constituents in the primary wastewater stream. Incoming wastes that have been found to contain significant amounts of oil or similar organic contamination can be processed through the Oil/Water Separator, which collects the organic waste stream for drumming and routing to the Oil Storage Facility. The separated wastewater component is routed into the downstream CPCF process to the Chemical Addition and Equalization Tank.

\subsubsection{4}

\section{Chemical Addition and Equalization Tank}

This tank collects the influent wastes (that have been treated as necessary for oil separation) for batch equalization and addition of chemicals for the precipitation/clarification process. Approximately 14,000 gallons of waste per batch is typically collected and treated as supply to the Reactor Clarifier Feed Tank.

\subsubsection{5 $\quad$ CPCF Storage Tanks}

The CPCF Storage Tanks provide an additional source of influent to the CPCF process. These tanks are utilized to collect and store the concentrated, highly acidic or basic (caustic) waste streams to be processed at the CPCF. There are both Acid Storage Tanks and Alkaline Storage Tanks. Depending on the $\mathrm{pH}$ of the incoming waste, the waste stream will be routed to the appropriate tank for holding until transfer to the $\mathrm{CPCF}$ process by introduction into the process reactors.

\subsubsection{Process Reactors}

The Process Reactors receive both acidic and caustic waste streams from the Storage Tanks, and react the wastes for neutralization and precipitation of contaminants. The reacted waste stream is transferred to the Reactor Settlers for further process development.

\subsubsection{7 $\quad$ Reactor Settlers}

The Reactor Settlers receive the $\mathrm{pH}$ adjusted waste stream from the Process Reactors and allows the settling/separation process to occur. The supernate from this process is routed to the Reactor Clarifier Feed Tank to be collected as the feed solution to the Reactor Clarifier. 
The sludges from this process are routed to the Sludge Dewatering Filters.

\subsubsection{Sludge Dewatering Filters}

Sludges from the Reactor Settlers are received and processed by the Sludge Dewatering Filters to remove the liquid components of the sludge. The solids are routed for shipment to the Waste Tank Farm. The liquid extract from the filtering process is transferred to the Polishing Filters.

\subsubsection{Polishing Filters}

The Polishing Filters associated with the Sludge Dewatering Filters are used to provide an extra cleanup step in the processing of the filtered supernate. These filters also may receive a liquid stream from the Reactor Settlers, if testing indicates this is appropriate. Effluent from the Polishing Filters is collected in the Sump Tank.

\subsubsection{Chromium Reduction Unit}

As an extra process step to be used as necessary, a Chromium Reduction Unit is provided for the Reactor Settlers. This unit may be used to eliminate chromium in batches of waste where chromium contamination is detected or known. Processed waste is recycled back to the Reactor Settlers.

\subsubsection{Reactor Clarifier Feed Tank}

The liquid waste streams from the Chemical Addition and Equalization Tank and the Reactor Settlers are collected in the Reactor Clarifier Feed Tank as a batch loading to the Clarifier Reactor. The Reactor Clarifier Feed Tank collects approximately 14,000 gallons. Also, a polymer antifoaming agent may be added as determined necessary at this process step.

\subsubsection{2 $\quad$ Reactor Clarifier}

The Reactor Clarifier serves as the vessel for precipitation, separation, and clarification processes. The clarified liquid waste is decanted and routed to the Sand Filter. 


\subsubsection{3 $\quad \underline{\text { Sand Filter }}$}

The Sand Filter unit is a granular media filtration system that is provided to provide a polishing step for the clarified waste stream by removal of the final low-level concentrations of suspended solids. The filtered effluent from this unit is collected in the Sump Tank.

\subsubsection{Sump Tank}

The Sump Tank collects the waste streams from the Sand Filter, the Polishing Filters for the sludge filtrate, and the effluent from the Plating Rinsewater Treatment Facility. These waste streams are collected and equalized in the Sump Tank prior to the final series of treatment processes.

\subsubsection{Carbon Adsorption Columns}

The Carbon Adsorption Columns were designed for removing organics from the waste stream. However, removal of other chemical contaminants (e.g., chelated nickel) is provided by the Carbon Adsorption Columns following sand filtration. The activated carbon removes the soluble organics and residual oils and grease not removed by the Oil/Water Separator, ensuring that the discharges can comply with $\mathrm{COD}$ release limits.

\subsubsection{Final Polishing Filter}

The processed effluent from the Carbon Adsorption Columns is passed through a final phase of polishing in the Final Polishing Filter. This is a filter apparatus that serves as the final processing stage prior to storage of the treated liquid effluent.

\subsubsection{Final pH Adjustment}

If required, the effluent from the Final Polishing Filter may be adjusted for $\mathrm{pH}$ to ensure that the subsequent discharge will comply with NPDES requirements. The adjusted waste discharge is routed to the NPDES 501 outfall at the East Fork of Poplar Creek.

An Effluent Basin once was used to collect the discharges prior to release at the NPDES Outfall. This basin is no longer in use. 


\subsubsection{Problems and Concerns}

- Key contaminants that have caused concerns under current or future permit scenarios are copper, cyanide and a single unusual fluoride occurrence.

- An alternative that has been recommended for CPCF/PRTF would be the addition to the existing plant of cyanide oxidation and supplemental $\mathrm{pH}$ control and filtration. This plant has suffered very few discharge violations in its entire history. The measures suggested are recommended as preemptive, safety measures to ensure $100 \%$ compliance under more stringent permit limitations. This approach includes a new ozone generator for cyanide destruction. The existing alkaline-chlorine oxidation unit is not being used because the cyanide wastes delivered here are reportedly not amenable to chlorination. Ozone may destroy sufficient non-amenable cyanide to provide compliance with a total cyanide limit, if such is imposed. If the cyanide limit contained in the permit is for amenable cyanide only, the ozone unit will not be required.

\subsection{OAK RIDGE WEST END TREATMENT FACILITY}

The West End Treatment Facility (WETF) in the Y-12 area uses pH control, metal precipitation, effluent polishing, and sludge dewatering to treat nitrate-bearing uranium-contaminated waste streams. This facility has a biodenitrification and biooxidation stage to process and reduce the nitrate levels in the wastewater stream.

\subsubsection{Wastewater Characterization}

Wastes are delivered to the WETF by Waste Tankers, Polytanks, and other smaller containers. (Refer to Figure A-7 of Appendix A.) Each waste shipment/container typically is accompanied by a summary of the analytical testing and composition analysis to provide information on the waste characteristics. This allows the operators at the WETF to prescribe the treatment scheme that will meet the requirements of the NPDES discharge limits.

There are four major characteristic waste streams that comprise the influent to the WETF. These are Acid-Nitrate Wastes, Caustic Wastes, Coolant Wastes, and Miscellaneous Wastes.

Acid-Nitrate Wastes include mop waters, evaporator condensate, and plating solutions. At least 25 waste streams have been identified in this category, and all have the common contaminant of soluble nitrate $\left(\mathrm{NO}_{3}\right)$. Individual waste streams may contain nitrate concentrations as high as $60 \%$ solutions, or as low 
as $100 \mathrm{mg} / \mathrm{L}$ as $\mathrm{NO}_{3}$. On the average, nitrate concentrations are approximately $25,000 \mathrm{mg} / \mathrm{L}$. In addition to the nitrates, these waste streams contain many metals (including heavy metals) in solution and/or suspension.

Although the Acid-Nitrate Waste streams primarily are inorganic, organic carbon has been detected in some of the streams at concentrations as high as $6,000 \mathrm{mg} / \mathrm{L}$ Total Organic Carbon (TOC). On the average, the TOC concentration of the composite waste typically has been approximately 1,000 $\mathrm{mg} / \mathrm{L}$ (which represents a BOD of 2,000 to $3,000 \mathrm{mg} / \mathrm{L}$ ).

The total influent volume of Acid-Nitrate Wastes is estimated at 750,000 gallons per year. The total amount of nitrate as $\mathrm{NO}_{3}$ is estimated at 120,000 pounds per year. Chemical analyses of the waste streams indicate the presence of aluminum, calcium, chromium, copper, iron, potassium, sodium, nickel, phosphorus, and silicon (silica). The most predominant metal contaminant (based on 1991-92 analyses) has been nickel, with an average concentration of more than $40 \mathrm{mg} / \mathrm{L}$. Chromium concentrations averaged more than $20 \mathrm{mg} / \mathrm{L}$. Copper, silver, lead, and zinc contaminants all averaged levels of approximately $5 \mathrm{mg} / \mathrm{L}$, cadmium averaged $1 \mathrm{mg} / \mathrm{L}$, and mercury near $0.1 \mathrm{mg} / \mathrm{L}$.

Suspended solids concentrations in individual batches of wastes range from 2 to $120,000 \mathrm{mg} / \mathrm{L}$ and dissolved solids concentrations vary from 8 to $870,000 \mathrm{mg} / \mathrm{L}$. Sulfate $\left(\mathrm{SO}_{4}\right)$ concentrations have ranged between 1 and $43,000 \mathrm{mg} / \mathrm{L}$. Acidity (expressed in ratio to $\mathrm{CaCO}_{3}$ ) has been present in concentrations ranging from 1 to $250,000 \mathrm{mg} / \mathrm{L}$. Other trace contaminants present in the WETF influent waste waters include aluminum, arsenic, barium, beryllium, calcium, cesium, cobalt, gallium, hafnium, lanthanum, lithium, magnesium, manganese, molybdenum, niobium, potassium, silicon, sodium, strontium, thallium, tin, titanium, tungsten, vanadium, and zirconium.

Caustic Wastes comprise at least six separate waste streams that have been identified as influent to the WETF. The estimated quantity of Caustic Wastes is 200,000 gallons per year, containing approximately 23,600 pounds of nitrate as $\mathrm{NO}_{3}$ per year. Chemical analyses of these waste streams indicate an average $\mathrm{pH}$ of 7.9, a Total Organic Carbon concentration of $90 \mathrm{mg} / \mathrm{L}$, and a mercury concentration ranging between zero (not detectable) and $0.005 \mathrm{mg} / \mathrm{L}$. Suspended solids have averaged $500 \mathrm{mg} / \mathrm{L}$, dissolved solids have averaged $36,200 \mathrm{mg} / \mathrm{L}$, and sodium has averaged $15,300 \mathrm{mg} / \mathrm{L}$.

Coolant Wastes are generated at the Waste Coolant Biodegradation Facility. A flow of 50,000 gallons was used as the design basis for this facility. Nitrate concentrations typically are approximately (or greater than) $2,000 \mathrm{mg} / \mathrm{L}$. The $\mathrm{pH}$ ranges between 6.0 and 9.0. The TOC concentration varies between 180 
to $300 \mathrm{mg} / \mathrm{L}$. The maximum phosphate concentration has been $20 \mathrm{mg} / \mathrm{L}$, while the suspended solids do not exceed $50,000 \mathrm{mg} / \mathrm{L}$.

Miscellaneous Wastes include other periodic waste streams such as groundwater and dike water, with an estimated maximum volume of $1,000,000$ gallons per year. The WETF typically receives small quantities of wastes transported by carboys, bottles, etc., including expired raw chemicals and reagents.

\subsubsection{Process Description}

At the WETF, waste materials are processed in three subsystems at the WETF Complex, namely, the Head End Treatment System (HETS), the West Tank Farm (WTF), and the Effluent Polishing System (EPS). A block flow diagram for the WETF is provided in Appendix A. The overall retention time for wastes from influent to the WETF to discharge via the NPDES outfall is approximately 175 days.

\subsubsection{Waste Receipt}

Waste is received at the WETF via 5,000 gallon Waste Tankers, 600 gallon Polytanks, and various small containers such as drums, carboys, bottles, etc. The content of each Waste Tanker is removed by a waste feed pump, and is transferred to the Waste Storage Tanks. Smaller containers are introduced into the WETF via the Slurry Box.

\subsubsection{Waste Storage Tanks}

The Waste Storage Tanks (designated as Tanks F-301, F-302, F-303, F-304, and F-305) are used to collect and store the liquid wastes transferred to the WETF. These tanks provide a means of equalizing and normalizing the waste stream feed to the downstream WETF operations by collecting batches of wastes in the tanks and allowing the wastes to blend in order to minimize the deviation of waste characterization of any single waste batch.

Presently the HETS portion of the WETF is operating under a strategy referred to as 'Head-End Modifications'. This strategy has been implemented to precipitate the metals components of the waste stream prior to (rather than following) the denitrification process, as had been the process with previous operations. This change in operations has significantly improved the quality of the effluent from the HETS, and from the WETF overall. The metals precipitation operation at the head end of the process involves the manual addition of lime to the five Waste Storage Tanks. The lime (calcium hydroxide, $\mathrm{Ca}(\mathrm{OH})_{2}$ ) is 
added to increase the $\mathrm{pH}$ to $10-12$, based on the results of 'jar tests' conducted to promote optimization of nickel carbonate removal (typically at $\mathrm{pH} 11.5$ ). Following lime addition, the tanks are decanted manually using air diaphragm pumps.

\subsubsection{Oil/Water Separator}

Incoming wastes that have been found to contain significant amounts of oil or similar organic contamination can be processed through the Oil/Water Separator, which collects the separated wastewater stream in Tank F-306.

\subsubsection{Cyanide Destruction Unit}

A Cyanide Destruction Unit was designed into the WETF operations. This unit would receive cyanide-bearing waste streams, and would collect the wastes in Tank F-307, which would be used as the reaction vessel. Chemical feed streams for sodium hydroxide $(\mathrm{NaOH})$, sulfuric acid $\left(\mathrm{H}_{2} \mathrm{SO}_{4}\right)$, and sodium hypochlorite $(\mathrm{NaOCl})$ support the cyanide removal process. However, because of safety concerns involving the use of sodium hypochlorite, this unit has never been used.

\subsubsection{Neutralization Tank}

The liquid waste stream from the Waste Storage Tanks (as well as the Oil/Water Separator and the Cyanide Destruction Unit, if used) is routed to the Neutralization Tank, where lime, acetic acid $\left(\mathrm{CH}_{3} \mathrm{COOH}\right)$, and triethylphosphate $\left(\left(\mathrm{C}_{2} \mathrm{H}_{5}\right)_{3} \mathrm{PO}_{4}\right)$ are added to adjust the $\mathrm{pH}$ and chemistry of the wastes.

\subsubsection{West Tank Farm I and Tank Farm II}

The treatment operations at the West End Tank Farm include the biological denitrification and biological oxidation processes conducted at the WTF Tank Farm I and Tank Farm II. These tanks are operated in sequencing batch-reactor mode, which involves the serial processes of empty/fill/settle/decant. At any given time, one tank will be empty, one tank will be receiving waste (which requires approximately 60 days to fill), and one tank will be either reacting (until the desired level of contents is reached) or settling, which takes approximately one week.

Nitrate concentrations of the waste stream in a selected Tank Farm reactor vessel at the end of the fill cycle typically are approximately $2000 \mathrm{mg} / \mathrm{L}$. The target nitrate concentration after reaction is less than $50 \mathrm{mg} / \mathrm{L}$. Approximately 300 gallons of acetic acid solution are added 
to each 500,000 gallon biodenitrification tank when the tank is almost full. Triethylphosphate is no longer utilized in this process. The reaction with the acetic acid solution typically is sufficient to reduce the nitrate loading to the desired target concentration. This reaction usually yields approximately 15,000 gallons of sludge per 500,000 gallons (one tank) of treated waste. The effluent is routed to Tanks F-10 and F-11 of the Tank Farm IV area.

Even with the Head-End Modification process for metals removal, some untreated metals contamination reaches Tank Farm I from sources within the system. When the Head-End Modifications are fully implemented, all waste streams will be routed through metal removal processes prior to the denitrification process.

\subsubsection{West Tank Farm III and Tank Farm IV}

The F-10 and F-11 Tanks of the WTF IV section are used to receive and collect the waste effluents from the biodenitrification process conducted in the WTF I and II area. These tanks serve as equalization tanks to facilitate continuous feed to the Effluent Polishing System. The remainder of the WTF III and IV sections (Tanks F-7, F-8, and F-9 in WTF III and F-12 and F-13 in WTF IV) are used for solids settling and storage. The decant from the WTF III and IV sections also is routed to the front end of the EPS.

\subsubsection{Solids Recirculation System}

The WETF is equipped with a system to recycle and recirculate the solids from the WTF Tank Farms III and IV. The solids are routed to a Solids Holding Tank where solids from other downstream operations may be introduced. The contents of the Solids Holding Tank are withdrawn by the Solids Transfer Pump, which may be set up to recirculate the solids slurry or to route the solids to the WTF for storage.

\subsubsection{Effluent Polishing System}

The Acid Mix Tank represents the front end of the Effluent Polishing System portion of the WETF. The primary function of the EPS treatment system as designed has been to remove solids and residual metals (primarily nickel and uranium). The EPS was designed to provide this removal process by utilizing acid for destruction of carbonate complexes, followed by chemical precipitation. However, under current operations, the bulk of nickel and uranium contamination is removed by the Head-End Modifications. 


\subsubsection{0 \\ $\underline{\text { Acid Mix Tank }}$}

The decant from the West Tank Farms is routed to the Acid Mix Tank for further treatment. The waste stream is mixed with sulfuric acid in the Acid Mix Tank, which prepares the waste solution for the process conducted in the Degasifier.

\subsubsection{Degasifier}

The Degasifier receives the acidified waste stream from the Acid Mix Tank, and strips the waste gasses from the solution. This unit was designed to destroy the carbonate complexes of contaminant metals in the waste stream, allowing effective chemical precipitation. However, with most of the metals contamination removed in the Head-End Modifications, very few metal carbonate complexes are formed. The acid Degasifier no longer seems to be required or essential to effective waste stream treatment.

The resulting liquid waste stream is fed to the Chemical Mix Tank.

\subsubsection{Chemical Mix Tank}

The liquid waste stream is routed to the Chemical Mix Tank, which is the first of a series of tanks used to treat the waste stream for the clarification process. Ferric sulfate $\left(\mathrm{Fe}_{2}\left(\mathrm{SO}_{4}\right)_{3}\right)$ is added to induce the precipitation reaction.

\subsubsection{3 $\mathrm{pH}$ Adjustment Tank}

From the Chemical Mix Tank, the treated waste stream is routed to the $\mathrm{pH}$ Adjustment Tank, where sodium hydroxide is added to adjust the $\mathrm{pH}$ to the desired range for precipitation.

\subsubsection{Flash Mix Tank}

The $\mathrm{pH}$-adjusted waste stream from the $\mathrm{pH}$ Adjustment Tank is transferred to the Flash Mix Tank, where a polymeric flocculent aid is added in a quick-mix process to induce the development of a largeparticle floc. This treated waste stream is routed immediately to the Clarifier. 


\subsubsection{5 $\quad$ Clarifier}

Solids removal from the chemically-treated waste stream is conducted in the Clarifier, where the flocculated contaminants are allowed to precipitate into the sludge bottoms. The Clarifier is designed for a detention time of approximately 36 hours (sufficient to accommodate 40,000 gallons). The clarified liquid supernate is drawn off via an overflow weir, and is routed to the Overflow Tank. Sludge bottoms are transferred for further treatment.

The treatment of the sludges from the Clarifier may involve dewatering in the Vacuum Filter, or the sludges may be transferred directly to the Solids Holding Tank.

\subsubsection{6 $\quad \underline{\text { Vacuum Filter }}$}

The Vacuum Filter receives the sludge slurry from the Clarifier. This sludge is dewatered via vacuum filtration, and the filtrate routed to the Acid Mix Tank. The filtered solids are passed through a Ribbon Blender, and then routed to the Solids Holding Tank for processing and ultimate transfer to storage.

\subsubsection{Overflow Tank}

The Overflow Tank receives the clarified supernate from the Clarifier, and collects the liquid waste stream as feed to the Tube Settler and Sand Filter. This tank serves to regulate the waste stream so that a continual, constant supply is fed to the downstream processing units.

\subsubsection{Tube Settler and Sand Filter}

The Sand Filter unit is a granular media filtration system that is provided to provide a polishing step for the clarified waste stream by removal of the final low-level concentrations of suspended solids. A polymer flocculent aid can be introduced to assist the filtration process. The filtered effluent from this unit is routed to the Carbon Absorption Columns for further polishing.

The Tube Settler portion of this system is not used.

\subsubsection{Carbon Absorption Columns}

The Carbon Absorption Columns consist of dual serial columns, each containing a two-ton charge of Calgon Filtrasorb 300 filtering material. These columns were initially designed for removing organics from the 
waste stream, with an anticipated carbon changeout frequency of once per 300,000 gallons of liquid waste processed. However, it has been determined that only 80,000 gallons of waste can be treated before the removal capacity for nickel is depleted.

Chelated nickel is of prime concern because the process for removal via chemical precipitation (in the Head-End Modifications and the Effluent Polishing System) is essentially ineffective. Therefore, removal of chelated nickel is provided by the Carbon Adsorption Columns following sand filtration. Chelated nickel waste streams currently are segregated and containerized in drums for permanent storage and/or disposal. For this reason, the primary source of chelated nickel in the WETF influent waste streams has been eliminated. However, there still are a number of miscellaneous dilute sources of nickel, including chelated nickel, that contribute to WETF contaminant loading.

Spent carbon filtering material is transferred for storage to the large tanks in the WTF Tank Farm IV.

\subsubsection{Final Filtration Unit}

The processed effluent from the Carbon Absorption Columns is passed through a final phase of polishing in the Final Filtration Unit. This is a standard 1-micron cartridge filter apparatus that serves as the final processing stage prior to storage of the treated liquid effluent.

\subsubsection{Effluent Hold Tank}

The polished effluent that has been processed through the Final Filtration Unit is collected and stored prior to release in the Effluent Hold Tank. This is a 6,000 gallon tank which stores the effluent for testing and analysis to confirm compliance with the NPDES discharge requirements. If the effluent is in compliance with the discharge limitations, it will be released to East Fork Poplar Creek via NPDES Outfall 502. Unacceptable batches of waste will be recycled into the WETF process (at the Sand Filter) for further cleanup.

\subsubsection{Problems and Concerns}

- $\quad$ As a result of the operating strategy referred to in Subsection 2.6.2.2, the effluent from HETS and WETF overall has significantly improved. Some untreated metals are still contributed to Tank Farm I from the slurry box, and in decant from sludge Storage Tanks F-7, F-8, F-9, F-12, and F-13. Head-End modifications will become fully 
implemented in the next two years, at which time, these latter two flows will also be directed through metal removal processes, prior to denitrification.

- The solids dewatering system has never, and may never, be used. Sludges stored in F-7, F-8, F-9, F-12, and F-13 are considered in permanent storage, unless the waste can be delisted or until a longterm solution for the mixed waste can be developed and approved. Decant from these sludge storage tanks is continuously fed to the WTF. Following the complete implementation of Head-End Modifications, the decant from these tanks will be treated with the influent wastewater.

- Key contaminants that have caused concerns under current or future permit scenarios are copper, nickel, and TSS.

- Three alternatives have been developed for modifying WETF:

A.

Head-End Mods (HEM) plus ozonation, insoluble starch exchange (ISX) precoat filtration, $\mathrm{pH}$ control, supplemental filtration, and effluent holding

B. HEM plus ozonation, $\mathrm{pH}$ control, ion exchange, and supplemental filtration, and effluent holding

C. HEM plus ozonation, FeS plus odor control, $\mathrm{pH}$ control, supplemental filtration, and effluent holding.

Alternative A capitalizes on the superior metals removal performance potentially available through the use of insoluble starch xanthates. In terms of achieving low effluent metals concentrations (including chelated metals), ISX is almost unmatched (down to $1 \mathrm{ppb}$ for most metals) and appears to be very reliable, especially when used in the precoat filter configuration. The excellent performance offered is, however, counterbalanced by specialized operating requirements (cold storage of ISX powder and fresh slurry preparations), and there are some uncertainties regarding by-products generated.

Alternative B capitalizes on ion exchange technology for metals polishing, and is probably the least preferred option, because the associated resins have not proven $100 \%$ reliable in removing chelated metals to low concentrations as would be targeted under the new permit. The resins selected for use in this application are Rhom and Haas Amberlite ISC-718 and DP-1. The manufacturer does not guarantee removal of chelated metals unless pilot tests confirm removal. The success of these resins is held in doubt because of 
MMES's previous pilot tests. There is universal agreement among MMES operators that ion exchange resins tested in the past were unsuccessful. Nevertheless, manufacturers maintain that possibilities for success are good.

Alternative $\mathrm{C}$ capitalizes on the insoluble sulfide process to reduce effluent metals concentrations to permitted levels (in the range $10 \mathrm{ppb}$ ). This is a well-proven technology and is the second-most preferred option for WETF but effluent concentrations of some metals are not as low as attainable with ISX. This process does not remove chelated nickel nearly as well as ISX and is also less efficient for removal of $\mathrm{Cr}$ and mercury.

\subsection{EXISTING DOE FACILITIES COSTS}

1. SRS ETF was constructed under two separate projects budgeted for $\$ 50,000,000$ and $\$ 4,600,000$. The $\$ 50,000,000$ project included the entire treatment plant, collection sewers, pump stations, cooling/storm water basins and effluent force main. The $\$ 4,600,000$ was to add the organic removal (carbon) columns to the plant with various support items.

The fiscal 1993 operating and maintenance costs were budgeted at approximately $\$ 18,000,000$. But from our review of the summary it appears that as much as $25-40 \%$ of this could encompass design and construction costs associated with the now discontinued filtration pilot program and does not provide a good representation of actual O\&M costs.

2. The Process Condensate Treatment Facility currently under construction at the Hanford Site is budgeted at $\$ 52,600,000$. This figure includes the treatment facility, service extensions, collection system and effluent disposal system, which disposes of the system final effluent into the ground. The treatment facility itself is subcontracted at approximately $\$ 30,000,000$ under a Design Build contract.

The plant is scheduled to start-up in June 1995 and therefore no real Operating and Maintenance (OPM) Cost numbers are available, but current projections have the annual O\&M Costs estimated at approximately $\$ 20,000,000$.

3. The Rocky Flats Liquid Radwaste Treatment Plant was originally constructed in the early 1970's and has went through two major renovations since its inception, one of which is currently ongoing. Therefore it was perceived that cost of construction figures are of very limited value and were not pursued. 
Annual O\&M Costs associated with the Rocky Flats facility were approximately $\$$ \%?\%: in fiscal ' 93 .

4. Capital and Operating \& Maintenance Costs associated with the two facilities at the Oak Ridge Site were perceived to be of limited value in that the facilities were radically different than the proposed facilities at LANL, therefore they were not pursued. Decontamination and Disposal cost figures associated with the Oak Ridge site were used in the cost evaluations and are presented in Chapter 8.3.4. 


\section{UNIT WASTE PROCESS CHARACTERIZATION FOR THE SRS EFFLUENT TREATMENT FACILITY}

\begin{tabular}{|c|c|c|c|c|c|c|c|c|c|c|c|c|c|c|c|c|c|c|}
\hline UNE NO. & 1 & 5 & 6 & 10 & 13 & 14 & 15 & 17 & 24 & 26 & 28 & 29 & 34 & 38 & 39 & 45 & 50 & 51 \\
\hline PROCESS MATERIAL & INFLUENT & $\begin{array}{c}\text { FACILITY } \\
\text { FEED }\end{array}$ & $\begin{array}{l}\text { FILTER } \\
\text { FEED }\end{array}$ & $\begin{array}{l}\text { FILTER } \\
\text { CONCEN- } \\
\text { TRATE }\end{array}$ & $\begin{array}{c}\text { RO } \\
\text { FEED }\end{array}$ & $\begin{array}{c}\text { RO } \\
\text { PERMEATE }\end{array}$ & $\begin{array}{l}\text { RO } \\
\text { CONCEN. } \\
\text { TRATE }\end{array}$ & $\underset{\text { FEED }}{\mathbb{I X}}$ & \begin{tabular}{|c|} 
IX \\
PRODUCT
\end{tabular} & $\begin{array}{l}\text { EVAP. } \\
\text { FEED }\end{array}$ & $\begin{array}{l}\text { EVAP. } \\
\text { BUTTONS }\end{array}$ & $\begin{array}{l}\text { CON- } \\
\text { DENSED } \\
\text { O'HEADS }\end{array}$ & \begin{tabular}{|l|} 
WASTE \\
CONCEN- \\
TRATE
\end{tabular} & $\begin{array}{c}\text { HG } \\
\text { COLUMN } \\
\text { FEED }\end{array}$ & $\begin{array}{c}\text { R/O } \\
\text { COOLER } \\
\text { FEED }\end{array}$ & $\begin{array}{l}\text { TREATED } \\
\text { WATER }\end{array}$ & $\begin{array}{l}\text { INFLUENT } \\
\text { CMAX. } \\
\text { CONC.) }\end{array}$ & $\begin{array}{c}\text { EFFLUENT } \\
\text { (MAX. } \\
\text { CONC.) }\end{array}$ \\
\hline FLOW, LBS/HR (Normal) & 82,500 & 98,500 & 98,600 & 800 & 97,800 & 88,000 & 9,800 & 93,500 & 98,500 & 11,300 & 810 & 10,500 & 810 & 97,810 & 97,800 & 98,500 & 82,500 & 98,300 \\
\hline SPECIFIC CAPACITY & 1.0 & 1.0 & 1.0 & 1.0 & 1.0 & 1.0 & 1.0 & 1.0 & 1.0 & 1.0 & 1.2 & 1.0 & 1.2 & 1.0 & 1.0 & 1.0 & 1.0 & 1.0 \\
\hline FLOW, GPM (Normal) & 165 & 197 & 197 & 1.6 & 196 & 176 & 20 & 197 & 197 & 23 & 1.3 & 21 & 1.3 & 196 & 196 & 197 & 165 & 197 \\
\hline FLOW, GPM (Maximum) & 235 & 295 & 300 & 100 & 300 & 270 & 30 & 270 & 270 & 34 & 2.6 & 40 & 2.6 & 300 & 300 & 300 & & \\
\hline $\begin{array}{l}\text { FLOW, GPM (Maximum } \\
\text { Hydraulic) }\end{array}$ & 1,000 & 295 & 300 & 50 & 300 & 270 & 30 & 270 & 270 & 50 & 50 & 40 & 50 & 300 & 300 & 300 & & \\
\hline $\begin{array}{l}\text { OPERATING TEMPERATURE } \\
{ }^{\circ} \mathrm{C}\end{array}$ & $5-50$ & 5-50 & 5.50 & $5-50$ & $5-35$ & 5-35 & $5-35$ & $5-35$ & $5 \cdot 35$ & $5-35$ & 104 & 60 & 104 & $5-35$ & $5-35$ & $5-35$ & & $5-35$ \\
\hline $\begin{array}{l}\text { OPERATING PRESSURE } \\
\text { P.S.I.G }\end{array}$ & ATM. & 37 & 50 & ATM. & 760 & ATM. & ATM. & 65 & ATM. & 22 & ATM. & 22 & 85 & 760 & 760 & 45 & & 45 \\
\hline COMPOSITION & & & & & & & & & & & & & & & & & & \\
\hline $\mathrm{pH}$ & $1.5-13$ & 1.5-13 & $7.5-8.0$ & & $6.0-6.5$ & & & $6.0-6.5$ & & $4.0-6.0$ & & & 12 & $6.0-6.9$ & $6.0-6.9$ & $6.0-7.0$ & & \\
\hline PPM & 74 & 63 & 83 & 10,000 & $<0.1$ & $<0.1$ & $<0.1$ & $<0.1$ & & 540 & 7,600 & & 8,000 & $<0.1$ & $<0.1$ & $<0.1$ & 510 & $<0.1$ \\
\hline TDS & 1600 & 1,300 & 1,400 & 1,400 & 1,500 & 160 & 13,000 & 160 & 160 & 21,000 & 300,000 & 5.0 & 300,000 & 1,500 & 1,500 & 150 & 7,000 & 610 \\
\hline ALUMINUM & 1.7 & 1.4 & 1.4 & 170 & 0.014 & $7.3 E-4$ & 0.14 & $7.9 \mathrm{E}-4$ & $8.0 \mathrm{E}-6$ & 12 & \begin{tabular}{|l|}
170 \\
\end{tabular} & 0.0033 & 170 & 0.014 & 0.014 & $3.5 \mathrm{E}-4$ & 12.4 & $2.5 E \cdot 3$ \\
\hline CHROMIUM & 0.035 & 0.029 & 0.029 & 0.029 & 0.029 & $3.3 E-3$ & $\overline{0.21}$ & $3.3 \mathrm{E}-3$ & $1.6 \mathrm{E}-5$ & 0.26 & 3.6 & $6.8 \mathrm{E}-5$ & 3.6 & 0.029 & 0.029 & $2.2 E-5$ & 7.28 & $4.5 E-3$ \\
\hline COPPER & 0.17 & 0.14 & 0.14 & 0.14 & 0.14 & $1.9 \mathrm{E}-3$ & 1.4 & $2.9 E-3$ & $8.0 \mathrm{E}-5$ & 1.3 & 17 & $3.3 \mathrm{E}-4$ & 17 & 0.14 & 0.14 & 1.1E-4 & 2.7 & $1.7 \mathrm{E}-3$ \\
\hline IRON & 3.0 & 2.6 & 26 & 320 & 0.026 & $1.5 \mathrm{E}-3$ & 0.25 & $1.5 \mathrm{E}-3$ & $1.5 E-5$ & 23 & 320 & $6.1 E-3$ & 320 & 0.026 & 0.026 & $6.4 E-4$ & 25 & $5.1 E-3$ \\
\hline MERCURY & 0.053 & 0.044 & 0.044 & 2.7 & 0.022 & $2.5 \mathrm{E}-3$ & 0.20 & $2.5 E-3$ & $2.5 E-5$ & 0.39 & 5.4 & $1.0 \mathrm{E}-4$ & 5.4 & 0.022 & 0.022 & $3.3 \mathrm{E}-5$ & 1.5 & $9.3 \mathrm{E} \cdot 4$ \\
\hline MANGANESE & 0.22 & 18 & 0.18 & 0.18 & 0.19 & 0.011 & 1.8 & 0.011 & $1.1 E-4$ & 1.6 & 23 & $4.3 E-4$ & 23 & 0.19 & 0.19 & $1.4 \mathrm{E}-4$ & 3.2 & $2.1 E-3$ \\
\hline NICKEL & 0.11 & 0.092 & 0.092 & 5.6 & 0.046 & $2.6 \mathrm{E}-3$ & 0.44 & $2.6 \mathrm{E}-3$ & $1.3 E-5$ & 0.81 & 11 & $2.1 E-4$ & 11 & 0.046 & 0.046 & $3.3 \mathrm{E}-5$ & 0.43 & $1.3 E-4$ \\
\hline LEAD & 0.14 & 0.12 & 0.12 & 12 & 0.024 & $1.3 \mathrm{E}-3$ & 0.23 & $1.3 \mathrm{E}-3$ & $1.3 \mathrm{E}-5$ & 1.0 & 14 & $2.7 E-4$ & 14 & 0.024 & 0.024 & $4.0 \mathrm{E}-5$ & 0.55 & $1.6 \mathrm{E}-4$ \\
\hline ZINC & 1.3 & 1.1 & 1.1 & 110 & 0.22 & 0.012 & 2.1 & 0.012 & $6.1 E-5$ & 9.5 & 130 & $2.5 E-3$ & 130 & 0.022 & 0.022 & $3.1 \mathrm{E}-4$ & 26.5 & $6.4 E-3$ \\
\hline SILICA & 6.8 & 7.6 & 7.6 & 7.6 & 7.7 & 0.45 & 73 & 0.45 & 0.45 & 6.4 & 890 & $1.7 \mathrm{E}-2$ & 890 & 7.7 & 7.7 & $4.0 \mathrm{E}-1$ & 39 & 1.8 \\
\hline AMMONIA & 18 & 15 & 15 & 15 & 15 & 0.89 & 140 & 0.89 & $8.9 E-3$ & 130 & 1,900 & 0.073 & 1900 & 15 & 15 & $1.5 E-2$ & 30 & $8.8 \mathrm{E}-2$ \\
\hline CHLORIDE & 1.2 & 2.1 & 2.1 & 2.1 & 2.9 & 0.32 & 26 & 0.32 & 0.32 & 23 & 330 & $5.9 E-3$ & 330 & 2.9 & 2.9 & $2.9 \mathrm{E}-1$ & 9.6 & \\
\hline SODIUM & 500 & 420 & 500 & 500 & 500 & 55 & 4,800 & 0.55 & 0.55 & 3,600 & 92,000 & 1.6 & 92,000 & 500 & 500 & 50 & 1,900 & \\
\hline NITRATE & 970 & 810 & 810 & 810 & 870 & $\overline{97}$ & 7,800 & 97 & 0.97 & 14,000 & 190,000 & 3.5 & 190,000 & 870 & 870 & 87 & 6,740 & \\
\hline HYPOCHLORITE & 7.4 & 6.2 & 6.2 & 6.2 & & & & & & 0.23 & 3.3 & $5.9 E-5$ & 3.3 & & & $6.0 E-6$ & & \\
\hline METABISUFITE & & 0.18 & 0.18 & 0.18 & 0.41 & 0.023 & 3.9 & 0.023 & $2.3 \mathrm{E}-2$ & 3.53 .5 & 50 & $9.0 \mathrm{E}-4$ & 50 & 0.41 & 0.41 & $2.1 \mathrm{E}-2$ & & \\
\hline URANIUM & 1.8 & 1.5 & 1.5 & 180 & 0.076 & $8.6 \mathrm{E}-4$ & 0.75 & $8.3 E-4$ & $8.3 E-5$ & 13 & 180 & $3.5 \mathrm{E}-3$ & 180 & 0.076 & 0.076 & $4.4 E-4$ & & \\
\hline OIL \& GAEASE & $0-70$ & & & & & & & & & & & & & & & & 70 & 70 \\
\hline PHOSPHATE & 1.6 & & 1.3 & 1.3 & 1.3 & 0.075 & 13 & 0.075 & 0.075 & 11 & 160 & $2.9 \mathrm{E}-3$ & 160 & $5-10$ & $<0.3$ & 6.7E-2 & 128 & .54 \\
\hline TOC & $0-510$ & & & & & & & & & & & & & & & & 510 & 510 \\
\hline
\end{tabular}


UNIT WASTE PROCESS CHARACTERIZATION FOR THE SRS EFFLUENT TREATMENT FACILITY (Continued)

\begin{tabular}{|c|c|c|c|c|c|c|c|c|c|c|c|c|c|c|c|c|c|c|}
\hline UNE NO. & 1 & 5 & 6 & 10 & 13 & 14 & 15 & 17 & 24 & 26 & 28 & 29 & 34 & 38 & 39 & 45 & $\mathbf{5 0}$ & 51 \\
\hline PROCESS MATERIAL & $\begin{array}{l}\text { INFLUENT } \\
\end{array}$ & $\begin{array}{l}\text { FACILITY } \\
\text { FEED }\end{array}$ & $\begin{array}{l}\text { FILTER } \\
\text { FEED }\end{array}$ & $\begin{array}{l}\text { FILTER } \\
\text { CONCEN. } \\
\text { TRATE }\end{array}$ & \begin{tabular}{|c|} 
RO \\
FEED
\end{tabular} & $\begin{array}{c}\text { RO } \\
\text { PERMEATE }\end{array}$ & $\begin{array}{c}\text { RO } \\
\text { CONCEN. } \\
\text { TRATE }\end{array}$ & $\begin{array}{c}\mathrm{IX} \\
\text { FEED }\end{array}$ & \begin{tabular}{|c|} 
IX \\
PRODUCT
\end{tabular} & $\begin{array}{l}\text { EVAP. } \\
\text { FEED }\end{array}$ & \begin{tabular}{|l|} 
EVAP. \\
BUTTONS
\end{tabular} & $\begin{array}{l}\text { CON- } \\
\text { DENSED } \\
\text { O'HEADS }\end{array}$ & $\begin{array}{l}\text { WASTE } \\
\text { CONCEN- } \\
\text { TRATE }\end{array}$ & $\begin{array}{c}\text { HG } \\
\text { COLUMN } \\
\text { FEED }\end{array}$ & $\begin{array}{c}R / O \\
\text { COOLER } \\
\text { FEED }\end{array}$ & $\begin{array}{l}\text { TAEATED } \\
\text { WATER } \\
\end{array}$ & $\begin{array}{l}\text { INFLUENT } \\
\text { MAX. } \\
\text { CONC.) }\end{array}$ & $\begin{array}{l}\text { EFFLUENT } \\
\text { (MAX. } \\
\text { CONC.) }\end{array}$ \\
\hline FLOW, LBS/HR (Normal) & 82,500 & 98,500 & 98,600 & 800 & 97,800 & 88,000 & 9,800 & 93,500 & 98,500 & 11,300 & 810 & 10,500 & 810 & 97,810 & 97,800 & 98,500 & 82,500 & 98,300 \\
\hline SPECIFIC CAPACITY & 1.0 & 1.0 & 1.0 & 1.0 & 1.0 & 1.0 & 1.0 & 1.0 & 1.0 & 1.0 & 1.2 & 1.0 & 1.2 & 1.0 & 1.0 & 1.0 & 1.0 & 1.0 \\
\hline \multirow{2}{*}{$\begin{array}{l}\text { FLOW, GPM (Normal) } \\
\text { FLOW, GPM (Maximum) }\end{array}$} & 165 & 197 & 197 & 1.6 & 196 & 176 & 20 & 197 & 197 & 23 & 1.3 & 21 & 1.3 & 196 & 196 & 197 & 165 & 197 \\
\hline & 235 & 295 & 300 & 100 & 300 & 270 & 30 & 270 & 270 & 34 & 2.6 & 40 & 2.6 & 300 & 300 & 300 & & \\
\hline $\begin{array}{l}\text { FLOW, GPM (Maximum } \\
\text { Hydraulic) }\end{array}$ & 1,000 & 295 & 300 & 50 & 300 & 270 & 30 & 270 & 270 & 50 & 50 & 40 & 50 & 300 & 300 & 300 & & \\
\hline $\begin{array}{l}\text { OPERATING TEMPERATURE } \\
{ }^{\circ} \mathrm{C}\end{array}$ & $5-50$ & $5-50$ & $5-50$ & $5-50$ & $5-35$ & $5-35$ & $5 \cdot 35$ & $5-35$ & $5-35$ & $5-35$ & 104 & 60 & 104 & $5-35$ & $5-35$ & $5-35$ & & $5-35$ \\
\hline $\begin{array}{l}\text { OPERATING PRESSURE } \\
\text { P.S.I.G }\end{array}$ & ATM. & 37 & 50 & ATM. & 760 & ATM. & ATM. & 65 & ATM. & 22 & ATM. & 22 & 85 & 760 & 760 & 45 & & 45 \\
\hline \multicolumn{19}{|l|}{ COMPOSITION } \\
\hline Ci/GAL & $5.8 E-8$ & $4.9 E-8$ & $4.9 E-8$ & $4.9 E-8$ & $4.9 E-8$ & $2.9 E-9$ & $4.5 \mathrm{E}-7$ & $2.9 E-9$ & $2.8 \mathrm{E}-11$ & $41 \mathrm{E}-7$ & $75 E-6$ & $47 E-11$ & $75 E-6$ & $49 E-8$ & $A 0=8$ & 30 & & \\
\hline $\mathrm{Ci} / \mathrm{GAL}$ & $1.1 E \cdot 8$ & $9.2 E-9$ & $9.2 \mathrm{E}-9$ & $9.2 \mathrm{E}-9$ & $9.2 \mathrm{E}-9$ & $5.5 \mathrm{E}-10$ & $8.8 E-8$ & $\begin{array}{c}5.5 E- \\
10\end{array}$ & $5.5 \mathrm{E}-11$ & $8.3 E-8$ & $1.4 \mathrm{E}-6$ & $8.9 \mathrm{E}-12$ & $1.4 \mathrm{E}-6$ & $\frac{9.9 C-0}{9.2 E-9}$ & $\frac{4.9 E-0}{9.2 E-9}$ & \begin{tabular}{|l|}
$5.0 E-11$ \\
$5.0 E-11$ \\
\end{tabular} & & \\
\hline $\mathrm{Ci} / \mathrm{GAL}$ & $1.1 \mathrm{E}-8$ & $9.2 \mathrm{E}-9$ & $9.2 \mathrm{E}-9$ & $9.2 \mathrm{E}-9$ & $9.2 \mathrm{E}-9$ & $5.5 \mathrm{E}-10$ & $8.8 \mathrm{E}-8$ & $\begin{array}{l}5.5 E- \\
10\end{array}$ & $5.5 E-11$ & $8.3 E-8$ & $1.4 \mathrm{E}-6$ & $8.9 \mathrm{E}-12$ & $1.4 E-6$ & $9.2 E-9$ & $9.2 E-9$ & $5.0 \mathrm{E}-11$ & & \\
\hline $\mathrm{Ci} / \mathrm{GAL}$ & $9.2 E-9$ & $7.7 E-9$ & $7.7 E-9$ & $7.7 E-9$ & $7.7 E-9$ & $5.1 \mathrm{E}-10$ & $7.1 \mathrm{E}-8$ & $\begin{array}{c}5.1 \mathrm{E} \cdot \\
10\end{array}$ & \begin{tabular}{|l|}
$5.1 E-12$ \\
\end{tabular} & $7.0 E-8$ & $1.2 E-6$ & \begin{tabular}{|l|}
$7.5 \mathrm{E}-12$ \\
\end{tabular} & $1.2 \mathrm{E}-6$ & $7.7 E-9$ & $7.7 E-9$ & $5.4 E-12$ & & \\
\hline $\mathrm{Ci} / \mathrm{GAL}$ & $5.8 \mathrm{E}-9$ & $4.9 E-9$ & $4.9 \mathrm{E}-9$ & $1.1 \mathrm{E}-7$ & $4.4 E-9$ & $2.5 \mathrm{E}-10$ & $4.1 E-8$ & $\begin{array}{c}2.5 \mathrm{E}- \\
10\end{array}$ & \begin{tabular}{|l|}
$2.5 \mathrm{E}-12$ \\
\end{tabular} & $4.3 E-8$ & $7.5 E-7$ & $4.7 \mathrm{E}-12$ & $7.5 E-7$ & $4.4 E-9$ & $4.4 \mathrm{E}-9$ & \begin{tabular}{|l|}
$2.7 E-12$ \\
\end{tabular} & & \\
\hline Ci/GAL & $1.6 \mathrm{E}-8$ & $1.3 E-8$ & $1.3 \mathrm{E}-8$ & $3.0 \mathrm{E}-7$ & $1.3 \mathrm{E}-8$ & $7.2 E-10$ & $1.2 \mathrm{E}-7$ & $\begin{array}{c}7.2 \mathrm{E}- \\
10\end{array}$ & $7.2 \mathrm{E}-12$ & $1.2 \mathrm{E}-7$ & $2.1 \mathrm{E}-6$ & \begin{tabular}{|l|}
$1.3 \mathrm{E}-11$ \\
\end{tabular} & $2.1 \mathrm{E}-6$ & $1.3 E-8$ & $1.3 \mathrm{E}-8$ & $7.8 \mathrm{E}-12$ & & \\
\hline Ci/GAL & $5.8 \mathrm{E}-8$ & $4.9 \mathrm{E}-8$ & $4.9 \mathrm{E}-8$ & $1.1 \mathrm{E}-5$ & $\begin{array}{l}2.5 \mathrm{E}-9 \\
\end{array}$ & $5.4 \mathrm{E}-11$ & $2.3 E-8$ & $\begin{array}{l}5.4 \mathrm{E}- \\
11\end{array}$ & $\overline{5.4 E-12}$ & $4.3 E-7$ & $7.5 E-6$ & $4.7 E-11$ & $7.5 E-6$ & $2.5 \mathrm{E}-9$ & $2.5 E-9$ & $9.7 \mathrm{E}-12$ & & \\
\hline Ci/GAL & $6.4 \mathrm{E}-8$ & $5.4 E-8$ & $5.4 \mathrm{E}-8$ & $1.2 \mathrm{E}-5$ & $2.7 \mathrm{E}-9$ & $5.9 \mathrm{E}-11$ & $2.6 \mathrm{E}-8$ & $\begin{array}{l}5.9 E- \\
11\end{array}$ & $5.9 \mathrm{E}-12$ & $4.8 \mathrm{E}-7$ & $8.2 E-6$ & \begin{tabular}{|l|}
$5.2 \mathrm{E}-11$ \\
\end{tabular} & $8.2 \mathrm{E}-6$ & $2.7 E-9$ & $2.7 E-9$ & \begin{tabular}{|l|}
$1.1 \mathrm{E}-11$ \\
\end{tabular} & & \\
\hline Ci/GAL & $4.0 E-8$ & $3.4 E-8$ & $3.4 E-8$ & $7.3 E-6$ & $3.4 \mathrm{E}-9$ & \begin{tabular}{|l|}
$7.7 E-11$ \\
\end{tabular} & $3.2 \mathrm{E}-8$ & $\begin{array}{c}7.7 E- \\
11\end{array}$ & $7.6 \mathrm{E}-12$ & $3.0 \mathrm{E}-7$ & $5.2 E-6$ & 3.3E-11 & $5.2 E-6$ & $3.4 \mathrm{E}-9$ & $3.4 \mathrm{E}-9$ & $1.0 \mathrm{E}-11$ & & \\
\hline $\mathrm{Ci} / \mathrm{GAL}$ & $3.1 \mathrm{E}-7$ & $2.6 \mathrm{E}-7$ & $2.6 \mathrm{E}-7$ & $5.7 E-5$ & $2.6 E-8$ & $5.8 \mathrm{E}-10$ & $2.5 \mathrm{E}-8$ & $\begin{array}{c}5.8 \mathrm{E}- \\
10\end{array}$ & $5.8 \mathrm{E}-11$ & $2.1 E-6$ & $4.0 \mathrm{E}-5$ & $2.5 \mathrm{E}-10$ & $4.0 \mathrm{E}-5$ & $2.6 E-8$ & $2.6 E-8$ & $7.8 \mathrm{E}-11$ & & \\
\hline $\mathrm{Sb}-124,125$ & $2.3 \mathrm{E}-9$ & $1.9 \mathrm{E}-9$ & $1.9 \mathrm{E}-9$ & $1.9 \mathrm{E}-9$ & $1.9 E-9$ & $1.9 \mathrm{E}-10$ & $1.8 \mathrm{E}-8$ & $\begin{array}{l}1.9 \mathrm{E}- \\
10\end{array}$ & $1.9 \mathrm{E}-10$ & $1.6 \mathrm{E}-8$ & $2.7 E-7$ & $1.7 \mathrm{E}-12$ & $2.7 E-7$ & $1.9 \mathrm{E}-9$ & $1.9 \mathrm{E}-9$ & \begin{tabular}{|l|}
$1.7 E-10$ \\
\end{tabular} & & \\
\hline $\mathrm{Ci} / \mathrm{GAL}$ & $1.4 \mathrm{E}-8$ & $1.2 \mathrm{E}-8$ & $1.2 \mathrm{E}-8$ & $1.2 E-8$ & $1.2 \mathrm{E}-8$ & $6.9 \mathrm{E}-10$ & $1.1 \mathrm{E}-7$ & $\begin{array}{c}6.9 \mathrm{E}- \\
10\end{array}$ & $6.9 E-10$ & $\overline{9.8 E-8}$ & $1.7 \mathrm{E}-6$ & $1.1 \mathrm{E}-11$ & $1.7 E-6$ & $1.2 E-8$ & $1.2 \mathrm{E}-8$ & $6.2 \mathrm{E}-10$ & & \\
\hline
\end{tabular}


UNIT WASTE PROCESS CHARACTERIZATION FOR THE SRS EFFLUENT TREATMENT FACILITY (Continued)

\begin{tabular}{|c|c|c|c|c|c|c|c|c|c|c|c|c|c|c|c|c|c|c|}
\hline LINE NO. & 1 & 5 & 6 & 10 & 13 & 14 & 15 & 17 & 24 & 26 & 28 & 29 & 34 & 38 & 39 & 45 & 50 & 51 \\
\hline PROCESS MATERIAL & INFLUENT & $\begin{array}{c}\text { FACILITY } \\
\text { FEED }\end{array}$ & $\begin{array}{l}\text { FILTER } \\
\text { FEED }\end{array}$ & $\begin{array}{c}\text { FILTER } \\
\text { CONCEN- } \\
\text { TRATE }\end{array}$ & $\begin{array}{l}\text { RO } \\
\text { FEED }\end{array}$ & $\begin{array}{c}\text { RO } \\
\text { PERMEATE }\end{array}$ & $\begin{array}{c}\text { RO } \\
\text { CONCEN. } \\
\text { TRATE }\end{array}$ & $\begin{array}{c}\text { IX } \\
\text { FEED }\end{array}$ & $\begin{array}{c}\text { IX } \\
\text { PRODUCT }\end{array}$ & $\begin{array}{l}\text { EVAP. } \\
\text { FEED }\end{array}$ & $\begin{array}{l}\text { EVAP. } \\
\text { BUTTONS }\end{array}$ & \begin{tabular}{c|} 
CON- \\
DENSED \\
O'HEADS
\end{tabular} & $\begin{array}{l}\text { WASTE } \\
\text { CONCEN- } \\
\text { TRATE }\end{array}$ & $\begin{array}{c}\text { HG } \\
\text { COLUMN } \\
\text { FEED }\end{array}$ & $\begin{array}{c}\text { R/O } \\
\text { COOLER } \\
\text { FEED }\end{array}$ & \begin{tabular}{|l|} 
TREATED \\
WATER \\
\end{tabular} & $\begin{array}{l}\text { INFLUENT } \\
\text { (MAX. } \\
\text { CONC.) }\end{array}$ & $\begin{array}{l}\text { EFFLUENT } \\
\text { (MAX. } \\
\text { CONC.) }\end{array}$ \\
\hline FLOW, LBS/HR (Normal) & 82,500 & 98,500 & 98,600 & 800 & 97,800 & 88,000 & 9,800 & 93,500 & 98,500 & 11,300 & 810 & 10,500 & 810 & 97,810 & 97,800 & 98,500 & 82,500 & 98,300 \\
\hline SPECIFIC CAPACITY & 1.0 & 1.0 & 1.0 & 1.0 & 1.0 & 1.0 & 1.0 & 1.0 & 1.0 & 1.0 & 1.2 & 1.0 & 1.2 & 1.0 & 1.0 & 1.0 & 1.0 & 1.0 \\
\hline FLOW, GPM (Normal) & 165 & 197 & 197 & 1.6 & 196 & 176 & 20 & 197 & 197 & 23 & 1.3 & 21 & 1.3 & 196 & 196 & 197 & 165 & 197 \\
\hline FLOW, GPM (Maximum) & 235 & 295 & 300 & 100 & 300 & 270 & 30 & 270 & 270 & 34 & 2.6 & 40 & 2.6 & 300 & 300 & 300 & & \\
\hline $\begin{array}{l}\text { FLOW, GPM (Maximum } \\
\text { Hydraulic) }\end{array}$ & 1,000 & 295 & 300 & 50 & 300 & 270 & 30 & 270 & 270 & 50 & 50 & 40 & 50 & 300 & 300 & 300 & & \\
\hline $\begin{array}{l}\text { OPERATING TEMPERATUAE } \\
{ }^{\circ} \mathrm{C}\end{array}$ & $5-50$ & $5-50$ & 5.50 & $5-50$ & $5-35$ & $5-35$ & $5-35$ & $5-35$ & $5-35$ & $5-35$ & 104 & 60 & 104 & $5-35$ & $5-35$ & $5-35$ & & $5-35$ \\
\hline $\begin{array}{l}\text { OPERATING PRESSURE } \\
\text { P.S.I.G }\end{array}$ & ATM. & 37 & 50 & ATM. & 760 & ATM. & ATM. & 65 & ATM. & 22 & ATM. & 22 & 85 & 760 & 760 & 45 & & 45 \\
\hline \multicolumn{19}{|l|}{ COMPOSITION } \\
\hline Cs-134 & $2.1 \mathrm{E}-8$ & $1.8 E-8$ & $1.8 \mathrm{E}-8$ & $3.9 \mathrm{E}-7$ & $1.6 \mathrm{E}-8$ & $9.3 E-10$ & $1.5 \mathrm{E}-7$ & $\begin{array}{c}9.3 \mathrm{E}- \\
10\end{array}$ & $9.4 \mathrm{E}-12$ & $1.6 \mathrm{E}-7$ & $2.7 \mathrm{E}-6$ & $1.7 \mathrm{E}-11$ & $2.7 E-6$ & $1.6 \mathrm{E}-8$ & $1.6 \mathrm{E}-8$ & $1.0 \mathrm{E}-11$ & & \\
\hline Ci/GAL & $1.5 \mathrm{E}-7$ & $1.3 \mathrm{E}-7$ & $1.3 \mathrm{E}-7$ & $2.8 E-6$ & $1.2 \mathrm{E}-7$ & $6.8 \mathrm{E}-9$ & $1.1 \mathrm{E}-6$ & $6.8 \mathrm{E}-9$ & $6.8 \mathrm{E}-11$ & 1.1E-6 & $1.9 \mathrm{E}-5$ & $1.2 \mathrm{E}-10$ & $1.9 \mathrm{E}-5$ & $1.2 \mathrm{E}-7$ & $1.2 \mathrm{E}-7$ & $7.4 \mathrm{E}-11$ & & \\
\hline $\mathrm{Ci} / \mathrm{GAL}$ & $5.8 \mathrm{E}-9$ & $4.9 E-9$ & $4.9 E-9$ & $1.1 \mathrm{E}-6$ & $\begin{array}{c}2.5 \mathrm{E}- \\
10\end{array}$ & $5.4 \mathrm{E}-12$ & $2.3 E-9$ & $\begin{array}{c}5.4 \mathrm{E}- \\
12\end{array}$ & $5.4 \mathrm{E}-13$ & $4.3 E-8$ & $7.5 \mathrm{E}-7$ & $4.7 \mathrm{E}-12$ & $7.5 E-7$ & 2.5E-10 & $2.5 \mathrm{E}-10$ & $9.7 \mathrm{E}-13$ & & \\
\hline $\mathrm{Ce}-144$ & $4.1 E-8$ & $3.4 \mathrm{E}-8$ & $3.4 \mathrm{E}-8$ & $7.9 \mathrm{E}-6$ & $1.8 \mathrm{E}-9$ & $3.9 \mathrm{E}-11$ & $1.8 \mathrm{E}-8$ & $\begin{array}{c}3.9 \mathrm{E}- \\
11\end{array}$ & $3.9 \mathrm{E}-12$ & $3.1 E-7$ & 5.3E-6 & 3.3E-11 & $5.3 E-6$ & $1.8 E-9$ & $1.8 \mathrm{E}-9$ & $7.0 \mathrm{E}-12$ & & \\
\hline$P m-147$ & $1.7 E-8$ & $1.4 \mathrm{E}-8$ & $1.4 \mathrm{E}-8$ & $1.4 \mathrm{E}-8$ & $1.4 \mathrm{E}-8$ & $8.4 \mathrm{E}-10$ & $1.4 \mathrm{E}-7$ & $\begin{array}{c}8.4 \mathrm{E}- \\
10\end{array}$ & $8.4 \mathrm{E}-11$ & $1.3 E-7$ & $2.2 E-6$ & $1.4 \mathrm{E}-11$ & $2.2 \mathrm{E}-6$ & $1.4 \mathrm{E}-8$ & $1.4 E-8$ & $7.7 E-11$ & & \\
\hline OTHER BETA GAMMACi/GAL & $1.5 \mathrm{E}-8$ & $1.3 \mathrm{E}-8$ & $1.3 E-8$ & $1.3 E-8$ & $1.3 \mathrm{E}-8$ & $7.4 \mathrm{E}-9$ & $1.2 \mathrm{E}-7$ & 7.4E-9 & $7.4 \mathrm{E}-11$ & $1.1 \mathrm{E}-7$ & $1.9 \mathrm{E}-6$ & $1.2 \mathrm{E}-11$ & $1.9 \mathrm{E}-6$ & $1.3 \mathrm{E}-8$ & $1.3 \mathrm{E}-8$ & $6.8 \mathrm{E}-11$ & & \\
\hline ALL ALPHA $\quad$ Ci/GAL & $8.1 \mathrm{E}-9$ & $6.8 \mathrm{E}-9$ & $6.8 \mathrm{E}-9$ & $1.5 \mathrm{E}-6$ & $\begin{array}{c}3.4 \mathrm{E}- \\
10\end{array}$ & $3.8 \mathrm{E}-12$ & $3.3 E-9$ & $\begin{array}{c}3.8 \mathrm{E}- \\
12\end{array}$ & $3.8 \mathrm{E}-13$ & $6.1 E-8$ & $1.0 \mathrm{E}-6$ & $6.6 \mathrm{E}-12$ & $1.0 \mathrm{E}-6$ & $3.4 \mathrm{E}-10$ & $3.4 \mathrm{E}-10$ & $1.0 \mathrm{E}-12$ & & \\
\hline TOTAL & 8.7E-7 & $7.3 \mathrm{E}-7$ & $7.3 \mathrm{E}-7$ & $1.0 \mathrm{E}-4$ & $3.1 E-7$ & $1.6 \mathrm{E}-8$ & $2.9 E-6$ & $1.6 \mathrm{E}-8$ & $1.3 \mathrm{E}-9$ & $6.3 E-6$ & $1.1 E \cdot 4$ & $7.0 \mathrm{E}-10$ & $1.1 E-4$ & 3.1E-7 & $3.1 E-7$ & $1.2 \mathrm{E}-9$ & & \\
\hline
\end{tabular}


Table 2-2 UNIT WASTE PROCESS CHARACTERIZATION FOR THE HANFORD SITE PROCESS CONDENSATE TREATMENT FACILITY

\begin{tabular}{|c|c|c|c|c|c|c|c|c|c|c|c|}
\hline \multirow{2}{*}{\multicolumn{2}{|c|}{ STREAM NAME }} & $\begin{array}{c}\text { PUREX } \\
\text { PLANT } \\
\text { PPD } \\
\end{array}$ & $\begin{array}{c}\text { PUREX } \\
\text { PLANT } \\
\text { ASD } \\
\end{array}$ & $\begin{array}{c}242 \\
\text { EVAPORATOR }\end{array}$ & $\begin{array}{l}\text { LRAF } \\
\text { BASINS }\end{array}$ & $\begin{array}{l}\text { FEED TO } \\
\text { FILTERS }\end{array}$ & $\begin{array}{l}\text { FEED TO U.V. } \\
\text { OXIDATION }\end{array}$ & $\begin{array}{l}\text { FEED TO } \mathrm{pH} \\
\text { ADJUSTMENT }\end{array}$ & $\begin{array}{c}\text { FEED TO } \\
\text { DEGASIFI- } \\
\text { CATION }\end{array}$ & $\begin{array}{l}\text { FEED TO } \\
\text { R.O. }\end{array}$ & $\begin{array}{l}\text { FEED TO } \\
\text { POLISHERS }\end{array}$ \\
\hline & & 1 & 2 & 3 & 4 & 5 & 6 & 7 & 8 & 9 & 10 \\
\hline $\begin{array}{l}\text { FLOWRATE } \\
\text { Max/min } \\
\text { Design }\end{array}$ & gpm & $\begin{array}{r}75 / \\
75 \\
\end{array}$ & $\begin{array}{r}75 / \\
75 \\
\end{array}$ & $\begin{array}{r}75 / \\
75 \\
\end{array}$ & $\begin{array}{c}150 / 0 \\
150 \\
\end{array}$ & $\begin{array}{c}150 / 40 \\
150 \\
\end{array}$ & 150 & 189 & 189 & 205.7 & 150 \\
\hline $\begin{array}{l}\mathrm{pH} \\
\text { Temp. }\end{array}$ & ${ }^{\circ} \mathrm{C}$ & $\begin{array}{c}2 \\
78.2 \\
\end{array}$ & $\begin{array}{c}10 \\
38.3 \\
\end{array}$ & & & $\begin{array}{l}10 \\
40 \\
\end{array}$ & $\begin{array}{l}10 \\
40 \\
\end{array}$ & $\begin{array}{l}10 \\
40 \\
\end{array}$ & $\begin{array}{l}4-6 \\
40 \\
\end{array}$ & $\begin{array}{l}4-6 \\
40 \\
\end{array}$ & $\begin{array}{r}6 \\
40 \\
\end{array}$ \\
\hline $\begin{array}{l}\text { TOTAL } B, y \\
\text { TOTAL } \alpha \\
H-3 \\
1-129 \\
\end{array}$ & $\begin{array}{l}\mathrm{Ci} / \mathrm{m}^{3} \\
\mathrm{Ci} / \mathrm{m}^{3} \\
\mathrm{Ci} / \mathrm{m}^{3} \\
\mathrm{Ci} / \mathrm{m}^{3}\end{array}$ & $\begin{array}{l}4.9 E-4 \\
2.2 E-4 \\
6.8 E-2 \\
7.8 E-7\end{array}$ & $\begin{array}{l}4.9 \mathrm{E}-4 \\
2.0 \mathrm{E}-6 \\
4.3 \mathrm{E}-3 \\
1.4 \mathrm{E}-7\end{array}$ & $\begin{array}{c}4.6 \mathrm{E}-6 \\
3.5 \mathrm{E}-7 \\
6.3 \mathrm{E}-3 \\
0.0 \\
\end{array}$ & & $\begin{array}{l}1.2 \mathrm{E}-4 \\
4.3 \mathrm{E}-5 \\
1.8 \mathrm{E}-2 \\
1.5 \mathrm{E}-7\end{array}$ & $\begin{array}{l}1.2 \mathrm{E}-4 \\
4.3 \mathrm{E}-5 \\
1.8 \mathrm{E}-2 \\
1.5 \mathrm{E}-7 \\
\end{array}$ & $\begin{array}{l}9.3 \mathrm{E}-5 \\
3.4 \mathrm{E}-5 \\
1.8 \mathrm{E}-2 \\
1.2 \mathrm{E}-7 \\
\end{array}$ & $\begin{array}{l}9.3 \mathrm{E}-5 \\
3.4 \mathrm{E}-5 \\
1.8 \mathrm{E}-2 \\
1.2 \mathrm{E}-7 \\
\end{array}$ & $\begin{array}{l}8.9 E-5 \\
3.2 E-5 \\
1.8 E-2 \\
1.2 E-7 \\
\end{array}$ & $\begin{array}{l}1.2 \mathrm{E}-6 \\
4.3 \mathrm{E}-7 \\
1.8 \mathrm{E}-2 \\
1.6 \mathrm{E}-9 \\
\end{array}$ \\
\hline $\begin{array}{l}\text { TSS } \\
\text { TDS } \\
\text { TOC }\end{array}$ & $\begin{array}{l}\text { ppm } \\
\text { ppm } \\
\text { ppm }\end{array}$ & $\begin{array}{c}10 \\
142 \\
130 \\
\end{array}$ & $\begin{array}{c}10 \\
216 \\
218 \\
\end{array}$ & $\begin{array}{c}10 \\
216 \\
213 \\
\end{array}$ & $\begin{array}{c}10 \\
0\end{array}$ & $\begin{array}{c}10 \\
615 \\
193 \\
\end{array}$ & $\begin{array}{l}0.1 \\
615 \\
193 \\
\end{array}$ & $\begin{array}{c}0.1 \\
485 \\
5 \\
\end{array}$ & $\begin{array}{c}0.1 \\
1270 \\
5 \\
\end{array}$ & $\begin{array}{c}0.1 \\
1160 \\
5 \\
\end{array}$ & $\begin{array}{c}0 \\
16 \\
0 \\
\end{array}$ \\
\hline $\begin{array}{l}\mathrm{Na} \\
\mathrm{K} \\
\mathrm{Ca} \\
\mathrm{NH}_{4} \\
\mathrm{Si} \\
\end{array}$ & $\begin{array}{l}\text { ppm } \\
\text { ppm } \\
\text { ppm } \\
\text { ppm } \\
\text { ppm }\end{array}$ & $\begin{array}{c}20 \\
1 \\
0.05 \\
0.038 \\
0.24 \\
\end{array}$ & $\begin{array}{c}4.5 \\
6.5 \\
2.8 \\
0 \\
24.3 \\
\end{array}$ & $\begin{array}{c}4.3 \\
6.3 \\
2.8 \\
0 \\
24.3 \\
\end{array}$ & & $\begin{array}{c}7.3 \\
3.2 \\
2.2 \\
425 \\
18.7 \\
\end{array}$ & $\begin{array}{r}7.3 \\
5.2 \\
2.2 \\
425 \\
18.7 \\
\end{array}$ & $\begin{array}{c}5.8 \\
4.1 \\
1.7 \\
337.3 \\
14.8 \\
\end{array}$ & $\begin{array}{c}5.8 \\
4.1 \\
1.7 \\
337.3 \\
14.8 \\
\end{array}$ & $\begin{array}{c}5.8 \\
4.0 \\
1.7 \\
323.1 \\
14.2 \\
\end{array}$ & $\begin{array}{l}0.1 \\
0.1 \\
0.0 \\
4.4 \\
0.2 \\
\end{array}$ \\
\hline $\begin{array}{l}\mathrm{NO}_{3} \\
\mathrm{SO}_{4} \\
\mathrm{CO}_{3} \\
\mathrm{Cl} \\
\mathrm{F}\end{array}$ & $\begin{array}{l}\text { ppm } \\
\text { ppm } \\
\text { ppm } \\
\text { ppm } \\
\text { ppm }\end{array}$ & $\begin{array}{c}119 \\
0 \\
0 \\
0 \\
1.2\end{array}$ & $\begin{array}{c}0.6 \\
0 \\
0 \\
2.3 \\
0\end{array}$ & $\begin{array}{c}2.3 \\
69 \\
104 \\
1.2 \\
1\end{array}$ & & $\begin{array}{c}25 \\
50 \\
80 \\
1 \\
1\end{array}$ & $\begin{array}{c}25 \\
50 \\
80 \\
1 \\
1\end{array}$ & $\begin{array}{c}19.8 \\
39.7 \\
63.5 \\
0.8 \\
0.8\end{array}$ & $\begin{array}{c}19.8 \\
821 \\
63.5 \\
0.8 \\
0.8\end{array}$ & $\begin{array}{c}19.0 \\
786.3 \\
5.0 \\
0.8 \\
0.8\end{array}$ & $\begin{array}{c}0.3 \\
10.8 \\
0.1 \\
0.0 \\
0.0\end{array}$ \\
\hline
\end{tabular}


UNIT WASTE PROCESS CHARACTERIZATION FOR THE HANFORD SITE PROCESS CONDENSATE TREATMENT FACILITY (Continued)

\begin{tabular}{|c|c|c|c|c|c|c|c|c|c|c|c|}
\hline \multirow{2}{*}{\multicolumn{2}{|c|}{ STEAM NAME }} & $\begin{array}{c}\text { FEED TO } \mathrm{pH} \\
\text { ADJUST- } \\
\text { MENT }\end{array}$ & $\begin{array}{l}\text { TREATED } \\
\text { LIQUID } \\
\text { WASTES }\end{array}$ & $\begin{array}{c}\text { FILTER BACK } \\
\text { WASH }\end{array}$ & $\begin{array}{c}\text { R/O } \\
\text { CONCEN- } \\
\text { TRATE }\end{array}$ & $\begin{array}{c}\text { FEED TO } \\
\text { EVAPORATO } \\
R\end{array}$ & $\begin{array}{c}\text { EVAPORATO } \\
\text { R CONCEN- } \\
\text { TRATE }\end{array}$ & $\begin{array}{c}\text { DRYER } \\
\text { CONDENSATE }\end{array}$ & $\begin{array}{c}\text { RECYCLED } \\
\text { CONDENSATE } \\
\mathrm{S}\end{array}$ & $\begin{array}{l}\text { POWDERED } \\
\text { WASTES }\end{array}$ & $\begin{array}{c}\text { REGENERA- } \\
\text { TION } \\
\text { WASTE } \\
\end{array}$ \\
\hline & & 11 & 12 & 13 & 14 & 15 & 16 & 17 & 18 & 19 & 20 \\
\hline $\begin{array}{l}\text { FLOWRATE } \\
\text { Max/min } \\
\text { Design }\end{array}$ & gpm & 150 & 150 & 1.5 & 39 & 41.05 & 0.5 & 0.3 & 40.5 & - & 0.55 \\
\hline $\begin{array}{l}\text { pH } \\
\text { Temp. } \\
\end{array}$ & ${ }^{\circ} \mathrm{C}$ & $\begin{array}{l}6-8 \\
40 \\
\end{array}$ & $\begin{array}{c}6-8 \\
40 \\
\end{array}$ & $\begin{array}{l}10 \\
40 \\
\end{array}$ & $\begin{array}{c}4 \\
40 \\
\end{array}$ & $\begin{array}{l}6-8 \\
40\end{array}$ & $\begin{array}{l}6-8 \\
110\end{array}$ & $\begin{array}{l}6-8 \\
50\end{array}$ & $\begin{array}{l}6-8 \\
30\end{array}$ & 40 & 40 \\
\hline $\begin{array}{l}\text { TOTAL } B, \gamma \\
\text { TOTAL } \alpha \\
H-3 \\
1-129\end{array}$ & $\begin{array}{l}\mathrm{Ci} / \mathrm{m}^{3} \\
\mathrm{Ci} / \mathrm{m}^{3} \\
\mathrm{Ci} / \mathrm{m}^{3} \\
\mathrm{Ci} / \mathrm{m}^{3}\end{array}$ & $\begin{array}{c}1.2 \mathrm{E}-8 \\
4.5 \mathrm{E}-9 \\
1.8 \mathrm{E}-2 \\
1.6 \mathrm{E}-11\end{array}$ & $\begin{array}{c}1.2 \mathrm{E}-8 \\
4.5 \mathrm{E}-9 \\
1.8 \mathrm{E}-2 \\
1.6 \mathrm{E}-11\end{array}$ & $\begin{array}{l}1.2 \mathrm{E}-4 \\
4.3 \mathrm{E}-5 \\
1.8 \mathrm{E}-2 \\
1.5 \mathrm{E}-7\end{array}$ & $\begin{array}{l}4.6 E-04 \\
1.7 E-04 \\
1.8 E-02 \\
6.2 E-07\end{array}$ & $\begin{array}{l}4.7 E-04 \\
1.7 E-04 \\
1.8 E-02 \\
6.2 E-07\end{array}$ & $\begin{array}{l}3.9 E-02 \\
1.4 E-02 \\
1.8 E-02 \\
5.1 E-05\end{array}$ & $\begin{array}{l}2.5 E-05 \\
9.4 E-06 \\
1.8 E-02 \\
3.4 E-08\end{array}$ & $\begin{array}{l}7.8 E-07 \\
2.8 E-07 \\
1.8 E-02 \\
1.0 E-09\end{array}$ & & $\begin{array}{l}3.3 E-04 \\
1.2 E-04 \\
1.8 E-02 \\
4.4 E-07\end{array}$ \\
\hline $\begin{array}{l}\text { TSS } \\
\text { TDS } \\
\text { TOC } \\
\end{array}$ & $\begin{array}{l}\mathrm{ppm} \\
\mathrm{ppm} \\
\mathrm{ppm}\end{array}$ & $\begin{array}{l}0 \\
0 \\
0\end{array}$ & $\begin{array}{l}0 \\
0 \\
0\end{array}$ & $\begin{array}{l}1,000 \\
613 \\
193 \\
\end{array}$ & $\begin{array}{c}0.5 \\
6,059 \\
25 \\
\end{array}$ & $\begin{array}{c}0.5 \\
6,882 \\
25 \\
\end{array}$ & $\begin{array}{c}41 \\
383,017 \\
\text { TRACE }\end{array}$ & $\begin{array}{l}0 \\
64\end{array}$ & $\begin{array}{c}0 \\
25 \\
\end{array}$ & & $\begin{array}{l}0 \\
0\end{array}$ \\
\hline $\begin{array}{l}\mathrm{Na} \\
\mathrm{K} \\
\mathrm{Ca} \\
\mathrm{NH}_{4} \\
\mathrm{Si}\end{array}$ & $\begin{array}{l}\text { ppm } \\
\text { ppm } \\
\text { ppm } \\
\text { ppm } \\
\text { ppm }\end{array}$ & $\begin{array}{l}0 \\
0 \\
0 \\
0\end{array}$ & $\begin{array}{l}0 \\
0 \\
0 \\
0\end{array}$ & $\begin{array}{l}7.3 \\
5.2 \\
2.2 \\
425 \\
18.7 \\
\end{array}$ & $\begin{array}{c}29.0 \\
20.6 \\
8.7 \\
1,685.9 \\
74.2 \\
\end{array}$ & $\begin{array}{c}230.1 \\
19.6 \\
8.3 \\
1,625 \\
70.3 \\
\end{array}$ & $\begin{array}{c}20,522 \\
1,610 \\
681 \\
133,412 \\
3,789 \\
\end{array}$ & & & & $\begin{array}{l}16,611 \\
1,667\end{array}$ \\
\hline $\begin{array}{l}\mathrm{NO}_{3} \\
\mathrm{SO}_{4} \\
\mathrm{CO}_{3} \\
\mathrm{Cl} \\
\mathrm{F}\end{array}$ & $\begin{array}{l}\text { ppm } \\
\text { ppm } \\
\text { ppm } \\
\text { ppm } \\
\text { ppm }\end{array}$ & $\begin{array}{l}0 \\
0 \\
0 \\
0 \\
0\end{array}$ & $\begin{array}{l}0 \\
0 \\
0 \\
0 \\
0\end{array}$ & $\begin{array}{c}25 \\
50 \\
80 \\
1 \\
1\end{array}$ & $\begin{array}{c}99.2 \\
4,108 \\
26.1 \\
4.0 \\
4.0\end{array}$ & $\begin{array}{c}94.3 \\
4,781.9 \\
24.8 \\
3.8 \\
3.8\end{array}$ & $\begin{array}{c}7,740 \\
392,597 \\
2,036 \\
310 \\
310\end{array}$ & & & & 37,000 \\
\hline
\end{tabular}





$$
\mid
$$



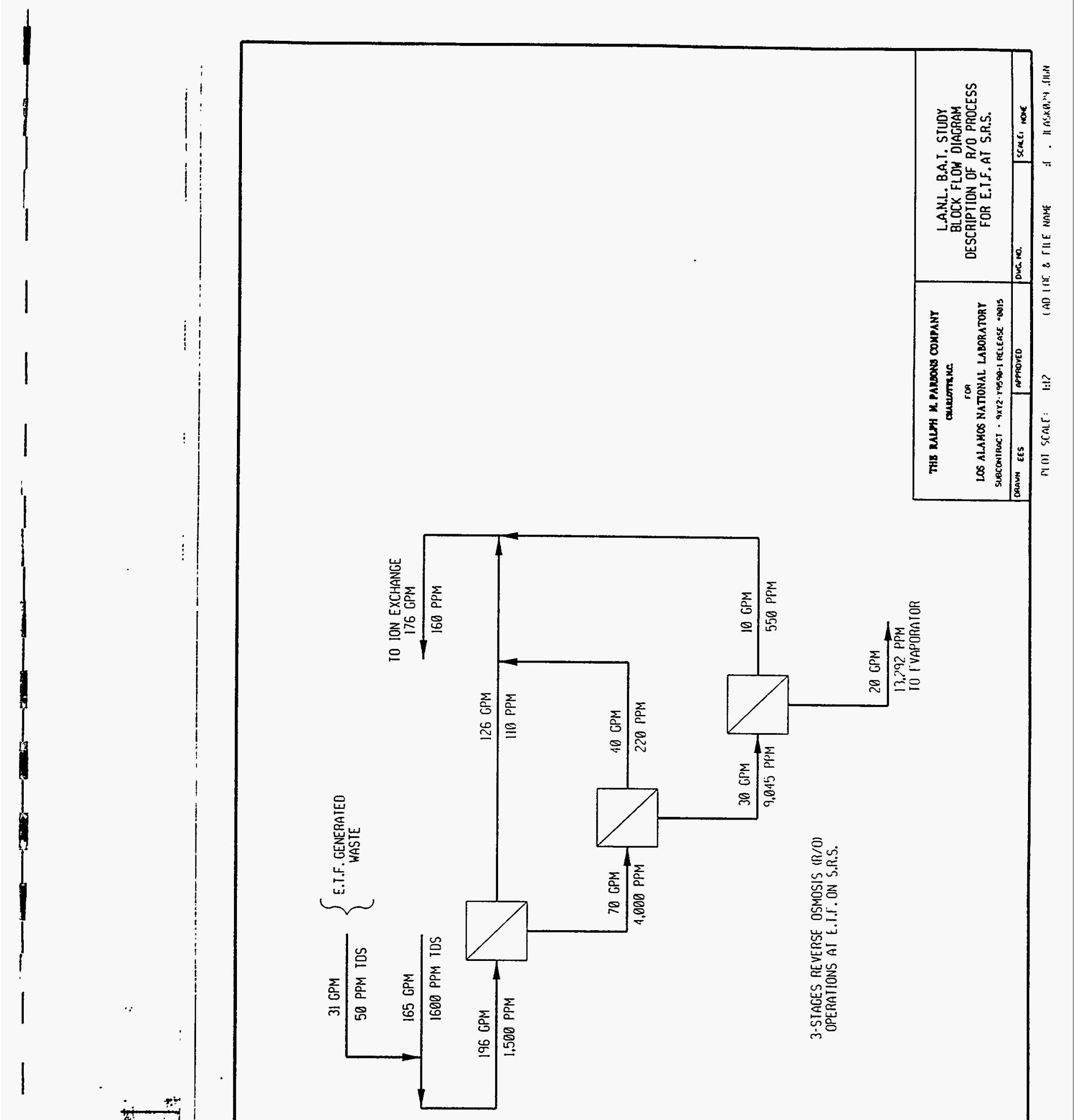


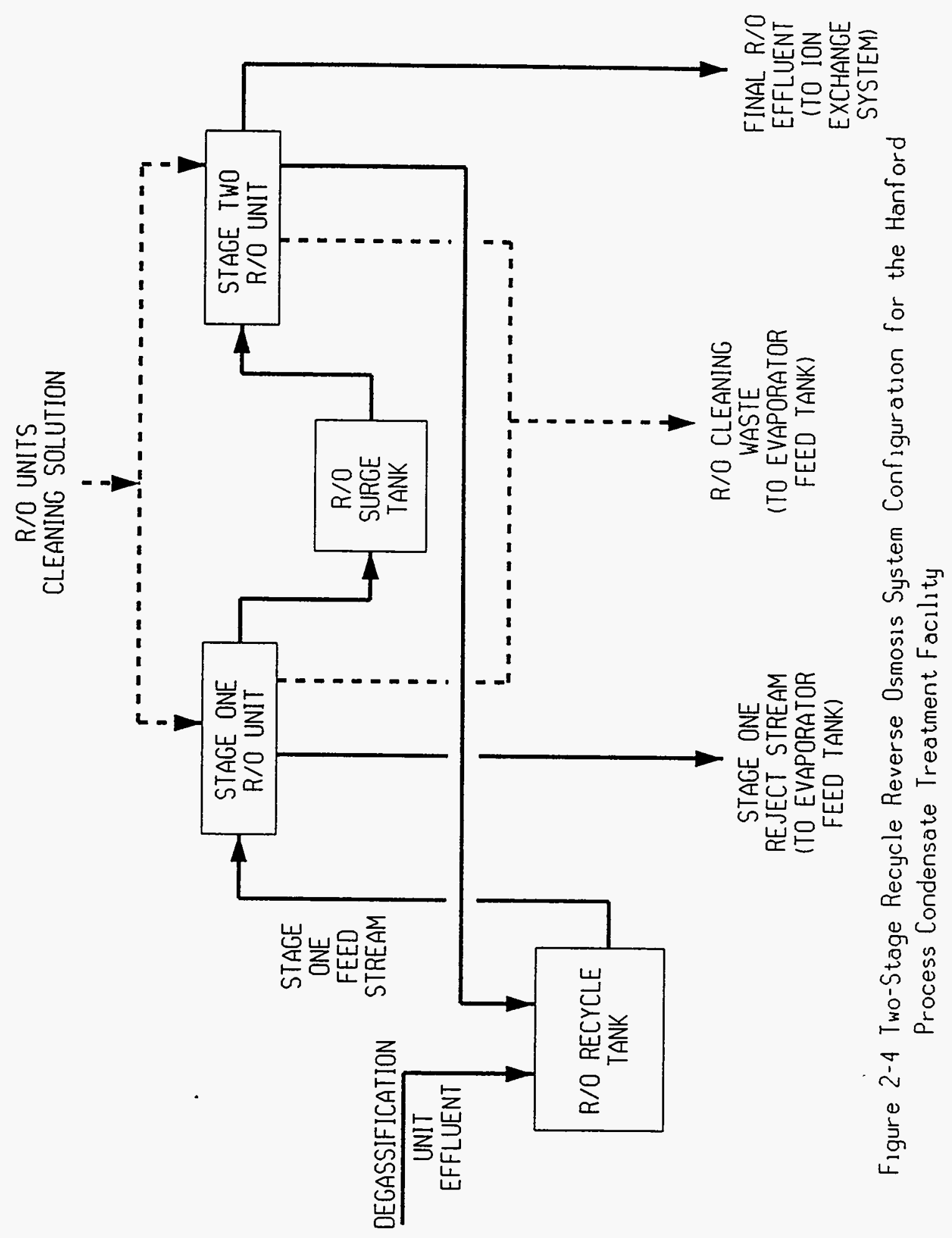

THB RALPH M PARSONS COMPANY challotinke

FOR

LOS ALAMOS NATIONAL LABORATORY

SUBCONTRACT - 9XY2-Y9590-1 RELEASE

BEST AYAILABLE TECHNOLOGY STUDY BLOCK FLOW DIAGRAM HANFORD SITE

PCTF OPERATIONS - R/O SYSTEM SHEET 1 OF 1 DRAWN LPG APPROVEO DWC. NO. SCALE: NONE 


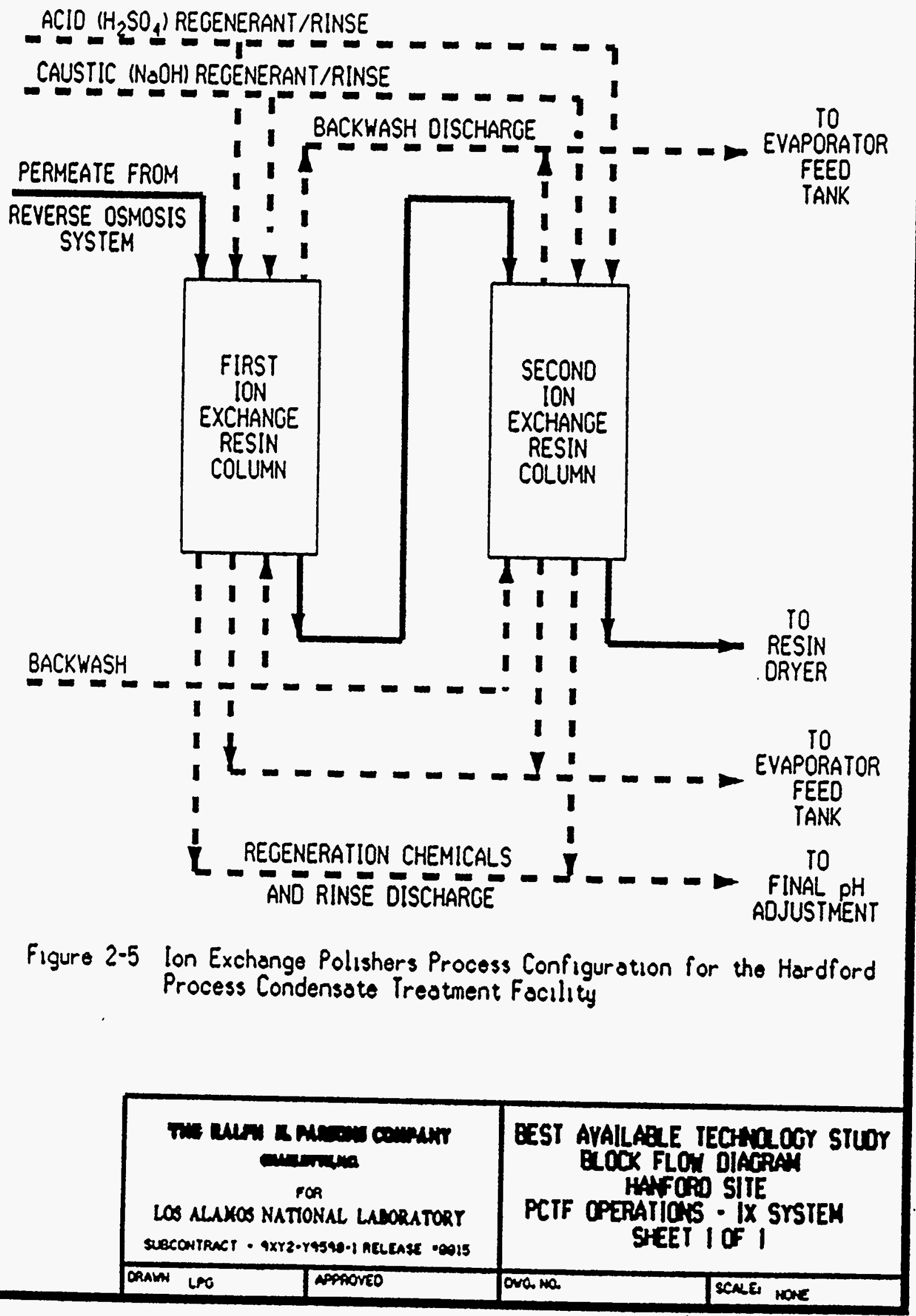




\subsection{SELECTION OF SUITABLE TREATMENT PROCESSES}

\subsection{BASES FOR THE EVALUATION OF UNIT PROCESSES}

\subsubsection{Understanding of the Evaluation Requirements}

The evaluation of the criteria and technologies involved in a BAT study is a complex and iterative process. The evaluation process is initiated by defining the problem or conditions to be remediated. Once this has been established, the desired result can be quantified, and a theoretical scheme to accomplish this result can be postulated. With the inputs and outputs of the process established, the intermediate engineering that will be required to accommodate these end points can be addressed.

In most instances, the characterization of the influent will dictate the treatment technologies and unit processes that must be applied for the desired (or required) results. This characterization can serve as the basis for elimination of many treatment technologies that obviously cannot accomplish the process objectives, or that are obviously a second choice to a more feasible process. The actual characterization of the influent waste may represent a difficult and time-consuming task. As an example, the influent characterization of the Savannah River Site Effluent Treatment Facility required approximately two years to complete.

The treatment processes that will be selected for the LANL Radioactive Liquid Waste Treatment Facility will process the same waste streams currently treated by the existing facility. Five years of operating chemical data was available for this study, providing a reasonable basis for the evaluation of candidate technologies (BAT selection). However, additional in-depth characterization efforts (which currently are in progress at LANL) will be needed if the long-range changes in the characteristics are realized due to potential changes in the activities at LANL. An allowance in the design must be made for the impact of these changes during design development.

The design basis waste characterization for the candidate treatment technologies is derived from the evaluation of existing data, which in summary identifies these waste streams as:

A. $\quad 180,000 \mathrm{~L} / \mathrm{yr}$ of combined wastes from TA-55

- approximately $80 \% \quad 3.5 \mathrm{~N} \mathrm{HNO}_{3}$ solution containing dissolved contaminants

- approximately $20 \% \quad 0.5 \mathrm{~N} \mathrm{NaOH}$ solution containing dissolved contaminants mixed with some $\mathrm{KOH}$ and neutralized $\mathrm{HCl}$ 
0

0

125-150 Ci/yr of alpha-emitting radionuclides, primarily plutonium and americium in a 2:1 ratio

After neutralization and dilution of these streams, an effluent of $250,000 \mathrm{~L} / \mathrm{yr}$ is generated containing $18.8 \%$ TDS

B. $20,500,000 \mathrm{~L} / \mathrm{yr}$ other wastes from multiple sources heavy metals

- $\quad 3.5 \mathrm{Ci} / \mathrm{yr}$ of alpha-emitting radionuclides

- TDS of approximately $1000 \mathrm{ppm}$ (before blending with the effluent from the TA-55 waste stream)

The current facility (RLWP) has a design capacity of $250 \mathrm{gpm}$, but over the years the actual loading has decreased to the point where RLWP is run 3 to $4 \mathrm{hrs}$ per day, 5 days a week to support LANL operations. The anticipated RLWTF influent rate of 20,500,000 liters per year would suggest one could provide a small (15 to $20 \mathrm{gpm}$ ) continuously operated facility or a larger batch type facility that would operate in campaigns. The size of the batch facility would be dependent on the up-front storage provided and a balancing of the on-line time with the capital equipment costs.

The choice between a small "round the clock" operation and a larger batch operation is an important consideration, and often difficult to make or to justify. For the purposes of this study the larger batch RLWTF was selected because of the following considerations:

1. Larger batch operations are typically much more flexible than small continuous operations and because of the anticipated wide variance in influent parameters at LANL this becomes an important trate.

2. Redundancy for reliable operations is easier to provide with the large batch-continuous type of operation.

3. The batch-continuous operation is much more compatible with the perceived "culture" established at TA-50 from past operations.

4. Much of the equipment that would be involved in this type of facility is not generally compatible with the small $(15-20 \mathrm{gpm})$ flow rates.

Once the operating scheme of a larger batch plant was selected, an influent rate also had to be established. For the purpose of this assessment an influent rate to the RLWTF of $100 \mathrm{gpm}$ was selected because it allowed reasonable down periods between campaigns and fit well with the commonly provided equipment sizing for this type of facility. It is to be noted that the final selection of an influent rate to RLWTF is an extremely important 
consideration and needs to be addressed in a more comprehensive fashion than it has been for this study. It is also to be noted that this study is concerned with relative comparisons of treatment schemes and therefore the selection of an influent rate should not effect the relative positioning of different treatment schemes.

Table 3-1 presents a characterization of the influent waste contaminant species and decontamination factors based on the evaluation of the five years of annual reporting data (1987-91). Table 3-2 presents the influent waste contaminant species as derived for the purposes of the BAT Study.

Using these ratios, the TA-55 stream comprises approximately $1.2 \%$ of the total waste stream volume. However, the TA-55 stream contains most of the radioactive contaminants that must be treated.

\subsubsection{Design Input for Technology Evaluation}

On August 26, 1992, a meeting was conducted between LANL and Parsons to determine the impact of newly developed requirements imposed upon LANL for the preparation of the BAT Study, and to discuss outstanding topics related to influent/effluent characterization. During this meeting, the following topics were discussed and were documented as important to design development.

1. If real (operating) data is not available from similar existing DOE facilities, bench-scale design data will be considered to be satisfactory for comparison of treatment methods.

2. Justification of design data is not required.

3. Organic contaminants (chelates, detergents, etc.) must be considered as a component of the influent wastewater because of their effects on treatment processes under consideration.

4. Definition of influent and effluent design basis characteristics is scheduled for completion in time for use as input to the BAT study.

5. The testing data used to generate process flow diagrams at the Savannah River Site should be researched and provided (if available).

6. Functional definition for preliminary process selection is to be discussed in the final BAT report.

7. Preliminary equipment selection will be made for costing purposes as an example of the implementation of functional definition. 
8. Bench-scale development work is to be identified in the final report to verify the selection process assumptions for the BAT unit processes and sludge disposal processes.

\subsubsection{Evaluation of the Existing Treatment Technology}

In the processes currently in use in Room 60 of the existing RLWP, the alpha-emitting radionuclides in the blended and neutralized TA-55 waste are removed effectively by two stages of contact and/or precipitation, sedimentation, sediment washing, and decantation. The thickened sludges that result from this process are fixed by a cementation process into a solid waste to be transferred for storage awaiting ultimate disposal. The effluent from this process is blended into the TA-50 influent.

The alpha-emitting radioactivity and the heavy metals in the TA-50 influent (which includes the processed Room 60/TA-55 waste stream) are removed by two stages of precipitation, flocculation, and sedimentation. The thickened sludge from this process is dewatered in a rotary vacuum precoated filter, with the filter cake routed for drumming and disposal. The clarified liquid from the sedimentation process is decanted and routed for release, while the filtrate from the sludge dewatering process is recycled into the process.

The annual production of filter cake is approximately 30055 -gallon drums per year (approximately $62,000 \mathrm{~kg} / \mathrm{yr}$ ). The total radioactivity content of transuranic isotopes in the solidified waste is approximately one-half of the maximum content limit that would require its classification as TRU waste.

The final effluent from this existing process in general has been in compliance with regulatory/permit (NPDES) release criteria on an average basis, with occasional short-duration excursions beyond release limits. Based on conversations with the involved regulatory agencies, LANL expects the NPDES permit release limits for the upcoming permit renewal to become more stringent. Since the existing system is working at near the technical capability of contaminant reduction, it is anticipated that the current operations will not be able to treat the current waste streams to a level of decontamination that would result in compliance with more stringent requirements.

Since there is some uncertainty in anticipating what will be the new NPDES criteria, it was decided that this BAT study would address both the State of New Mexico Environmental Department 'Wild Life and Live Stock Watering' criteria and the best estimation of September 11, 1992 NPDES release limits. 


\subsubsection{Unit Treatment Technologies Considered}

In any type of consideration of alternatives, a judgement must be made on the criteria that will be used to narrow the list of all possible alternatives, options, and configurations to a list of alternatives that are reasonable, feasible, and are capable of being implemented. Since it would be vastly more expensive and time-consuming to consider every possible alternative and configuration that could possibly be imagined, it is preferable to using engineering experience, existing information, and professional judgement to winnow down the list of all possible alternatives to those that are generally accepted as realistic. Once this has been accomplished, screening criteria may be applied to eliminate candidate technologies down to a workable list that can be analyzed in depth to determine the preferred alternative.

In considering the entire spectrum of treatment technologies that could be utilized in planning the technology schemes for alternatives, the following technology processes were evaluated to determine if further evaluation was warranted:

\section{Thermal Treatment}

$\begin{array}{ll}\circ & \text { Incineration } \\ \circ & \text { Molten salt destructor } \\ \circ & \text { Pyrolysis } \\ \circ & \text { Wet air oxidation } \\ \circ & \text { Calcination } \\ \circ & \text { Microwave discharge } \\ \circ & \text { Cement kiln } \\ \circ & \text { Lime kiln }\end{array}$

\section{Chemical Treatment}

$\begin{array}{ll}\circ & \text { Absorption mound/field } \\ \circ & \text { Absorption bed/columns } \\ \circ & \text { Chemical fixation } \\ \circ & \text { Chemical oxidation } \\ \circ & \text { Chemical precipitation } \\ \circ & \text { Chemical reduction } \\ \circ & \text { Chlorination } \\ \circ & \text { Chlorinolysis } \\ \circ & \text { Cyanide destruction } \\ \circ & \text { Ion Exchange } \\ \circ & \text { Neutralization } \\ \circ & \text { Ozonation } \\ \circ & \text { Photolysis }\end{array}$


- UV irradiation

Physical Treatment - Separation of Components

- Centrifugation

- Clarification

- Coagulation

- Decanting

- Encapsulation

$\circ \quad$ Filtration

- Flocculation

- Flotation

- Foaming

- Sedimentation

- Thickening

$\circ \quad$ Ultrafiltration

\section{Physical Treatment - Removal of Specific Components}

- Activated carbon

- Blending

- Catalysis

- Crystallization

- Distillation

- Electrodialysis

- Electrolysis

- Evaporation

- High gradient magnetic separation

- Leaching

- Liquid-liquid extraction

- Molecular sieve adsorption

- Reverse osmosis

- Solvent recovery

- Stripping

- Sand filter

Sanitary Waste/Biological Treatment
- Activated sludge
- Aerobic lagoon
- Composting
- Septic tank
- Spray irrigation
- Thickening filter
- Trickling filter 
The process of screening technologies and unit processes for possible treatment schemes requires a knowledge of the type and variability of the waste streams to be treated. Very specific technology can be applied to welldefined waste streams of consistent composition. When variability in composition is a consideration, as it is in the design of the RLWTF, a certain degree of flexibility must be inherent in the selected processes. Since the TA-50 and TA-55 waste streams may be characterized in general as aqueous wastes containing radionuclides and heavy metals, the processes to be utilized must provide optimum treatment for these parameters.

\subsection{SUMMARY AND PRELIMINARY SCREENING OF TREATMENT PROCESSES}

The first screening test was to eliminate treatment technologies that were not applicable to the waste characterization and contaminant species involved in the TA-50 and TA-55 waste streams. The unit processes that were eliminated on the basis of non-applicability are addressed in the following summaries.

\subsubsection{Thermal Treatment}

Thermal treatment, destruction, and/or conversion technology typically is used for liquid, gaseous, and solid organic wastes, including hazardous wastes. As an example, high-efficiency incineration may be utilized for the destruction of dioxins or polychlorinated biphenyls as long as the organic substances can be destroyed or removed to an efficiency (DRE) or $99.9999 \%$ Thermal waste treatment usually involves air pollution control devices/measures for treatment of the process emissions.

Since the TA-50 and TA-55 waste streams do not have significant organic constituents, the initial screening indicated that there is little value to utilizing expensive, complex technology such as thermal treatment systems when there is little or no combustible material present. If used, a thermal treatment system would do little more than serve as an evaporator. Based on this premise, all of the thermal treatment systems were eliminated from further consideration, which included incineration, molten salt destruction, pyrolysis, wet air oxidation, calcination, microwave discharge, cement kilns, and lime kilns.

\subsubsection{Chemical Treatment}

The field of chemical waste treatment technology encompasses the unit processes that typically are among most effective for the treatment of waste streams with contaminants and characteristics similar to the TA-50 and 
Room 60/TA-55 wastes. With this consideration, it was evident that each generic process in this category should be carefully analyzed and evaluated for practical application to the treatment of LANL wastes. Each of the identified processes were addressed individually for consideration.

\subsubsection{Absorption Mound/Field}

The absorption mound and/or absorption field process involves the use of an open area, such as a field, for the treatment of liquid waste streams. Due to the presence of radionuclides in the LANL wastes, this method clearly was not a viable option.

\subsubsection{Absorption Bed/Column}

Absorption beds and columns would provide some treatment value, but would generate large volumes of secondary waste. In comparison to other similar technologies in this grouping, it was evident that there was no clear advantage for the use of this technology, nor any substantiable reason for further consideration.

\subsubsection{Chemical Fixation}

Chemical fixation represents a means of stabilizing waste, and typically is utilized for sludge or sediment disposal. Due to the volumes of waste that would be generated, fixation is not a technology to be considered for disposal of a large volume of liquid wastes (such as the LANL waste streams). However, chemical fixation does provide an attractive option for the disposal of secondary sludges and semi-solid wastes from other processes that were applicable for LANL. This technology warranted further consideration.

\subsubsection{Chemical Oxidation}

Chemical oxidation is used primarily for treatment of cyanide and of dilute solutions containing oxidizable organics. Chlorine, calcium or sodium hypochlorites, chlorine dioxide, hydrogen peroxide, and potassium permanganate are common oxidizing chemicals (NOTE: although ozonation may be considered a chemical oxidation process for this study, it was treated as a separate class of organic destruction process and is addressed in later discussions). This process would be inappropriate for the metallic salts in the LANL waste streams, which are unaffected to any appreciable degree by oxidation. This process was eliminated from further examination. 


\subsubsection{5}

Chemical Precipitation

Chemical precipitation is a widely-used technology for the removal of metals in aqueous wastes. Common precipitation agents include lime, caustic $(\mathrm{NaOH})$ solutions, and sulfide compounds. The process of chemical precipitation already has been demonstrated in the existing LANL liquid waste treatment facility as an effective unit within an overall treatment scheme. This process was selected as viable for further development.

\subsubsection{Chemical Reduction}

Chemical reduction is used primarily for treatment of wastes containing hexavalent chromium, mercury, and lead. Common reducing agents include sulfur dioxide, sulfite salts, and ferrous sulfate. These contaminants are not of primary concern for the treatment of LANL wastes. However, in a similar type of reduction reaction, certain oxidizer species such as nitrates $\left(\mathrm{NO}_{x}\right)$ can be eliminated by use of a basic oxidation/reduction action. This chemical process was determined to warrant additional consideration.

\subsubsection{Chlorination and Chlorinolysis}

Chlorine is used as a disinfecting agent in the form of free chlorine or as hypochlorites. In either form it acts as a potent oxidizing agent and often dissipates itself in side reactions so rapidly that little disinfection is accomplished until amounts in excess of the chlorine demand have been achieved. However, since bacteriological contamination is not a concern, chlorination was eliminated as a favored process. For these same reasons, chlorinolysis also was eliminated.

\subsubsection{Cyanide Destruction}

Cyanide destruction involves systems designed destroy the cyanidebearing salts (usually potassium or sodium cyanide) in wastes from -specialized processes, such as in the Plating Rinsewater Treatment Facility at the Oak Ridge Central Pollution Control Facility. However, since cyanides are not contaminants of concern at LANL, this technology is not applicable and was eliminated from further analysis.

\subsubsection{Ion Exchange}

Ion exchange water and wastewater treatment has become a useful technology for treatment of many specific ionic species. Typical ion exchange methods include sodium cycle, split stream, and 
demineralization. In instances where very pure (or ultrapure) effluent is required, ion exchange typically is a technology of choice. Ion exchange is most effective when dealing with a well-characterized waste stream of very consistent composition. For this reason, these units have been of preference in facilities such as the SRS Effluent Treatment Plant. However, it is important to consider that the composition of the influent waste stream may have a marked effect on the capacity and leakage frequency of the ion exchange resins. Also, the resins in ion exchange systems must be regenerated with acidic and basic solutions, creating a significant secondary waste stream. Perhaps the most important consideration is that using a simple technology such as the precipitation of radionuclides and heavy metals in the front end of the LANL process minimizes the usefulness and practicality of ion exchange as a 'polishing' process step. An additional factor is that ion exchange provides the most effective results when incorporated in a continuous, dynamic system rather than one that would require the continual startup and shutdown of the RLWTF batch process. Given these criteria, it would seem feasible to dismiss ion exchange from further consideration due to the availability of more simple and flexible technology, creation of significant secondary waste volumes, the uncertain nature of the waste streams to be processed, batch process concerns, and unnecessary treatment/decontamination that provides no obvious benefits for the considerable costs. Since the treatment of the LANL effluents will require reduction of contaminants to levels such as the Wildlife and Livestock Drinking regulations or the anticipated stringent NPDES requirements, the incorporation of ion exchange may be appropriate. This technology was carried forward for further consideration.

\subsubsection{Neutralization}

Neutralization (adjustment of the $\mathrm{pH}$ of the waste solution to neutral value by the addition of acidic or basic chemicals) may be considered an important element of the larger process of precipitation, flocculation, and clarification. For this reason, neutralization was carried forward in this analysis as a viable unit process.

\subsubsection{Ozonation}

Ozonation has been used extensively in recent years as a preferable alternative to chlorine for disinfection. The high oxidation potential of ozone makes it the equal of chlorine for destruction of bacteria, and it may be superior in the destruction of viruses. Detriments have been the relatively high capital and operating costs of ozonation systems, and the lack of residual protection. However, for use as an organic 
destruction agent, ozone is a unsurpassed as a treatment technology. Based on this evaluation, ozonation was considered worthy of further evaluation as an element of an organics destruction process.

\subsubsection{2 $\underline{\text { Photolysis }}$}

Photolysis is considered an exotic treatment for certain chemical species that are photoreactive. Since this does not apply to the LANL wastes, this technology was eliminated.

\subsubsection{Ultraviolet Irradiation}

UV irradiation has been considered an accepted alternative for disinfection and organic destruction. The process is simple in principle, involving the exposure of the waste stream to UV light sources that radiate energy in the wavelength range of 2500 to 2600 angstroms. Since this requires no chemical addition (similar to ozone), there is no secondary waste. A method that is commonly used in current waste treatment is to combine UV irradiation and ozonation units into an organic destruction unit, which provides superior combined destruction of oxidizable and sensitive organics. With this combined system as a consideration, UV irradiation was selected as a technology for further analysis.

\subsubsection{Summary of Technology Evaluations}

Within the category of chemical treatment, the technologies and unit processes that were eliminated as not applicable or clearly not an optimal choice were absorption mound/fields, absorption bed/columns, chemical oxidation, chlorination, chlorinolysis, cyanide destruction, and photolysis. The processes that were retained for further consideration were chemical fixation, chemical reduction, chemical precipitation, ion exchange, neutralization, ozonation, and UV irradiation.

\subsubsection{Physical Treatment - Separation of Components}

The separation of chemical components by physical treatment utilizes the physical properties of the materials in the waste as a means of separating unwanted components from the waste stream. Typically processes and/or actions are involved that accentuate the physical difference in substances to be separated, making the process of separation easier. Physical separation processes are very effective for the treatment of waste streams such as the TA-50 and Room 60/TA-55 wastes. For this reason, each generic process in this category was analyzed and evaluated for practical application to the 
treatment of LANL wastes. Each of the identified processes were addressed individually for consideration.

\subsubsection{1 $\quad$ Centrifugation}

Centrifugation is utilized as a method for separation of materials of differing densities by use of centrifugal force. For example, centrifuges have been used effectively for oil/water separation. In theory, centrifuges can be used for sludge thickening, but high costs and maintenance requirements have limited their use in this application. The use of centrifuge units typically has been in applications where space is limited or when other sludge thickening methods are ineffective. Since oil/water separation is not an issue for LANL wastes, and space would not be limited for more effective sludge thickening or dewatering systems, centrifuges were not included in the further consideration of technologies.

\subsubsection{2 $\quad$ Clarification}

Clarification is an element of the integrated precipitation, flocculation, and sedimentation/clarification process which involves the removal of particulate matter, chemical floc, and precipitates from suspension via gravity settling. In a typical application, a liquid process stream is mixed with flocculating agents and coagulants, and is fed into a mixing and settling chamber. Solid particles accumulate and increase in size and weight and subsequently, the floc is allowed to settle. Clarification occurs when the particulates settle out of the feed solution, leaving a clarified supernate. Contemporary systems integrate the processes of mixing, flocculation, and setting into a single unit referred to as a solids contact unit. This technology is attractive because of its simplicity, low cost, low maintenance requirements, and reliability. Since this technology already has been demonstrated to be effective for partial treatment of the LANL waste streams, the clarification process was included in unit processes warranting further consideration.

\subsubsection{Coagulation}

As with clarification, coagulation also is an integral part of the unit process involving settling of particulate matter. Coagulation involves the chemical process in which particles in solution are induced to combine into larger particles by the addition to the feed solution of a coagulation-inducing agent (commonly referred to as a 'coagulant aid'). This process increases the particle size of suspended solids, thereby increasing their settling velocity. The larger particles settle out by gravity more readily, providing a clearer supernate. Since this process 
step is instrumental to the overall clarification process that already has been selected as having potential for further consideration, the coagulation process also was selected.

\subsubsection{Decanting}

Decanting is simply the action of removing the supernate from a clarified liquid in the clarification process. This process step also was selected to be carried forth as a possible process for further evaluation.

\subsubsection{Encapsulation}

Encapsulation is usually the formation of a capsule, a small descrite volume of one material encased in another to seal the first material off from the environs. This is a specialized technology that typically is employed for the disposal of solid or semi-solid wastes by sealing the wastes (or mixture of wastes with an inert stabilizing media) in a capsular containment system or unit. This process usually is utilized for wastes of higher specific activity than the sludges and filtrants expected from the LANL process, and more basic (and less costly) processes will serve to manage the secondary wastes equally well. For these reasons, encapsulation was deleted from the list of technologies under consideration.

\subsubsection{Filtration}

When the removal of low levels of suspended solids is required, filtration provides a proven, reliable, and effective technology. Filtration involves the removal of suspended solids by passing a liquid or liquified waste stream through a bed of porous material. The solids are removed by a screening action of the media, or entrapment within the pores of the media. The media may be contained in an gravityflow container or an enclosed pressurized vessel.

A typical gravity filtration process may consist of a common underdrain collection and backwash distribution system, a layer of graded, coarse supporting medium, and a layer of filter medium. Types of filter media include clean, uniform-sized sand and anthracite coal. Dual media filters may be used, where a top coarser medium allows deep bed filtration and an increase in filtration capacity.

In applications where the bulk of the filter media is reused, provisions must be made for cleaning the media by backwashing. However, a significant design consideration is the treatment of filter backwash water which may contain high concentrations of contaminants, and the 
disposal of expended filter material. Given these considerations, and understanding how effective filtration can be in conjunction with other processes proposed (e.g., clarification, sludge dewatering) for further consideration for the LANL wastes, filtration was considered to be a viable basic technology for further assessment.

\subsubsection{Flocculation}

As mentioned with the discussion on clarification, flocculation is an integral part of the clarification process. This unit step in gravity separation is included in the technology slated for further consideration.

\subsubsection{8 $\quad$ Flotation}

Flotation is a density separation process based on the use of several stages of controlled water elutriation, heavy liquids, and dense media. This technology is used for processes such as nonferrous metal recovery, but has no application to the LANL waste treatment requirements. This process was deleted from further consideration.

\subsubsection{Foaming}

Foaming, or creating density separation by foam formation, is used in industrial processes such as materials recovery, but has no applicability to the waste treatment schemes required for LANL. Other than inoperability, the process of creating the foam could induce the aerosolization of radioactive particles, which would become a radiological protection concern. This technology was eliminated from the list of potential processes.

\subsubsection{0 $\quad$ Sedimentation}

As mentioned in the discussion on clarification, sedimentation is a fundamental step in the gravity separation process. This process step was included in the processes to be analyzed further for applicability to the LANL treatment requirements.

\subsubsection{Thickening}

Thickening is the process of water removal from sludges and/or process bottoms by any of a number of physical processes, including filtration and drying. Since filtration already has been selected as a technology for further consideration, thickening follows as a process step that also will be evaluated. 


\subsubsection{Ultrafiltration}

Ultrafiltration is one of many subgroups of the process of filtration. This process is typically used to remove and concentrate low initial concentrations of colloidal particals. In tube ultrafiltration systems, process liquid is forced through tubular filter media, leaving a concentrated reject stream of significantly lesser volume. However, this technology is not required for the particular species in the LANL wastes, since the particulate loading can be reduced easily by common process filtration. There is no significant gain to be achieved by use of this process, so that ultrafiltration was not selected for further consideration. Also, other advanced technology filtration systems such as cross-flow filters were eliminated on the basis of inappropriate application for this particular waste stream. However since ultrafiltration type technologies offer the benefit of reduced secondary waste production, it is recommended that additional efforts be conducted to determine whether the technology should warrant additional consideration.

\subsubsection{Summary of Technology Evaluations}

From the category of physical treatment/separation processes, the technologies and unit processes that were eliminated as not applicable or clearly not an optimal choice were centrifugation, encapsulation, flotation, foaming, and ultrafiltration. The processes that were retained for further consideration were clarification, coagulation, decanting, filtration, flocculation, sedimentation, and thickening.

\subsubsection{Physical Treatment - Removal of Specific Components}

As discussed in the preceding section, separation of chemical components by physical treatment utilizes the physical properties of the chemicals in the waste as a means of separating unwanted components from the waste stream. Evaluation of the applicability of these processes to the LANL treatment requirements involved the analysis of each generic process in this category.

\subsubsection{1 $\quad$ Activated Carbon}

Certain organic compounds are resistant to conventional wastewater treatment methods at low concentrations. Activated carbon has an affinity for various organics, and is widely used for organic contaminant removal, especially in drinking water supply programs. However, the depleted carbon adsorption media comprises a large secondary waste disposal problem, especially since the carbon will absorb toxic and hazardous constituents. In order to avoid secondary waste disposal 
problems, alternate technology involving organic destruction by ozonation and UV irradiation is preferable for the LANL applications. Activated carbon was eliminated from further consideration.

\subsubsection{Blending}

Blending of waste streams with nonwaste streams to reduce the net concentration of wastes per unit volume no longer is an acceptable method of disposal with regulatory agencies. This process was not considered for LANL wastes.

\subsubsection{Catalysis}

Catalysis involves the use of catalytic agents to induce or speed up reactions that will destroy or alter the compounds of concern. However, there is no practical application of this technology that is effective for heavy metals or radionuclides. This process was eliminated from further consideration.

\subsubsection{Crystallization}

Crystallization utilizes crystal-forming processes for selective elimination of contaminant species. This method would have limited use for the LANL waste streams, since none of the primary contaminants can be separated selectively by this process to the level of decontamination required. Purification of waste water by freeze crystallization is under development, but the efficiencies and applications of such processes is, as yet, indeterminate. No further evaluation was afforded to this process.

\subsubsection{Distillation}

Referring to the production of clean water or waste water disposal, single stage distillation and evaporation are synonymous. Distillation is frequently used as a method for the production of portable water by evaporation of sea water by desalination. Primary systems and processes that commonly are utilized include long tube vertical, multistage flash, and vapor compression. This technique is very effective for reducing salt content to an acceptably low concentration. For the removal of trace waste constituents, however this technology reaches the point of diminishing returns, and may not be competitive with newer technologies for cleanup of low level contaminants. For high TDS levels and minimization of a highly concentrated waste stream distillation competes with the newer membrane processes. In desalinization, a 50 volume percent reject stream can be easily 
returned to the ocean, but a similar stream from waste clean up entails high disposal costs. Distillation serves its most useful function when recovery of the primary process stream is the object of the treatment. Evaporation provides similar benefits without the need to recovery the decontaminated process stream. This technology was considered appropriate for further consideration in the LANL applications.

\subsubsection{Electrodialysis}

Electrodialysis was developed originally as an alternative to distillation for desalination of salt water and for removing acids from water. This process uses specialized membranes and an applied electrical current to selectively remove ionic species against the osmotic gradient. As with distillation, this process is effective when a large population of ionic species is present in the process stream. Dissolved solids are not treated by chemical reaction, but rather are concentrated in a secondary waste stream by electrical separation.

Anion-permeable and cation-permeable membranes are utilized for electrodialysis. The membranes are assembled in stacks in alternating order, forming compartments between the membrane surfaces. Electrodes with the same area as the membranes are placed on opposite ends of each membrane. When a direct current potential is applied across the electrodes, the anionic and cationic species in the waste stream migrate toward the oppositely-charged electrode. The electrical driving force induces the ions to cross the membranes and become concentrated in the intermembrane compartments in a manner where alternate compartments contain the processed waste stream (effluent) and the concentrated secondary stream. Electrodialysis can concentrate up to $90 \%$ of the dissolved ions in a secondary stream comprising approximately $10 \%$ of the total process flow, leaving $90 \%$ of the waste process stream as a treated effluent from the electrodialysis unit.

In the electrodialysis process, the electrical requirements increase dramatically as the ionic concentration diminishes, making removal of trace contaminants very difficult and costly. Although electrodialysis is not as susceptible as is reverse osmosis to silica or carbonate fouling, all membrane technologies require some pretreatment for mitigation of fouling by certain contaminants. In addition the capital and annual costs for electrodialysis systems are comparable to those for reverse osmosis, with less expected maintenance and the volume of the reject stream could be less. Based on the overall technology evaluation and considering the tentative characterization of the waste streams and the release criteria which must be met, this process was deleted from the 
list of technologies to be considered for treatment of LANL wastes. However, since electrodialysis has some desirable benefits, it is recommended that additional efforts be made to determine whether this technology should warrant additional consideration, particularly if the feed or release criteria changes.

\subsubsection{Electrolysis}

Electrolysis utilizes electric current for the dissociation and collection of contaminant species. However, this technology suffers from the same energy requirements as electrodialysis, and was considered inappropriate for application in LANL waste treatment scenarios.

\subsubsection{Evaporation}

Evaporation involves the reduction of liquid volumes by conversion of the liquid phase to the gaseous phase by the application of thermal loading. A distinction is made between evaporation without condensation and distillation, where the vapors are condensed and recovered as a clean product stream. Evaporation can occur as a natural process, such as with an open evaporation pond, or as a mechanical process, with heating systems to expedite the phase conversion process. Since open waste ponds at DOE sites have received strong negative response from regulatory and special-interest agencies, mechanical evaporation systems provide a more attractive alternative for LANL applications. This treatment technology was selected for further consideration.

\subsubsection{High Gradient Magnetic Separation}

High gradient magnetic separation utilizes an induced magnetic field for the separation and concentration of contaminants susceptible to a magnetic gradient. Since this type of separation technology would have little application for the materials and concentrations involved with the LANL waste streams, this process was eliminated from further consideration.

\subsubsection{Leaching}

Leaching typically is applicable to the treatment of small amounts of contamination from solid wastes. This has no application for the LANL waste streams and was eliminated from the technology list. 


\subsubsection{Liquid-Liquid Extraction}

Liquid-liquid extraction involves the concentration of contaminants into a secondary liquid phase extraction solution by selective extraction from the process liquid stream. This technology is effective for certain contaminants, but is inappropriate to the mixtures found in the LANL waste streams. This process was eliminated from further consideration.

\subsubsection{Molecular Sieve Adsorption}

Molecular sieve absorption utilizes size segregation at a molecular level for waste decontamination. This is useful for elimination of small contaminant particles, and produces a high-quality effluent. However, with a large volume waste stream, fouling of the molecular sieve could become a problem. Also, other technologies exist that provide elimination of the wastes of concern more effectively and more efficiently, and at lower installation and maintenance costs. For these reasons, this technology was considered to be not applicable for the LANL study.

\subsubsection{Reverse Osmosis}

Reverse osmosis utilizes a semipermeable membrane system to concentrate contaminants into a reject stream, while removing dissolved solids from the principal wastewater flow. Osmosis is the tendency of a solvent, or weak solution, to diffuse across the wall of a semipermeable membrane into a solution of higher concentration. The pressure, or driving force, exerted by the solvent is osmotic pressure. By exerting an outside pressure greater than the osmotic pressure on the concentrated solution, solute can be forced through the membrane against the normal osmotic gradient (from the high concentration side to the low concentration side), or in the reverse of the normal osmosis event.

In most instances, the use of reverse osmosis to reduce concentrations of dissolved organic and inorganic solids is limited. In hazardous waste treatment, reverse osmosis is an alternative for removal of highly toxic contaminants (such as metals and pesticides) from low-volume waste streams. Extensive pretreatment is necessary to protect the unit from excessive suspended solids loading, extreme $\mathrm{pH}$ values, oils and grease, and other wastewater characteristics that might impede operation or damage the membrane. Also, problems involving significant levels of silica and possible membrane damage due to intermittent operations would have to be addressed. An even more restrictive consideration is that reverse osmosis systems function best when utilized in a 
continuous, dynamic process rather than an intermittent batch process because of concerns for membrane fouling and ion migration. Recirculation systems create additional problems including energy use and chemical consumption. Even with a number of reasons for the dismissal of this technology from further consideration, reverse osmosis presents a viable option for production of high quality process water, and was carried forward as an option for this study.

\subsubsection{Solvent Recovery}

The solvent recovery process has no application for LANL wastes. No solvents are used or involved in the current or planned processes.

\subsubsection{Stripping}

Stripping is a method of removing volatile components from a waste stream. In this process, a contaminated waste stream is mixed with air, and intimate contact causes a mass transfer process by which volatile contaminants are transferred to the gas phase. However, since volatile components are minimal in the LANL wastes, this technology was considered as not applicable for process scenarios to be considered.

\subsubsection{6 $\quad \underline{\text { Sand Filter }}$}

Sand filtration is another method of removal of suspended solids from a waste stream. However, with processes such as those that would be involved in predicted treatment scenarios for LANL wastes, sand filtration would be an unfavorable choice compared to other filtration technologies such as those previously discussed in this report. For this reason, sand filtration was eliminated from further consideration in favor of more advanced filtration methods.

\subsubsection{Summary of Technology Evaluations}

From the category of physical treatment process for removal of specific components, the technologies and unit processes that were eliminated as not applicable or clearly not an optimal choice were activated carbon, blending, catalysis, crystallization, distillation, electrodialysis, electrolysis, high gradient magnetic separation, leaching, liquid-liquid extraction, molecular sieve adsorption, reverse osmosis, solvent recovery, stripping, and sand filtration. The process from this grouping that was retained for further consideration was evaporation. 


\subsubsection{Sanitary Waste/Biological Treatment}

Biological processes, in most application, are aerobic treatment processes used for removal of organic matter. Proven process technology offers a wide variety of treatment schemes, such as activated sludge, aerobic lagoons, composting, septic tank/systems, spray irrigation, thickening filtration, trickling filtration, and waste stabilization ponds. However, due to the characteristics of the LANL wastes, none of these technologies can provide significant treatment potential. Biological processes as a group were eliminated from further consideration.

\subsubsection{Selection of Preferred Unit Processes}

Based on the composition and quantities of the existing waste streams and the assumed release restrictions, a number of functional technical requirements must be considered in order to conceptualize an appropriate treatment scheme:

1. It is necessary to significally reduce the concentration of certain soluble salts that are not precipitable. In the absence of a selective process for these specific salts, treatment would require the reduction of TDS.

2. The elevated silica levels in the influent waste stream requires that treatment must include silica removal or reduction prior to the concentration and removal of TDS by use of treatment technologies such as $\mathrm{R} / \mathrm{O}$. If the high silica content is not reduced prior to these units, there is a high likelihood that as the silicates are concentrated colloidal silica will foul the membranes involved in the process.

3. Silica contamination removed by typical precipitation processes, such as hot magnesium hydroxide precipitation, will most likely coprecipitate the alpha-emitting radionuclides. The radioactive components of the waste should be precipitated selectively (to the extent possible) to minimize the amount of TRU waste formed as a result of the process.

4. Based on information from Savannah River Site, the present levels of TDS in the existing LANL Plant effluent would generate approximately 1000 barrels/yr of waste if fixed in a saltstone matrix. This would be in addition to the present waste generation of filter cake and cement paste.

5. Proper operation of a contact process such as surface entrapment or adsorption requires a working volume of reasonable size and appropriate holdup times. An arbitrary holdup time of one hour was selected (to reduce short-circuiting of the process), with a feed rate of 
$100 \mathrm{gpm}$ and a recycle of $25 \mathrm{gpm}$ associated with a working volume of 7500 gallons. This would represent an appropriate size for a contact vessel.

6. At $100 \mathrm{gpm}$, the $20,500,000 \mathrm{~L} / \mathrm{yr}$ of influent can be processed in approximately 900 hours of operation. This represents less than half of the time of a normal operation period ( 8 hours/day, 5 days/week) but this type of operation is consistent with historic and present TA-50 operations.

It was necessary to analyze these criteria in order to make decisions regarding the type of operations to be performed and the acceptable technologies to support these operations. Some of the potential waste treatment technology processes work very well under a program of continuous operation, and with an influent with relatively consistent waste characteristics. Examples of these processes are some of the unit operations utilized at the SRS Effluent Treatment Plant, which include cross-flow filtration, reverse osmosis, and ion exchange.

The SRS ETF is a 24 hour/day, 7 day/week, 52 weeks/year operation that treats a waste stream of typically consistent characteristics. The process utilizes a large-volume upstream waste collection system to blend out any variations in waste composition and to equalize flow feed rates. However, these same unit processes which are so effective for SRS operations are poor technologies for use with a waste treatment process scheme where daily or even weekly shutdowns or interruptions of operations may occur. For example, if unit processes using membrane technology are not drained, flushed, and placed in a standby mode upon cessation of waste stream processing, the result usually will be membrane fouling. After consideration of these criteria, R/O was eliminated from further consideration as a preferred unit process.

Ion exchange units that are inactivated intermittently are subject to ionic migration and premature ion breakthrough or leakage. If it is a requirement to design an IX system for a finite level/concentration of ionic species in the waste stream which cannot be exceeded, then the system design must be based on the maximum expected influent concentrations rather than averages. The data on the LANL waste influent indicates a 4:1 ratio of the peak to average concentration on an annual basis for many significant ionic species, and as much as a 10:1 variation over the five years of data used as the basis for this study. Unless these wide variations in ion concentrations can be normalized (which might involve an additional waste characterization program of significant effort), a considerable margin of system overdesign would be required. These reasons led to elimination of ion exchange from the list of preferred unit processes for further evaluation. 
For similar reasons such as ion migration and potential for fouling, other more sophisticated technologies are not optimal for a batch process mode, and work better with a more dynamic process. Systems such as cross-flow filtration, $\mathrm{R} / \mathrm{O}$, and electrodialysis are not easily placed in a standby mode without additional considerations such as treatment and recirculation, which can be costly. These operations can be very labor-intensive, and are not consistent with a plan of operation that includes intermittent processing. Since maintenance and repairs can be considerable with minimum operability, these technologies must be dismissed as having potential, but being inappropriate for this application.

An additional consideration for these types of unit processes is the generation of significant secondary wastes, such as reject or regeneration streams. Even with these unit operations, it is very likely that approximately $20 \%$ of the waste stream will require treatment by evaporation. Also many supporting functions are required, such as pretreatment, backpulsing, recirculation, chemical cleaning, antifouling and antibacterial treatment, backwashing, rinsing, and chemical regeneration. At only $1600 \mathrm{ppm}$ TDS, SRS recycles $32 \mathrm{gpm}$ by evaporation from the R/O rejects and IX regenerations (Table 2.2). If the inlet TDS increases the quantity of recycles will increase.

The simplest batch treatment plant involves the collecting of waste volumes or 'batches' in receiving tanks, and processing the waste volume in an evaporator system. However, this simple process is energy intensive and has the drawback that all of the solid wastes generated from the evaporation process would be TRU wastes, which would result in very large disposal costs. However, the evaporation technology in conjunction with other pretreatment processes could result in the most effective treatment scheme with respect to NPDES discharge restrictions, since very little contamination would remain in the discharge stream.

The current Room 60 operations on the TA-55 waste stream effectively remove and segregate the alpha-emitting radiological contaminants. This process is not selective for the radiological contaminants, but the total volume of secondary TRU waste is not excessive. This process operation can be utilized to treat the TA-50 influent to remove most of the radiological contamination from the wastes, allowing the remaining liquids to be evaporated. With this scheme, only the sludge from the precipitation process step would have the potential to be classified as TRU waste. The evaporator bottoms would be either nonradiological or (at most) low-level radiological wastes from remaining soluble radionuclides.

Since precipitation is a desirable first process step for removal of radiological contamination regardless of subsequent treatment, the technology decision is focused on the treatment of the decant or supernate from the precipitation 
reaction. The requirement to comply with stringent environmental regulations would narrow the choices to two primary technologies. These are the combination of $\mathrm{R} / \mathrm{O}$ and ion exchange (with approximately $20 \%$ evaporation), and evaporation of $100 \%$ of the supernate. After weighing the advantages and disadvantages of short-term intermittent batch processing runs versus the requirements for continuous operation, the scheme using total evaporation becomes more favorable.

After elimination of the most unlikely treatment processes, the remaining basic treatment technologies for the TA-50 and TA-55 waste streams that were considered relevant, appropriate, feasible, viable, reliable, operable, and maintainable included chemical fixation, chemical reduction, chemical precipitation, neutralization, ozonation, UV irradiation, clarification, coagulation, decanting, filtration, flocculation, sedimentation, thickening, and evaporation. Once compiled into operable systems, these unit processes yielded five basic treatment schemes to be utilized for the development and analysis of the final Candidate Technologies to be reviewed in depth. These five processes were Nitrate Removal (by chemical reduction), Gravimetric Physical Separation (which includes the integrated processes of neutralization, chemical precipitation, coagulation, flocculation, sedimentation, clarification, decanting, and filtration), Evaporation, Organics Destruction (by use of combined ozonation and UV irradiation), and Solids/Sludge Disposal (which includes thickening, filtration, and chemical fixation).

\subsubsection{Nitrate Removal}

Nitrate removal would be conducted by the addition of reactive chemicals and the application of heat to convert and volatilize nitrogen-bearing chemical components. The removal of nitrates from the TA-55 waste stream is highly desirable, but is not required for the neat TA-50 waste stream, since nitrate levels in this stream are not excessive. In consideration of the entire treatment scheme, nitrate removal is not a mandatory treatment process for operability, but is a consideration for compliance with strict regulations and for secondary waste volume reduction. It will be necessary to segregate and fix the TDS from the waste stream. Nitrate removal will reduce the TDS to be removed and fixed by almost $50 \%$.

The adjustment of the $\mathrm{pH}$ by use of a strong acid such as sulfuric acid will serve to eliminate ammonia-form nitrogen $\left(\mathrm{NH}_{3}\right)$ on the back end of the process, prior to evaporation. 
The combined processes of neutralization, chemical precipitation, coagulation, flocculation, sedimentation, clarification, decanting, and filtration are required to remove the alpha-emitting radionuclides from the TDS waste content in order to minimize the volume of TRU waste produced as a secondary waste stream.

\subsubsection{Organics Destruction}

Destruction or removal of organic compounds will be required to comply with release limits for COD. Destruction via the combination of ozonation and UV irradiation was selected based on principles of minimization of secondary wastes.

\subsubsection{Evaporation}

The evaporation of the final treated waste stream is an efficient and cost-effective means of reducing the TDS in the final effluent. This means of TDS reduction will ensure that the concentration of regulated contaminants in the final discharge will be below prescribed limits.

The main stream evaporator proposed for each of the Candidate Technologies is a mechanical vapor recompression type, that employees multiple tubes with a thin film flowing down the interior of each tube. Water is driven off as steam from the surface of this thin film in each tube and the concentrate discharges to a sump at the base of the evaporator. The concentrate is recirculated to the top of the evaporator, while the steam is compressed and circulated to the exterior surface of the tubes. Water condenses on the exterior of the tubes and is collected for discharge. The concentrate is continuously recirculated and the influent and waste rates are set to maintain predetermined TDS and TSS concentrations in the recirculated stream. The precipitating crystals, ie silica and other salts, that have an affinity for hard surfaces are left in the concentrate to provide a surface for the newly forming crystals to adhere to rather than sealing on the tubes.

This type of evaporator is common, efficient ( 4 to $5 \mathrm{lbs}$ or product per $\mathrm{lb}$ of steam) and has proven effective in preventing sealing on streams with silica levels similar to these anticipated at RLWTF. 


\subsubsection{Solids/Sludge Disposal}

The processing and handling of treatment sludges is a major concern for existing operations. The processes currently in service at TA-50 are not in alignment with current DOE and regulatory requirements for such operations.

It is anticipated that some form of stabilization/fixation will be required for all of the secondary wastes, solids, sludges, and concentrated salt solutions produced by the treatment processes. Based on evaluation of comparable processes and technologies in use, reduction of liquid volumes, or thickening, by filtration followed by the fixation of these secondary wastes in saltstone was selected as the preferred technology. However, if further information warrants additional evaluation, other selections may be considered accordingly.

\subsubsection{Suggested Additional Studies}

Since the selection of unit processes was conducted based on limited waste stream characterization and assumptions that were made to address indefinite parameters, the following studies (which should be conducted prior to or in conjunction with conceptual design) are suggested in order to specify the most effective and efficient unit processes for a proposed treatment scheme:

1. A detailed characterization of the expected constituents and concentrations of the waste streams to be processed by the RLWTF must be completed in order to optimize the processing configuration. The study under development by the LANL EM-8 group is intended to address these requirements.

In addition to the characterization, related activities that should be considered are the development of a formal sampling plan, analytical data collation, and independent data analysis and validation.

2. An analysis is required that will define the proportion of contaminant constituents that are present as dissolved solids, and those that are present as suspended solids. A study is currently in progress that is intended to provide the required information.

3. Bench-scale treatability studies must be conducted to evaluate and confirm the removal efficiencies of unit processes and the proposed process configuration.

Some of the necessary bench-scale studies currently are in progress. These include: 


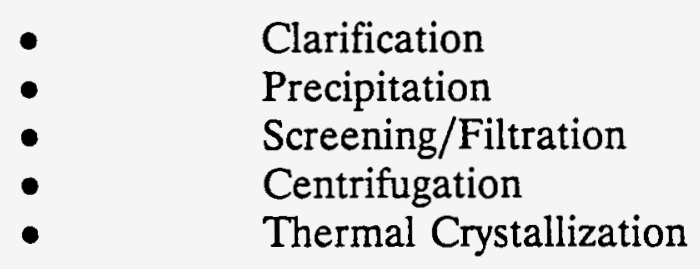

4. Larger-scale (pilot-scale or demonstration-level) tests will be required subsequent to the bench-scale studies in order to evaluate uncertainties associated with process scale-up factors.

5. Further investigation should be conducted regarding the unit treatment processes that were dismissed during this study as unfavorable or not optimum, but were not totally eliminated. These unit processes include $\mathrm{R} / \mathrm{O}$, ultrafiltration, ion exchange, freeze crystallization, centrifugation, thermal crystallization, and electrodialysis.

6. Consideration has been given to the process of precontacting or recontacting the influent waste stream with generated sludge, in what would amount to a counter-current operation similar to that currently utilized on the caustic wastes in Room 60. Some questions exist as to the amount of reduction of chemical usage and secondary with production that could be expected if such an operation were applied to the main TA-50 influent. It is not clear that adequate data exists to indicate how beneficial this process would be when applied to the TA-50 influent. In order to determine the applicability of this process, it is likely that bench-scale testing will be required.

\subsection{PROCESS CONFIGURATIONS}

The unit processes that were selected by the preliminary screening process were arranged into logical optimum working configurations which were responsive to the regulatory requirements imposed by the Wildlife and Livestock Watering regulations, and for the anticipated more stringent NPDES requirements to be required by NMED. These arrangements provide the basis for the Process Configurations (as depicted in the diagrams in Appendix B), and are represented by four basic groupings of 3 cases each:

- Individual treatment scenarios for the TA-50 (neat), the Room 60/TA-55, and blended waste streams that involve upgrading the existing facility and compliance with the Wildlife and Livestock Watering regulations

- Individual treatment scenarios for the TA-50 (neat), the Room 60/TA-55, and blended waste streams that involve the construction of a new treatment facility and compliance with the Wildlife and Livestock Watering regulations 
- Individual treatment scenarios for the TA-50 (neat), the Room 60/TA-55, and blended waste streams that involve the construction of a new treatment facility and compliance with anticipated stringent NPDES release requirements by NMED

- Individual treatment scenarios for the TA-50 (neat), the Room 60/TA-55, and blended waste streams that involve upgrading the existing facility and compliance with anticipated stringent NPDES release requirements by NMED

\subsubsection{Evaluation of TA-55 Treatment Options}

The design basis liquid waste volume for the TA-55 operations is based on the data of the past five years, and is estimated at 250,000 liters per year. At this low-volume rate, any proposed process would be measurably more effective if conducted in a batch mode, where a quantity of waste is collected prior to treatment. This also would serve to homogenize the waste batches, and eliminate transient processing problems due to irregular composition of waste influent.

The primary treatment concerns for the TA-55 waste stream are $\mathrm{pH}$ (acidic and caustic waste constituents), alpha-emitting radionuclides, and TDS. The annual averages of the particular contaminant species in the influent have been relatively consistent over the past five-year period, although there are indications that variance in composition is possible for any given waste batch. Prescribed unit processes must have the capacity to treat the possible range of contaminants.

As presented in the discussion in Section 3.1, the unit processes that are considered to be most appropriate for treatment of the specific contaminants in a batch treatment mode are discussed below.

1. Nitrogen control by chemical reduction, which would be used to remove nitrates (as an oxidizer species) by means of a basic oxidation/reduction action. This chemical process was determined to warrant additional consideration. Since a batch process mode is required, it was determined that chemical reduction would offer superior performance to biological nitrogen control systems.

Gaseous components of the reaction process would be managed by an off-gas treatment system.

2. Neutralization, which would involve the blending of acidic and caustic influent waste streams in a reaction vessel/tank, and further chemical adjustment of the $\mathrm{pH}$ as necessary to achieve a neutral $\mathrm{pH}$ waste solution. 
3. Gravimetric physical separation, which will include flocculation, coagulation, flocculation, precipitation, and sedimentation of the radionuclides and TSS contaminants, and subsequent clarification and decanting of the primary waste stream.

4. Filtration, for final removal of filterable solids in the primary waste stream, and a second filtration system for sludge thickening and dewatering.

5. Evaporation, with sequential condensation, for reduction of contaminants in the primary waste stream to levels that will comply with strict environmental standards.

6. Solids/sludge disposal, which includes thickening, filtration, and chemical fixation. Fixation would occur in conjunction with two process stages: 1) to fix the sediments from the Gravimetric Physical Separation process into a TRU waste cement paste; and 2) to solidify the evaporator bottoms (which should not contain sufficient activity to warrant handling as a TRU waste) as saltstone.

\subsubsection{Evaluation of TA-50 Treatment Options}

As with the TA-55 waste stream, the design basis liquid waste volume for the TA-50 operations is based on the data of the past five years, and is estimated at $20,500,000$ liters per year. At this rate, a process volume capacity of 250 gpm should be adequate to accommodate any fluctuations in waste handling requirements that could be anticipated. This is considering an estimated condition of approximately half-time for a 40-hour work week. At this operating level, the average flow rate for TA-50 would be approximately 86 gpm (325 liters/min.).

The primary treatment concerns for the TA-50 waste streams (without the TA-55 blending) are heavy metals, a small quantity of alpha-emitting radionuclides, and TDS. The annual averages of the particular contaminant species in the influent have been relatively consistent over the past five-year period. Prescribed unit processes must have the capacity to treat these contaminants and volumes.

Occasional variations in composition can be managed by the use of front-end collection tanks. Typical current operations involve collecting of a volume of waste in the Waste Holding Tanks, processing the accumulated waste volume on a batch/continuous flow basis, and then placing the facility in standby awaiting the accumulation of the next batch. This same method of equalization would be utilized in any process upgrade scenario. 
As presented in the discussion in Section 3.1, the unit processes that are considered to be most appropriate for treatment of the specific contaminants at the specified volumes in a batch treatment mode are:

1. Gravimetric physical separation, which will involve neutralization of possible $\mathrm{pH}$ variations, flocculation, coagulation, flocculation, precipitation, and sedimentation of the metals, radionuclides, and TDS contaminants, and clarification and decanting of the primary waste stream.

2. Filtration, for final removal of filterable solids in the primary waste stream, and a second filtration system for sludge thickening and dewatering.

3. Evaporation, with sequential condensation, for reduction of contaminants in the primary waste stream to levels that will comply with strict environmental standards.

4. Organics destruction by use of combined ozonation and UV irradiation to remove residual organic compounds and/or COD contaminants.

5. Solids/sludge disposal, which includes thickening, filtration, and chemical fixation.

\subsubsection{Evaluation of Blended Waste Treatment Options}

The blending of the TA-55 and TA-50 waste streams offers the option of economizing on equipment and construction costs by utilizing common unit processes where feasible. However, based on the DOE directives to minimize production of TRU wastes, pretreatment of the TA-55 waste stream to remove radionuclides is considered to be an indispensable part of the treatment scenario. Therefore, the blending of wastes would occur after the pretreatment of the TA-55 waste stream for radionuclide removal, but would eliminate the need for duplicate evaporation systems.

An argument can be made for the introduction of the TA-55 waste stream both at the influent of the TA-50 process, or as a tie-in prior to the evaporation unit process. However, since the volume of the TA-55 waste stream is small and further decontamination can be achieved (if needed) by utilizing the entire TA- 50 process, it was decided to route the pretreated TA-55 waste stream to the TA-50 influent.

Since the TA-55 waste stream would comprise slightly more than $1 \%$ of the total combined waste influent to TA-50, the maximum process volume 
throughput capacity of $250 \mathrm{gpm}$ would be more than adequate to accommodate this additional volume.

Based on the evaluation of the separated treatment options for the TA-55 and TA-50 waste streams (Sections 3.1.1 and 3.1.2), the combined unit processes that would be required are:

1. Pretreatment of the TA-55 waste, which would include nitrogen control by chemical reduction, neutralization, gravimetric physical separation, filtration, and chemical fixation of the sediments from the gravimetric physical separation process.

2. Blending of the TA-55 and TA-50 waste streams in holding tanks.

3. Gravimetric physical separation.

4. Filtration, for final removal of filterable solids in the primary waste stream, and a second filtration system for sludge thickening and dewatering.

5. Evaporation, with sequential condensation.

6. Organics destruction.

7. Solids/sludge disposal, which includes thickening, filtration, and chemical fixation of the evaporator bottoms.

\subsubsection{Development of Treatment Process Configurations}

Based on the consideration of feasible process units and configurations that had the capability to provide the required treatment results, the following list of alternative arrangement of unit processes, or Process Configurations, was developed:

1. Process Configuration \#1: Upgrade Existing TA-50 Operations, TA-50 Waste Only - Wildlife and Livestock Watering Release Limits. This would involve retrofit/upgrading of the existing TA-50 unit processes for gravimetric physical separation and filtration, as well as the addition of new systems for evaporation, organics destruction, and solids/sludge disposal for the clarifier solids and the evaporator bottoms.

2. Process Configuration \#2: Upgrade Existing Room 60 Operations, TA-55 Waste Only - Wildlife and Livestock Watering Release Limits. This would include retrofit and upgrading of the existing 
TA-55/Room 60 unit processes for neutralization, gravimetric physical separation, filtration, and solids/sludge disposal for the clarifier, with the addition of a new front-end unit process for nitrogen control by chemical reduction and the addition of new final treatment unit processes for evaporation and solids/sludge disposal for the evaporator bottoms.

3. Process Configuration \#3: Upgrade Existing TA-50 Operations, Blended TA-55 and TA-50 Wastes - Wildlife and Livestock Watering Release Limits. For this scenario, actions would include retrofit and upgrading of the existing TA-55/Room 60 unit processes for neutralization, gravimetric physical separation, filtration, and solids/sludge disposal for the clarifier, with the addition of a new front-end unit process for nitrogen control by chemical reduction. The treated TA-55 waste stream would be routed to the influent of the TA-50 operations. Modifications to the TA-50 operations would include retrofit/upgrading of the existing unit processes for gravimetric physical separation and filtration, as well as the addition of new systems for evaporation, organics destruction, and solids/sludge disposal for the clarifier solids and the evaporator bottoms.

4. Process Configuration \#4: New Facility, TA-50 Waste Only - Wildlife and Livestock Watering Release Limits. This scenario involves the construction of a totally separate new facility for the management of the TA-50 waste stream. The unit processes involved in the treatment scheme are neutralization, physical separation by sludge contacting, gravimetric physical separation (via use of a flash mixer, flocculator and a lamella plate clarifier), evaporation (using a two-part process with a mechanical vapor recompression evaporator with cooler, and a thin-film finishing evaporator/compressor for the concentrate from the first evaporator), and organics destruction. Solids and sludge disposal will include dewatering, thickening, and concentrating the sediments from the clarifier, mixing with the bottoms from the evaporation unit, and fixation in solid form.

5. Process Configuration \#5: New Facility, TA-55 Waste Only - Wildlife and Livestock Watering Release Limits. This involves the construction of a totally separate new facility for the management of the TA-55 waste stream. The unit processes involved in the proposed process are Nitrate Destruction (for the acidic influent stream), neutralization, physical separation by sludge contacting, gravimetric physical separation, evaporation (using a two-part process with a mechanical vapor recompression evaporator with cooler, and a thin-film finishing evaporator/compressor for the concentrate from the first evaporator), and organics destruction. Solids and sludge disposal will include 
dewatering, thickening, and concentrating the sediments from the clarifier, mixing with the bottoms from the evaporation unit, and fixation in solid form.

6. Process Configuration \#6: New Facility, Blended TA-55 and TA-50 Wastes - Wildlife and Livestock Watering Release Limits. This configuration would entail a new facility that combines the general features discussed in Process Configurations \#5 and \#6. The TA-55 acidic waste stream would be treated by a nitrate destruction process, and combined with the TA-55 caustic waste stream in a neutralization process. The neutralized TA-55 waste stream would be combined with the TA-50 waste stream, which would be routed through the unit processes of physical separation by sludge contacting, gravimetric physical separation, evaporation (using a two-part process with a mechanical vapor recompression evaporator with cooler, and a thin-film finishing evaporator/compressor for the concentrate from the first evaporator), and organics destruction. Solids and sludge disposal will include dewatering, thickening, and concentrating the sediments from the clarifier, mixing with the bottoms from the evaporation unit, and fixation in solid form.

7. Process Configuration \#7: New Facility, TA-50 Waste Only September 11, 1992 NMED Release Limits. For this scenario, the final effluent from the processing of the TA-50 waste stream would be required to comply with release standards more stringent than those for Wildlife and Livestock watering. To ensure that there was no opportunity for exceeding the most stringent limits conceivable, this process scenario assumed a zero release criterion, and configured unit processes to meet this requirement.

The basic unit processes for this configuration would be the same as that for the TA-50 Wildlife and Livestock watering scenario in Process Configuration \#4. The treatment scheme would involve neutralization, physical separation by sludge contacting, gravimetric physical separation, evaporation (using a two-part process with a mechanical vapor recompression evaporator with cooler, and a thin-film finishing evaporator/compressor for the concentrate from the first evaporator), and organics destruction. However, rather than discharging the effluent from the organics destruction unit to an NPDES outfall, an additional evaporation process would be involved using a solar pond. Settled solids from the pond would be routed back to the thin-film finishing evaporator. Solids and sludge disposal will include dewatering, thickening, and concentrating the sediments from the clarifier, mixing with the bottoms from the evaporation unit, and fixation in solid form. 
8. Process Configuration \#8: New Facility, TA-55 Waste Only September 11, 1992 NMED Release Limits. As with Process Configuration \#7, in this scheme the final effluent from the processing of the TA-55 waste stream would be required to comply with release standards more stringent than those for Wildlife and Livestock watering. A zero release scenario is proposed.

The basic unit processes for this configuration would be the same as that for the TA-55 Wildlife and Livestock watering scenario in Process Configuration \#5. the treatment scheme would involve nitrate destruction for the acidic influent, neutralization involving blending of the acidic and caustic TA-55 waste streams, physical separation by sludge contacting, gravimetric physical separation, evaporation (using a two-part process with a mechanical vapor recompression evaporator with cooler, and a thin-film finishing evaporator/compressor for the concentrate from the first evaporator), and organics destruction. The effluent from the organics destruction unit would be routed to a solar pond. Settled solids from the pond would be routed back to the thinfilm finishing evaporator. Solids and sludge disposal will include dewatering, thickening, and concentrating the sediments from the clarifier, mixing with the bottoms from the evaporation unit, and fixation in solid form.

9. Process Configuration \#9: New Facility, Blended TA-55 and TA-50 Wastes - September 11, 1992 NMED Release Limits. This configuration combines the zero release scenarios from Process Configurations \#7 and \#8 into a single blended process. The TA-55 acidic waste stream would be treated by a nitrate destruction process, and combined with the TA-55 caustic waste stream in a neutralization process. The neutralized TA-55 waste stream would be combined with the TA-50 waste stream, which would be routed through the unit processes of physical separation by sludge contacting, gravimetric physical separation, evaporation (using a two-part process with a mechanical vapor recompression evaporator with cooler, and a thinfilm finishing evaporator/compressor for the concentrate from the first evaporator), and organics destruction. The effluent from the organics destruction unit would be routed to a solar pond. Settled solids from the pond would be routed back to the thin-film finishing evaporator. Solids and sludge disposal will include dewatering, thickening, and concentrating the sediments from the clarifier, mixing with the bottoms from the evaporation unit, and fixation in solid form.

10. Process Configuration \#10: Upgrade Existing TA-50 Operations, TA-50 Waste Only - September 11, 1992 NMED Release Limits. As 
with Process Configuration \#1, this selection would involve retrofit/upgrading of the existing TA-50 unit processes for gravimetric physical separation and filtration, as well as the addition of new systems for evaporation (using a two-part process with a mechanical vapor recompression evaporator with cooler, and a thin-film finishing evaporator/compressor for the concentrate from the first evaporator) and organics destruction. The effluent from the organics destruction unit would be routed to a solar pond. Settled solids from the pond would be routed back to the thin-film finishing evaporator. Solids and sludge disposal will include dewatering, thickening, and concentrating the sediments from the clarifier, mixing with the bottoms from the evaporation unit, and fixation in solid form.

11. Process Configuration \#11: Upgrade Existing Room 60 Operations, TA-55 Waste Only - September 11, 1992 NMED Release Limits. As with Process Configuration \#2, this would include retrofit and upgrading of the existing TA-55/Room 60 unit processes for neutralization, gravimetric physical separation, filtration, and solids/sludge disposal for the clarifier, with the addition of a new frontend unit process for nitrogen control by chemical reduction and the addition of new systems for evaporation (using a two-part process with a mechanical vapor recompression evaporator with cooler, and a thinfilm finishing evaporator/compressor for the concentrate from the first evaporator) and organics destruction. The effluent from the organics destruction unit would be routed to a solar pond. Settled solids from the pond would be routed back to the thin-film finishing evaporator. Solids and sludge disposal will include dewatering, thickening, and concentrating the sediments from the clarifier, mixing with the bottoms from the evaporation unit, and fixation in solid form.

12. Process Configuration \#12: Upgrade Existing TA-50 operations, Blended TA-55 and TA-50 Wastes - September 11, 1992 NMED Release Limits. This scenario combines the treatment systems from Process Configurations \# 10 and \#11, in a manner similar to that in Process Configuration \#3. This would include retrofit and upgrading of the existing TA-55/Room 60 unit processes for neutralization, gravimetric physical separation, filtration, and solids/sludge disposal for the clarifier, with the addition of a new front-end unit process for nitrogen control by chemical reduction. The treated TA-55 waste stream would be routed to the influent of the TA-50 operations. Modifications to the TA-50 operations would include retrofit/upgrading of the existing unit processes for gravimetric physical separation and filtration, as well as the addition of new systems for evaporation (using a two-part process with a mechanical vapor recompression evaporator with cooler, and a thin-film finishing 
evaporator/compressor for the concentrate from the first evaporator) and organics destruction. The effluent from the organics destruction unit would be routed to a solar pond. Settled solids from the pond would be routed back to the thin-film finishing evaporator. Solids and sludge disposal will include dewatering, thickening, and concentrating the sediments from the clarifier, mixing with the bottoms from the evaporation unit, and fixation in solid form.

These twelve Process Configurations are depicted in the Block Flow Diagrams provided in Appendix B.

The Process Configurations represent the evaluation of the most feasible technology systems configurations that will provide compliance with the specified discharge limitations. The matrix presented as Table 3-1 indicates the general influent waste configuration and the release criteria used as the basis for comparison of treatment effectiveness. Of these twelve cases, those associated with the 'TA-50 blended waste stream' represent technologies for the treatment of the waste stream that currently are treated by existing operations. In the other options, the TA-55 waste stream is not blended with the neat TA-50 waste stream prior to treatment, but rather is treated as a separate waste stream.

In Process Configuration 1,4,7, and 10, the unblended (neat) TA-50 treatment process is addressed separately from the existing process of treating the two waste streams (the TA-50 influent with the Room 60/TA-55 components). In Process Configuration 2,5,8, and 11, the Room 60/TA-55 waste stream is treated as a separate concern. In Process Configuration 3, 6, 9, and 12, blending of the TA-55/Room 60 waste stream into the primary treatment process occurs after the radiological contamination from the TA-55 waste stream has been treated and removed. The effluent from this process is to be blended into the TA-50 process influent. The TA- 50 blended case is a combination of the TA-55 effluent after removal of radionuclides with the primary TA-50 influent stream.

\subsubsection{Additional Considerations for Process Configurations}

The twelve Process Configurations as specified were developed based on existing information, assumptions that were made to accommodate incomplete or unavailable information, comparison with existing schemes for similar facilities, knowledge of process mechanics, and interactions and agreements with LANL. Additional considerations that should be addressed within the development of conceptual design include: 
The caustic waste stream routed to Room 60 has the highest loading of alpha radioactivity, and an appreciable fraction of the alpha contamination may be expected to precipitate in insoluble hydroxide compounds by reaction with residual calcium hydroxide, and/or be adsorbed on other sludge particles. These particles would be eliminated via gravity separation by entrainment in the settling mass. However, it is not evident that removal by this process would eliminate contamination that would not be removed in the TA-50 clariflocculation process. Although the decontamination factors are large, a significant amount of radioactive materials are left in the process effluent. At the current level of $50 \mathrm{nCi} / \mathrm{L}$, an additional decontamination factor of 1667 would be required to meet the release level of $30 \mathrm{pCi} / \mathrm{L}$.

\section{Reconfiguration to Optimize Front-End Organics Destruction}

Detergents and other surfactants may be components of influent waste streams. These organic constituents could have significant effects on the types and orientation of the unit processes selected in the overall waste treatment scenario. If these organic contaminants are a valid concern, modifications will be required in the processing systems to accommodate and treat such compounds. Technologies that have been used in similar situations include specific filtration mechanisms, selective filtration materials (e.g., activated carbon), and $\mathrm{R} / \mathrm{O}$.

Consideration was given to the relocation of the proposed organics destruction unit to the front end of the process, thereby eliminating organics prior to the primary unit processes. This would represent a trade-off, since it would be expected that the organics destruction unit would have an easier duty if the process is utilized on the evaporator concentration immediately prior to discharge. All of the heavier organic molecules would remain in the evaporator bottoms either due to higher boiling points or to adhesion to the sludge solids. Locating the unit in this process position would be optimal for organics destruction for purposes of COD removal. If the unit is relocated upstream of the other unit processes, it is likely that this would improve removal of the radionuclides and heavy metals, the degree of precipitant formation and separation, and would reduce the likelihood of foaming (among other contributions). However, in the untreated waste stream, it is possible that complete organics removal would not be achieved, resulting in elevated discharge COD. Also, it would not be unreasonable to expect that the organic materials remaining in the waste stream as feed to the evaporator would remain in the evaporator bottoms rather than be carried over in the condensate. Based on this analysis, the only predictable benefit of relocating the organics destruction unit upstream would be the probability of a more efficient process operation versus an unknown (but most likely small) increase 
of COD in the discharge stream. Since this type of trade-off cannot readily be evaluated quantitatively on chemical theory principles, further testing is required to validate such reconfiguration of unit processes.

\section{Consideration of Electrodialysis}

Of particular interest would be further investigation into the utilization of electrodialysis technology. This technology was not selected in this evaluation of alternatives based on the nature of the planned process (e.g., batch processing) and the related problems with the intermittent startup, shutdown, and offline maintenance for such systems. It is possible, however, that under certain circumstances and operational parameters that electrodialysis could be a viable technical candidate.

In addition to concerns involving operational variability of electrodialysis systems, a question has been posed regarding the potential for reduction of effectiveness by the presence of elevated silica levels in the waste stream. Although the silica concentration level in the TA-50 bulk waste stream fluid is not expected to exceed the solubility level in the electrodialysis process, silica problems still may be expected at the predicted waste stream levels. The potential for these problems exists because in the process of diffusing certain electrochemical species through the membranes at the cell walls, a countercurrent diffusional gradient may be established at these cell walls that acts to increase the silica concentration in the area adjacent to the membrane, which is the most undesirable location. It is uncertain that the silica would precipitate and foul the membranes, but to establish this would require additional testing. However, in industrial practices, a typical process scheme may include treatment for silica removal upstream of the electrodialysis unit when treating waste streams with silica levels similar to those anticipated for TA-50 wastes. In addition, it is possible that elevated silica levels will create additional problems in situations where the electrodialysis unit is place in a hold/standby mode, especially if the wastewater stream is left in contact with the treatment unit membranes.

It would seem that the advantages of a technology such as electrodialysis warrant further investigations with equipment manufacturers, and possibly could include bench-scale/pilot-scale testing.

\section{Consideration of Ultrafiltration}

Ultrafiltration is a somewhat generic term for a pressure driven membrane liquid-solid separation process on a molecular scale. The membrane discriminates on the basis of molecular size, retaining feed solids larger than the membrane pores and allowing smaller solids to pass through. The term ultrafilter applies to a mechanical separation of solids down to a range of 
0.001 to 0.02 micron in size. The membrane is constructed to have the smallest pore size at the surface, where the liquid interface is to occur, and somewhat larger pores below in the substrate. This will permit any particles passing through the initial layer to be discharged with the filtrate and not retained in the substrate. The most common configuration of ultrafilters used on RLWTF type waste is the cross flow type. This configuration uses a tangential velocity to scour the membrane surface to prevent the solids from collecting in a layer at the surface during separation and plugging the membrane.

The SRS-ETF during early stages of design hypothesed the use of ultrafilters and even purchased pilot scale units for the now discontinued filter improvement program. The initial decision not to use ultrafilters at SRS was not based on their viability in the process, but on the opinion that the additional solids removal afforded by ultrafilters was not required in the overall process scheme and their increased cost relative to ceramic filters.

Therefore the use of ultrafilters at the RLWTF for the initial removal of precipitated solids should be considered as viable and merits further investigation. Additionally it should be noted that the key to this process lies in the selection of the membrane and to a lessor extent the equipment configuration. This selection needs to be based on a good characterization of the waste and bench/pilot scale testing, as expert opinions in this area of limited experience are not adequate by themselves to guarantee a successful application of this technology.

Some of the potential problems that may be encountered with the application of ultrafiltration at RLWTF are listed below:

1) Some of the solids formed by precipitation and organic solids in the waste stream may be in a gel like state and therefore may tend to smear on the surface of the membrane rather the remaining suspended in the flow stream. This will increase the require cleaning cycles and thereby introduce additional recycle into the plant.

2) The effects or organics and radiation on the membrane material will have to be considered as there may be a degradation of the membrane due to short or long term exposure to either or both.

3) Ultrafilters are better suited to a continuously operated type service in that after completion of each run the ultrafilter must be cleaned and placed in wet storage. This includes the addition of biological growth inhibitors to the solution storing the membrane. In addition at start-up the unit must be flushed to eliminate the biocide and readied for 
service. This reduces the available operating time of the units and also contributes to the secondary waste stream.

4) Both SRS and Hanford have found, with the use of microfiltration type units that are similar in principle to ultrafiltration, that additive chemicals are required to aid in filtering their wastes. SRS uses a 20-30 ppm of Aluminum Nitrate as a precipitant aid or body feed and Hanford anticipates use of a precoat. Therefore it can be reasonably assumed that this may also have to be applied at RLWTF for use of ultrafilters. This of course would increase the secondary waste load and must be consider in any economic analysis of an ultrafiltration unit.

5) The large amount of solids in the TA-55 supernate stream would seem to preclude the use of ultrafilters without some form of pretreatment for solids removed. Therefore while it may be practicle for the TA-50 stream or a blended stream, it does not seem practicable to use a two step approach to solids removal on the TA-55 stream.

The filtration subgroup of ultrafiltration, which includes cross flow filters, hollow fiber filters, and tubular precoat filters as well as ultrafiltration, has been successfully employed at other DOE sites and therefore needs to be investigated further. The selected process configuration of sedimentation followed by evaporation of the main stream, does not benefit from a further reduction of TSS prior to evaporation and the effluent stream from the filters would not be suitable for direct discharge. But the ultrafiltration process in conjunction with precipitation may offer a reduction of secondary wastes with an accompanying decrease in the flexibility to handle varied or changing waste streams. Please note this reduction in secondary waste may also produce another TRU waste stream and therefore may actually increase disposal costs even though the actual stream volume is less. Because of the potential savings in secondary waste production this type of treatment should be further investigated after characterization of the streams is complete. 
Table 3-1 LANL RLWP Waste Analysis Data Compiled for the Design Basis Waste Characterization

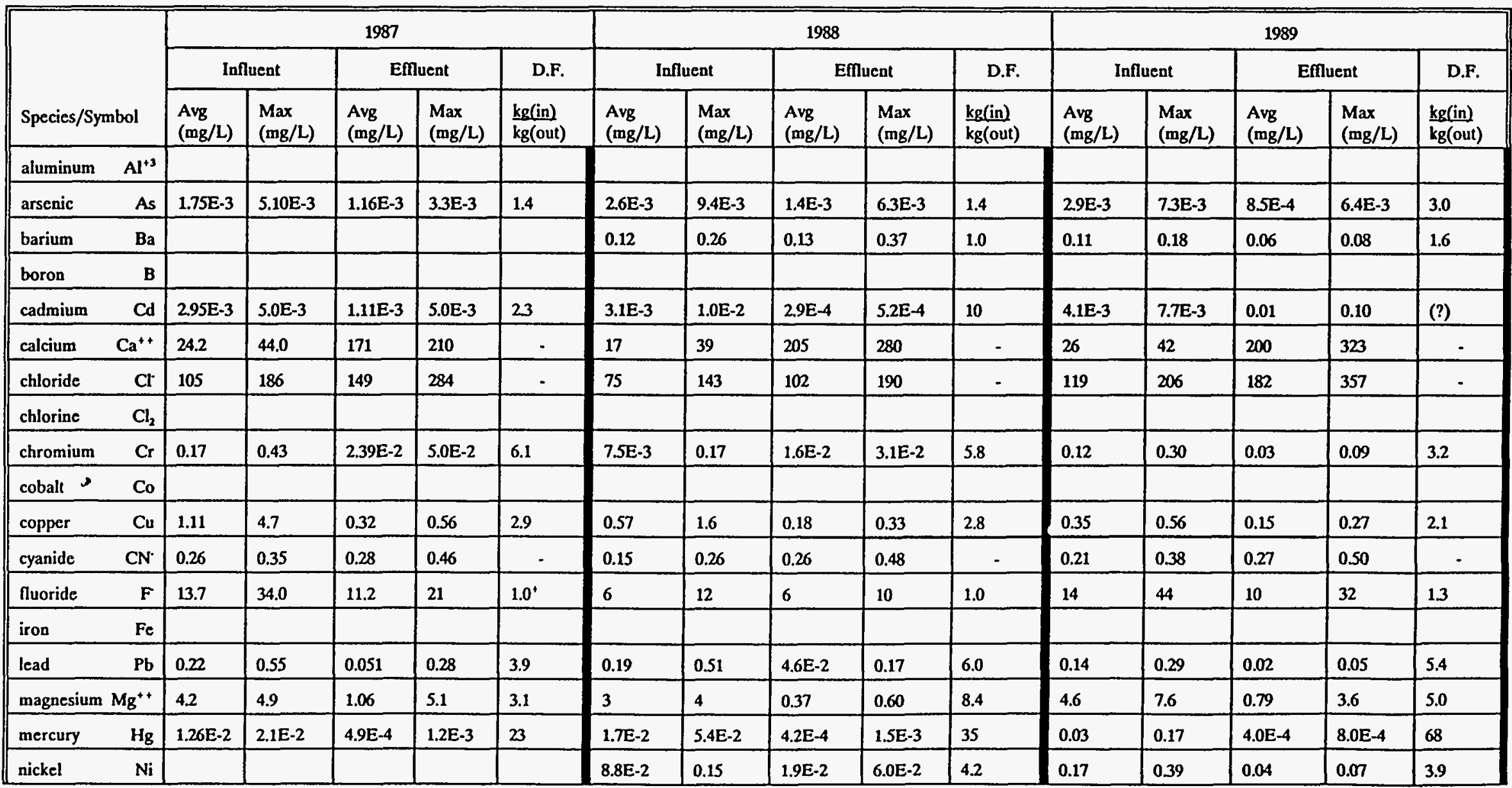


Table 3-1 LANL RLWP Waste Analysis Data Compiled for the Design Basis Waste Characterization (Cont)

\begin{tabular}{|c|c|c|c|c|c|c|c|c|c|c|c|c|c|c|c|}
\hline \multirow{3}{*}{$\begin{array}{c}-\cdot \\
\text { Species/Symbol }\end{array}$} & \multicolumn{5}{|c|}{1987} & \multicolumn{5}{|c|}{1988} & \multicolumn{5}{|c|}{1989} \\
\hline & \multicolumn{2}{|c|}{ Influent } & \multicolumn{2}{|c|}{ Effluent } & \multirow{2}{*}{$\begin{array}{c}\text { D.F. } \\
\frac{\mathrm{kg}_{\text {Q (in) }}}{\mathrm{kg} \text { (out) }} \\
\end{array}$} & \multicolumn{2}{|c|}{ Influent } & \multicolumn{2}{|c|}{ Emluent } & \multirow{2}{*}{$\frac{\text { D.F. }}{\frac{\mathrm{kg} \text { (in) }}{\mathrm{kg} \text { (out) }}}$} & \multicolumn{2}{|c|}{ Influent } & \multicolumn{2}{|c|}{ Effluent } & \multirow{2}{*}{$\begin{array}{c}\text { D.F. } \\
\mathrm{kg} \text { (in) } \\
\mathrm{kg} \text { (out) }\end{array}$} \\
\hline & $\begin{array}{c}\text { Avg } \\
(\mathrm{mg} / \mathrm{L})\end{array}$ & $\begin{array}{c}\operatorname{Max} \\
(\mathrm{mg} / \mathrm{L}) \\
\end{array}$ & $\begin{array}{c}\text { Avg } \\
(\mathrm{mg} / \mathrm{L})\end{array}$ & $\begin{array}{c}\operatorname{Max} \\
(\mathrm{mg} / \mathrm{L}) \\
\end{array}$ & & $\begin{array}{c}\text { Avg } \\
(\mathrm{mg} / \mathrm{L})\end{array}$ & $\begin{array}{c}\text { Max } \\
(\mathrm{mg} / \mathrm{L})\end{array}$ & $\begin{array}{c}\text { Avg } \\
(\mathrm{mg} / \mathrm{L})\end{array}$ & $\begin{array}{c}\mathrm{Max} \\
(\mathrm{mg} / \mathrm{L})\end{array}$ & & $\begin{array}{c}\text { Avg } \\
(\mathrm{mg} / \mathrm{L})\end{array}$ & $\begin{array}{c}\operatorname{Max} \\
(\mathrm{mg} / \mathrm{L}) \\
\end{array}$ & $\begin{array}{c}\text { Avg } \\
(\mathrm{mg} / \mathrm{L})\end{array}$ & $\begin{array}{c}\mathrm{Max} \\
(\mathrm{mg} / \mathrm{L}) \\
\end{array}$ & \\
\hline nitrogen as $\mathrm{NH}_{3}$ & 6.28 & 10.3 & 14.5 & 55 & - & 5 & 7 & 7 & 12 & - & 5.6 & 12 & 11.9 & 37 & - \\
\hline nitrogen as $\mathrm{NO}_{2}^{-}$ & 0.38 & 0.74 & 2.61 & 17.9 & - & 0.30 & 0.54 & 0.98 & 2 & - & 0.25 & 0.50 & 1.7 & 5.0 & - \\
\hline nitrogen & 267 & 415 & 476 & 1700 & - & 192 & 317 & 384 & 785 & - & 287 & 507 & 488 & 1280 & - \\
\hline phosphate $\mathrm{PO}_{4}^{*}$ & 13.3 & 124 & 1.51 & 12 & 7.5 & 3 & 11 & 0.24 & 0.39 & 9.0 & 5.8 & 18.5 & 0.29 & 0.62 & 17 \\
\hline potassium $\quad \mathbf{K}^{*}$ & 267 & 720 & 325 & 1200 & - & 123 & 270 & 137 & 190 & - & 168 & 310 & 164 & 330 & - \\
\hline sclenium & & 4.6E-3 & & $6.6 \mathrm{E}-3$ & $(?)$ & $9.8 \mathrm{E}-4$ & $2.7 E-3$ & $1.2 \mathrm{E}-3$ & $2.8 \mathrm{E}-3$ & $(?)$ & $1.7 \mathrm{E}-3$ & $8.5 \mathrm{E}-3$ & $4.9 \mathrm{E}-3$ & 0.03 & $(?)$ \\
\hline \multicolumn{16}{|l|}{ silica $\quad \mathrm{SiO}_{2}$} \\
\hline silver & & & & & & $28 \mathrm{E}-2$ & $6.2 \mathrm{E}-2$ & $5.4 \mathrm{E}-3$ & $1.0 \mathrm{E}-2$ & 4.3 & 0.02 & 0.05 & $8.2 \mathrm{E}-3$ & 0.03 & 2.3 \\
\hline sodium & 405 & 700 & 917 & 3400 & - & 246 & 460 & 693 & 1900 & - & 396 & 713 & 933 & 2260 & - \\
\hline sulfate $\quad \mathrm{SO}_{4}^{-}$ & 49.6 & 246 & 240 & 490 & - & 23 & 34 & 169 & 675 & - & 27 & 37 & 194 & 1180 & - \\
\hline \multicolumn{16}{|l|}{ vanadium $\quad \mathrm{V}$} \\
\hline zinc & & & & & & 0.33 & 0.53 & 8.1E-2 & 0.12 & 3.0 & 0.24 & 0.43 & 0.11 & 0.36 & 1.8 \\
\hline $\mathrm{pH}$ & & 11.9 & & 7.77 & - & & 9.23 & & 7.91 & - & & 10.1 & & 7.85 & - \\
\hline C.O.D. & 167 & 271 & 101 & 178 & 1.3 & 78 & 124 & 38 & 68 & 1.8 & 99 & 145 & 44 & 86 & 2.0 \\
\hline T.D.S. & 2140 & 3230 & 4150 & 13,000 & - & 1480 & 2330 & 3120 & 5140 & - & 2040 & 3120 & 4070 & 7960 & - \\
\hline T.S.S. & 59.1 & 167 & - & - & - & 33 & 95 & - & - & - & 34 & 99 & - & - & - \\
\hline
\end{tabular}


Table 3-1 LANL RLWP Waste Analysis Data Compiled for the Design Basis Waste Characterization (Cont)

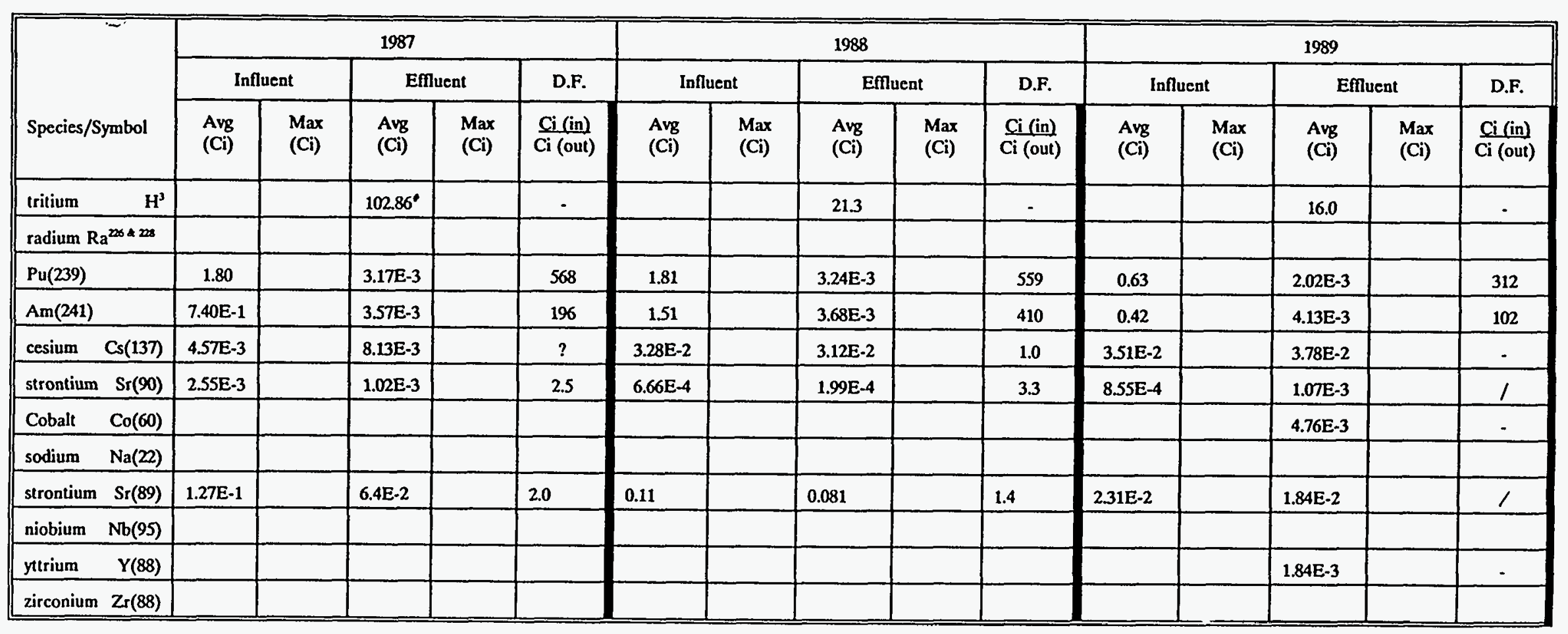

'known error due to non-representative sampling after caustic additions

- not used in averaging

() High Decontamination Factors (D.F.'s) possibly reflect changes in operations 
3

Table 3-1 LANL RLWP Waste Analysis Data Compiled for the Design Basis Waste Characterization (Cont)

\begin{tabular}{|c|c|c|c|c|c|c|c|c|c|c|c|c|}
\hline \multirow[b]{3}{*}{ Species/Symbol } & \multicolumn{5}{|c|}{1990} & \multicolumn{5}{|c|}{1991} & \multicolumn{2}{|c|}{ 5-yr average } \\
\hline & \multicolumn{2}{|c|}{ Influent } & \multicolumn{2}{|c|}{ Effluent } & \multirow{3}{*}{$\frac{\text { D.F. }}{\frac{\mathrm{kg}(\text { in })}{\mathrm{kg}(\text { out })}}$} & \multicolumn{2}{|c|}{ Influent } & \multicolumn{2}{|c|}{ Effluent } & \multirow{3}{*}{$\begin{array}{c}\text { D.F. } \\
\mathrm{kg} \text { (in) } \\
\mathrm{kg} \text { (out) }\end{array}$} & \multirow{3}{*}{$\begin{array}{l}\text { Em. } \\
(\mathrm{mg} / \mathrm{L})\end{array}$} & \multirow{2}{*}{$\begin{array}{l}\text { Current } \\
\text { D.F. }\end{array}$} \\
\hline & $\begin{array}{l}\text { Avg } \\
(\mathrm{mg} / \mathrm{L})\end{array}$ & $\begin{array}{l}\text { Max } \\
(\mathrm{mg} / \mathrm{L})\end{array}$ & $\begin{array}{l}\text { Avg } \\
(\mathrm{mg} / \mathrm{L})\end{array}$ & $\begin{array}{l}\text { Max } \\
(\mathrm{mg} / \mathrm{L})\end{array}$ & & $\begin{array}{l}\text { Avg } \\
\text { (mg/L) }\end{array}$ & $\begin{array}{l}\operatorname{Max} \\
(\mathrm{mg} / \mathrm{L})\end{array}$ & $\begin{array}{l}\text { Avg } \\
\text { (mg/L) } \\
\end{array}$ & $\begin{array}{l}\operatorname{Max} \\
(\mathrm{mg} / \mathrm{L})\end{array}$ & & & \\
\hline aluminum & & & & & & & & & & & & \\
\hline arsenic & $3.4 \mathrm{E}-3$ & $1.3 \mathrm{E}-2$ & $1.2 \mathrm{E}-3$ & 4.1E-3 & 2.0 & 3.7E-3 & 0.02 & $1.9 \mathrm{E}-3$ & $8.6 \mathrm{E}-3$ & $(20) ?$ & $13 \mathrm{E}-3$ & $1.4 / 3$ \\
\hline barium & 0.2 & 0.3 & 0.06 & 0.09 & 2.3 & 0.1 & 0.3 & 0.03 & 0.06 & 3.3 & 0.07 & $1 / 3$ \\
\hline boron & & & & & & & & & & & - & - \\
\hline cadmium & 0.02 & 0.2 & $4.3 E-4$ & $1.6 \mathrm{E}-3$ & 5.8 & 0.01 & 0.04 & $3.3 E-4$ & $20 \mathrm{E}-3$ & $(26) ?$ & $2.4 \mathrm{E}-03$ & $\sim 10$ \\
\hline calcium & 34 & 137 & 241 & 333 & - & 24 & 149 & 290 & 475 & - & 221 & - \\
\hline chloride & 100 & 263 & 97 & 167 & - & 74 & 179 & 82 & 113 & - & 122 & - \\
\hline chlorine & & & & & & & & & & & - & - \\
\hline chromium & 0.1 & 0.4 & 0.02 & 0.04 & 4.8 & 0.1 & 0.3 & $4.0 \mathrm{E}-3$ & $9.0 \mathrm{E}-3$ & (21) & 0.015 & $3 / 21$ \\
\hline cobalt & & & & & & & & & & & - & - \\
\hline copper & 1.0 & 8.2 & 0.2 & 0.3 & 4.6 & 1.2 & 6.1 & 0.2 & 0.3 & 5.5 & 0.17 & $2 / 5$ \\
\hline cyanide & 0.2 & 0.4 & 0.2 & 0.3 &. & 0.2 & 0.3 & 0.2 & 0.2 & . & 0.24 & . \\
\hline nuoride & 14.9 & 39 & 113 & 16.7 & 1.2 & 4.0 & 14 & 3.3 & 6.7 & 1.1 & 8.4 & - \\
\hline iron & & & & & & & & & & & & \\
\hline lead & 0.2 & 1.1 & 0.02 & 0.05 & 8.6 & 0.3 & 0.9 & $7.1 \mathrm{E}-3$ & 0.03 & (24) & 0.029 & $4 / 24$ \\
\hline magnesium $\mathrm{Mg}^{+*}$ & 4.8 & 23.5 & 6.3 & 44.3 & & 4.8 & 32 & 0.2 & 1.1 & $(21)$ & 1.7 & $3 / 21$ \\
\hline mercury & 0.01 & 0.03 & $3.6 \mathrm{E}-4$ & $2.2 \mathrm{E}-3$ & 21.7 & 0.01 & 0.03 & $1.6 \mathrm{E}-4$ & $4.5 \mathrm{E}-4$ & $(60)$ & $3.7 \mathrm{E}-4$ & $22 / 68$ \\
\hline nickel & 0.3 & 1.1 & 0.08 & 0.1 & 3.2 & 0.3 & 1.0 & 0.04 & 0.06 & 6.3 & 0.045 & $3 / 6$ \\
\hline nitrogen as $\mathrm{NH}_{3}$ & 8.0 & 42.1 & 73 & 15.8 & - & 63 & 15.9 & 6.9 & 10.9 & - & 11.5 & - \\
\hline
\end{tabular}


Table 3-1 LANL RLWP Waste Analysis Data Compiled for the Design Basis Waste Characterization (Cont)

\begin{tabular}{|c|c|c|c|c|c|c|c|c|c|c|c|c|c|}
\hline \multirow{3}{*}{\multicolumn{2}{|c|}{ Species/Symbol }} & \multicolumn{5}{|c|}{1990} & \multicolumn{5}{|c|}{1991} & \multicolumn{2}{|c|}{ 5-yr average } \\
\hline & & \multicolumn{2}{|c|}{ Influent } & \multicolumn{2}{|c|}{ Effluent } & \multirow{2}{*}{$\frac{\text { D.F. }}{\frac{\mathrm{kg} \text { (in) }}{\mathrm{kg}(\text { out })}}$} & \multicolumn{2}{|c|}{ Influent } & \multicolumn{2}{|c|}{ Efluent } & \multirow{2}{*}{$\begin{array}{c}\text { D.F. } \\
\frac{\mathrm{kg} \text { (in) }}{\mathrm{kg}(\text { out })}\end{array}$} & \multirow[b]{2}{*}{$\begin{array}{l}\text { Eft. } \\
(\mathrm{mg} / \mathrm{L})\end{array}$} & \multirow{2}{*}{$\begin{array}{l}\text { Current } \\
\text { D.F. }\end{array}$} \\
\hline & & $\begin{array}{l}\text { Avg } \\
(\mathrm{mg} / \mathrm{L})\end{array}$ & $\begin{array}{l}\mathrm{Max} \\
(\mathrm{mg} / \mathrm{L})\end{array}$ & $\begin{array}{l}\text { Avg } \\
(\mathrm{mg} / \mathrm{L}) \\
\end{array}$ & $\begin{array}{l}\operatorname{Max} \\
(\mathrm{mg} / \mathrm{L})\end{array}$ & & $\begin{array}{l}\text { Avg } \\
\text { (mg/L) }\end{array}$ & $\begin{array}{l}\operatorname{Max} \\
(\mathrm{mg} / \mathrm{L})\end{array}$ & $\begin{array}{l}\text { Avg } \\
(\mathrm{mg} / \mathrm{L})\end{array}$ & $\begin{array}{l}\operatorname{Max} \\
(\mathrm{mg} / \mathrm{L})\end{array}$ & & & \\
\hline \multicolumn{2}{|c|}{ nitrogen as $\mathrm{NO}_{2}$} & 0.09 & 0.5 & 0.6 & 1.6 & - & $1.7 \mathrm{E}-3$ & 0.02 & 0.3 & 0.7 & - & 3.9 & \\
\hline nitrogen & $\mathrm{NO}_{3}$ & 306 & 515 & 297 & 475 & - & 119 & 283 & 164 & 274 & - & 1603 & - \\
\hline phosphate & $\mathrm{PO}_{4}^{-1}$ & 4.4 & 13.6 & 0.2 & 0.4 & 17.8 & 5.7 & 14.3 & 0.9 & 6.6 & 6.6 & 0.63 & $6 / 18$ \\
\hline potassium & $\mathbf{K}^{+}$ & 148 & 244 & 161 & 296 & - & 34.4 & 98 & 51.3 & 108 & - & 168 & - \\
\hline selenium & Se & $1.8 \mathrm{E}-3$ & $6.1 \mathrm{E}-3$ & 2.1E-3 & $6.5 \mathrm{E} 3$ & (?) & $3.7 \mathrm{E} 3$ & 0.02 & $1.9 \mathrm{E}-3$ & $8.6 \mathrm{E}-3$ & (?) & $2.5 E-3$ & (?) \\
\hline silica & $\mathrm{SiO}_{2}$ & & & & & & & & & & & -70 & \\
\hline silver & $\mathrm{Ag}$ & 0.02 & 0.05 & $1.2 \mathrm{E}-3$ & $3.4 \mathrm{E}-3$ & 25 & 0.02 & 0.05 & $2.0 \mathrm{E}-3$ & $5.0 \mathrm{E}-3$ & 7.5 & $4.2 \mathrm{E}-3$ & $2 / 25$ \\
\hline sodium & $\mathrm{Na}^{+}$ & $1660^{\circ}$ & $9170^{\circ}$ & 591 & 918 & - & $2100^{\circ}$ & $6680^{\circ}$ & 397 & 766 & - & 706 & - \\
\hline sulfatc & So: $_{4}^{-1}$ & 39.9 & 12.1 & 194 & 501 & . & 323 & 101 & 128 & 288 & - & 185 & - \\
\hline vanadium & $\mathbf{v}$ & & & & & & & & & & & (?) & \\
\hline & $\mathrm{Zn}$ & 0.3 & 1.1 & 0.1 & 0.2 & 2.8 & 0.5 & 1.4 & 0.08 & 0.1 & 5 & 0.093 & $2 / 5$ \\
\hline $\mathrm{pH}$ & & & 13.2 & & 7.8 & - & & 13.2 & & 7.7 & - & & \\
\hline C.O.D. & & 116 & 210 & 33 & 76 & 3.2 & 110 & 166 & 29 & 51 & 35 & 49 & $1 / 4$ \\
\hline T.D.S. & & $3680^{\circ}$ & $11,100^{\prime}$ & 2550 & 3570 & - & $3570^{\circ}$ & $10,000^{\circ}$ & 1810 & 2370 & - & 3140 & \\
\hline T.S.S. & & 58.8 & 206 & - & - & - & 70.4 & 163 & - & - & - & 0 & \\
\hline
\end{tabular}


Table 3-1 LANL RLWP Waste Analysis Data Compiled for the Design Basis Waste Characterization (Cont)

\begin{tabular}{|c|c|c|c|c|c|c|c|c|c|c|c|c|}
\hline \multirow[b]{3}{*}{ Species/Symbol } & \multicolumn{5}{|c|}{1990} & \multicolumn{5}{|c|}{1991} & \multicolumn{2}{|c|}{ 5-yr average } \\
\hline & \multicolumn{2}{|c|}{ Influent } & \multicolumn{2}{|c|}{ Emuent } & \multirow{2}{*}{$\frac{\text { D.F. }}{\frac{\text { Cilin) }}{\text { Ci(out) }}}$} & \multicolumn{2}{|c|}{ Influent } & \multicolumn{2}{|c|}{ Effluent } & \multirow{2}{*}{$\begin{array}{l}\text { D.F. } \\
\frac{\text { Ci(in) }}{\text { Ci (out) }} \\
\end{array}$} & \multirow[b]{2}{*}{$\begin{array}{l}\text { Em. } \\
(\mathrm{C} i)\end{array}$} & \multirow[b]{2}{*}{$\begin{array}{l}\text { Current } \\
\text { D.F. }\end{array}$} \\
\hline & $\begin{array}{l}\text { Avg } \\
\text { (Ci) }\end{array}$ & $\begin{array}{l}\text { Max } \\
\text { (Ci) }\end{array}$ & $\begin{array}{l}\text { Avg } \\
\text { (Ci) }\end{array}$ & $\begin{array}{l}\text { Max } \\
\text { (Ci) }\end{array}$ & & $\begin{array}{l}\text { Avg } \\
\text { (Ci) }\end{array}$ & $\begin{array}{l}\text { Max } \\
\text { (Ci) }\end{array}$ & $\begin{array}{l}\text { Avg } \\
\text { (Ci) }\end{array}$ & $\begin{array}{l}\text { Max } \\
\text { (Ci) }\end{array}$ & & & \\
\hline tritium & & & 12.2 & & - & & & 10.60 & & - & $6.8 \times 10^{3}$ & \\
\hline \multicolumn{13}{|l|}{ radium $\mathrm{Ra}^{200 \times 2 a}$} \\
\hline Pu(239) & 0.58 & & $5.82 \mathrm{E}-4$ & & 1000 & 0.547 & & $9.41 \mathrm{E}-4$ & & 581 & $90.5 \times 10^{3}$ & 604 \\
\hline $\operatorname{Am}(241)$ & 0.19 & & $2.68 \mathrm{E}-3$ & & 71 & 0.185 & & $1.07 \mathrm{E}-3$ & & 173 & $138 \times 10^{3}$ & 190 \\
\hline cesium $\quad \operatorname{Cs}(137)$ & $1.1 \mathrm{E}-2$ & & $1.17 \mathrm{E}-2$ & & 1 & 0.114 & & $6.69 \mathrm{E}-2$ & & 1.7 & 1.42 & - \\
\hline strontium $\operatorname{Sr}(90)$ & $1.5 \mathrm{E}-3$ & & $2.92 \mathrm{E}-4$ & & 5 & 0.148 & & $8.06 \mathrm{E}-2$ & & 1.8 & 0.76 & $1 / 5$ \\
\hline$C_{0}(60)$ & & & $9.5 E-4$ & & - & & & - & & & & \\
\hline sodium $\quad \mathrm{Na}(22)$ & & & & & & & & & & & & \\
\hline strontium $\operatorname{Sr}(89)$ & 0.12 & & $7.26 \mathrm{E}-2$ & & 1.6 & $9.26 \mathrm{E}-3$ & & $5.16 \mathrm{E}-3$ & & 1.8 & & \\
\hline niobium $\mathrm{Nb}(95)$ & & & & & & $7.3 \mathrm{E}-2$ & & & & & & \\
\hline yttrium & & & $4.0 \mathrm{E}-4$ & & - & $7.5 \mathrm{E}-2$ & & $6.4 \mathrm{E}-4$ & & 117 & & \\
\hline zirconium $\operatorname{Zr}(88)$ & & & & & & $7.4 \mathrm{E}-2$ & & & & & & \\
\hline
\end{tabular}

known error due iu non-representative sampling after caustic additions

- not used in averaging

() High Decontamination Factors (D.F.'s) possibly reflect changes in operations 
TABLE 3.2 Influent Characteristics Compiled for BAT Study

\begin{tabular}{|c|c|c|c|}
\hline $\begin{array}{c}\text { Influent } \\
\text { Component }\end{array}$ & $\begin{array}{c}\text { TA-50-1 Influents } \\
\text { (Neat) } \\
\mathrm{mg} / \mathrm{L} \\
\end{array}$ & $\begin{array}{c}\text { TA-50-1 Influents } \\
\text { (Blended) } \\
\mathrm{mg} / \mathrm{L}\end{array}$ & $\begin{array}{c}\text { TA-55 Effluent } \\
\text { (Supernate) } \\
\text { mg/L }\end{array}$ \\
\hline As & $.002 / .003$ & $.002 / .003$ & 0.77 \\
\hline $\mathrm{Ba}$ & $<0.5$ & $<0.5$ & 0.2 \\
\hline $\mathrm{Cd}$ & $.005 / 0.10$ & $.005 / 0.10$ & 0 \\
\hline $\mathrm{Ca}$ & $24 / 40$ & 40 & 8.1 \\
\hline $\mathrm{Cl}$ & $30 / 84$ & $200 / 500$ & 8834 \\
\hline Total $\mathrm{Cr}$ & $.02 / 0.25$ & $.02 / 0.25$ & $0.44 / 45$ \\
\hline $\mathrm{Cu}$ & $0.15 / 0.30$ & $0.15 / 0.30$ & $<.003$ \\
\hline $\mathrm{CN}$ & $0.2 / 0.6$ & $0.2 / 0.6$ & 6.15 \\
\hline $\mathrm{F}$ & $1 / 8$ & $1 / 8$ & 350 \\
\hline $\mathrm{Fe}$ & $0.5 / 1.0$ & $0.5 / 1.0$ & .005 \\
\hline $\mathrm{Pb}$ & $<.01 / 0.2$ & $<0.01$ & 0.33 \\
\hline $\mathrm{Mg}$ & $3 / 4$ & $3 / 4$ & 0.57 \\
\hline $\mathrm{Hg}$ & $.0004 / .005$ & $.0004 / .005$ & .0035 \\
\hline $\mathrm{Ni}$ & $0.1 / 1.0$ & $0.1 / 1.0$ & $<.02 / 31$ \\
\hline $\mathrm{NH}_{3}-\mathrm{N}$ & $3 / 15$ & 10 & 4.6 \\
\hline $\mathrm{NO}_{2}-\mathrm{N}$ & $.07 / 1.0$ & $.07 / 1.0$ & .05 \\
\hline $\mathrm{NO}_{3}-\mathrm{N}$ & $90 / 325$ & 1603 & 28,000 \\
\hline $\mathrm{PO}_{4}-\mathrm{P}$ & $0.3 / 40$ & $0.3 / 40$ & 0.11 \\
\hline $\mathbf{K}$ & $28 / 76$ & 240 & 9100 \\
\hline $\mathrm{Se}$ & $<0.1 / 1.0$ & 1.0 & 1.6 \\
\hline $\mathrm{SiO}_{2}$ & $80 / 87$ & 80 & 8 \\
\hline $\mathrm{Ag}$ & $<.006 / 0.03$ & $<.006$ & 0.44 \\
\hline $\mathrm{SO}_{4}$ & $0 / 26$ & $18 / 26$ & 0 \\
\hline
\end{tabular}

LANL/Table3.2-1 
Table 3.2 Influent Characteristics Compiled for BAT Study (Cont)

\begin{tabular}{|c|c|c|c|}
\hline $\begin{array}{c}\text { Influent } \\
\text { Component }\end{array}$ & $\begin{array}{c}\text { TA-50-1 Influents } \\
\text { (Neat) } \\
\text { mg/L } \\
\end{array}$ & $\begin{array}{c}\text { TA-50-1 Influents } \\
\text { (Blended) } \\
\text { mg/L }\end{array}$ & $\begin{array}{c}\text { TA-55 Emuent } \\
\text { (Supernate) } \\
\text { mg/L }\end{array}$ \\
\hline $\mathrm{Na}$ & $275 / 512$ & 1508 & 2,332 \\
\hline $\mathrm{Zn}$ & $0.25 / 0.3$ & $<.002$ & $<.001$ \\
\hline Total Alkalinity & $8 / 150$ & 45 & 3,010 \\
\hline P-Alkalinity & -- & -- & -- \\
\hline Conductivity (Micromho/cm) & $840 / 2680$ & 7176 & 188,680 \\
\hline $\mathrm{pH}$ & $4 / 10$ & 7.3 & 12 \\
\hline \multicolumn{4}{|l|}{ Total Hardness } \\
\hline COD & $4 / 123$ & 81 & 424 \\
\hline$\sum$ Cations (meg/l) & -- & -- & -- \\
\hline$\sum$ Anions $(\mathrm{meg} / \mathrm{l})$ & -- & $\cdots$ & -- \\
\hline TDS & 967 & 2500 & 188,000 \\
\hline TSS & $4 / 45$ & $?$ & 226 \\
\hline $\mathrm{Be}$ & -- & -- & -- \\
\hline $\mathrm{Al}$ & -- & -- & -- \\
\hline B & -- & -- & -- \\
\hline Co & -- & -- & -- \\
\hline$V \mathbf{n}$ & -- & -- & -- \\
\hline Tritium & -- & -- & -- \\
\hline $\mathrm{Ra}^{226+228}$ & -- & -- & -- \\
\hline $\mathrm{Pu}^{299}$ & $1 / 100 \mathrm{nCi} / \mathrm{L}$ & -- & -- \\
\hline $\mathrm{Am}^{241}$ & $1 / 20 \mathrm{NCi} / \mathrm{L}$ & - & -- \\
\hline $\mathrm{Cs}^{137}$ & $0.7 / 11 \mathrm{nCi} / \mathrm{L}$ & - & -- \\
\hline $\mathrm{Sr}^{90}$ & $0.55 / 3.4 \mathrm{nCi} / \mathrm{L}$ & - & -- \\
\hline $\mathrm{Co}^{60}$ & $0.7 / 0.8 \mathrm{nCi} / \mathrm{L}$ & -- & -- \\
\hline $\mathrm{Na}^{22}$ & $0.96 \mathrm{nCi} / \mathrm{L}$ & -- & -- \\
\hline $\mathrm{Sr}^{88}$ & $0.5 / 0.75 \mathrm{nCi} / \mathrm{L}$ & -- & -- \\
\hline
\end{tabular}

LANL/Table3.2-1 
Table 3.2 Influent Characteristics Compiled for BAT Study (Cont)

\begin{tabular}{|c|c|c|c|}
\hline $\begin{array}{c}\text { Influent } \\
\text { Component }\end{array}$ & $\begin{array}{c}\text { TA-50-1 Influents } \\
\text { (Neat) } \\
\mathrm{mg} / \mathrm{L}\end{array}$ & $\begin{array}{c}\text { TA-50-1 Influents } \\
\text { (Blended) } \\
\mathrm{mg} / \mathrm{L}\end{array}$ & $\begin{array}{c}\text { TA-55 Emuent } \\
\text { (Supernate) } \\
\text { mg/L }\end{array}$ \\
\hline \hline $\mathrm{Nb}^{95}$ & $0.9 \mathrm{nCi} / \mathrm{L}$ & -- & -- \\
\hline $\mathrm{Y}^{88}$ & $0.5 / 13 \mathrm{nCi} / \mathrm{L}$ & -- & -- \\
\hline $\mathrm{Zr}^{88}$ & $0.4 / 6 \mathrm{nCi} / \mathrm{L}$ & -- & - \\
\hline
\end{tabular}

$\begin{array}{lll}\quad & = & \text { unable to evaluate } \\ -- & = & \text { no value stated }\end{array}$

LANL/Table3.2-1 


\subsection{ANALYSIS OF ENVIRONMENTAL ISSUES}

\subsection{ANALYSIS OF ENVIRONMENTAL/REGULATORY ISSUES}

\subsubsection{Current Environmental Discharge Restrictions}

The design bases and requirements for the RLWTF have varied due to changing regulatory perceptions regarding the selection of applicable discharge quality parameters. LANL presently is operating under an NPDES permit, and has maintained compliance utilizing the present/existing process technology. It is expected that the renewal of this permit will include the imposition by EPA of more stringent release restrictions that cannot be met by the existing systems and processes.

The LANL discharges from TA-50-1 currently are in compliance with the existing NPDES permit. However, according to the requirements specified in DOE Order 5400.5, a BAT Study is required since the sum of the Derived Concentration Guidelines (DCGs) is being exceeded. The sum of the DCGs is defined in DOE Order 5400.5 as the combined concentrations of radionuclides in air or water that, under conditions of continuous exposure for one year would result in an effective dose equivalent of $100 \mathrm{mrem}(1 \mathrm{mSv})$.

The current NPDES Permit for the LANL site (Permit No. NM0028355), regulates wastewater discharges from the site to ephemeral streams which produce little or no flow to Segment No. 2-111 and Segment No. 2-118 of the Rio Grande Basin. The appropriate designated stream uses to be associated with these ephemeral streams has come into question. EPA has stated the position that permit conditions consistent with wildlife watering use (as a minimum) must be implemented.

EPA Region 6 has included the State of New Mexico as a reviewer of the NPDES. The New Mexico Environmental Department (NMED) was invited to provide, as a condition of certification of the NPDES, any additional specific permit limitations necessary to meet State requirements (including appropriate designated stream uses) in accordance with the provisions of 40 CFR 124.53(e)(1-3).

There are varying opinions among regulatory groups as to the appropriate application of discharge water quality parameters. LANL currently is in compliance with the release requirements of the existing NPDES permit. The more stringent permit requirements proposed by EPA would result in noncompliance on certain parameters under the current operations. EPA has identified the point of discharge for TA-50 as appropriate for livestock and wildlife watering. The NMED requirements for this use category are slightly more stringent than those promulgated by EPA. In addition, the NMED has 
requested even more restrictive release requirements, which would entail production of a high-purity effluent or even potentially a zero release configuration.

\subsubsection{Requirements for Wildlife Consumption and Livestock Watering}

As stated in Public Notice of Draft NPDES Permit for LANL (issued as Advertising Order No. 2T-3299-NNLX, EPA Region 6 in consultation with the State of New Mexico, May 16, 1992), the proposed new NPDES permit includes the application of water quality limits for wildlife consumption. This means that EPA has identified the point of discharge of the current RLWP as appropriate for 'livestock and wildlife watering'. However, due to the location and siting of the LANL site, only wildlife have access to the water.

The general and specific stream standards that are relevant to waters of the State of New Mexico are provided in Water Ouality Standards for Interstate and Intrastate Streams in New Mexico, by the New Mexico Waste Quality Control Commission, amended May 22, 1991.

Standards for livestock and wildlife consumption were derived or calculated by NMED for LANL discharges based on existing criteria and guidance. As an example, for total metals discharge limits, the metals partition coefficients (per cent dissolved) and the associated total metals calculations for various TSS levels were derived from New Mexico Water Quality Standards numerical criteria for Livestock and Wildlife Watering. These calculations and derived data were based on the New Mexico Interim Guidance for Implementation of Water Quality Standards, dated June 25, 1991, and Technical Guidance Manual for Performing Wasteload Allocations, Book II, Streams and Rivers, Chapter 3, Toxic Substances (EPA-440/4-84-022, June 1984).

\subsubsection{Predicted NMED NPDES Requirements}

As stated in the draft NPDES Permit Public Notice, NMED was invited to provide any additional permit limitations deemed necessary for compliance with State requirements. Under this allowance, it was possible that LANL would have to provide for additional treatment expenses such as total retention and evaporation at individual outfalls, if the increased permit stringency for Water Quality Standards imposed by NMED so dictated.

A letter dated January 31, 1991, was transmitted from NMED to EPA Region 6, providing preliminary comments and recommendations for the LANL NPDES permit. This letter stated that " . the State will not hesitate 
to deny any permit that does not adequately address all concerns for the protection of the receiving streams, water quality and the environment in New Mexico."

On August 9, 1991, NMED issued a Denial of the Draft LANL NPDES Permit on the grounds that ". . compliance with the terms and conditions of the permit as proposed will not provide reasonable assurance that the permitted activities will be conducted in a manner which will not violate applicable water quality standards or be compatible with appropriate State law." This denial initiated a series of meetings and transmittals of correspondence between LANL, EPA, and the State, which included the request by EPA that NMED develop "permit conditions and references to appropriate state laws, regulations, and standards for conditional certification" of the permit. On November 27, 1991, NMED restated that the permit had been denied by the State. On December 13, 1991, a meeting was held between EPA, LANL, and NMED, where it was stated that NMED will require (as a minimum) wildlife watering uses under State Water Quality Standards in a reissued short-term permit.

Although agreeing that the RLWP discharge is appropriate for wildlife and livestock watering, NMED has stated the position that the discharge limits should be even more stringent. If the most stringent requirements are selected and enforced, it is anticipated that the only process configuration that could guarantee consistent compliance would be a Zero Release scenario. Also, NMED has imposed a groundwater contaminant standard of $10 \mathrm{mg} / \mathrm{L}$ for nitrate-nitrogen. All of these 1991 release requirements proposed by NMED are considerably more stringent than those promulgated in the federal regulations.

\subsubsection{Analysis of Potential Compliance Limits}

Since the ultimate release criteria and limits have not been established by a final decision, the BAT Study includes a comparison of remedial technology for both the discharge limits associated with the use of releases as a source of drinking water for wildlife or for watering livestock, and for the limits based on the most restrictive anticipated values to be imposed by the new (currently under regulatory evaluation) NPDES permit.

The Permit Notice of May 16, 1992 provided specific effluent limitations and conditions that were calculated or otherwise derived for the specific contaminants of concern for the LANL waste streams. The substantiation for these limits and conditions included a citation of the applicable effluent limitation guidance or performance standard provisions as required under 40 CFR 122.44 and 122.45 , and an explanation of the applicability. These criteria and evaluations included: 
- $\quad$ Total metals limitations under Water Quality Standards for Livestock and Wildlife Watering

- Water Quality Standards effluent limits

- Applicable daily maximum levels reported for Water Quality parameters

- Application daily maximum levels reported for other State-regulated parameters

- Effluent limitations based on technology considerations

The matrix presented in Appendix $C$ provides a summary of the various effluent discharge limits that have been evaluated for applicability to the LANL RLWP effluent.

\subsection{DESIGN BASIS CHARACTERIZATION OF THE INFLUENT WASTE STREAM}

\subsubsection{Availability of Waste Characterization Data}

Although there have been annual summaries of the influent and effluent waste constituents pertinent to the TA-50 operations, there has been no definitive characterization of each waste stream that is received by the facility. Parsons was instructed (ref. Change Order Proposal for Work Release 0015, EM-7RLW-92-060, August 18, 1992) to prepare the BAT study with existing information (best estimates of waste characterization), assuming no additional information will be provided by bench-scale or pilot-scale studies.

\subsubsection{Waste Characterization Parameters Used for Design}

In lieu of a definitive waste characterization for all existing and anticipated waste streams to be processed by the RLWTF, the averages calculated from the annual reports were used as a basis for this study. The documents used to establish the influent wastewater characterization included four annual reports ('Summary of Operating Data' annual reports for the years 1987-90), five analyses of RLWP influent from early 1991, taken during different times in the weekly RLWP operating cycle, and a summation by LANL of the five analyses.

The results of the waste characterization efforts that were based on the analysis of five weekly samplings are presented in Table 4-1. In this table, the five groupings show the variance that can be expected for the TA-50 influent. Although the overall waste characterization tends to be uniform, an occasional pretreatment (Room 60) operation will produce a very concentrated supernate, which historically has been blended with the primary TA-50 influent. These occasional concentrated batches of TA-55 waste create spikes in the analytical data for the RLWP influent. As shown in Table 4-1, 
it was the position of LANL that Analyses 1,2, and 4 represent the range of unblended influent concentrations, Analysis 5 represents the strong Room 60 supernate, and Analysis 3 represents a short-term mixture of the two streams. Examination of the concentration values for $\mathrm{K}, \mathrm{Na}$, and $\mathrm{NO}_{3}$ indicates that the spike in concentration represents a mixture of $>3 \%$, rather than the typical $1.2 \%$ mixture typical of annual reports.

Decontamination factors were derived from the ratios of the expected influent contaminants to the required discharge contaminant levels. These factors were developed in order to define the required efficiencies for the unit processes to be considered in candidate treatment scenarios. Decontamination factors required to meet selected representative release limits are presented in Table 4-2.

Based on the data from the five analyses and a historical knowledge of the conditions that prevailed when each of the five samplings were taken, LANL compiled an estimate of concentration which divided the influent waste into three categories:

1. An unblended influent, where only the TA-50 waste influent is considered

2. An unblended waste influent for the TA-55 wastes as a separate stream 3. A blended TA-50/TA-55 influent stream

Evaluations produced by LANL provided an estimate of concentration ranges that might be expected for these three categories. These evaluations that are contained in Table 4-3 indicate the constituents in the TA-50 waste streams which approach or exceed the limits anticipated by regulatory guidance and/or directives. These are the primary contaminants that must be addressed by any proposed treatment scheme.

The chemical analysis of the influent presented in the four annual reports in terms of maximum and minimum values for each contaminant species provides adequate correlation with the values calculated by LANL. This suggests that the influent composition has been relatively consistent both in characteristic and in volume over the period of 1987 to 1991.

These data and analyses represent current operations. However, the information provided in Table 4-4 provided by LANL and derived from their ongoing characterization effort implies that in excess of 20 new sources of influent to the RLWTF may be planned for the future. The impact of these changes to the TA-50 influent cannot be assessed at this time, and will require specific characterization for appropriate design. At this point in design development, a flexible technology is appropriate if not an absolute necessity, in order to most effectively accommodate the possible variations in treatment 
requirements. This flexibility was considered as primary design and evaluation criteria for the BAT study.

Given the present estimated composition of the influent stream, there is no single waste constituent that drives the requirements for process development. In current operations, the combined processes of flocculation, precipitation, and filtration remove most of the radiological contamination, and also are utilized to remove heavy metal contamination. In addition, the same types of technology that could be utilized to reduce the non-precipitating radioactivity could be utilized to remove the non-radioactive ionic species. This logic indicates that a very general approach to TA-50 wastewater treatment should be adopted.

\subsubsection{Waste Stream Considerations and Assumptions}

Since the waste characterization efforts are ongoing at LANL it was not possible at this time to obtain definitive waste characteristics and contaminants, the following considerations and assumptions were made relative to the RIWTF process waste streams:

1. Tritium removal (accelerator-produced tritium) will not be required. Administrative controls via Waste Acceptance Criteria for the RLWTF will be used to moderate tritium content and levels.

2. The high concentrations of tritium in the influent preclude the reuse of treated water (e.g., in a cooling tower) for virtually any reuse scenario. Therefore, the concept of zero release becomes questionable.

3. The loading of organic contaminants would be of the concentration levels that could be managed by an organics destruction to reduce COD levels prior to discharge, but would not be of a concentration high enough to affect upstream treatment operations.

\subsection{ENVIRONMENTAL BASES FOR THE PROCESS CONFIGURATIONS}

The twelve process configurations (PCs) selected for evaluation in this BAT study represent four groupings of treatment alternatives.

\subsubsection{Construction of a New Plant to Comply with Wildlife and Livestock Watering Release Limits}

In this group, a combined process is proposed using two stages of precipitation/flocculation, evaporation, and a finishing evaporator which can meet the requirements for processing the wastewater and discharging an 
effluent in compliance with the NMED release criteria for livestock and wildlife watering. COD and nitrate destruction are provided to remove volatile organics and to reduce the secondary waste volume but these processes are not crucial to the base operations. This addresses PC \#6, the construction of a new plant to treat the blended stream in compliance with the prescribed release criteria.

PC \#4, which involves the construction of a new plant to treat the TA-50 unblended ('neat') influent, is essentially the same design as PC \#6, with a little less operating time required and a little less waste produced. The TA-50 'neat' stream includes all the present influent to TA-50 minus the present Room 60 effluent.

PC \#5, which addresses construction of a new plant to treat only the TA-55 effluent for compliance with the release criteria, would use the same process steps but on a much reduced scale. Actually, PC \#4 and PC \#5 represent only partial solutions to the plant concerns since each treats a segregated element of the current influent waste stream. The one RLWTF for PC \#6 is proposed to handle both streams (as with current operations).

\subsubsection{Modification of the Existing Plant to Comply with Wildlife and Livestock Watering Release Limits}

The proposed new plant and existing operations have essentially the same precipitation/flocculation/sedimentation unit processes. If evaporation is added to the existing plant, the released effluent would be able to meet the new discharge requirements.

According to calculations of required decontamination factors, COD removal (in terms of $\mathrm{mg} / \mathrm{L}$ concentration) is not required under the livestock and wildlife watering criteria. However, certain constituents that contribute to the COD loading may be objectionable, particularly chelates and surfactants. Therefore, COD removal should be considered as an addition to the present plant. Similarly, while nitrate destruction on the TA-55 waste is not essential to meet release requirements, the high concentration of nitrates in the waste stream presents the opportunity via nitrate destruction for a major secondary waste volume reduction.

Accordingly:

PC \#3 Includes addition of a nitrate destruction unit (for TA-55 effluent only), COD removal, and evaporation to treat the blended (TA-55/TA-50) stream. 
PC \#1

PC \#2
Includes the same arrangement as PC \#3, but treats only the TA-50 "neat" influent without modification of the Room 60 effluent.

Includes the same arrangement as PC \#3, but with modifications to the Room 60 operations, with no internal modifications for the base TA-50 operations.

\subsubsection{Construction of a New Plant to Comply with the September 11, 1992 Proposed NPDES Limits}

PC \#7, PC \#8, and PC \#9 are very similar to the scenarios in PC \#4, PC \#5, and $\mathrm{PC} \# 6$, respectively, the difference being the addition of solar evaporation ponds to provide a zero release configuration. This arrangement would eliminate any NPDES requirements, including the need for a NPDES permit for the TA-50 effluent. An evaporative cooling tower was considered as an alternative to the solar ponds, but this system is not considered to be energy efficient.

\subsubsection{Modification of the Existing Plant to Comply with the September 11, 1992 Proposed NPDES Limits}

PC \#10, PC \#11, and PC \#12 use the same configurations as PC \#1, PC \#2, and $\mathrm{PC} \# 3$, respectively, with the addition of solar evaporation ponds.

\subsection{ENVIRONMENTAL ISSUES FOR PC \#1}

The treatment scenario proposed as $\mathrm{PC} \# 1$ involves the upgrading of the existing TA-50 Operations to handle only the TA-50 influent minus the TA-55/Room 60 supernate.

\subsubsection{Regulatory Compliance}

The upgrade of process equipment would be designed to comply with the NMED regulations promulgated for the release of effluents considered suitable for wildlife and livestock consumption. Without the contaminants from the TA-55 supernate, the contaminants in the waste stream are composed primarily of heavy metals and TDS. The existing processes of clarification/flocculation and filtration are particularly efficient for removal of these constituents and the addition of an evaporation process unit would ensure regulatory compliance.

Since only the primary TA-50 waste stream is considered in this scenario, the proposed upgrades and modifications can be designed to effectively achieve 
the desired decontamination factors required for compliance with discharge limits.

\subsubsection{Comparison with Derived Concentration Guide Values}

The allowable concentrations of radionuclide contaminants in liquid waste discharges are established by the DCG values derived from the criteria in DOE Order 5400.5. Since the TA-55 waste stream, which contains by far the largest volume of radioactive contamination, is not included in this PC the remaining radioactivity in the TA-50 influent is of minor concern in the treatment process. The proposed upgrades and additions should effectively remove radioactivity to acceptable discharge levels.

\subsubsection{Contaminants and Concentrations in Discharge Streams}

The proposed modifications would provide specific treatment technology for the anticipated influent contaminant species for the base TA-50 operations. An analysis of the expected influent to effluent processing specifications for this $\mathrm{PC}$ is provided in the table for PC \#1 in Appendix D.

\subsection{ENVIRONMENTAL ISSUES FOR PC \#2}

The treatment scheme for PC \#2 involves the upgrading of the existing Room 60 operations only for continued treatment of the TA-55 waste stream. The design basis discharge parameters would involve compliance with the NMED requirements for wildlife and livestock watering release limits.

\subsubsection{Regulatory Compliance}

The upgrade and addition of process equipment would be designed to comply with the NMED regulations promulgated for the release of effluents considered suitable for wildlife and livestock consumption. With a low-volume waste stream that would not be blended with the TA-50 influent, the contaminants in the waste stream would comprise acidic and caustic influents, significant quantities of radionuclides, and lesser levels of TDS. The existing processes of clarification/flocculation and filtration are particularly efficient for removal of these constituents and the addition of an evaporation process unit would ensure regulatory compliance. A nitrate removal processing step would be included to reduce the nitrate contaminant loading.

Since only the primary TA-50 waste stream is considered in this scenario, the proposed upgrades and modifications can be designed to effectively achieve the desired decontamination factors required for compliance to discharge limits. 


\subsubsection{Comparison with Derived Concentration Guide Values}

The allowable concentrations of radionuclide contaminants in liquid waste discharges are established by the DCG values derived from the criteria in DOE Order 5400.5 . Since the TA-55 waste stream contains by far the largest volume of radioactive contamination, removal of radionuclides is an essential element of any proposed treatment scheme. Alpha-emitting radioactivity is reduced effectively by coagulation/precipitation processes. The proposed upgrades and additions should effectively remove radioactivity to acceptable discharge levels.

\subsubsection{Contaminants and Concentrations in Discharge Streams}

The proposed modifications would provide specific treatment technology for the anticipated contaminant species introduced into the Room 60/TA-50 operations. An analysis of the expected influent to effluent processing specifications for this PC is provided in the table for PC \#2 in Appendix D.

\subsection{ENVIRONMENTAL ISSUES FOR PC \#3}

The PC \#3 treatment scenario involves the upgrade of existing TA-50 operations to accommodate both the TA-55 and TA-50 wastes, which is representative of the current operations. Modifications and additional unit processes are to be included to ensure compliance with wildlife and livestock watering release limits.

\subsubsection{Regulatory Compliance}

The primary reason for this BAT study is to evaluate alternatives for modification or replacement of the existing TA-50 operations because the facility as it is currently configured is not capable of providing compliance with more demanding discharge regulations. The upgrade and addition of process equipment would be necessary to meet the NMED wildlife and livestock watering requirements.

To treat the combined TA-50 and TA-55 wastes, required modifications would include retrofit and upgrading of the existing TA-55/Room 60 unit processes, with the addition of a new front-end unit process for nitrogen control. Modifications to the TA-50 operations would include retrofit/upgrading of the existing unit processes, as well as the addition of new systems for evaporation, organics destruction, and solids/sludge disposal. With these proposed upgrades and modifications, the TA-50 operations can be retrofitted to achieve the desired decontamination factors required for compliance with the stated discharge limits. 


\subsubsection{Comparison with Derived Concentration Guide Values}

In this proposed scheme, the TA- 55 waste stream is pretreated to remove most of the radioactive constituents prior to blending with the TA-50 influent. The remaining radioactivity in the primary TA-50 waste stream is of minor concern in the treatment process. The proposed upgrades and additions should effectively remove radioactivity to acceptable discharge levels.

\subsubsection{Contaminants and Concentrations in Discharge Streams}

The proposed modifications would provide separate specific treatment technologies for the TA-55 and TA-50 influent contaminant species, with the supernate from Room 60 receiving a second cleanup processing via blending into the TA-50 influent. An analysis of the expected influent to effluent processing specifications for this PC is provided in the table for PC \#3 in Appendix D.

\subsection{ENVIRONMENTAL ISSUES FOR PC \#4}

PC \#4 involves the construction of a new facility to treat only the TA-50 waste stream. This new facility would be designed to provide compliance with the NMED wildlife and livestock watering release limits.

\subsubsection{Regulatory Compliance}

The selection of new process equipment specifically for the anticipated influent contaminant species would allow to be optimized for compliance with the wildlife and livestock watering requirements. The contaminants in the TA-50 waste stream (without the TA-55 supernate) are composed primarily of heavy metals and TDS. The proposed precipitation process units include a flash mixer, flocculator and a lamella plate clarifier, which would be especially effective in reducing heavy metals and TDS over a range of species. Evaporation and organics destruction should ensure continuing regulatory compliance.

Since only the primary TA-50 waste stream is considered in this scenario, the proposed unit processes can be designed to effectively achieve the desired decontamination factors required for compliance with discharge limits.

\subsubsection{Comparison with Derived Concentration Guide Values}

The DCG values from DOE Order 5400.5 can be achieved with little difficulty by the selection of appropriate unit processes. Since the TA- 55 waste stream is not included in this PC, the remaining radioactivity in the TA-50 influent 
is of minor concern in the treatment process. The proposed treatment scheme should effectively reduce radioactivity to acceptable discharge levels.

\subsubsection{Contaminants and Concentrations in Discharge Streams}

The proposed new facility would provide separate specific treatment technology for the anticipated influent contaminant species for the base TA-50 operations. An analysis of the expected influent to effluent processing specifications for this PC is provided in the table for PC \#4 in Appendix D.

\subsection{ENVIRONMENTAL ISSUES FOR PC \#5}

In this PC, a new facility is proposed to treat only the TA-55 waste stream. This new process facility would be designed to ensure compliance of the Room 60 supernate with wildlife and livestock watering release limits. Proposed new unit processes include nitrate destruction, flash mixing, flocculation, clarification, and evaporation.

\subsubsection{Regulatory Compliance}

The designation of new process equipment selected specifically to attain compliance with the NMED wildlife and livestock watering limits would ensure optimal contamination removal. With a low-volume waste stream generally composed of acidic and caustic influents, radionuclides, nitrates, and TDS, the selection of flexible processing equipment is a simple task.

Since only the TA-55 waste stream is considered in this scenario, the proposed unit processes can be designed to effectively achieve the desired decontamination factors required for compliance to discharge limits, and also can be sized to properly accommodate the predicted waste volumes.

\subsubsection{Comparison with Derived Concentration Guide Values}

Since the TA-55 waste stream contains the largest volume of radioactive contamination, removal of radionuclides is the primary function of this proposed treatment scheme. Alpha-emitting radioactivity is reduced effectively by coagulation/precipitation processes. The selected unit processes should effectively remove radioactivity to acceptable discharge levels.

\subsubsection{Contaminants and Concentrations in Discharge Streams}

The proposed new facility and equipment would provide specific treatment technology for the anticipated contaminant species introduced into the Room 60 operations. An analysis of the expected influent to effluent processing specifications for this PC is provided in the table for PC \#5 in Appendix D. 


\subsection{ENVIRONMENTAL ISSUES FOR PC \#6}

In PC \#6 a new facility is proposed to treat the blended TA-55 and TA-50 wastes to produce an effluent that would be in compliance with NMED wildlife and livestock watering release limits. This configuration would entail a new facility that combines the general features discussed in PCs \#5 and \#6.

\subsubsection{Regulatory Compliance}

The selection of specific process equipment for the new facility would be essential for compliance with the NMED wildlife and livestock watering requirements. To treat the combined TA-50 and TA-55 wastes, integrated processes have been proposed for nitrate destruction, neutralization, physical separation, evaporation, and organics destruction. The TA-50 and TA-55 waste streams are the combined facility influent, with no proposed pretreatment of the TA-55 wastes.

With the construction of an all-new facility designed specifically for handling of the anticipated waste streams, the desired decontamination factors required for compliance with the stated discharge limits can be achieved by design requirements.

\subsubsection{Comparison with Derived Concentration Guide Values}

In this proposed scheme, the TA-55 waste stream is not pretreated prior to blending with the TA-50 influent. The total radioactivity from both waste streams is introduced into the primary treatment process. Radiological removal is conducted by a precipitation step that is effective for alpha-emitting radionuclides. This integrated radionuclide removal process should be acceptable for reducing contamination to stated discharge levels.

\subsubsection{Contaminants and Concentrations in Discharge Streams}

The proposed new configuration offers a single, integrated treatment process for removal of all contaminants of concern, including radionuclides, TDS, organics, nitrates, heavy metals, and $\mathrm{pH}$. An analysis of the expected influent to effluent processing specifications for this $\mathrm{PC}$ is provided in the table for PC \#6 in Appendix D.

\subsection{ENVIRONMENTAL ISSUES FOR PC \#7}

PC \#7 involves the construction of a new facility to treat TA-50 waste only, and to achieve the stringent NMED release limits proposed September 11, $1992 \mathrm{draft}$ NPDES. For this scenario, the final effluent from the processing of the TA-50 waste 
stream would be required to comply with release standards more stringent than those for wildlife and livestock watering. The only process configuration that can ensure compliance with the most stringent release limits would be a zero release design.

The basic unit processes for this configuration would be the same as those for the TA-50 wildlife and livestock watering scenario in PC \#4. The difference is the inclusion of an additional evaporation process using a solar pond. No liquid wastes would be routed to an NPDES outfall.

\subsubsection{Regulatory Compliance}

The selection of new process equipment for a zero release would allow design to be optimized for contamination removal. Since there would be no liquid discharge released to the environment, regulatory compliance would not be a consideration. However, if fluctuations in the evaporation processes result in the creation of airborne contamination, other regulatory restrictions could be pertinent. Also, the placement of solar ponds for the evaporation of the final effluent may require regulatory scrutiny.

\subsubsection{Comparison with Derived Concentration Guide Values}

The DCG values from DOE Order 5400.5 may have little bearing on this scenario. If it can be demonstrated that the evaporator/solar pond scenario has little risk of impact on human health or the environment, the DCGs from 5400.5 would not apply.

\subsubsection{Contaminants and Concentrations in Discharge Streams}

The proposed new facility would provide specific treatment technology for the removal and/or concentration of anticipated influent contaminant species for the base TA-50 operations. An analysis of the expected influent and effluent (solar pond influent) processing specifications for this $\mathrm{PC}$ is provided in the table for PC \#7 in Appendix D.

\subsection{ENVIRONMENTAL ISSUES FOR PC \#8}

The proposed scenario for PC \#8 is the construction of a new facility only for the treatment of TA-55 wastes. This new facility would be designed to meet the September 11, 1992 NMED release limits. As with PC \#7, a zero release scenario is proposed.

The basic unit processes for this configuration would be the same as that for the TA-55 wildlife and livestock watering scenario in PC \#5. The difference between the two configurations is the routing of the final treatment effluent to a solar pond. 


\subsubsection{Regulatory Compliance}

New process equipment selected for a zero release would allow design to be optimized for radiological contamination removal. Since there would be no liquid discharge released to the environment, regulatory compliance would not be a consideration.

\subsubsection{Comparison with Derived Concentration Guide Values}

The DCG values from DOE Order 5400.5 may have little bearing on this scenario. If it can be demonstrated that the evaporator/solar pond scenario has little risk of impact on human health or the environment, the DCGs from 5400.5 would not apply.

\subsubsection{Contaminants and Concentrations in Discharge Streams}

The proposed new facility would provide specific treatment technology for the removal and/or concentration of anticipated influent contaminant species for the Room 60 operations. An analysis of the expected influent and effluent (solar pond influent) processing specifications for this $\mathrm{PC}$ is provided in the table for PC \#8 in Appendix D.

\subsection{ENVIRONMENTAL ISSUES FOR PC \#9}

The treatment scheme in $\mathrm{PC} \# 9$ involves the construction of a new facility to treat the blended TA-55 and TA-50 waste streams. This new facility design is based on compliance with the stringent NMED release limits. Also, this configuration combines the zero release scenarios from PCs \#7 and \#8 into a single blended process.

\subsubsection{Regulatory Compliance}

With the initial premise of a zero release configuration, the design of processes and equipment would be optimized for contamination reduction and/or removal. Since there would be no liquid discharge released to the environment, regulatory compliance would not be a consideration.

\subsubsection{Comparison with Derived Concentration Guide Values}

The DCG values from DOE Order 5400.5 would have little bearing on this scenario. If it can be demonstrated that the evaporator/solar pond scenario has little risk of impact on human health or the environment, the DCGs from 5400.5 would not apply. 


\subsubsection{Contaminants and Concentrations in Discharge Streams}

The proposed new facility would provide specific treatment technology for the removal and/or concentration of anticipated influent contaminant species from both the current primary TA-50 influent and the influent stream currently routed to the Room 60 operations. An analysis of the expected influent and effluent (solar pond influent) processing specifications for this $\mathrm{PC}$ is provided in the table for PC \#9 in Appendix D.

\subsection{ENVIRONMENTAL ISSUES FOR PROCESS CONFIGURATION \#10}

For PC \#10, the scenario includes the upgrading of existing TA-50 operations for only the primary TA-50 waste stream. This upgrade would be to meet the September 11, 1992 NMED release limits. As with PC \#1, this selection would involve retrofit/upgrading of the existing TA-50 unit processes, as well as the addition of new systems for evaporation and organics destruction. The final effluent would be routed to a solar pond in a zero release configuration.

\subsubsection{Regulatory Compliance}

The upgrading and modification of process equipment to provide a zero release scenario would allow design to be optimized for contamination removal. Since there would be no liquid discharge released to the environment, regulatory compliance would not be a consideration.

\subsubsection{Comparison with Derived Concentration Guide Values}

The DCG values from DOE Order 5400.5 may have little bearing on this scenario. If it can be demonstrated that the evaporator/solar pond scenario has little risk of impact on human health or the environment, the DCGs from 5400.5 would not apply.

\subsubsection{Contaminants and Concentrations in Discharge Streams}

The proposed modifications would provide specific treatment technology for the removal and/or concentration of anticipated influent contaminant species for the base TA-50 operations. An analysis of the expected influent and effluent (solar pond influent) processing specifications for this $\mathrm{PC}$ is provided in the table for PC \#10 in Appendix D.

\subsection{ENVIRONMENTAL ISSUES FOR PC \#11}

The scenario proposed as PC \#11 is for the upgrade of existing Room 60 operations for the treatment of the TA-55 waste stream only, in order to comply with the September 11, 1992 NMED release limits. As with PC \#2, this would include 
retrofit and upgrading of the existing TA-55/Room 60 unit processes, and the addition of a new front-end unit processes for nitrogen control, evaporation, and organics destruction. The effluent from the organics destruction unit would be routed to a solar pond for zero release.

\subsubsection{Regulatory Compliance}

The upgrading and modification of process equipment to provide a zero release scenario would allow design to be optimized for contamination removal. Since there would be no liquid discharge released to the environment, regulatory compliance would not be a consideration.

\subsubsection{Comparison with Derived Concentration Guide Values}

If it can be demonstrated that the evaporator/solar pond scenario has little risk of impact on human health or the environment, the DCGs from DOE Order 5400.5 would not apply.

\subsubsection{Contaminants and Concentrations in Discharge Streams}

The proposed modifications would provide specific treatment technology for the removal and/or concentration of anticipated influent contaminant species for the Room 60 operations. An analysis of the expected influent and effluent (solar pond influent) processing specifications for this PC is provided in the table for PC \#11 in Appendix D.

\subsection{ENVIRONMENTAL ISSUES FOR PC \#12}

PC \#12 involves the upgrading of the existing TA-50 operations to accommodate blended TA-55 and TA-50 wastes for compliance with the September 11, 1992 NMED release limits. This scenario combines the treatment systems from PCs \# 10 and \#11, in a manner similar to that in PC \#3. This would include retrofit and upgrading of the existing TA-50 operations to a zero release configuration, and the integration of the Room 60 influent into the primary TA-50 influent waste stream.

\subsubsection{Regulatory Compliance}

With the initial premise of a zero release configuration, the design of processes and equipment would be optimized for contamination reduction and/or removal. Since there would be no liquid discharge released to the environment, regulatory compliance would not be a consideration. 


\subsubsection{Comparison with Derived Concentration Guide Values}

The DCG values from DOE Order 5400.5 would have little bearing on this scenario. If it can be demonstrated that the evaporator/solar pond scenario has little risk of impact on human health or the environment, the DCGs from 5400.5 would not apply.

\subsubsection{Contaminants and Concentrations in Discharge Streams}

The proposed modifications would provide specific treatment technology for the removal and/or concentration of anticipated influent contaminant species from both the current primary TA-50 influent and the influent stream currently routed to the Room 60/TA-55 operations. An analysis of the expected influent and effluent (solar pond influent) processing specifications for this PC is provided in the table for PC \#12 in Appendix D.

\subsection{EVALUATION AND ELIMINATION OF PCS BASED ON ENVIRONMENTAL ISSUES}

\subsubsection{Comparative Analysis of Environmental Issues}

The deciding factors for the application of environmental concerns to the evaluation of PCs center on whether or not the treatment scenarios can comply with the prescribed liquid waste discharge contamination limits. In the twelve proposed PCs, there are essentially two issues that are the focus of regulatory compliance:

1. Compliance with the concentration limits as established by NMED for wildlife and livestock watering

2. Compliance with the very conservative and stringent concentration limits proposed by NMED on September 11, 1992 for the new NPDES permit

In conversations with LANL on the application of criteria more stringent than any required by existing promulgated standards (i.e., that would require a zero release configuration), it was agreed that a detailed analysis of the waste processing data should be performed. Upon examination of the data, it became evident that an appropriate evaporator system design could meet the discharge criteria in almost all instances. In [potentially] only a few cases would the decontamination factors required for strict regulatory compliance be exceeded. Based on the accumulated waste characterization data (refer to Table 4-2) the highest requirements for decontamination are:

- $\quad \mathrm{DF}=850$ to meet the $\mathrm{Cu}^{+2}$ criteria of NMED-051 
- $\quad \mathrm{DF}=384$ to meet the $\mathrm{NH}_{3}$ criteria of the draft NPDES

- $\quad \mathrm{DF}=160$ to meet the $\mathrm{NO}_{3}^{-}$criteria for LS\&WS and NMED-051

- $\mathrm{DF}=115$ to meet the $\mathrm{NH}_{3}$ criteria of NMED-051

- $\mathrm{DF}=42$ to meet the $\mathrm{Ag}^{+}$criteria of NMED-051

The decontamination factors for removal of all other contaminant species are less than these.

In evaluation of these parameters, it is evident that overdesign to meet the most stringent conceivable requirements (without basis for this level of compliance) is unnecessarily costly. Practical applications and experience in evaporator operations indicate that all of the proposed NPDES discharge limits (with the possible exceptions of those for $\mathrm{NH}_{3}$ and $\mathrm{NO}_{3}{ }^{-}$) could be met by proper design and operation of an evaporator system as the final decontamination unit process. The nitrate compounds can be eliminated by the proposed chemical destruction unit. Therefore, a zero release scenario no longer is required.

Although the necessity for a zero release configuration (e.g., addition of a solar pond, or consideration of an evaporative cooling tower) cannot be demonstrated by practicality, it may be desirable to consider this option if regulatory agencies maintain that release requirements for LANL must be more stringent than those for other industrial operations. Other than this consideration, the same evaporator scenario used for compliance with livestock and wildlife watering restrictions can be utilized to comply with the intent of the new NPDES.

\subsubsection{Process Configurations Eliminated}

Based on the discussion presented in Section 4.16.1, there can no longer be a valid position for the development of a zero release scenario. If this postulate is acceptable, the following PCs (which represent the utilization of solar ponds for zero release) can be eliminated from further consideration:

- PC \#7: New Facility, TA-50 Waste Only - September 11, 1992 NMED Release Limits

- PC \#8: New Facility, TA-55 Waste Only - September 11, 1992 NMED Release Limits

- PC \#9: New Facility, Blended TA-55 and TA-50 Wastes September 11, 1992 NMED Release Limits 
- $\quad$ PC \#10: Upgrade Existing TA-50 Operations, TA-50 Waste Only September 11, 1992 NMED Release Limits

- PC \#11: Upgrade Existing Room 60 Operations, TA-55 Waste Only September 11, 1992 NMED Release Limits

- $\quad$ PC \#12: Upgrade Existing TA-50 operations, Blended TA-55 and TA-50 Wastes - September 11, 1992 NMED Release Limits

\subsubsection{PCs Sustained}

In consideration of the elimination of PCs \#7 through \#12 from further consideration, the following PCs represent those that will be sustained for further evaluation in this BAT study:

- PC \#1: Upgrade Existing TA-50 Operations, TA-50 Waste Only Wildlife and Livestock Watering Release Limits

- $\quad$ PC \#2: Upgrade Existing Room 60 Operations, TA-55 Waste Only Wildlife and Livestock Watering Release Limits

- $\quad$ PC \#3: Upgrade Existing TA-50 Operations, Blended TA-55 and TA-50 Wastes - Wildlife and Livestock Watering Release Limits

- PC \#4: New Facility, TA-50 Waste Only - Wildlife and Livestock Watering Release Limits

- PC \#5: New Facility, TA-55 Waste Only - Wildlife and Livestock Watering Release Limits

- PC \#6: New Facility, Blended TA-55 and TA-50 Wastes - Wildlife and Livestock Watering Release Limits

The subsequent sections of this document will develop additional criteria for the examination of these alternative technologies. 
Table 4-1 Chemical Analysis of Selected 1991 Samplings

\begin{tabular}{|c|c|c|c|c|c|c|}
\hline SPECIES & SYMBOL & $\begin{array}{c}\text { Analysis } 1 \\
3 / 22 / 91, \mathrm{mg} / \mathrm{L}^{(1)}\end{array}$ & $\begin{array}{c}\text { Analysis } 2 \\
3 / 25 / 91, \mathrm{mg} / \mathrm{L}^{(2)}\end{array}$ & $\begin{array}{c}\text { Analysis } 3 \\
4 / 12 / 91, \mathrm{mg} / \mathrm{L}^{(3)}\end{array}$ & $\begin{array}{c}\text { Analysis } 4 \\
4 / 15 / 91, \mathrm{mg} / \mathrm{L}^{(4)}\end{array}$ & $\begin{array}{c}\text { Analysis } 5 \\
\text { no date, } \mathrm{mg} / \mathrm{L}^{(5)}\end{array}$ \\
\hline arsenic & As & $.002 \pm .001$ & .003 & $<.02$ & $<.02$ & .77 \\
\hline barium & $\mathrm{Ba}$ & $<0.5$ & $<.5$ & .047 & .045 & .21 \\
\hline cadmium & $\mathrm{Cd}$ & $.001 \pm .001$ & .002 & $<.005$ & $<.005$ & $<0.00$ \\
\hline calcium & $\mathrm{Ca}$ & $28.9 \pm 2.9$ & 29. & 39.4 & 24. & 8.1 \\
\hline chloride & $\mathrm{Cl}$ & $51.6 \pm 5.2$ & 37.3 & 229. & 84.2 & 6.3 \\
\hline chromium & Tot $\mathrm{Cr}$ & $.037 \pm .022$ & .026 & .22 & 0.11 & .44 \\
\hline copper & $\mathrm{Cu}$ & $.224 \pm .002$ & .154 & .21 & 0.13 & $<.003$ \\
\hline cyanide & $\mathrm{Cn}$ & $.212 \pm .02$ & .087 & .59 & 0.255 & 6.15 \\
\hline fluoride & $\mathbf{F}$ & $2.66 \pm .27$ & 1.96 & 7.18 & 4.56 & 250. \\
\hline iron & $\mathrm{Fe}$ & $.995 \pm .10$ & .576 & .85 & 0.54 & $<.005$ \\
\hline lead & $\mathrm{Pb}$ & $.207 \pm .04$ & .138 & $<.009$ & 0.03 & .33 \\
\hline magnesium & $\mathrm{Mg}$ & $3.91 \pm 0.4$ & 4.41 & 3.3 & 3.7 & .57 \\
\hline mercury & $\mathrm{Hg}$ & $.0037 \pm .0004$ & .0036 & .0016 & .0013 & .004 \\
\hline nickel & $\mathrm{Ni}$ & $.225 \pm .04$ & 1.08 & .22 & 0.10 & .02 \\
\hline $\begin{array}{l}\text { nitrogen as } \\
\mathrm{NH}_{3}\end{array}$ & $\mathrm{NH}_{3}-\mathrm{N}$ & $13.8 \pm 1.4$ & 6.8 & 9.8 & 3.67 & 4.6 \\
\hline $\begin{array}{l}\text { nitrogen as } \\
\mathrm{NO}_{2}\end{array}$ & $\mathrm{NO}_{2}-\mathrm{N}$ & $.692 \pm .07$ & .322 & .125 & 0.068 & .05 \\
\hline $\begin{array}{l}\text { nitrogen as } \\
\mathrm{NO}_{3}\end{array}$ & $\mathrm{NO}_{3}-\mathrm{N}$ & $160 \pm 16$ & 89.7 & 980. & 325.0 & 28,000 \\
\hline phosphate & $\mathrm{PO}_{4}-\mathrm{P}$ & $1.90 \pm .4$ & 1.23 & 4.04 & 0.275 & .115 \\
\hline potassium & $\mathbf{K}$ & $55.2 \pm 5.5$ & 28.0 & 238. & 76.1 & 6500 \\
\hline selenium & $\mathrm{Se}$ & $.0006 \pm .0001$ & .0004 & 1.0 & $<0.01$ & 1.6 \\
\hline silica & $\mathrm{SiO}_{2}$ & $83 \pm 8$ & 84 & 80. & 87 & 8.0 \\
\hline silver & $\mathrm{Ag}$ & $.028 \pm .01$ & .014 & .006 & $<.006$ & .44 \\
\hline sodium & $\mathrm{Na}$ & $275 \pm 28$ & 280.0 & 1508. & 512 & 46.280 \\
\hline
\end{tabular}




\begin{tabular}{|c|c|c|c|c|c|c|}
\hline SPECIES & SYMBOL & $\begin{array}{c}\text { Analysis } 1 \\
3 / 22 / 91, \mathrm{mg} / \mathrm{L}^{(1)}\end{array}$ & $\begin{array}{c}\text { Analysis } 2 \\
3 / 25 / 91, \mathrm{mg} / \mathrm{L}^{(2)}\end{array}$ & $\begin{array}{c}\text { Analysis } 3 \\
4 / 12 / 91, \mathrm{mg} / \mathrm{L}^{(3)}\end{array}$ & $\begin{array}{c}\text { Analysis } 4 \\
4 / 15 / 91, \mathrm{mg} / \mathrm{L}^{(4)}\end{array}$ & $\begin{array}{c}\text { Analysis } 5 \\
\text { no date, } \mathrm{mg} / \mathrm{L}^{(s)}\end{array}$ \\
\hline sulfate & $\mathrm{SO}_{4}$ & $29.5 \pm 2.6$ & 17.8 & 18.4 & 0 & 0 \\
\hline \multirow[t]{3}{*}{ zinc } & $\mathrm{Zn}$ & $.316 \pm .032$ & .254 & .002 & 0.26 & $<.001$ \\
\hline & Tot-ALK & $127 \pm 13$ & 142 & 45 & 8 & 2150 \\
\hline & P-ALK & $30 \pm 5$ & 55 & $<5$ & $<5$ & 1970 \\
\hline Conductivity & $\mathrm{M} \mathrm{mho} / \mathrm{cm}$ & $1440 \pm 72$ & 840 & 7176 & 2682 & 188,600 \\
\hline $\mathrm{pH}$ & $\mathrm{pH}$ & $8.58 \pm .1$ & 9.48 & 7.28 & 6.24 & 12.4 \\
\hline $\begin{array}{l}\text { Total } \\
\text { Hardness }\end{array}$ & T Hard & $123.97 \pm 12.4$ & 106.8 & 88.5 & 75.2 & 22.7 \\
\hline \multirow{3}{*}{$\begin{array}{l}\text { Chemical } \\
\text { Oxygen } \\
\text { Demand }\end{array}$} & $\begin{array}{r}\mathrm{COD} \\
(\mathrm{meq} / \mathrm{L})\end{array}$ & $66 \pm 13$ & 68. & 81. & 36 & 303 \\
\hline & $\begin{array}{l}\sum \text { cations } \\
\text { (meq/L) }\end{array}$ & $16.01 \pm 1.6$ & 10.45 & 74.6 & 25.92 & 2179. \\
\hline & $\sum$ Anions & $16.07 \pm 1.6$ & 10.77 & 78.1 & 25.97 & 2255. \\
\hline $\begin{array}{l}\text { Total } \\
\text { Dissolved } \\
\text { Solids }\end{array}$ & $\begin{array}{c}\text { TDS } \\
\text { (filtered) }\end{array}$ & $1186 \pm 119$ & 916 & 6200. & 2400 & 188,000 \\
\hline $\begin{array}{l}\text { Total } \\
\text { Suspended } \\
\text { Solids } \\
\end{array}$ & TSS & $0 \pm 1$ & 4 & $<1.0$ & $<1.0$ & 226 \\
\hline
\end{tabular}

(1) Thursday influent - fairly delute

(2) Monday influent - very delute from weekend flow

(3) Friday influent - fairly concentrated

(4) Monday Sample - slightly deluted over weekend

() Room 60 superutant 
Table 4-2 Calculated Additional Decontamination Factors Required for Compliance with Selected Release Limits

\begin{tabular}{|c|c|c|c|c|c|c|c|c|c|c|c|}
\hline & & \multicolumn{2}{|c|}{ 5-Yr Averages } & \multicolumn{8}{|c|}{$\begin{array}{l}\text { Various Release Limits } \\
\text { And Additional Required D.F.'s To Meet The Release Requirements }\end{array}$} \\
\hline \multicolumn{2}{|c|}{ Species/Symbol } & $\begin{array}{l}\text { Current } \\
\text { Effluent } \\
\text { (mg/L) }\end{array}$ & $\begin{array}{l}\text { Current } \\
\text { D.F.'s }\end{array}$ & $\begin{array}{c}\text { Old } \\
\text { NPDES }\end{array}$ & $\begin{array}{c}\text { Required } \\
\text { Additional } \\
\text { D.F. }\end{array}$ & $\begin{array}{l}\text { L.S. \& W.L. } \\
\text { (Task 015) }\end{array}$ & $\begin{array}{c}\text { Required } \\
\text { Additional } \\
\text { D.F. } \\
\end{array}$ & $\begin{array}{l}\text { N.M.E.D. } \\
-051\end{array}$ & $\begin{array}{c}\text { Required } \\
\text { Additional } \\
\text { D.F. }\end{array}$ & $\begin{array}{l}\text { Sept. 11, } 1992 \\
\text { NPDES }\end{array}$ & $\begin{array}{c}\text { Required } \\
\text { Additional } \\
\text { D.F. } \\
\end{array}$ \\
\hline aluminum & $\mathrm{Al}^{+3}$ & & & 5.0 & & 5.0 & & 5.0 & & 0.087 & \\
\hline arsenic & As & $1.3 \mathrm{E}-3$ & $1.4 / 3$ & 0.03 & - & 0.02 & - & 0.074 & - & 0.040 & - \\
\hline barium & $\mathrm{Ba}$ & 0.07 & $1 / 3$ & - & & - & & - & & - & \\
\hline boron & $\mathbf{B}$ & - & - & 5.0 & & 5.0 & & 0.75 & & 0.74 & \\
\hline cadmium & $\mathrm{Cd}$ & $2.4 \mathrm{E}-03$ & $\sim 10$ & 0.17 & - & 0.05 & - & 0.0005 & 4.8 & 0.0022 & 1.1 \\
\hline calcium & $\mathrm{Ca}^{++}$ & 221 & - & - & & & & & & - & \\
\hline chloride & $\mathrm{Cl}^{-}$ & 122 & - & & & & & & & - & \\
\hline chlorine & $\mathrm{Cl}_{2}$ & - & - & - & - & - & & - & & 0.004 & $(?)$ \\
\hline chromium & $\mathrm{Cr}$ & 0.015 & $3 / 21$ & 4.0 & - & 1.0 & - & 0.0023 & 6.3 & 0.51 & - \\
\hline cobalt & Co & 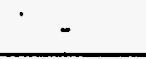 & - & 1.0 & & 1.0 & $(?)$ & 0.05 & $(?)$ & 0.05 & $(?)$ \\
\hline copper & $\mathrm{Cu}$ & 0.17 & $2 / 5$ & 1.0 & - & 0.5 & - & 0.0002 & 850 & 0.0178 & 9.6 \\
\hline cyanide & $\mathrm{CN}^{-}$ & 0.24 & - & - & & & & 0.008 & 30 & 0.0052 & 47 \\
\hline fluoride & $\mathrm{F}$ & 8.4 & - & & & & & & & & \\
\hline iron & $\mathrm{Fe}$ & & & & & & & & & & \\
\hline
\end{tabular}


Table 4-2 Calculated Decontamination Factors Required for Compliance with Selected Release Limits (Cont)

\begin{tabular}{|c|c|c|c|c|c|c|c|c|c|c|}
\hline \multirow[b]{2}{*}{ Species/Symbol } & \multicolumn{2}{|c|}{ 5-Yr Averages } & \multicolumn{8}{|c|}{$\begin{array}{l}\text { Various Release Limits } \\
\text { And Additional Required D.F.'s To Meet The Release Requirements }\end{array}$} \\
\hline & $\begin{array}{l}\text { Current } \\
\text { Effluent } \\
\text { (mg/L) }\end{array}$ & $\begin{array}{c}\text { Current } \\
\text { D.F.'s }\end{array}$ & $\begin{array}{c}\text { Old } \\
\text { NPDES }\end{array}$ & $\begin{array}{c}\text { Required } \\
\text { Additional } \\
\text { D.F. }\end{array}$ & $\begin{array}{l}\text { L.S. \& W.L. } \\
\text { (Task 015) }\end{array}$ & $\begin{array}{c}\text { Required } \\
\text { Additional } \\
\text { D.F. }\end{array}$ & $\begin{array}{l}\text { N.M.E.D. } \\
-051\end{array}$ & $\begin{array}{c}\text { Required } \\
\text { Additional } \\
\text { D.F. }\end{array}$ & $\begin{array}{l}\text { Sept. 11, } 1992 \\
\text { NPDES }\end{array}$ & $\begin{array}{c}\text { Required } \\
\text { Additional } \\
\text { D.F. }\end{array}$ \\
\hline lead & 0.029 & $4 / 24$ & 0.13 & - & 0.1 & - & 0.0045 & 6.4 & 0.0041 & 7.0 \\
\hline magnesium $\mathrm{Mg}^{++}$ & 1.7 & $3 / 21$ & & & & & & & & \\
\hline mercury & 3.7E-4 & $22 / 68$ & 0.03 & - & 0.01 & - & 0.00003 & 12.2 & 0.000012 & 30.5 \\
\hline nickel & 0.045 & $3 / 6$ & - & & - & & 0.0131 & 3.4 & 0.198 & - \\
\hline nitrogen as $\mathrm{NH}_{3}$ & 11.5 & - & $\mathrm{N} / \mathrm{A}$ & & - & & 0.10 & 115 & 0.030 & 384 \\
\hline nitrogen as $\mathrm{NO}_{2}^{-}$ & 3.9 & & & & & & & & & \\
\hline nitrogen as $\mathrm{NO}_{3}^{-}$ & 1,603 & - & - & & 10.0 & 160 & 10.0 & 160 & - & - \\
\hline phosphate & 0.63 & $6 / 18$ & & & & & & & & \\
\hline potassium & 168 & - & & & & & & & & \\
\hline selenium & $2.5 \mathrm{E}-3$ & $(?)$ & 0.05 & & 0.05 & & 0.0086 & - & 0.005 & - \\
\hline $\mathrm{SiO}_{2}$ & $\sim 70$ & & & & & & & & & \\
\hline silver & $4.2 \mathrm{E}-3$ & $2 / 25$ & - & & - & & 0.0001 & 42 & 0.00012 & 35 \\
\hline sodium & 706 & - & & & & & & & & \\
\hline sulfate & 185 & - & & & & & & & & \\
\hline vanadium & $(?)$ & & 0.10 & & 0.1 & & 0.05 & & 0.10 & \\
\hline zinc & 0.093 & $2 / 5$ & 63. & - & 5.0 & - & 0.0036 & 2.5 & 0.197 & - \\
\hline
\end{tabular}


Table 4-2 Calculated Decontamination Factors Required for Compliance with Selected Release Limits (Cont)

\begin{tabular}{|c|c|c|c|c|c|c|c|c|c|c|}
\hline \multirow[b]{2}{*}{ Species/Symbol } & \multicolumn{2}{|c|}{ 5-Yr Averages } & \multicolumn{8}{|c|}{$\begin{array}{l}\text { Various Release Limits } \\
\text { And Additional Required D.F.'s To Meet The Release Requirements }\end{array}$} \\
\hline & $\begin{array}{l}\text { Current } \\
\text { Effluent } \\
\text { (mg/L) }\end{array}$ & $\begin{array}{l}\text { Current } \\
\text { D.F.'s }\end{array}$ & $\begin{array}{c}\text { Old } \\
\text { NPDES }\end{array}$ & $\begin{array}{c}\text { Required } \\
\text { Additional } \\
\text { D.F. }\end{array}$ & $\begin{array}{l}\text { L.S. \& W.L. } \\
\text { (Task 015) }\end{array}$ & $\begin{array}{c}\text { Required } \\
\text { Additional } \\
\text { D.F. } \\
\end{array}$ & $\begin{array}{l}\text { N.M.E.D. } \\
-051 \\
\end{array}$ & $\begin{array}{c}\text { Required } \\
\text { Additional } \\
\text { D.F. }\end{array}$ & $\begin{array}{l}\text { Sept. 11, } 1992 \\
\text { NPDES }\end{array}$ & $\begin{array}{c}\text { Required } \\
\text { Additional } \\
\text { D.F. }\end{array}$ \\
\hline $\mathrm{pH}$ & & & $6-9$ & & $6-9$ & & $6-9$ & & & \\
\hline C.O.D. & 49 & $1 / 4$ & & & 125 & - & 125 & - & & \\
\hline T.D.S. & 3140 & & & & & & & & & \\
\hline T.S.S. & 0 & & & & & & 0 & & & \\
\hline \multicolumn{11}{|c|}{ Concentrations In $\mathrm{pCi} / \mathrm{L}$} \\
\hline tritium & $6.8 \times 10^{5}$ & & $3 \times 10^{6}$ & - & $3 \times 10^{6}$ & - & $2 \times 10^{4}$ & 34 & $2 \times 10^{4}$ & 34 \\
\hline radium $\quad \mathrm{Ra}^{225 \& 228}$ & & & 300 & & 30 & & 30 & & 30 & \\
\hline \multicolumn{11}{|c|}{ (Concentration in $\mathrm{nCi} / \mathrm{L}$ ) } \\
\hline plutonium $\mathrm{Pu}(239)$ & $90.5 \times 10^{-3}$ & 604 & & & $2.0 \times 10^{-3}$ & 4.5 & $30 \times 10^{-3}$ & 3.0 & & \\
\hline americium $\operatorname{Am}(241)$ & $138 \times 10^{-3}$ & 190 & & & $2.0 \times 10^{-3}$ & 6.9 & $30 \times 10^{-3}$ & 4.6 & & \\
\hline cesium $\quad \mathrm{Cs}(137)$ & 1.422 & - & & & 1.0 & 1.4 & 3.0 & - & & \\
\hline strontium $\quad \operatorname{Sr}(90)$ & 0.76 & $1 / 5$ & & & 0.5 & 1.5 & 1.0 & - & & \\
\hline $\mathrm{Co}(60)$ & & & & & 3.0 & & 5.0 & & & \\
\hline sodium $\quad \mathrm{Na}(22)$ & & & & & 6.0 & & 10.0 & & & \\
\hline strontium $\quad \operatorname{Sr}(89)$ & & & & & & & 70.0 & & & \\
\hline
\end{tabular}


Table 4-2 Calculated Decontamination Factors Required for Compliance with Selected Release Limits (Cont)

\begin{tabular}{|c|c|c|c|c|c|c|c|c|c|c|c|}
\hline \multirow{2}{*}{\multicolumn{2}{|c|}{ Species/Symbol }} & \multicolumn{2}{|c|}{ 5-Yr Averages } & \multicolumn{8}{|c|}{$\begin{array}{l}\text { Various Release Limits } \\
\text { And Additional Required D.F.'s To Meet The Release Requirements }\end{array}$} \\
\hline & & $\begin{array}{l}\text { Current } \\
\text { Effluent } \\
\text { (mg/L) } \\
\end{array}$ & $\begin{array}{l}\text { Current } \\
\text { D.F.'s }\end{array}$ & $\begin{array}{c}\text { Old } \\
\text { NPDES }\end{array}$ & $\begin{array}{c}\text { Required } \\
\text { Additional } \\
\text { D.F. }\end{array}$ & $\begin{array}{l}\text { L.S. \& W.L. } \\
\text { (Task 015) }\end{array}$ & $\begin{array}{c}\text { Required } \\
\text { Additional } \\
\text { D.F. } \\
\end{array}$ & $\begin{array}{l}\text { N.M.E.D. } \\
-051\end{array}$ & $\begin{array}{c}\text { Required } \\
\text { Additional } \\
\text { D.F. }\end{array}$ & $\begin{array}{l}\text { Sept. 11, } 1992 \\
\text { NPDES }\end{array}$ & $\begin{array}{c}\text { Required } \\
\text { Additional } \\
\text { D.F. } \\
\end{array}$ \\
\hline niobium & $\mathrm{Nb}(95)$ & & & & & 30.0 & & 60.0 & & & \\
\hline yttrium & $Y(88)$ & & & & & 10.0 & & 30.0 & & & \\
\hline zirconium & $\operatorname{Zr}(88)$ & & & & & 50.0 & & 100.0 & & & \\
\hline
\end{tabular}

$?=$ unable to evaluate

- = no value stated 
TABLE 4-3 RLWTF Influent Characteristics Compiled for BAT Study

\begin{tabular}{|c|c|c|c|}
\hline $\begin{array}{c}\text { Influent } \\
\text { Component }\end{array}$ & $\begin{array}{c}\text { TA-50-1 Influents } \\
\text { (Neat) } \\
\text { mg/L }\end{array}$ & $\begin{array}{c}\text { TA-50-1 Influents } \\
\text { (Blended) } \\
\mathrm{mg} / \mathrm{L}\end{array}$ & $\begin{array}{c}\text { TA-55 Emuent } \\
\text { (Supernate) } \\
\text { mg/L }\end{array}$ \\
\hline As & $.002 / .003$ & $.002 / .003$ & 0.77 \\
\hline $\mathrm{Ba}$ & $<0.5$ & $<0.5$ & 0.2 \\
\hline $\mathrm{Cd}$ & $.005 / 0.10$ & $.005 / 0.10$ & 0 \\
\hline $\mathrm{Ca}$ & $24 / 40$ & 40 & 8.1 \\
\hline $\mathrm{Cl}$ & $30 / 84$ & $200 / 500$ & 6310 \\
\hline Total $\mathrm{Cr}$ & $.02 / 0.25$ & $.02 / 0.25$ & $0.44 / 45$ \\
\hline $\mathrm{Cu}$ & $0.15 / 0.30$ & $0.15 / 0.30$ & $<.003$ \\
\hline $\mathrm{CN}$ & $0.2 / 0.6$ & $0.2 / 0.6$ & 6.15 \\
\hline F & $1 / 8$ & $1 / 8$ & 250 \\
\hline $\mathrm{Fe}$ & $0.5 / 1.0$ & $0.5 / 1.0$ & .005 \\
\hline $\mathrm{Pb}$ & $<.01 / 0.2$ & $<0.01$ & 0.33 \\
\hline $\mathrm{Mg}$ & $3 / 4$ & $3 / 4$ & 0.57 \\
\hline $\mathrm{Hg}$ & $.0004 / .005$ & $.0004 / .005$ & .0035 \\
\hline $\mathrm{Ni}$ & $0.1 / 1.0$ & $0.1 / 1.0$ & $<.02 / 31$ \\
\hline $\mathrm{NH}_{3}-\mathrm{N}$ & $3 / 15$ & 10 & 4.6 \\
\hline $\mathrm{NO}_{2}-\mathrm{N}$ & $.07 / 1.0$ & $.07 / 1.0$ & .05 \\
\hline $\mathrm{NO}_{3}-\mathrm{N}$ & $90 / 325$ & 980 & 28,000 \\
\hline $\mathrm{PO}_{4}-\mathrm{P}$ & $0.3 / 40$ & $0.3 / 40$ & 0.11 \\
\hline $\mathbf{K}$ & $28 / 76$ & 240 & 6500 \\
\hline $\mathrm{Se}$ & $<0.1 / 1.0$ & 1.0 & 1.6 \\
\hline $\mathrm{SiO}_{2}$ & $80 / 87$ & 80 & 8 \\
\hline $\mathrm{Ag}$ & $<.006 / 0.03$ & $<.006$ & 0.44 \\
\hline $\mathrm{SO}_{4}$ & $0 / 26$ & $18 / 26$ & 0 \\
\hline
\end{tabular}


Table 4-3 RLWTF Influent Characteristics Compiled for BAT Study (Cont)

\begin{tabular}{|c|c|c|c|}
\hline $\begin{array}{c}\text { Influent } \\
\text { Component }\end{array}$ & $\begin{array}{c}\text { TA-50-1 Influents } \\
\text { (Neat) } \\
\mathrm{mg} / \mathrm{L} \\
\end{array}$ & $\begin{array}{c}\text { TA-50-1 Influents } \\
\text { (Blended) } \\
\mathrm{mg} / \mathrm{L}\end{array}$ & $\begin{array}{c}\text { TA-55 Emluent } \\
\text { (Supernate) } \\
\text { mg/L }\end{array}$ \\
\hline $\mathrm{Na}$ & $275 / 512$ & 1508 & 46,280 \\
\hline $\mathrm{Zn}$ & $0.25 / 0.3$ & $<.002$ & $<.001$ \\
\hline Total Alkalinity & $8 / 150$ & 45 & 2150 \\
\hline P-Alkalinity & -- & -- & -- \\
\hline Conductivity (Micromho/cm) & $840 / 2680$ & 7176 & 188,680 \\
\hline $\mathrm{pH}$ & $4 / 10$ & 7.3 & 12 \\
\hline \multicolumn{4}{|l|}{ Total Hardness } \\
\hline $\mathrm{COD}$ & $4 / 123$ & 81 & 303 \\
\hline$\sum$ Cations (meg/l) & -- & -- & -- \\
\hline$\sum$ Anions (meg/l) & - & - & - \\
\hline TDS & $900 / 2400$ & 6200 & 188,000 \\
\hline TSS & $4 / 45$ & $?$ & 226 \\
\hline $\mathrm{Be}$ & -- & - & -- \\
\hline Al & -- & -- & -- \\
\hline B & -- & - & -- \\
\hline Co & - & - & - \\
\hline Vn & $\cdots$ & -- & -- \\
\hline Tritium & -- & - & -- \\
\hline $\mathrm{Ra}^{225+228}$ & -- & -- & -- \\
\hline $\mathrm{Pu}^{239}$ & $1 / 100 \mathrm{nCi} / \mathrm{L}$ & - & - \\
\hline $\mathrm{Am}^{2 / 1}$ & $1 / 20 \mathrm{NCi} / \mathrm{L}$ & -- & - \\
\hline $\mathrm{Cs}^{137}$ & $0.7 / 11 \mathrm{nCi} / \mathrm{L}$ & -- & - \\
\hline $\mathrm{Sr}^{90}$ & $0.55 / 3.4 \mathrm{nCi} / \mathrm{L}$ & $\cdots$ & - \\
\hline $\mathrm{Co}^{60}$ & $0.7 / 0.8 \mathrm{nCi} / \mathrm{L}$ & - & - \\
\hline $\mathrm{Na}^{22}$ & $0.96 \mathrm{nCi} / \mathrm{L}$ & -- & - \\
\hline $\mathrm{Sr}^{88}$ & $0.5 / 0.75 \mathrm{nCi} / \mathrm{L}$ & -- & -- \\
\hline
\end{tabular}


Table 4-3 RLWTF Influent Characteristics Compiled for BAT Study (Cont)

\begin{tabular}{|c|c|c|c||}
\hline $\begin{array}{c}\text { Influent } \\
\text { Component }\end{array}$ & $\begin{array}{c}\text { TA-50-1 Influents } \\
\text { (Neat) } \\
\mathrm{mg} / \mathrm{L}\end{array}$ & $\begin{array}{c}\text { TA-50-1 Influents } \\
\text { (Blended) } \\
\mathrm{mg} / \mathrm{L}\end{array}$ & $\begin{array}{c}\text { TA-55 Emuent } \\
\text { (Supernate) } \\
\mathrm{mg} / \mathrm{L}\end{array}$ \\
\hline \hline $\mathrm{Nb}^{95}$ & $0.9 \mathrm{nCi} / \mathrm{L}$ & - & -- \\
\hline $\mathrm{Y}^{88}$ & $0.5 / 13 \mathrm{nCi} / \mathrm{L}$ & -- & - \\
\hline $\mathrm{Zr}^{88}$ & $0.4 / 6 \mathrm{nCi} / \mathrm{L}$ & -- & - \\
\hline
\end{tabular}

? = unable to evaluate

-. $\quad=$ no value stated 


\begin{tabular}{|c|c|c|c|c|c|}
\hline Program/Process & $\begin{array}{l}\text { Volume } \\
(1 / y r)\end{array}$ & $\begin{array}{l}\text { Activity } \\
\text { (uCi/liter) }\end{array}$ & $\begin{array}{l}\text { Type of Waste } \\
\text { (Isotope) }\end{array}$ & $\begin{array}{l}\text { Life Span } \\
\text { (yr) }\end{array}$ & $\begin{array}{l}\text { Start Up } \\
\text { date }\end{array}$ \\
\hline Omega West Reactor & 550,000 & $3 \mathrm{uCi} / \mathrm{l}$ & $\begin{array}{l}\text { Fission products, } \\
\text { tritium, Map isotopes }\end{array}$ & 50 & $01 \mathrm{Jan}, 95$ \\
\hline $\begin{array}{l}\text { ATW, Accelerator Transmutation } \\
\text { Waste }\end{array}$ & "TBD" & "TBD" & $\begin{array}{l}\text { Fission products, TRU, } \\
\text { \& MAP Isotopes }\end{array}$ & 10 & $01 \mathrm{Jan}, 02$ \\
\hline $\begin{array}{l}\text { Center for Material Science } \\
\text { Laboratory, TA3 }\end{array}$ & 5000 & $20 \mathrm{nCi} /$ liter & $\mathrm{Na}-22, \mathrm{Zr}-95$ & 40 & $01 \mathrm{Jul}, 93$ \\
\hline $\begin{array}{l}\text { Solid State Synthesis } \\
\text { Electronic \& Magnetic } \\
\text { Materials, TA3, SM29 }\end{array}$ & 5 & 10 & Uranium \& Thorium & 10 & $15 \mathrm{Mar}, 93$ \\
\hline Moly 99 & 100 & 5 & Moly 99 & 10 & $01 \mathrm{Jan}, 94$ \\
\hline $\begin{array}{l}\text { Ulysses, U235 recovery } \\
\& \text { recycle TA3, SM } 29\end{array}$ & 20,000 & 5 & Uranium & 50 & 01 Feb, 97 \\
\hline $\begin{array}{l}\text { Hanford Tank chemistry, } \\
\text { TA3, SM29 }\end{array}$ & 500 & $.5 \mathrm{uCi} /$ liter & $\begin{array}{l}\text { Uranium, fission } \\
\text { products, TRU }\end{array}$ & 10 & 01 Sep, 92 \\
\hline U238 Processing, TA3, SM66 & 20,000 & $2 \mathrm{nCi} / \mathrm{liter}$ & $\mathrm{U}-238$ & 25 & $01 \mathrm{Jan}, 93$ \\
\hline $\begin{array}{l}\text { Weapons Engineering Tritium } \\
\text { Facility (WETF), TA16 }\end{array}$ & 30,000 & $2.5 \mathrm{uCi} / \mathrm{L}$ & Tritium & 50 & $01 \mathrm{Jan}, 93$ \\
\hline $\begin{array}{l}\text { Hanford Tank Safety Issues, } \\
\text { TA21 }\end{array}$ & 50 & $.5 \mathrm{uCi} /$ liter & $\begin{array}{l}\text { Uranium, fission } \\
\text { products, TRU }\end{array}$ & 10 & 01 Sep, 92 \\
\hline $\begin{array}{l}\text { Tritium Systems Tests } \\
\text { Assembly, TSTA, TA21 }\end{array}$ & 8000 & $10 \mathrm{uCi} / \mathrm{L}$ & Tritium & 10 & 01 Jan, 93 \\
\hline $\begin{array}{l}\text { Tritium Science and Fabrication } \\
\text { Facility, TA21 }\end{array}$ & 8000 & $10 \mathrm{uCi} / \mathrm{L}$ & Tritium & 10 & $01 \mathrm{Jan}, 93$ \\
\hline $\begin{array}{l}\text { ICF, AGEX II Traget Fabrication, } \\
\text { TA35 }\end{array}$ & 50 & $5 \mathrm{uCi}$ & $\begin{array}{l}\text { Tritium \& Uranium } \\
\text { D38 }\end{array}$ & 25 & $01 \mathrm{Jan}, 93$ \\
\hline Fission product separation, TA-48 & 200 & $.5 \mathrm{uCi} / \mathrm{liter}$ & $\begin{array}{l}\text { Uranium, fission } \\
\text { products, TRU }\end{array}$ & 10 & 01 Sep, 92 \\
\hline Actinide Separation, TA- 44 , PF-4 & 200 & $.5 \mathrm{uCi} /$ liter & $\begin{array}{l}\text { Uranium, fission } \\
\text { products, TRU }\end{array}$ & 10 & $01 \mathrm{Sep}, 92$ \\
\hline $\begin{array}{l}\text { Actinide Organametalic } \\
\text { chemistry, TA21 }\end{array}$ & 2000 & $.10 \mathrm{uCi}$ & U-235, Thorium & 20 & 01 Jan, 93 \\
\hline
\end{tabular}


Table 4-4 Future Waste Streams to the RLWTF (cont.)

Program/Process

Uranium processing chemistry, TA21

Condensed Matter Physics, TA3-34

Medical Isotope production, TA48, Radiochemistry site

Deuterium-Tritium Fuel Cycle, $\quad 2100$ TA21

Mechanical Fabrication, TA3, SM102

2000

100

\section{Volume \\ $(1 / \mathbf{y r})$ \\ Type of Waste \\ (Isotope)}

1000

750,000
U-235, Thorium

U238, Thorium

Activated Materials

Tritium

U238, U235
Life Span

(yr)

20

50

01 Jan, 93

01 Jan, 93

10

01 Jan, 93

50

Start Up date

$01 \mathrm{Jan}, 93$

20

01 Jan, 93 


\subsection{SELECTION OF CANDIDATE TECHNOLOGIES}

\subsection{DEVELOPMENT OF CANDIDATE TECHNOLOGIES}

\subsubsection{Evaluation of Selected Treatment Configurations}

The PCs presented in Section 3 represent the arrangement of the most feasible technologies and systems that can provide the desired level of contaminant removal necessary for compliance with prescribed discharge limits. Of the twelve configuration scenarios, those associated with the 'TA-50 blended waste stream' represent technologies that will provide treatment of the waste stream that is the combined TA-50/Room 60 influent to existing plant operations. In the other configurations, either the unblended TA-50 waste stream is addressed, or the TA-55 waste stream is presented as a process stream not blended with the 'neat' TA-50 waste stream prior to treatment, but rather is treated as a separate waste stream.

\subsubsection{Development of Integrated Treatment Scenarios}

Upon examination of the twelve PCs it becomes evident that some of the scenarios address the treatment of only part of the waste stream currently processed in the existing TA-50 operations. This is consistent with the directives from LANL to develop both combined and separated options for the treatment of the TA-50 and Room 60/TA-55 waste streams.

In PCs $\# 1,4,7$, and 10 , the unblended (neat) TA-50 treatment process is addressed separately from the existing process of treating the two waste streams (the TA-50 influent with the Room 60/TA-55 components). In PCs \#2, 5, 8, and 11, the Room 60/TA-55 waste stream is treated as a separate concern. In PCs \#3, 6, 9, and 12, blending of the TA-55/Room 60 waste stream into the primary treatment process occurs after the radiological contamination from the TA-55 waste stream has been treated and removed so that the effluent from this process is to be blended into the TA-50 process influent. The TA-50 blended case is a combination of the TA-55 effluent after removal of radionuclides with the primary TA-50 influent stream. The summary of this evaluation is provided as Table 5-1.

In order to provide a basis for comparison of technical alternatives, it was necessary to develop process scenarios that included the integrated treatment of both the TA-50 and TA-55 waste streams in a single scenario, even if the proposed treatment involved totally separate processing for both waste streams. Only by addressing the treatment of both waste streams in a single proposed scenario could a common, comprehensive basis for equitable comparison be established. 


\subsubsection{Development of Candidate Technologies}

After evaluating the various remaining PCs (without consideration of the zero release option eliminated in Section 4) and combining the similar options, the following four Candidate Technologies may be postulated for further technical evaluation:

1. Candidate Technology \#1: Upgrade both the TA-50 waste stream processes and the Room 60/TA-55 processes to meet Wildlife and Livestock Watering Limits;

2. Candidate Technology \#2: Upgrade the TA-50 waste stream processes to meet Wildlife and Livestock Watering Limits, and construct a new facility for the Room 60/TA-55 processes;

3. Candidate Technology \#3: Construct a new facility for the TA-50 waste streams, and upgrade the Room 60/TA-55 processes to meet Wildlife and Livestock Watering Limits; and

4. Candidate Technology \#4: Construct a new facility to accommodate both the TA-50 waste stream and the Room 60/TA-55 waste stream to meet Wildlife and Livestock Watering Limits.

The block/process flow diagrams for these four Candidate Technologies are presented in Appendix E.

\subsection{DISCUSSION OF CANDIDATE TECHNOLOGIES SELECTED}

\subsubsection{Candidate Technology \#1: Upgrade the TA-50 and TA-55 Operations}

In Candidate Technology \#1 the proposed scenario includes upgrades to both of the existing TA-50 and the Room 60/TA-55 waste stream processes to ensure compliance with the appropriate regulatory discharge criteria (e.g., wildlife and livestock watering limits). This scenario is analogous PC \#3 from Section 3, which represents the combined process schemes for PC \#1 and PC \#2.

Candidate Technology \#1 would involve the retrofit and upgrading of the existing TA-55/Room 60 unit processes in order to provide pretreatment of the primary source of radioactivity routed to the RLWTF. These unit processes would include nitrate removal on the acidic waste stream, and combination of the acidic and caustic waste streams (with additional $\mathrm{pH}$ adjustment chemical addition) in order to accomplish neutralization. The neutralized waste would be transferred into a gravimetric physical separation process, which would include chemical addition and precipitation, flocculation, 
and sedimentation. The clarified liquid would be routed through a filtration unit, while the solids and sludges from the sedimentation process would be transferred for fixation as a cement paste and subsequent disposal. The filtered supernate from this pretreatment process would be routed to the primary influent of the main TA-50 operations.

This scenario also would involve retrofit/upgrading of the existing TA-50 unit processes. The combined ('blended') influent would be transferred into a gravimetric physical separation process (similar to the smaller pretreatment system for TA-55), which would involve precipitation, flocculation, and sedimentation. The clarified liquid would be routed to a filtration unit. The sediment sludges and solids would be transferred to a decanting unit for further liquids removal. The thickened sludge would be transferred to a rotary vacuum precoat filter for final dewatering, and the dewatered filter cake sent to a fixation unit for processing into a final waste form as saltstone.

The filtered waste stream would be transferred to an evaporation unit for final removal of solid contaminants, and the bottoms from this evaporator would be routed to the saltstone fixation process. The evaporator process stream (overheads) would be sent to a condenser for conversion to liquid form, routed through a $\mathrm{UV} / \mathrm{O}_{3}$ organics destruction process, and collected in a holdup tank for analysis prior to discharge at a permitted outfall.

\subsubsection{Candidate Technology \#2: Upgrade the TA-50 Operations, and Construct a New Room 60/TA-55 Facility}

Candidate Technology \#2 proposes the upgrading of the existing TA-50 operations, while constructing a new facility or enclosed area for the Room 60/TA-55 waste stream. This scenario represents a combination of the primary process steps presented in PC \#1 and PC \#5 from Section 3.

In the initial pretreatment process for Candidate Technology \#2, a new facility would be constructed to provide a totally separated treatment scheme for the TA-55 waste stream. This arrangement would involve collection tanks for both the acidic and caustic influent streams. A nitrate destruction unit would be used for nitrates reduction via a batch process for the acidic wastes. The two influent streams would be neutralized by blending and $\mathrm{pH}$ adjustment in a neutralization/holding tank, which would accumulate a volume of waste for batch pretreatment processing. The collected waste would be routed to a sludge contact separator for treatment (initial removal of TSS) by sludge contacting. The primary pretreatment process of gravimetric physical separation would be conducted using a flash mixer, flocculator and clarifier. The thickened sludge from this operation would be transferred to a sludge concentration unit, and the concentrated bottoms from this operation routed to a chemical fixation unit for disposal preparation. The clarified liquid from 
the gravimetric separation process would be collected in a surge tank that would route the TA-55 supernate to the influent of the TA-50 primary operations.

The proposed upgrades and final processing scheme for the main TA-50 operations would be identical to those presented for this portion of Candidate Technology \#1.

\subsubsection{Candidate Technology \#3: Construct a New TA-50 Facility, and Upgrade the Room 60/TA-55 Operations}

Candidate Technology \#3 proposes the upgrading of the existing TA-55/Room 60 operations, while constructing a new facility for the primary TA-50 waste treatment operations. This scenario represents a combination of the primary process steps presented in PC \#2 and PC \#4 from Section 3.

In Candidate Technology \#3 the modifications, retrofits, and upgrades to the TA-55 operations would be identical to those presented for this portion of the treatment scheme in Candidate Technology \#1. The final pretreatment effluent/supernate would be routed to the TA-50 influent.

The proposed TA-50 portion of this scenario involves the construction of a totally separate new facility for the management of the TA-50 waste stream. The combined ('blended') influent to the new TA-50 facility would be collected in an influent storage tank system for batch processing, and neutralization could be effected as necessary in this operation. The collected liquid waste would be routed to a sludge contact separator as the first processing step of solids separation. The main unit process involved in the gravimetric physical separation of solids utilizes a flash mixer, flocculator and clarifier. The sediments and sludges from this separation process would be routed to a sludge concentrator, and the bottoms of this operation sent to a fixation unit for processing into saltstone and to subsequent ultimate disposal. The clarified liquid from the separation process would be collected in a surge tank as feed to an evaporation unit. The evaporation process would involve a two-part process with a mechanical vapor recompression evaporator with cooler, and a thin-film finishing evaporator/compressor for the concentrate from the first evaporator. The bottoms of this operation would be sent to the saltstone fixation process. The evaporator process stream (overheads) would be routed through a cooler for condensation, and on to a $\mathrm{UV} / \mathrm{O}_{3}$ organics destruction unit. The final effluent would be collected in a holdup tank for analysis prior to release at a permitted outfall. 


\subsubsection{Candidate Technology \#4: Construct a New Facility for the TA-50 and Room 60/TA-55 Operations}

In the final scenario, Candidate Technology \#4 a new facility is proposed for the combined TA-55/Room 60 and TA-50 waste treatment operations. This scenario is analogous to PC \#6 from Section 3, which incorporates process elements of PC \#4 and PC \#5.

The basis of Candidate Technology \#4 is a configuration that would entail a new facility to combine the general features discussed in process configurations for optimum treatment based on the current waste characterization. The TA-55 acidic waste stream would be collected in a receiving tank treated via batch processing in a nitrate destruction process. The treated acidic waste stream would be combined with the TA-55 caustic waste stream in a neutralization and holding tank, which would include an additional $\mathrm{pH}$ adjustment process. The neutralized TA-55 waste stream would be retained in the Neutralization/Holding Tank until a predetermined volume could be collected to initiate a batch or campaign of processing the TA-55 waste stream through the proposed facility. During this period, the normal TA-50 influent would be accumulated in a designated storage tank until processing of the TA-55 waste was completed. TA-50 influent processing could then be resumed until such time as another "batch" of TA-55 waste had accumulated. Each of these waste streams would be separately routed through a sludge contact separator for initial solids treatment, and subsequently to the primary gravimetric physical separation process of flash mixing, flocculation, and clarification. The thickened sludge from the separation process would be routed to a sludge concentrator, and the concentrated sludge transferred to a saltstone fixation process. The clarified liquid from the separation process would be routed to a surge tank to be held as feed to the downstream evaporation processes.

Evaporation would be conducted using a two-part process, which would include a mechanical vapor recompression evaporator with cooler, and a thinfilm finishing evaporator/compressor for the concentrate from the first evaporator. The bottom from the final evaporation process would be routed to the saltstone fixation process. The process stream (overheads) from the recompression evaporator would be sent through a $\mathrm{UV} / \mathrm{O}_{3}$ organics destruction unit, and on to a holdup tank for analysis prior to discharge via a permitted outfall. 


\subsection{ANALYSIS OF ENVIRONMENTAL ISSÜES FOR CANDIDATE TECHNOLOGIES}

Although the twelve Process Configurations were screened in Section 4 based on environmental and regulatory compliance considerations, it is important that the Candidate Technologies be evaluated for relative ranking based on consideration of environmental issues. This analysis focuses on the aspects of the environment that may be affected by the liquid effluent discharge, as well as the operational and regulatory considerations.

The environmental issues that were considered for this evaluation reflect the specific concerns discussed in previous sections, and the information derived from site documents and references.

\subsubsection{Environmental Releases}

Considerations in this category can include the concentration of significant radionuclides at the point of discharge, the accumulated quantity of activity in environmental media, total annual discharge of contaminants, the potential for radiological dose to the environmental and human pathways, and ecological impacts.

\subsubsection{Regulatory Compliance}

Regulatory Compliance topics include compliance with proposed discharge limits such as those established by the NMED Wildlife and Livestock Watering guidelines and the predicted NPDES requirements, as well as the flexibility of the technology to comply with potential future regulatory requirements and restrictions.

Also, compliance with Derived Concentration Guidelines of DOE Order 5400.5 should be addressed.

\subsubsection{Waste Generation and Disposal}

Waste generation and disposal factors become increasingly important as the radiological content, type and waste form vary. Issues of importance include the minimization of radiological, TRU, and mixed wastes, the control of secondary wastes, and the minimization and waste form management of sludges and stabilized wastes.

\subsubsection{Environmental Analysis of Candidate Technology \#1}

As presented in Section 5.2.1, the configuration for Candidate Technology \#1 involves the upgrading of both of the existing TA-50 and the Room 60/TA-55 
waste stream processes to ensure compliance with the appropriate regulatory discharge criteria. Since the primary purpose of these upgrades would be to achieve compliance with the regulatory directives, it would be inherent in design that controls be included to regulate environmental releases. The processing technologies would be designed to remove the contaminants of concern from the waste stream and to immobilize these contaminants in a form acceptable for disposal. New processing equipment could be expected to be more efficient than the existing technology in the decontamination of waste streams. Radiological exposures could be minimized by improved design.

Ecological concerns would be addressed in two ways (1) since the effluent stream would be treated to comply with strict discharge regulations, there would be no anticipated environmental impacts as a result of the regulated release of the final effluent, and (2) since no new facilities are to be constructed, there are no impacts on land or surrounding environs.

With regulatory compliance as a design basis, it would be expected that permit discharge limits and DCG requirements would be met as a matter of routine operations. However, since the existing processing facility and support systems would be utilized, there would be limited flexibility for future process changes without significant major renovations and expense.

It is anticipated that the improved treatment processes will aid in the management of waste generation and disposal. The Candidate Technology \# 1 scenario provides retrofit and upgrading of the existing TA-55/Room 60 unit processes in order to provide pretreatment of the primary source of radioactivity routed to the RLWTF. This should minimize TRU waste generation. Benefits also would be anticipated (based on improved process efficiency and equipment configuration) for mixed waste minimization, limiting of generation of secondary wastes, and reducing the volumes of sludges to be stabilized.

\subsubsection{Environmental Analysis of Candidate Technology \#2}

As presented in Section 5.2.2, the configuration for Candidate Technology \#2 involves the upgrading of the primary TA-50 waste stream processes, and the construction of a new facility for the pretreatment of the Room 60 influent. Design controls would be included to regulate environmental releases and the processing technologies would be designed to remove the contaminants of concern from the waste stream and to immobilize these contaminants in a form acceptable for disposal. The new processing facility and upgraded process equipment could be expected to be more efficient than the existing technology in the decontamination of waste streams. Radiological exposures could be minimized by improved design. 
There would be no anticipated ecological/environmental impacts as a result of the regulated release of the final effluent from this option. And although the Room 60/TA-55 waste stream would be routed to a new facility, it is anticipated that this facility could be collocated with the existing TA-50 operation, creating a minimal (if any) impact on land and surrounding environs.

The regulatory compliance design basis would serve to ensure that permit discharge limits and DCG requirements would be met as a matter of routine operations. However, since most of the existing processing facility and support systems would be utilized, there would be limited flexibility for future process changes without significant major renovations and expense.

It is anticipated that the improved treatment processes will aid in the management of waste generation and disposal. The Candidate Technology \#2 scenario provides a new processing facility for the TA-55/Room 60 waste stream, which will provide pretreatment of the primary source of radioactivity routed to the RLWTF. This should minimize TRU waste generation. Benefits also would be anticipated (based on improved process efficiency and equipment configuration) for mixed waste minimization, limiting of generation of secondary wastes, and reducing the volumes of sludges to be stabilized.

\subsubsection{Environmental Analysis of Candidate Technology \#3}

The discussion of Candidate Technology \#3 in Section 5.2.3 addresses the construction of a new facility to replace the present TA-50 operations, with an upgrade of the existing Room 60 processes. As with previous options, design controls would be included to regulate environmental releases. The processing technologies would be designed to remove the contaminants of concern from the waste stream and to immobilize these contaminants in a form acceptable for disposal. The new TA-50 processing facility and upgraded process equipment for Room 60 could be expected to be more efficient than the existing technology in the decontamination of waste streams. In particular, the design of a all-new primary process should provide an extra level of decontamination efficiency. Radiological exposures could be minimized by improved facility and equipment design.

There would be no anticipated ecological/environmental impacts as a result of the regulated release of the final effluent from this option. However, with the construction of a new facility, there will be inundation of previously unused land, which may be perceived as an ecological detriment.

The regulatory compliance design basis would serve to ensure that permit discharge limits and DCG requirements would be met as a matter of routine operations. Also, with the construction of a new primary processing facility 
and support systems, there would be a significant amount of design flexibility for future process changes.

It is anticipated that the improved treatment processes will aid in the management of waste generation and disposal. The Candidate Technology \#3 scenario provides a new processing facility for the main TA-50 waste stream, and also provides pretreatment of the primary source of radioactivity routed to the RLIT. This should minimize TRU waste generation. Benefits also would be anticipated (based on improved process efficiency and equipment configuration) for mixed waste minimization, limiting of generation of secondary wastes, and reducing the volumes of sludges to be stabilized.

\subsubsection{Environmental Analysis of Candidate Technology \#4}

The analysis of Candidate Technology \#4 in Section 5.2.4 addresses the scenario for the construction of an all-new facility to replace both the TA-50 and Room 60/TA-55 operations. As with previous options, design controls would be included to regulate environmental releases. The processing technologies would be designed for maximum effectiveness in the removal of contaminants of concern from the waste stream and immobilization of these contaminants in a form acceptable for disposal. The new facility could be expected to be the most efficient option for the decontamination of waste streams. Radiological exposures could be minimized by improved facility and equipment design.

There would be no anticipated ecological/environmental impacts as a result of the regulated release of the final effluent from this option. However, with the construction of a new facility to replace the existing operations, there will be inundation of previously unused land, which may be perceived as an ecological detriment.

The regulatory compliance design basis would serve to ensure that permit discharge limits and DCG requirements would be met as a matter of routine operations. Also, with the construction of a new facility and support systems, there would be a maximum amount of design flexibility for future process changes.

It is anticipated that the improved treatment processes will aid in the management of waste generation and disposal. The Candidate Technology \#4 scenario provides a new single processing facility for the main TA-50 waste stream and also provides separate treatment of the primary source of radioactivity the TA-55 waste stream. This should also minimize the TRU waste generation. Benefits could be anticipated (based on improved process efficiency and equipment configuration) for mixed waste minimization, limiting 
of generation of secondary wastes, and reducing the volumes of sludges to be stabilized.

\subsubsection{Summary Environmental Evaluation of Candidate Technologies}

Since the primary focus for the evaluation of alternatives to the existing TA-50 operations was for reasons of compliance with environmental regulations and pollution control requirements, it would be expected that all alternatives identified as Candidate Technologies would address at the least the minimum requirements for these issues.

Based on the generalized evaluations provided in Section 5.3.5 through 5.3.7 and information from previous sections, a summary analysis is presented in Table 5-2, which categorizes each primary topic related to environmental issues and criteria. The rankings of each Candidate Technology (based on the referenced evaluations and review of related materials specified in Section 10) are presented in order of perceived conformance to operational requirements.

\subsubsection{Status of Candidate Technologies}

Since the environmental compliance issues are inherent in the configuration and selection of technology for each of the four Candidate Technologies, each of these options is relatively comparable in conformance to environmental compliance. None of the Candidate Technologies can be eliminated strictly on the basis of environmental issues and criteria. In general, the ranking of the four options (from most favorable to least favorable, based on Table 5-2) for the issues and considerations addressed in this section is:

1. Candidate Technology \#4

2. Candidate Technology \#3

3. Candidate Technology \#2

4. Candidate Technology \#1

The differences in these rankings are small, and do not permit the selection of any single Candidate Technology alone on the basis of environmental issues and criteria. 


\begin{tabular}{|c|c|c|c|}
\hline \multicolumn{4}{|c|}{$\begin{array}{l}\text { TABLE 5-1 Evaluation of the Selected Process Configurations } \\
\text { for Comparative RLWTE Waste Stream Treatment }\end{array}$} \\
\hline Process Configuration & $\begin{array}{l}\text { Treats } \\
\text { TA-50 Stream }\end{array}$ & $\begin{array}{l}\text { Treats } \\
\text { TA-55 Stream }\end{array}$ & $\begin{array}{r}\text { Level of } \\
\text { Treatment }\end{array}$ \\
\hline$\# 1$ & YES & NO & PARTIAL \\
\hline$\# 2$ & NO & YES & PARTIAL \\
\hline \#3 & YES & YES & COMPLETE \\
\hline$\# 4$ & YES & NO & PARTIAL \\
\hline$\# 5$ & NO & YES & PARTIAL \\
\hline$\# 6$ & YES & NO & COMPLETE \\
\hline$\# 7$ & YES & NO & PARTIAL \\
\hline$\# 8$ & NO & YES & PARTIAL \\
\hline$\# 9$ & YES & YES & COMPLETE \\
\hline$\# 10$ & YES & NO & PARTIAL \\
\hline$\# 11$ & NO & YES & PARTIAL \\
\hline \#12 & YES & NO & COMPLETE \\
\hline
\end{tabular}


TABLE 5-2

Comparative Analysis of Environmental Issues and Criteria ${ }^{1}$

ISSUES/CRITERIA

$\underline{\mathrm{CT} 1}$

$\underline{C T 2}$

$\underline{\mathrm{CT} 3}$

$\underline{\mathrm{CT} 4}$

1. Environmental Releases

Release of Radionuclides

1

1

1

1

Total Annual Discharge of

2

2

1

1

Contaminants

Dose Contributions

4

3

2

1

Ecological Impacts

1

1

2

2

2. Regulatory Compliance

Compliance with Proposed

1

.1

1

1

Discharge Limits

Flexibility for Future

4

3

2

1

Compliance

Compliance with Derived

Concentration Guidelines

1

1

1

1

3. Waste Generation and Disposal

Minimization of TRU Waste 1

1

1

1

Minimization of Mixed Waste 2

2

1

1

Secondary Liquid Wastes 1

1

1

1

Sludge/Sediment Volumes $\underline{2}$

OVERALL

$\underline{2}$

$\underline{2}$

1

1

3

2

1

1 Rankings provided in numerical order of most favorable (1) to least favorable (4) 


\subsection{ANALYSIS OF OPERATIONAL ISSUES}

\subsection{OPERATIONAL EVALUATION OF THE EXISTING FACILITY}

The design and construction of the 30-year old LANL Radioactive Liquid Waste Plant (RLWP), TA-50 do not meet criteria currently in effect primarily because the construction of the RLWP predates many of these requirements and regulations. Relevant requirements that have been promulgated subsequent to RLWP construction include consideration for design of radiological treatment facilities, seismic qualification, safety-class emissions monitors, primary radiation control, multiple confinement barriers, ventilation, primary/secondary containment of tanks and transfer lines, underground storage tank management, and radiological health/ALARA controls.

It is not surprising that many of the systems and structures associated with the RLWP have deteriorated over the past 30 years. This aging and deterioration is responsible for the result that the facility as it currently exists not only does not comply with the standards and criteria of today, but also has deteriorated past the standards in effect during the time of original construction. LANL has conducted several studies that indicate that it is neither feasible nor practical to upgrade the existing facility to a condition that is comparable (as much as possible) to a new facility in terms of operability and maintainability.

The present design uses the flocculation and clarification processes to remove both the largest portion of alpha-emitting radionuclides and TSS, as components of the precipitated sludge. However, this system does not have the capacity to remove TDS to the extent that would be required to meet the anticipated water quality standards.

Some of the issues that make a no-action approach unacceptable are:

- The concrete-walled clarifier tanks have deteriorated, and cannot comply with design requirements for waste containment

- The existing facility has no safety zoning for ventilation systems that would allow appropriate management of airborne radionuclide emissions

- The underground piping associated with the facility does not comply with design and regulatory codes

- Due to the deterioration of equipment and systems, the maintenance and operational costs for the existing facility have become excessive

- Due to the nature of the demands and requirements for waste processing, the TA-50 operations cannot be shut down for the length of time that would be required for extensive repairs and upgrades. This means that an temporary treatment system would be required for the interim/transition period. 
Any scenario that involves the upgrading of the existing facility must consider the retrofit engineering of these items as well as the replacement of the basic unit processes.

\subsection{DEFINITION OF FUNCTIONAL REQUIREMENTS}

As a comparative design basis, the proposed RLWTF operations are similar to that of the present operations at the Rocky Flats facility. In fact, it is very likely that the Rocky Flats Plant could effectively process the LANL wastes. However, a great deal of additional information (e.g., waste characterization) would be required in order to establish this parallel with a more complex facility such as the ETF at SRS. The information currently available seems to indicate that a facility such as ETF could not be successfully applied to the LANL waste streams, without extensive process changes.

\subsubsection{Operational Parameters}

In terms of functional requirements, the required operational parameters are straightforward:

1. Selective removal of alpha-emitting radionuclides, and minimization of the volume of TRU waste with heavy metals and TSS removed in the same process.

2. Utilization of processes to treat the TDS in order to remove heavy metals, radioactivity, and nitrates. Residual TSS and other components of the TDS will be removed simultaneously. There is no proven technology or process that has the selectivity to remove only the chemical species of concern for regulatory permitting.

3. Volatile contaminants remaining in the purified condensate (because of their volatility and potential for carry-over) must be removed or destroyed. This treatment is required for the volatile COD fraction.

4. All materials removed from the wastewater by non-destructive means (secondary waste) must be packaged for final disposal.

\subsubsection{Basic Functional Requirements}

The above four functional requirements are the basics required for a functional system, and could be implemented in a number of different ways. As explained in Section 3, the evaluation of unit processes has resulted in the selection of: 
1. Precipitation/flocculation/sedimentation to remove precipitation products, TSS and radioactivity

2. Evaporation to remove residual TSS and TDS

3. Oxidation using UV and ozone to destroy volatile contaminants

4. Fixation in saltstone for final disposal of the waste generated.

These basic process units were chosen to meet the functional requirements. A supporting operation of nitrate destruction is used to reduce the volume of contaminants that could increase the generation of secondary waste and would create the potential for violation of discharge limitations for nitrates.

\subsubsection{Deficiencies in Functional Requirements}

A number of deficiencies in the definition of functional parameters and requirements were identified. These deficiencies required the implementation of certain assumptions in order to proceed with the BAT study. The most important deficiencies are listed to indicate the type of information that may be required to support additional design development:

1. Insufficient information on the TA-55 effluent/supernate because there is only a single compositional analysis of Room 60 effluent.

2. Process chemical information is lacking on several NPDES species.

3. Information has not been provided on the chemical addition volumes and treatment dosages for the Room 60 operations or the TA- 50 flocculator treatment.

4. The composition of the Room 60 sludge is unknown.

5. The composition of the TA-50 filter cake is unknown.

6. The composition of the COD compounds is unknown, as is the means of reduction of this contaminant loading in present operations.

7. Fixation requirements have not been defined.

8. The maximum limits for TRU waste $(\mathrm{Pu})$ constituent content are estimated.

9. Decontamination factors are based on averaged numbers that have exhibited variability over the 1987-1991 period. There is a question as 
to whether the averages or the most recent values are more representative of actual operations.

10. The same concerns in item 9 hold true for quantities of individual contaminant species.

11. A loss of total radioactivity through the facilities (influent vs secondary waste) was noted in the annual reports and attributed to sampling and measurement errors. This needs to be investigated and confirmed.

\subsection{OPERATIONAL ASSUMPTIONS}

In order to establish a common basis for comparison of the operational criteria (e.g, operability, reliability, availability, and maintainability requirements) for the selected Candidate Technologies, the study required the formulation of certain assumptions. These assumptions reflect the preliminary stage of development for the BAT Study and related Candidate Technologies. Many of these concerns would be eliminated by further design and engineering development (e.g, development of conceptual design criteria). The most significant assumptions are:

1. Interim modifications can be considered, as long as the existing plant could be kept on-line for 10 to 12 more years.

2. Due to the critical and essential nature of the RLWP operations, any scenario to be considered must include provisions for a smooth transition of operations to minimize downtime and to restore treatment capabilities as soon as possible.

3. Disposal of secondary waste streams, and in particular sludges and sediments, is of prime importance as an operational and cost factor.

4. Past operational difficulties have resulted in the inactivation of some of the existing unit processes (e.g., polishing filters and ion exchange units). It is important that future technology applications be selected with this in mind.

5. Operational parameters evaluated for the Candidate Technologies will be based on working knowledge and general industrial experience with commercially available and proven technologies.

6. Although radiation levels in the waste stream are low, the potential for the presence of alpha-emitting radionuclides (e.g., plutonium) that could be released via an aerosol mechanism requires some consideration for personnel safety. 
7. Radiation dosage via external irradiation is not a concern.

8. There are no specific operational directives or mandates that favor the reuse of existing equipment and facilities rather than the construction of new facilities.

9. Chemical and occupational hazards associated with the existing RLWP and planned RLWTF operations present no more risk than in standard industrial applications.

10. Equipment inspectability and verifiability is essential in all configurations.

11. It is assumed that $\mathrm{Ca}(\mathrm{OH})_{2}$ and $\mathrm{Fe}_{2}\left(\mathrm{SO}_{4}\right)_{3}$ are currently used as flocculating agents although other chemical systems may be more effective and/or result in less secondary waste.

12. The 5-year average TDS concentration in TA-50 influent is $3,140 \mathrm{mg} / \mathrm{L}$, which is not in agreement with the $2,500 \mathrm{mg} / \mathrm{L}$ from the 1990 value, the assumed design basis. An arbitrary reduction of each chemical species by 25 percent is questionable and probably would not be representative.

13. $\mathrm{UV} / \mathrm{O}_{3}$ destruction of $\mathrm{COD}$ rather than chemical destruction methods is speculative because there is insufficient information on chemical dosages and efficiencies on which to base a choice.

14. Chemical destruction of nitrates is feasible, but the details of chemical addition/dosage, efficiency and off-gases are inadequate for material balance and removal/decontamination factor evaluations.

An additional consideration (discussed in previous sections) is that there is insufficient waste characterization data and process data information to attempt a meaningful material or energy balance on the existing processes. However, for the type of processes involved and the approach taken in this study, the lack of information does not create a significant problem, nor does it affect the type of processes that must be chosen. The approach taken is that the influent waste streams, over the years, have been subject to some degree of short-term variability and that the RIETF should be designed to accommodate this variability. Also, a design is required such that future capabilities can be reasonably implemented. 


\subsection{OPERATIONAL ISSUES AND CRITERIA}

Operational issues (as defined by DOE/EH-263T) are those other than environmental and regulatory concerns that affect the daily operations of the process facility and associated liquid effluent treatment systems. This would include any aspects of systems, equipment, and process operations, along with associated maintenance and repair activities.

Of the eight BAT evaluation factors presented in DOE Order 5400.5, six may be considered to be based on operational considerations. These six general evaluation factors are:

1. Age of the equipment and facilities involved

2. Processes employed

3. Engineering aspects of the application of various types of control techniques;

4. Process changes

5. Safety considerations

6. Public policy considerations

Other issues that may be considered are availability and ease of maintenance of the equipment, changes in throughput, alteration of process flow streams, operational safety, occupational exposure, and impacts on program mission.

Operational issues that are primary to BAT evaluation may be grouped into two general categories: (1) issues that determine the cost of operating the technology, and (2) issues that determine how well the technology complies with functional requirements. Other issues typically are relevant for the long term, and may not be developed in sufficient detail to support BAT analysis. These long-term considerations include the ability of the technology to provide reassurance to the public regarding safety and environmental concerns related to the facility, to adapt to expected changes in future functional requirements (e.g., expanded or reduced processing needs) while still satisfying the overall objectives of the operations to incorporate personnel safety considerations, and to allow for upgrading to include future improvements in the technology.

\subsubsection{Cost and Performance}

For the comparison of Candidate Technologies for waste processing and control, the following topics related to cost and performance were used as evaluation criteria:

1. Existing Facility Constraints - Each Candidate Technology was reviewed to determine facility constraints that would negatively impact or preclude implementation. As an example, existing facility constraints may include limited space, inability to reconfigure buildings, 
and incompatibility between existing technology and proposed treatment systems.

2. Throughput Capability - For each process stream, the ability of the proposed Candidate Technology to comply with the throughput capacity of functional design requirements was assessed. This assessment includes the general topics of reliability, availability, and maintainability of the system.

3. Equipment Maintenance Needs and Available Support - an qualitative estimation of preventive maintenance and repair for the Candidate Technologies was included. The purpose of this effort was to assist in identifying (1) the relative maintenance costs for the Candidate Technologies, and 2) the maintenance support required in terms of personnel, spare components, and maintenance contracts.

4. Expected Equipment Availability and Reliability - In most cases, the equipment selected was part of a larger system or unit process. Therefore, each Candidate Technology was evaluated in terms of overall expected availability and reliability. These parameters were assessed to ensure that the availability and reliability of any single unit process or system would not be compromised by the implementation of the overall process technology.

5. Resources Required for Operation - This evaluation involved assessment of requirements for operating personnel. This also provides support for the economic evaluation.

6. Human Factors and Training Considerations - The role of human factors in the operation and maintenance of Candidate Technologies was reviewed. This included the analysis of general tasks required to be performed by operators and maintenance staff, and consideration of whether the technologies could be designed to minimize both human errors and the results of such errors, should they occur (i.e., consideration of operational safety requirements). This analysis provided elements common to the evaluations of reliability, availability, safety, and throughput capability. Human factors review also considers training requirements (both initial and continuing) based on the complexity of operations.

\subsubsection{Long-Term Technology Effectiveness}

The comparison of Candidate Technologies for long-term effective wastewater treatment included: 
1. Response to Public Policy Issues - Public policy issues associated with public and local regulatory perception of LANL and the RLWTF were considered. The primary issues were associated with discharge of specified levels of contaminants and the perception of contamination control problems.

2. Flexibility of Technology to Adapt to Changing Requirements - This criterion was included to address the potential for changes in facility operations that could have a bearing on the operability of a selected Candidate Technology, such as changes in composition of the influent waste stream.

3. Ability of Technology to Comply with Safety Requirements - The operational aspects of each Candidate Technology were examined for associated safety and health protection problems. Safety received a high priority for BAT evaluation.

4. Flexibility of Technology to Meet Advances in Technology - A review of the ability of the Candidate Technologies to incorporate anticipated improvements was conducted.

\subsection{OPERATIONAL ISSUES FOR CANDIDATE Technology \# 1}

Candidate Technology \# 1 requires that upgrades to both of the existing TA-50 and the Room 60/TA-55 waste stream processes be made in a manner that will ensure operability for the life of the facility. The retrofit and upgrading of the existing Room 60 unit processes would be provided for pretreatment of the TA-55 waste stream, which is the primary source of radioactivity routed to the RLWTF. This scenario also would involve retrofit/upgrading of the existing TA-50 unit processes in order to restore reliable, operable process capacity.

\subsubsection{General Considerations}

\section{A. Existing Conditions}

As previously stated, the age of the equipment and facilities is the primary cause of the deterioration of existing operations. It is expected that a facility will deteriorate over a period of time, eventually reaching the point where continued operations (at any level of maintenance) are threatened. Such is the case with the existing RLWP. Since it is not clear that any equipment or unit operations could be salvaged, an upgrade would most likely involve the replacement of each unit process with a modern equivalent, with only the infrastructure remaining in place. Even with these modifications, strict compliance to the design 
specifications of DOE Order 6430.1A and related codes and standards cannot be expected.

The actual unit processes employed within the facility function very well for the intended purposes and waste types, as is evidenced by the years of compliance with past discharge limits. However, since the technology is working at the limit of effectiveness, the existing arrangement would need augmentation (e.g., the proposed evaporator system) in order to meet more stringent compliance requirements. If not for the obvious operational deterioration at the RLWP it is conceivable that the addition of the planned evaporator system to the existing TA-50 operations would provide the final treatment step required for proposed regulatory compliance requirements.

The engineering aspects of current types of control techniques are effective, but the equipment is no longer capable of functioning as designed. New equipment which utilizes the more efficient and effective design of contemporary systems is probably needed for continued operation.

\section{B. Functional Requirements}

The functional requirements of the existing operations are based on 30-year old design criteria. Although upgrades and replacement of unit processes may restore a certain level of operability, the existing facility cannot be revised to comply with the functional requirements of a modern DOE treatment facility without an effort that would be equivalent to a new construction.

C. Theoretical Mass Balance of Unit Operations

The required decontamination values that have been calculated for the overall process provide an indication of the required mass transfers and overall mass balance required for this Candidate Technology. Specific mass balances will be calculated by use of the Aspen + model, and will be issued as a separate attendant report in conjunction with this BAT study.

D. Decontamination Requirements

In recent years, the mission of many of the existing DOE facilities at various sites have changed from operations to remediation, including decontamination and decommissioning (D\&D). These changes in mission have allowed the initiation of a DOE data base intended to provide cost and labor factors for D\&D activities. However, the 
development of the data base is a nascent effort, and firm factors that might apply to any given type of effort are not fully developed.

All of the Candidate Technologies have a common factor in that the existing RLWP eventually must be subjected to D\&D efforts so that the final D\&D of the existing RLWP is considered not to be a factor. Candidate Technology \#1 represents the scenario with the lowest decontamination requirements and costs, since this option involves the least amount of new construction that would be subject to contamination. Since the task requires that most of the existing equipment be removed and decontaminated, but not the facility itself, final D\&D would be delayed until final facility closure. All other Candidate Technologies propose new facilities in addition to the RLWTF that would pose an additional level of D\&D for future consideration.

\subsubsection{Cost and Performance}

A. Existing Facility Constraints

One of the most restrictive problems with Candidate Technology \#1 is the limited space within which to remove and replace equipment. Building TA-50-1 is a three-level, $37,417 \mathrm{ft}^{2}$ building which currently houses the wastewater treatment processes as well as a D\&D section, laboratories, administrative support personnel, and analytical chemistry personnel. Almost any conceivable scenario for the reuse of this facility would involve the construction of additional space.

Also associated with the considerations of limited space is the inability to effectively reconfigure the existing buildings for process and personnel. Due to the years of operation of the facility, it would require additional significant relocation of equipment and support systems for any meaningful process reconfiguration. This explains the significant variation in estimation of RLWTF space requirements in former upgrade studies (see Table 6-1).

The potential also exists for design of incompatibility between the existing technology and proposed treatment systems. For example, the existing piping and electrical systems may be inadequate to support upgraded or replacement systems and equipment.

B. Throughput Capability

The RLWP as constructed has sufficient design capacity to accommodate even the largest influent volumes that can be predicted 
for the facility. The proposed upgrades for Candidate Technology \#1 would maintain this additional process capacity. One of the considerations that would be improved by Candidate Technology \#1 is the adaptability to change. The flexibility of the proposed treatment system can handle variations in the specific waste constituents to be processed. Also, the process of batching waste collected and held for treatment would serve to normalize transient fluctuations from the normal waste composition.

Perhaps the areas of greatest concern for Candidate Technology \#1 (or for any scenario that evaluates upgraded construction against new facilities) are those associated with reliability, availability, and maintainability. Whenever a project involves integrating new systems and components with older, existing systems, the potential for operational limitations and incompatibility is much greater than for a typical all-new construction based on optimized design.

\section{Maintenance Requirements}

While the maintenance of the new unit process equipment to be installed in the Candidate Technology \#1 upgrades would not be expected to be different than that for new construction involving the same equipment, the systems that contain the new equipment may require more attention. Often it is the system rather than the individual components or equipment that creates high maintenance considerations, and increasing systems/facilities age typically has a direct correlation to increased maintenance requirements. Associated with an increase in maintenance demands is an increase in the correlated costs.

A more sitewide consideration is brought to bear when considering the availability of support for maintenance. It is not uncommon on many older DOE sites to see only a few facilities requiring the vast majority of the maintenance personnel and associated costs. Although retrofitting and upgrading older facilities mitigates the maintenance requirements, it does not reduce the effort levels to those of a new operation.

Other maintenance considerations (other that the availability of adequately-trained personnel) are the availability of spare components for portions of the facility operations that would not be upgraded by Candidate Technology \#1, and the costs to sustain maintenance contracts for the older equipment. 


\section{Equipment Availability and Reliability}

The overall expected availability of the Candidate Technology \#1 upgrades option could be considered to be good, especially since the treatment processes are not continuous operations. The overall expected reliability of the processes should be good, although intermittent problems (particularly in subsystems and support systems that would not be upgraded or replaced) are probable.

\section{E. Resources Required for Operation}

In order to operate the new/upgraded equipment and systems, trained operating personnel will be required that are familiar with the operational processes. Proper accommodations for these personnel would be inherent in all Candidate Technologies.

Administrative controls would be required to support operations. Along with the upgrading of existing facilities, additional administrative controls are required to direct worker safety and health, since it is unlikely that the operational controls for the existing facility could be upgraded to the level of compliance required for contemporary DOE health and safety directives.

\section{F. Human Factors}

The evaluation of human factors on operations should be considered in the evaluation of Candidate Technologies. Since more administrative controls are anticipated to manage the health and safety concerns for Candidate Technology \#1, there is a higher risk and potential for human failure in this area. The new unit processes can be designed to minimize complexity and to mitigate potential accidents, but the integration of new and existing technologies provides a possible mechanism for breakdown of operational controls.

Training considerations are important to minimize failures and accidents due to human factors. Initial and continuing training should be considered as a consistent factor in all of the Candidate Technologies.

\subsubsection{Long-Term Technology Effectiveness}

\section{A. Public Policy Issues and Considerations}

For all DOE sites, the considerations of the public and intervening organizations for the protection of personnel, the population at large, 
and the environment are topics that warrant close scrutiny. For the RUVTF, the primary public concerns are focused on the discharge of specified levels of contaminants and the perception of contamination control problems. The ongoing negotiations regarding the finalizing of LANL NPDES permit discharge limits is a testimony to the amount of time and effort that can be expended in order to satisfy pressures from concerned organizations. It is anticipated that the proposed processes for the Candidate Technology \# 1 scenario will be adequate to satisfy the discharge issues of public concern.

\section{B. Flexibility to Accommodate Process Changes}

The nature of the unit processes selected for the Candidate Technology \#1 upgrades provides for flexible operation and capacity to accommodate variation in waste composition. Process input changes within expected limitations should not create long-term problems for this scenario.

\section{Compliance with Safety Requirements}

Safety concerns typically increase with the age of a process or facility. Operational safety requirements would be more stringent for Candidate Technology \#1 due to the inability to upgrade the entire operation to DOE Order 6430.1A standards.

Although hazardous chemicals are handled during TA-50 operations, the chemical safety hazards associated with these operations are no different that those acceptable for other industrial practices.

Since the installation of various upgrades and replacement of equipment is the basis for Candidate Technology \#1, occupational safety would be an elevated concern. Not only is space minimal for retrofit activities, but some of the areas to be utilized are radiologically contaminated and would require special precautions and protection.

Although radiological contamination is present in the existing RLWP worker safety is not considered to be a problem that cannot be handled with proper precautions. However, the potential for contact with plutonium-bearing wastes exists, so that a high level of consideration for safety measures during renovation and operation must be maintained. 


\section{Flexibility to Address Advances in Technology}

While it will be possible to update the technology of the new unit processes installed as a result of implementation of Candidate Technology \#1, consideration must be given to systems configurations into which these processes are installed. It is anticipated that Candidate Technology \#1 will be able to adapt to basic advances in technology, but there may be instances in which the combination of new and older technology will restrict further technology upgrading.

\subsection{OPERATIONAL ISSUES FOR CANDIDATE Technology \#2}

Candidate Technology \#2 is based on the upgrading of the existing TA-50 operations, while constructing a new facility or enclosed area for the Room 60/TA-55 waste stream. In the initial pretreatment process for Candidate Technology \#2, a new facility would be constructed to provide a totally separated treatment scheme for the TA-55 waste stream. The proposed upgrades and final processing scheme for the main TA-50 operations would be identical to those presented for this portion of Candidate Technology \#1. This scenario would provide operability advantages by effectively eliminating the bulk of radioactivity from the primary process stream by the utilization of the new systems planned for the Room 60 replacement operations.

\subsubsection{General Considerations}

\section{A. Existing Conditions}

For the new TA-55/Room 60 facilities, the conditions can be optimized to ensure operability and process efficiency. The upgrade of the existing TA-50 operations would involve the replacement of the existing unit process with a modern equivalent, with the infrastructure and support systems remaining in place. Although the new TA-55/Room 60 facility can be built to comply with contemporary standards and design codes, strict compliance by the upgraded TA-50 facility to the design specifications of DOE Order 6430.1A and related codes and standards cannot be expected.

The anticipated increased effectiveness in the removal of the TA-55 waste stream radionuclides (as well as nitrates destruction) will lessen significantly the treatment demands on the primary waste stream. Since the system has maintained years of compliance with past discharge limits, it is expected that with new equipment and a decreased demand to process radioactivity, the system should be even more effective at reducing contaminant levels. The addition of the 
proposed evaporator system should ensure that the Candidate Technology \#2 configuration can meet the more stringent compliance requirements.

The engineering of the TA-55/Room 60 replacement operations can provide state-of-the-art treatment technology. However, the equipment in the existing TA-50 operations is no longer capable of functioning as designed. New equipment which utilizes the more efficient and effective design of contemporary systems is needed for continued operation.

\section{B. Functional Requirements}

The functional requirements of the existing operations are based on 30-year old design criteria. Although upgrades and replacement of unit processes may restore a certain level of operability, the existing facility cannot be revised to comply with the functional requirements of a modern DOE treatment facility without an effort that would be equivalent to new construction. However, the functional requirements for the proposed new TA-55/Room 60 facility can be established at a level that will ensure maximum operability.

\section{Theoretical Mass Balance of Unit Operations}

The required decontamination values that have been calculated for the overall process provide an indication of the required mass transfers and overall mass balance required for this Candidate Technology. Specific mass balances will be calculated by use of the Aspen + model, and will be issued as a separate attendant report in conjunction with this BAT study.

\section{Decontamination Requirements}

As previously stated, the DOE data base to accumulate cost and labor factors for D\&D activities is being established, and factors that might apply to any given type of effort are not fully developed.

This option would result in slightly higher decontamination requirements and costs than Candidate Technology \#1, since it involves the $\mathrm{D} \& \mathrm{D}$ of the current Room 60 operations and the construction of a new facility that later would be subject to contamination and subsequent $D \& D$. Also, the task requires that most of the existing equipment from the TA-50 operations be removed and decontaminated prior to installation of the new or upgraded equipment. 


\subsubsection{Cost and Performance}

A. Existing Facility Constraints

As with Candidate Technology \#1, there is limited space within which to remove and replace equipment. Scenarios for the reuse of the TA-50 facility would involve the construction of additional space. Also associated with the considerations of limited space is the inability to reconfigure the existing buildings for process and personnel considerations.

While the entirely new processing equipment for the Room 60/TA-55 replacement facility should create no problems involving incompatibility, the potential exists for design incompatibility between the existing technology and proposed treatment systems for the TA-50 upgrades.

B. Throughput Capability

The RLWP as constructed has sufficient design capacity to accommodate even the largest volumes that can be predicted as influent to the facility. The proposed new facility and upgrades for Candidate Technology \#2 would maintain this additional process capacity.

The new TA-55 treatment facility and proposed upgrading of TA-50 operations will provide a certain degree of adaptability to change. The flexibility of the proposed treatment system can handle variations in the specific waste constituents to be processed. The process of batching waste collected and held for treatment would serve to normalize transient fluctuations from the normal waste composition.

While reliability, availability, and maintainability parameters can be optimized by design for the new Room 60/TA-55 replacement facility, the problems referenced for Candidate Technology \#1 involving the upgrading of the TA-50 operations also exist with Candidate Technology \#2. A project involving the integration of new systems and components with older, existing systems creates a greater potential for operational than a typical all-new construction based on optimized design. 


\section{Maintenance Requirements}

In all typical process applications, new equipment (with proper design and installation) provides a great maintenance advantage over older, upgraded, or integrated replacement equipment. Actualized cost savings typically can be calculated to verify this advantage. For the new TA-55 waste stream treatment facility, a minimal maintenance requirement can be expected, especially with equipment that has been selected as proven technology.

The maintenance of the new unit process equipment to be installed in the Candidate Technology \#2 upgrades would not be expected to be different from that for new construction involving the same equipment. However, the systems that contain the new equipment may require more attention. Associated with the increase in maintenance demands is the increase in correlated costs.

Since Candidate Technology \#2 involves a new facility, it is anticipated that the demands for the availability of maintenance support may be slightly less than those for Candidate Technology \#1. Also, the availability of spare components for portions of the facility operations that would not be upgraded in the Candidate Technology \#2 would be a consideration, as would the costs to sustain maintenance contracts for the older equipment.

D. Equipment Availability and Reliability

The overall expected availability of the Candidate Technology \#2 new construction and upgrades option is considered to be good, and slightly better than for Candidate Technology \#1. The overall expected reliability of the processes should be good, although intermittent problems (particularly in subsystems and support systems that would not be upgraded or replaced) for TA-50 operations are probable.

\section{E. Resources Required for Operation}

In order to operate the new/upgraded equipment and systems, trained operating personnel will be required that are familiar with the operational processes. Proper accommodations for these personnel would be inherent in all Candidate Technologies.

Administrative controls would be required to govern operations. Since operational controls can be built into the new TA-55/Room 60 facility, the requirements for administrative controls for direction of worker safety and health should be fewer than with Candidate Technology \# 1. 


\section{F. Human Factors}

Fewer administrative controls will required to manage the health and safety concerns for Candidate Technology \#2 that were required for Candidate Technology \#1, so there should be a correlated lowering in the risk and potential for human failure in this area. The new facility and unit processes can be designed to minimize complexity and to mitigate potential accidents, but the integration of new and existing technologies provides a possible mechanism for breakdown of operational controls.

Training considerations are important to minimize failures and accidents due to human factors. Initial and continuing training should be considered as a consistent factor in all of the Candidate Technologies.

\subsubsection{Long-Term Technology Effectiveness}

\section{A. Public Policy Issues and Considerations}

The primary public concerns for Candidate Technology \#2 are focused on the discharge of specified levels of contaminants and the perception of contamination control problems. It is anticipated that the proposed processes for the Candidate Technology \#2 scenario will be adequate to satisfy the discharge issues of public concern, and that the new facility to treat radionuclides would receive a slightly more favorable consideration than would the upgrades in Candidate Technology \#1.

B. Flexibility to Accommodate Process Changes

The nature of the unit processes selected for the Candidate Technology \#2 new construction and upgrades is for flexible operation and capacity to accommodate variation is waste composition. Process input changes within expected limitations should not create long-term problems for this scenario.

\section{Compliance with Safety Requirements}

It is expected that the new TA-55/Room 60 treatment facility would be constructed in full compliance with the safety and health protection criteria currently in effect for DOE operations. Operational safety requirements for the TA-50 facilities would be more stringent due to the inability to upgrade the entire operation to DOE Order 6430.1A standards. 
Although hazardous chemicals are handled during TA-50 operations, the chemical safety hazards associated with these operations are no different than those acceptable for other industrial practices.

Since the installation of various upgrades and replacement of equipment is part of the basis for Candidate Technology \#2, occupational safety would be an elevated concern. Not only is space minimal for retrofit activities, but some of the areas to be utilized are radiologically contaminated and would require special precautions and protection. Since the Room 60 facility represents only a small portion of the overall operation, this concern would be approximately equivalent between Candidate Technologies \#1 and \#2.

The potential for contact with plutonium-bearing wastes can be greatly mitigated or eliminated by the proper design of the new TA-55/Room 60 replacement facility. This should result in a somewhat lower level of safety risk during renovation and operations.

D. Flexibility to Address Advances in Technology

The construction of the new TA-55/Room 60 replacement facility should provide maximum flexibility to incorporate any appropriate advances in technology that could be of use in the future. As for the TA-50 operational upgrades, it is anticipated that unit processes will be able to adapt to basic advances in technology, but there may be instances in which the combination of new and older technology will restrict further technology upgrading.

\subsection{OPERATIONAL ISSUES FOR CANDIDATE Technology \#3}

The options presented as Candidate Technology \#3 propose upgrading the existing TA-55/Room 60 operations, while constructing a new facility for the primary TA-50 waste treatment operations. The modifications, retrofits, and upgrades to the TA-55 operations would be identical to those presented for this portion of the treatment scheme in Candidate Technology \#1. The final pretreatment effluent/supernate would be routed to the TA-50 influent. The proposed TA-50 portion of this scenario involves the construction of a totally separate new facility for the management of the TA-50 waste stream.

\subsubsection{General Considerations}

\section{A. Existing Conditions}

For this Candidate Technology, the new treatment facilities for the main TA-50 operations can be optimized to ensure operability and 
process efficiency. The upgrade of the existing TA-55/Room 60 operations would involve the replacement of the existing unit processes with a modern equivalent, with the minor infrastructure and support systems required for this small operation remaining in place. Although the new TA-50 processing facility can be built to comply with contemporary standards and design codes, strict compliance by the upgraded TA-55/Room 60 facility to the design specifications of DOE Order $6430.1 \mathrm{~A}$ and related codes and standards cannot be expected.

Although less than the credit to Candidate Technology \#2 can be taken for the increased effectiveness in the removal of the TA-55 waste stream radionuclides, the pretreatment operations in the existing configuration are very effective at reducing the overall radiological loading influent to TA-50. Any reduction in pretreatment should assist in minimizing the radiological and mixed wastes generated as secondary waste streams by TA-50 operations.

With proper equipment selection and installation, a slight increase in effectiveness may be expected in the Room 60 operations over the existing process, similar to the increase expected for Candidate Technology \#1.

The engineering of the TA-50 replacement facility and operations can provide treatment technology that is fully compliant with applicable codes and standards. However, the equipment in the existing TA-55/Room 60 operations is no longer capable of functioning fully within original design capabilities. New equipment which utilizes the more efficient and effective design of contemporary systems is needed for continued operation.

\section{B. Functional Requirements}

The construction of a new TA-50 facility will ensure that contemporary functional requirements will be implemented as a portion of preliminary design. These functional requirements for the proposed new TA-50 facility can be established at a level that will ensure maximum operability. However, the functional requirements of the existing TA-55/Room 60 operations are based on old design criteria, and compliance with the requirements of a modern DOE treatment facility would involve significant facility modifications.

\section{Theoretical Mass Balance of Unit Operations}

The required decontamination values that have been calculated for the overall process provide an indication of the required mass transfers 
and overall mass balance required for this Candidate Technology. Specific mass balances will be calculated by use of the Aspen + model, and will be issued as a separate attendant report in conjunction with this BAT study.

D. Decontamination Requirements

As previously stated, the DOE data base to accumulate cost and labor factors for D\&D activities is being established and factors that might apply to any given type of effort are not fully developed.

Candidate Technology \#3 would require decontamination requirements and costs that would be higher than those for Candidate Technology \#2, since it involves the D\&D of a major portion of the existing RLWP and the construction of a new facility that later would be subject to contamination and subsequent D\&D. Also, the task requires that most of the existing equipment from the TA-55/Room 60 operations be removed and decontaminated prior to installation of the new or upgraded equipment.

\subsubsection{Cost and Performance}

A. Existing Facility Constraints

The problem with limited space that was discussed for Candidate Technologies \#1 and \#2 would not apply with the exception of the small area for Room 60 operations. Additional space for ancillary operations can be planned by design for the new facility. Also, the design of the new TA-50 facility can incorporate the ability to reconfigure the buildings to accommodate future process and personnel considerations.

The construction of an entirely new TA-50 replacement facility should resolve any problems involving incompatibility of new and older process equipment and systems. The potential exists for incompatibility between the existing technology and proposed treatment systems for the TA-55/Room 60 upgrades, but this should be a minor concern.

B. Throughput Capability

The new construction of a TA-50 facility would include sufficient design capacity to accommodate the largest volumes that can be predicted as influent to the facility. 
The new TA-50 treatment facility and proposed upgrading of TA-55/Room 60 operations will provide some of adaptability for change. The flexibility of the proposed treatment system can handle variations in the specific waste constituents to be processed. The process of batching waste collected and held for treatment would serve to normalize transient fluctuations from the normal waste composition.

Reliability, availability, and maintainability parameters can be optimized by the design for the new TA-50 replacement facility. The potential for problems involving the upgrading of the TA-55/Room 60 operations still exist, but should be of minor impact based on the small size and volume of the Room 60 operations.

\section{Maintenance Requirements}

For the new TA-50 treatment facility, a minimal maintenance requirement can be expected, especially with the equipment that has been selected as proven technology. The upgraded Room 60 operations should show a reduction in maintenance requirements from past operations, but would still have higher requirements for this portion of the treatment operations than would Candidate Technology \#2. Since Candidate Technology \#3 involves a new facility, it is anticipated that the demands for the availability of maintenance support may be significantly less than those for Candidate Technology \#2.

Spare parts and components should not be a concern with the new TA-50 facility and related processes. The availability of spare components for the upgraded Room 60 operations may present a minor consideration, but with new unit process equipment, should be negligible. Equipment maintenance contracts should involve minimal costs.

D. Equipment Availability and Reliability

The overall expected availability of the Candidate Technology \#3 new construction and upgrades option is considered to be very good, and significantly better than that for Candidate Technology \#2. The overall expected reliability of the processes should be very good, although minor intermittent problems (particularly in subsystems and support systems that would not be upgraded or replaced) for Room 60 operations are probable. 
E. Resources Required for Operation

In order to operate the new/upgraded equipment and systems, trained operating personnel will be required that are familiar with the operational processes. Proper accommodations for these personnel would be inherent in all Candidate Technologies.

Administrative controls would be required to govern operations. Since operational controls can be built into the new TA-50 facility, there should be no significant requirements for administrative controls for direction of worker safety and health based on operational hazards or risks.

\section{F. Human Factors}

The new construction of TA-50 should minimize the potential for human risk and/or failure. Fewer administrative controls will required to manage the health and safety concerns than were required for Candidate Technology \#2. The new facility and unit processes can be designed to minimize complexity and to mitigate potential accidents.

Training considerations are important to minimize failures and accidents due to human factors. Initial and continuing training should be considered as a consistent factor in all of the Candidate Technologies.

\subsubsection{Long-Term Technology Effectiveness}

A. Public Policy Issues and Considerations

The primary public concerns for all Candidate Technologies are focused on the discharge of specified levels of contaminants and the perception of contamination control problems. It is anticipated that the proposed processes for the Candidate Technology \#3 scenario will be adequate to satisfy the discharge issues of public concern, and that the new TA-50 facility would receive a slightly more favorable consideration than would the upgrades in Candidate Technology \#2, with the exception of potential concern involving additional project costs.

\section{B. Flexibility to Accommodate Process Changes}

The unit processes selected for the Candidate Technology \#3 new construction and upgrades will be designed for flexible operation and capacity to accommodate variations is waste composition. Process 
input changes within expected limitations should not create long-term problems.

C. Compliance with Safety Requirements

It is expected that the new TA-50 treatment facility would be constructed in full compliance with the safety and health protection criteria currently in effect for DOE operations. It is possible that additional operational safety requirements may be required for the TA-55/Room 60 operations, but it is not anticipated that these additional requirements will have a significant impact on overall operations.

Although hazardous chemicals are handled during TA-50 operations, the chemical safety hazards associated with these operations are no different than those acceptable for other industrial practices.

The concern for occupational safety (other than construction safety) would minimized because the number of uncontrolled areas that would be radiologically contaminated could be reduced and managed more effectively by proper design considerations. Since the Room 60 facility represents only a small portion of the overall operation, the concerns for occupational safety should be fewer for Candidate Technology \#3 than for either Candidate Technology \#1 or Candidate Technology \#2.

The potential for contact with plutonium-bearing wastes can be greatly mitigated by the new equipment selected for the TA-55/Room 60 upgrades and the precautions taken for the TA-50 replacement facility. This should result in a low level of safety risk during renovation and operations.

D. Flexibility to Address Advances in Technology

The construction of the new TA-50 replacement facility should provide maximum flexibility to incorporate any appropriate advances in technology that could be of use in the future. As for the TA-55/Room 60 operational upgrades, it is anticipated that unit processes will be able to adapt to basic advances in technology, but there may be instances in which the combination of new and older technology will restrict further technology upgrading. 


\subsection{OPERATIONAL ISSUES FOR CANDIDATE Technology \#4}

Candidate Technology \#4 represents the construction of an all-new facility for the treatment of combined TA-55/Room 60 and TA-50 wastes. The existing TA-50 operations (including Room 60) would be submitted for D\&D.

\subsubsection{General Considerations}

\section{A. Existing Conditions}

Candidate Technology \#4, the design and construction of all-new treatment facilities, represents the most desirable scenario in terms of optimization of engineering features and conditions to ensure operability and process efficiency. The new RLWTF could be built to comply with all of the appropriate and applicable contemporary standards and design codes, and can provide strict compliance with the design specifications of DOE Order 6430.1A.

With the selected proven unit processes and technologies, maximum effectiveness may be expected in comparison with other Candidate Technologies.

The engineering and construction of the new RLWTF could provide treatment technology that is fully compliant with operational requirements, and not constrained by limitations caused by aging equipment or facilities.

\section{B. Functional Requirements}

The construction of a new RLWTF will ensure that contemporary functional requirements will be implemented as a portion of preliminary design. These functional requirements for the proposed RIXTH can be established at a level that will ensure maximum operability.

\section{Theoretical Mass Balance of Unit Operations}

The required decontamination values that have been calculated for the overall process provide an indication of the required mass transfers and overall mass balance required for this Candidate Technology. Specific mass balances will be calculated by use of the Aspen + model, and will be issued as a separate attendant report in conjunction with this BAT study. 
D. Decontamination Requirements

Candidate Technology \#4 would require the most demanding decontamination requirements and costs of the four Candidate Technologies, since this option involves the closure and D\&D of the entire existing TA-50 operations, and the construction of a new facility that later would be subject to contamination and subsequent D\&D.

\subsubsection{Cost and Performance}

A. Existing Facility Constraints

The problems with space limitations discussed primarily for Candidate Technologies \#1 and \#2 would not be applicable. Additional space for ancillary operations can be planned by design for the new facility. Also, the design of the new TA-50 facility can incorporate the ability to reconfigure the buildings to accommodate future process and personnel considerations.

The construction of an entirely new RLWTF would resolve any problems involving incompatibility of new and older process equipment and systems.

B. Throughput Capability

The new construction of a new RLWTF would include sufficient design capacity to accommodate the largest volumes that can be predicted as influent to the facility.

The new RLWTF could also provide design-inclusive adaptability for change. The flexibility of the proposed treatment system and unit processes could be designed so that it would be easy to adapt to variations in the specific waste constituents to be processed.

Reliability, availability, and maintainability parameters would be optimum for the design for a new facility in comparison with any combination of upgrades.

\section{Maintenance Requirements}

For the new RLWTF, the comparative minimum of maintenance requirements could be expected, especially with the equipment that has been selected as proven technology. Also, it would be anticipated that the demands for the maintenance support would be the least of the Candidate Technologies. 
Availability of spare parts and components should not be a concern with an all-new RLWTF. Equipment maintenance contracts should reflect only the design life of the new facility rather than the extended periods required for the upgrade options.

D. Equipment Availability and Reliability

The overall expected availability of the Candidate Technology \#4 new construction option should be the best of the options under evaluation. The overall expected reliability of the processes should be optimum.

\section{E. Resources Required for Operation}

In order to operate the new/upgraded equipment and systems, trained operating personnel will be required that are familiar with the operational processes. Proper accommodations for these personnel would be inherent in all Candidate Technologies.

For a new facility, the minimum of administrative controls would be required to govern operations. This is because design can take full advantage of engineered safety features to minimize the requirements for administrative controls subject to operator error.

\section{F. Human Factors}

With optimized design for safety, the new RLWTF should minimize the potential for human risk and/or failure. Only essential administrative controls will be required to manage the health and safety concerns. The new facility and unit processes can be designed to minimize complexity and to mitigate potential accidents.

Training considerations are important to minimize failures and accidents due to human factors. Initial and continuing training should be considered as a consistent factor in all of the Candidate Technologies.

\subsubsection{Long-Term Technology Effectiveness}

\section{A. Public Policy Issues and Considerations}

The primary public concerns for Candidate Technology \#4 are the same as for other options. The new RLWTF should satisfy the discharge issues of public concern, and should receive the most favorable consideration for environmental protection and safety. 
However, the potential concern involving project costs is greatest with this option.

\section{B. Flexibility to Accommodate Process Changes}

The unit processes selected for new construction will be designed for flexible operation and capacity to accommodate variation waste composition. Process input changes within expected limitations should not create long-term problems.

\section{Compliance with Safety Requirements}

It is expected that the new RLWTF would be constructed in full compliance with the safety and health protection criteria currently in effect for DOE operations.

Although hazardous chemicals are handled during TA-50 operations, the chemical safety hazards associated with these operations are no different from those acceptable for other industrial practices.

For Candidate Technology \#4, the concern for occupational safety (other than construction safety) would be minimal because the number of uncontrolled areas that would be radiologically contaminated could be reduced and managed more effectively by proper design considerations.

The potential for contact with plutonium-bearing wastes can be minimized by the new equipment and processes selected for the RLWTF. This should result in a low level of safety risk during operations.

\section{Flexibility to Address Advances in Technology}

The construction of the new RLWTF will provide maximum flexibility to incorporate any appropriate advances in technology that could be of use in the future.

\subsection{FINAL EVALUATION OF CANDIDATE TECHNOLOGIES}

\subsubsection{Comparative Analysis of Operational Issues}

The evaluations provided in Sections 6.4 through 6.8 are the basis of the analysis presented in Table 6-2, which summarizes each primary topic related to operations issues and criteria. The ranking of each Candidate Technology (based on the referenced evaluations and review of related materials specified 
in Section 10) is presented in order of relative conformance to operational requirements.

\subsubsection{Status of Candidate Technologies}

Based on the merits attributed to each of the four Candidate Technologies, none can be eliminated strictly on the basis of operational issues and criteria. In general, the ranking of the four options (from most favorable to least favorable) for the issues and considerations addressed in this section is:

1. Candidate Technology \#4

2. Candidate Technology \#3

3. Candidate Technology \#2

4. Candidate Technology \#1

It should be noted that the differences in these rankings are not equally spaced, nor are the rankings of significant enough difference to eliminate or favorably select any single Candidate Technology on the basis of the operational issues and criteria considered. 
TABLE 6-1 - Project Space Allotment/Requirements for New RLWTF Facilities

\begin{tabular}{|c|c|c|c|}
\hline Facility Area & $\begin{array}{r}\text { Existing } \\
\text { Space, } \mathrm{ft}^{2}\end{array}$ & $\begin{array}{c}12 / 89 \\
\text { Estimate, } \mathrm{ft}^{2}\end{array}$ & $\begin{array}{l}02 / 91 \\
\text { Estimate, } \mathrm{ft}^{2}\end{array}$ \\
\hline Technical Support Areas & 6,964 & 11,535 & 9,000 \\
\hline Office Space & 4,235 & 3,800 & \\
\hline HSE-9 Operations & 380 & 425 & \\
\hline Library & 336 & 450 & \\
\hline Shipping & 281 & 375 & \\
\hline Lunch Room & 381 & 500 & \\
\hline Women's Change Room & 273 & 390 & \\
\hline Men's Change Room & 393 & 390 & \\
\hline Computer Area & 342 & 3,455 & \\
\hline Exercise Room & 343 & -.. & \\
\hline HSE-7 Operations & -.. & 1,750 & \\
\hline Laboratories & 9,560 & 6,880 & 9,560 \\
\hline HSE-9 Lab & 6,520 & 2.440 & \\
\hline Other Lab Space & 3,040 & 4,440 & \\
\hline Warehouse & $\ldots$ & 5,000 & ..- \\
\hline
\end{tabular}


TABLE 6-1 - Project Space Allotment/Requirements for New RCWTT Facilities (Cont.)

\begin{tabular}{|c|c|c|c|}
\hline Facility Area & $\begin{array}{r}\text { Existing } \\
\text { Space, } \mathrm{ft}^{2}\end{array}$ & $\begin{array}{r}12 / 89 \\
\text { Estimate, } \mathrm{ft}^{2} \\
\end{array}$ & $\begin{array}{r}02 / 91 \\
\text { Estimate } \mathrm{ft}^{2}\end{array}$ \\
\hline Process RLWTT & 15,860 & 35,800 & 28,860 \\
\hline Process Area & 13,940 & 26,800 & \\
\hline Vehicle Decontamination & 1,920 & 2,500 & \\
\hline Workspace & --- & 6,000 & \\
\hline Equipment Storage & -- & 500 & \\
\hline Shops/Maintenance & 1,640 & 10,000 & 36,850 \\
\hline Mechanical Space & 1,640 & 10,000 & \\
\hline Process Area & included & included & \\
\hline Shop/Maintenance & included & included & \\
\hline Other Areas & 2,128 & 2,005 & 8,329 \\
\hline Chemical Storage & 1,067 & --. & \\
\hline General Storage & 411 & -... & \\
\hline Miscellaneous & 650 & --- & \\
\hline Unaccounted For & $\underline{1265}$ & - & $\ldots$ \\
\hline TOTAL & 37,417 & 71,220 & 92,599 \\
\hline
\end{tabular}


TABLE 6-2

TABLE 6-2 - Comparative Analysis of Operational Issues and Criteria ${ }^{1}$

Candidate Technologies

ISSUES/CRITERIA

1. General Considerations

Existing Conditions

Functional Requirements

Theoretical Mass Balance of Unit Operations

Decontamination Requirements

2. Cost and Performance

Existing Facility Constraints
$\# 3$

\#4

$\# 1$

$\# 2$

3

2

2

$\mathrm{n} / \mathrm{a}$

n/a

$\mathrm{n} / \mathrm{a}^{1}$

$\mathrm{n} / \mathrm{a}$

2

3

4

1 1 1 
TABLE 6-2 - Comparative Analysis of Operational Issues and Criteria ${ }^{1}$ (Cont.)

\begin{tabular}{|c|c|c|c|c|}
\hline \multirow[b]{2}{*}{ ISSUES/CRITERIA } & \multicolumn{4}{|c|}{ Candidate Technologies } \\
\hline & $\# 1$ & $\# 2$ & $\# 3$ & $\# 4$ \\
\hline Throughput Capability & 2 & 2 & 1 & 1 \\
\hline Maintenance Requirements & 4 & 3 & 2 & 1 \\
\hline $\begin{array}{l}\text { Equipment Availability and } \\
\text { Reliability }\end{array}$ & 4 & 3 & 2 & 1 \\
\hline $\begin{array}{l}\text { Resources Required for } \\
\text { Operation }\end{array}$ & 2 & 2 & 1 & 1 \\
\hline Human Factors & 4 & 3 & 2 & 1 \\
\hline \multicolumn{5}{|l|}{$\begin{array}{l}\text { Long-Term Technology } \\
\text { Effectiveness }\end{array}$} \\
\hline $\begin{array}{l}\text { Public Policy Issues and } \\
\text { Considerations }\end{array}$ & $\mathrm{n} / \mathrm{c}^{3}$ & $\mathrm{n} / \mathrm{c}$ & $\mathrm{n} / \mathrm{c}$ & $\mathrm{n} / \mathrm{c}$ \\
\hline $\begin{array}{l}\text { Flexibility to Accommodate } \\
\text { Process Changes }\end{array}$ & 4 & 3 & 2 & 1 \\
\hline $\begin{array}{l}\text { Compliance with Safety } \\
\text { Requirements }\end{array}$ & 4 & 3 & 2 & 1 \\
\hline
\end{tabular}


TABLE 6-2 - Comparative Analysis of Operational Issues and Criteria ${ }^{1}$ (Cont.)

Candidate Technologies

$\begin{array}{lrrrr}\text { ISSUES/CRITERIA } & \# 1 & \# 2 & \# 3 & \# 4 \\ \begin{array}{l}\text { Flexibility to Address } \\ \text { Advances in Technology }\end{array} & 4 & 3 & 2 & 1 \\ \text { Overall } & - & - & - & -\end{array}$

1 Rankings provided in numerical order of most favorable (1) to least favorable (4)

$2 \quad \mathrm{n} / \mathrm{a}=$ not available at this time

3 All relevant issues not clear at this time 


\subsection{ANALYSIS OF ENERGY AND RESOURCE ISSUES}

\subsection{EVALUATION OF THE ENERGY AND RESOURCES USE BY THE EXISTING FACILITY}

The current TA-50 operations involve treatment of liquid radioactive wastewaters via selected unit processes. The impacts of current operations on the utilization of energy and the consumption of resources is evaluated to be in agreement with expected requirements for a non-consumptive treatment facility.

\subsubsection{Site Location and Resources}

The current RLWP is located within the very well developed section of the LANL site, and presents minimal additional area for expansion. A number of line item project proposals for use of facilities and areas adjacent to the TA-50-1 building have required revisions to the plans for future land use in the TA-50 and TA-63 areas. The TA-50 site is almost fully developed and the burial areas to the south have been restricted as undevelopable in the near future due to environmental restoration constraints. Expansion land for waste management functions is needed in close proximity to the existing TA-50 facility if the use of existing operations is to be considered.

If the area around TA-50 cannot be used for facility expansion, the primary target area for expansion would be in the TA- 63 area, immediately southeast of the existing site. It has been stated by LANL that this would be the only suitable land for the required expansion.

\subsubsection{Existing Energy and Utility Resources}

As a combined process plant and technical support facility, TA-50-1 requires several types of utility services. Electrical power is required for operation of the process equipment, powering the facility lighting and heating/cooling systems, and electrical supply support for technical and administrative functions (e.g, office equipment and computer use). Telecommunications, natural gas, water supply, sanitary sewers, and an industrial wastewater line are other utility services required for TA-50 operations.

Electric power for building TA-50-1 is rated at a primary voltage of $13.2 \mathrm{kV}$ and a secondary voltage of $480 \mathrm{~V}$. The power is supplied via circuit S-9, and is provided first through the $15 \mathrm{kV}$ switchgear at manhole 50-110, and subsequently through a $750 \mathrm{kVA}$ transformer.

Telephone communications are routed to TA-50-1 via a three-inch conduit line. 
Natural gas is supplied to the building by a two-inch, 100 psi supply line. This line ties into a three-inch gas main subsurface along Pecos Drive.

Water is provided to the building by two eight-inch transite pipes, which provide both domestic and fire protection water. These lines connect to a twelve-inch cast-iron water main subsurface near Pecos Drive.

Domestic sewage is transported to treatment lagoons at TA-35 via a four-inch ductile iron force main line that ties into an eight-inch subsurface line along Pecos Drive. Lift stations are required to transfer the sewage from the existing facility to the eight-inch line.

Radioactive wastewaters are transported to the TA-50 facility for processing primarily via two pathways. The largest volume of wastewater is routed to TA-50 via a double-encased polyethylene waste line connected to the various waste generating areas. Other radioactive wastewater is transferred via truck to TA-50 from technical areas that are not connected to the industrial waste collection line. Currently an approximate flow rate of 7.9 million gal/yr is treated by TA-50, with an average flow rate of approximately $15 \mathrm{gpm}$.

\subsubsection{Energy and Resource Utilization for Existing Unit Process}

Although a quantitative evaluation of the energy use of each unit process in the current TA-50 operations was not available for this BAT study, the follow unit processes can be identified as the primary consumers of electrical power and material/chemical resources:

A. TA-55/Room 60 Operations

1. Neutralization

2. Precipitation/Flocculation/Clarification

3. Sludge Contacting and Decanting

4. Supernate Filtration

5. Sludge Washing

6. Cementation

B. TA-50 Primary Operations

1. $\mathrm{pH}$ Adjustment

2. Stage 1 Precipitation/Flocculation/Clarification

3. Stage 2 Precipitation/Flocculation/Clarification

4. Mixed Media Filtration

5. Sludge Settling and Decanting

6. Rotary Vacuum Precoat Filtration

7. Sludge/Solids Stabilization 
These unit processes were used as the basis for the comparative analysis of the alternative process configurations of the Candidate Technologies.

\subsection{TECHNICAL CONSIDERATIONS FOR ENERGY MANAGEMENT AND RESOURCE UTILIZATION}

\subsubsection{LANL Site Characteristics}

The LANL is located on the eastern slopes of the Jemez Mountains in north central New Mexico between the altitudes of $6,500 \mathrm{ft}(1980 \mathrm{~m})$ and $9,000 \mathrm{ft}$ $(2700 \mathrm{~m})$. Annual rainfall is approximately 20 inches $(0.5 \mathrm{~m})$. Rainfall and snowmelt drains to the east to the Rio Grande river. The ground water table is more than $1,000 \mathrm{ft}(300 \mathrm{~m})$ below the elevation of 7,200 $\mathrm{ft}(2200 \mathrm{~m})$.

\subsubsection{Siting Considerations for New Land Use}

For optimal consideration, new land resources should include the following criteria:

- The distance from the liquid radioactive waste line required to transfer wastes from the existing TA-50 facility

- The ability to gravity-feed the wastes from TA-50

- Developability and expandability of the site, including size, shape, and slope

- Potential (or lack of) for future adjacent area utilization near TA-50

- Development and redevelopment costs

- $\quad$ Proximity to compatible operations

\subsubsection{Interdependencies with Other Projects}

The project is functionally related to the proposed Hazardous Waste Treatment Facility, and can be collocated in the area approved for the Mixed Waste Receiving and Storage Facility (ref. the 1992 LANL Site Development Plan), which would then house all three waste management operations.

A map indicating this collocation of facilities is provided as Figure 7-1.

\subsubsection{Energy Utilization and Evaporation Processes}

All of the recommended Candidate Technologies incorporate an evaporation unit process as a part of the proposed design. In each scenario, this single unit process would be the operational unit that would require the highest energy consumption in order to perform as designed. Since this will be a common, constant factor among the Candidate Technologies, there will be no clear advantage or disadvantage for any one scenario based on this operation. 
In many applications, operation of a single-effect evaporator may be considered inefficient in terms of energy use. Steam is utilized to effect evaporation in the basic single-effect system, a ratio of $1.2 \mathrm{lbs}$. of steam may be required for the evaporation of each pound of water. This inefficiency can be due to operational factors such as heat loss, mechanical inefficiency, boiling point rise, and other criteria.

In order to increase the unit operation efficiency, a multiple-effect evaporation system is often used. This type of operation uses sequential stages to economize the utilization of steam, and exhibits a correlated efficiency rating for the additional stages. As an example, a triple-stage (triple-effect) evaporator might operate at an efficiency of 3 pounds of evaporated wastewater for every pound of steam consumption.

Since the volume of the current TA-50 waste treatment operations is not large enough to warrant a multiple-stage evaporation system, a mechanical vapor recompression evaporator has been recommended. This system utilizes an engine (driven most likely by natural gas) to compress the steam to a higher pressure. This will allow condensation at a higher temperature, which will conserve energy in the steam recycle. Proper design of this evaporation system should allow an efficiency similar to that for a four-effect evaporator, although higher efficiencies may be achieved.

\subsection{ENERGY AND RESOURCE USE ASSUMPTIONS}

Since there was very little quantitative data available for this study on issues related to energy and resource consumptions, the following assumptions have been utilized for the analysis of energy and resource usage as a part of this BAT study:

- The current operations at TA-50 have developed a normalized energy use cost over the past thirty years that could be normalized to current dollars for the sake of comparison. This qualitative value has been considered as a constant for the comparison of energy costs.

- The current operations and the proposed Candidate Technologies do not require the use or depletion of scarce materials, chemicals, or other resources.

\subsection{ENERGY AND RESOURCE USE ISSUES AND CRITERIA}

Energy and resource issues (as defined by DOE/EH-263T) are those involving energy consumption and use of limited or scarce resources. The impacts of these utilizations are to be considered as a part of the BAT impact analysis process. 


\subsubsection{Energy Usage}

As an attribute affecting selection of alternatives, energy usage may influence technology selection in two significant ways:

1. Energy Costs - Cost estimates should be considered that include the costs of energy associated with operating different technologies. Therefore, those options that have high operating costs because of increased energy requirements and consumption can be identified.

2. Energy Conservation - Considerations should be given to the conservation of energy, especially if strategic fuels (e.g., fossil fuels) will be consumed in large quantities in order to produce the required energy.

Any energy usage and conservation issues related to a specific candidate technology (including the no-action scenario) should be evaluated as elements of the decision process for BAT selection.

\subsubsection{Resource Usage}

As with energy usage, the cost of the resources that would be depleted by the implementation of a given technology should be considered when evaluating candidate technologies. This cost also is a part of the economic impact analysis.

Resource commitments, including irreversible commitments of resources (except for those considered under energy usage) should be included in the evaluation. These may include direct or indirect commitments, such as use of precious metals in effluent removal technology, or use of scarce commodities or services that may impact other operations onsite or in the surrounding communities. Additional potential resource impacts that may be considered are land use and land planning issues, and possible socioeconomic effects of uses of specific resources. Positive effects, such as recovery and recycling of materials or chemicals should be included.

\subsection{ENERGY AND RESOURCE USE AND CONSERVATION ISSUES FOR CANDIDATE Technology \#1}

\subsubsection{Energy Use Issues}

The proposed technology associated with Candidate Technology \#1 involves upgrading the existing TA-55/Room 60 and TA-55 operations by replacing the unit process equipment with new units. 


\section{A. Energy Costs}

The unit processes proposed for Candidate Technology \#1 represent proven, established technologies that have been utilized for numerous industrial applications. The unit processes are similar to those currently in use for Room 60 and TA-50 operations, with the significant addition of:

1. Nitrate destruction (with off-gas treatment)

2. Organic destruction $\mathrm{UV} / \mathrm{O}_{3}$

3. Evaporation

Of these three processes, it is expected that energy consumption for evaporation will be the greatest. Evaporation typically is considered a high energy demand process. The input of energy to provide steam (and steam recompression) to drive the evaporation process will represent the largest energy usage item. The production of ozone for the organic destruction unit will also be a major contributor to energy consumption.

B. Energy Conservation

With the selection and design of new equipment for the Room 60 and TA-50 operations, an increase in efficiency per unit process can be anticipated. However, Candidate Technology \# 1 includes several new unit processes in the treatment scheme when compared to existing operations, so it is expected that the energy requirements will increase over those for the existing RLWP.

By selection of a mechanical vapor recompression evaporator as an additional unit process for Candidate Technology \#1, an optimum level of energy conservation and operational efficiency can be achieved by effective evaporator design.

For the proposed Candidate Technologies associated with this study, the use and consumption of strategic fuels is not a factor that warrants specific consideration.

\subsubsection{Resource Use Issues}

The resources required for operation of the Candidate Technology \#1 scenario are those that would be common to a typical industrial waste treatment operation. 


\section{A. Resource Costs}

For the RLWTF Candidate Technologies, the consideration of expenditure of resources is limited primarily to the consumption of treatment chemicals for the various unit processes. No particularly valuable or irreplaceable resources will be required, so resource costs can be expected to be commensurate with a typical industrial operation.

\section{B. Resource Commitments}

Candidate Technology \#1 requires no special commitment of resources, either direct or indirect, that are not commercially-available items. The consideration of use and requirements for scarce commodities is not applicable.

\section{Land Utilization}

For Candidate Technology \#1, the requirements for land use and planning are minimal. The existing facility site will be utilized, with expansion as allowable into areas adjacent to the existing TA-50-1 building. Only minor adjustments would be required to site development and land utilization plans for LANL.

D. Socioeconomic Effects

There are no applicable socioeconomic effects predicted as the result of the implementation of Candidate Technology \#1.

\section{E. Recovery and Recycling}

There are no current plans at this stage of development of Candidate Technology \#1 for the recycling or reuse of chemicals or materials.

\subsection{ENERGY AND RESOURCE USE AND CONSERVATION ISSUES FOR CANDIDATE Technology \#2}

\subsubsection{Energy Use Issues}

The proposed technology associated with Candidate Technology \#2 involves upgrading the existing TA-50 operations by replacing the unit process equipment with new units, and the construction of a new facility for the pretreatment of TA-55/Room 60 wastes. 


\section{A. Energy Costs}

The unit processes proposed for Candidate Technology \#2 represent proven, established technologies that have been utilized for numerous industrial applications and are similar to those currently in use for Room 60 and TA-50 operations, with the significant addition of:

1. Nitrate destruction (with off-gas treatment)

2. Clarification

3. Organic destruction $\mathrm{UV} / \mathrm{O}_{3}$

4. Evaporation

It is expected that energy consumption for evaporation will be the greatest, with the production of ozone also being a major contributor.

B. Energy Conservation

With the construction of a new facility for Room 60 operations and the selection and design of new equipment for the TA-50 operations, an increase in efficiency per unit process for the Candidate Technology \#2 scenario can be anticipated. It is expected that the energy requirements will increase over those for the existing RLWP, but since an all-new facility is to be constructed to augment the modification of the TA-50 operations, the overall energy demand may be slightly higher than that for Candidate Technology \#1.

By selection of a mechanical vapor recompression evaporator as an additional unit process for Candidate Technology \#2, an optimum level of energy conservation and operational efficiency can be achieved by effective evaporator design.

For the proposed Candidate Technologies associated with this study, the use and consumption of strategic fuels is not a factor that warrants specific consideration.

\subsubsection{Resource Use Issues}

The resources required for operation of the Candidate Technology \#2 scenario are those that would be common to a typical industrial waste treatment operation.

\section{A. Resource Costs}

For the RLWTF Candidate Technologies, the consideration of expenditure of resources is limited primarily to the consumption of 
treatment chemicals for the various unit processes. No particularly valuable or irreplaceable resources will be required, so resource costs can be expected to be commensurate with a typical industrial operation.

\section{B. Resource Commitments}

Candidate Technology \#2 requires no special commitment of resources, either direct or indirect, that are not commercially available items. The consideration of use and requirements for scarce commodities is not applicable.

C. Land Utilization

For Candidate Technology \#2 the requirements for land use and planning are limited, but exceed that for Candidate Technology \#1, because a new facility for Room 60 operations is proposed. The existing facility site will be utilized for the TA-50 operations and an ideal expansion would place the new Room 60 facility adjacent to the existing TA-50-1 building. With these land and facility requirements, minor adjustments would be required to site development and land utilization plans for LANL.

D. Socioeconomic Effects

There are no applicable socioeconomic effects predicted as the result of the implementation of Candidate Technology \#2.

E. Recovery and Recycling

There are no current plans at this stage of development of Candidate Technology \#2 for the recycling or reuse of chemicals or materials.

\subsection{ENERGY AND RESOURCE USE AND CONSERVATION ISSUES FOR CANDIDATE Technology \#3}

\subsubsection{Energy Use Issues}

Candidate Technology \#3 involves upgrading the existing Room 60/TA-55 operations by replacing the unit process equipment with new units, and the construction of a new facility to replace the existing TA-50 operations. 
A. Energy Costs

The proposed unit processes represent proven, established technologies that are of common use in industry and similar to those currently in use for Room 60 and TA-50 operations, with the significant addition of:

1. Nitrate destruction (with off-gas treatment)

2. Clarification

3. Organic destruction $\mathrm{UV} / \mathrm{O}_{3}$

4. Two evaporation processes

It is expected that energy consumption for evaporation will be the greatest, with the production of ozone also being a major contributor.

\section{B. Energy Conservation}

With the construction of a new facility for TA-50 operations and selection and design of new equipment for the Room 60/TA-55 operations, an increase in efficiency per unit process for the Candidate Technology \#3 scenario can be anticipated. As with previous scenarios, it is expected that the energy requirements will increase over those for the existing RLWP. However, since an all-new facility for TA-50 operations is to be constructed to augment the modification of the Room 60/TA-55 operations, the overall energy demand may be expected to be higher than that for previous Candidate Technologies.

By selection of a mechanical vapor recompression evaporator as an additional unit process for Candidate Technology \#3, an optimum level of energy conservation and operational efficiency can be achieved by effective evaporator design. However, the addition of a thin-film finishing evaporator for treatment of the mechanical vapor recompression evaporator bottoms, and additional high energy demand unit process has been included that will increase the overall energy usage for Candidate Technology \#3.

For the proposed Candidate Technologies associated with this study, the use and consumption of strategic fuels is not a factor that warrants specific consideration.

\subsubsection{Resource Use Issues}

As with previous Candidate Technologies, the resources required for operation of the Candidate Technology \#3 scenario are those that would be common to a typical industrial waste treatment operation. 


\section{A. Resource Costs}

For the RCWTF Candidate Technologies, the consideration of expenditure of resources is limited primarily to the consumption of treatment chemicals for the various unit processes. No particularly valuable or irreplaceable resources will be required, so resource costs can be expected to be commensurate with a typical industrial operation.

\section{B. Resource Commitments}

Candidate Technology \#3 requires no special commitment of resources, either direct or indirect, that are not commercially-available items. The consideration of use and requirements for scarce commodities is not applicable.

\section{C. $\quad \underline{\text { Land Utilization }}$}

For Candidate Technology \#3, the requirements for land use and planning exceed those for previous scenarios. The construction of a new major facility to replace the existing TA-50 operations will require coordination with site land use planning organization and appropriate adjustments to site development and land utilization plans for LANL.

There are no specific land utilization considerations associated with the upgrading of the Room 60 operations.

D. Socioeconomic Effects

There are no applicable socioeconomic effects predicted as the result of the implementation of Candidate Technology \#3.

\section{E. Recovery and Recycling}

There are no current plans at this stage of development of Candidate Technology \#3 for the recycling or reuse of chemicals or materials.

\subsection{ENERGY AND RESOURCE USE AND CONSERVATION ISSUES FOR CANDIDATE Technology \#4}

\subsubsection{Energy Use Issues}

Candidate Technology \#4 involves the construction of a new facility to replace the existing TA-50 operations. This facility would incorporate treatment for 
both the primary TA-50 influent and the influent to the existing Room 60 operations.

\section{A. - Energy Costs}

The proposed unit processes represent proven, established technologies that are of common use in industry and similar to those currently in use for Room 60 and TA-50 operations, with the significant addition of:

1. Nitrate destruction (with off-gas treatment)

2. Clarification

3. Organic destruction $\mathrm{UV} / \mathrm{O}_{3}$

4. Two evaporation processes

It is expected that energy consumption for evaporation will be the greatest, with the production of ozone also being a major contributor.

\section{B. Energy Conservation}

With the construction of an all-new facility to replace current TA-50 operations, an increase in efficiency per unit process for the Candidate Technology \#4 scenario can be anticipated. As with previous scenarios, it is expected that the energy requirements will increase over those for the existing RLWP. However, since an all-new facility is to be constructed to replace the existing facility, the overall energy demand may be expected to be higher than that for previous Candidate Technologies.

By selection of a mechanical vapor recompression evaporator as an additional unit process for Candidate Technology \#4, an optimum level of energy conservation and operational efficiency can be achieved by effective evaporator design. However, with the addition of a thinfilm finishing evaporator for treatment of the mechanical vapor recompression evaporator bottoms, high energy demand unit process has been included that will increase the overall energy usage for Candidate Technology \#4.

For the proposed Candidate Technologies associated with this study, the use and consumption of strategic fuels is not a factor that warrants specific consideration. 


\subsubsection{Resource Use Issues}

As with previous Candidate Technologies, the resources required for operation of the Candidate Technology \#4 scenario are those that would be common to a typical industrial waste treatment operation.

\section{A. Resource Costs}

For the RLWTF Candidate Technologies, the consideration of expenditure of resources is limited primarily to the consumption of treatment chemicals for the various unit processes. No particularly valuable or irreplaceable resources will be required, so resource costs can be expected to be commensurate with a typical industrial operation.

B. Resource Commitments

Candidate Technology \#4 requires no special commitment of resources, either direct or indirect, that are not commercially-available items. The consideration of use and requirements for scarce commodities is not applicable.

\section{Land Utilization}

For Candidate Technology \#4 the requirements for land use and planning require the largest amount of consideration and coordination of all of the scenarios. The construction of a new major facility to replace the existing TA-50 operations will require coordination with site land use planning organization and appropriate adjustments to site development and land utilization plans for LANL.

\section{Socioeconomic Effects}

There are no applicable socioeconomic effects predicted as the result of the implementation of Candidate Technology \#4.

E. Recovery and Recycling

There are no current plans at this stage of development of Candidate Technology \#4 for the recycling or reuse of chemicals or materials. 


\subsection{EVALUATION AND ELIMINATION OF PROCESS CONFIGURATIONS BASED ON ENERGY AND RESOURCE USE ISSUES}

\subsubsection{Comparative Analysis of Energy and Resource Use Issues}

The evaluations provided in Sections 7.4 to 7.8 are the basis of the analysis presented in Table 7-1, which summarizes each primary topic related to operations issues and criteria. The rankings of each Candidate Technology (based on the referenced evaluations and review of related materials specified in Section 10) are presented in order of perceived conformance to energy and resource use requirements.

\subsubsection{Status of Candidate Technologies}

The information and criteria for evaluation of energy and resource issues is not well-defined at the BAT study stage of engineering development, so none of the Candidate Technologies can be eliminated strictly on the basis of energy and resource use issues and criteria. In general, the ranking of the four options (from most favorable to least favorable) for the issues and considerations addressed in this section is:

1. Candidate Technology \#1

2. Candidate Technology \#2

3. Candidate Technology \#3

4. Candidate Technology \#4

It should be noted that the differences in these rankings are not equally spaced, nor are the rankings significant enough to eliminate or favorably select any single Candidate Technology on the basis of energy and resource use issues and criteria. 
TABLE 7-1

Comparative Analysis of Energy and Resource Use Issues and Criteria ${ }^{1}$

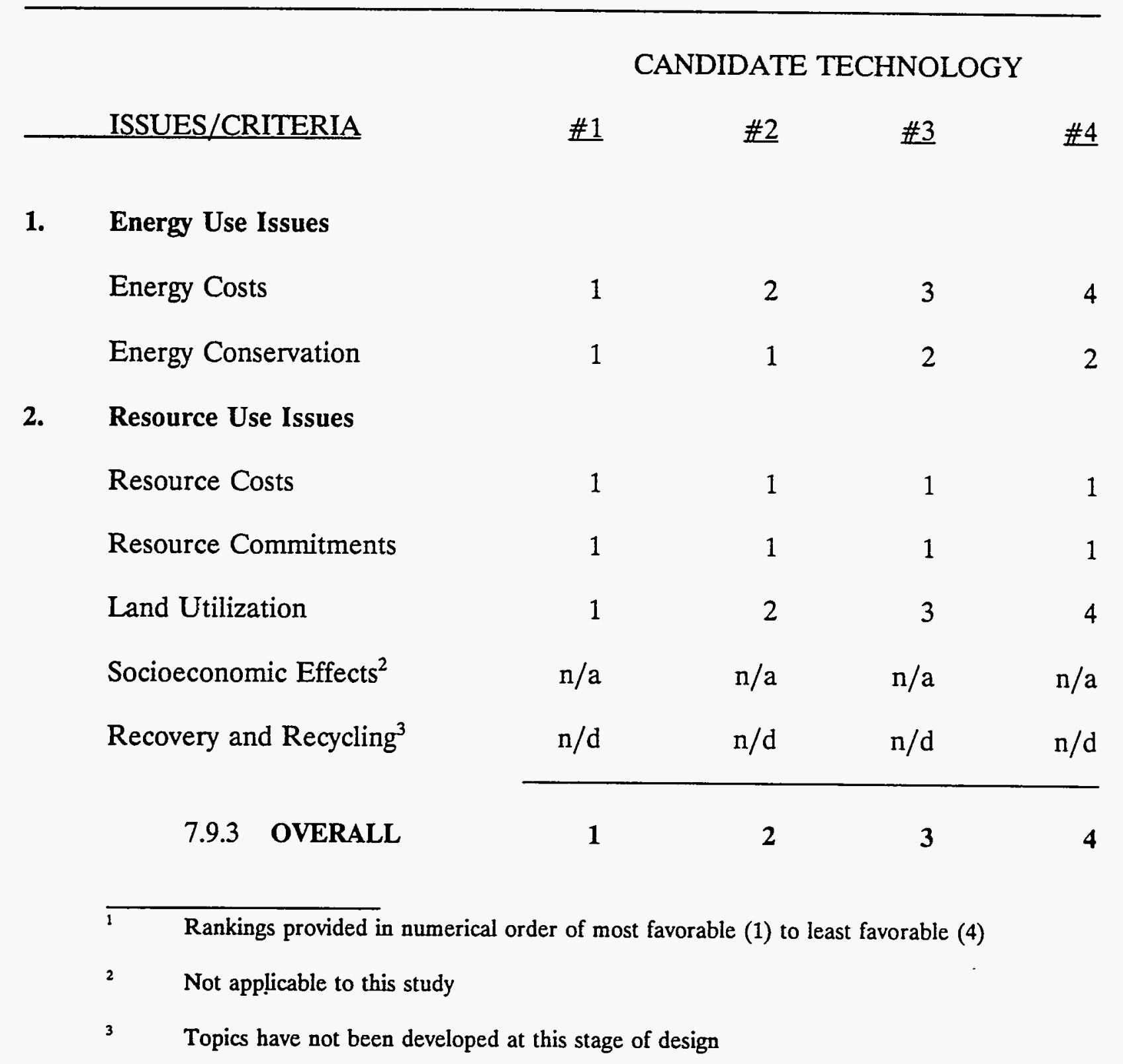




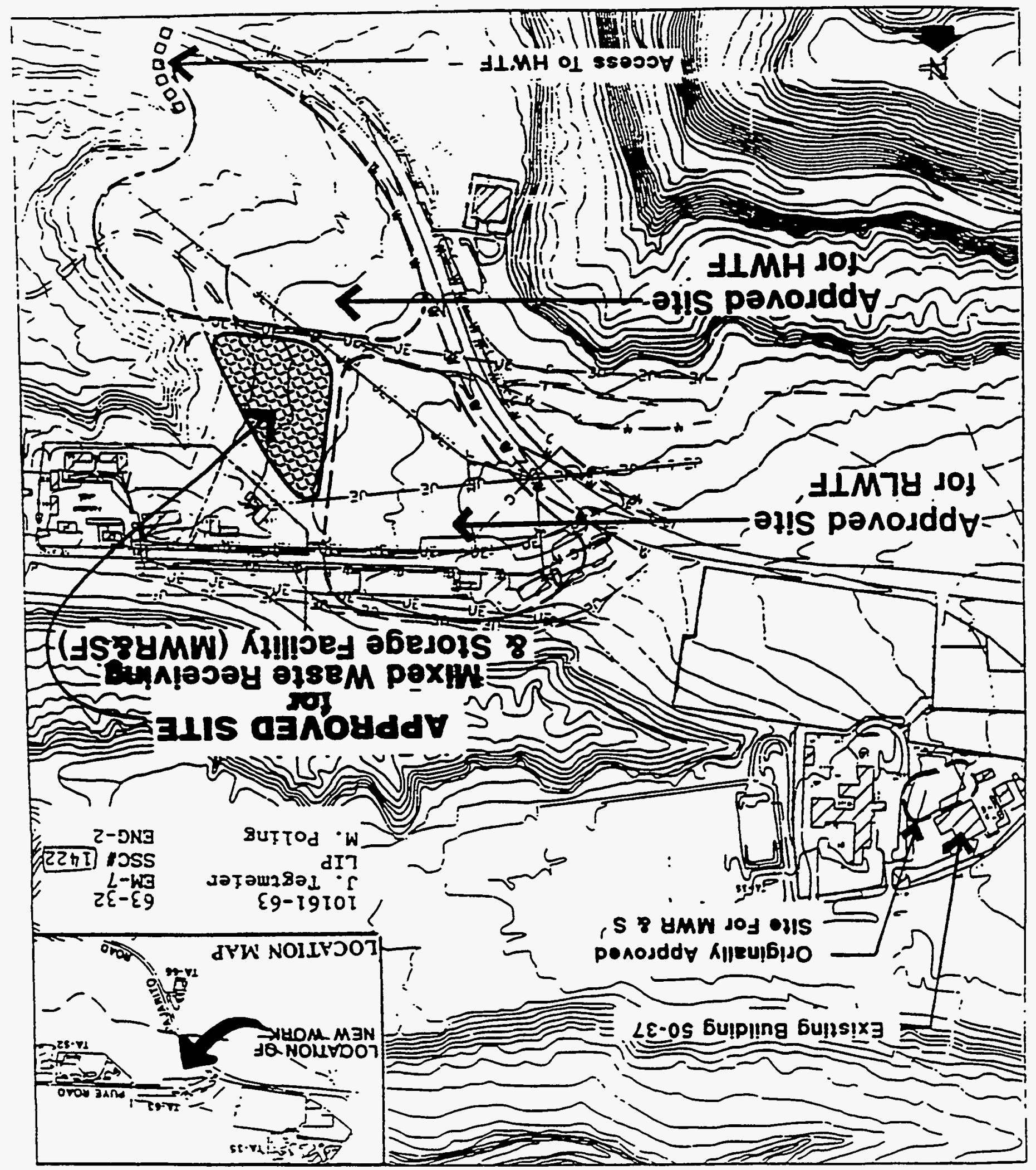

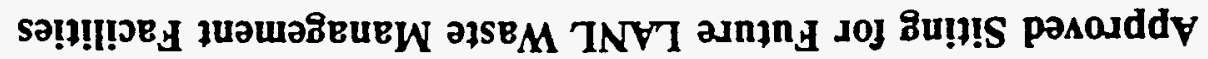
I-L G8חDIA 


\subsection{ANALYSIS OF ECONOMIC/COST FEASIBILITY}

The evaluation of economic feasibility is an area of paramount importance to the selection process for a BAT alternative. Regardless of how favorable (or unfavorable) an alternative might be, the associated costs of construction and operation have a direct bearing on the likelihood of implementation of a given technical option.

\subsection{FIGURE-OF-MERIT CALCULATIONS}

Unlike other categories for evaluation of the potential impacts of alternatives, economic merits are evaluated using 'figures-of-merit'. Economic figures-of-merit are compiled from projections of the cash flows associated with construction and operation of a control system. These estimates provide a basis for the determination of the economic feasibility of the control technology under consideration.

This method of economic analysis requires that the Candidate Technologies be characterized in sufficient detail to allow capital and operating costs to be determined accurately enough to reveal real differences among the competing technologies. The performance analysis must also be detailed enough that the required inputs can be generated with a reasonable level of accuracy. This method requires the consideration of general project information, general economic assumptions, and estimates of project costs.

In order to provide this level of cost analysis, a significant amount of design and operating information is required upon which to make assumptions and to base quantity and unit cost evaluations.

\subsection{ELEMENTS OF ECONOMIC ANALYSIS}

The basis of the economic analysis of Candidate Technologies is the calculation of annual cash flows for four categories of cost. These are the initial (construction and start-up) capital costs, subsequent interim (annual) capital costs, operation and maintenance costs, and decommissioning costs. A final calculation, for net present value, is a combination of all previous cash flow calculations. Four different economic figures-of-merit may be calculated from this information and used for the economic analysis portion of technology comparisons. These are the net present value, the annualized cost, the levelized life-cycle cost of radionuclide removal, and the levelized life-cycle cost per unit of throughput.

\subsubsection{Determination of Capital Costs}

Initial capital costs are any costs that occur at the beginning of the project, and during construction and facility start-up. Interim capital costs may occur at any time during the life of the facility. These costs are typically associated with major modifications or process improvements, and are expected to occur 
infrequently. DOE requires that a 'betterments' provision be met for improvements or modifications to be classified as capital expense, which requires that:

1. The total cost of the improvement or modification must be at least $20 \%$ of the initial cost.

2. The improvement or modification must extend the useful life of the facility.

Topics for consideration in the determination of capital costs include:

- Facilities (e.g, building structure, support facilities, storage facilities, waste management facilities, etc.)

- Equipment (e.g., unit process equipment, tanks, monitoring and sampling equipment, etc.)

- Original complement (i.e., items required to outfit the facility for occupancy)

- Other capital costs (e.g., engineering, design, start-up and testing, documentation, etc.)

\subsubsection{Determination of Operating and Maintenance Costs}

Operation and maintenance (O\&M) costs are regularly-occurring costs that can be estimated on an annual basis. Such costs are generally for labor and materials but other annual costs such as increased downtime also are included. Changes in O\&M labor costs should be considered.

Increased labor costs generally can be attributed to one of three factors:

1. Increases in the number of O\&M hours required by the technology

2. Increases in labor rates for O\&M personnel

3. Decreases in the labor hours available from each worker due to health protection requirements (e.g., increases in exposure)

Major O\&M cost headings and evaluation elements may include:

- $\quad$ Routine operations (e.g., operating procedures, waste handling, dose monitoring, inspection and testing, etc.)

- Maintenance (e.g., for new facilities or increased requirements for existing facilities, increased equipment technology, testing and monitoring, etc.)

- Utilities (e.g., electrical, water, gas/air, etc.)

- $\quad$ Supplies (e.g., filters, chemicals, spare parts, etc.)

- Downtime (i.e., unscheduled outages and increases in scheduled downtime) 
- Other O\&M costs (e.g., new hiring, training, document preparation, administration, etc.)

\subsubsection{Determination of Decommissioning Costs}

At the end of the useful life of the facility, the facility and control process equipment must be decommissioned and transported for disposal. The costs associated with these requirements consist primarily of labor to decontaminate the facility and equipment and disposal costs. Any potential revenue from recycle or recovery activities should be weighed against decommissioning costs.

Major decommissioning costs may be grouped into topics such as:

- Decontamination (e.g., decontamination systems and equipment, secondary waste management, etc.)

- Disposal (e.g., disposal site costs, waste type and form evaluation, transportation, temporary storage, etc.)

\subsection{ECONOMIC CONSIDERATIONS FOR CANDIDATE TECHNOLOGIES}

In order to calculate the detailed cost analyses recommended by DEO/EH-263T, a level of design for each viable Candidate Technology must be completed that will provide the cost analysis with sufficient information upon which to justify the elements of the cost estimations. For this BAT, the level of design for Candidate Technologies were sufficient for generalized estimations of capital costs, but information was lacking that would have allowed the accurate assessment of costs associated with operations and maintenance and decommissioning. In order to weigh these factors with the contexts of economic impact, relative cost comparisons were compiled.

\subsubsection{Bases of the BAT Cost Estimates}

The estimates for this study were based on the four technology options, with each option having specific building requirements. The buildings are classified as moderate hazard structures (as a worst-case estimate, without the benefit of a hazards analysis) for the sake of design criteria comparison. For Candidate Technology \#1 \#2, and \#3 a portion of the existing buildings is used to house process equipment.

The costs of upgrading the existing buildings to comply with DOE Order 6430.1A standards (if this could be accomplished) is not included in these estimates. The information supplied for this study regarding such factors as structural conditions or contamination levels and amounts is insufficient to attempt any meaningful estimations based on these criteria. 
Areas where new buildings are to be constructed are considered to be 'green field' design, with no area contamination to be considered. Therefore, no costs have been included for site decontamination or remedial preparation.

\subsubsection{Cost Factors and Allowances}

An allowance of $\$ 750,000$ is included in each option to cover the costs of laboratory equipment. Also included in each option is funding for original complement items (e.g., furniture) based on the building sizes. A budget of $\$ 75,000$ is included in each option for safety analysis.

A $15 \%$ labor inefficiency factor is included to cover the time lost for facility access through security checkpoints, and to accommodate time loss for health protection practices such as dress-out and showering for work in contaminated areas.

The cost for instrumentation and piping, and electrical requirements was based on factors developed for a recent similar project at the DOE Oak Ridge Reservation. The building, equipment foundations, and site costs also were calculated based on the cost factors derived from this project.

The costs for engineering, construction management, and facility operations were based on percentages of the construction value.

Escalation was included at $10 \%$, and a contingency was added of $25 \%$ of the project cost.

The detailed cost analysis sheets for this study are provided as Appendix G.

\subsubsection{Special Considerations for Cost Evaluation}

The lack of detailed information on many cost-related topics required that estimates be made on comparative experience and engineering judgement. The cost/economic estimates for this BAT represent a reasonably accurate assessment of cost relative to the economics among the four options. However, caution should be utilized when comparing these costs with those prepared for similar operations outside the context of this evaluation.

The available information provided for economic analysis was insufficient for calculation of a realistic value for interim capital costs. In order to provide some basis of relative economic comparison, quantitative estimates of the anticipated interim costs for the four Candidate Technologies were made.

O\&M costs were calculated with a constant factor for operations and maintenance based on the complexity of operations rather than condition of 
the facilities to be utilized. The existing facilities were not characterized in a level of detail that would allow an accurate cost estimate for O\&M purposes.

Table 8-1 provides a summary of the economic evaluation factors.

\subsubsection{Estimation of D\&D Costs}

Within the DOE complex, D\&D costs vary greatly from site to site, and often from facility to facility within a site. Factors such as the complexity of operations and the degree and levels of contamination can create vast disparities in estimates. Without the advantage of a more detailed design and approved figures for $D \& D$ unit costs, a realistic $D \& D$ calculation was not possible.

In order to obtain some estimation of potential $D \& D$ costs, a totally subjective calculation was performed. The assumptions used for this estimate were:

1. D\&D costs are directly related to the number of pieces of equipment in a facility.

2. D\&D costs may be very high, in some instances higher than the construction cost of a facility.

3. Worst-case contamination can be assumed.

4. Factors from other DOE sites can be used as general guidance.

The factors mentioned in Item 4 were derived from two major D\&D projects, one at the Oak Ridge site and one at the Fernald Environmental Management Project. The assumption used was that there is a correlation between the square footage of roof area and the costs for facilities of comparable complexity (this holds true only for one-story construction).

For the Oak Ridge estimate, values ranging from $\$ 1225 / \mathrm{ft}^{2}$ to $\$ 4134 / \mathrm{ft}^{2}$ were calculated, depending on the range of contamination and methodologies for cleanup. For the Fernald site, an estimate of $\$ 1677 / \mathrm{ft}^{2}$ was calculated for a number of facilities housing low-level contamination. Since only low-level contamination is expected at the RIWTF, a median value of $\$ 1700 / \mathrm{ft}^{2}$ was used for this study. While this number generated D\&D costs that appear to be high, it represents application of a consistent factor for evaluation basis. These factors are summarized in Table 8-2.

It should be noted that the $D \& D$ costs for this project were calculated only for the facilities to be decommissioned at the end of the life cycle starting with implementation of an alternative. Existing facilities not included for use in a specific scenario were not included in D\&D considerations. 


\subsection{COST ANALYSIS OF CANDIDATE Technology \#1}

\subsubsection{Capital/Construction Cost Estimates}

With the least amount of proposed new construction, Candidate Technology \#1 represents the lowest cost alternative. Based on the criteria and assumptions discussed in Section 8.3, a capital cost figure of $\$ 69,515,000$ was calculated.

Although available information was insufficient for calculation of a realistic value for interim capital costs, a quantitative estimate of the anticipated interim costs for Candidate Technology \#1 provided a relative ranking of 1 (highest), reflecting the use of aging and deteriorated existing facilities.

\subsubsection{Relative Operating and Maintenance (O\&M) Costs}

O\&M costs were based on experience with similar types of facilities and operations, using factors estimated for related engineering tasks. Based on an arbitrary analysis of the level and complexity of the equipment and operations to be conducted for Candidate Technology \#1, an annual cost of $\$ 1,123,200$ was calculated for operations along with a cost of $\$ 507,100$ for maintenance, for a total O\&M cost of $\$ 1,630,300$ per year.

Although this represents a relative small comparative figure, some items that might be considered maintenance were included in the uncertainty associated with interim capital costs. The amount of maintenance cost per operating unit is expected to be highest for this option.

\subsubsection{Relative Decommissioning Costs}

Insufficient information was available for a detailed estimation of $D \& D$ costs. Based on comparison to projects conducted at other DOE sites, and assuming significant contamination of facilities and equipment, a speculative D\&D cost estimate of $\$ 144,883,000$ was calculated.

\subsection{COST ANALYSIS OF CANDIDATE Technology \#2}

\subsubsection{Relative Capital/Construction Costs}

Candidate Technology \#2 involves upgrading TA-50 and the construction of a new facility to replace Room 60 operations. A capital cost figure of $\$ 77,319,000$ was assigned for Candidate Technology \#2.

With insufficient information to calculate interim capital costs, a quantitative estimation placed a relative ranking of 2 for interim costs for this option. 


\subsubsection{Relative Operating and Maintenance (O\&M) Costs}

Based on experience and factors from similar tasks, an annual cost of $\$ 1,123,200$ was calculated for operations along with a cost of $\$ 536,600$ for maintenance, for a total O\&M cost of $\$ 1,659,800$ per year. However, some items that were considered as potential interim capital cost items may actually be managed under O\&M operations.

\subsubsection{Relative Decommissioning Costs}

Insufficient information was available for a detailed estimation of $D \& D$ costs. Based on comparison to projects conducted at other DOE sites, and assuming significant contamination of facilities and equipment, a speculative D\&D cost estimate of $\$ 166,388,000$ was calculated.

\subsection{COST ANALYSIS OF CANDIDATE Technology \#3}

\subsubsection{Relative Capital/Construction Costs}

Candidate Technology \#3 requires a construction of a major facility to replace the primary TA-50 operations, and also requires the most equipment for new and upgraded system. This option has the highest capital costs, which were estimated at $\$ 144,684,000$.

With insufficient information to calculate interim capital costs, a quantitative estimation placed a relative ranking of 3 for interim costs for this option.

\subsubsection{Relative Operating and Maintenance (O\&M) Costs}

Based on experience and factors from similar tasks, an annual cost of $\$ 1,123,200$ was calculated for operations along with a cost of $\$ 1,007,700$ for maintenance, for a total O\&M cost of $\$ 2,130,900$ per year.

Although this is the highest predicted annual O\&M cost among the Candidate Technologies, it should be considered that this option also includes the most new pieces of equipment. O\&M per unit operation should be comparatively low.

\subsubsection{Relative Decommissioning Costs}

Insufficient information was available for a detailed estimation of $D \& D$ costs. Based on comparison to projects conducted at other DOE sites, and assuming significant contamination of facilities and equipment, a speculative D\&D cost estimate of $\$ 242,038,000$ was calculated. 


\subsection{COST ANALYSIS OF CANDIDATE Technology \#4}

\subsubsection{Relative Capital/Construction Costs}

Candidate Technology \#4 involves the highest level of new facility construction. However, the upgrades planned for Candidate Technology \#3 involve more equipment. The estimated capital costs for Candidate Technology \#4 were \$138,235,000.

With insufficient information to calculate interim capital costs, a quantitative estimation placed a relative ranking of 4 (lowest) for interim costs for this option.

\subsubsection{Relative Operating and Maintenance (O\&M) Costs}

Based on experience and factors from similar tasks, an annual cost of $\$ 1,123,200$ was calculated for operations along with a cost of $\$ 962,800$ for maintenance, for a total O\&M cost of $\$ 2,086,000$ per year.

The high O\&M cost for this option is indicative of the quantity of new equipment. O\&M per unit operation should be comparatively low.

\subsubsection{Relative Decommissioning Costs}

Insufficient information was available for a detailed estimation of $D \& D$ costs. Based on comparison to projects conducted at other DOE sites, and assuming significant contamination of facilities and equipment, a speculative D\&D cost estimate of $\$ 196,563,000$ was calculated.

\subsection{ESTIMATED RELATIVE PROJECT COST COMPARISONS FOR SELECTED CANDIDATE TECHNOLOGIES}

Since many of the variables needed to produce more accurate cost evaluations were not available for this study, relative total cost comparisons were prepared with the following assumptions:

1. The calculated capital costs in this study are accurate.

2. Interim capital costs can be estimated as an arbitrary percent of capital costs by applying a factor of 5\% times the relative ranking in Table 8-1.

3. Total O\&M costs can be calculated by multiplying the annual costs from Table 8-1 times a 20-year period.

4. $\mathrm{D} \& \mathrm{D}$ costs are speculative, and are not included.

Since the D\&D costs follow a general trend of construction costs, the elimination of this factor should not change the relative total cost rankings. 
The final cost analysis is provided in Table 8-3. The total estimated project costs for the four Candidate Technologies are:

Candidate Technology \#1 - $\$ 116,024,000$

Candidate Technology \#2 - \$122,113,000

Candidate Technology \#3 - \$201,731,000

Candidate Technology \#4 - \$186,867,000 
TABLE 8-1

Economic Evaluation of the Candidate Technologies ${ }^{2}$

\begin{tabular}{|c|c|c|c|c|c|}
\hline CT\# & CAPITAL COSTS & $\begin{array}{r}\text { CAPITAL } \\
\text { COST RANKING } \\
\end{array}$ & $\begin{array}{l}\text { INTERIM CAPITAL } \\
\text { COST RANKING } \\
\end{array}$ & $\begin{array}{r}\text { ANNUAL } \\
\text { O\&M COSTS } \\
\end{array}$ & $\begin{array}{l}\text { O\&M COST } \\
\text { RANKING } \\
\end{array}$ \\
\hline 1 & $\$ 69,515,000$ & 1 & 4 & $\$ 1,630,300$ & 1 \\
\hline 2 & $\$ 77,319,000$ & 2 & 3 & $\$ 1,659,800$ & 2 \\
\hline 3. & $\$ 144,648,000$ & 4 & 2 & $\$ 2,130,900$ & 4 \\
\hline 4. & $\$ 138,235,000$ & 3 & 1 & $\$ 2,086,000$ & 3 \\
\hline
\end{tabular}

2 Rankings provided in numerical order of most favorable (1) to least favorable (4) 
TABLE 8-2

Speculative Estimation of D\&D Costs for Candidate Technologies

\begin{tabular}{|c|c|c|c|}
\hline CT\# & $\begin{array}{l}\text { TOTAL PIECES } \\
\text { OF EOUIPMENT }\end{array}$ & $\underline{\text { ROOF AREA }}$ & $\begin{array}{l}\text { ESTIMATED } \\
\text { D\&D COSTS }\end{array}$ \\
\hline 1 & 95 & $\begin{array}{l}E-13,000 \\
N-72,225 \\
T-85,225\end{array}$ & $\$ 144,883,000$ \\
\hline 2 & 113 & $\begin{array}{l}E-13,000 \\
\text { N }-84,875 \\
T-97,875\end{array}$ & $\$ 166,388,000$ \\
\hline 3 & 154 & $\begin{array}{r}E-13,000 \\
N-129,375 \\
T-172,375\end{array}$ & $\$ 242,038,000$ \\
\hline 4 & 133 & $N-115,625$ & $\$ 196,563,000$ \\
\hline
\end{tabular}

3 Area expressed in square feet 
TABLE 8-3

Estimation of Total Project Costs for the Candidate Technologies

\begin{tabular}{lrrrr}
\hline CT \# & CAPITAL COSTS & $\begin{array}{r}\text { INTERIM CAPITAL } \\
\text { COST ESTIMATES }\end{array}$ & $\begin{array}{r}\text { O\&M COST } \\
\text { ESTIMATES }^{5}\end{array}$ & $\begin{array}{r}\text { TOTAL ESTIMATED } \\
\text { PROJECT COSTS }^{6}\end{array}$ \\
1 & $\$ 69,515,000$ & $\$ 13,903,000$ & $\$ 32,606,000$ & $\$ 116,024,000$ \\
2 & $\$ 77,319,000$ & $\$ 11,597,850$ & $\$ 33,196,000$ & $\$ 122,113,000$ \\
3 & $\$ 144,648,000$ & $\$ 14,464,800$ & $\$ 42,618,000$ & $\$ 201,731,000$ \\
4 & $\$ 138,235,000$ & $\$ 6,911,750$ & $\$ 41,720,000$ & $\$ 186,867,000$
\end{tabular}

4 Calculated at $5 \%$ of the capital costs times the relative ranking

5 Calculated as 20 years times the annual O\&M costs

6 Does not include estimates for D\&D 


\subsection{SELECTION OF THE BEST AVAILABLE TECHNOLOGY}

The final assessment of the BAT (as defined by the process in DOE/EH-263T) is a three-step process that utilizes compiled data and professional judgement to reach objective conclusions. This process provides a structured approach that encourages objective evaluation and accountability of the analyses. Each Candidate Technology is evaluated relative to the existing (no-action) technology for each of the key issues, thereby providing a common base of reference. Final BAT selection may then be conducted impartially with examination of costs and the impacts of each selected technology.

\subsection{TECHNOLOGY ISSUES}

The technical evaluation of Candidate Technologies involves the combined qualitative and quantitative comparison of proposed options in order to determine which of the options is optimum for the site-specific application. The main areas that form the basis for comparative analysis are environmental, operational, energy and resources, and economic (as addressed in Sections 4 through 8 of this study).

For the issues that have been identified as significant, the comparative analysis should include an evaluation against the no-action scenario in order to establish a relative value of the proposed configuration. The criteria used as the basis for the comparisons should be weighted by applying a 'value factor' to each criterion, based on an evaluation and estimate of the impact of the criterion in relation to technical feasibility, sensitivity, parties involved, and level of concern. Since a given Candidate Technology potentially may be either better or worse than the existing no-action alternative, the no-action case is valued in the center of the range of value factors. In accordance with the guidance provided by DOE/EH-263T, the working range for a BAT values is 0 to 10 , with the no-action case rated at the value of 5 . All other Candidate Technologies are to be rated in relation to this median. The general criteria system for establishing value factors is presented in Table 9-1.

\subsubsection{Relative Comparison of Technologies}

The comparative analysis should focus on those factors that will assist in discriminating among the Candidate Technologies. The evaluations are to be as objective as possible but in many instances, lack of information requires that judgement be used based on professional experience, good engineering practices, general knowledge, and whatever site-specific information that is available. Although evaluation factors (especially those expressed as costs) may vary considerably from those that would be calculated for a more detailed phase of design, the important issue is that all factors be objective and applied consistently to provide a true basis for relative comparison among the selected technologies. 


\subsubsection{Technology Comparison Matrix}

The Technology Comparison Matrices are the basis of the overall BAT study. A Technology Comparison Matrix has been prepared for each of the primary technical evaluation areas, which are:

1. Environmental Issues (Table 9-2, based on the information and analyses from Sections 4 and 5)

2. Operational Issues (Table 9-3, based on the information and analyses from Section 6)

3. Energy and Resource Issues (Table 9-4, based on the information and analyses from Section 7)

Each matrix provides a means for comparing and evaluating the four Candidate Technologies on a relative basis using the same criteria. The value factors for each technical category are averaged, and summary value factors for the major technological topics are prepared for further evaluation. The summary value factors for this study are presented in Table 9-5.

\subsubsection{Summary Comparison Matrix}

The Summary Comparison Matrix summarizes the information in the Technology Comparison Matrices, and provides a weighted evaluation of the impact of each technology. Two types of information are used for the Summary Comparison Matrix.

The first information required is gathered from the evaluations of the Technology Comparison Matrices. The issues identified during the evaluation process and the value factors determined for each Candidate Technology for each of those issues are the foundation of the matrix. Also included is information on impact evaluations that is common or applicable to all of the technology options.

The second information category required is a determination of the relative importance of each of the technology issues. A weighting factor is assigned to each issue based on the relative importance of the issue to all other issues. The weighting factors used in this study are presented in Table 9-6 and are consistent for all Candidate Technologies.

Once all value factors and weighting factors have been established and entered into the matrix, the total weighted value factor is calculated for each Candidate Technology. Examining each technology individually, the value factor and the weighting factor are multiplied for each issue in order to 
quantify the ranking of the technology. The weighted values for each technical issue are summed to produce a total weighted value factor for each Candidate Technology. This final technical evaluation is presented in the Summary Comparison Matrix in Table 9-7.

\subsection{ECONOMIC EVALUATION}

The discussion in Section 8 outlines the approach taken in this study for economic analysis, and the figures and parameters estimated for the analysis. Due to the preliminary development of the Candidate Technologies and the need for additional process and cost information for the existing operations, it was not possible to provide economic calculations for levelized costs, investment and cash flow, present value, figures-of-merit, or detailed life-cycle costs. The figures provided at total project costs in Table 8-3 represent a lump sum total expected cash outlay for operations, without inclusion of $D \& D$ costs.

\subsection{FINAL COST-EFFECT ANALYSIS AND TECHNOLOGY SELECTION}

The final step in the BAT process is to assemble the technical and economic information into a format where an overall comparison can be made. This is to facilitate the selection of the Candidate Technology rated to be the BAT by the study.

\subsubsection{Basis for the BAT Selection}

As directed by the guidance in DOE/EH-263T, each of the Candidate Technologies were evaluated carefully, initially based on technical merit (e.g., the option with the greatest positive effect). The primary guidance for technical evaluation is the Total Weighted Value Factors, which combine the analysis of environmental, operational, and energy and resource use elements. As stated in DOE/EH-263T, economic calculations are secondary considerations in the decision-making process.

\subsubsection{Determination of the BAT}

Upon consideration of the significant superior technical advantages versus the relative cost factors, it becomes evident that there is much to be gained by implementation of the scenario in Candidate Technology \#4. Based on the documents, information, and client input to this study, it seems that this option presents the greatest opportunity for reliable operations and compliance with regulatory requirements, while maintaining a reasonably comparable cost.

The criteria that were compiled for the evaluation and selection of the BAT are presented in the cost-effect summary in Table 9-8. 
TABLE 9-1

General Criteria for Establishing Value Factors

for Evaluating Candidate Technologies

\begin{tabular}{|c|l|}
\hline Value Factor (VF) & \multicolumn{1}{|c|}{ Criteria } \\
\hline \hline 0 & $\begin{array}{l}\text { Inferior (i.e., the candidate technology is not appropriate } \\
\text { for this issue) }\end{array}$ \\
\hline $1-2$ & $\begin{array}{l}\text { Substantially deficient, definite negative effect (i.e., the } \\
\text { candidate technology is significantly worse for this issue } \\
\text { than the existing technology) }\end{array}$ \\
\hline $3-4$ & $\begin{array}{l}\text { Slightly deficient, slight negative effect (i.e., the candidate } \\
\text { technology is somewhat worse for this issue than the } \\
\text { existing technology) }\end{array}$ \\
\hline 5 & $\begin{array}{l}\text { No change (i.e., the candidate technology does not offer any } \\
\text { change from the existing, baseline technology) }\end{array}$ \\
\hline $6-7$ & $\begin{array}{l}\text { Minimal improvement, slight positive effect (i.e., the } \\
\text { candidate technology improves on this issue only slightly) }\end{array}$ \\
\hline $8-9$ & $\begin{array}{l}\text { Substantial improvement, definite positive effect (i.e., the } \\
\text { candidate technology improves on the issue quite well) }\end{array}$ \\
\hline 10 & $\begin{array}{l}\text { Excellent improvement, significant positive effect (i.e., the } \\
\text { candidate technology improves on the issue extremely well, } \\
\text { even if it does not totally resolve the issue) }\end{array}$ \\
\hline
\end{tabular}


TABLE 9-2

Technology Comparison Matrix for Environmental Issues

\begin{tabular}{|c|c|c|c|c|c|}
\hline & $\begin{array}{l}\text { No-Action } \\
\text { Alternative }\end{array}$ & Option 1 & Option 2 & Option 3 & Option 4 \\
\hline Issues & VF & VF & VF & VF & VF \\
\hline \multicolumn{6}{|l|}{ 1. Environmental Releases } \\
\hline Release of Radionuclides & 5 & 7 & 7 & 7 & 7 \\
\hline $\begin{array}{l}\text { Total Annual Discharge of } \\
\text { Contaminants }\end{array}$ & 5 & 8 & 8 & 9 & 9 \\
\hline Dose Contributions & 5 & 6 & 6 & 9 & 10 \\
\hline Ecological Impacts & 5 & 5 & 5 & 4 & 4 \\
\hline \multicolumn{6}{|l|}{ 2. Regulatory Compliance } \\
\hline $\begin{array}{l}\text { Compliance with Proposed } \\
\text { Discharge Limits }\end{array}$ & 5 & 9 & 9 & 9 & 9 \\
\hline Flexibility for Future Compliance & 5 & 6 & 6 & 9 & 10 \\
\hline $\begin{array}{l}\text { Compliance with Derived } \\
\text { Concentration Guidelines }\end{array}$ & 5 & 9 & 9 & 9 & 9 \\
\hline \multicolumn{6}{|l|}{ 3. Waste Generation and Disposal } \\
\hline Minimization of TRU Waste & 5 & 7 & 7 & 7 & 7 \\
\hline Minimization of Mixed Waste & 5 & 8 & 8 & 9 & 9 \\
\hline Secondary Liquid Wastes & 5 & 7 & 7 & 7 & 7 \\
\hline Sludge/Sediment Volumes & 5 & 7 & 7 & 8 & 8 \\
\hline
\end{tabular}


TABLE 9-3

Technology Comparison Matrix for Operational Issues

\begin{tabular}{|c|c|c|c|c|c|}
\hline & $\begin{array}{l}\text { No-Action } \\
\text { Alternative }\end{array}$ & Option 1 & Option 2 & Option 3 & Option 4 \\
\hline Issues & VF & VF & VF & VF & VF \\
\hline \multicolumn{6}{|l|}{ 1. General Considerations } \\
\hline Existing Conditions & 5 & 6 & 7 & 8 & 10 \\
\hline Functional Requirements & 5 & 6 & 7 & 8 & 10 \\
\hline $\begin{array}{l}\text { Theoretical Mass Balance of Unit } \\
\text { Operations }\end{array}$ & 5 & N/A & N/A & N/A & $\mathrm{N} / \mathrm{A}$ \\
\hline Decontamination Requirements & 5 & 4 & 3 & 2 & 1 \\
\hline \multicolumn{6}{|l|}{ 2. Cost and Performance } \\
\hline Existing Facility Constraints & 5 & 6 & 7 & 9 & 10 \\
\hline Throughput Capability & 5 & 7 & 7 & 9 & 10 \\
\hline Maintenance Requirements & 5 & 8 & 8 & 9 & 10 \\
\hline $\begin{array}{l}\text { Equipment Availability and } \\
\text { Reliability }\end{array}$ & 5 & 8 & 8 & 9 & 10 \\
\hline $\begin{array}{l}\text { Resources Required for } \\
\text { Operation }\end{array}$ & 5 & 6 & 7 & 9 & 10 \\
\hline Human Factors & 5 & 6 & 7 & 9 & 10 \\
\hline \multicolumn{6}{|l|}{$\begin{array}{l}\text { 3. Long-Term Technology } \\
\text { Effectiveness }\end{array}$} \\
\hline $\begin{array}{l}\text { Public Policy Issues and } \\
\text { Considerations }\end{array}$ & 5 & $\mathrm{~N} / \mathrm{C}$ & $\mathrm{N} / \mathrm{C}$ & $\mathrm{N} / \mathrm{C}$ & $\mathrm{N} / \mathrm{C}$ \\
\hline $\begin{array}{l}\text { Flexibility to Accommodate } \\
\text { Process Changes }\end{array}$ & 5 & 6 & 6 & 9 & 10 \\
\hline $\begin{array}{l}\text { Compliance with Safety } \\
\text { Requirements }\end{array}$ & 5 & 5 & 6 & 9 & 10 \\
\hline $\begin{array}{l}\text { Flexibility to Address Advances in } \\
\text { Technology }\end{array}$ & 5 & 7 & 8 & 9 & 10 \\
\hline
\end{tabular}

N/A - Information not available at this time

$\mathrm{N} / \mathrm{C}$ - Issues associated with this topic are not clear at this time 
TABLE 9-4

Technology Comparison Matrix for Energy and Resource Issues

\begin{tabular}{||l|c|c|c|c|c||}
\hline & $\begin{array}{c}\text { No-Action } \\
\text { Alternative }\end{array}$ & Option 1 & Option 2 & Option 3 & Option 4 \\
\hline \multicolumn{1}{|c|}{ Issues } & VF & VF & VF & VF & VF \\
\hline \hline 1. Energy Use Issues & & & & & \\
\hline Energy Costs & 5 & 5 & 5 & 4 & 4 \\
\hline \hline Energy Conservation & 5 & 5 & 5 & 4 & 4 \\
\hline 2. Resource Use Issues & & & & & \\
\hline Resource Costs & 5 & 6 & 6 & 7 & 7 \\
\hline Resource Commitments & 5 & 5 & 5 & 5 & 5 \\
\hline Land Utilization & 5 & 5 & 5 & 4 & 4 \\
\hline Socioeconomic Effects & 5 & N/A & N/A & N/A & N/A \\
\hline Recovery and Recycling & 5 & N/A & N/A & N/A & N/A \\
\hline
\end{tabular}

N/A - This issue is either not applicable to this study, or insufficient information is available 
TABLE 9-5

Summary Value Factor Matrix for the Candidate Technologies

\begin{tabular}{||l|c|c|c|c|c||}
\hline \multicolumn{1}{|c|}{ Issues } & $\begin{array}{c}\text { No-Action } \\
\text { Alternative }\end{array}$ & Option 1 & Option 2 & Option 3 & Option 4 \\
\hline \hline \multicolumn{1}{|c|}{ 1. Environmental Issues } & VF & VF & VF & VF & VF \\
\hline \hline Environmental Releases & 5 & 6 & 6 & 7 & 7 \\
\hline Regulatory Compliance & 5 & 8 & 8 & 9 & 9 \\
\hline Waste Generation and Disposal & 5 & 7 & 7 & 8 & 8 \\
\hline \multicolumn{1}{|c|}{ 2. Operational Issues } & & & & & \\
\hline \hline General Considerations & 5 & 5 & 6 & 6 & 7 \\
\hline Cost and Performance & 5 & 7 & 7 & 9 & 10 \\
\hline $\begin{array}{l}\text { Long-Term Technology } \\
\text { Effectiveness }\end{array}$ & 5 & 6 & 7 & 9 & 10 \\
\hline \hline $\begin{array}{l}\text { 3. Energy and Resource Use } \\
\text { Issues }\end{array}$ & & & & & \\
\hline \hline Energy Use & 5 & 5 & 5 & 4 & 4 \\
\hline Resource Use Issues & 5 & 5 & 5 & 5 & 5 \\
\hline \hline
\end{tabular}


TABLE 9-6

Weighting Factors for the Analysis of Candidate Technologies

ISSUES/CRITERIA

WEIGHTING FACTOR

1. Environmental Issues

$\begin{array}{ll}\text { Environmental Release } & 10\end{array}$

$\begin{array}{ll}\text { Regulatory Compliance } & 20\end{array}$

Waste Generation and Disposal 20

2. Operational Issues

$\begin{array}{ll}\text { General Considerations } & 10\end{array}$

$\begin{array}{ll}\text { Cost and Performance } & 15\end{array}$

Long-Term Technology Effectiveness 15

3. Energy and Resource Use Issues

Energy Use 5

Resource Use Issues 5

TOTAL OF WEIGHTING FACTORS $\quad 100$ 
TABLE 9.7

Summary Comparison Matrix for the Candidate Technologies

\begin{tabular}{|c|c|c|c|c|c|c|c|c|c|c|c|}
\hline \multirow{2}{*}{$\begin{array}{c}\text { Technology Issues Matrix } \\
\text { Issues }\end{array}$} & \multirow[b]{2}{*}{ WF } & \multicolumn{2}{|c|}{$\begin{array}{l}\text { Existing } \\
\text { No-Action } \\
\text { Alternative }\end{array}$} & \multicolumn{2}{|c|}{ Option 1} & \multicolumn{2}{|c|}{ Option 2} & \multicolumn{2}{|c|}{ Option 3} & \multicolumn{2}{|c|}{ Option 4} \\
\hline & & $\mathbf{V F}$ & WVF & $\mathbf{V F}$ & WVF & VF & WVF & VF & WVF & VF & WVF \\
\hline \multicolumn{12}{|l|}{ 1. Environmental Issues } \\
\hline Environmental Releases & 10 & 5 & 50 & 6 & 60 & 6 & 60 & 7 & 70 & 7 & 70 \\
\hline Regulatory Compliance & 20 & 5 & 100 & 8 & 160 & 8 & 160 & 9 & 180 & 9 & 180 \\
\hline Waste Generation and Disposal & 20 & 5 & 100 & 7 & 140 & 7 & 140 & 8 & 160 & 7 & 140 \\
\hline \multicolumn{12}{|l|}{ 2. Operational Issues } \\
\hline General Consideration & 10 & 5 & 50 & 5 & 50 & 6 & 60 & 6 & 60 & 7 & 70 \\
\hline Cost and Performance & 15 & 5 & 75 & 7 & 105 & 7 & 105 & 9 & 135 & 10 & 150 \\
\hline Long-Term Technology Effectiveness & 15 & 5 & 75 & 6 & 90 & 7 & 105 & 9 & 135 & 10 & 150 \\
\hline \multicolumn{12}{|l|}{ 3. Energy and Resource Use Issues } \\
\hline Energy Use & 5 & 5 & 25 & 5 & 25 & 5 & 25 & 4 & 20 & 4 & 70 \\
\hline Resource Use Issues & 5 & 5 & 25 & 5 & 25 & 5 & 25 & 5 & 25 & 5 & 25 \\
\hline \multicolumn{2}{|c|}{$\begin{array}{l}\text { Total Weighted Value Factor (TWVF) for } \\
\text { each option }-\Sigma(\text { WVF) }\end{array}$} & \multicolumn{2}{|c|}{500} & \multicolumn{2}{|c|}{655} & \multicolumn{2}{|c|}{680} & \multicolumn{2}{|c|}{785} & \multicolumn{2}{|c|}{805} \\
\hline
\end{tabular}

WF $\quad=\quad$ weight factor, Table 9-6

$\mathrm{VF} \quad=\quad$ value factor, Table 9-1

WVF $=$ WF $\times$ VF

TWVF $=$ Sum of WVFS for an option 
TABLE 9-8

Cost-Effect Summary for the Candidate Technologies

\begin{tabular}{lrrr}
\hline CT\# & $\begin{array}{r}\text { TOTAL WEIGHTED } \\
\text { VALUE FACTOR }\end{array}$ & $\begin{array}{r}\text { TOTAL ESTIMATED } \\
\text { PROJECT COSTS }\end{array}$ & $\begin{array}{r}\text { OVDERALL } \\
\text { BDATANKING }\end{array}$ \\
1 & 655 & $\$ 116,024,000$ & 3 \\
2 & 680 & $\$ 122,122,850$ & 4 \\
3 & 785 & $\$ 201,730,800$ & 2 \\
4 & 835 & $\$ 186,866,750$ & 1
\end{tabular}




\subsection{REFERENCES}

10.1 Annual Report, Summary of Operating_Data; Liquid Waste Management Group HSE-7, Los Alamos National Laboratory, Los Alamos, NM; Calendar Year 1987.

10.2 Annual Report, Summary of Operating Data; Liquid Waste Management Group HSE-7, Los Alamos National Laboratory, Los Alamos, NM; Calendar Year 1988.

10.3 Annual Report, Summary of Operating Data; Liquid Waste Management Group HSE-7, Los Alamos National Laboratory, Los Alamos, NM; Calendar Year 1989.

10.4 Annual Report, Summary of Operating Data; Liquid Waste Management Group HSE-7, Los Alamos National Laboratory, Los Alamos, NM; Calendar Year 1990.

10.5 Annual Report, Summary of Operating Data; Liquid Waste Management Group HSE-7, Los Alamos National Laboratory, Los Alamos, NM; Calendar Year 1991.

10.6 DOE Order 5400.5, Radiation Protection of the Public and the Environment; U.S. Department of Energy, Washington D.C.; February 8, 1990; Change 1, June 5, 1990.

10.7 DOE Order 6430.1A, General Design Criteria; U.S. Department of Energy, Washington, D.C.; April 6, 1989.

10.8 DOE/EH-263T, Implementation Manual for Application of Best-Available Technology Processes for Radionuclides in Liquid Effluents, Interim Final Report; U.S. Department of Energy, Assistant Secretary for Environment, Safety and Health, Office of Environmental Guidance; June 1992.

10.9 DOE/EV/1830-T5, A Guide to Reducing Radiation Exposure to As Low As Reasonably Achievable (ALARA); U.S. Department of Energy, Assistant Secretary for Environment, Division of Operational and Environmental Safety; April 1980.

10.10 DOE/LLW-13Tc, Low-Level Radioactive Waste Treatment Technology; LowLevel Radioactive Waste Management Handbook Series, National Low-Level Radioactive Waste Management Program, July 1984. 
10.11 EM-7-RLW-92-019, A Study of Radioactive Liquid Waste Treatment Options for Los Alamos National Laboratory; Black and Veatch Engineers-Architects for Los Alamos National Laboratory, Los Alamos, NM; April 04, 1987.

10.12 EM-7-RLW-92-022, Radioactive Liquid Waste Treatment Facility Lab Job 10411-50; ICF Kaisers Engineers for Los Alamos National Laboratory, Los Alamos, NM; December 1989.

10.13 EM-7-RLW-92-031, Radioactive Liquid Waste Treatment Facility Retrofit Lab Job 10411-50; ICF Kaisers Engineers for Los Alamos National Laboratory, Los Alamos, NM; February 1991.

10.14 EM-7-RLW-92-060, Change Order Proposal for Work Release 0015; Los Alamos National Laboratory, August 18, 1992.

10.15 EPA-440/4-84-022, Technical Guidance Manual for Performing Wasteload Allocations; Book II, 'Streams and Rivers', Chapter 3, 'Toxic Substances'; June 1984.

10.16 Letter, K.E. Pallesen to B. Mahaffey, Contract 9-XY2-Y9591-1 Work Release \#0015; Los Alamos National Laboratory, Los Alamos, NM; May 8, 1992.

10.17 Letter, B.F. Shelton to W. MIdkiff, RLWTT BAT Assessment Minutes of Meeting. MM-L0004-1; Ralph M. Parsons Company, Charlotte, NC; September 16, 1992.

10.18 LW-EM7-OI-06, ROO, Operating Instructions for Main Radioactive Liquid Waste Treatment Plant; Los Alamos National Laboratory HSE-7, Los Alamos, NM; August 1991.

10.19 LW-HSE7-OI-01, R05, Detailed Operating Instructions, Room 60 Pretreatment Plant; Los Alamos National Laboratory HSE-7, Los Alamos, NM; April 1991.

10.20 LW-HSE7-SPO-01, RO2, Standard Operating Procedure, TA-50-1 Pretreatment Plant; Los Alamos National Laboratory HSE-7, Los Alamos, NM; March 1991.

10.21 Radionuclide Release Data 1990; Memorandum, Buchholz to Lincoln, Los Alamos National Laboratory, March 28, 1991.

10.22 National Pollution Discharge Elimination System (NPDES) Permit No. MN0028355 (present permit), modified for the Los Alamos National Laboratory on January 30, 1990. 
10.23 National Pollution Discharge Elimination System (NPDES) Permit No. MN0028355 (reapplication), received from the Los Alamos National Laboratory dated September 1, 1990.

10.24 National Pollution Discharge Elimination System (NPDES) Permit No. MN0028355 (draft permit), for the Los Alamos National Laboratory, issued for Public Notice on May 11, 1991.

10.25 New Mexico Interim Guidance for Implementation of Water Quality Standards Through Implementation of National Pollutant Discharge Elimination System Permits, New Mexico Water Quality Control Commission, June 25, 1991.

10.26 Preconceptual Design Executive Summary, Radioactive Liquid Waste Treatment Facility; P.I. 10411, Los Alamos National Laboratory, Los Alamos, NM; April 1992.

10.27 SAR 86-2F, Approval for Safety Analysis Report for Radioactive Liquid Waste Treatment Facility TA-50-01; Los Alamos National Laboratory HSE-7, Los Alamos, NM; August 1988.

10.28 Water Quality Standards for Interstate and Intrastate Streams in New Mexico, New Mexico Water Quality Control Commission, June 29, 1991.

10.29 WQCC 82-1, Water Quality Control Commission Regulations, State of New Mexico, September 20, 1982. 


\section{APPENDICES}

APPENDIX A - BLOCK FLOW DIAGRAMS FOR EXISTING DOE FACILITIES APPENDIX B - BLOCK FLOW DIAGRAMS FOR PROCESS CONFIGURATIONS APPENDIX C - REGULATORY DISCHARGE GUIDELINES FOR SPECIFIC EFFLUENT COMPONESTS

APPENDIX D - ANTICIPATED INFLUENT/EFFLUENT CONTAMINANT CONCENTRATIONS FOR PROPOSED PROCESS CONFIGURATIONS

APPENDIX E - BLOCK FLOW DIAGRAMS FOR CANDIDATE TECHNOLOGIES

APPENDIX F - PROCESS LAYOUTS FOR CANDIDATE TECHNOLOGIES

APPENDIX G - DETAILED COST ESTIMATES FOR THE CANDIDATE TECHNOLOGIES

APPENDIX H - PROCESS CALCULATIONS 


\section{APPENDIX A}

BLOCK FLOW DIAGRAMS FOR EXISTING DOE FACILITIES 


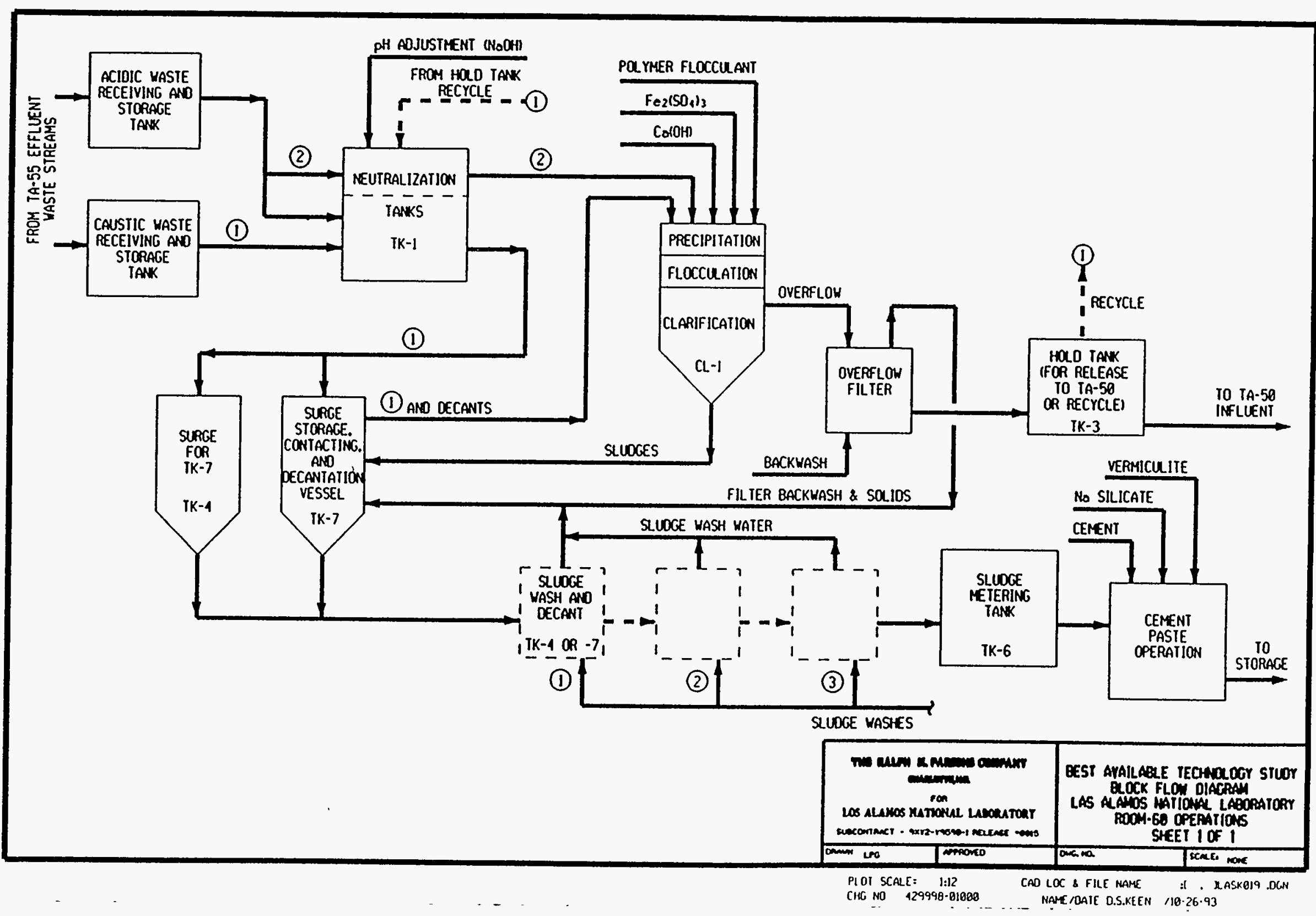




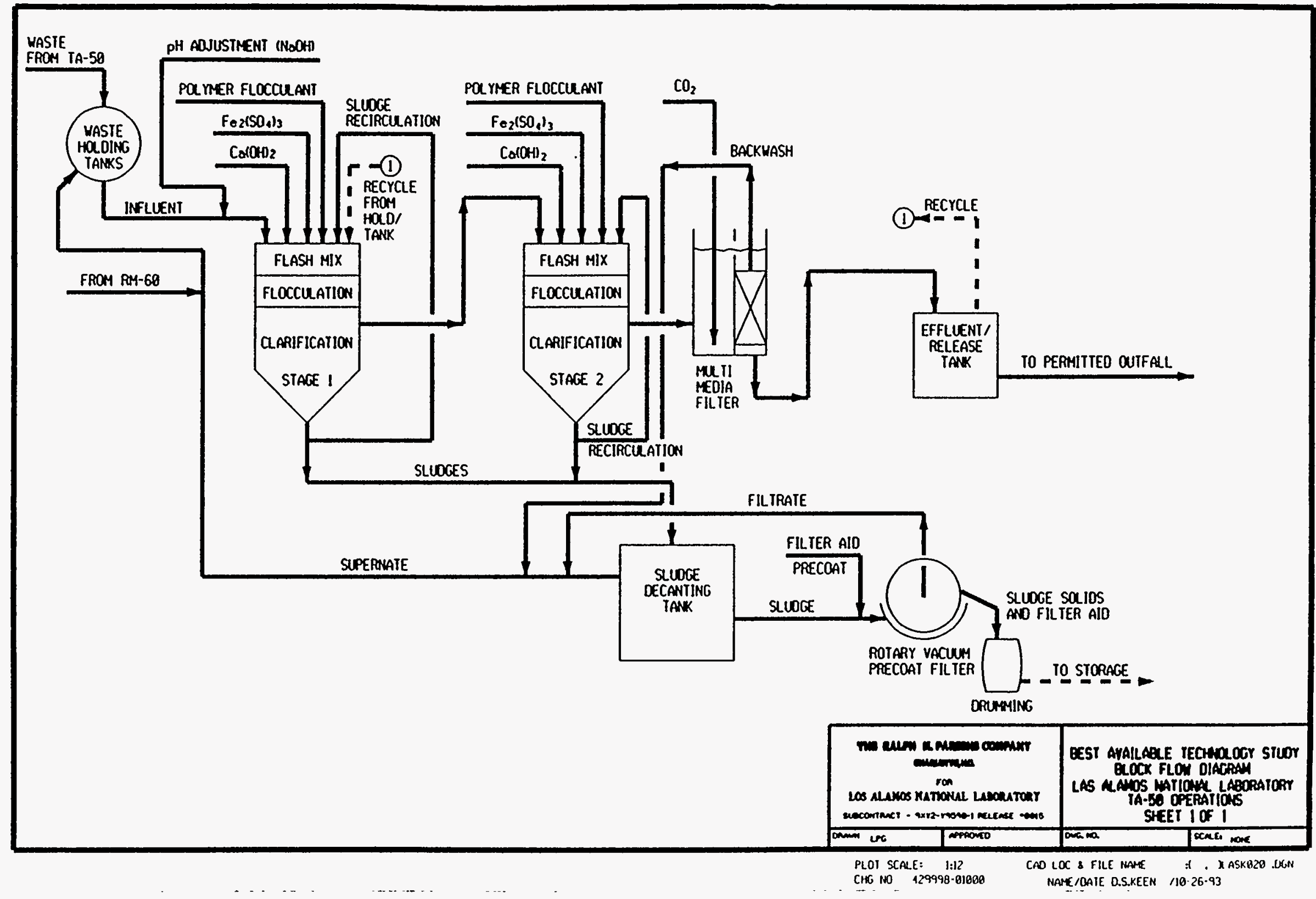




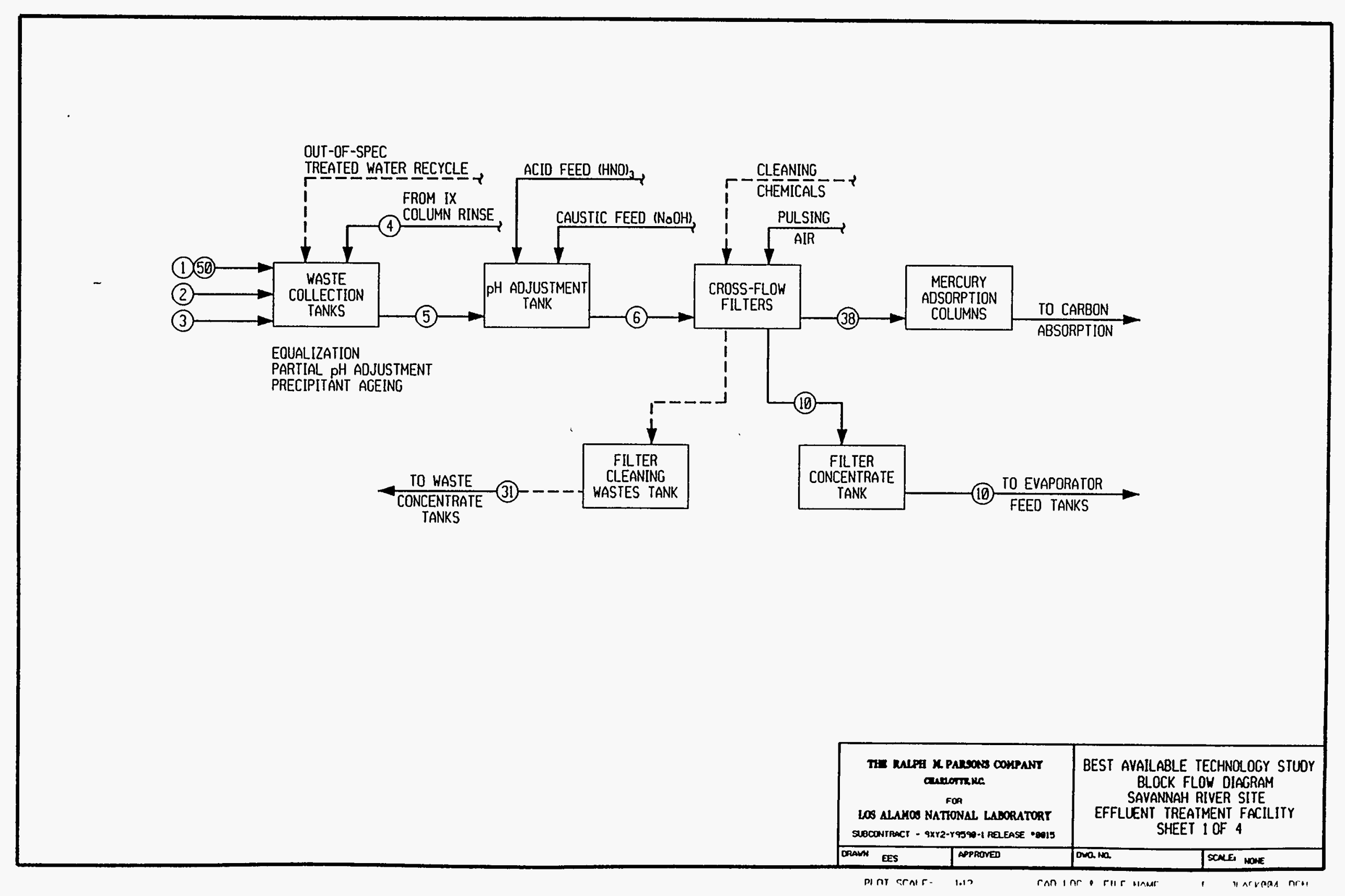




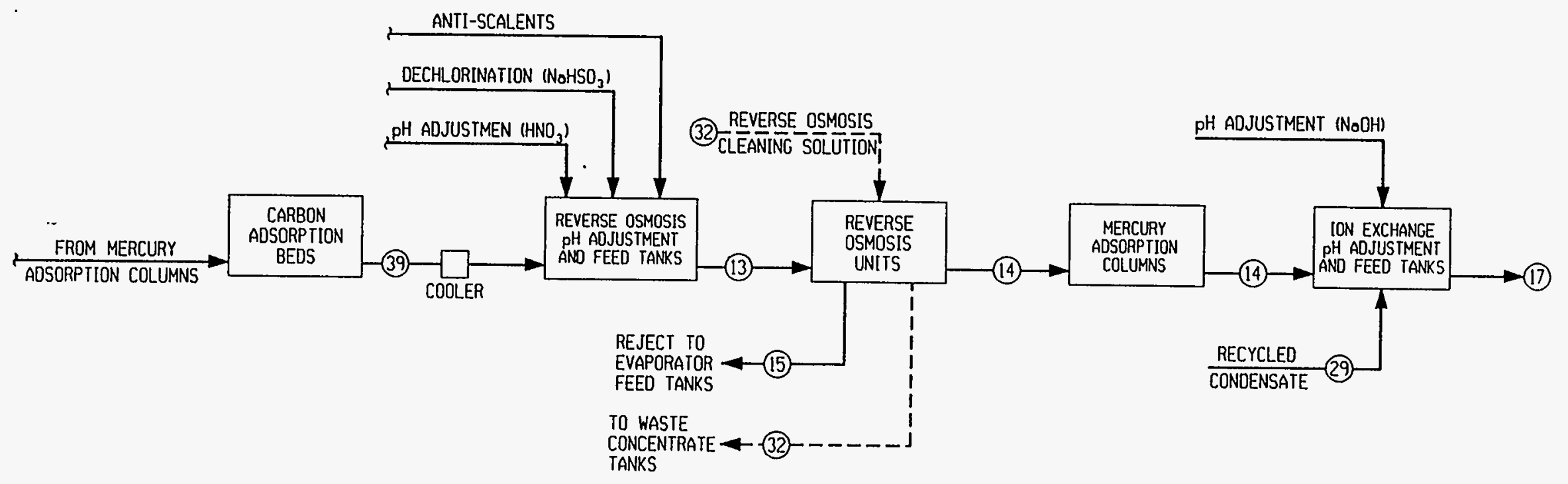

\begin{tabular}{|c|c|c|}
\hline \multicolumn{2}{|c|}{ 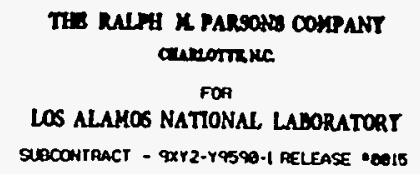 } & $\begin{array}{c}\text { BEST AVAILABLE TECHNOLOGY STUDY } \\
\text { BLOCK FLOH OIAGRAM } \\
\text { SAVANNAH RIVER SITE } \\
\text { EFFLUENT TREATMENT FACILITY } \\
\text { SHEET } 2 \text { OF } 4\end{array}$ \\
\hline EES & APFoved & scalle mane \\
\hline
\end{tabular}

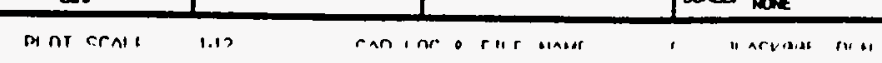




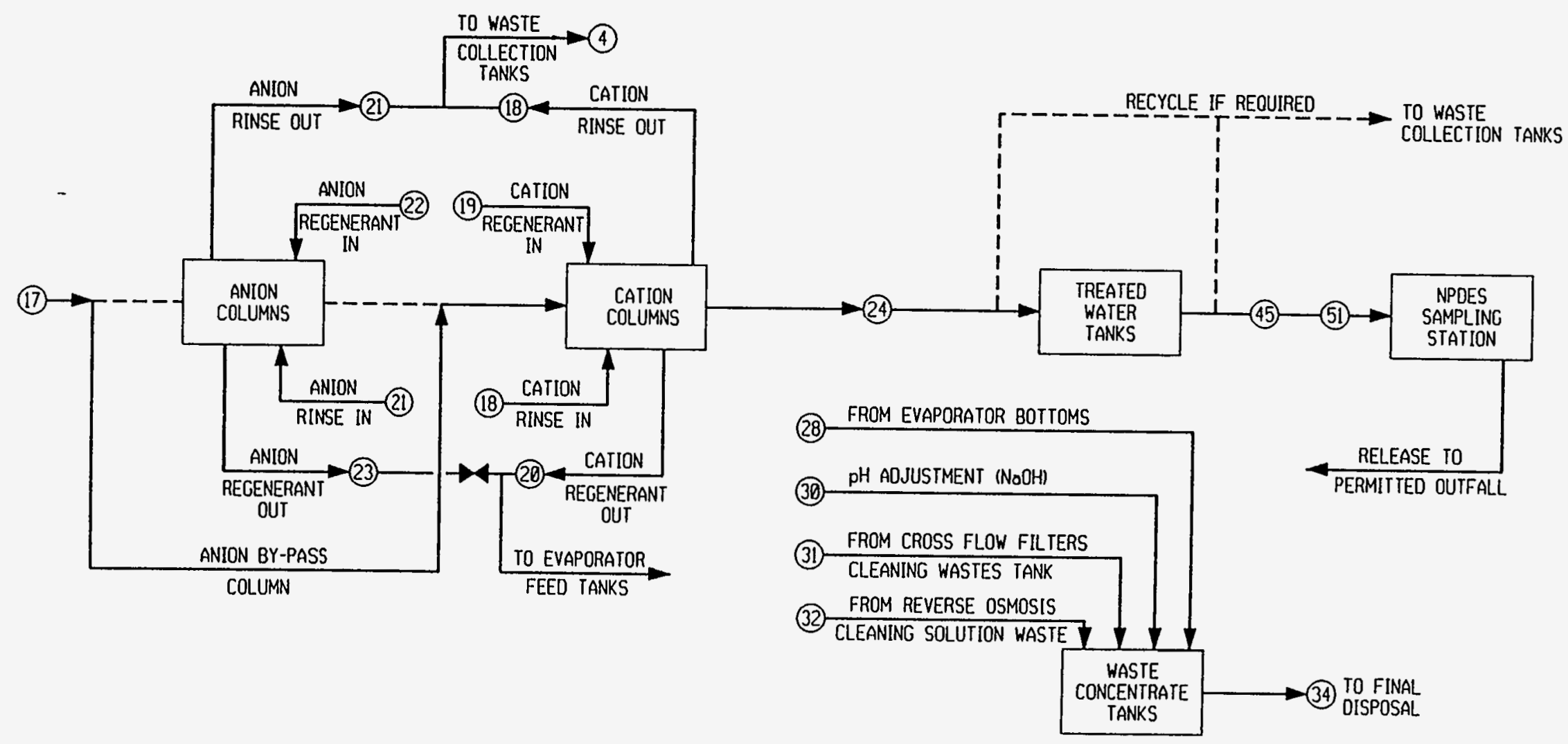

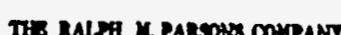
areorrese

For

LOS ALAMOS NATIONAL LABORATORT SLOCONIRACI - QXV2-Y9590-1 RELEASE - DeO15
BEST AVAILABLE TECHNOLOGY STUDY BLOCK FLOW DIAGRAM SAVANNAH RIVER SITE EFFLUENT TREATMENT FACILITY SHEET 3 of 4 
s

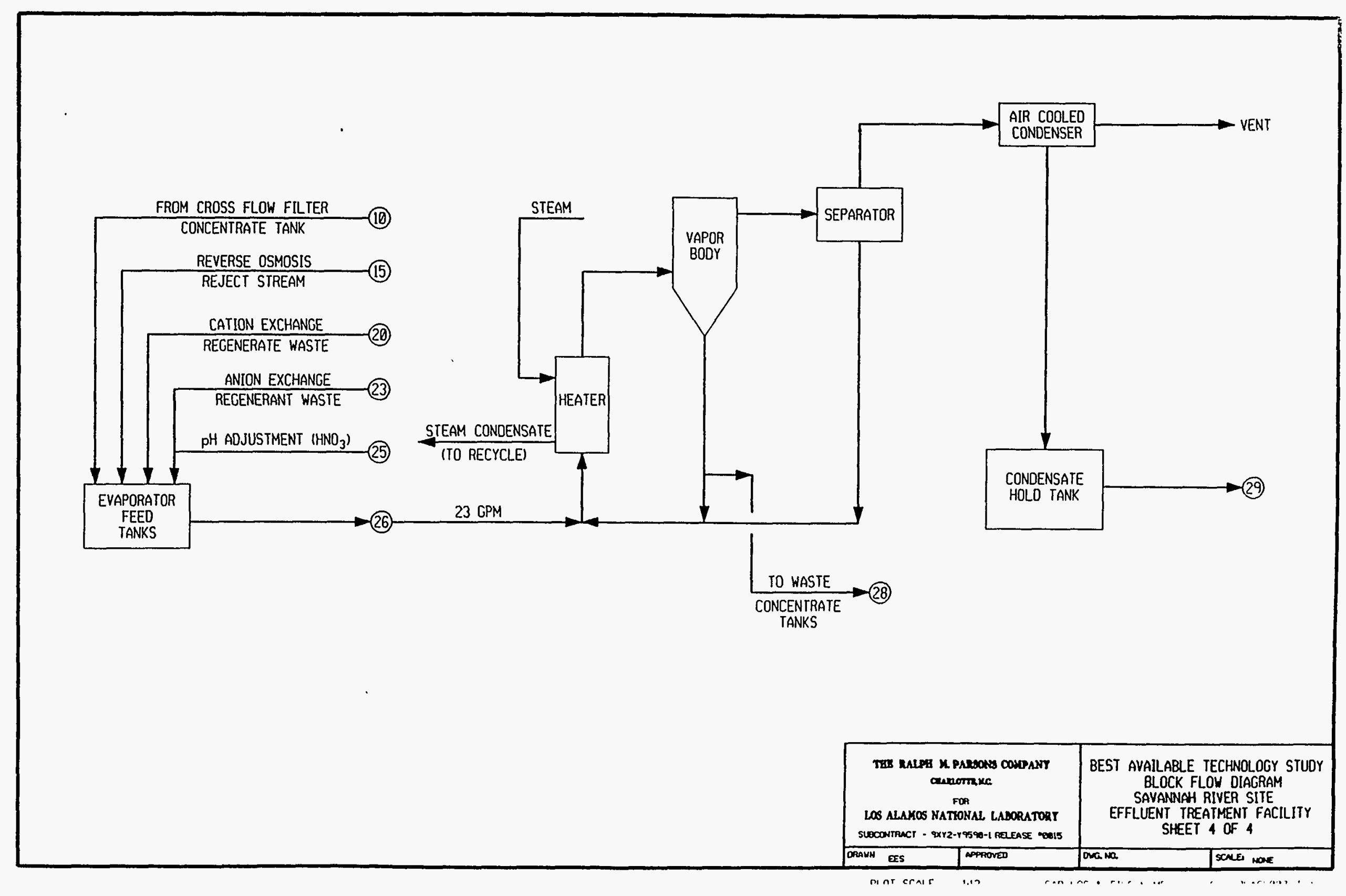




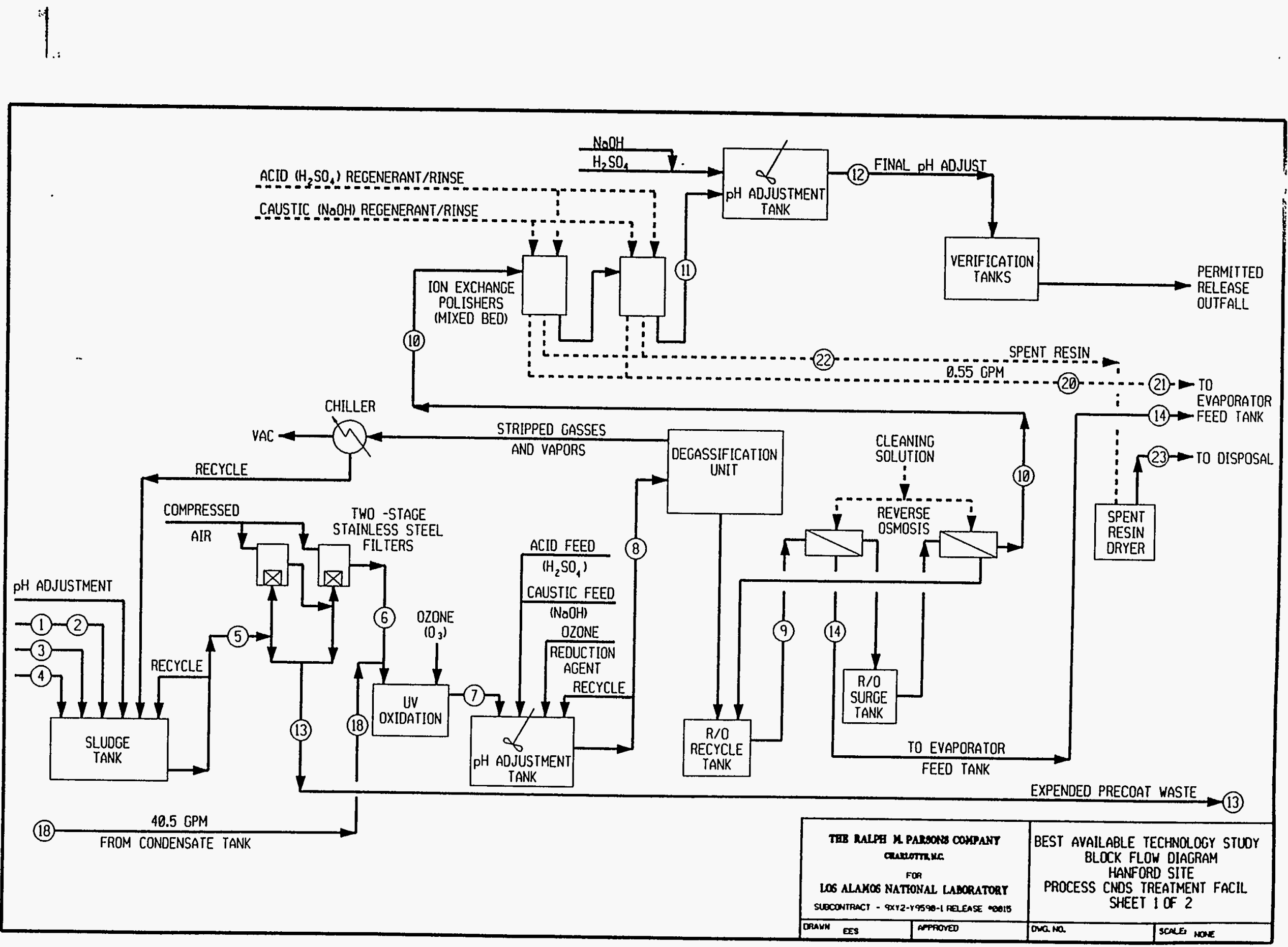




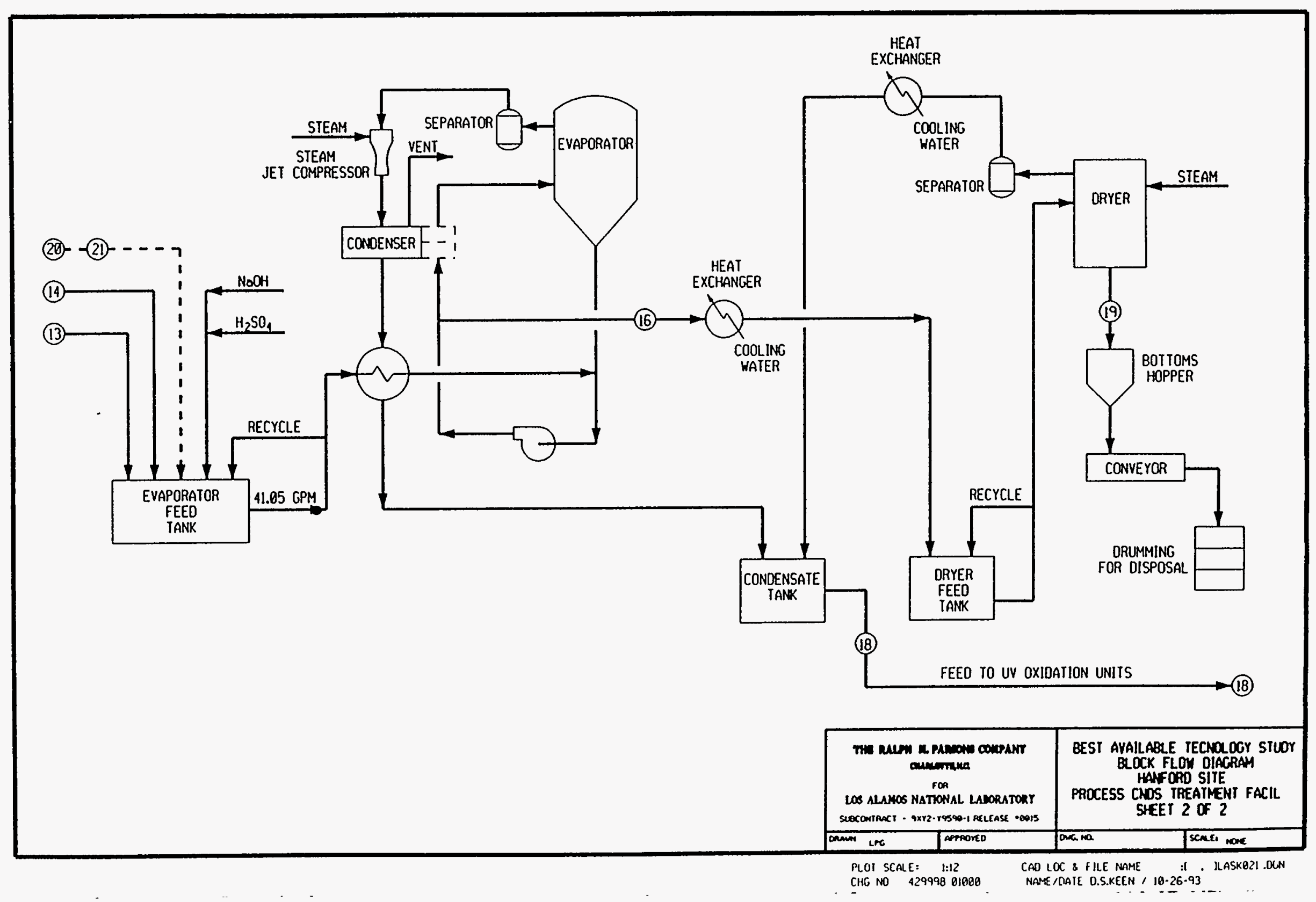




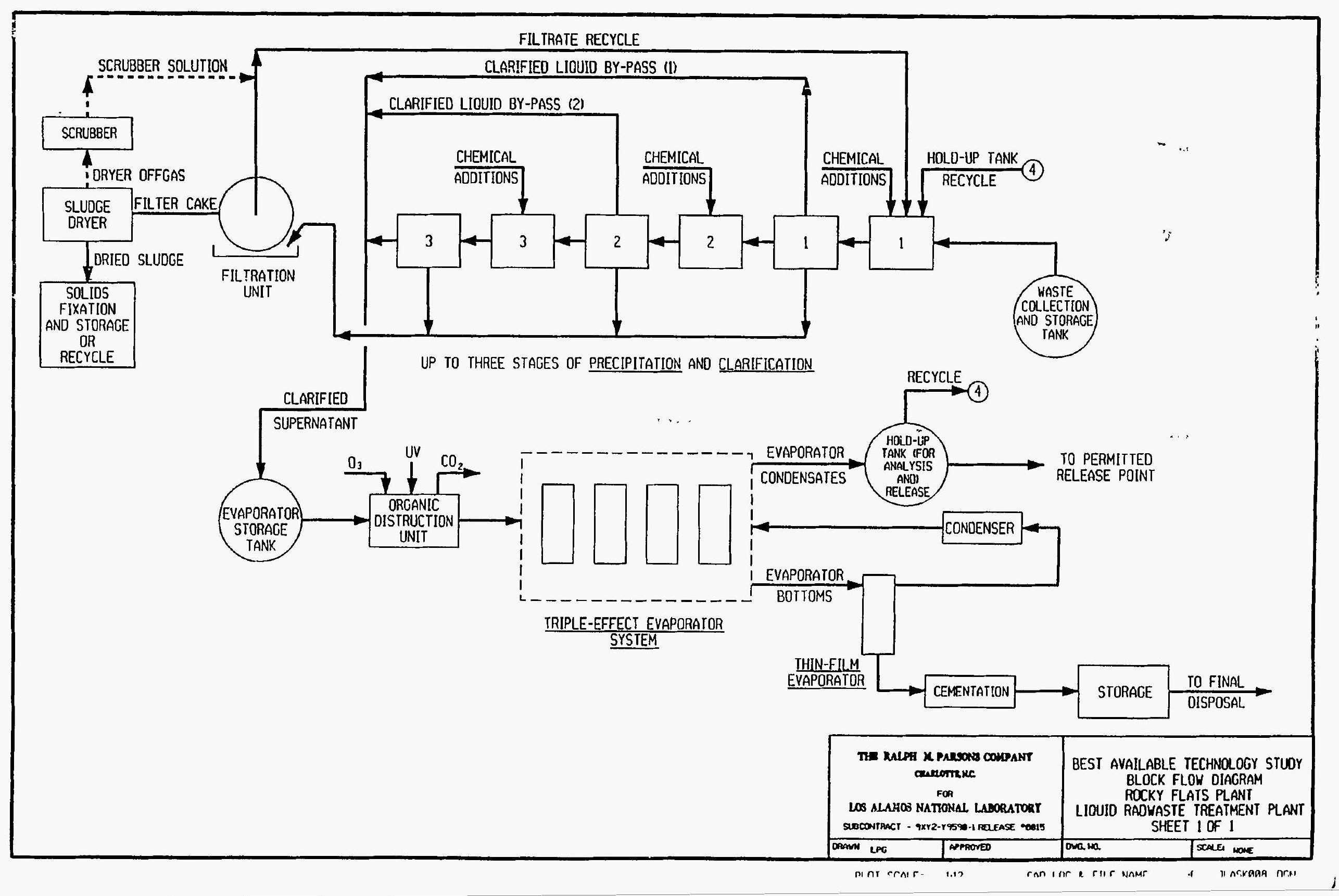




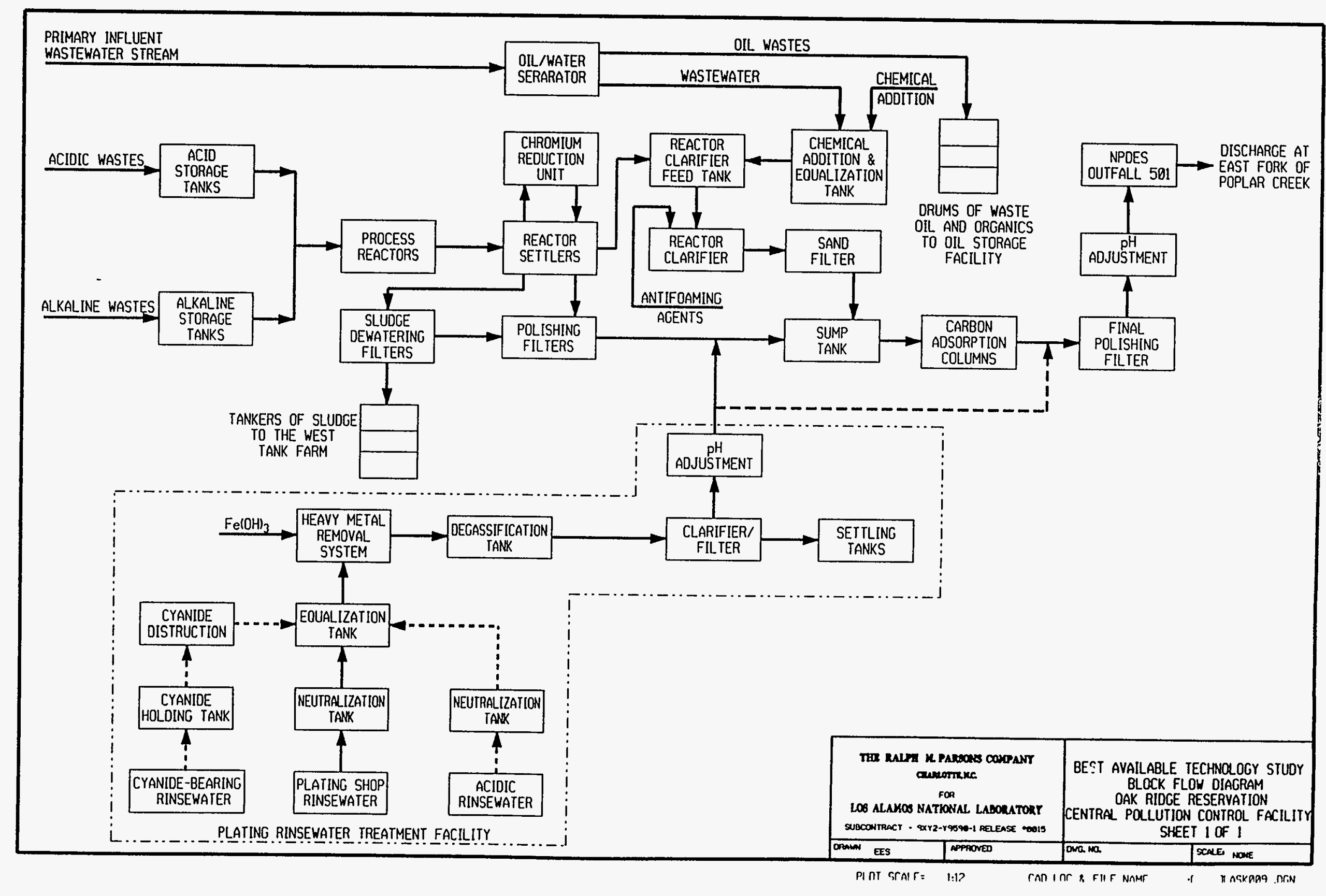




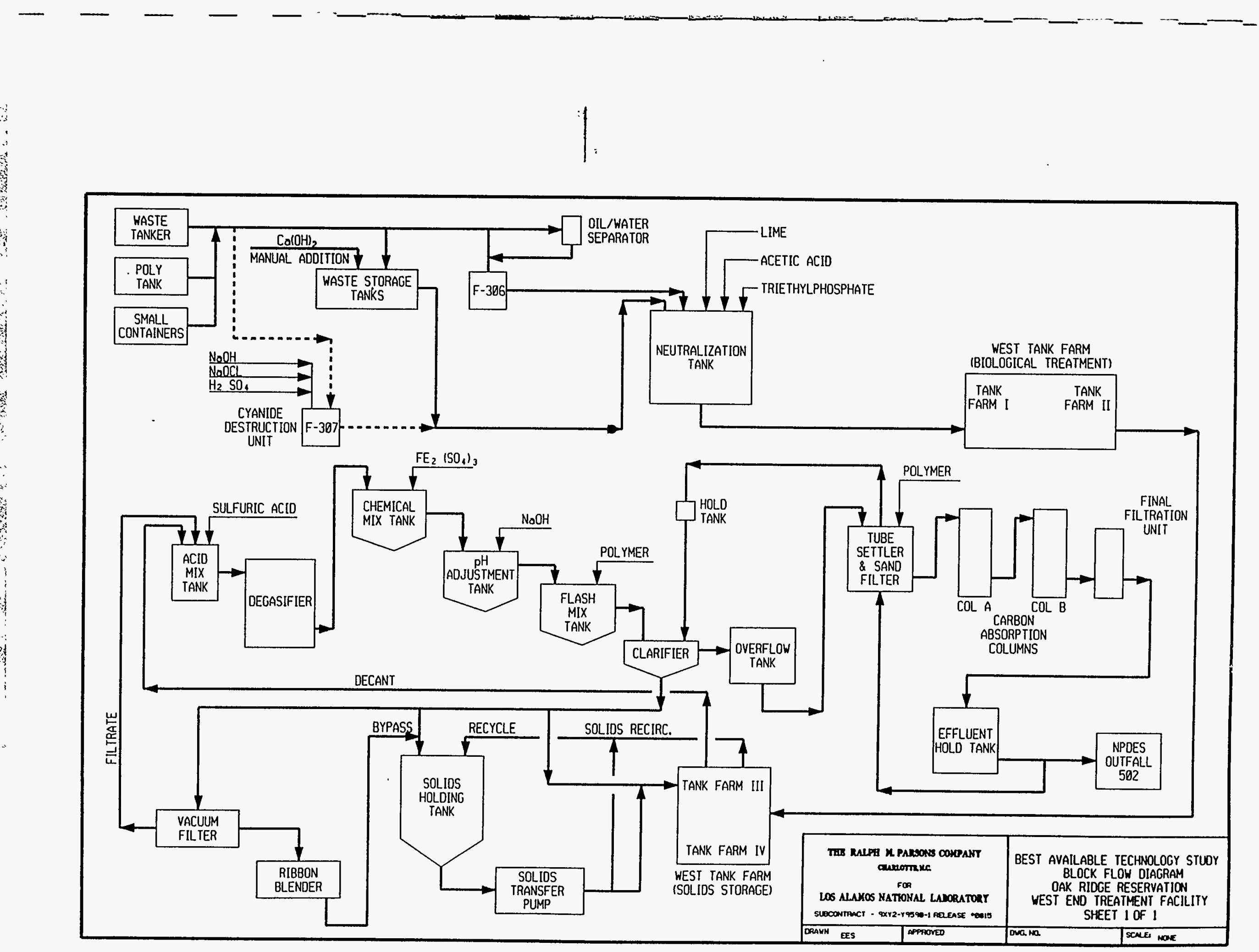




\section{APPENDIX B}

BLOCK FLOW DIAGRAMS FOR PROCESS CONFIGURATIONS 


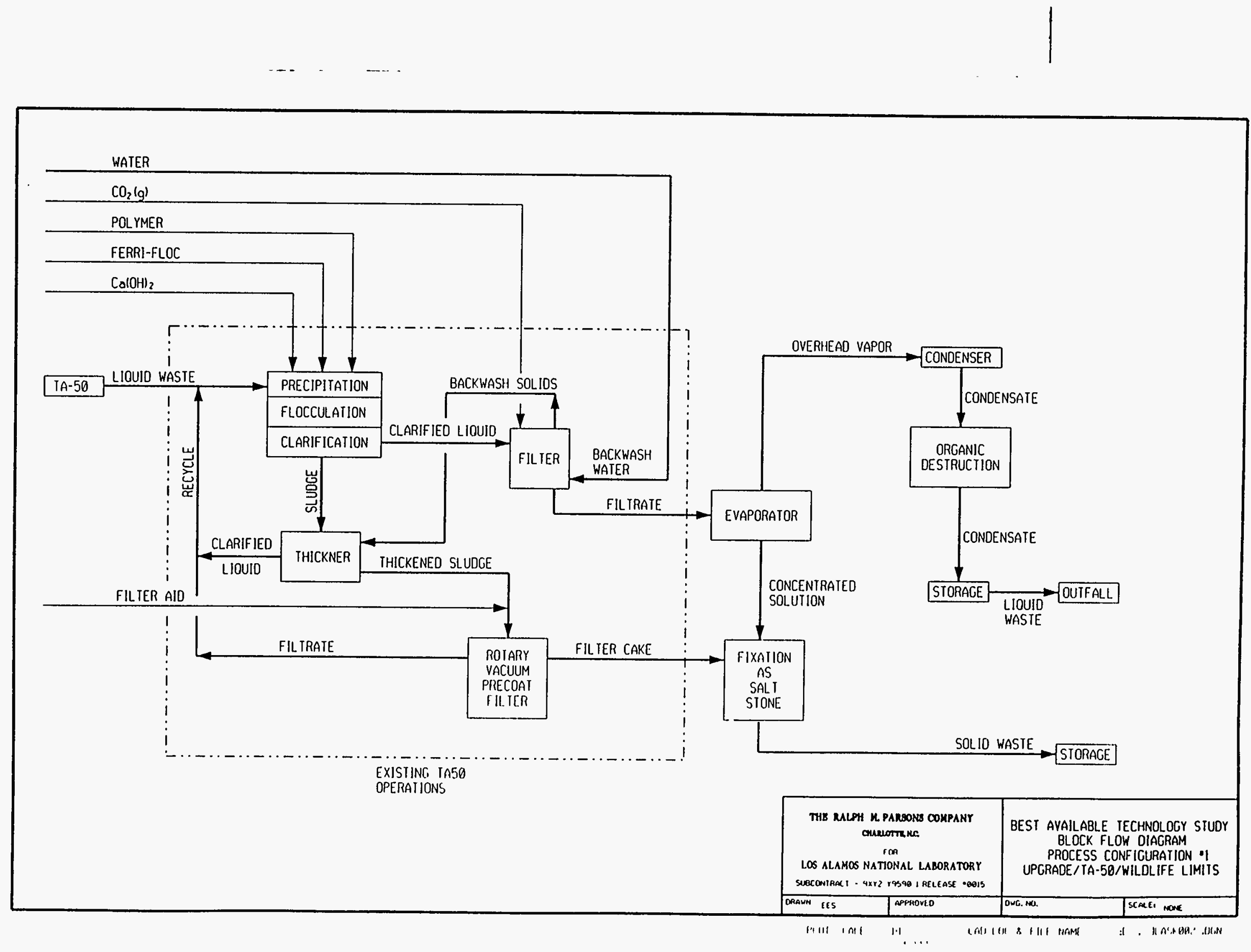





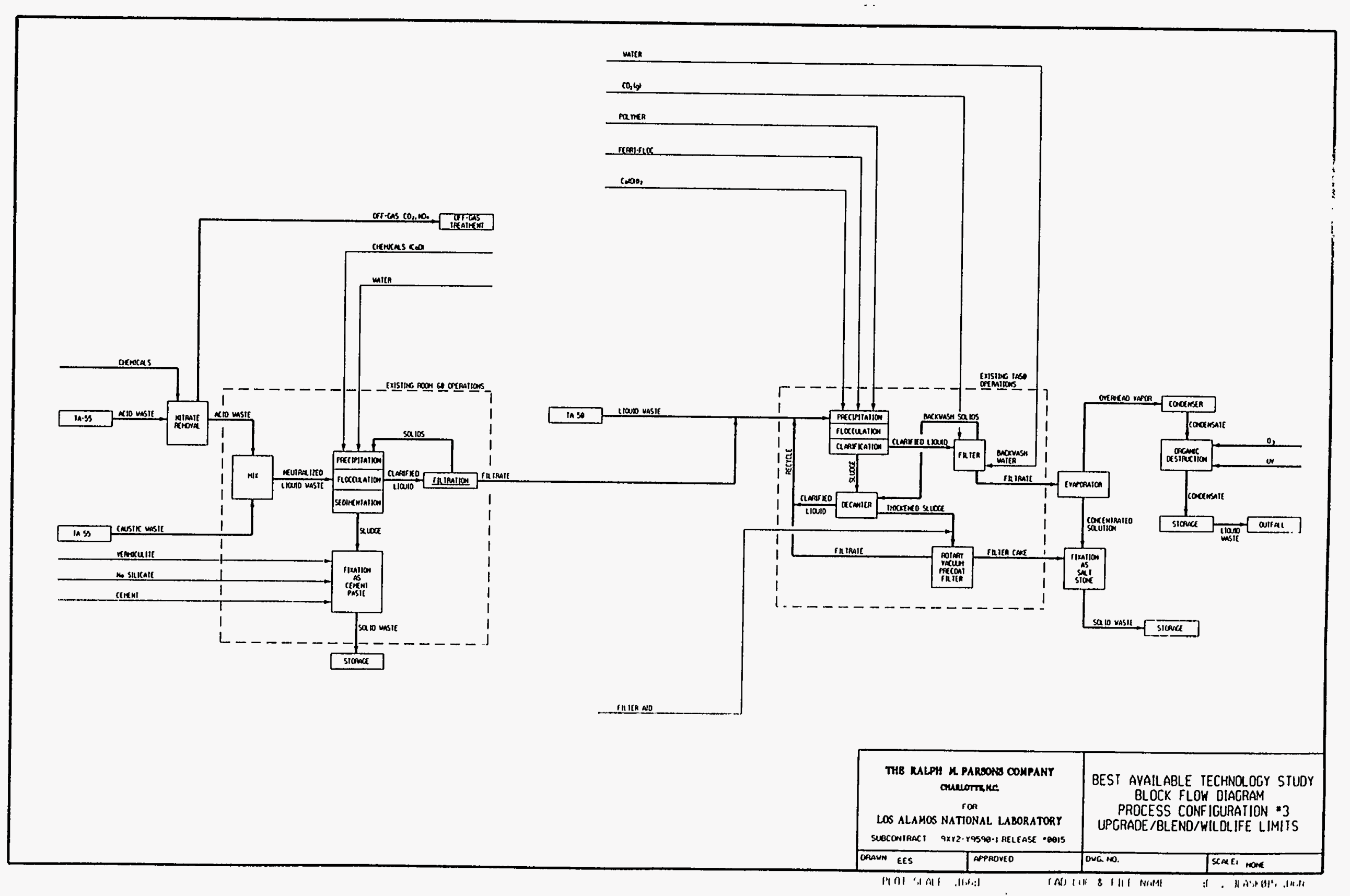




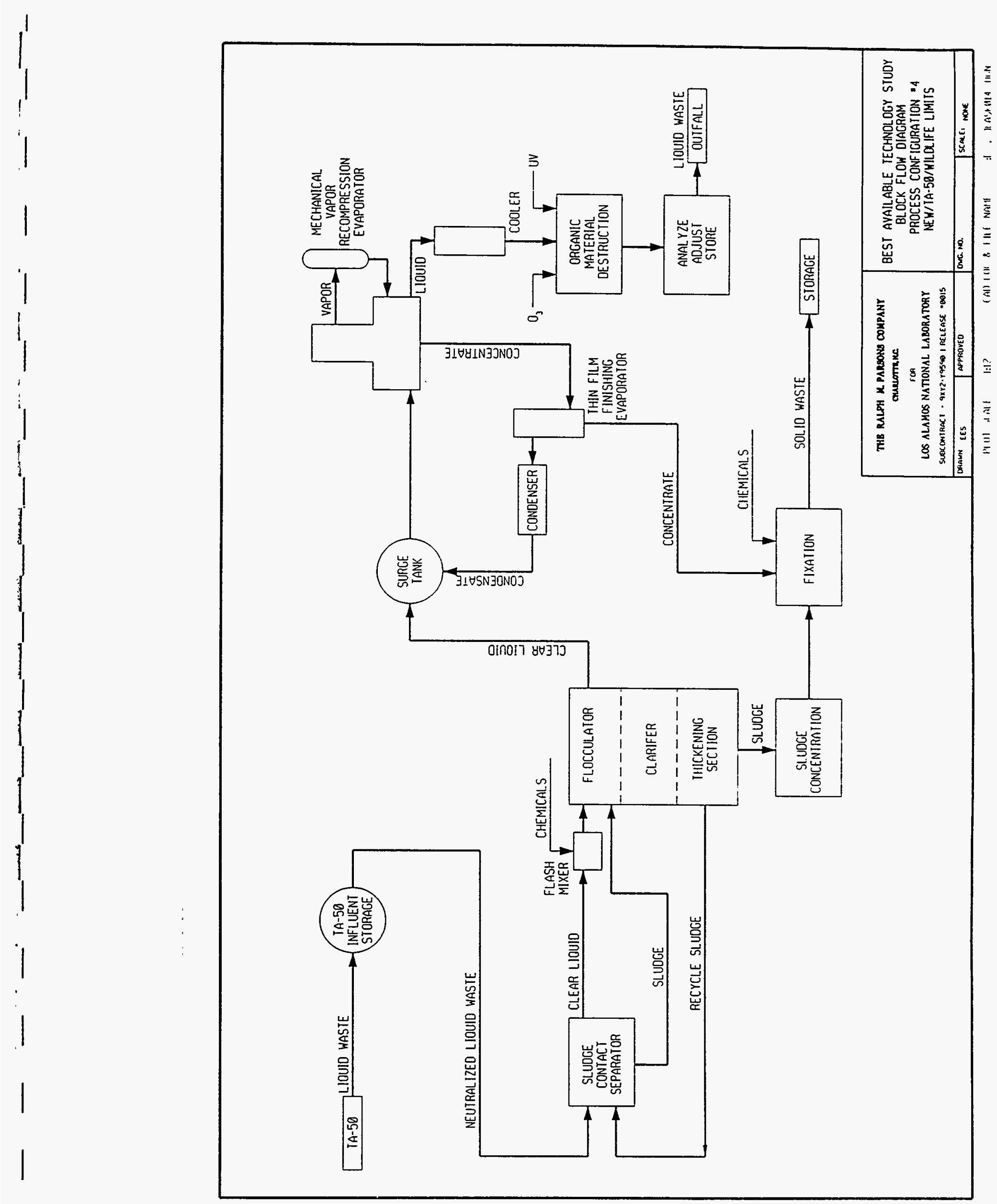





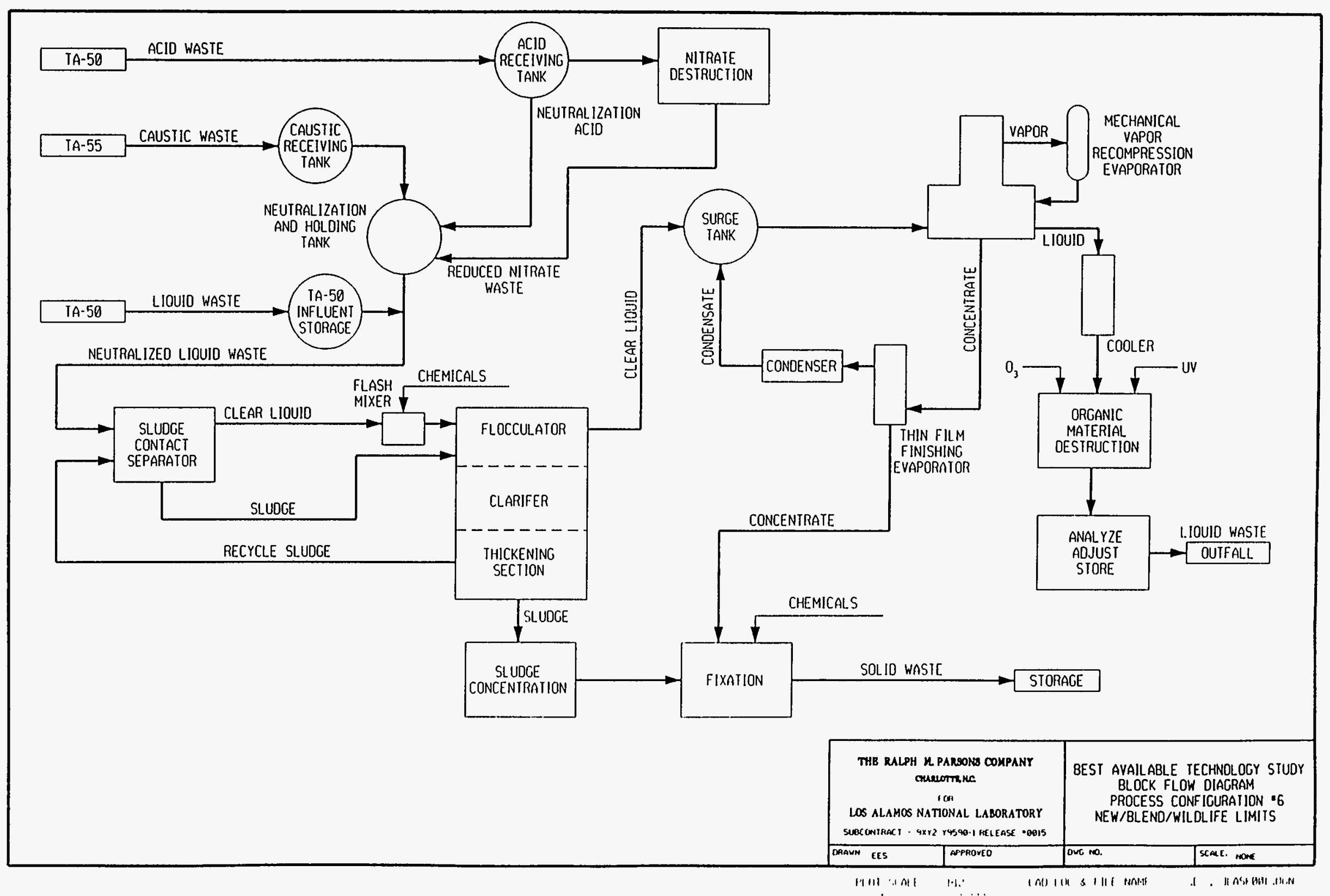




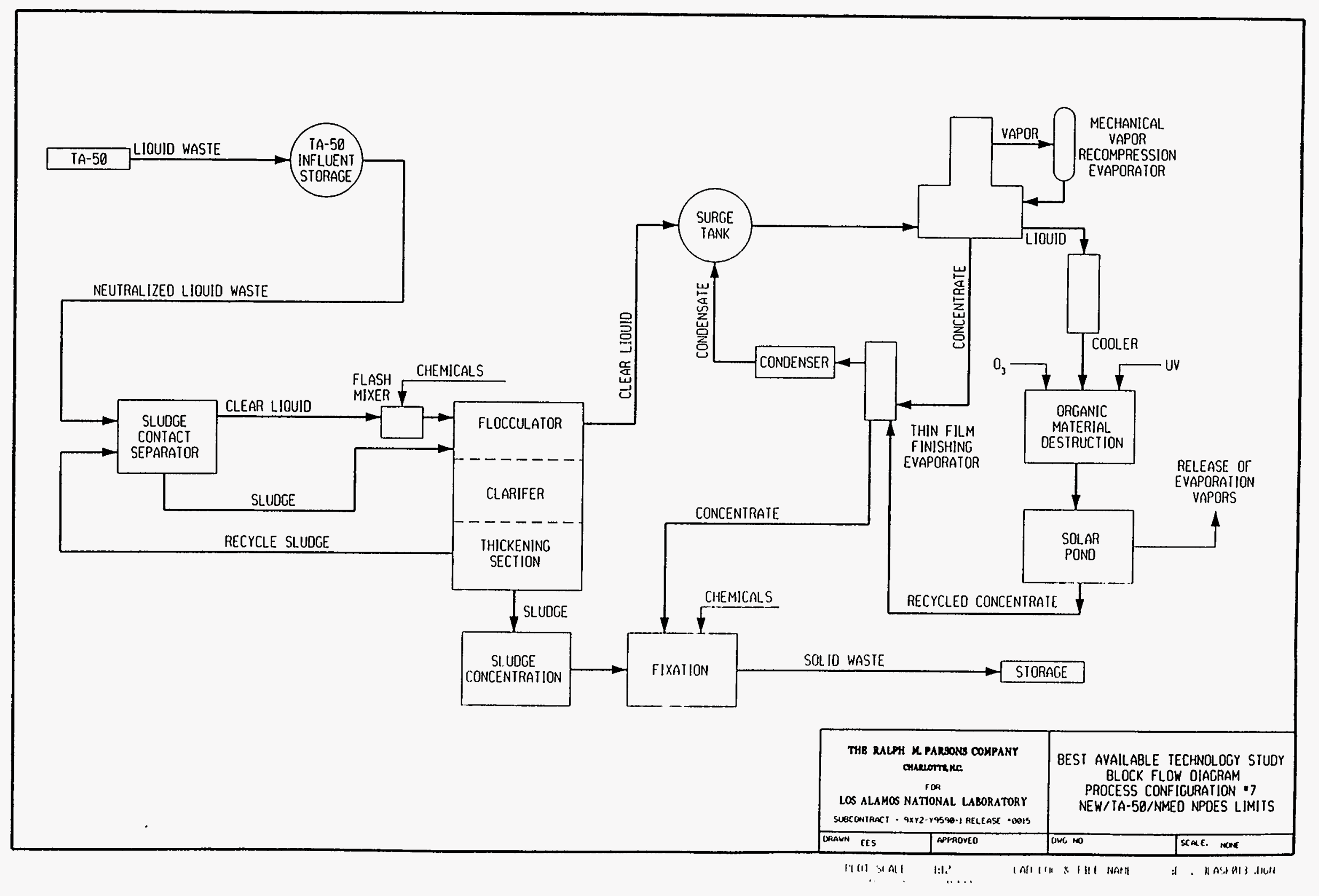




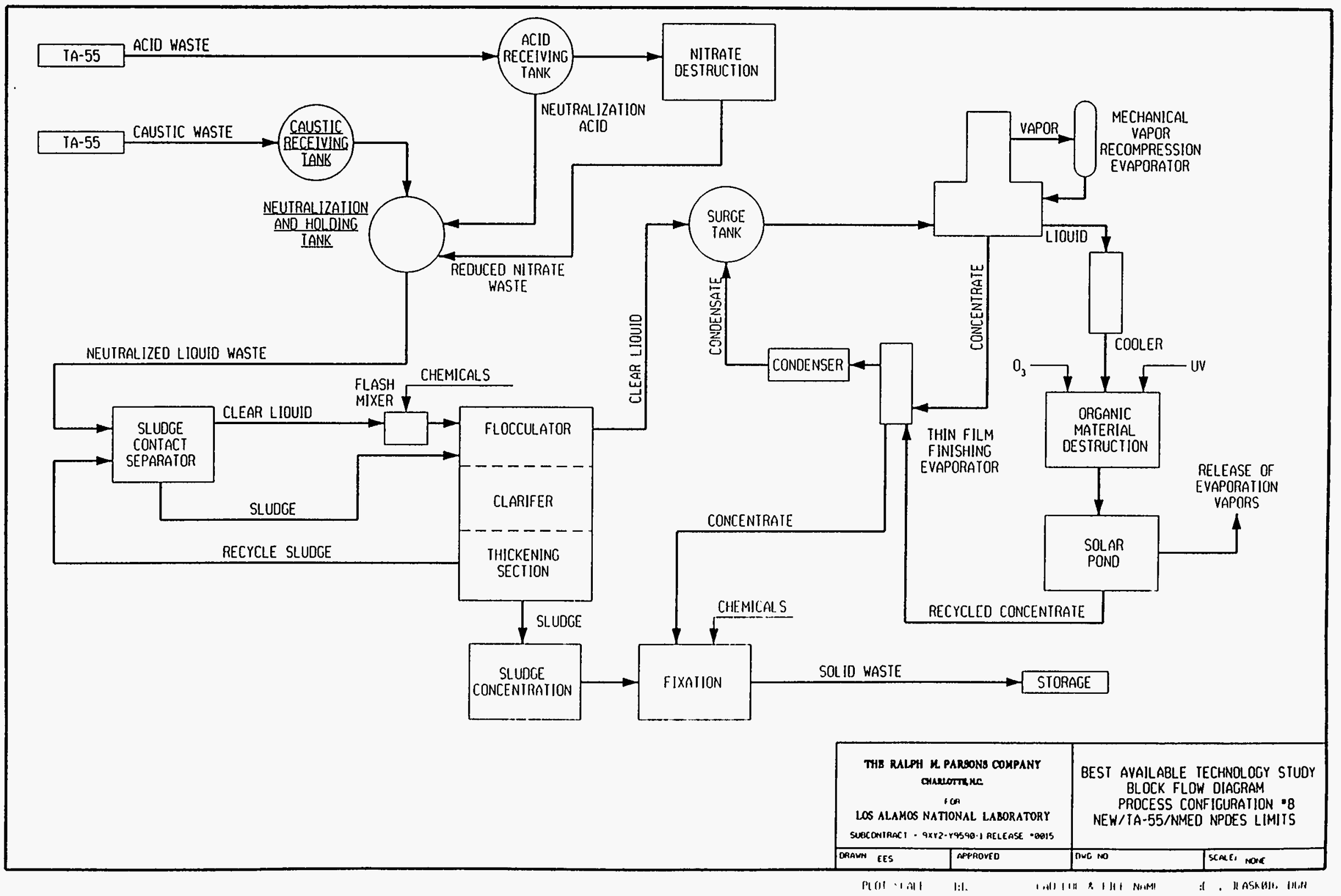





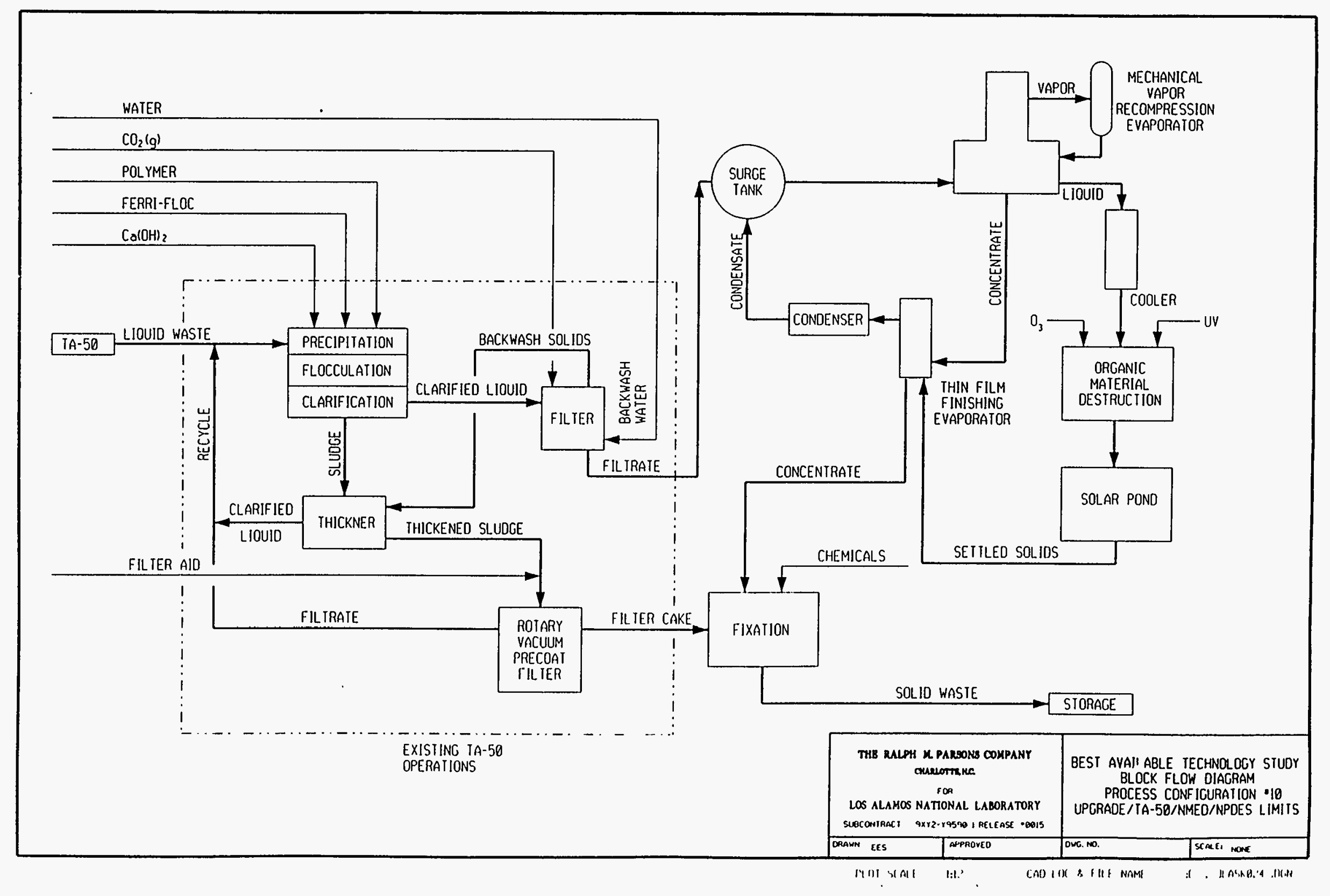




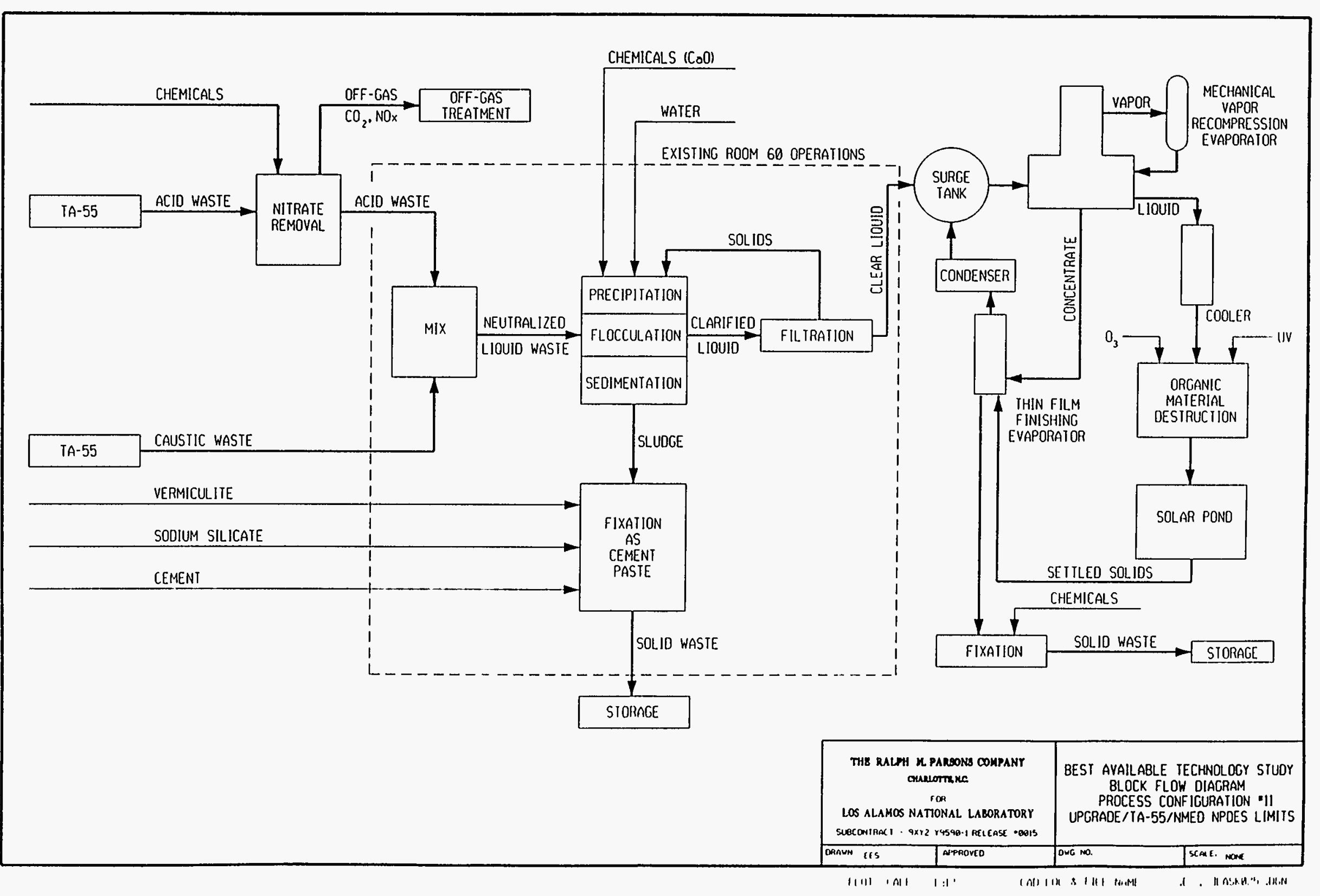





\section{APPENDIX C}

REGULATORY DISCHARGE GUIDELINES

FOR SPECIFIC EFFLUENT COMPONENTS 


\section{APPENDIX C \\ REGULATORY DISCHARGE GUIDELINES FOR SPECIFIC EFFLUENT COMPONENTS}

The tables in this appendix provide a matrix of the effluent guidelines that were researched as a part of this BAT Study. It was necessary to identify all of the promulgated standards that could have possible bearing on the effluent waste streams generated by the potential design configurations in order to ensure that compliance requirements could be attained with a minimum of expensive and unnecessary overdesign. Also, the following tables provide a perspective of the proposed NPDES standards in comparison to those promulgated for environmental compliance and protection under other regulatory authorities.

The following abbreviations have been used in the tables in this appendix:

DCG, DOE 5400.5 Derived Concentration Guides for radionuclide emissions to the environment established by the criteria in DOE Order 5400.5 .

DWP, DOE 5400.5

Maximum concentration guidelines for radionuclide uptake in human via the drinking water pathway established by the criteria in DOE Order 5400.5.

EG\&S, 40 CFR 413 Effluent guidelines and standards for wastewater contaminants regulated under 40 CFR 413 for the electroplating industry (provided for comparison).

LDR, 40 CFR 268 Restrictions on contaminant levels in wastes intended for land disposal under the requirements of 40 CFR 268.

LS \& WL

New Mexico Environmental Division contaminant concentration limits derived from the Water Quality Standards for wildlife and livestock watering/consumption.

NMED-051/1991

New Mexico Environmental Division maximum concentration effluent limits established for LANL Outfall 051 for Calendar Year 1991.

NMED-051/1992

New Mexico Environmental Division maximum concentration effluent limits established on July 16, 1992 for LANL Outfall 051.

NPDES (current)

Discharge effluent concentration limits established in the current (implemented) LANL NPDES permit. 
NPDES, Part 1 (day)

NPDES, Part 1 (max)

NPDES, TSS

NPDES, WQS

NPDRW, 40 CFR 141

NSDRW, 40 CFR 143

RPS, 10 CFR 20

TCLP, 40 CFR 261

WQCC, Part 2

WQCC, Part 3 .
Discharge limits from the draft NPDES permit for LANL, issued by the Environmental Protection Agency (Region 6) on May 16, 1992; Effluent Limitations and Monitoring Requirements, daily average.

Discharge limits from the draft NPDES permit for LANL, issued by the Environmental Protection Agency (Region 6) on May 16, 1992; Effluent Limitations and Monitoring Requirements, daily maximum.

Effluent concentration limits established by the Environmental Protection Agency (Region 6) for LANL in the May 16, 1992 draft NPDES permit; TSS levels of metals for livestock and wildlife watering.

Effluent concentration limits established by the Environmental Protection Agency (Region 6) for LANL in the May 16, 1992 draft NPDES permit; Water Quality Standards effluent limits.

Contaminant limits established for human consumption under the National Primary Drinking Water Regulations in 40 CFR 141.

Contaminant limits established for human consumption under the National Secondary Drinking Water Regulations in 40 CFR 143.

Radiation protection standards considered to be protective of human health as promulgated under 10 CFR 20.

Contaminant concentration limits established by the Toxicity Characteristic Leachate Procedure promulgated in 40 CFR 261.

New Mexico Water Quality Control Commission Regulations, Section 82-1: Part 2, Water Quality Control (non-NPDES), general.

New Mexico Water Quality Control Commission Regulations, Section 82-1: Part 3, Discharges Onto or Below the Surface of the Ground. 


\begin{tabular}{|c|c|c|c|}
\hline $\begin{array}{c}\text { Effluent } \\
\text { Component }\end{array}$ & $\begin{array}{c}\text { Component } \\
\text { Symbol } \\
\end{array}$ & $\begin{array}{l}\text { Effluent } \\
\text { Limits }\end{array}$ & $\begin{array}{l}\text { Source of Effluent } \\
\text { Limit Guidance }\end{array}$ \\
\hline arsenic & As & $\begin{array}{l}0.074 \mathrm{mg} / \mathrm{L} \\
5.0 \mathrm{mg} / \mathrm{L} \\
0.5 \mathrm{mg} / \mathrm{L} \\
5.0 \mathrm{mg} / \mathrm{L} \\
1.4 \mathrm{mg} / \mathrm{L} * * \\
\text { no } \mathrm{req} \\
0.02 \\
0.0014 \\
0.1 \mathrm{mg} / \mathrm{L} \\
0.03 \mathrm{mg} / \mathrm{L} \\
0.03 \mathrm{mg} / \mathrm{L} \\
0.03 \mathrm{mg} / \mathrm{L} \\
0.03 \mathrm{mg} / \mathrm{L}\end{array}$ & $\begin{array}{l}\text { NंMED-051/1992 } \\
\text { TCLP, } 40 \text { CFR } 261 \\
\text { NPDWR, 40 CFR } 141 \\
\text { LDR, } 40 \text { CFR } 268 \\
\text { LDR, 40 CFR } 268 \\
\text { NPDES (current) } \\
\text { LS \& WL } \\
\text { NMED-051/1991 } \\
\text { WQCC, Pt. } 3 \\
\text { NPDES, TSS } \\
\text { NPDES, WQS } \\
\text { NPDES, Pt. } 1 \text { (day) } \\
\text { NPDES, Pt. } 1 \text { (max) }\end{array}$ \\
\hline barium & $\mathrm{Ba}$ & $\begin{array}{l}100.0 \mathrm{mg} / \mathrm{L} \\
1.0 \mathrm{mg} / \mathrm{l} \\
100.0 \mathrm{mg} / \mathrm{L} \\
1.2 \mathrm{mg} / \mathrm{L}^{* *} \\
\text { no req } \\
\text { no req } \\
\text { no req } \\
\text { no req } \\
0.06 \\
1.0 \mathrm{mg} / \mathrm{L}\end{array}$ & $\begin{array}{l}\text { TCLP, } 40 \text { CFR } 261 \\
\text { NPDWR, 40 CFR } 141 \\
\text { LDR, } 40 \text { CFR } 268 \\
\text { LDR, } 40 \text { CFR } 268 \\
\text { NPDES (current) } \\
\text { EPA-92 } \\
\text { LS \& WL } \\
\text { NMED-051/1992 } \\
\text { NMED-051/1991 } \\
\text { WQCC, Pt. } 3\end{array}$ \\
\hline
\end{tabular}




\begin{tabular}{|c|c|c|c|}
\hline $\begin{array}{c}\text { Effluent } \\
\text { Component }\end{array}$ & $\begin{array}{l}\text { Component } \\
\text { Symbol }\end{array}$ & $\begin{array}{l}\text { Effluent } \\
\text { Limits }\end{array}$ & $\begin{array}{l}\text { Source of Emluent } \\
\text { Limit Guidance }\end{array}$ \\
\hline cadmium & $\mathrm{Cd}$ & $\begin{array}{l}0.0005 \mathrm{mg} / \mathrm{L} \\
1.0 \mathrm{mg} / \mathrm{l} \\
0.010 \mathrm{mg} / \mathrm{L} \\
0.7 \mathrm{mg} / \mathrm{L} \\
1.2 \mathrm{mg} / \mathrm{L}^{*} \\
1.0 \mathrm{mg} / \mathrm{L} \\
0.2 \mathrm{mg} / \mathrm{L}^{* *} \\
\text { no } \mathrm{req} \\
0.05 \\
0.002 \\
0.01 \mathrm{mg} / \mathrm{L} \\
0.17 \mathrm{mg} / \mathrm{L} \\
0.17 \mathrm{mg} / \mathrm{L}\end{array}$ & $\begin{array}{l}\text { NMED-051/1992 } \\
\text { TCLP, 40 CFR } 261 \\
\text { NPDWR, } 40 \text { CFR } 141 \\
\text { EG\&S, } 40 \text { CFR } 413 \\
\text { EG\&S, } 40 \text { CFR } 413 \\
\text { LDR, } 40 \text { CFR } 268 \\
\text { LDR, } 40 \text { CFR } 268 \\
\text { NPDES (current) } \\
\text { LS \& WL } \\
\text { NMED-051/1991 } \\
\text { WQCC, Pt. 3 } \\
\text { NPDES, TSS } \\
\text { NPDES, WQS }\end{array}$ \\
\hline calcium & $\mathrm{Ca}$ & $\begin{array}{l}\text { no req } \\
475.0\end{array}$ & $\begin{array}{l}\text { NPDES (current) } \\
\text { NMED-051/1991 }\end{array}$ \\
\hline chloride & $\mathrm{Cl}$ & $\begin{array}{l}250 \mathrm{mg} / \mathrm{L} \\
0.2 \mathrm{mg} / \mathrm{L} \\
0.5 \mathrm{mg} / \mathrm{L}^{*} \\
\text { no req } \\
113.0 \\
\text { no req } \\
113.0 \\
250 \mathrm{mg} / \mathrm{L}\end{array}$ & $\begin{array}{l}\text { NSDWR, } 40 \text { CFR } 143 \\
\text { EG\&S, } 40 \text { CFR } 423 \\
\text { EG\&S, } 40 \text { CFR } 423 \\
\text { NPDES (current) } \\
\text { NMED-051/1991 } \\
\text { NPDES (current) } \\
\text { NMED-051/1991 } \\
\text { WQCC, Pt. } 3\end{array}$ \\
\hline chlorine (free available & $\mathrm{Cl}$ & $\begin{array}{l}0.2 \mathrm{mg} / \mathrm{L} \\
0.5 \mathrm{mg} / \mathrm{L}\end{array}$ & $\begin{array}{l}\text { NPDES, Pt. } 1 \text { (day) } \\
\text { NPDES, Pt. } 1 \text { (mac) }\end{array}$ \\
\hline chlorine (total residual) & $\mathrm{Cl}$ & $\begin{array}{l}1 \mathrm{mg} / \mathrm{L} \\
1 \mathrm{mg} / \mathrm{L}\end{array}$ & $\begin{array}{l}\text { NPDES, Pt. } 1 \text { (day) } \\
\text { NPDES, Pt. } 1 \text { (max) }\end{array}$ \\
\hline
\end{tabular}




\begin{tabular}{|c|c|c|c|}
\hline $\begin{array}{c}\text { Effluent } \\
\text { Component }\end{array}$ & $\begin{array}{c}\text { Component } \\
\text { Symbol }\end{array}$ & $\begin{array}{c}\text { Effluent } \\
\text { Limits }\end{array}$ & $\begin{array}{c}\text { Source of Emluent } \\
\text { Limit Guidance }\end{array}$ \\
\hline chromium (total) & Total $\mathrm{Cr}$ & $\begin{array}{l}0.00023 \mathrm{mg} / \mathrm{L} \\
5.0 \mathrm{mg} / \mathrm{L} \\
0.05 \mathrm{mg} / \mathrm{L} \\
7.0 \mathrm{mg} / \mathrm{L} \\
4.0 \mathrm{mg} / \mathrm{L}^{*} \\
0.2 \mathrm{mg} / \mathrm{L} \\
5.0 \mathrm{mg} / \mathrm{L} \\
0.37 \mathrm{mg} / \mathrm{L}^{* *} \\
\text { no req } \\
1.0 \\
0.009 \\
0.05 \mathrm{mg} / \mathrm{L} \\
4.00 \mathrm{mg} / \mathrm{L} \\
4.00 \mathrm{mg} / \mathrm{L} \\
1.00 \mathrm{mg} / \mathrm{L} \\
1.00 \mathrm{mg} / \mathrm{L} \\
\end{array}$ & $\begin{array}{l}\text { NMED-051/1992 } \\
\text { TCLP, } 40 \text { CFR } 261 \\
\text { NPDWR, } 40 \text { CFR } 141 \\
\text { EG\&S, } 40 \text { CFR } 413 \\
\text { EG\&S, } 40 \text { CFR } 413 \\
\text { EG\&S, } 40 \text { CFR } 423 \\
\text { LDR, } 40 \text { CFR } 268 \\
\text { LDR, } 40 \text { CFR } 268 \\
\text { NPDES (current) } \\
\text { LS \& WL } \\
\text { NMED-51/1991 } \\
\text { WQCC, Pt. } 3 \\
\text { NPDES, TSS } \\
\text { NPDES, WQS } \\
\text { NPDES, Pt. } 1 \text { (day) } \\
\text { NPDES, Pt. } 1 \text { (max) }\end{array}$ \\
\hline copper & $\mathrm{Cu}$ & $\begin{array}{l}1.0 \mathrm{mg} / \mathrm{L} \\
2.7 \mathrm{mg} / \mathrm{L} \\
4.5 \mathrm{mg} / \mathrm{L}^{*} \\
1.0 \mathrm{mg} / \mathrm{L} \\
1.3 \mathrm{mg} / \mathrm{L}^{* *} \\
0.0002 \mathrm{mg} / \mathrm{L} \\
\text { no } \mathrm{req} \\
0.5 \\
0.3 \\
1.0 \mathrm{mg} / \mathrm{L} \\
1.00 \mathrm{mg} / \mathrm{L} \\
1.00 \mathrm{mg} / \mathrm{L} \\
1.0 \mathrm{mg} / \mathrm{L} \\
1.0 \mathrm{mg} / \mathrm{L}\end{array}$ & $\begin{array}{l}\text { NSDWR, } 40 \text { CFR } 143 \\
\text { EG\&S, } 40 \text { CFR } 413 \\
\text { EG\&S, } 40 \text { CFR } 413 \\
\text { EG\&S, 40 CFR } 423 \\
\text { LDR, 40 CFR } 268 \\
\text { NMED-051/1992 } \\
\text { NPDES (current) } \\
\text { LS \& WL } \\
\text { NMED-051/1991 } \\
\text { WQCC, Pt. } 3 \\
\text { NPDES, TSS } \\
\text { NPDES, WQS } \\
\text { NPDES, Pt. } 1 \text { (day) } \\
\text { NPDES, Pt. } 1 \text { (max) }\end{array}$ \\
\hline cyanide & $\mathrm{CN}$ & $\begin{array}{l}0.008 \mathrm{mg} / \mathrm{L} \\
1.0 \mathrm{mg} / \mathrm{L} \\
1.9 \mathrm{mg} / \mathrm{L} \\
1.2 \mathrm{mg} / \mathrm{L}^{* *} \\
\text { no req } \\
\text { no req } \\
0.2 \\
0.2 \mathrm{mg} / \mathrm{L}\end{array}$ & $\begin{array}{l}\text { NMED-051/1992 } \\
\text { EG\&S, } 40 \text { CFR } 413 \\
\text { EG\&S, } 40 \text { CFR } 413 \\
\text { LDR, } 40 \text { CFR } 268 \\
\text { NPDES (current) } \\
\text { LS \& WL } \\
\text { NMED-051/1991 } \\
\text { WQCC, Pt. } 3\end{array}$ \\
\hline
\end{tabular}




\begin{tabular}{|c|c|c|c|}
\hline $\begin{array}{l}\text { Effluent } \\
\text { Component }\end{array}$ & $\begin{array}{l}\text { Component } \\
\text { Symbol }\end{array}$ & $\begin{array}{l}\text { Effluent } \\
\text { Limits }\end{array}$ & $\begin{array}{l}\text { Source of Effluent } \\
\text { Limit Guidance }\end{array}$ \\
\hline fluoride & $\mathrm{F}$ & $\begin{array}{l}4.0 \mathrm{mg} / \mathrm{L} \\
2.0 \mathrm{mg} / \mathrm{L} \\
35 \mathrm{mg} / \mathrm{L}^{* *} \\
\text { no req } \\
6.7 \\
1.6 \mathrm{mg} / \mathrm{L}\end{array}$ & $\begin{array}{l}\text { NPDWR, } 40 \text { CFR } 141 \\
\text { NSDWR, } 40 \text { CFR } 143 \\
\text { LDR, } 40 \text { CFR } 268 \\
\text { NPDES (current) } \\
\text { NMED-051/1991 } \\
\text { WQCC, Pt. } 3\end{array}$ \\
\hline iron & $\mathrm{Fe}$ & $\begin{array}{l}0.3 \mathrm{mg} / \mathrm{L} \\
1.0 \mathrm{mg} / \mathrm{L} \\
\text { no req } \\
1.0 \mathrm{mg} / \mathrm{L} \\
10 \mathrm{mg} / \mathrm{L} \\
40 \mathrm{mg} / \mathrm{L}\end{array}$ & $\begin{array}{l}\text { NSDWR, } 40 \text { CFR } 143 \\
\text { EG\&S, } 40 \text { CFR } 423 \\
\text { NPDES (current) } \\
\text { WQCC, Pt. } 3 \\
\text { NPDES, Pt. } 1 \text { (day) } \\
\text { NPDES, Pt. } 1 \text { (max) }\end{array}$ \\
\hline lead & $\mathrm{Pb}$ & $\begin{array}{l}0.0045 \mathrm{mg} / \mathrm{L} \\
5.0 \mathrm{mg} / \mathrm{L} \\
0.05 \mathrm{mg} / \mathrm{L} \\
0.4 \mathrm{mg} / \mathrm{L} \\
0.6 \mathrm{mg} / \mathrm{L}^{*} \\
5.0 \mathrm{mg} / \mathrm{L} \\
0.28 \mathrm{mg} / \mathrm{L}^{* *} \\
\text { no req } \\
0.1 \\
0.03 \\
0.05 \mathrm{mg} / \mathrm{L} \\
0.13 \mathrm{mg} / \mathrm{L} \\
0.13 \mathrm{mg} / \mathrm{L} \\
\end{array}$ & $\begin{array}{l}\text { NMED-051/1992 } \\
\text { TCLP, } 40 \text { CFR } 261 \\
\text { NPDWR, } 40 \text { CFR } 141 \\
\text { EG\&S, } 40 \text { CFR } 413 \\
\text { EG\&S, 40 CFR } 413 \\
\text { LDR, } 40 \text { CFR } 268 \\
\text { LDR, } 40 \text { CFR } 268 \\
\text { NPDES (current) } \\
\text { LS \& WL } \\
\text { NMED-051/1991 } \\
\text { WQCC, Pt. } 3 \\
\text { NPDES, TSS } \\
\text { NPDES, WQS }\end{array}$ \\
\hline magnesium & $\mathrm{Mg}$ & $\begin{array}{l}\text { no req } \\
1.1\end{array}$ & $\begin{array}{l}\text { NPDES (current) } \\
\text { NMED-051/1991 }\end{array}$ \\
\hline manganese & $\mathrm{Mn}$ & $0.2 \mathrm{mg} / \mathrm{L}$ & WQCC, Pt. 3 \\
\hline
\end{tabular}




\begin{tabular}{|c|c|c|c|}
\hline $\begin{array}{c}\text { Effluent } \\
\text { Component }\end{array}$ & $\begin{array}{c}\text { Component } \\
\text { Symbol }\end{array}$ & $\begin{array}{l}\text { Effluent } \\
\text { Limits }\end{array}$ & $\begin{array}{l}\text { Source of Effluent } \\
\text { Limit Guidance }\end{array}$ \\
\hline mercury & $\mathrm{Hg}$ & $\begin{array}{l}0.00003 \mathrm{mg} / \mathrm{L} \\
0.2 \mathrm{mg} / \mathrm{L} \\
0.002 \mathrm{mg} / \mathrm{L} \\
0.20 \mathrm{mg} / \mathrm{L} \\
0.15 \mathrm{mg} / \mathrm{L}^{* *} \\
\text { no req } \\
0.01 \\
0.00045 \\
0.002 \mathrm{mg} / \mathrm{L} \\
0.03 \mathrm{mg} / \mathrm{L} \\
0.03 \mathrm{mg} / \mathrm{L} \\
\end{array}$ & $\begin{array}{l}\text { NMED-051/1992 } \\
\text { TCLP, } 40 \text { CFR } 261 \\
\text { NPDWR, 40 CFR } 141 \\
\text { LDR, } 40 \text { CFR } 268 \\
\text { LDR, } 40 \text { CFR } 268 \\
\text { NPDES (current) } \\
\text { LS \& WL } \\
\text { NMED-051/1991 } \\
\text { WQCC, Pt. } 3 \\
\text { NPDES, TSS } \\
\text { NPDES, WQS } \\
\end{array}$ \\
\hline molybdenum & Mo & $1.0 \mathrm{mg} / \mathrm{L}$ & WQCC, Pt. 3 \\
\hline nickel & $\mathrm{Ni}$ & $\begin{array}{l}0.013 \mathrm{mg} / \mathrm{L} \\
2.6 \mathrm{mg} / \mathrm{L} \\
4.1 \mathrm{mg} / \mathrm{L}^{*} \\
0.55 \mathrm{mg} / \mathrm{L}^{* *} \\
\text { no req } \\
\text { no req } \\
0.06 \\
0.2 \mathrm{mg} / \mathrm{L}\end{array}$ & $\begin{array}{l}\text { NMED-051/1992 } \\
\text { EG\&S, } 40 \text { CFR } 413 \\
\text { EG\&S, } 40 \text { CFR } 413 \\
\text { LDR, } 40 \text { CFR } 268 \\
\text { NPDES (current) } \\
\text { LS \& WL } \\
\text { NMED-051/1991 } \\
\text { WQCC, Pt. } 3\end{array}$ \\
\hline ammonia nitrogen & $\mathrm{NH}_{3}-\mathrm{N}$ & $\begin{array}{l}0.1 \mathrm{mg} / \mathrm{L} \\
\text { no req } \\
\text { no req } \\
10.9\end{array}$ & $\begin{array}{l}\text { NMED-051/1992 } \\
\text { NPDES (current) } \\
\text { LS \& WL } \\
\text { NMED-051/1991 }\end{array}$ \\
\hline nitrite nitrogen & $\mathrm{NO}_{2}-\mathrm{N}$ & $\begin{array}{l}\text { no req } \\
0.7\end{array}$ & $\begin{array}{l}\text { NPDES (current) } \\
\text { NMED-051/1992 }\end{array}$ \\
\hline nitrate nitrogen & $\mathrm{NO}_{3}-\mathrm{N}$ & $\begin{array}{l}10 \mathrm{mg} / \mathrm{L} \\
20 \mathrm{mg} / \mathrm{L}^{*} \\
\text { no req } \\
\text { no req } \\
10.0 \\
274.0 \\
10.0 \mathrm{mg} / \mathrm{L}\end{array}$ & $\begin{array}{l}\text { NPDWR, } 40 \text { CFR } 141 \\
\text { NPDWR, } 40 \text { CFR } 141 \\
\text { NPDES (current) } \\
\text { LS \& WL } \\
\text { NMED-051/1992 } \\
\text { NMED-051/1991 } \\
\text { WQCC, Pt. } 3\end{array}$ \\
\hline
\end{tabular}




\begin{tabular}{|c|c|c|c|}
\hline $\begin{array}{c}\text { Effluent } \\
\text { Component }\end{array}$ & $\begin{array}{c}\text { Component } \\
\text { Symbol }\end{array}$ & $\begin{array}{c}\text { Effluent } \\
\text { Limits }\end{array}$ & $\begin{array}{l}\text { Source of Effluent } \\
\text { Limit Guidance }\end{array}$ \\
\hline phosphate phosporus & $\mathrm{PO}_{4}-\mathrm{P}$ & $\begin{array}{l}\text { no req } \\
6.6\end{array}$ & $\begin{array}{l}\text { NPDES (current) } \\
\text { NMED-051/1991 }\end{array}$ \\
\hline phosphorus (total) & $\mathrm{P}$ & $\begin{array}{l}20 \mathrm{mg} / \mathrm{L} \\
40 \mathrm{mg} / \mathrm{L} \\
\end{array}$ & $\begin{array}{l}\text { NPDES, Pt } 1 \text { (day) } \\
\text { NPDES, Pt. } 1 \text { (max) }\end{array}$ \\
\hline potassium & $\mathrm{K}$ & $\begin{array}{l}\text { no req } \\
108.0\end{array}$ & $\begin{array}{l}\text { NPDES (current) } \\
\text { NMED-051/1991 }\end{array}$ \\
\hline selenium & $\mathrm{Se}$ & $\begin{array}{l}0.0086 \mathrm{mg} / \mathrm{L} \\
1.0 \mathrm{mg} / \mathrm{L} \\
0.01 \mathrm{mg} / \mathrm{L} \\
1.0 \mathrm{mg} / \mathrm{L} \\
0.82 \mathrm{mg} / \mathrm{L}^{* *} \\
\text { no req } \\
0.05 \\
0.0086 \\
0.05 \mathrm{mg} / \mathrm{L} \\
0.05 \mathrm{mg} / \mathrm{L} \\
\end{array}$ & $\begin{array}{l}\text { NMED-051/1992 } \\
\text { TCLP, } 40 \text { CFR } 261 \\
\text { NPDWR, 40 CFR } 141 \\
\text { LDR, } 40 \text { CFR } 268 \\
\text { LDR, } 40 \text { CFR } 268 \\
\text { NPDES (current) } \\
\text { LS \& WL } \\
\text { NMED-051/1991 } \\
\text { WQCC, Pt. 3 } \\
\text { NPDES, WQS }\end{array}$ \\
\hline silica & $\mathrm{SiO}_{2}$ & no req & NPDES (current) \\
\hline silver & $\mathrm{Ag}$ & $\begin{array}{l}0.0001 \\
5.0 \mathrm{mg} / \mathrm{L} \\
0.1 \mathrm{mg} / \mathrm{L} \\
0.7 \mathrm{mg} / \mathrm{L} \\
1.2 \mathrm{mg} / \mathrm{L}^{*} \\
0.29 \mathrm{mg} / \mathrm{L}^{* *} \\
\text { no } \mathrm{req} \\
\text { no } \mathrm{req} \\
0.005 \\
0.05 \mathrm{mg} / \mathrm{L} \\
0.5 \mathrm{mg} / \mathrm{L} \\
1.0 \mathrm{mg} / \mathrm{L} \\
\end{array}$ & $\begin{array}{l}\text { NMED-051/1992 } \\
\text { TCLP, } 40 \text { CFR } 261 \\
\text { NPDWR, } 40 \text { CFR } 141 \\
\text { EG\&S, } 40 \text { CFR } 413 \\
\text { EG\&S, } 40 \text { CFR } 413 \\
\text { LDR, } 40 \text { CFR } 268 \\
\text { NPDES (current) } \\
\text { LS \& WL } \\
\text { NMED-051/1991 } \\
\text { WQCC, Pt. } 3 \\
\text { NPDES, Pt. } 1 \text { (day) } \\
\text { NPDES, Pt. } 1 \text { (max) }\end{array}$ \\
\hline sulfate & $\mathrm{SO}_{4}^{-2}$ & $\begin{array}{l}250 \mathrm{mg} / \mathrm{L} \\
\text { no req } \\
288.0 \\
600 \mathrm{mg} / \mathrm{L}\end{array}$ & $\begin{array}{l}\text { NPDWR, } 40 \text { CFR } 143 \\
\text { NPDES (current) } \\
\text { NMED-051/1991 } \\
\text { WQCC, Pt. } 3\end{array}$ \\
\hline sulfite & $\mathrm{SO}_{3}^{-2}$ & $\begin{array}{l}35 \mathrm{mg} / \mathrm{L} \\
70 \mathrm{mg} / \mathrm{L}\end{array}$ & $\begin{array}{l}\text { NPDES, Pt. } 1 \text { (day) } \\
\text { NPDES, Pt. } 1 \text { (max) }\end{array}$ \\
\hline
\end{tabular}




\begin{tabular}{|c|c|c|c|}
\hline $\begin{array}{c}\text { Effluent } \\
\text { Component }\end{array}$ & $\begin{array}{c}\text { Component } \\
\text { Symbol }\end{array}$ & $\begin{array}{c}\text { Effluent } \\
\text { Limits }\end{array}$ & $\begin{array}{l}\text { Source of Effluent } \\
\text { Limit Guidance }\end{array}$ \\
\hline sodium & $\mathrm{Na}$ & $\begin{array}{l}\text { no req } \\
766.0\end{array}$ & $\begin{array}{l}\text { NPDES (current) } \\
\text { NMED-051/1991 }\end{array}$ \\
\hline zinc & $\mathrm{Zn}$ & $\begin{array}{l}0.0036 \mathrm{mg} / \mathrm{L} \\
5.0 \mathrm{mg} / \mathrm{L} \\
2.6 \mathrm{mg} / \mathrm{L} \\
4.2 \mathrm{mg} / \mathrm{L}^{*} \\
1.0 \mathrm{mg} / \mathrm{L} \\
1.0 \mathrm{mg} / \mathrm{L} \\
\text { no req } \\
5.0 \\
0.1 \\
10.0 \mathrm{mg} / \mathrm{L} \\
63 \mathrm{mg} / \mathrm{L} \\
63 \mathrm{mg} / \mathrm{L}\end{array}$ & $\begin{array}{l}\text { NMED-051/1992 } \\
\text { NPDWR, } 40 \text { CFR } 143 \\
\text { EG\&S, } 40 \text { CFR } 413 \\
\text { EG\&S, } 40 \text { CFR } 413 \\
\text { EG\&S, } 40 \text { CFR } 423 \\
\text { LDR, } 40 \text { CFR } 268 \\
\text { NPDES (current) } \\
\text { LS \& WL } \\
\text { NMED-051/1991 } \\
\text { WQCC, Pt. } 3 \\
\text { NPDES, TSS } \\
\text { NPDES, WQS }\end{array}$ \\
\hline total alkalinity & T-alk & no req & NPDES (current) \\
\hline conductivity & $\Omega$ & no req & NPDES (current) \\
\hline $\mathrm{pH}$ & & $\begin{array}{l}6.5-8.5 \\
7.7-10.0 \\
6.0-9.0 \\
6.0-9.0 \\
7.2-7.7 \\
6.6-8.6 \\
6.0-9.0\end{array}$ & $\begin{array}{l}\text { NPDWR, } 40 \text { CFR } 143 \\
\text { EG\&S, } 40 \text { CFR } 413 \\
\text { EG\&S, } 40 \text { CFR } 423 \\
\text { NPDES (current) } \\
\text { NMED-051/1991 } \\
\text { WQCC, Pt. } 2 \\
\text { WQCC, Pt. } 3\end{array}$ \\
\hline total hardness & T Hard & no req & NPDES (current) \\
\hline chemical oxygen demand & COD & $\begin{array}{l}\text { no req } \\
\text { no req } \\
125.0 \\
51.0 \\
>125 \mathrm{mg} / \mathrm{L} \\
125 \mathrm{mg} / \mathrm{L} \\
125 \mathrm{mg} / \mathrm{L}\end{array}$ & $\begin{array}{l}\text { NPDES (current) } \\
\text { LS \& WL } \\
\text { NMED-051/1992 } \\
\text { NMED-051/1991 } \\
\text { WQCC, Pt. } 2 \\
\text { NPDES, Pt. } 1 \text { (day) } \\
\text { NPDES, Pt. } 1 \text { (max) }\end{array}$ \\
\hline biochemical oxygen demand & BOD & $>30 \mathrm{mg} / \mathrm{L}$ & WQCC, Pt. 2 \\
\hline
\end{tabular}




\begin{tabular}{|c|c|c|c|}
\hline $\begin{array}{c}\text { Emluent } \\
\text { Component }\end{array}$ & $\begin{array}{l}\text { Component } \\
\text { Symbol }\end{array}$ & $\begin{array}{l}\text { Effluent } \\
\text { Limits }\end{array}$ & $\begin{array}{l}\text { Source of Effluent } \\
\text { Limit Guidance }\end{array}$ \\
\hline total dissolved solids & TDS & $\begin{array}{l}500 \mathrm{mg} / \mathrm{L} \\
\text { no req } \\
2370.0 \\
1000 \mathrm{mg} / \mathrm{L} \\
\end{array}$ & $\begin{array}{l}\text { NPDWR, } 40 \text { CFR } 143 \\
\text { NPDES (current) } \\
\text { NMED-051/1991 } \\
\text { WQCC, Pt. } 3 \\
\end{array}$ \\
\hline $\begin{array}{l}\text { total suspended solids } \\
\text { (settleable) }\end{array}$ & TSS & $\begin{array}{l}13.4 \mathrm{mg} / \mathrm{L} \\
20.0 \mathrm{mg} / \mathrm{L}^{*} \\
30.0 \mathrm{mg} / \mathrm{L} \\
50.0 \mathrm{mg} / \mathrm{L}^{*} \\
100.0 \mathrm{mg} / \mathrm{L}^{*} \\
\text { no } \mathrm{req} \\
0.0 \\
0.0 \\
30 \mathrm{mg} / \mathrm{L} \\
45 \mathrm{mg} / \mathrm{L} \\
100 \mathrm{mg} / \mathrm{L} \\
>0.5 \mathrm{mg} / \mathrm{L}\end{array}$ & $\begin{array}{l}\text { EG\&S, } 40 \text { CFR } 413 \\
\text { EG\&S, } 40 \text { CFR } 413 \\
\text { EG\&S, } 40 \text { CFR } 423 \\
\text { EG\&S, 40 CFR } 423 \\
\text { EG\&S, } 40 \text { CFR } 423 \\
\text { NPDES (current) } \\
\text { NMED-051/1992 } \\
\text { NMED-051/1991 } \\
\text { NPDES, Pt. } 1 \text { (day) } \\
\text { NPDES, Pt. } 1 \text { (max) } \\
\text { NPDES, Pt. } 1 \text { (max) } \\
\text { WQCC, Pt. } 2\end{array}$ \\
\hline beryllium & $\mathrm{Be}$ & $\begin{array}{l}0.052 \mathrm{mg} / \mathrm{L} \\
0.82 \mathrm{mg} / \mathrm{L}^{* *} \\
\text { no req }\end{array}$ & $\begin{array}{l}\text { NMED-051/1992 } \\
\text { LDR, } 40 \text { CFR } 268 \\
\text { current }\end{array}$ \\
\hline aluminum & $\mathrm{Al}$ & $\begin{array}{l}5.0 \mathrm{mg} / \mathrm{L} \\
\text { no req } \\
5.0 \\
5.0 \mathrm{mg} / \mathrm{L} \\
5.0 \mathrm{mg} / \mathrm{L}\end{array}$ & $\begin{array}{l}\text { NMED-051/1992 } \\
\text { current } \\
\text { LS \& WL } \\
\text { WQCC, Pt. } 3 \\
\text { NPDES, WQS }\end{array}$ \\
\hline boron & B & $\begin{array}{l}0.75 \mathrm{mg} / \mathrm{L} \\
\text { no } \mathrm{req} \\
5.0 \\
0.75 \mathrm{mg} / \mathrm{L} \\
5.0 \mathrm{mg} / \mathrm{L}\end{array}$ & $\begin{array}{l}\text { NMED-051/1992 } \\
\text { current } \\
\text { LS \& WL } \\
\text { WQCC, Pt. } 3 \\
\text { NPDES, WQS }\end{array}$ \\
\hline cobalt & Co & $\begin{array}{l}\text { no req } \\
1.0 \\
0.05 \\
0.05 \mathrm{mg} / \mathrm{L} \\
1.00 \mathrm{mg} / \mathrm{L}\end{array}$ & $\begin{array}{l}\text { current } \\
\text { LS \& WL } \\
\text { NMED-051/1992 } \\
\text { WQCC, Pt. } 3 \\
\text { NPDES, WQS }\end{array}$ \\
\hline
\end{tabular}




\begin{tabular}{|c|c|c|c|}
\hline $\begin{array}{c}\text { Effluent } \\
\text { Component }\end{array}$ & $\begin{array}{l}\text { Component } \\
\text { Symbol }\end{array}$ & $\begin{array}{l}\text { Effluent } \\
\text { Limits }\end{array}$ & $\begin{array}{l}\text { Source of Effluent } \\
\text { Limit Guidance }\end{array}$ \\
\hline vanadium & $\mathrm{Vn}$ & $\begin{array}{l}0.042 \mathrm{mg} / \mathrm{L}^{* *} \\
0.05 \mathrm{mg} / \mathrm{L} \\
\text { no req } \\
0.1 \\
0.10 \mathrm{mg} / \mathrm{L}\end{array}$ & $\begin{array}{l}\text { LDR, } 40 \text { CFR } 268 \\
\text { NMED-051/1992 } \\
\text { current } \\
\text { LS \& WL } \\
\text { NPDES, WQS }\end{array}$ \\
\hline uranium & $\mathrm{U}$ & $5.0 \mathrm{mg} / \mathrm{L}$ & WQCC, Pt. 3 \\
\hline tritium & $\mathrm{H}^{3}$ & $\begin{array}{l}20,000 \mathrm{pCi} / \mathrm{L} \\
2,000,000 \mathrm{pCi} / \mathrm{L} \\
20,000 \mathrm{pCi} / \mathrm{L} \\
1,000,000 \mathrm{pCi} / \mathrm{L} \\
3.0 \\
1.91 \\
3,000,000 \mathrm{pCi} / \mathrm{L}\end{array}$ & $\begin{array}{l}\text { NMED-051/1992 } \\
\text { DCG, DOE 5400.5 } \\
\text { NPDWR, } 40 \text { CFR } 141 \\
\text { RPS, 10 CFR } 20 \\
\text { LS \& WL } \\
\text { NMED-051/1991 } \\
\text { NPDES, WQS }\end{array}$ \\
\hline radium-226 \& -228 & $\mathrm{Ra}^{226+228}$ & $\begin{array}{l}5 \mathrm{pCi} / \mathrm{L} \\
100 \mathrm{pCi} / \mathrm{L} \\
5 \mathrm{pCi} / \mathrm{L} \\
60 \mathrm{pCi} / \mathrm{L} \\
30.0 \\
30.0 \\
30.0 \mathrm{pCi} / \mathrm{L} \\
30.0 \mathrm{pCi} / \mathrm{L}\end{array}$ & $\begin{array}{l}\text { DWP, DOE } 5400.5 \\
\text { DCG, DOE } 5400.5 \\
\text { NPDWR, } 40 \text { CFR } 141 \\
\text { RPS, } 10 \text { CFR } 20 \\
\text { LS \& WL } \\
\text { NMED-051/1992 } \\
\text { WQCC, Pt. } 3 \\
\text { NPDES, WQS }\end{array}$ \\
\hline plutonium-239 & $\mathrm{Pu}^{239}$ & $\begin{array}{l}30 \mathrm{pCi} / \mathrm{L} \\
20 \mathrm{pCi} / \mathrm{L}\end{array}$ & $\begin{array}{l}\text { DCG, DOE } 5400.5 \\
\text { RPS, } 10 \text { CFR } 20\end{array}$ \\
\hline americium-241 & $\mathrm{Am}^{241}$ & $\begin{array}{l}30 \mathrm{pCi} / \mathrm{L} \\
20 \mathrm{pCi} / \mathrm{L}\end{array}$ & $\begin{array}{l}\text { DCG, DOE } 5400.5 \\
\text { RPS, } 10 \text { CFR } 20\end{array}$ \\
\hline cesium-137 & $\mathrm{Cs}^{137}$ & $\begin{array}{l}3000 \mathrm{pCi} / \mathrm{L} \\
1000 \mathrm{pCi} / \mathrm{L}\end{array}$ & $\begin{array}{l}\text { DCG, DOE } 5400.5 \\
\text { RPS, } 10 \text { CFR } 20\end{array}$ \\
\hline strontium-90 & $\mathrm{Sr}^{90}$ & $\begin{array}{l}1000 \mathrm{pCi} / \mathrm{L} \\
8 \mathrm{pCi} / \mathrm{L} \\
500 \mathrm{pCi} / \mathrm{L}\end{array}$ & $\begin{array}{l}\text { DCG, DOE } 5400.5 \\
\text { NPDWR, } 40 \text { CFR } 141 \\
\text { RPS, } 10 \text { CFR } 20\end{array}$ \\
\hline cobalt- 60 & $\mathrm{Co}^{60}$ & $\begin{array}{l}5000 \mathrm{pCi} / \mathrm{L} \\
3000 \mathrm{pCi} / \mathrm{L}\end{array}$ & $\begin{array}{l}\text { DCG, DOE } 5400.5 \\
\text { RPS, } 10 \text { CFR } 20 \\
\end{array}$ \\
\hline sodium-22 & $\mathrm{Na}^{22}$ & $\begin{array}{l}10,000 \mathrm{pCi} / \mathrm{L} \\
6000 \mathrm{pCi} / \mathrm{L} \\
\end{array}$ & $\begin{array}{l}\text { DCG, DOE } 5400.5 \\
\text { RPS, } 10 \text { CFR } 20 \\
\end{array}$ \\
\hline strontium- 88 & $\mathrm{Sr}^{88}$ & $70,000 \mathrm{pCi} / \mathrm{L}$ & DCG, DOE 5400.5 \\
\hline
\end{tabular}




\begin{tabular}{||c|c|l|l||}
\hline $\begin{array}{c}\text { Effluent } \\
\text { Component }\end{array}$ & $\begin{array}{c}\text { Component } \\
\text { Symbol }\end{array}$ & \multicolumn{1}{|c|}{$\begin{array}{c}\text { Effluent } \\
\text { Limits }\end{array}$} & \multicolumn{1}{|c|}{$\begin{array}{c}\text { Source of Effluent } \\
\text { Limit Guidance }\end{array}$} \\
\hline \hline niobium-95 & $\mathrm{Nb}^{95}$ & $\begin{array}{l}60,000 \mathrm{pCi} / \mathrm{L} \\
30,000 \mathrm{pCi} / \mathrm{L}\end{array}$ & $\begin{array}{l}\text { DCG, DOE 5400.5 } \\
\text { RPS, 10 CFR 20 }\end{array}$ \\
\hline yttrium-88 & $\mathrm{Y}^{88}$ & $\begin{array}{l}30,000 \mathrm{pCi} / \mathrm{L} \\
10,000 \mathrm{pCi} / \mathrm{L}\end{array}$ & $\begin{array}{l}\text { DCG, DOE 5400.5 } \\
\text { RPS, 10 CFR 20 }\end{array}$ \\
\hline zirconium-88 & $\mathrm{Zr}^{88}$ & $\begin{array}{l}100,000 \mathrm{pCi} / \mathrm{L} \\
50,000 \mathrm{pCi} / \mathrm{L}\end{array}$ & $\begin{array}{l}\text { DCG, DOE 5400.5 } \\
\text { RPS, 10 CFR 20 }\end{array}$ \\
\hline Total Alpha & - & $30 \mathrm{pCi} / \mathrm{L}$ & NMED-051/1992 \\
\hline
\end{tabular}

* conditional limit guidelines

** under Waste Code F039

Note: Concentration is $\mathrm{mg} / \mathrm{L}$ unless noted 


\section{APPENDIX D}

ANTICIPATED INFLUENT/EFFLUENT CONTAMINANT CONCENTRATIONS FOR PROPOSED PROCESS CONFIGURATIONS 
Table for Process Configuration \# 1, Upgrade Existing TA-50 Operations, TA-50 Waste Only - Wildlife and Livestock Watering Release Limits

\begin{tabular}{|c|c|c|}
\hline \multirow[t]{2}{*}{ COMPONENT } & INFLUENT & EFFLUENT \\
\hline & $T A-50-1$ & $\begin{array}{l}\text { WILDLIFE } \\
\text { LIMITS }\end{array}$ \\
\hline & $\mathrm{mg} / \mathrm{h}$ & $\mathrm{mg} / \mathrm{h}$ \\
\hline ARSENIC & 0.003 & 0.02 \\
\hline BARIUM & 0.5 & $1.0^{*}$ \\
\hline CADMIUM & 0.10 & 0.05 \\
\hline CALCIUM & 40.0 & - \\
\hline CHLORIDE & 84 & $250 *$ \\
\hline CHROMIUM & 0.25 & 1.0 \\
\hline COPPER & 0.30 & 0.5 \\
\hline CYANIDE & 0.6 & 0.2 * \\
\hline FLUORIDE & 8.0 & 1.6 * \\
\hline IRON & 1.0 & $1.0 *$ \\
\hline LEAD & 0.2 & 0.1 \\
\hline MAGNESIUM & 4.0 & - \\
\hline MERCURY & 0.005 & 0.01 \\
\hline NICKEL & 1.0 & $0.2^{*}$ \\
\hline AMMONIA & 15.0 & - \\
\hline (as N) & & \\
\hline NITRITE & 1.0 & - \\
\hline (as N) & & \\
\hline NITPATE & 325 & 10.0 \\
\hline (as N) & & \\
\hline PHOSPHATE & 40.0 & - \\
\hline POTASSIUM & 76.0 & - \\
\hline SELENIUM & 1.0 & 0.05 \\
\hline SILICA & 87.0 & - \\
\hline SILVER & 0.03 & $0.05 *$ \\
\hline SODIUM & 512 & - \\
\hline SULFATE & 26.0 & $600 *$ \\
\hline ZINC & 0.3 & 5.0 \\
\hline $\mathrm{pH}$ & 10.0 & $6.0-9.0$ \\
\hline COD & 123 & 125.0 \\
\hline TDS & 2000 & $1000 *$ \\
\hline TSS & 45.0 & $45.0^{*}$ \\
\hline BERYLLIUM & & - \\
\hline ALUMINUM & & 5.0 \\
\hline BORON & & 5.0 \\
\hline COBALT & & 1.0 \\
\hline VANADIUM & & 0.1 \\
\hline
\end{tabular}

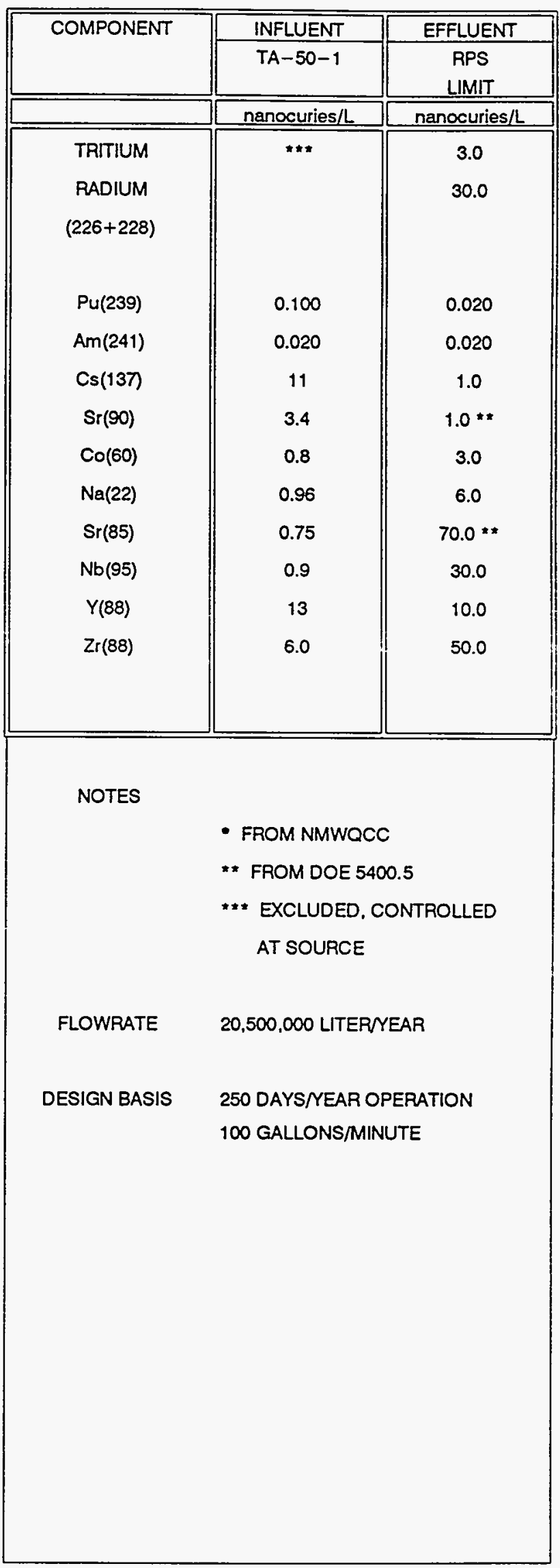


Table for Process Configuration \# 2, Upgrade Existing Room 60 Operations, TA-55 Waste Only - Wildlife and Livestock Watering Release Limits

\begin{tabular}{|c|c|c|c|}
\hline \multirow[t]{2}{*}{ COMPONENT } & INFLUENT & SUPERNATE & EFFLUENT \\
\hline & $R M-60$ & $\mathrm{RM}-60$ & $\begin{array}{l}\text { WILDLIFE } \\
\text { LIMITS }\end{array}$ \\
\hline & $\mathrm{mg} / \mathrm{l}$ & $\mathrm{mg} / \mathrm{l}$ & $\mathrm{mg} / \mathrm{l}$ \\
\hline ARSENIC & & 0.77 & 0.02 \\
\hline BARIUM & & 0.2 & $1.0 *$ \\
\hline CADMIUM & 0 & 0 & 0.05 \\
\hline CALCIUM & 8.1 & 8.1 & - \\
\hline CHLORIDE & 42,974 & 6,310 & $250^{\circ}$ \\
\hline CHROMIUM & 1,350 & 45.0 & 1.0 \\
\hline COPPER & 0.09 & $<.003$ & 0.5 \\
\hline CYANIDE & & 15.0 & $0.2^{*}$ \\
\hline FLUORIDE & 250 & 250 & $1.6^{*}$ \\
\hline IRON & & .005 & $1.0 *$ \\
\hline LEAD & 9.9 & 0.33 & 0.1 \\
\hline MAGNESIUM & & 0.57 & - \\
\hline MERCURY & 0.11 & 0.0035 & 0.01 \\
\hline NICKEL & 930 & $<.02 / 31$ & $0.2^{\star}$ \\
\hline $\begin{array}{c}\text { AMMONIA } \\
\text { (as N) }\end{array}$ & 4.6 & 4.6 & - \\
\hline $\begin{array}{l}\text { NITRITE } \\
\text { (as N) }\end{array}$ & & .05 & - \\
\hline $\begin{array}{l}\text { NITRATE } \\
\text { (as N) }\end{array}$ & 1400 & 28,000 & 10.0 \\
\hline PHOSPHATE & & 0.11 & - \\
\hline POTASSIUM & 6.500 & 6,500 & - \\
\hline SELENIUM & 48 & 1.6 & 0.05 \\
\hline SILICA & & 8 & - \\
\hline SILVER & 13.2 & 0.44 & $0.05 *$ \\
\hline SODIUM & 24,140 & 46,280 & - \\
\hline SULFATE & 0 & 0 & $600 *$ \\
\hline ZINC & & $<.001$ & 5.0 \\
\hline $\mathrm{pH}$ & & 12 & $6.0-9.0$ \\
\hline COD & & 303 & 125.0 \\
\hline TDS & & 188,000 & $1000 *$ \\
\hline TSS & & 226 & $45.0^{*}$ \\
\hline BERYLLIUM & & & - \\
\hline ALUMINUM & & & 5.0 \\
\hline BORON & & & 5.0 \\
\hline COBALT & & & 1.0 \\
\hline VANADIUM & & & 0.1 \\
\hline
\end{tabular}

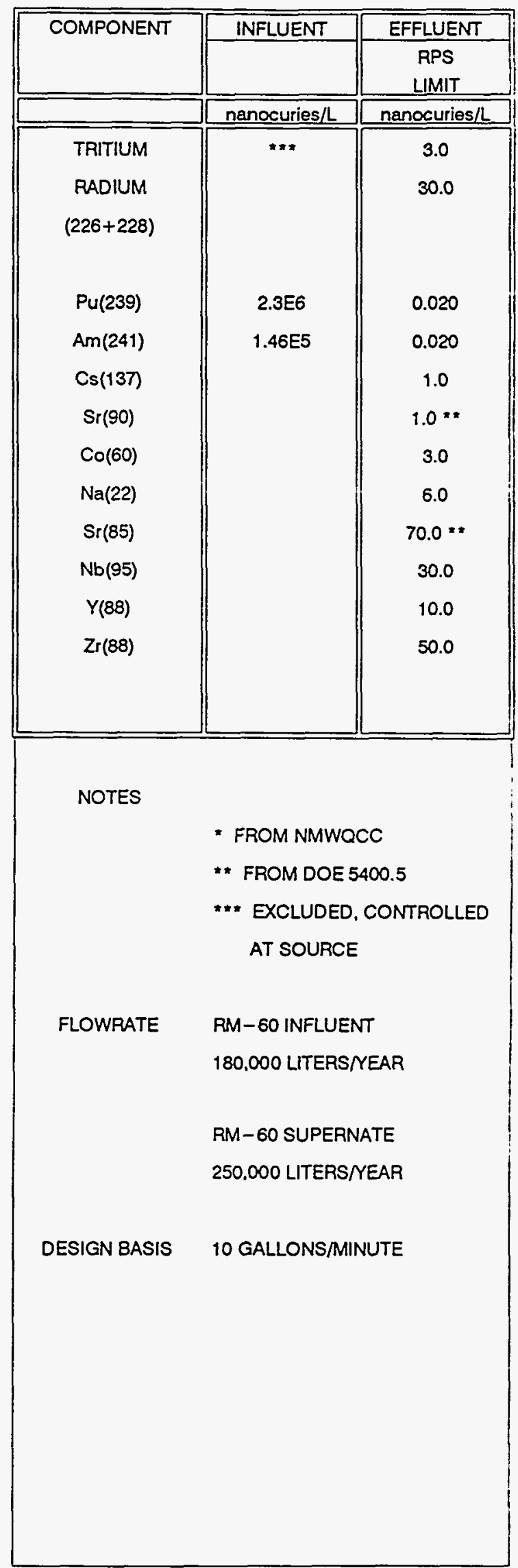


Table for Process Configuration \# 3, Upgrade Existing TA-50 Operations, Blended TA-55 and TA-50 Wastes - Wildlife and Livestock Watering Release Limits

\begin{tabular}{|c|c|c|}
\hline \multirow[t]{2}{*}{ COMPONENT } & INFLUUENT & EFFLUENT \\
\hline & $\begin{array}{l}\text { TA-50-1 } \\
\text { BLENDED }\end{array}$ & $\begin{array}{l}\text { WILDLIFE } \\
\text { LIMITS }\end{array}$ \\
\hline & $\mathrm{mg} / \mathrm{A}$ & $\mathrm{mg} / \mathrm{A}$ \\
\hline ARSENIC & 0.003 & 0.02 \\
\hline BARIUM & $<0.5$ & $1.0=$ \\
\hline CADMIUM & 0.10 & 0.05 \\
\hline CALCIUM & 40.0 & - \\
\hline CHLORIDE & 500 & 250 * \\
\hline CHROMIUM & 0.25 & 1.0 \\
\hline COPPER & 0.30 & 0.5 \\
\hline CYANIDE & 0.6 & $0.2 *$ \\
\hline FLUORIDE & 8.0 & $1.6^{\circ}$ \\
\hline IRON & 1.0 & $1.0^{*}$ \\
\hline LEAD & 0.2 & 0.1 \\
\hline MAGNESIUM & 4.0 & - \\
\hline MERCURY & 0.005 & 0.01 \\
\hline NICKEL & 1.0 & 0.2 * \\
\hline $\begin{array}{c}\text { AMMONIA } \\
\text { (as N) }\end{array}$ & 10 & - \\
\hline $\begin{array}{l}\text { NITRITE } \\
\text { (as N) }\end{array}$ & 1.0 & - \\
\hline $\begin{array}{c}\text { NITHATE } \\
\text { (as N) }\end{array}$ & 350 & 10.0 \\
\hline PHOSPHATE & 4.0 & - \\
\hline POTASSIUM & 240 & - \\
\hline SELENIUM & 1.0 & 0.05 \\
\hline SILICA & 80 & - \\
\hline SILVER & $<.006$ & 0.05 * \\
\hline SODIUM & 1508 & - \\
\hline SULFATE & 26.0 & $600 *$ \\
\hline ZINC & $<.002$ & 5.0 \\
\hline $\mathrm{pH}$ & 7.3 & $6.0-9.0$ \\
\hline$C O D$ & 81 & 125.0 \\
\hline TDS & 2500 & $1000 *$ \\
\hline TSS & 45.0 & $45.0 *$ \\
\hline BERYLLIUM & & - \\
\hline ALUMINUM & & 5.0 \\
\hline BORON & & 5.0 \\
\hline COBALT & & 1.0 \\
\hline VANADIUM & & 0.1 \\
\hline
\end{tabular}

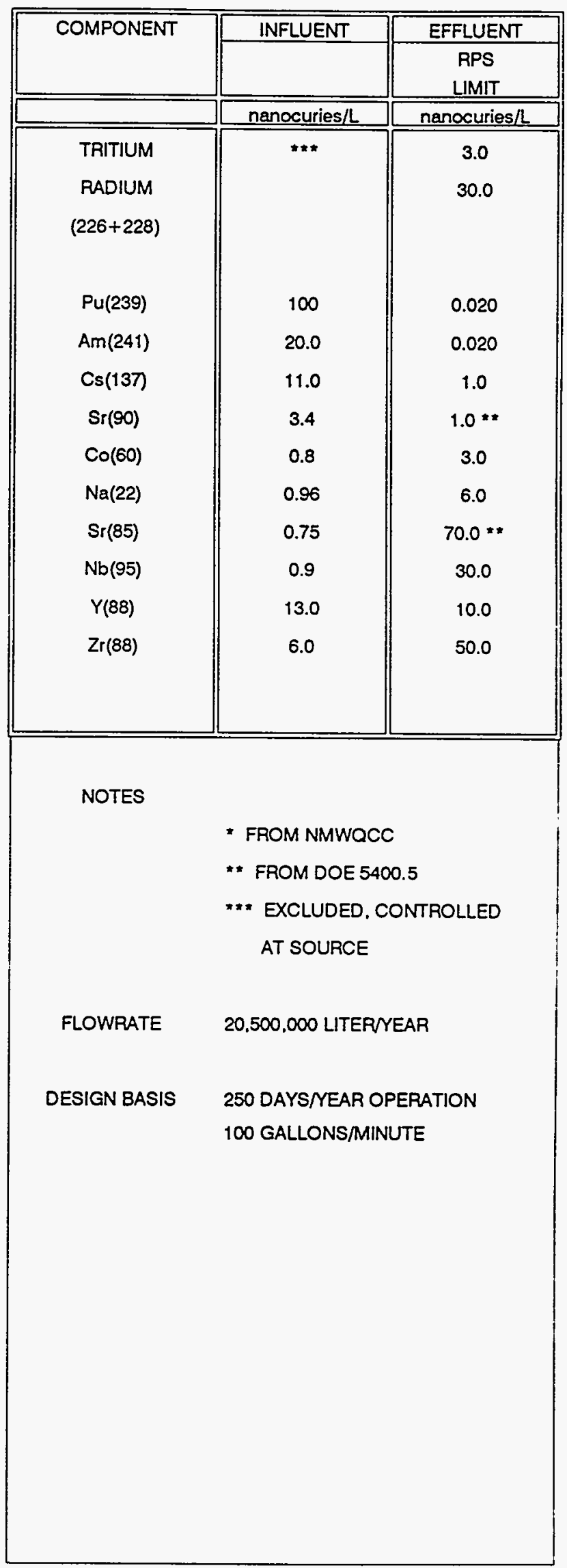


Table for Process Configuration \# 4, New Facility TA-50 Waste Only

- Wildlife and Livestock Watering Release Limits

\begin{tabular}{|c|c|c|}
\hline \multirow[t]{2}{*}{ COMPONENT } & INFLUENT & EFFLUENT \\
\hline & $T A-50-1$ & $\begin{array}{l}\text { WILDLIFE } \\
\text { LIMITS }\end{array}$ \\
\hline & $\mathrm{mg} \Lambda$ & $\mathrm{mg} / \mathrm{l}$ \\
\hline ARSENIC & 0.003 & 0.02 \\
\hline BARIUM & 0.5 & $1.0^{*}$ \\
\hline CADMIUM & 0.10 & 0.05 \\
\hline CALCIUM & 40.0 & - \\
\hline CHLORIDE & 84 & $250 *$ \\
\hline CHROMIUM & 0.25 & 1.0 \\
\hline COPPER & 0.30 & 0.5 \\
\hline CYANIDE & 0.6 & 0.2 \\
\hline FLUORIDE & 8.0 & $1.6^{*}$ \\
\hline IRON & 1.0 & $1.0^{*}$ \\
\hline LEAD & 0.2 & 0.1 \\
\hline MAGNESIUM & 4.0 & - \\
\hline MERCURY & 0.005 & 0.01 \\
\hline NICKEL & 1.0 & 0.2 * \\
\hline $\begin{array}{c}\text { AMMONIA } \\
\text { (as N) }\end{array}$ & 15.0 & - \\
\hline $\begin{array}{l}\text { NITRITE } \\
\text { (as N) }\end{array}$ & 1.0 & - \\
\hline $\begin{array}{c}\text { NITRATE } \\
\text { (as N) }\end{array}$ & 325 & 10.0 \\
\hline PHOSPHATE & 40.0 & - \\
\hline POTASSIUM & 76.0 & - \\
\hline SELENIUM & 1.0 & 0.05 \\
\hline SILICA & 87.0 & - \\
\hline SILVER & 0.03 & $0.05 *$ \\
\hline SODIUM & 512 & - \\
\hline SULFATE & 26.0 & $600 *$ \\
\hline ZINC & 0.3 & 5.0 \\
\hline $\mathrm{pH}$ & 10.0 & $6.0-9.0$ \\
\hline$C O D$ & 123 & 125.0 \\
\hline TDS & 2000 & $1000 *$ \\
\hline TSS & 45.0 & 45.0 * \\
\hline BERYLLIUM & & - \\
\hline ALUMINUM & & 5.0 \\
\hline BORON & & 5.0 \\
\hline COBALT & & 1.0 \\
\hline VANADIUM & & 0.1 \\
\hline
\end{tabular}

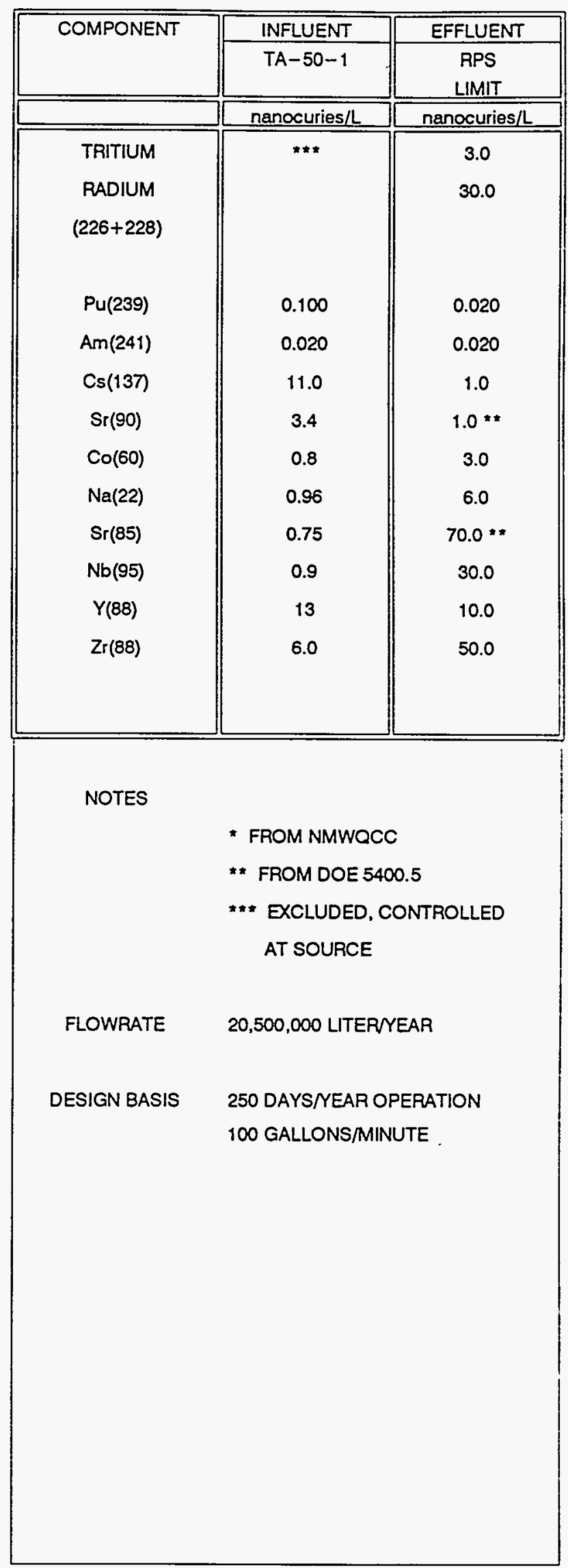


Table for Process Configuration \# 5, New Facility, TA-55 Waste Only

- Wildlife and Livestock Watering Release Limits

\begin{tabular}{|c|c|c|c|c|c|c|}
\hline \multirow[t]{2}{*}{ COMPONENT } & INFLUENT & SUPERNATE & EFFLUENT & \multirow[t]{2}{*}{ COMPONENT } & INFLUENT & \multirow{2}{*}{$\begin{array}{c}\text { EFFLUUNT } \\
\text { RPS } \\
\text { LIMIT }\end{array}$} \\
\hline & $\mathrm{RM}-60$ & $R M-60$ & $\begin{array}{c}\text { WILDLIFE } \\
\text { LIMITS } \\
\end{array}$ & & & \\
\hline & $\mathrm{mg} \Omega$ & $\mathrm{mg} \Lambda$ & $\mathrm{mg} / \mathrm{l}$ & & nanocuries $/ L$ & nanocuries $/ \mathrm{L}$ \\
\hline ARSENIC & & 0.77 & 0.02 & TRITIUM & $* * *$ & 3.0 \\
\hline BARIUM & & 0.2 & $1.0^{*}$ & RADIUM & & 30.0 \\
\hline CADMIUM & 0 & 0 & 0.05 & $(226+228)$ & & \\
\hline CALCIUM & 8.1 & 8.1 & - & & & \\
\hline CHLORIDE & 42,974 & 6,310 & 250 * & $\mathrm{Pu}(239)$ & 2.3E6 & 0.020 \\
\hline CHROMIUM & 1.350 & 45.0 & 1.0 & $\operatorname{Am}(241)$ & $1.46 E 5$ & 0.020 \\
\hline COPPER & 0.09 & $<.003$ & 0.5 & $\operatorname{Cs}(137)$ & & 1.0 \\
\hline CYANIDE & & 15.0 & 0.2 & $\operatorname{Sr}(90)$ & & $1.0^{* *}$ \\
\hline FLUORIDE & 250 & 250 & $1.6^{*}$ & $\mathrm{Co}(60)$ & & 3.0 \\
\hline IRON & & .005 & $1.0^{*}$ & $\mathrm{Na}(22)$ & & 6.0 \\
\hline LEAD & 9.9 & 0.33 & 0.1 & $\operatorname{Sr}(85)$ & & $70.0 * *$ \\
\hline MAGNESIUM & & 0.57 & - & $\mathrm{Nb}(95)$ & & 30.0 \\
\hline MERCURY & 0.11 & 0.0035 & 0.01 & $Y(88)$ & & 10.0 \\
\hline NICKEL & 930 & $<.02 / 31$ & 0.2 & $\operatorname{Zr}(88)$ & & 50.0 \\
\hline $\begin{array}{c}\text { AMMONIA } \\
\text { (as N) }\end{array}$ & 4.6 & 4.6 & - & & & \\
\hline NITRITE & & .05 & - & & & \\
\hline (as N) & & & & NOTES & & \\
\hline NITAATE & 1400 & 28,000 & 10.0 & & - FROM NMWC & \\
\hline (as N) & & & & & ** FROM DOE & 00.5 \\
\hline PHOSPHATE & & 0.11 & - & & $* * *$ EXCLUDED & CONTROLLED \\
\hline POTASSIUM & 6.500 & 6,500 & - & & AT SOURC & \\
\hline SELENIUM & 48 & 1.6 & 0.05 & & & \\
\hline SILICA & & 8 & - & FLOWRATE & RM-60 INFLUE & \\
\hline SILVER & 13.2 & 0.44 & 0.05 * & & 180,000 LTERS & EAR \\
\hline SODIUM & 24,140 & 46,280 & - & & & \\
\hline SULFATE & 0 & 0 & $600^{\circ}$ & & RM-60 SUPER & \\
\hline ZINC & & $<.001$ & 5.0 & & 250,000 LITERS & EAR \\
\hline $\mathrm{pH}$ & & 12 & $6.0-9.0$ & & & \\
\hline$C O D$ & & 303 & 125.0 & DESIGN BASIS & 20 DAYS $/$ EAAR & 'ERATION \\
\hline TOS & & 188,000 & $1000 *$ & & 10 GALLONSM & UTE \\
\hline TSS & & 226 & $45.0^{*}$ & & & \\
\hline BERYLLIUM & & & - & & & \\
\hline ALUMINUM & & & 5.0 & & & \\
\hline BORON & & & 5.0 & & & \\
\hline COBALT & & & 1.0 & & & \\
\hline VANADIUM & & & 0.1 & & & \\
\hline
\end{tabular}


Table for Process Configuration \# 6, New Facility, Blended TA-55 and TA-50 Wastes

- Wildlife and Livestock Watering Release Limits

\begin{tabular}{|c|c|c|}
\hline \multirow[t]{2}{*}{ COMPONENT } & INFLUENT & EFFLUENT \\
\hline & $\begin{array}{l}\text { TA-50-1 } \\
\text { BLENDED }\end{array}$ & $\begin{array}{l}\text { WILDLIFE } \\
\text { LIMITS }\end{array}$ \\
\hline & $\mathrm{mg} / \mathrm{I}$ & $\mathrm{mg} / \mathrm{l}$ \\
\hline ARSENIC & 0.003 & 0.02 \\
\hline BARIUM & $<0.5$ & $1.0^{*}$ \\
\hline CADMIUM & 0.10 & 0.05 \\
\hline CALCIUM & 40.0 & - \\
\hline CHLORIDE & 500 & 250 * \\
\hline CHROMIUM & 0.25 & 1.0 \\
\hline COPPER & 0.30 & 0.5 \\
\hline CYANIDE & 0.6 & $0.2^{\bullet}$ \\
\hline FLUORIDE & 8.0 & $1.6^{*}$ \\
\hline IRON & 1.0 & $1.0^{*}$ \\
\hline LEAD & 0.2 & 0.1 \\
\hline MAGNESIUM & 4.0 & - \\
\hline MERCURY & 0.005 & 0.01 \\
\hline NICKEL & 1.0 & $0.2^{*}$ \\
\hline $\begin{array}{l}\text { AMMONIA } \\
\text { (as N) }\end{array}$ & 10 & - \\
\hline $\begin{array}{c}\text { NITRITE } \\
\text { (as N) }\end{array}$ & 1.0 & - \\
\hline $\begin{array}{c}\text { NITRATE } \\
\text { (as N) }\end{array}$ & 350 & 10.0 \\
\hline PHOSPHATE & 4.0 & - \\
\hline POTASSIUM & 240 & - \\
\hline SELENIUM & 1.0 & 0.05 \\
\hline SILICA & 80 & - \\
\hline SILVER & $<.006$ & $0.05^{*}$ \\
\hline SODIUM & 1508 & - \\
\hline SULFATE & 26.0 & 600. \\
\hline ZINC & $<.002$ & 5.0 \\
\hline $\mathrm{pH}$ & 7.3 & $6.0-9.0$ \\
\hline$C O D$ & 81 & 125.0 \\
\hline TDS & 2500 & $1000 *$ \\
\hline TSS & 45.0 & 45.0 * \\
\hline BERYLLIUM & & - \\
\hline ALUMINUM & & 5.0 \\
\hline BORON & & 5.0 \\
\hline COBALT & & 1.0 \\
\hline VANADIUM & & 0.1 \\
\hline
\end{tabular}

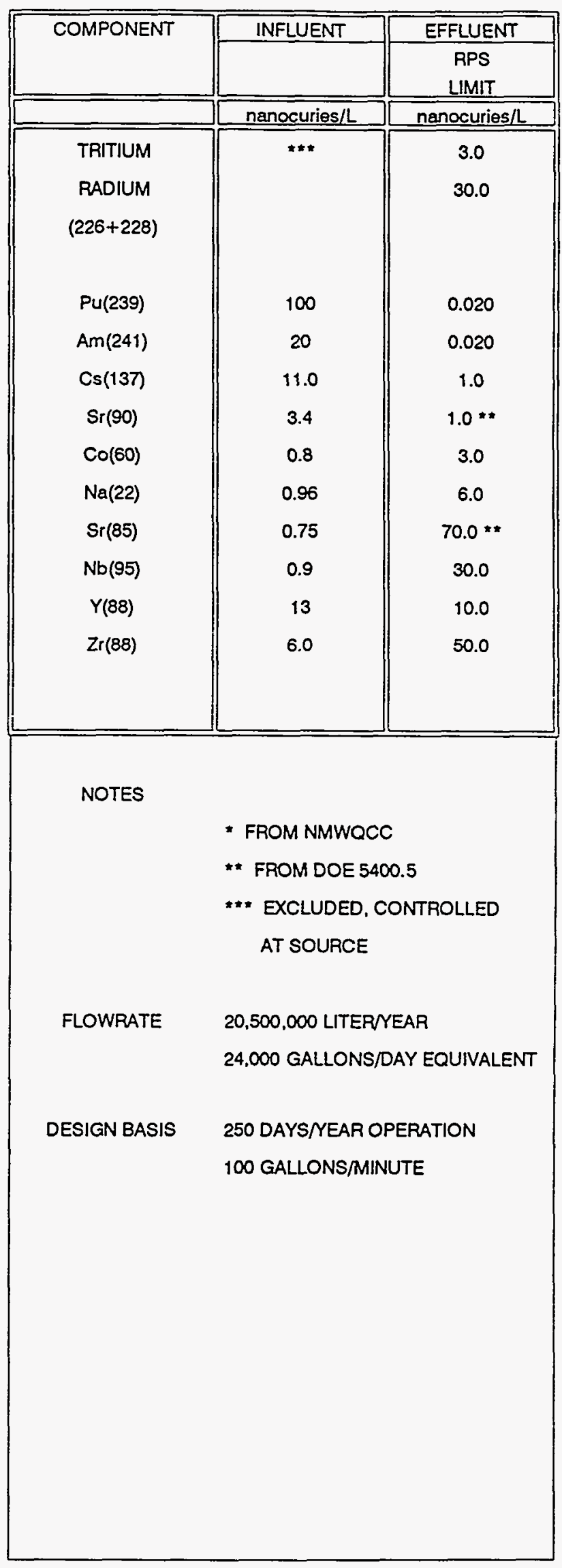


Table for Process Configuration \# 7, New Facility, TA-50 Waste Only

- September 11, 1992 Proposed NPDES Limits

\begin{tabular}{|c|c|c|}
\hline \multirow[t]{3}{*}{ COMPONENT } & INFLUENT & EFFLUENT \\
\hline & $T A-50-1$ & NMED-051 \\
\hline & $\mathrm{mg} / \mathrm{l}$ & $\mathrm{mg} / \mathrm{l}$ \\
\hline ARSENIC & 0.003 & 0.04 \\
\hline BARIUM & 0.5 & $1.0^{*}$ \\
\hline CADMIUM & 0.10 & 0.0022 \\
\hline CALCIUM & 40.0 & - \\
\hline CHLORIDE & 84 & $250 *$ \\
\hline CHROMIUM & 0.25 & 0.51 \\
\hline COPPER & 0.30 & 0.0178 \\
\hline CYANIDE & 0.6 & 0.0052 \\
\hline FLUORIDE & 8.0 & $1.6 *$ \\
\hline IRON & 1.0 & $1.0 *$ \\
\hline LEAD & 0.2 & 0.0041 \\
\hline MAGNESIUM & 4.0 & - \\
\hline MERCUAY & 0.005 & 0.000012 \\
\hline NICKEL & 1.0 & 0.198 \\
\hline AMMONIA & 15.0 & 0.003 \\
\hline$(\operatorname{as~N})$ & & \\
\hline NITRITE & 1.0 & - \\
\hline (as N) & & \\
\hline NITRATE & 325 & 10.0 \\
\hline (as N) & & \\
\hline PHOSPHATE & 40.0 & - \\
\hline POTASSIUM & 76.0 & - \\
\hline SELENIUM & 1.0 & 0.005 \\
\hline SILICA & 87.0 & - \\
\hline SILVER & 0.03 & 0.00012 \\
\hline SODIUM & 512 & - \\
\hline SULFATE & 26.0 & $600 *$ \\
\hline ZINC & 0.3 & 0.197 \\
\hline $\mathrm{pH}$ & 10.0 & $6.0-9.0$ \\
\hline COD & 123 & 125.0 \\
\hline TDS & 2000 & 1000 * \\
\hline TSS & 45.0 & $45.0^{*}$ \\
\hline BERYLLIUM & & 0.00523 \\
\hline ALUMINUM & & 0.087 \\
\hline BORON & & 0.75 \\
\hline COBALT & & 0.05 \\
\hline VANADIUM & & 0.10 \\
\hline CHLORINE & & 400 \\
\hline
\end{tabular}

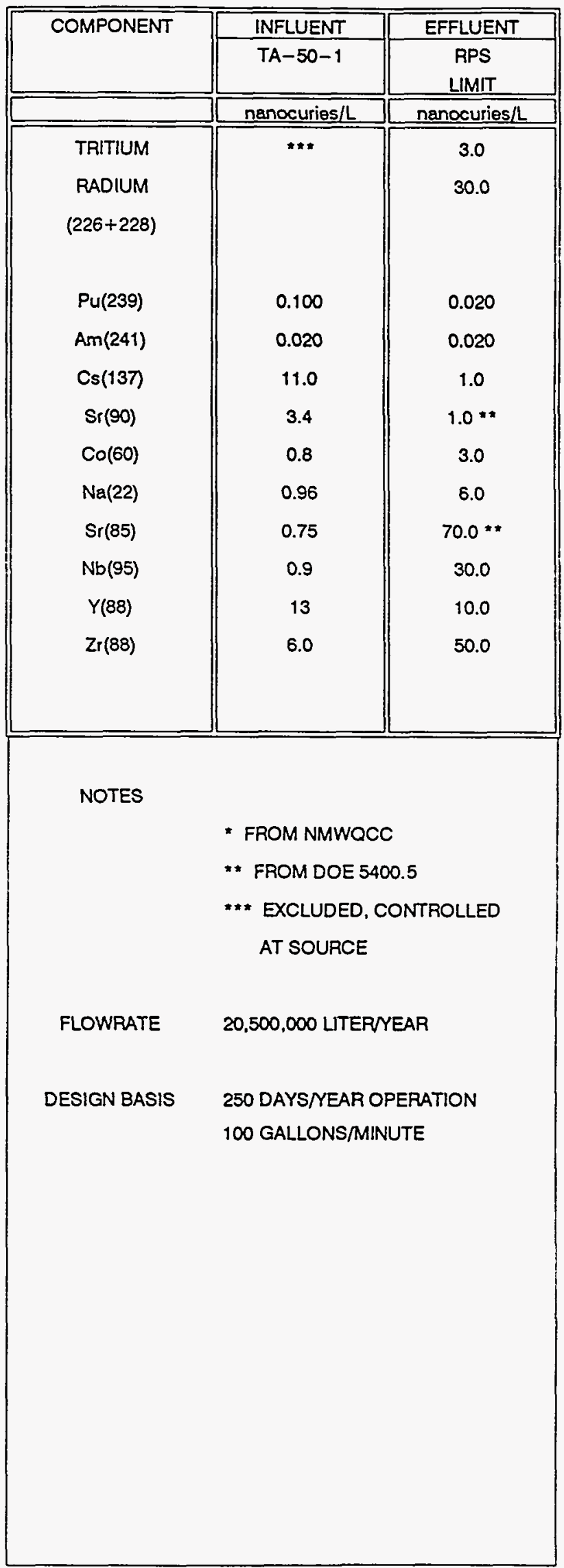


Table for Process Configuration \# 8, New Facility, TA-55 Waste Only

- September 11, 1992 Proposed NPDES Limits

\begin{tabular}{|c|c|c|c|}
\hline \multirow[t]{3}{*}{ COMPONENT } & INFLUENT & SUPERNATE & EFFLUENT \\
\hline & $R M-60$ & $R M-60$ & NMED - 051 \\
\hline & $\mathrm{mg} /$ & $\mathrm{mg} /$ & $\mathrm{mg} \Omega$ \\
\hline ARSENIC & & 0.77 & 0.04 \\
\hline BARIUM & & 0.2 & $1.0 *$ \\
\hline CADMIUM & 0 & 0 & 0.0022 \\
\hline CALCIUM & 8.1 & 8.1 & - \\
\hline CHLORIDE & 42,974 & 6,310 & $250^{*}$ \\
\hline CHROMIUM & 1.350 & $.44 / 45$ & 0.51 \\
\hline COPPER & 0.09 & $<.003$ & 0.0178 \\
\hline CYANIDE & & 15.0 & 0.0052 \\
\hline FLUORIDE & 250 & 250 & $1.6^{\bullet}$ \\
\hline IRON & & .005 & $1.0^{*}$ \\
\hline LEAD & 9.9 & 0.33 & 0.0041 \\
\hline MAGNESIUM & & 0.57 & - \\
\hline MERCURY & 0.11 & 0.0035 & 0.000012 \\
\hline NICKEL & 930 & $<.02 / 31$ & 0.198 \\
\hline $\begin{array}{c}\text { AMMONIA } \\
\text { (as N) }\end{array}$ & 4.6 & 4.6 & 0.003 \\
\hline $\begin{array}{c}\text { NITRITE } \\
\text { (as N) }\end{array}$ & & .05 & - \\
\hline $\begin{array}{c}\text { NITRATE } \\
\text { (as N) }\end{array}$ & 1400 & 28,000 & 10.0 \\
\hline PHOSPHATE & & 0.11 & - \\
\hline POTASSIUM & 6.500 & 6.500 & - \\
\hline SELENIUM & 48 & 1.6 & 0.005 \\
\hline SILICA & & 8 & - \\
\hline SILVER & 13.2 & 0.44 & 0.00012 \\
\hline SODIUM & 24,140 & 46.280 & - \\
\hline SULFATE & 0 & 0 & $600^{\circ}$ \\
\hline ZINC & & $<.001$ & 0.197 \\
\hline $\mathrm{pH}$ & & 12 & $6.0-9.0$ \\
\hline$C O D$ & & 303 & 125.0 \\
\hline TOS & & 188,000 & $1000 *$ \\
\hline TSS & & 226 & $45.0^{*}$ \\
\hline BERYLLIUM & & & 0.00523 \\
\hline ALUMINUM & & & 0.087 \\
\hline BORON & & & 0.75 \\
\hline COBALT & & & 0.05 \\
\hline VANADIUM & & & 0.10 \\
\hline CHLORINE & & & 400 \\
\hline
\end{tabular}

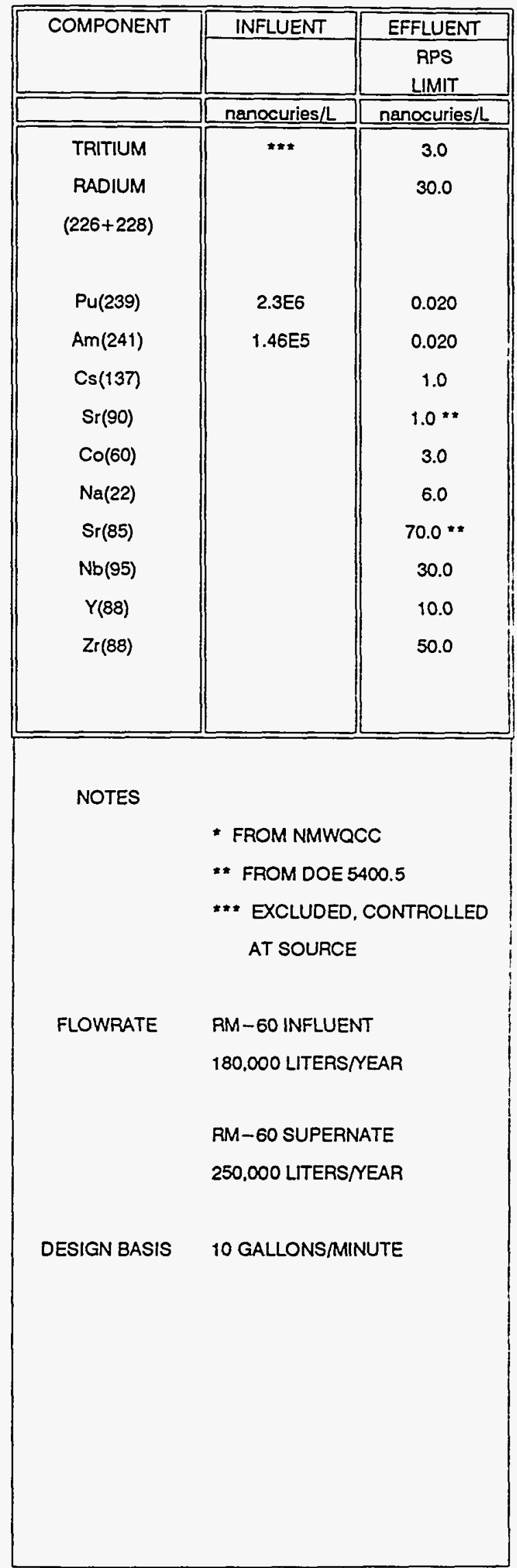


Table for Process Configuration \# 9, New Facility, Blended TA-55 and TA-50 Wastes - September 11, 1992 Proposed NPDES Limits

\begin{tabular}{|c|c|c|}
\hline \multirow[t]{2}{*}{ COMPONENT } & INFLUENT & EFFLUENT \\
\hline & $\begin{array}{l}\text { TA-50-1 } \\
\text { BLENDED }\end{array}$ & NMED - 051 \\
\hline & $\mathrm{mg} / \mathrm{l}$ & $\mathrm{mg} / \mathrm{l}$ \\
\hline ARSENIC & 0.003 & 0.04 \\
\hline BARIUM & $<0.5$ & $1.0^{*}$ \\
\hline CADMIUM & 0.10 & 0.0022 \\
\hline CALCIUM & 40.0 & - \\
\hline CHLORIDE & 500 & $250^{\bullet}$ \\
\hline CHROMIUM & 0.25 & 0.51 \\
\hline COPPER & 0.30 & 0.0178 \\
\hline CYANIDE & 0.6 & 0.0052 \\
\hline FLUORIDE & 8.0 & $1.6 *$ \\
\hline IRON & 1.0 & $1.0^{*}$ \\
\hline LEAD & 0.2 & 0.0041 \\
\hline MAGNESIUM & 4.0 & - \\
\hline MERCURY & 0.005 & 0.000012 \\
\hline NICKEL & 1.0 & 0.198 \\
\hline $\begin{array}{l}\text { AMMONIA } \\
\text { (as N) }\end{array}$ & 10 & 0.003 \\
\hline $\begin{array}{l}\text { NITRITE } \\
\text { (as N) }\end{array}$ & 1.0 & - \\
\hline $\begin{array}{c}\text { NITRATE } \\
\text { (as N) }\end{array}$ & 350 & 10.0 \\
\hline PHOSPHATE & 4.0 & - \\
\hline POTASSIUM & 240 & - \\
\hline SELENIUM & 1.0 & 0.005 \\
\hline SILKA & 80 & - \\
\hline SILVER & $<.006$ & 0.00012 \\
\hline SODIUM & 1508 & - \\
\hline SULFATE & 26.0 & $600^{*}$ \\
\hline ZINC & $<.002$ & 0.197 \\
\hline $\mathrm{pH}$ & 7.3 & $6.0-9.0$ \\
\hline$C O D$ & 81 & 125.0 \\
\hline TDS & 2500 & 1000 * \\
\hline TSS & 45.0 & $45.0^{\circ}$ \\
\hline BERYLLIUM & & 0.00523 \\
\hline ALUMINUM & & 0.087 \\
\hline BORON & & 0.75 \\
\hline COBALT & & 0.05 \\
\hline VANADIUM & & 0.10 \\
\hline
\end{tabular}

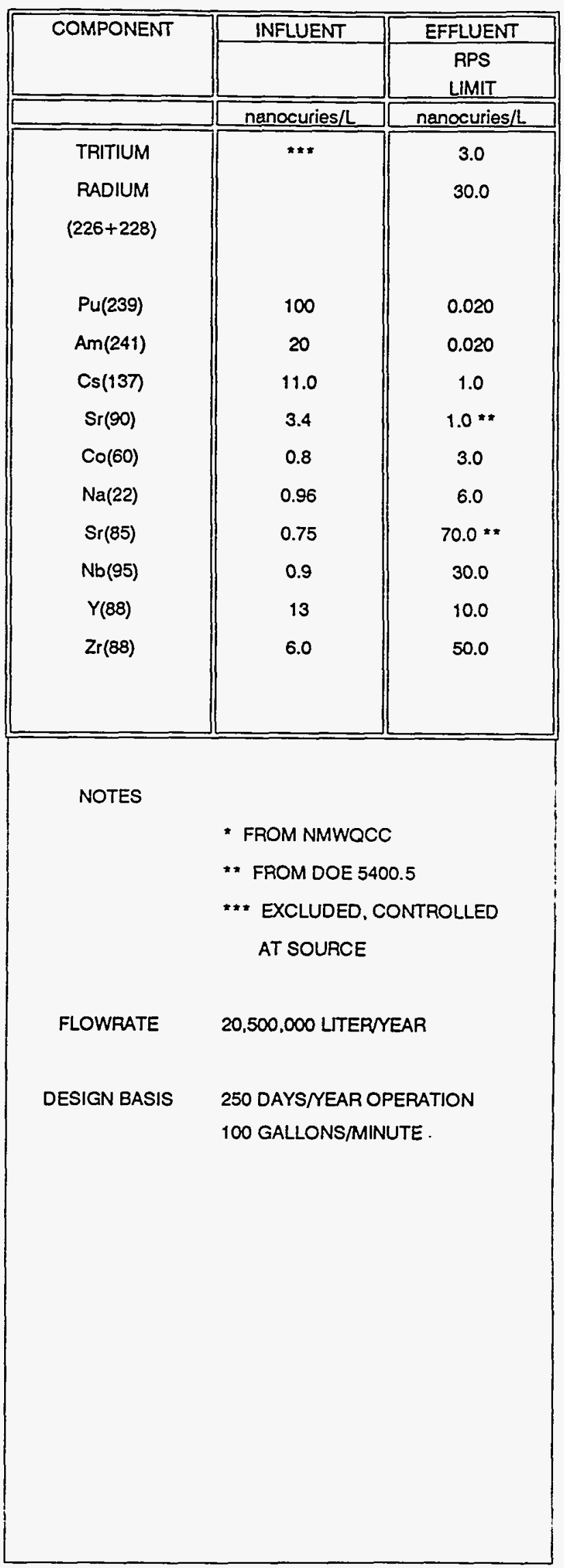


Table for Process Configuration \# 10, Upgrade Existing TA-50 Operations, TA-50 Wastes Only - September 11, 1992 Proposed NPDES Limits

\begin{tabular}{|c|c|c|c|c|c|}
\hline \multirow[t]{2}{*}{ COMPONENT } & INFLUENT & EFFLUENT & \multirow[t]{2}{*}{ COMPONENT } & \multirow{2}{*}{$\begin{array}{l}\text { INFLUENT } \\
\text { TA-50-1 }\end{array}$} & \multirow{2}{*}{$\begin{array}{c}\text { EFFLUENT } \\
\text { RPS } \\
\text { LIMIT }\end{array}$} \\
\hline & $T A-50-1$ & NMED - 051 & & & \\
\hline & $\mathrm{mg} \Omega$ & $\mathrm{mg} / \mathrm{l}$ & & nanocuries $/ L$ & nanocuries $/ \mathrm{L}$ \\
\hline ARSENIC & 0.003 & 0.04 & TRITIUM & $m *$ & 3.0 \\
\hline BARIUM & 0.5 & $1.0^{\circ}$ & RADIUM & & 30.0 \\
\hline CADMIUM & 0.10 & 0.0022 & $(226+228)$ & & \\
\hline CALCIUM & 40.0 & - & & & \\
\hline CHLORIDE & 84 & $250^{*}$ & $\mathrm{Pu}(239)$ & 0.100 & 0.020 \\
\hline CHROMIUM & 0.25 & 0.51 & $\operatorname{Am}(241)$ & 0.020 & 0.020 \\
\hline COPPER & 0.30 & 0.0178 & $\operatorname{Cs}(137)$ & 11.0 & 1.0 \\
\hline CYANIDE & 0.6 & 0.0052 & $\operatorname{sr}(90)$ & 3.4 & $1.0 * *$ \\
\hline FLUORIDE & 8.0 & $1.6^{\bullet}$ & $\mathrm{Co}(60)$ & 0.8 & 3.0 \\
\hline IRON & 1.0 & $1.0 *$ & $\mathrm{Ne}(22)$ & 0.96 & 6.0 \\
\hline LEAD & 0.2 & 0.0041 & $\operatorname{Sr}(85)$ & 0.75 & $70.0 * *$ \\
\hline MAGNESIUM & 4.0 & - & $\mathrm{Nb}(95)$ & 0.9 & 30.0 \\
\hline MERCURY & 0.005 & 0.000012 & $Y(88)$ & 13 & 10.0 \\
\hline NICKEL & 1.0 & 0.198 & $\operatorname{Zr}(88)$ & 6.0 & 50.0 \\
\hline $\begin{array}{c}\text { AMMONIA } \\
\text { (as N) }\end{array}$ & 15.0 & 0.003 & & & \\
\hline NITRITE & 1.0 & - & & & \\
\hline (as N) & & & NOTES & & \\
\hline NITRATE & 325 & 10.0 & & * FROM NMWC & \\
\hline$(\operatorname{as~N})$ & & & & ** FROM DOE & \\
\hline PHOSPHATE & 40.0 & - & & *** EXCLUDED & NTROLLED \\
\hline POTASSIUM & 76.0 & - & & AT SOURCE & \\
\hline SELENIUM & 1.0 & 0.005 & & & \\
\hline SILKA & 87.0 & - & FLOWRATE & 20.500 .000 LITE & \\
\hline SILVER & 0.03 & 0.00012 & & & \\
\hline SODIUM & 512 & - & DESIGN BASIS & 250 DAYSNEAR & PATION \\
\hline SULFATE & 26.0 & $600^{*}$ & & 100 GALLONS $/$ & \\
\hline ZINC & 0.3 & 0.197 & & & \\
\hline pH & 10.0 & $6.0-9.0$ & & & \\
\hline$C O D$ & 123 & 125.0 & & & \\
\hline TDS & 2000 & $1000 *$ & & & \\
\hline TSS & 45.0 & $45.0^{\circ}$ & & & \\
\hline BERYLLIUM & & 0.00523 & & & \\
\hline ALUMINUM & & 0.087 & & & \\
\hline BORON & & 0.75 & & & \\
\hline COBALT & & 0.05 & & & \\
\hline VANADIUM & & 0.10 & & & \\
\hline CHLORINE & & 400 & & & \\
\hline
\end{tabular}


Table for Process Configuration \# 11, Upgrade Existing Room 60 Operations, TA-55 Waste Only - September 11, 1992 Proposed NPDES Limits

\begin{tabular}{|c|c|c|c|}
\hline \multirow[t]{3}{*}{ COMPONENT } & INFLUENT & SUPERNATE & EFFLUENT \\
\hline & $\mathrm{RM}-60$ & $\mathrm{RM}-60$ & NMED-051 \\
\hline & $\mathrm{mg} / \mathrm{l}$ & $m g / 1$ & $\mathrm{mg} / \mathrm{l}$ \\
\hline ARSENIC & & 0.77 & 0.04 \\
\hline BARIUM & & 0.2 & $1.0 *$ \\
\hline CADMIUM & 0 & 0 & 0.0022 \\
\hline CALCIUM & 8.1 & 8.1 & - \\
\hline CHLORIDE & 42,974 & 6,310 & $250 *$ \\
\hline CHROMIUM & 1,350 & 45.0 & 0.51 \\
\hline COPPER & 0.09 & $<.003$ & 0.0178 \\
\hline CYANIDE & & 15.0 & 0.0052 \\
\hline FLUORIDE & 250 & 250 & $1.6 *$ \\
\hline IRON & & .005 & $1.0 *$ \\
\hline LEAD & 9.9 & 0.33 & 0.0041 \\
\hline MAGNESIUM & & 0.57 & - \\
\hline MERCURY & 0.11 & 0.0035 & 0.000012 \\
\hline NICKEL & 930 & $<.02 / 31$ & 0.198 \\
\hline $\begin{array}{l}\text { AMMONIA } \\
\text { (as N) }\end{array}$ & 4.6 & 4.6 & 0.003 \\
\hline $\begin{array}{c}\text { NITRITE } \\
\text { (as N) }\end{array}$ & & .05 & - \\
\hline $\begin{array}{c}\text { NITRATE } \\
\text { (as N) }\end{array}$ & 1400 & 28.000 & 10.0 \\
\hline PHOSPHATE & & 0.11 & - \\
\hline POTASSIUM & 6.500 & 6,500 & - \\
\hline SELENIUM & 48 & 1.6 & 0.005 \\
\hline SILICA & & 8 & - \\
\hline SILVER & 13.2 & 0.44 & 0.00012 \\
\hline SODIUM & 24,140 & 46,280 & - \\
\hline SULFATE & 0 & 0 & $600^{*}$ \\
\hline ZINC & & $<.001$ & 0.197 \\
\hline $\mathrm{pH}$ & & 12 & $6.0-9.0$ \\
\hline COD & & 303 & 125.0 \\
\hline TDS & & 188,000 & $1000^{\circ}$ \\
\hline TSS & & 226 & $45.0 *$ \\
\hline BERYLLIUM & & & 0.00523 \\
\hline ALUMINUM & & & 0.087 \\
\hline BORON & & & 0.75 \\
\hline COBALT & & & 0.05 \\
\hline VANADIUM & & & 0.10 \\
\hline CHLORINE & & & 400 \\
\hline
\end{tabular}

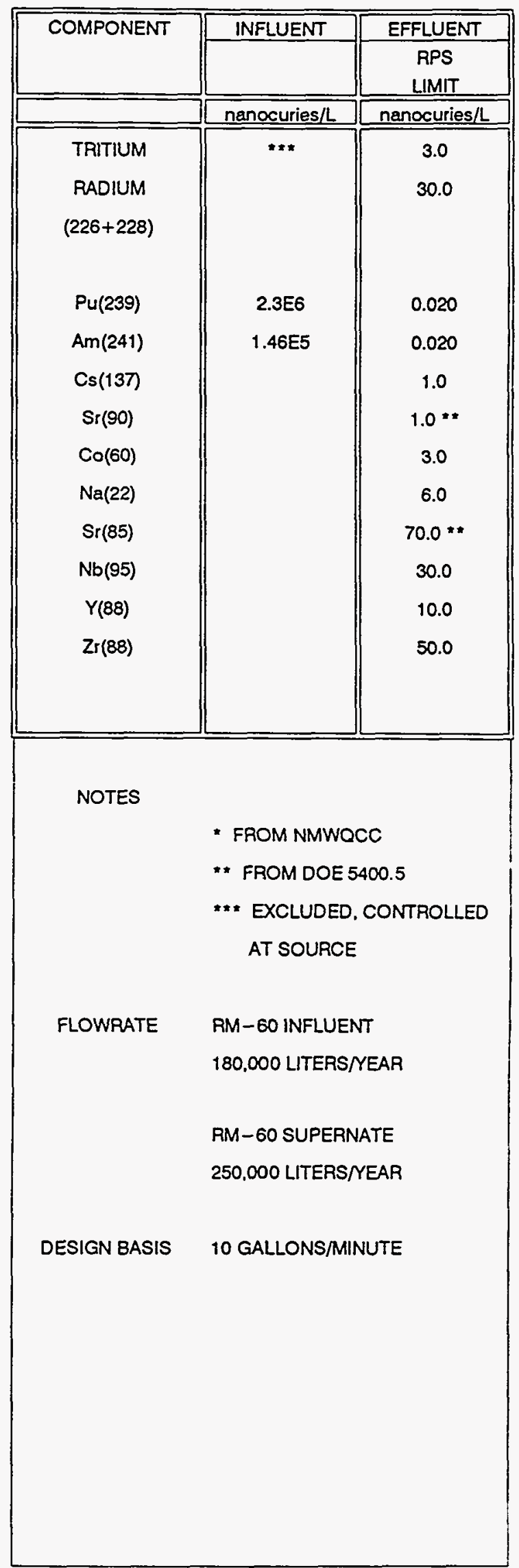


Table for Process Configuration \# 12, Upgrade Existing TA-50 Operations, Blended TA-55 and TA-50 Wastes - September 11, 1992 Proposed NPDES Limits

\begin{tabular}{|c|c|c|}
\hline \multirow[t]{2}{*}{ COMPONENT } & INFLUENT & EFFLUENT \\
\hline & $\begin{array}{l}\text { TA-50-1 } \\
\text { BLENDED }\end{array}$ & NMED -051 \\
\hline & $\mathrm{mg} / \mathrm{l}$ & $\mathrm{mg} / \mathrm{l}$ \\
\hline ARSENIC & 0.003 & 0.04 \\
\hline BARIUM & $<0.5$ & $1.0^{*}$ \\
\hline CADMIUM & 0.10 & 0.0022 \\
\hline CALCIUM & 40.0 & - \\
\hline CHLORIDE & 500 & $250 *$ \\
\hline CHROMIUM & 0.25 & 0.51 \\
\hline COPPER & 0.30 & 0.0178 \\
\hline CYANIDE & 0.6 & 0.0052 \\
\hline FLUORIDE & 8.0 & $1.6^{\bullet}$ \\
\hline IRON & 1.0 & $1.0^{\circ}$ \\
\hline LEAD & 0.2 & 0.0041 \\
\hline MAGNESIUM & 4.0 & - \\
\hline MERCURY & 0.005 & 0.000012 \\
\hline NICKEL & 1.0 & 0.198 \\
\hline $\begin{array}{l}\text { AMMONIA } \\
\text { (as N) }\end{array}$ & 10 & 0.003 \\
\hline $\begin{array}{c}\text { NITRITE } \\
\text { (as N) }\end{array}$ & 1.0 & - \\
\hline $\begin{array}{l}\text { NITRATE } \\
\text { (as N) }\end{array}$ & 350 & 10.0 \\
\hline PHOSPHATE & 4.0 & - \\
\hline POTASSIUM & 240 & - \\
\hline SELENIUM & 1.0 & 0.005 \\
\hline SILICA & 80 & - \\
\hline SILVER & $<.006$ & 0.00012 \\
\hline SODIUM & 1508 & - \\
\hline SULFATE & 26.0 & $600 *$ \\
\hline ZINC & $<.002$ & 0.197 \\
\hline pH & 7.3 & $6.0-9.0$ \\
\hline$C O D$ & 81 & 125.0 \\
\hline TDS & 2500 & $1000^{*}$ \\
\hline TSS & 45.0 & $45.0^{*}$ \\
\hline BERYLLIUM & & 0.00523 \\
\hline ALUMINUM & & 0.087 \\
\hline BORON & & 0.75 \\
\hline COBALT & & 0.05 \\
\hline VANADIUM & & 0.10 \\
\hline
\end{tabular}

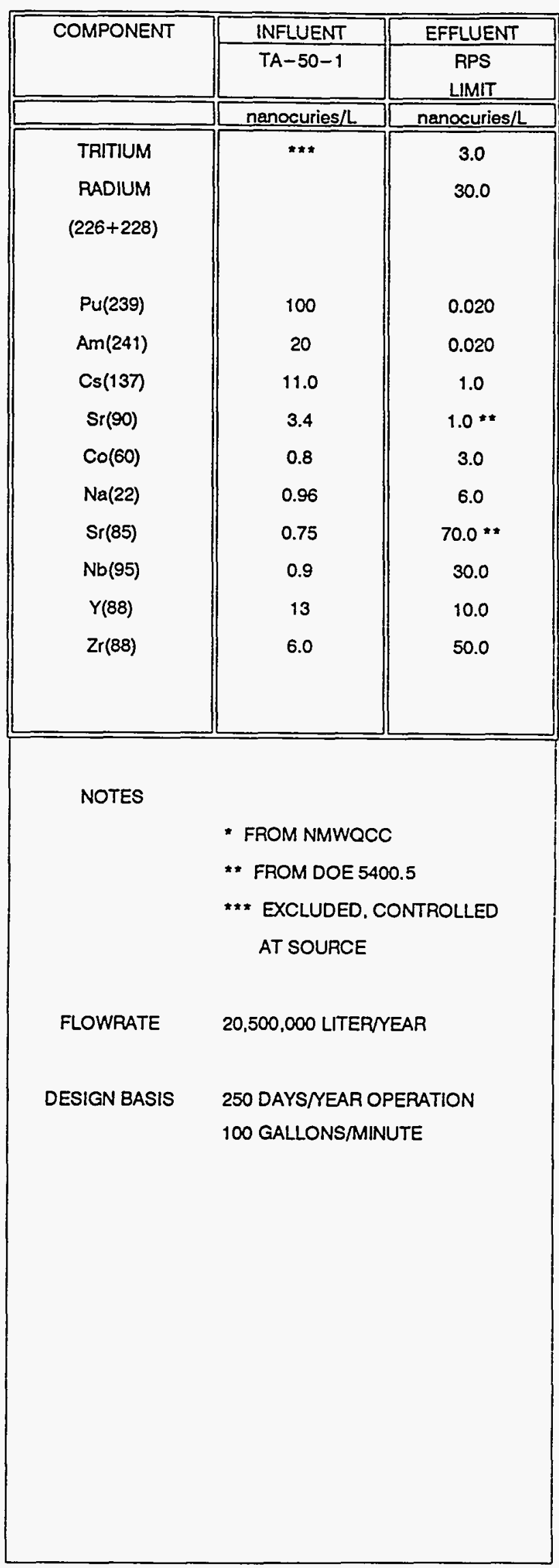




\section{APPENDIX E}

\section{BLOCK FLOW DIAGRAMS FOR CANDIDATE TECHNOLOGIES}




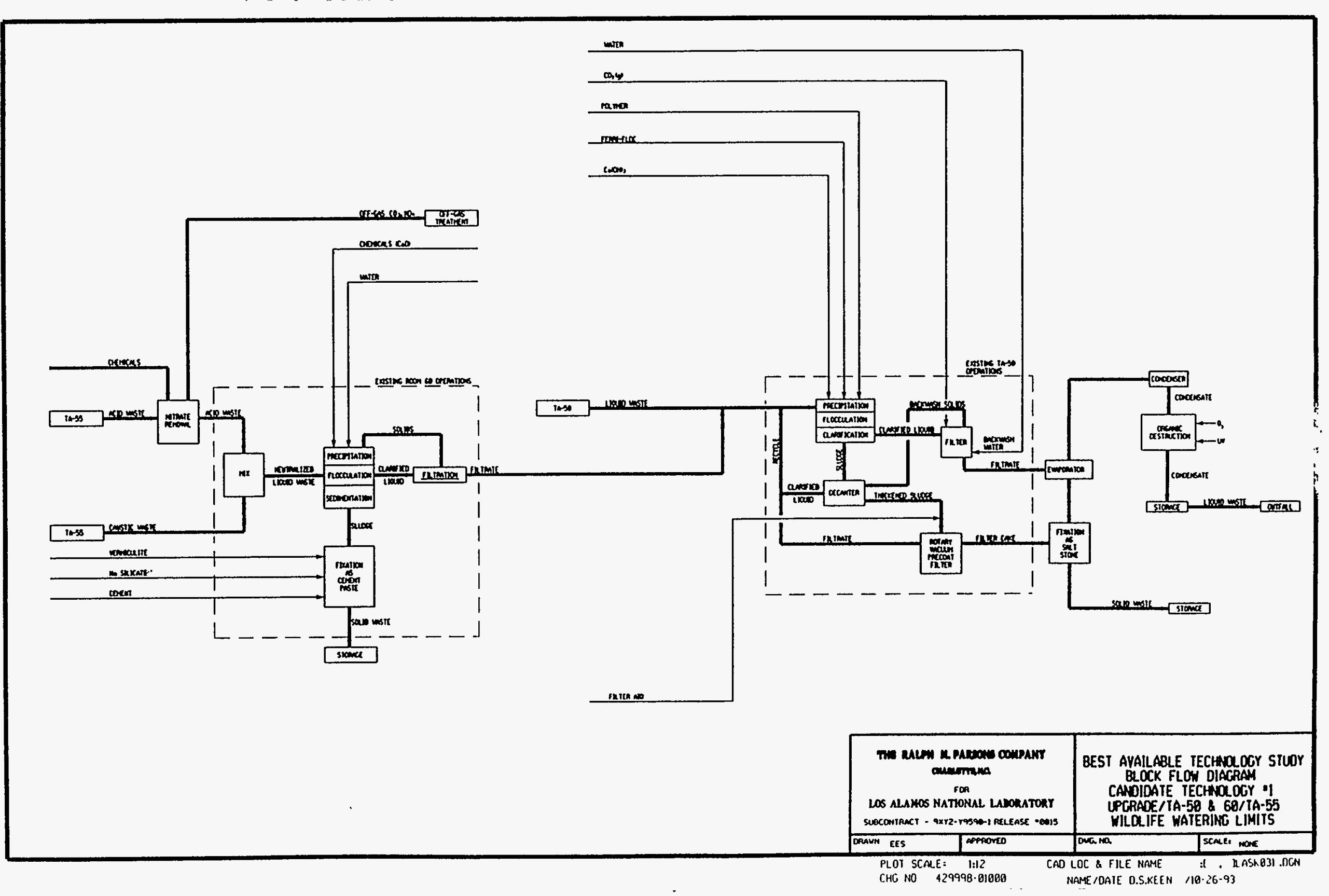




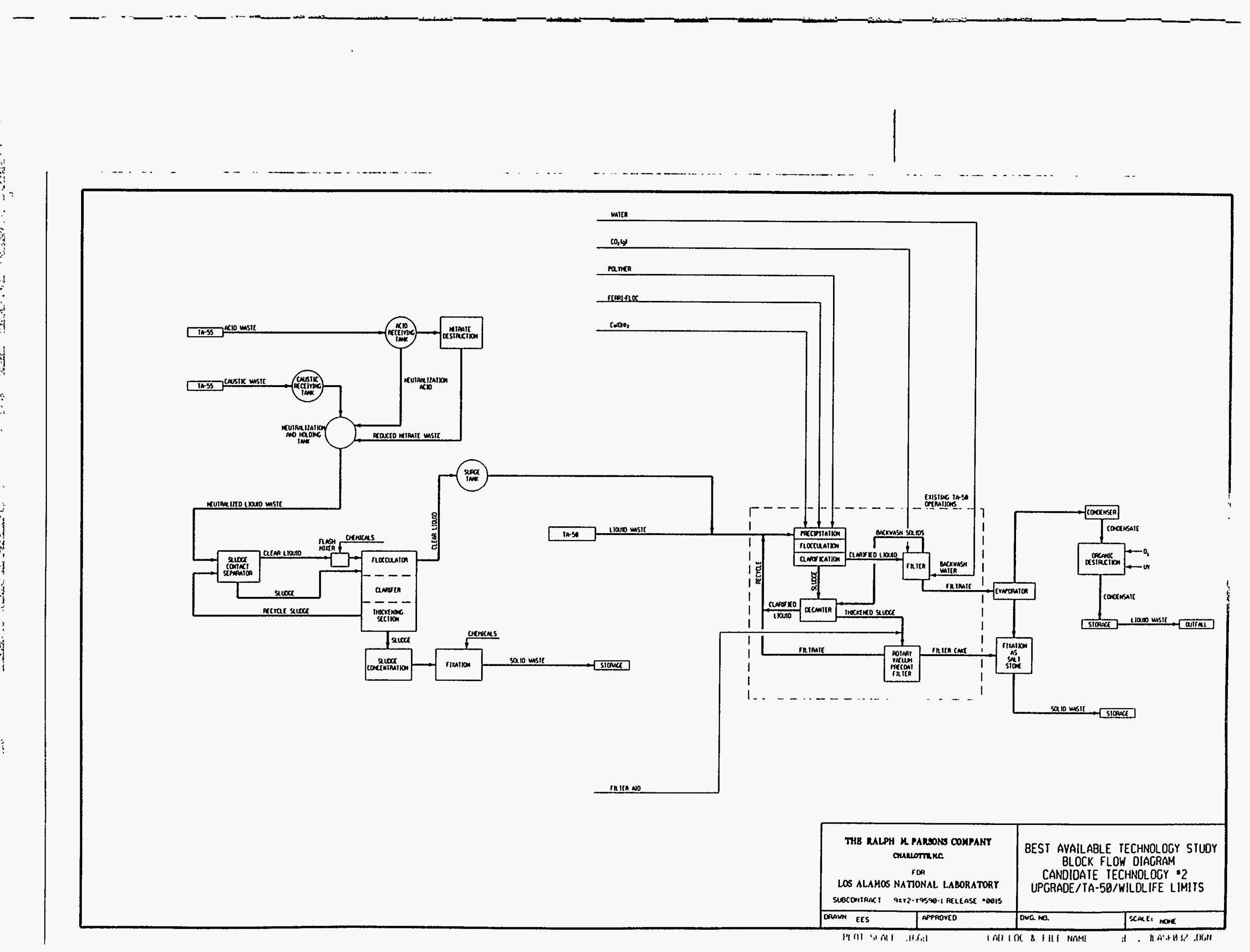




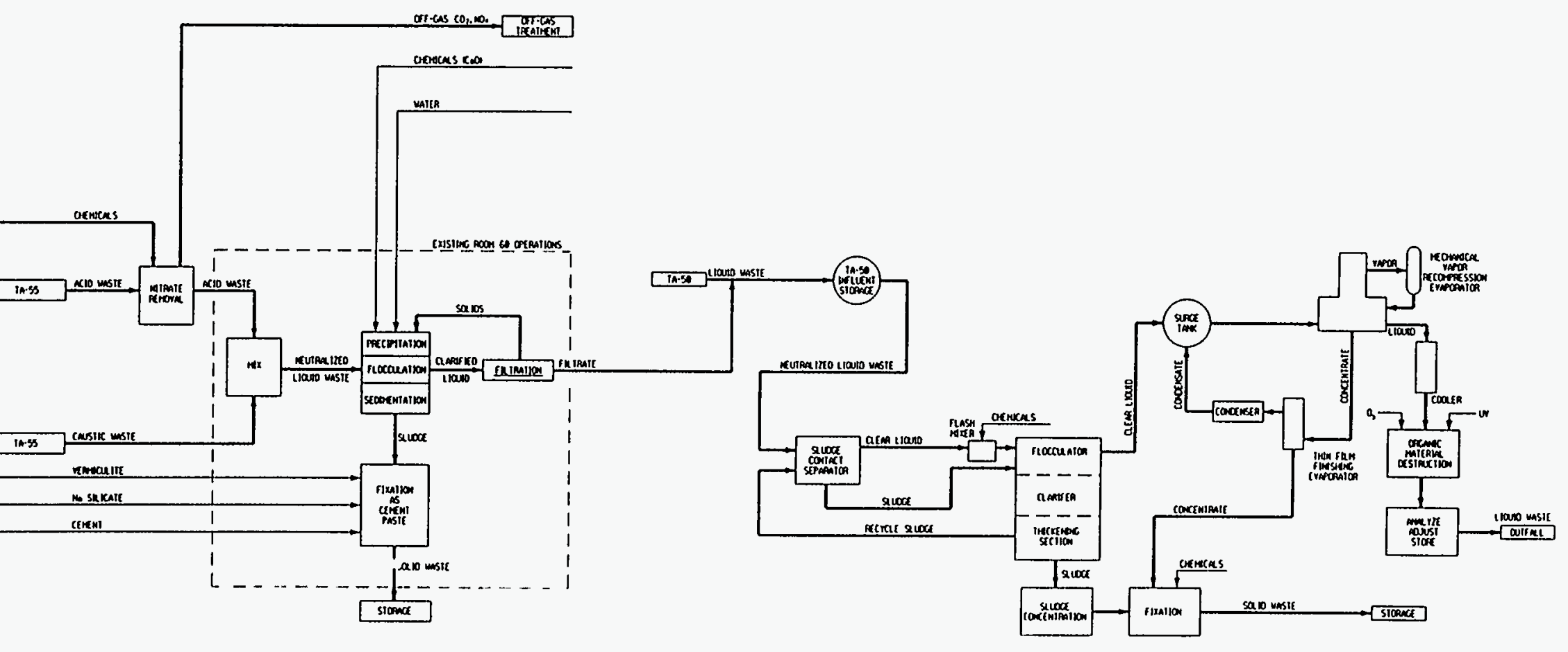

\begin{tabular}{|c|c|c|c|}
\hline \multicolumn{2}{|c|}{ 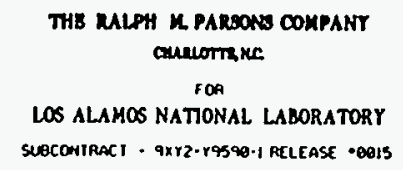 } & \multicolumn{2}{|c|}{$\begin{array}{l}\text { BEST AYAILABLE TECHNOLOGY STUDY } \\
\text { BLOCK FLOW DIACRAM } \\
\text { CANDIDATE IECHNOLOCY }-3 \\
\text { UPCRADE/TA-50 \& 60/IA-55 } \\
\text { WILDLIFE WATERING LIMITS }\end{array}$} \\
\hline DaAW EES & APPROVEO & OWGano. & SCACE, nore \\
\hline
\end{tabular}




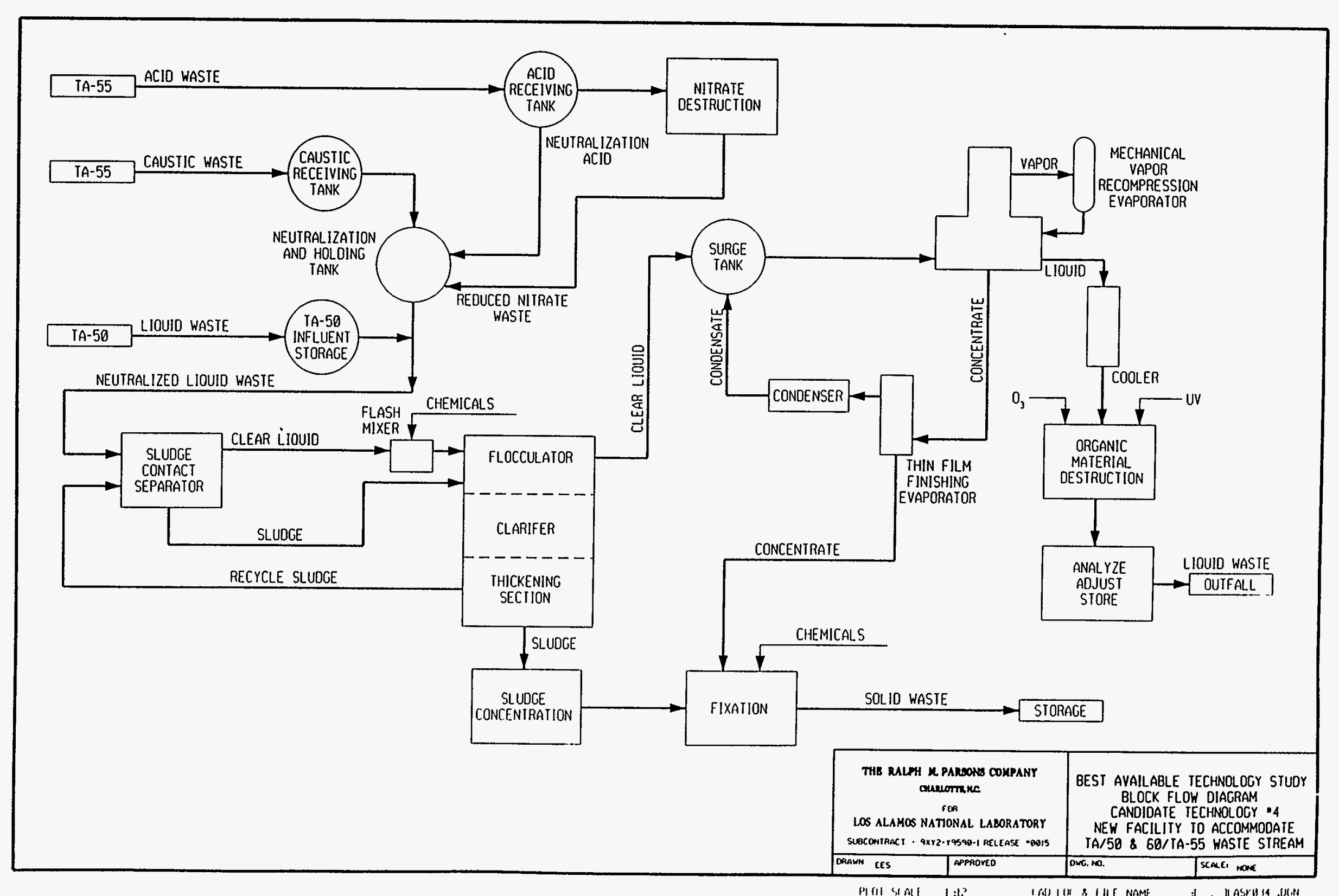


APPENDIX F

\section{PROCESS LAYOUTS}

\section{FOR CANDIDATE TECHNOLOGIES}






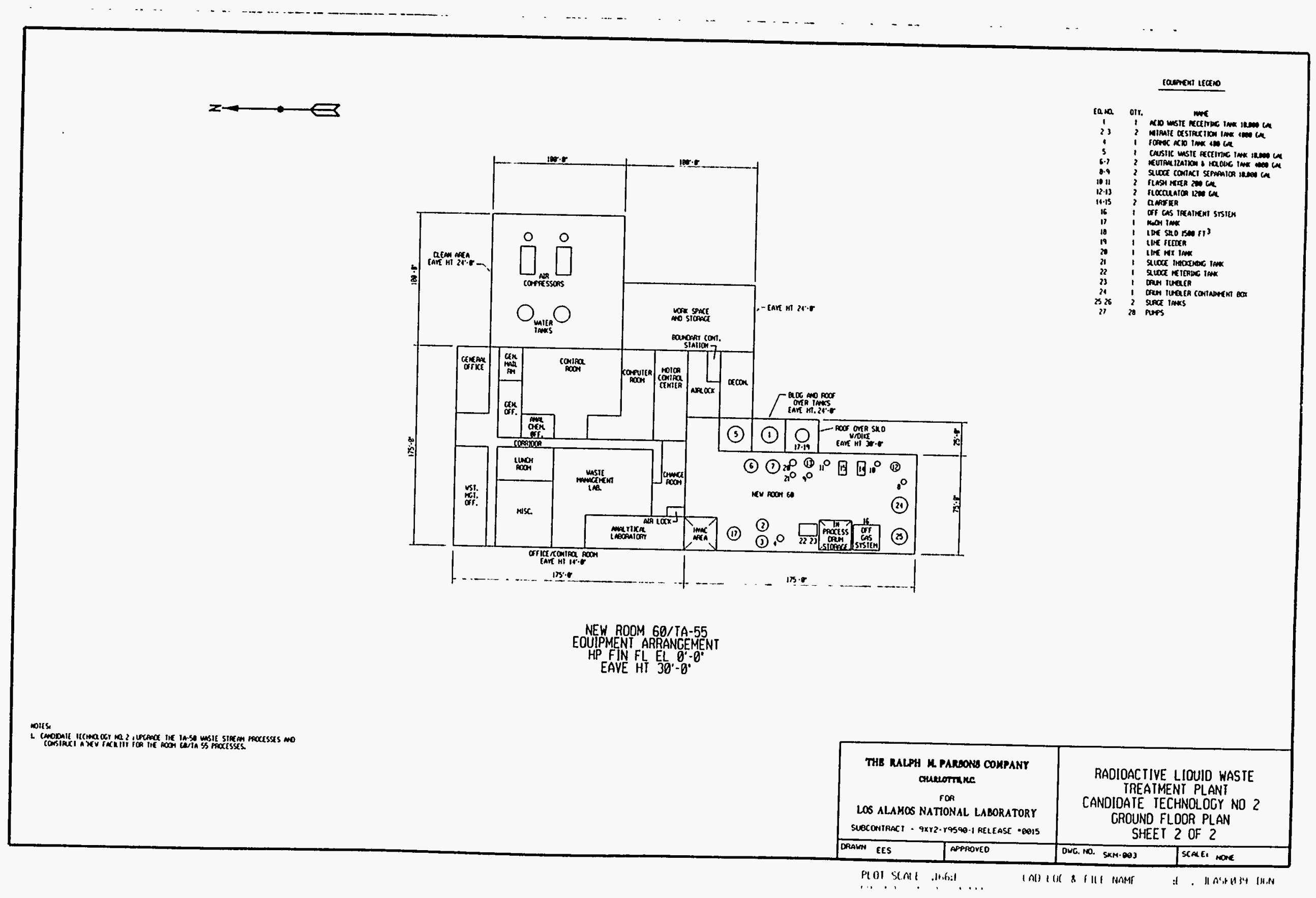


NभI zดQYSE II - ]:

JWUN 37158301007

"

\begin{tabular}{|c|c|c|c|}
\hline Dow & seo-HXs on Jno & onoussos & 533 menter \\
\hline
\end{tabular}

2 $501133 \mathrm{HS}$

$\varepsilon$ ON $23070 \mathrm{NH}$ J3 J1 JOIONG

IN甘ר INJWI

31S甘M 0INOI 7 3Al13 $3010 \forall 4$

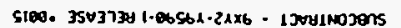

dHOLVนO9V7 7VNOLLV SOHV7V SOI

M⿻上丨𣥂

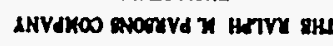

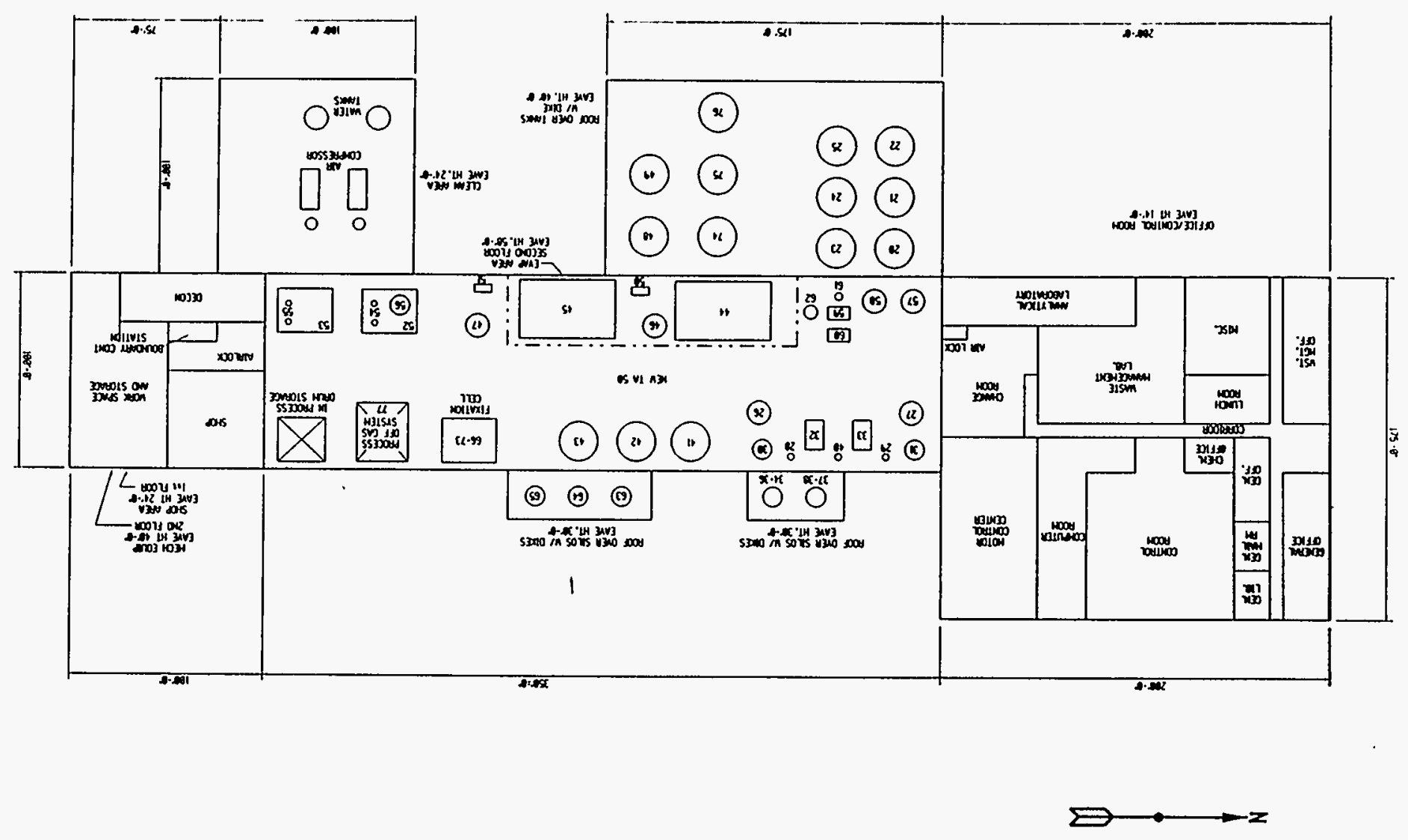

axn torums
$00-.0611370 \forall 3$

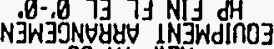

QG $\forall 1$ M

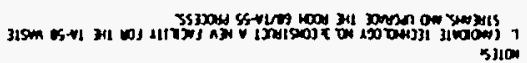

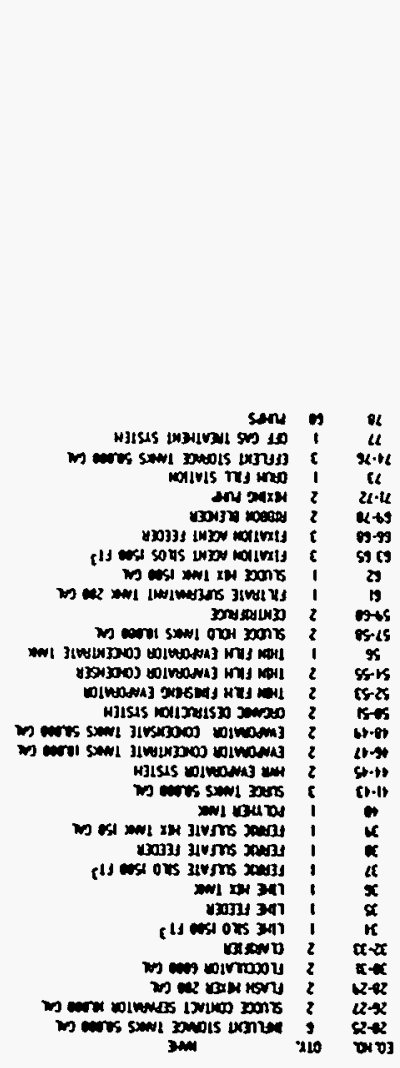




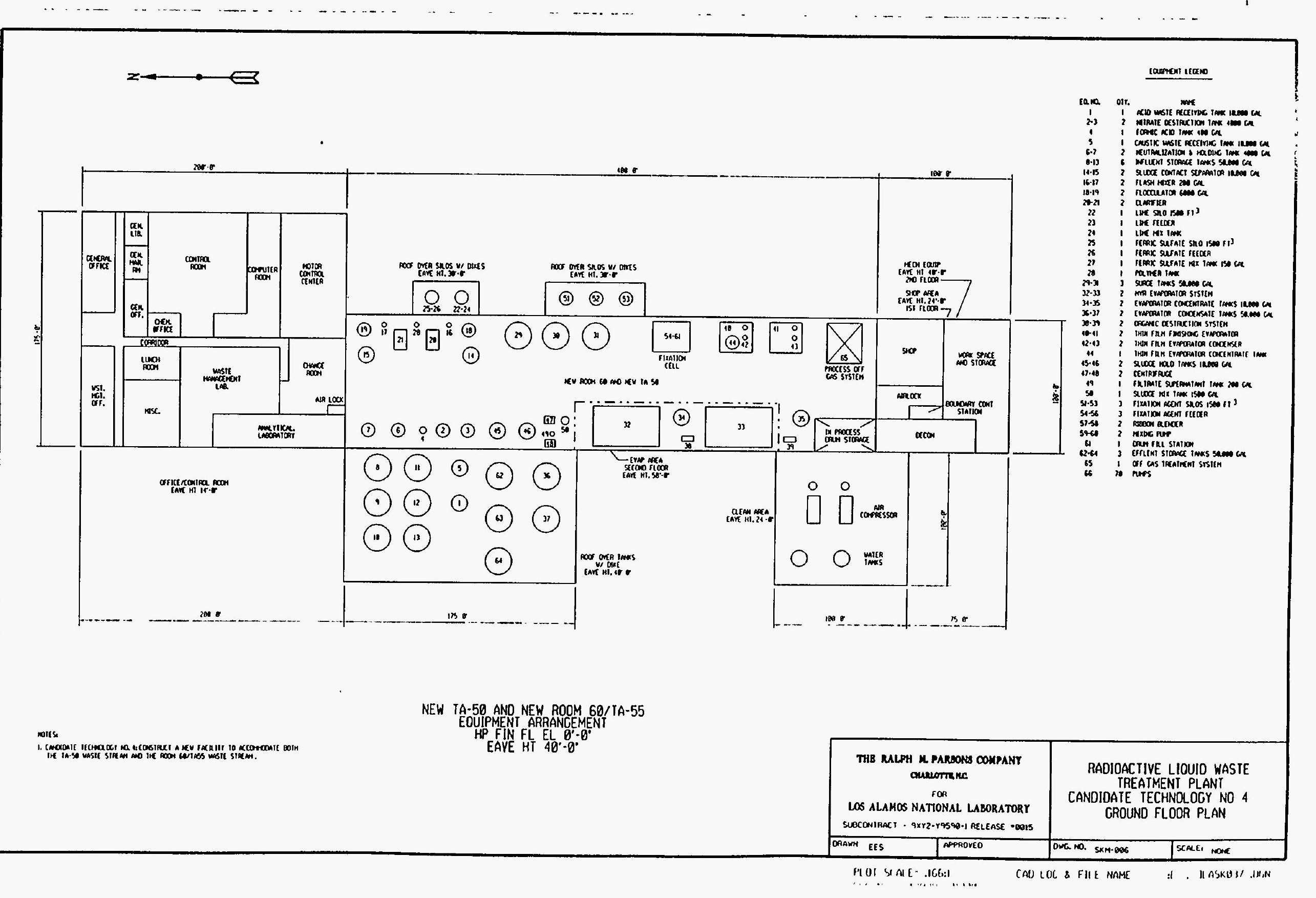




\section{APPENDIX G}

\section{DETAILED COST ESTIMATES FOR THE CANDIDATE TECHNOLOGIES}


LOS ALAMOS NATIONAL LABORATORY

BEST AVAILABLE TECHNOLOGY STUDY

\section{ESTIMATE QUALIFICATIONS}

The estimate was based on four (4) equipment options with each option having its own building requirements. The buildings are classified as moderate hazard structures. For Best Available Technology (BAT) options $\# 1$ and $\# 2$ a portion of the existing buildings are used to house part of the process equipment. The cost of up-grading the existing buildings to meet DOE order $6430.1 \mathrm{a}$ is not included in the cost estimates. Not enough information is known about the existing structure or, the amount of contamination that might have to be dealt with, if any, to make any attempt to estimate the costs involved.

Areas where new building are to be constructed are considered to be "green-field" and no contamination is present, and therefore no money is included in the estimate for decommissioning and decontamination.

An allowance of $\$ 750,000$ is included in each option to cover the cost of laboratory equipment. Also included in each option is monies for furniture based on the building sizes. A $\$ 75,000$ budget is also included for a safety analysis.

A $15 \%$ labor inefficiency factor is included to cover the time lost for going through security check points, and to cover the time lost in showering and "dressing-out".

The cost for instrumentation, piping, and electrical was based on factors developed for a similar project that was done by parsons at DOE's Oak Ridge site. The building, equipment foundations, and site costs were also obtained based on factors developed from the cost estimate of this same Oak Ridge project.

The costs for engineering, construction management, and the facility operator were based on percentages of the construction value.

Escalation was included at $10 \%$ and a contingency of $25 \%$ of the project cost was also included. 
BASED ON LABOA OF: $\$ 3.933 .00000$ PRENIUMTTIME

HEIGHTAELOW GRADE

WEATHER

INDIRECTCOSTS

Bes

4 WORKING IN RC AREAS

SHOW-Ur TIME

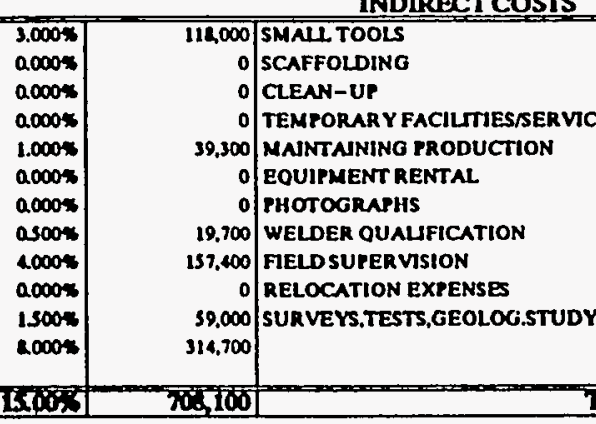

\section{Hol EOFFCE}

7 OPERATNO INTERFERENCES

WELDER QUAUFICATION

CLEAN-UP

10. STRINGENT TESTING \& INSPECTION

11. MAINTANN \& SERVICE EOUIPMENT

12 NON-FAOD. TIME

T.

\begin{tabular}{|c|c|c|}
\hline $3.000 \pi$ & 118,000 & \\
\hline $1.000 \%$ & 39.300 & PROJECT MANAGEMENT \\
\hline $2.000 \%$ & 78.700 & PROCUREMENT \\
\hline $5.000 \%$ & 16,700 & ESTIMATING \\
\hline $0.000 \%$ & & SCHEDULUNG \\
\hline $12.000 \%$ & 708.100 & PROJECT CONTROLS \\
\hline $0.100 *$ & 3,900 & PERMTTS \\
\hline $0500 \%$ & 19,700 & TRAVEL, POSTAGE, PRINTS, PHONE ETC. \\
\hline $18000 \%$ & 708,100 & QA \\
\hline $3.000 \%$ & 118,000 & \\
\hline $1500 \%$ & $\$ 9,000$ & SUBTOTAL \\
\hline & & PROFIT (TOS \\
\hline
\end{tabular}

\begin{tabular}{|c|c|}
\hline $3.000 \%$ & $118 \mathrm{cms}$ \\
\hline $1.000 \%$ & $34 \mathrm{lmon}$ \\
\hline $1.500 \%$ & $54,1 \mathrm{~nm}$ \\
\hline $1.000 \%$ & $34.34 \times 1$ \\
\hline $1500 \%$ & sy (nx) \\
\hline $1.500 \%$ & $39(x \times)$ \\
\hline $2000 \%$ & (2). $(n)$ \\
\hline $0.00 \%$ & {$[y, r(x)$} \\
\hline $3.000 x$ & $23(1+4 \times 1$ \\
\hline $17.000 \times$ & 793,600 \\
\hline
\end{tabular}




\begin{tabular}{|c|c|c|c|c|c|c|c|c|c|c|c|c|c|c|}
\hline & $\begin{array}{l}\text { THE AALPH M. PARSONS COMPAN } \\
\text { PRO } \\
\text { WES: } 1.0\end{array}$ & LOGY & & & & & & & & & & & & $\begin{array}{r}10-A p 1-93 \\
0003 \text { AM }\end{array}$ \\
\hline $\begin{array}{l}\text { COST } \\
\text { CODE }\end{array}$ & DESCRIPNON & oTY & UM & \begin{tabular}{c|} 
UNIT \\
MH \\
\end{tabular} & \begin{tabular}{c|c} 
TOTAL \\
MH
\end{tabular} & $\begin{array}{l}\text { LABOR } \\
\text { UNIT }\end{array}$ & $\begin{array}{l}\text { TOTAL } \\
\text { UABOR } \\
\end{array}$ & $\begin{array}{c}\text { UNIT } \\
\text { EQUIPMENT } \\
\end{array}$ & $\begin{array}{c}\text { TOTAL } \\
\text { EQUIPMENT } \\
\end{array}$ & $\begin{array}{c}\text { UNIT } \\
\text { MATERIAL }\end{array}$ & \begin{tabular}{|c|} 
TOTAL \\
MATERIAL \\
\end{tabular} & $\begin{array}{l}\text { UNIT } \\
\text { S/C }\end{array}$ & $\begin{array}{l}\text { TOTAL } \\
\text { S/C }\end{array}$ & TOTAL \\
\hline 6.000 & ACID WASTE RECEIVNQ TK & 1 & EA & 40.000 & 40 & 17.41 & 090 & $30,000.00$ & 30.000 & & 0 & & 0 & 31.580 \\
\hline 0.000 & CAUSTC WASTE RECEMNG TK & 1 & EA & 40.000 & 40 & 17.41 & 000 & $20,600.00$ & 20.600 & & o & & o & 21.290 \\
\hline 0.000 & NITRATE DESTRUCTON TK & 1 & EA & 30.000 & 30 & 17.41 & 522 & 25.750 .00 & 25.750 & & of & & of & 20.272 \\
\hline 0,000 & FORMIC ACID TK & 1 & EA & 10.000 & 10 & 17.41 & 174 & $2,000.00$ & 2,080 & & 0 & & o & 2.234 \\
\hline 0.000 & MIX TANK & 1 & EA & 10.000 & 10 & 17.41 & 174 & $2,060.00$ & 2,060 & & 0 & & o & 2.234 \\
\hline 0,000 & FLASH MIXER & 1 & EA & 30.000 & 30 & 17.41 & 522 & 2.000 .00 & 2,000 & & 0 & & 0 & 2.582 \\
\hline 0.000 & FLOCCULATOR & 1 & EA & 40.000 & 40 & 17.41 & 000 & $10,300.00$ & 10,300 & & 0 & & o & 10.000 \\
\hline 0,000 & LAMELLA CLARIFIER & 1 & EA & 200.000 & 200 & 17.41 & 3,482 & $51,500.00$ & 51,500 & & o & & 0 & 54.082 \\
\hline 0,000 & OFF-GAS TREATMENT SYSTEM & $1]$ & EA & 320.000 & 320 & 17.41 & 5,571 & $25,750.00$ & 25,750 & & o & & o) & 31,321 \\
\hline 0,000 & NaOH TK & 1 & EA & 40.000 & 40 & 17.41 & 000 & $15,450.00$ & 15,450 & & 0 & & 0 & 10.140 \\
\hline 0,000 & LIME SILO & 1 & EA & 80.000 & 80 & 17.41 & 1,303 & $30,050.00$ & 36,050 & & o & & 0 & 37.443 \\
\hline 0,000 & LIME FEEDER & 1 & EA & 20.000 & 20 & 17.41 & 348 & 5.150 .00 & 5.150 & & o & & o & 3,408 \\
\hline 0,000 & LIME MIX TK & 1 & EA & 10.000 & 10 & 17.41 & 174 & 5.150 .00 & 5.150 & & o & & 0 & 5,324 \\
\hline 0,000 & GRAVITY FILTER & 1 & EA & 240.000 & 240 & 17.41 & 4,178 & $41,200.00$ & 41,200 & & o & & o & 45.378 \\
\hline 0.000 & SLUDGE CONTACT SEPARATOR & 1 & EA & 20.000 & 20 & 17.41 & 348 & $5,150.00$ & 5.150 & & 0 & & o & 5.408 \\
\hline 6.000 & SLUDGE METERING TK & 1 & EA & 10.000 & 10 & 17.41 & 174 & $2,060.00$ & 2.060 & & $\mathbf{0}$ & & o) & 2.234 \\
\hline 0,000 & DRUM TUMBLEA & 1 & EA & 80.000 & 80 & 17.41 & 1,303 & $10,300.00$ & 10.300 & & $\mathbf{0}$ & & o) & 11.003 \\
\hline 0,000 & DRUM TUMBLER CONTANMENT BOX & 1 & EA & 30.000 & 30 & 17.41 & 522 & 5.150 .00 & 5,150 & & 0 & & o] & 5,072 \\
\hline 0,000 & PUMPS & 18 & EA & 30.000 & 540 & 17.41 & 0,401 & $5,150.00$ & 92.700 & & $\mathbf{0}$ & & of & 102.101 \\
\hline 0,000 & INFLUENT STORAGE TK, 75K QAL & 1 & EA & & o & & 0 & & & & 0 & 150.000 .00 & 150,000 & 150.000 \\
\hline 0,000 & INFLUENT STORAGE TK, 25K GAL & 1 & EA & & 0 & & o & & of & & 0 & 50.000 .00 & 50,000 & 50.000 \\
\hline 0,000 & FLASH MIXEA & 1 & EA & 20.000 & 20 & 17.41 & 348 & $2,000.00$ & 2.000 & & $\mathbf{0}$ & & o & 2.408 \\
\hline 0,000 & FLOCCULATOA & i) & EA & $\$ 0.000$ & 40 & 17.41 & 000 & $20,000.00$ & 20,600 & & 0 & & 0 & 21.290 \\
\hline 0,000 & LAMELLA CLARIFIER & 1 & EA & 100.000 & 400 & 17.41 & 0,004 & $87,550.00$ & 87,550 & & 0 & & 0 & 94,514 \\
\hline 0,000 & LIME SILO & 1 & EA & 80.000 & 80 & 17.41 & 1,393 & 30.050 .00 & 30.050 & & 0 & & 0 & 37.413 \\
\hline 0,000 & LIME FEEDER & 1 & EA & 20.000 & 20 & 17.41 & 348 & $5,150.00$ & 5.150 & & 0 & & 0 & 5.498 \\
\hline 0,000 & LIME MIX TK & 1 & EA & 10.000 & 10 & 17.41 & 174 & 5.150 .00 & 5.150 & & 0 & & 0 & 3.324 \\
\hline 0,000 & FERRIC SULFATE TK & 1 & EA & 80.000 & 80 & 17.41 & 1,303 & $30,050.00$ & 36,050 & & 0 & & 0 & 37.443 \\
\hline 0,000 & FERRIC SULFATE FEEDER & 1 & EA & 20.000 & 20 & 17.41 & 348 & 5.150 .00 & 5.150 & & 0 & & 0 & 5.498 \\
\hline 0,000 & FAERAIC SULFATE MIX TK & 1 & EA & 10.000 & 10 & 17.41 & 174 & $5,150.00$ & 5.150 & & 0 & & 0 & 5,324 \\
\hline 0,000 & POLYMER TK & 1 & EA & 10.000 & 10 & 17.41 & 174 & 5.150 .00 & 5.150 & & 0 & & 0 & 5,324 \\
\hline 0,000 & GRAVITY FILTER & 1 & EA & 360.000 & 360 & 17.41 & 0,208 & $72,100.00$ & 72.100 & & 0 & & 0 & 78,368 \\
\hline 0.000 & SLUDGE DECANT TK & 1 & EA & 40.000 & 40 & 17.41 & 690 & $20,600.00$ & 20,000 & & o & & 0 & 21.290 \\
\hline j,000 & ROTARY VACUUM PRECOAT FILTER & 1 & EA & 360.000 & 360 & 17.41 & 0,208 & $103,000.00$ & 103,000 & & 0 & & 0 & 109.268 \\
\hline 0,000 & PAECOAT TK & 1 & EA & 10.000 & 10 & 17.41 & 174 & $2,060.00$ & 2.000 & & 0 & & 0 & 2.234 \\
\hline 0.000 & EVAPORATOR FEED TK & 1 & EA & & o & & 0 & & & & 0 & 40,00000 & 40.000 & 40.000 \\
\hline 0.000 & EVAPORATOR HEATER \& BODY & 1 & EA & 400.000 & 400 & 17.41 & 0.064 & $1,236,000.00$ & $1,236,000$ & & 0 & & 0 & 1.242 .064 \\
\hline 0.000 & CONDENSER & 1 & EA & 40.000 & 40 & 17.41 & 000 & $20,600.00$ & 20.000 & & 0 & & 0 & 21.206 \\
\hline 0,000 & CONDENSATE TK & 1 & EA & 30.000 & 30 & 17.41 & 522 & $10,300.00$ & 10,300 & & 0 & & 0 & 10.822 \\
\hline 0.000 & ORGANIC DESTRUCTION SYSTEM & 1 & EA & 360.000 & 360 & 17.41 & 0,208 & $103,000.00$ & 103,000 & & 0 & & 0 & 100.268 \\
\hline 0,000 & FIXATION AGENT SILOS & 3 & EA & 00.000 & 240 & 17.41 & 4.178 & $36,050.00$ & 108,150 & & o & & 0 & 112,328 \\
\hline 0,000 & FIXATION AGENT FEEDERS & 3. & EA & 20.000 & 00 & 17.41 & 1.045 & 5.150 .00 & 15.450 & & o & & o & 16.405 \\
\hline 0,000 & RIBBON BLENDEA & 1 & EA & 60.000 & $\infty 0$ & 17.41 & 1.046 & $51,500.00$ & 51,500 & & 0 & & of & 32.545 \\
\hline 0,000 & SLUDGE MIX TK & 1 & EA & 20.000 & 20 & 17.41 & 348 & 5.150 .00 & 5,150 & & 0 & & 0 & 5.408 \\
\hline 0.000 & MIXING PUMP & 1 & EA & 10.000 & 40 & 17.41 & 090 & 30,900 & 30,900 & & 0 & & o] & 31.500 \\
\hline 0,000 & DAUM FILL STATION & i] & EA & 240.000 & 240 & 17.41 & 4,178 & 20,000 & 20.000 & & 0 & & 0 & 24.778 \\
\hline 0.000 & EFFLUENT STORAGE TKS & 2 & EA & & 0 & & & & & & 0 & 200,000 & 400,000 & 400,000 \\
\hline 0.000 & PUMPS & 20 & EA & 30.000 & 780 & 17.41 & 13,580 & 5.150 & 133,000 & & 0 & & 0 & 147,480 \\
\hline & SUBTOTAL & & & & $\frac{0}{6,520}$ & & & & $\frac{0}{20307 \pi}$ & & $\frac{0}{0}$ & & 040.000 & $3,200,813$ \\
\hline 0,000 & MISCELLANEOUS MATERIAL & 1 & LS & & $\begin{array}{r}6,520 \\
270\end{array}$ & 17.41 & $\begin{array}{r}06,103 \\
4,805\end{array}$ & & $\begin{array}{r}2,530,710 \\
0\end{array}$ & & 03,300 & & & 08,105 \\
\hline 0.000 & VENDOR REPRESENTATIVE & 1 & is & & & & & & 38,000 & & & & & 38,000 \\
\hline 0,000 & EQUIPMENT INSULATION & 500 & SF & & & & & & & & & 10.00 & 5,000 & 5.000 \\
\hline 0.000 & EQUIPMENT TOUCH - UP PAINTING & 1 & LS & & 83 & 1450 & 1.211 & & 0 & & 1.300 & & & 2311 \\
\hline 0,000 & TOTAL PAOCESS EQUIP MENT & & & & 5,870 & & 102.110 & & $2,568,710$ & & 64,000 & & 045,000 & $3.380,420$ \\
\hline & & & & & & & & & & & & & & \\
\hline
\end{tabular}




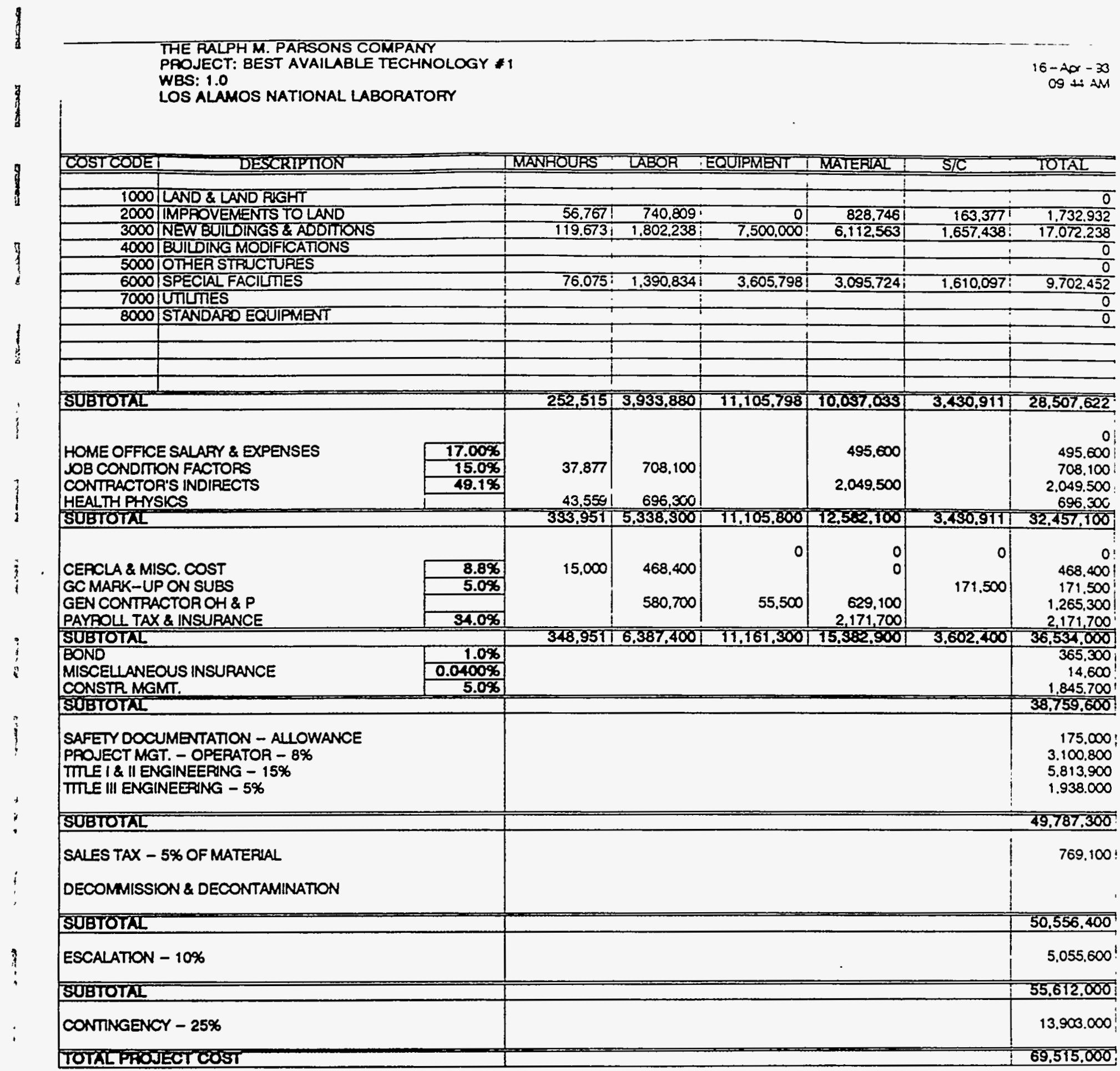


THE RALPH M. PARSONS COMPANY

PROJECT: BEST AVAILABLE TECHNOLOGY *1

WBS: 1.0

LOS ALAMOS NATIONAL LABORATORY

\begin{tabular}{|c|c|c|c|c|c|c|c|c|}
\hline \multirow[b]{2}{*}{ DESCRIPTION } & \multirow[b]{2}{*}{ QTY } & \multicolumn{4}{|c|}{ LABOA } & \multicolumn{2}{|c|}{ MATERIAL } & \multirow[b]{2}{*}{$\begin{array}{l}\text { TÓTAL } \\
\text { COST }\end{array}$} \\
\hline & & $\begin{array}{c}\text { UNIT } \\
\text { HOURS }\end{array}$ & $\begin{array}{l}\text { TOTAL } \\
\text { HOURS } \\
\end{array}$ & $\begin{array}{c}\text { AVERAGE } \\
\text { RATE }\end{array}$ & $\begin{array}{l}\text { TOTAL } \\
\text { LABOR } \\
\end{array}$ & $\begin{array}{l}\text { UNIT } \\
\text { PRICE }\end{array}$ & $\begin{array}{l}\text { TOTAL } \\
\text { MATL }\end{array}$ & \\
\hline $\begin{array}{l}\text { 1. SAFETY TRAINING } \\
\text { 2. ENTRY/ EXIT MEDICAL EXAMS (EXAMS BY LANL STAFF) } \\
\text { 3. BIOASSAY TESTING ( } 1 \text { TEST/MANMEEK) } \\
\text { 4. ANNUAL MEDICAL EXAMS (EXAMS BY LANL STAFF) } \\
\text { 5. ANNUAL 8 HR REFRESHER TRAINING (1 YR) } \\
\text { 6. ATTRITION (20\% OF ITEMS } 1,2 \& 3 \text { ) }\end{array}$ & $\begin{array}{r}141 \\
141 \\
9620 \\
141 \\
141 \\
1\end{array}$ & $\begin{array}{r}64.00 \\
4.00 \\
0.16 \\
4.00 \\
8.00\end{array}$ & $\begin{array}{r}0 \\
9,008 \\
563 \\
1,539 \\
563 \\
1,126 \\
2,200 \\
0 \\
\end{array}$ & $\begin{array}{l}31.22 \\
31.22 \\
31.22 \\
31.22 \\
31.22 \\
31.22\end{array}$ & $\begin{array}{r}0 \\
281,200 \\
17,600 \\
48,100 \\
17,600 \\
35,200 \\
68,700 \\
0 \\
\end{array}$ & $\begin{array}{l}0.00 \\
0.00 \\
0.00\end{array}$ & $\begin{array}{l}0 \\
0 \\
0 \\
0 \\
0 \\
0 \\
0 \\
0 \\
\end{array}$ & $\begin{array}{r}281,200 \\
17,600 \\
48,100 \\
17,600 \\
35,200 \\
68,700 \\
0\end{array}$ \\
\hline SUBTOTAL - CERCLA & & & 15,000 & & 468,400 & & $\$ 0$ & 468.400 \\
\hline PEASONNEL PAOTECTION & & & & & & & & \\
\hline 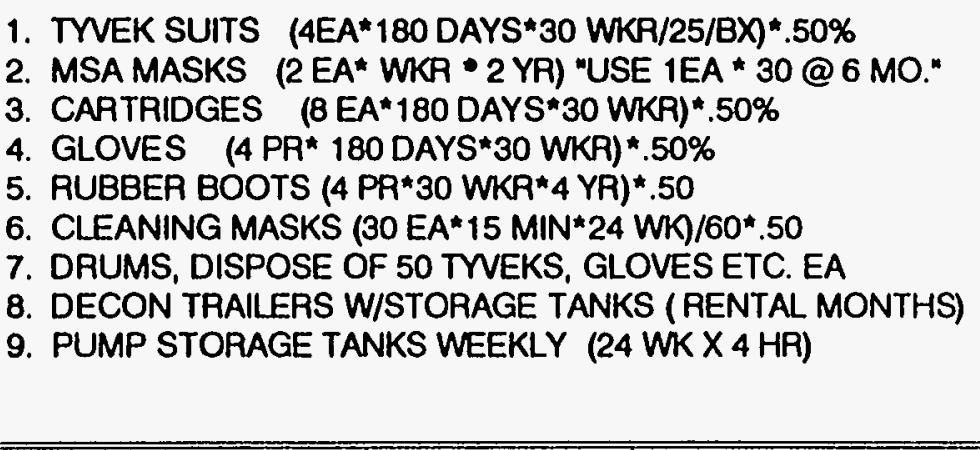 & & $\begin{array}{l}0.00 \\
4.00\end{array}$ & $\begin{array}{l}0 \\
0 \\
0 \\
0 \\
0 \\
0 \\
0 \\
0 \\
0 \\
0 \\
0 \\
\end{array}$ & & $\begin{array}{l}0 \\
0 \\
0 \\
0 \\
0 \\
0 \\
0 \\
0 \\
0 \\
0 \\
0\end{array}$ & $\begin{array}{r}85.00 \\
25.00 \\
1.00 \\
4.65 \\
20.00 \\
35.00 \\
35.00 \\
2,500.00 \\
0.00\end{array}$ & $\begin{array}{l}0 \\
0 \\
0 \\
0 \\
0 \\
0 \\
0 \\
0 \\
0 \\
0 \\
0\end{array}$ & $\begin{array}{l}0 \\
0 \\
0 \\
0 \\
0 \\
0 \\
0 \\
0 \\
0 \\
0 \\
0\end{array}$ \\
\hline SUBTOTAL - PERSONNEL PROTECTION & & & $\overline{0}$ & & 0 & & 0 & \\
\hline TOTAL CERCLA \& MISC COST & & & 15,000 & & 468,400 & & & 468,400 \\
\hline
\end{tabular}




\begin{tabular}{|c|c|c|c|c|c|c|c|c|c|c|c|c|c|c|}
\hline & $\begin{array}{l}\text { THE RALPH W. PARSONS COMPAN } \\
\text { PROJECT: BEST AVAILBLE TECHN } \\
\text { WBS: } 1.0\end{array}$ & loLogr : & & & & & & & & & & & & $\begin{array}{r}16-\mathrm{Apr}-93 \\
0003 \mathrm{AM}\end{array}$ \\
\hline $\begin{array}{l}\text { COST } \\
\text { CODE }\end{array}$ & DESCRIPTION & QTY & UM & $\begin{array}{l}\text { UNIT } \\
\text { MH }\end{array}$ & $\begin{array}{c}\text { TOTAL } \\
\text { MH }\end{array}$ & $\begin{array}{l}\text { LABOR } \\
\text { UNIT }\end{array}$ & $\begin{array}{l}\text { TOTAL } \\
\text { LABOR } \\
\end{array}$ & $\begin{array}{c}\text { UNIT } \\
\text { EQUIPMENT }\end{array}$ & $\begin{array}{c}\text { TOTAL } \\
\text { EQUIPMENT }\end{array}$ & $\begin{array}{c}\text { UNIT } \\
\text { MATERINL }\end{array}$ & $\begin{array}{c}\text { TOTAL } \\
\text { MATERIAL }\end{array}$ & $\begin{array}{l}\text { UNIT } \\
\text { S/C }\end{array}$ & $\begin{array}{l}\text { TOTAL } \\
\text { S/C }\end{array}$ & TOIAL \\
\hline 6,000 & INSTAUMENTATION & 1 & LS & & 8,460 & 18.00 & 157,408 & 110.00 & 031.260 & 32.00 & 270,012 & & & 1.350 .040 \\
\hline 0,000 & PROCESS PIPING & 1 & LS & & 52,011 & 18.24 & 905,097 & & & 45.00 & $2,380,095$ & 1.00 & 905,097 & 4.311 .188 \\
\hline 0,000 & PAOCESS ELECTRICAL & 1 & Ls & & 8.810 & 18.84 & 106,150 & 12.00 & 105,828 & 13.00 & 370.217 & & & 051,195 \\
\hline 6,000 & TOTAL INBTA. PIPE, \&ELECT. & & & & 70.100 & & $1,268,714$ & 1.00 & $1,037,088$ & 00.00 & $3,031.124$ & & 905,097 & $0,322,023$ \\
\hline $\begin{array}{l}3,000 \\
3,000\end{array}$ & $\begin{array}{l}\text { BUILDINGS } \\
\text { LABORATORY EQUIPMENT-ALLOW } \\
\text { BUILDING }\end{array}$ & 1.491 .280 & $\begin{array}{l}\text { LS } \\
\text { CF }\end{array}$ & 0.050 & 74,563 & 15.07 & 1.123 .004 & $7,500,000.00$ & $7,500,000$ & 205 & 4300188 & 0.75 & 1118 & 7.500 .000 \\
\hline 3.000 & BUILDING MECH/ELECT SERVICES & $1,401,260$ & CF & 0.028 & 37,281 & 18.07 & 501,025 & & 0 & 0.75 & $1,118,438$ & 0.35 & 521,037 & $2.202,200$ \\
\hline $\begin{array}{l}3.000 \\
3.000\end{array}$ & $\begin{array}{l}\text { COVERED AREAS } \\
\text { FURNITURE ALLOWANCE }\end{array}$ & 4,878 & $\begin{array}{l}\text { BF } \\
\text { LS }\end{array}$ & 0.400 & $\begin{array}{r}1,050 \\
0\end{array}$ & 16.07 & $\begin{array}{r}29,307 \\
0\end{array}$ & & $\begin{array}{l}0 \\
0\end{array}$ & 12.50 & $\begin{array}{r}00,038 \\
430,000\end{array}$ & 3.50 & $\begin{array}{r}17.003 \\
0\end{array}$ & 107.387 \\
\hline 3,000 & $\begin{array}{l}\text { TOTAL BUILPA, } \\
\text { EQUIPMENT FDNS } \\
\text { EQUIPMENT FOUNDATIONS }\end{array}$ & Th & 노 & & \begin{tabular}{r|}
113,704 \\
0 \\
0 \\
0 \\
0 \\
5,870 \\
\end{tabular} & 14.80 & \begin{tabular}{r|}
$, 714,070$ \\
0 \\
0 \\
0 \\
0 \\
87,302 \\
\end{tabular} & & $\begin{array}{r}7.600,000 \\
0 \\
0 \\
0 \\
0 \\
0 \\
\end{array}$ & & \begin{tabular}{r|}
$0.018,403$ \\
0 \\
0 \\
0 \\
0 \\
94,100
\end{tabular} & & \begin{tabular}{r|}
$1,057,438$ \\
0 \\
0 \\
0 \\
0 \\
0
\end{tabular} & $\begin{array}{r}16,000,770 \\
0 \\
0 \\
0 \\
0 \\
181.402\end{array}$ \\
\hline 2,000 & $\begin{array}{l}\text { TOTAL FOUNDATIONS } \\
\text { SITE } \\
\text { SITE AND UTLITIES }\end{array}$ & 1 & LS & & \begin{tabular}{r|}
5,070 \\
0 \\
0 \\
0 \\
0 \\
56.767 \\
\end{tabular} & 13.05 & $\begin{array}{r}87,302 \\
0 \\
0 \\
0 \\
0 \\
740,800 \\
\end{array}$ & & $\begin{array}{l}0 \\
0 \\
0 \\
0 \\
0 \\
0 \\
\end{array}$ & & \begin{tabular}{r|r|}
94.100 \\
0 \\
0 \\
0 \\
0 \\
828.740 \\
\end{tabular} & & $\begin{array}{r}0 \\
0 \\
0 \\
0 \\
0 \\
163,377 \\
\end{array}$ & $\begin{array}{r}181.402 \\
0 \\
0 \\
0 \\
0 \\
1,732.932\end{array}$ \\
\hline 2,000 & TOTAL SITE & & & & \begin{tabular}{r|}
50.707 \\
0 \\
0 \\
0 \\
0 \\
0 \\
0 \\
0 \\
0 \\
0
\end{tabular} & & $\begin{array}{r}740,800 \\
0 \\
0 \\
0 \\
0 \\
0 \\
0 \\
0 \\
0 \\
0\end{array}$ & & $\begin{array}{l}0 \\
0 \\
0 \\
0 \\
0 \\
0 \\
0 \\
0 \\
0 \\
0\end{array}$ & & $\begin{array}{r}828.746 \\
0 \\
0 \\
0 \\
0 \\
0 \\
0 \\
0 \\
0 \\
0\end{array}$ & & $\begin{array}{r}163.377 \\
0 \\
0 \\
0 \\
0 \\
0 \\
0 \\
0 \\
0 \\
0\end{array}$ & $\begin{array}{r}1.732 .032 \\
0 \\
0 \\
0 \\
0 \\
0 \\
0 \\
0 \\
0 \\
0\end{array}$ \\
\hline \multicolumn{2}{|c|}{ TOTAL DIRECT 8} & & & & 252,516 & & $3,033,020$ & & $11,105,700$ & & $10,037,033$ & & 3.430 .011 & 28.507 .622 \\
\hline
\end{tabular}




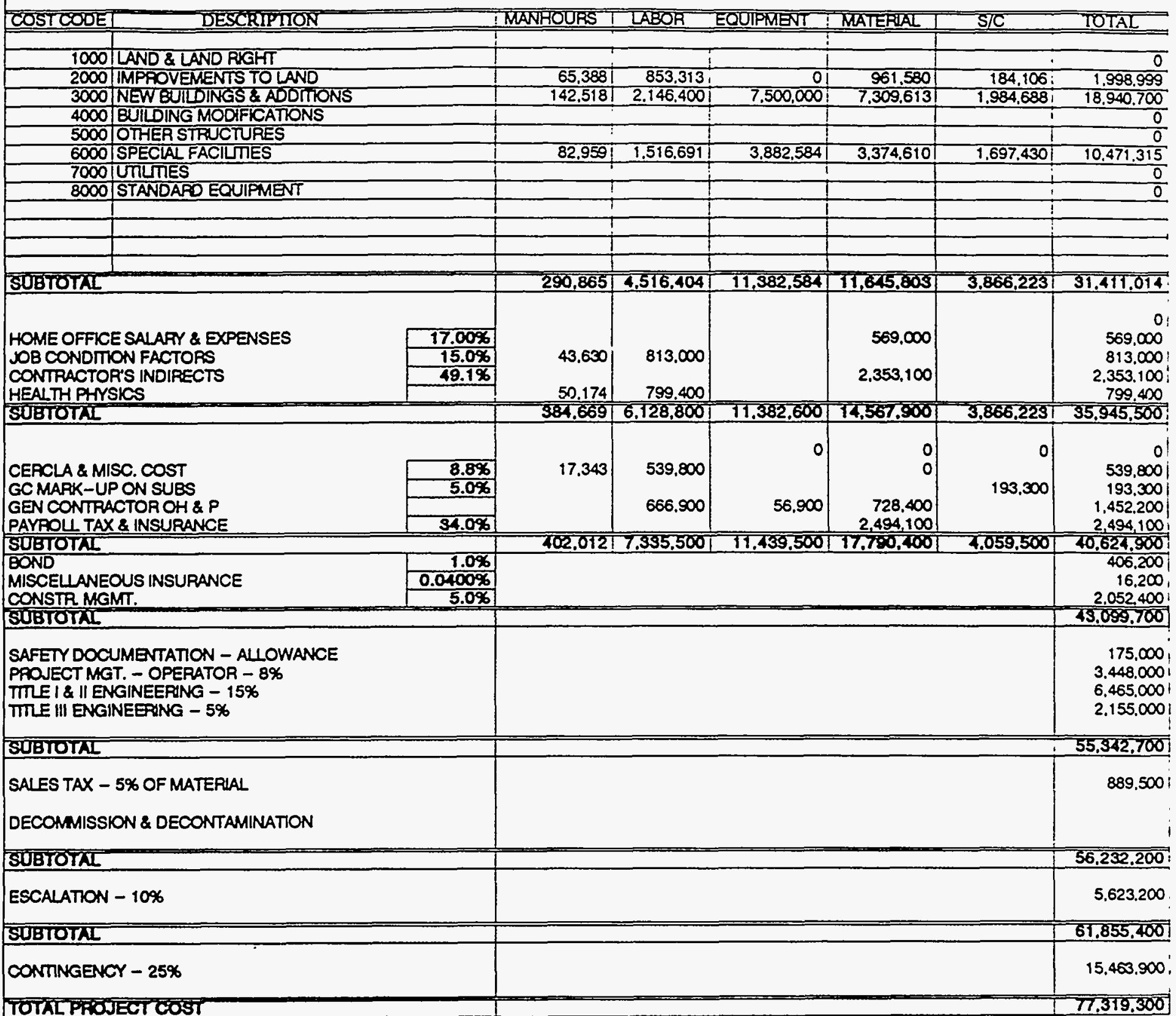




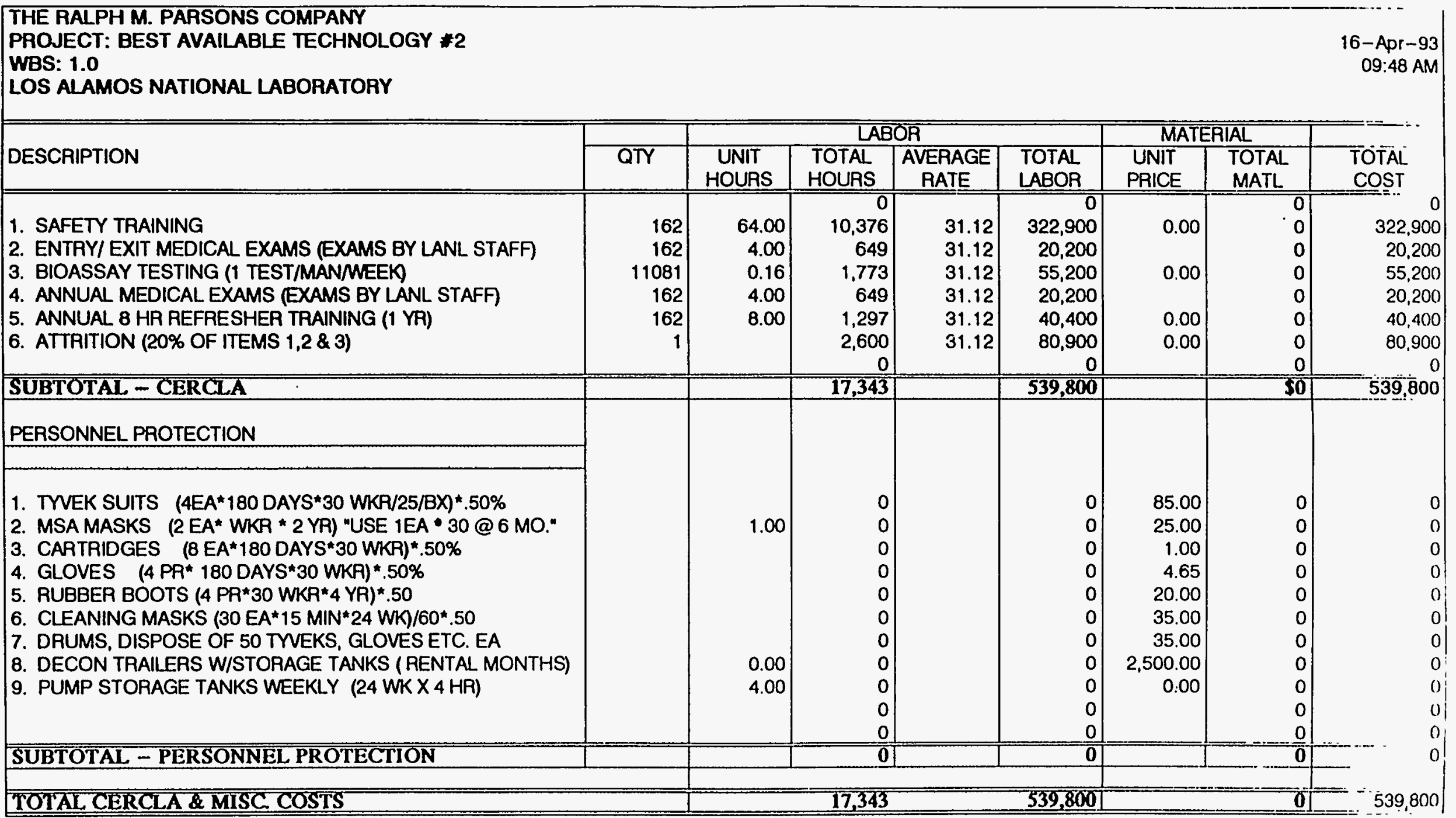




TOOHT

$3.000 \%$
$0.000 \%$
$0.000 \%$
$0.000 \%$
$1.000 \%$
$0.000 \%$
$0.000 \%$
$0.000 \%$
$1000 \%$
$0.000 \%$
$1.500 \%$
$1000 \%$
INDIRECTCOSTS
135S00 SMAILTOOLS
0 SCAFFOLOING

o SCAFFoldine

- clean-ur

- TEMPORARY FACILTIESSERVICE

45.200 MANTAAINING PRODUCTION

- EQUIPMENT RENTAL.

- HotOGRAPHS

22,600 WELDER QUALITICATION

120,700 FELDSUPER VISION

- RELOCATION EXPENSES
67,700 SURVEYS,TESTS,GEOLOG.STUDY 361,300

73,000

BASED ON LABOA OF: $\$ 1510$

$3.000 \%$
$1.000 \%$
$2.000 \%$
$5.000 \%$
$0.000 \%$
$10.00 \%$
$0.100 \%$
$0.500 \%$
$18.000 \%$
$3.000 \%$
$1.500 \%$

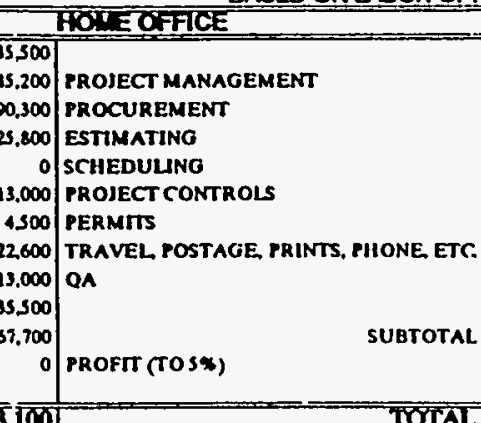

TOTAL

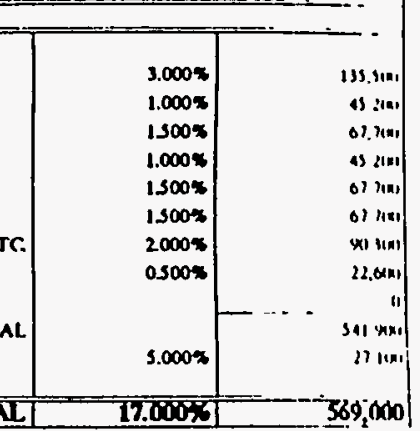




\begin{tabular}{|c|c|c|c|c|c|c|c|c|c|c|c|c|c|c|}
\hline & $\begin{array}{l}\text { THE RALPH W. PARSONS COMPAN } \\
\text { PROJECT: BEST AVALABLE TECHN } \\
\text { WBS: } 1.0\end{array}$ & Logr: & & & & & & & & & & & & $\begin{array}{r}10-A p r-03 \\
0048 \text { AM }\end{array}$ \\
\hline $\begin{array}{l}\text { COST } \\
\text { CODE }\end{array}$ & DESCRIPTION & aTr & UM & $\begin{array}{c}\text { UNTT } \\
\text { MH }\end{array}$ & $\begin{array}{c}\text { TOTAL } \\
\text { MH }\end{array}$ & $\begin{array}{l}\text { LABOR } \\
\text { UNIT }\end{array}$ & \begin{tabular}{l|} 
TOTAL \\
LABOR
\end{tabular} & \begin{tabular}{c|} 
UNIT \\
EQUIPMENT
\end{tabular} & $\begin{array}{c}\text { TOTAL } \\
\text { EQUIPMENT }\end{array}$ & $\begin{array}{c}\text { UNIT } \\
\text { MATERIAL }\end{array}$ & $\begin{array}{c}\text { TOTAL } \\
\text { MATERIAL }\end{array}$ & $\begin{array}{l}\text { UNIT } \\
\text { S/C }\end{array}$ & $\begin{array}{l}\text { TOTAL } \\
\text { S/C }\end{array}$ & TOTAL \\
\hline 0,000 & ACID WASTE RECEMNG TK & 1 & EA & 40.000 & 40 & 17.41 & 600 & 30.000 .00 & 30,000 & & 0 & & of & 31,590 \\
\hline 0,000 & NITRATE DESTRUCTION TKS & 2 & EA & 30.000 & $\infty$ & 17.41 & 1,045 & $25,750.00$ & 51,500 & & o & & o) & 32,545 \\
\hline 0,000 & FORMIC ACID TK & 1 & EA & 10.000 & 10 & 17.41 & 174 & 2.000 .00 & 2,080 & & o & & o] & 2.234 \\
\hline 0,000 & CAUSTIC WASTE RECEMNG TK & $i$ & EA & 40.000 & 40 & 17.41 & 800 & $20,000.00$ & 20,000 & & 0 & & 0 & 21.200 \\
\hline 0,000 & NEUTRALIZATION \& HOLDING TKS & 2 & EA & 30.000 & -00 & 17.41 & 1.045 & $15,450.00$ & 30,000 & & o & & 0 & 31.045 \\
\hline 0,000 & SLUDGE CONTACT SEPARATORS & 2 & EA & 10.000 & 20 & 17.41 & 348 & $5,180.00$ & 10,300 & & 0 & & 0 & 10.048 \\
\hline 0.000 & FLASH MIXERS & 2 & EA & 30.000 & $\infty$ & 17.41 & 1,045 & $2,000.00$ & 4,120 & & 0 & & ol & 5,105 \\
\hline 0.000 & FLOCULATORS & 2 & EA & 40.000 & 80 & 17.41 & $\$, 303$ & 10.300 .00 & 20,000 & & o & & o & 21,003 \\
\hline 0.000 & LAMELLA CLARIFIERS & 2 & EA & 200.000 & 100 & 17.41 & 0,004 & $51,500.00$ & 103,000 & & 0 & & ol & 100.004 \\
\hline 0.000 & OFF-GAS TAEATMENT SYSTEM & 1 & EA & 320.000 & 320 & 17.41 & 5.571 & 25.750 .00 & 25,750 & & 0 & & 0 & 31,321 \\
\hline 0.000 & NaOH TANK & 1 & EA & 40.000 & 40 & 17.41 & 000 & $15,450,00$ & 15,450 & & 0 & & 0 & 10.140 \\
\hline 0.000 & LIME SILO & 1 & EA & 80.000 & 80 & 17.41 & 1,303 & 30.050 .00 & 36,050 & & 0 & & o) & 37.443 \\
\hline 0,000 & LIME FEEDER & 1 & EA & 20.000 & 20 & 17.41 & 348 & $5,150.00$ & 5,150 & & 0 & & o & 5.408 \\
\hline 0,000 & LIME MIX TK & 1 & EA & 10.000 & 10 & 17.41 & 174 & $5,150.00$ & 5,150 & & o] & & o & 3.324 \\
\hline 0,000 & SLUDGE THICKENING TK & 1 & EA & 10.000 & 10 & 17.41 & 174 & $3,150.00$ & 5.150 & & o & & ol & 3.324 \\
\hline 0,000 & SLUDGE METERING TK & 1. & EA & 10.000 & 10 & 17.41 & 174 & $2,000.00$ & 2,000 & & 0 & & 0 & 2,234 \\
\hline 0,000 & DRUM TUMBLER & 1 & EA & 80.000 & 80 & 17.41 & 1,303 & $10,300.00$ & 10,300 & & 0 & & o & 11.003 \\
\hline 0,000 & DRUM TUMBLER CONTANMENT BOX & 1 & EA & 30.000 & 30 & 17.41 & 522 & $0,150.00$ & 5,150 & & 0 & & o. & 5.072 \\
\hline 0,000 & SURGE TANKS & 2 & EA & 40.000 & 80 & 17.41 & 1,393 & $20,000.00$ & 11,200 & & $\mathbf{0}$ & & 0 & 42.593 \\
\hline 0,000 & PUMPS & 28 & EA & 30.000 & 840 & 17.41 & 14,021 & $5,150.00$ & 144,200 & & 0 & & 0 & 158,824 \\
\hline 6,000 & INFLUENT STORAGE TK, 75K GAL & 1 & EA & & o & & 0 & & & & o & $150,000.00$ & 150,000 & 150.000 \\
\hline 6,000 & INFLUENT STORAGE TK, 25K GAL & 1 & EA & & 0 & & o & & 0 & & o] & $50,000.00$ & 50.000 & 50.000 \\
\hline 0,000 & FLASH MIXEA & 1 & EA & 20.000 & 20 & 17.41 & 348 & $2,060.00$ & 2,000 & & 0 & & 0 & 2.408 \\
\hline 0,000 & FLOCCULATOR & 1 & EA & 10.000 & 40 & 17.41 & 000 & $20,000.00$ & 20,000 & & 0 & & 0 & 21.280 \\
\hline 0,000 & LAMELLA CLARIFIEA & 1 & EA & 400.000 & 400 & 17.41 & 0,004 & $87,550.00$ & 87,550 & & 0 & & of & 84.514 \\
\hline 0,000 & LIME SILO & 1 & EA & 80.000 & 80 & 17.41 & 1,303 & $36,050.00$ & 36,050 & & 0 & & 0 & 37.443 \\
\hline 0.000 & LIME FEEDER & 1 & EA & 20.000 & 20 & 17.41 & 348 & $5,150.00$ & 5,150 & & 0 & & 0 & 5,408 \\
\hline 0.000 & LIME MIX TK & 1 & EA & 10.000 & 10 & 17.41 & 174 & $5,150.00$ & 5.150 & & 0 & & 0 & 5.324 \\
\hline 0,000 & FERRIC SULFATE TK & 1 & EA & 80.000 & BO & 17.41 & 1,303 & $36,050.00$ & 36,050 & & o) & & o & 37.443 \\
\hline 0.000 & FERAIC SULFATE FEEDER & 1 & EA & 20.000 & 20 & 17.41 & 348 & $5,150.00$ & 5.150 & & 0 & & 0 & 5,488 \\
\hline 0,000 & FAERAIC SULFATE MIX TK & 1 & EA & 10.000 & 10 & 17.41 & 174 & 5.150 .00 & 5,150 & & 0 & & o & 5,324 \\
\hline 0,000 & POLYMEA TK & 1 & EA & 10.000 & 10 & 17.41 & 174 & $5,150.00$ & 5.150 & & o) & & ol & 5,324 \\
\hline 0,000 & GRAVITY FILTER & 1 & EA & 360.000 & 360 & 17.41 & 0.268 & $72,100.00$ & 72.100 & & 0 & & o) & $78.36 \mathrm{H}$ \\
\hline 0.000 & SLUDGE DECANT TK & 1 & EA & 40.000 & 10 & 17.41 & ODO & $20,600.00$ & 20,000 & & o) & & 0 & 21.206 \\
\hline 0,000 & ROTARY VACUUM PAECOAT FILTER & 1 & EA & 360.000 & 360 & 17.41 & 0.200 & 103.000 .00 & 103,000 & & 0 & & 0 & 100.208 \\
\hline 0.000 & PRECOAT TK & 1 & EA & 10.000 & 10 & 17.41 & 174 & $2,060.00$ & 2,080 & & 0 & & o & 2,234 \\
\hline 0.000 & EVAPORATOR FEEO TK & 1 & EA & & o & & & & & & o & $40,000.00$ & 40,000 & 40,000 \\
\hline 0.000 & EVAPORATOR HEATER \& BODY & 1 & EA & 100.000 & 400 & 17.41 & 0,004 & $1,236,000.00$ & $1,236.000$ & & o & & 0 & $1,242,064$ \\
\hline 0,000 & CONDENSER & 1 & EA & 40.000 & 40 & 17.41 & ODO & 20.000 .00 & 20.000 & & 0 & & 0 & 21,296 \\
\hline 0.000 & CONDENSATE TK & 1 & EA & 30.000 & 30 & 17.41 & 322 & $10,300.00$ & 10,300 & & 0 & & 0 & $10,82:$ \\
\hline 0,000 & ORGANIC DESTRUCTION SYSTEM & 1 & EA & 360.000 & 360 & 17.41 & 0.208 & $103,000.00$ & 103,000 & & 0. & & 0 & 100,208 \\
\hline 0.000 & FIXATION AGENT SILOS & 3 & EA & 80,000 & 240 & 17.41 & 4,178 & 30.050 .00 & 108,150 & & o) & & 0 & 112,328 \\
\hline 0,000 & FIXATION AGENT FEEDERS & 3 & EA & 20.000 & 60 & 17.41 & 1.045 & 5,15000 & 15,450 & & o) & & 0 & 10.405 \\
\hline 0,000 & RIBBON BLENDER & 1 & EA & 00.000 & oo & 17.41 & 1.045 & $51,500.00$ & $\$ 1,500$ & & 0 & & o) & 52.515 \\
\hline 0,000 & SLUDGE MIX TK & 1 & EA & 20.000 & 20 & 17.41 & 340 & 5.150 .00 & 5,150 & & o & & 0 & 5.408 \\
\hline 0,000 & MIXING PUMP & 1 & EA & 40.000 & 40 & 17.41 & 000 & 30,000 & 30,000 & & o & & 0 & 31.500 \\
\hline 0,000 & DRUM FILL STATION & 1 & EA & 240.000 & 210 & 17.41 & 4.170 & 20,000 & 20,000 & & o & & 0 & 24.778 \\
\hline 0,000 & EFFLUENT STORAGE TKS & 2 & EA & & 0 & & & & & & 0 & 200,000 & 400,000 & 400.000 \\
\hline 0,000 & PUMPS & 20 & EA & 30.000 & 780 & 17.41 & 13.580 & 5.150 & 133,000 & & o & & 0 & 147480 \\
\hline 6.000 & SUBTOTAL & & & & 6,020 & & 104,008 & & $2,710,000$ & & & & 040,000 & $3,455,708$ \\
\hline 0,000 & MISCELLANEOUS MATERIML & 1 & LS & & 301 & 17.41 & 5,240 & & & & 67,800 & & 0 & 73.040 \\
\hline 0.000 & VENDOR REPRESENTATIVE & 1 & LS & & & & & & 40,700 & & & & & 40.700 \\
\hline 0.000 & EQUIPMENT INSULATON & 500 & SF & & & & & & & & & 1000 & 5,000 & 5000 \\
\hline 0.000 & EQUIPMENT TOUCH-UP PAINTING & 1 & LS & & $\infty 0$ & 14.50 & 1,313 & & 0 & & 1.400 & & & 2.113 \\
\hline 6,000 & TOTAL PAOCESS EQUIPMENT & & & & 0.411 & & 111,302 & & $2,751,600$ & & 00,200 & & 045,000 & 3.577 .222 \\
\hline
\end{tabular}




\begin{tabular}{|c|c|c|c|c|c|c|c|c|c|c|c|c|c|c|}
\hline & $\begin{array}{l}\text { THE RALPH M. PARSONS COMPAN } \\
\text { PRO JECT: BEST AVAILABLE TECHA } \\
\text { WBS: } 1.0\end{array}$ & OLOeY & & & & & & & & & & & & 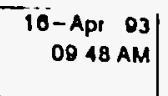 \\
\hline $\begin{array}{l}\text { COST } \\
\text { CODE }\end{array}$ & DESCAIPTION & an & UM & $\begin{array}{l}\text { UNIT } \\
\text { MH }\end{array}$ & $\begin{array}{c}\text { TOTAL } \\
\text { MH }\end{array}$ & \begin{tabular}{|c|} 
UABOA \\
UNIT \\
\end{tabular} & $\begin{array}{l}\text { TOTAL } \\
\text { LABOR }\end{array}$ & $\begin{array}{c}\text { UNIT } \\
\text { EQUIPMENT }\end{array}$ & $\begin{array}{c}\text { TOTAL } \\
\text { EQUIPMENT }\end{array}$ & $\begin{array}{c}\text { UNIT } \\
\text { MATERIAL } \\
\end{array}$ & $\begin{array}{l}\text { TOTAL } \\
\text { MATERIAL }\end{array}$ & $\begin{array}{l}\text { UNIT } \\
\text { S/C }\end{array}$ & $\begin{array}{l}\text { TOTAL } \\
\text { S/C }\end{array}$ & TOTAL \\
\hline 0,000 & INSTRUMENTATION & & LS & & 9.232 & 18.60 & 171.715 & 110.00 & $1,015.520$ & 32.00 & 295,424 & & & $1,482.050$ \\
\hline 0,000 & PROCESS PIPING & 1 & Ls & & 57.000 & 18.24 & $1,052,430$ & & & 45.00 & $2,500,455$ & 1.00 & $1,052,430$ & 4.701 .315 \\
\hline 0.000 & PROCESS ELECTRICAL & 1 & LS & & 0.017 & 18.84 & 181.184 & 12.00 & 115.404 & 13.00 & 413,531 & & & 710.110 \\
\hline 0,000 & TOTAL INBTR.. PIPE, \& ELECT. & & & & 70,540 & & $1,405,320$ & & $1,130,024$ & & $\mathbf{3 , 3 0 5 , 4 1 0}$ & & 1.052 .430 & $0,804.003$ \\
\hline $\begin{array}{l}3,000 \\
3,000 \\
3.000 \\
3,000 \\
3,000\end{array}$ & $\begin{array}{l}\text { BUILDINGS } \\
\text { LABORATORY EQUIPMENT-ALLOW } \\
\text { BUILDING } \\
\text { BUILDING MECHIELECT SERVICES } \\
\text { COVERED AREAS } \\
\text { FURNITURE ALLOWANCE }\end{array}$ & $\begin{array}{r}1 \\
1.788 .780 \\
1.788 .780 \\
4,078 \\
1\end{array}$ & $\begin{array}{l}\text { LS } \\
\text { CF } \\
\text { CF } \\
\text { SF } \\
\text { LS }\end{array}$ & $\begin{array}{l}0.050 \\
0.025 \\
0.400\end{array}$ & $\begin{array}{r}80,436 \\
44,710 \\
1,050 \\
0\end{array}$ & $\begin{array}{l}15.07 \\
15.07 \\
15.07\end{array}$ & $\begin{array}{r}1,347,831 \\
073,015 \\
29,387 \\
0\end{array}$ & $7,500,000.00$ & $\begin{array}{r}7.500,000 \\
0 \\
0 \\
0 \\
0\end{array}$ & $\begin{array}{r}2.05 \\
0.75 \\
12.50\end{array}$ & $\begin{array}{r}5,270,813 \\
1,341,503 \\
00,938 \\
527.700\end{array}$ & $\begin{array}{l}0.75 \\
0.35 \\
3.50\end{array}$ & $\begin{array}{r}1,341,503 \\
020,003 \\
17,003 \\
0\end{array}$ & $\begin{array}{r}7.500000 \\
7.960 .200 \\
2.641 .540 \\
107.387 \\
527,700\end{array}$ \\
\hline 3,000 & $\begin{array}{l}\text { TOTAL BUILDINA } \\
\text { EQUIPMENT FDNS } \\
\text { EQUIPMENT FOUNDATIONS }\end{array}$ & & LS & & \begin{tabular}{r|}
36,107 \\
0 \\
0 \\
0 \\
0 \\
0.411 \\
\end{tabular} & 14.80 & \begin{tabular}{r|}
2.081 .132 \\
0 \\
0 \\
0 \\
0 \\
05,207 \\
\end{tabular} & & $\begin{array}{r}7,500,000 \\
0 \\
0 \\
0 \\
0 \\
0\end{array}$ & & \begin{tabular}{r|r|}
$7,207.013$ \\
0 \\
0 \\
0 \\
0 \\
102,600
\end{tabular} & & \begin{tabular}{r|r}
1.084 .088 \\
0 \\
0 \\
0 \\
0 \\
0
\end{tabular} & $\begin{array}{r}18.742 .832 \\
0 \\
0 \\
0 \\
0 \\
197.807\end{array}$ \\
\hline 3,000 & \begin{tabular}{|} 
TOTAL FOUNDATIONS \\
SITE \\
SITE AND UTLITIES
\end{tabular} & & |LS & & \begin{tabular}{r|}
6.411 \\
0 \\
0 \\
0 \\
0 \\
65,388
\end{tabular} & 13.05 & $\begin{array}{r}96,207 \\
0 \\
0 \\
0 \\
0 \\
853,313\end{array}$ & & $\begin{array}{l}0 \\
0 \\
0 \\
0 \\
0 \\
0\end{array}$ & & $\begin{array}{r}102,000 \\
0 \\
0 \\
0 \\
0 \\
861,580\end{array}$ & & $\begin{array}{r}0 \\
0 \\
0 \\
0 \\
0 \\
184,106\end{array}$ & $\begin{array}{r}107,807 \\
0 \\
0 \\
0 \\
0 \\
1.998,999\end{array}$ \\
\hline 2,000 & TOTAL SITE & & & & \begin{tabular}{r|}
65.388 \\
0 \\
0 \\
0 \\
0 \\
0 \\
0 \\
0 \\
0 \\
0
\end{tabular} & & $\begin{array}{r}63.313 \\
0 \\
0 \\
0 \\
0 \\
0 \\
0 \\
0 \\
0 \\
0\end{array}$ & & $\begin{array}{l}0 \\
0 \\
0 \\
0 \\
0 \\
0 \\
0 \\
0 \\
0 \\
0\end{array}$ & & $\begin{array}{r}801.580 \\
0 \\
0 \\
0 \\
0 \\
0 \\
0 \\
0 \\
0 \\
0\end{array}$ & & $\begin{array}{r}184.106 \\
0 \\
0 \\
0 \\
0 \\
0 \\
0 \\
0 \\
0 \\
0\end{array}$ & $\begin{array}{r}1.998,909 \\
0 \\
0 ! \\
0 \\
0 \\
0 \\
0 \\
0 \\
0 \\
0\end{array}$ \\
\hline TOTAL & DDTECTS & & & & 290.005 & & 4.510 .404 & & $11,982,664$ & & 11.045 .803 & & 3.806 .223 & $31,411,0$ \\
\hline
\end{tabular}




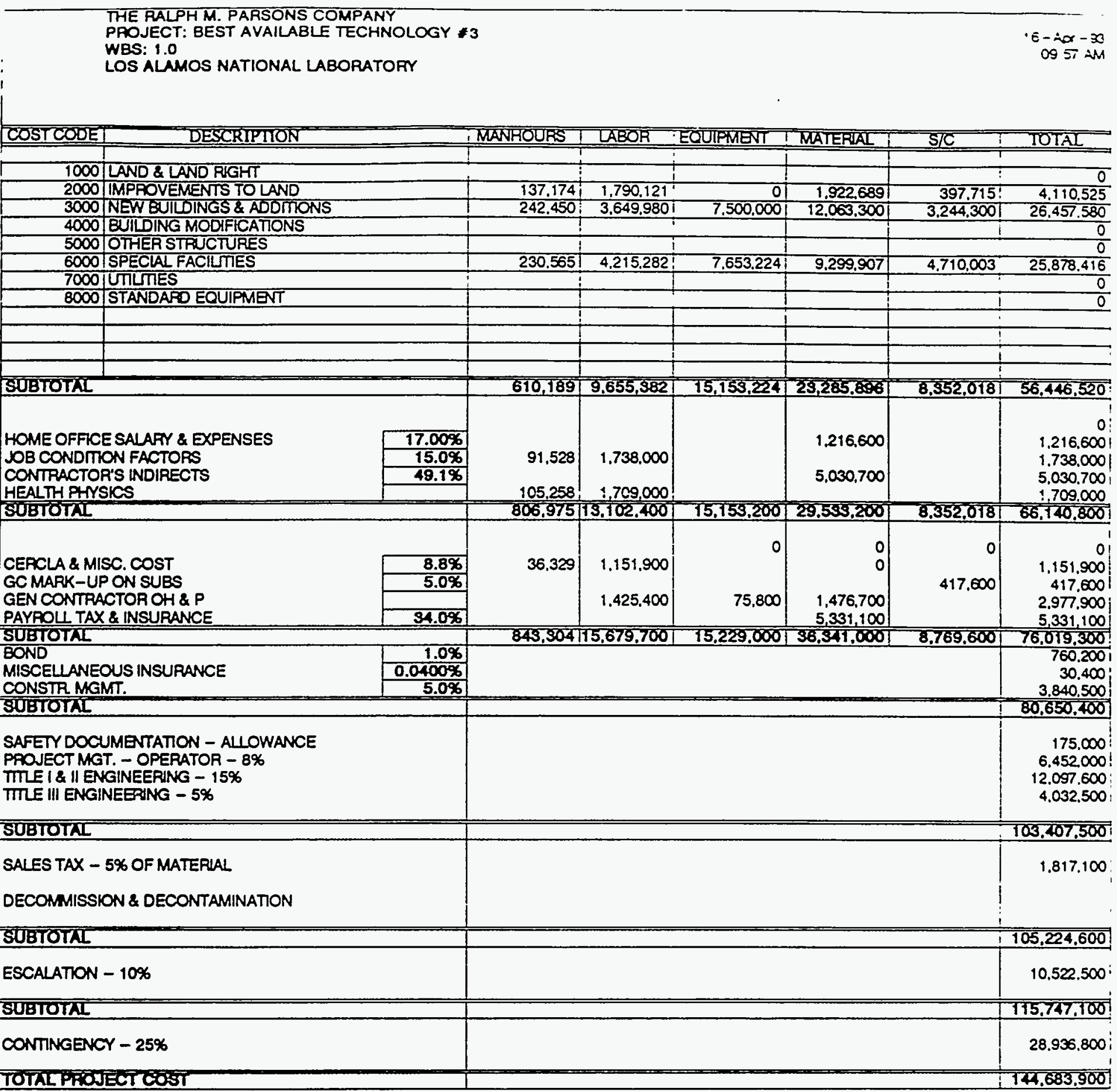




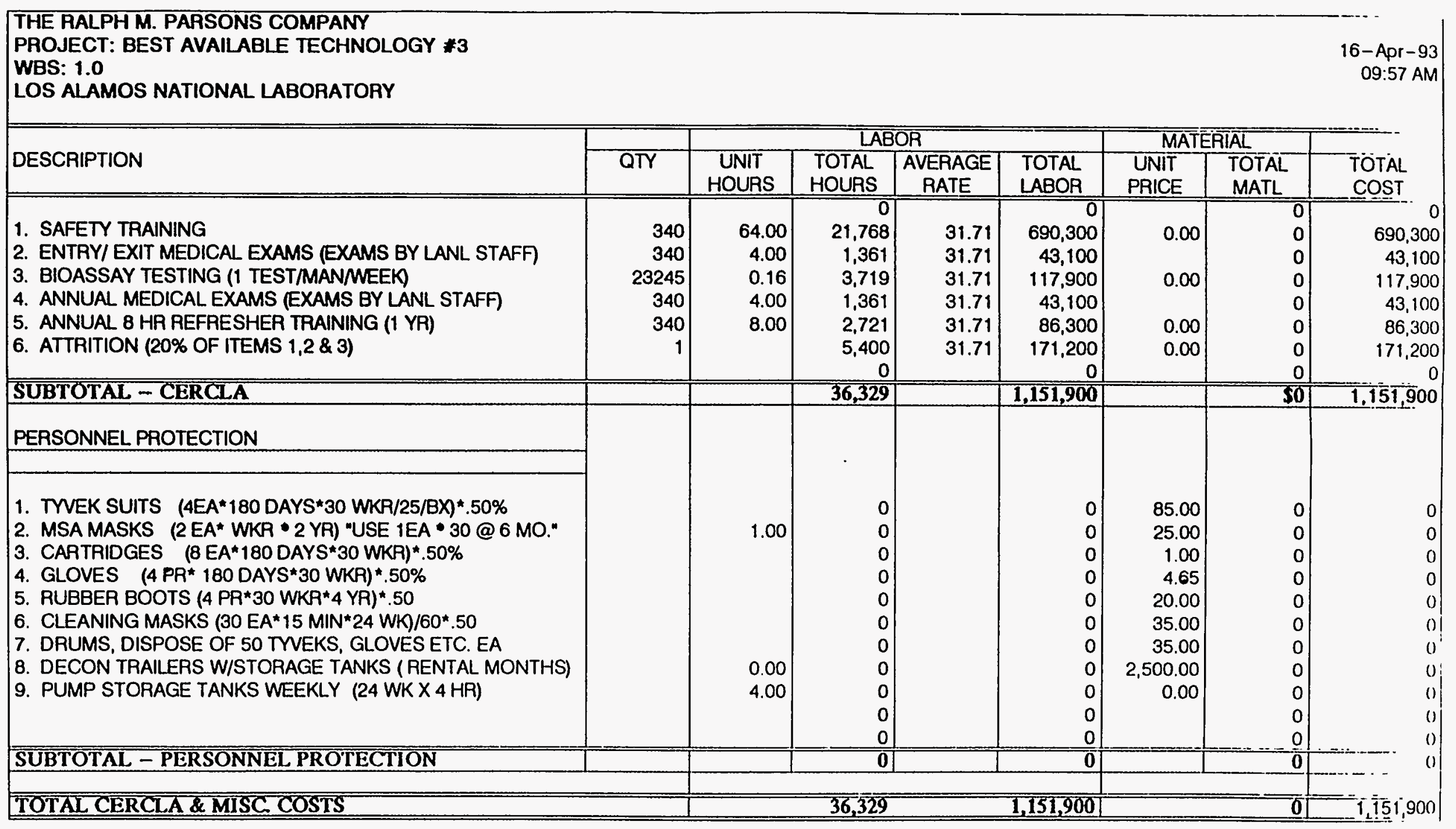




\begin{tabular}{|c|c|c|c|c|c|c|c|c|c|c|c|c|c|c|}
\hline & $\begin{array}{l}\text { THE RALPH M. PARSONS COMPAN } \\
\text { PROJECT: BEST AVALABLE TECH } \\
\text { WBS: } 1.0\end{array}$ & oar & & & & & & & & & & & & $\begin{array}{rl}10-A p 1 & 03 \\
0057 & \text { AM }\end{array}$ \\
\hline $\begin{array}{l}\text { COST } \\
\text { CODE }\end{array}$ & DESCRIPTION & OTY & UM & $\begin{array}{c}\text { UNIT } \\
\text { MH }\end{array}$ & $\begin{array}{c}\text { TOTAL } \\
\text { MH } \\
\end{array}$ & $\begin{array}{l}\text { LABOA } \\
\text { UNIT }\end{array}$ & $\begin{array}{l}\text { TOTAL } \\
\text { LABOR }\end{array}$ & $\begin{array}{c}\text { UNIT } \\
\text { EQUIPMENT } \\
\end{array}$ & $\begin{array}{c}\text { TOTAL } \\
\text { EQUIPMENT }\end{array}$ & $\begin{array}{c}\text { UNIT } \\
\text { MATERIAL }\end{array}$ & $\begin{array}{c}\text { TOTAL } \\
\text { MATEAIAL }\end{array}$ & $\begin{array}{l}\text { UNIT } \\
\text { S/C }\end{array}$ & $\begin{array}{l}\text { TOTAL } \\
\text { S/C }\end{array}$ & TOTAL \\
\hline 0.000 & ACID WASTE RECEIVNG TK & 1 & EA & 40.000 & 40 & 17.41 & 090 & $30,000.00$ & 30,000 & & 0 & & 0 & 31.580 \\
\hline 0.000 & CAUSTIC WASTE RECEIMNG TK & 1 & EA & 40.000 & 40 & 17.41 & 890 & $20,000.00$ & 20,000 & & o & & 0 & 21.200 \\
\hline 0.000 & NITRATE DESTRUCTION TK & 1 & EA & 30.000 & 30 & 17.41 & 522 & 25.750 .00 & 25.750 & & o & & o & 20.272 \\
\hline 0.000 & FORMIC ACID TK & & EA & 10.000 & 10 & 17.41 & 174 | & $2,080.00$ & 2,080 & & o & & 0 & 2.234 \\
\hline 0,000 & MIX TANK & 1 & EA & 10.000 & 10 & 17.41 & 174 & $2,000.00$ & 2,000 & & 0 & & 0 & 2,234 \\
\hline 0,000 & FLASH MIXER & 1 & EA & 30.000 & 30 & 17.41 & 522 & $2,000.00$ & 2,080 & & 0 & & 0 & 2.582 \\
\hline 0.000 & FLOCCULATOR & 1 & EA & 40.000 & 40 & 17.41 & 000 & $10,300.00$ & 10,300 & & 0 & & o & 10.000 \\
\hline 0,000 & |LAMELLA CLARIFIER & 1 & EA & 200.000 & 200 & 17.41 & 3,482 & $51,500.00$ & 51,500 & & o & & 0 & 54.982 \\
\hline 0,000 & OFF-GAS TREATMENT SYSTEM & 1 & EA & 320.000 & 320 & 17.41 & 5,571 & $25,750.00$ & 25,750 & & o & & 0 & 31,321 \\
\hline 0.000 & NaOH TK & 1 & EA & 10.000 & 40 & 17.41 & 000 & 15.450 .00 & 15,450 & & 0 & & o & 16146 \\
\hline 0,000 & LIME SILO & 1 & EA & 80.000 & 80 & 17.41 & 1,393 & $30,050.00$ & 36.050 & & o & & 0 & 37,443 \\
\hline 0,000 & UME FEEDER & 1 & EA & 20.000 & 20 & 17.41 & 348 & $5,150.00$ & 5,150 & & 0 & & o & 5490 \\
\hline 0,000 & LIME MIX TK & 1 & EA & 10.000 & 10 & 17.41 & 174 & 5.150 .00 & 5.150 & & 0 & & 0 & $532+$ \\
\hline 0,000 & GAAVITY FILTEA & 1 & EA & 240.000 & 240 & 17.41 & 4.178 & $41,200.00$ & 41,200 & & 0 & & 0 & 45,378 \\
\hline 0.000 & SLUDGE CONTACT SEPARATOR & 1 & EA & 20.000 & 20 & 17.41 & 348 & $5,150.00$ & $\mathbf{5 , 1 5 0}$ & & 0 & & 0 & 5.490 \\
\hline 0,000 & SLUDGE METEAING TK & 1 & EA & 10.000 & 10 & 17.41 & 174 & $2,000.00$ & 2,060 & & 0 & & 0 & 2.234 \\
\hline 0,000 & DRUM TUMBLER & 1 & EA & 80.000 & 80 & 17.41 & 1,393 & $10,300.00$ & 10,300 & & 0 & & 0 & 11.003 \\
\hline 0.000 & [DRUM TUMBLEA CONTANMENT BOX] & 1 & EA & 30.000 & 30 & 17.41 & 322 & 5.150 .00 & 5.150 & & 0 & & 0 & 5,072 \\
\hline 0.000 & SLUDGE CONTACT SEPARATORS & 2 & EA & 40.000 & 80 & 17.41 & 1,303 & $30,000.00$ & 01,800 & & 0 & & 0 & 63.103 \\
\hline 0,000 & | INFLUENT/EFFLUENT STQ TKS & - & EA & & 0 & & of & & & & 0 & $100,000.00$ & 800,000 & 900.000 \\
\hline 0.000 & FLASH MIXERS & 2 & EA & 20.000 & 40 & 17.41 & 600 & 2.060 .00 & 4.120 & & 0 & $50,000.00$ & 100,000 & 104.810 \\
\hline 0,000 & FLOCCULATORS & 2 & EA & 40.000 & 80 & 17.41 & 1,303 & 20.000 .00 & 41,200 & & 0 & & 0 & 42.503 \\
\hline 0,000 & LAMELLA CLARIFIERS & 2 & EA & 400.000 & 800 & 17.41 & 13,028 & 87.550 .00 & 175.100 & & 0 & & o & 180,028 \\
\hline 0.000 & LIME SILO & 1 & EA & 80.000 & Bo & 17.41 & 1,303 & $36,050.00$ & 36.050 & & 0 & & 0 & 37.443 \\
\hline 6.000 & LIME FEEDER & 1 & EA & 20.000 & 20 & 17.41 & 348 & 5.150 .00 & 5.150 & & o & & 0 & 3480 \\
\hline 0,000 & LIME MIX TK & 1 & EA & 10.000 & 10 & 17.41 & 174 & 5.150 .00 & 3.150 & & 0 & & 0 & 532.4 \\
\hline 6.000 & FERAIC SULFATE SILO & 1 & EA & 80.000 & 80 & 17.41 & 1,393 & 36,05000 & 36,050 & & 0 & & 0 & 37443 \\
\hline 0.000 & FERAIC SULFATE FEEDER & 1 & EA & 20.000 & 20 & 17.41 & 348 & $5,150.00$ & 5.150 & & 0 & & of & 5,408 \\
\hline 0.000 & FERRIC SULFATE MIX TK & 1 & EA & 10.000 & 10 & 17.41 & 174 & 5.150 .00 & 5.150 & & 0 & & o) & 5,324 \\
\hline 0,000 & POLYMER TANK & 1 & EA & 10.000 & 10 & 17.41 & 174 & 5.150 .00 & 5,150 & & o & & of & 5,324 \\
\hline 0.000 & SURGE TANKS, 50K GAL & 3 & EA & & 0 & & 0 & & & & o & 100.000 .00 & 300.000 & 300,000 \\
\hline 0.000 & MVA EVAPORATOR SYSTEMS & 2 & EA & 4600.000 & 0,200 & 17.41 & 160.172 & $1,272,050.00$ & $2,544,100$ & & 0 & & o & $2.704 .27 i$ \\
\hline 0,000 & MVA EVAP. CONCENTRATE TKS & 2 & EA & 40.000 & 80 & 17.41 & 1,303 & $30,800.00$ & 61,800 & & o & & 0 & $63.10 \mathrm{~s}$ \\
\hline 0.000 & MVR EVAP. CONDENSATE TKS & 2 & EA & & 0 & 17.41 & & & & & 0 & $100,000.00$ & 200.000 & 200.000 \\
\hline 0,000 & ORGANIC DESTRUCTION SYSTEMS & 2 & EA & 360.000 & 720 & 17.41 & 12.535 & $103,000.00$ & 206.000 & & o & & 0 & 218335 \\
\hline 0,000 & THIN FILM FINISHING EVAPORATORS & 2 & EA & 120.000 & 240 & 17.41 & 4.178 & $56,650.00$ & 113,300 & & o & $40,000.00$ & 80,000 & 197.478 \\
\hline 0,000 & THIN FILM EVAP. CONOENSERS & 2 & EA & 60.000 & 100 & 17.41 & 2.780 & $5,150.00$ & 10,300 & & 0 & & 0 & 13.086 \\
\hline 0,000 & THIN FILM EVAP. CONCENTRATE TK & 1 & EA & 40.000 & 40 & 17.41 & 600 & $15,450.00$ & 15,450 & & o & & o. & 16.148 \\
\hline 0,000 & SLUDGE HOLOING TKS & 2 & EA & 40.000 & 80 & 17.41 & 1,393 & $25,750.00$ & 51,500 & & 0 & & 0 & 52,893 \\
\hline 0,000 & CENTRIFUGES & 2 & EA & 100.000 & 320 & 17.41 & 5,671 & $10,300.00$ & 20,000 & & o & & o) & 26.171 \\
\hline 0,000 & SUPERNATANT TK & 1 & EA & 10.000 & 10 & 17.41 & 174 & $2,000.00$ & 2,000 & & 0 & & 0 & 2,231 \\
\hline 0,000 & SLUDGE MX TK & 1 & EA & 20.000 & 20 & 17.41 & 348 & 8.150 .00 & 5,150 & & 0 & & 0 & 5.408 \\
\hline 0,000 & FIXATION AGENT SILOS & 3 & EA & 80.000 & 240 & 17.41 & 4,178 & $30,050.00$ & 108,150 & & o & & 0 & 112,328 \\
\hline 0.000 & FIXATION AGENT FEEDERS & 3 & EA & 20.000 & $\infty$ & 17.41 & 1,045 & $8,150.00$ & 15,450 & & 0 & & of & 10,405 \\
\hline 0.000 & AIBBON BLENDERS & 2 & EA & 60.000 & 120 & 17.41 & 2,000 & 51,500 & 103,000 & & o & & o & 105.000 \\
\hline 0,000 & MIXING PUMPS & 2 & EA & 30.000 & $\infty 0$ & 17.41 & 1.045 & 30,000 & 01,800 & & o & & 0 & 02,045 \\
\hline 0.000 & DRUM FILL STATON & 1 & EA & 240.000 & 240 & 17.41 & 4.178 & 20,000 & 20,000 & & o & 200,000 & 200,000 & 224.778 \\
\hline 0.000 & OFF-QAS TREATMENT SYSTEM & 1 & EA & 320.000 & 320 & 17.41 & 5,671 & 25,750 & 25,750 & & o) & & o & 31,321 \\
\hline 0.000 & PUMPS & 78 & EA & 30.000 & 2,340 & 17.41 & 10,730 & 5.150 & 401,700 & & o. & & $\mathbf{0}$ & $\ldots 42.439$ \\
\hline 0,000 & SUBTOTAL & & & & 10,730 & & 201.200 & & 4.443 .420 & & 0 & & $1,780,000$ & $0,514.080$ \\
\hline 0,000 & MISCELLANEOUS MATEAIAL & 1 & LS & & 837 & 17.41 & 14,572 & & & & 111,100 & & & 125012 \\
\hline 0.000 & VENDOA REPRESENTATIVE & 1 & LS & & & & & & 00.700 & & & & & 00.700 \\
\hline 0,000 & EQUIPMENT INSULATION & 500 & $\mathbf{S F}$ & & & & & & & & & 1000 & 5,000 & 5000 \\
\hline 0.000 & EQUIPMENT TOUCH-UP PANTING & 1 & LS & & 251 & 1450 & 3.002 & & 0 & & 2.200 & & & $38 \pi:$ \\
\hline 6.000 & TOTAL PAOCESS EQUTPMENT & & & & 17.818 & & 300,604 & & $4.510,120$ & & 113,300 & & 1.785 .000 & 0.717 .024 \\
\hline
\end{tabular}




\begin{tabular}{|c|c|c|c|c|c|c|c|c|c|c|c|c|c|c|}
\hline & $\begin{array}{l}\text { THE RALPH M. PARSONS COMPAN } \\
\text { PRO JECT: BEST AVALABLE TECH } \\
\text { WBS: } 1.0\end{array}$ & LLOQY & & & & & & & & & & & & $\begin{array}{rl}10-A p t-03 \\
0057 & 0 M\end{array}$ \\
\hline $\begin{array}{l}\text { COST } \\
\text { CODE }\end{array}$ & DESCRIPTION & atr & UM & $\begin{array}{l}\text { UNIT } \\
\text { MH }\end{array}$ & $\begin{array}{c}\text { TOTAL } \\
\text { MH }\end{array}$ & $\begin{array}{l}\text { LABOR } \\
\text { UNIT }\end{array}$ & $\begin{array}{l}\text { TOTAL } \\
\text { LABOA }\end{array}$ & $\begin{array}{c}\text { UNIT } \\
\text { EQUIPMENT } \\
\end{array}$ & $\begin{array}{c}\text { TOTAL } \\
\text { EOUIPMENT }\end{array}$ & $\begin{array}{l}\text { UNIT } \\
\text { MATERIAL }\end{array}$ & $\begin{array}{l}\text { TOTAL } \\
\text { MATERIAL }\end{array}$ & $\begin{array}{l}\text { UNIT } \\
\text { S/C }\end{array}$ & $\begin{array}{l}\text { TOTAL } \\
\text { S/C }\end{array}$ & IOETAL \\
\hline 0.000 & INSTRUMENTATION & & LS & & 25,058 & 18.00 & 477,230 & 110.00 & $2,822,380$ & 32.00 & 821,050 & & & 4.120 .073 \\
\hline 0,000 & PROCESS PIPING & & LS & & 100,302 & 18.24 & $2,025,003$ & & & 45.00 & $7,210,200$ & 1.00 & $2,028,003$ & $13,000,200$ \\
\hline 0.000 & PROCESS ELECTRICAL & & LS & & 20,727 & 18.84 & $503, .337$ & 12.00 & 320.724 & 43.00 & 1.140 .201 & & & $\ldots 1.073 .522$ \\
\hline 6.000 & TOTAL WSTH.. PIPE. E ELECT. & & & & 212.747 & & $3,005,77$ & & $3.143,104$ & & 0.180 .007 & & $2,025,003$ & 10.100 .482 \\
\hline $\begin{array}{l}3,000 \\
3,000\end{array}$ & $\begin{array}{l}\text { BUILOINGS } \\
\text { LABORATORYEQUIPMENT - ALLOW } \\
\text { BUILDING }\end{array}$ & $2,881,750$ & $\begin{array}{l}\text { LS } \\
\text { CF }\end{array}$ & 0.050 & 144.088 & 15.07 & $2.171,400$ & $7,500.000 .00$ & $\begin{array}{r}7.500 .000 \\
0\end{array}$ & 2.95 & $8,501,103$ & 0.75 & $2,161,313$ & $\begin{array}{r}7,500000 \\
12,833,801\end{array}$ \\
\hline 3,000 & BUILDING MECH/ELECT SERVICES & $2,081,780$ & CF & 0.025 & 72,044 & 15.07 & $1,085,703$ & & 0 & 0.75 & $2,101,313$ & & $1,008,012$ & $4.255,028$ \\
\hline $\begin{array}{l}3,000 \\
3,000\end{array}$ & COVERED AREAS & 21,250 & SF & 0.400 & $\begin{array}{r}8,500 \\
0\end{array}$ & 15.07 & $\begin{array}{r}128,095 \\
0\end{array}$ & & $\begin{array}{l}0 \\
0 \\
0\end{array}$ & 12.50 & $\begin{array}{l}265,025 \\
850,100\end{array}$ & 3.50 & $\begin{array}{r}74.375 \\
0\end{array}$ & $\begin{array}{l}408.005 \\
850,00\end{array}$ \\
\hline 3,000 & $\begin{array}{l}\text { TOTAL BUILDING } \\
\text { EQUIPMENT FDNS } \\
\text { EQUIPMENT FOUNDATIONS } \\
\end{array}$ & & LS & & \begin{tabular}{r|}
224,032 \\
0 \\
0 \\
0 \\
0 \\
17.818 \\
\end{tabular} & 14.00 & $\begin{array}{r}3,385,204 \\
0 \\
0 \\
0 \\
0 \\
204.775 \\
\end{array}$ & & \begin{tabular}{r|}
7.500 .000 \\
0 \\
0 \\
0 \\
0 \\
0 \\
\end{tabular} & & \begin{tabular}{r|}
$11.778,200$ \\
0 \\
0 \\
0 \\
0 \\
285,100 \\
\end{tabular} & & \begin{tabular}{r|}
$3,244,300$ \\
0 \\
0 \\
0 \\
0 \\
0 \\
\end{tabular} & $\begin{array}{r}25,907.704 \\
0 \\
0 \\
0 \\
0 \\
548,875\end{array}$ \\
\hline 3.000 & $\begin{array}{l}\text { TOTAL FOUNDATIONS } \\
\text { SITE } \\
\text { SITE AND UTILITIES }\end{array}$ & & LS & & $\begin{array}{r}17,018 \\
0 \\
0 \\
0 \\
0 \\
137,174\end{array}$ & 13.05 & \begin{tabular}{r|}
264.775 \\
0 \\
0 \\
0 \\
0 \\
$1.700,121$ \\
\end{tabular} & & $\begin{array}{l}0 \\
0 \\
0 \\
0 \\
0 \\
0\end{array}$ & & $\begin{array}{r}285,100 \\
0 \\
0 \\
0 \\
0 \\
1,022,680 \\
\end{array}$ & & $\begin{array}{r}0 \\
0 \\
0 \\
0 \\
0 \\
397.715 \\
\end{array}$ & $\begin{array}{r}549,075 \\
0 \\
0 \\
0 \\
0 \\
-4.110525\end{array}$ \\
\hline 2,000 & TOTAL SITE & & & & \begin{tabular}{r|}
137,174 \\
0 \\
0 \\
0 \\
0 \\
0 \\
0 \\
0 \\
0 \\
0
\end{tabular} & & $\begin{array}{r}1.700 .121 \\
0 \\
0 \\
0 \\
0 \\
0 \\
0 \\
0 \\
0 \\
0\end{array}$ & & $\begin{array}{l}0 \\
0 \\
0 \\
0 \\
0 \\
0 \\
0 \\
0 \\
0 \\
0\end{array}$ & & $\begin{array}{r}1,022,680 \\
0 \\
0 \\
0 \\
0 \\
0 \\
0 \\
0 \\
0 \\
0\end{array}$ & & $\begin{array}{r}397.715 \\
0 \\
0 \\
0 \\
0 \\
0 \\
0 \\
0 \\
0 \\
0\end{array}$ & $\begin{array}{r}4.110 .523 \\
0 \\
0 \\
0 \\
0 \\
0 \\
0 \\
0 \\
0\end{array}$ \\
\hline TOTAI & DIRECT & & & & 010.100 & & $0,055,302$ & & $15,153,224$ & & 23.285 .800 & & $8,352,010$ & $50.410,520$ \\
\hline
\end{tabular}




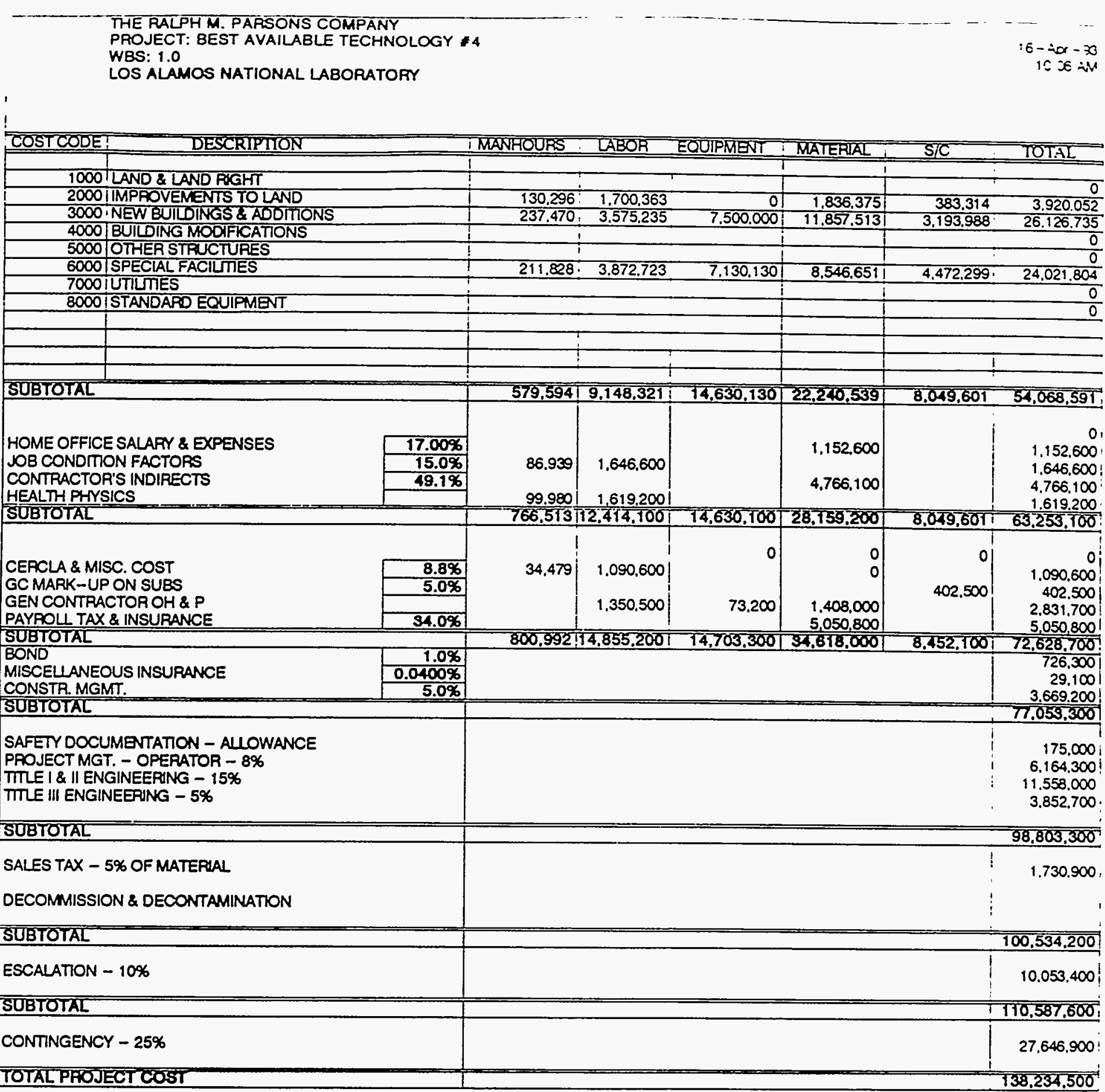




\begin{tabular}{|c|c|c|c|c|c|c|c|c|}
\hline $\begin{array}{l}\text { THE RALPH M. PARSONS COMPANY } \\
\text { PROJECT: BEST AVAILABLE TECHNOLOGY } * 4 \\
\text { WBS: } 1.0 \\
\text { LOS ALAMOS NATIONAL LABORATORY }\end{array}$ & & & & & & & & $\begin{array}{r}16-\mathrm{Apr}-93 \\
1006 \mathrm{AM}\end{array}$ \\
\hline & & & $\mathrm{LAB}$ & & & MÄTE & AIAL & \\
\hline DESCAIPTION & QTY & $\begin{array}{c}\text { UNIT } \\
\text { HOURS }\end{array}$ & $\begin{array}{l}\text { TOTAL } \\
\text { HOUAS } \\
\end{array}$ & $\begin{array}{c}\text { AVERAGE } \\
\text { AATE }\end{array}$ & $\begin{array}{l}\text { TOTAL } \\
\text { LABOR }\end{array}$ & $\begin{array}{l}\text { UNIT } \\
\text { PRICE }\end{array}$ & $\begin{array}{l}\text { TOTAL } \\
\text { MATL }\end{array}$ & $\begin{array}{l}\text { TOTAL } \\
\text { COST }\end{array}$ \\
\hline $\begin{array}{l}\text { 1. SAFETY TRAINING } \\
\text { 2. ENTRY/ EXIT MEDICAL EXAMS (EXAMS BY LANL STAFF) } \\
\text { 3. BIOASSAY TESTING ( } 1 \text { TEST/MANMEEK) } \\
\text { 4. ANNUAL MEDICAL EXAMS (EXAMS BY LANL STAFF) } \\
\text { 5. ANNUAL } 8 \text { HR REFRESHER TRAINING (1 YR) } \\
\text { 6. ATTRITION (20\% OF ITEMS } 1,2 \& 3 \text { ) }\end{array}$ & $\begin{array}{r}323 \\
323 \\
22080 \\
323 \\
323 \\
1\end{array}$ & $\begin{array}{r}64.00 \\
4.00 \\
0.16 \\
4.00 \\
8.00\end{array}$ & $\begin{array}{r}0 \\
0,677 \\
1,292 \\
3,533 \\
1,292 \\
2,585 \\
5,100 \\
0 \\
\end{array}$ & $\begin{array}{l}31.63 \\
31.63 \\
31.63 \\
31.63 \\
31.63 \\
\approx 1.63\end{array}$ & $\begin{array}{r}0 \\
654,000 \\
40,900 \\
111,700 \\
40,900 \\
81,800 \\
161,300 \\
0 \\
\end{array}$ & $\begin{array}{l}0.00 \\
0.00 \\
0.00 \\
0.00\end{array}$ & $\begin{array}{l} \\
\end{array}$ & $\begin{array}{r}05 \\
654,000 \\
40,900 \\
111,700 \\
40,900 \\
81,800 \\
161,300 \\
0\end{array}$ \\
\hline SUBTOTAL - CERCLA & & & 34,479 & & $1,090,600$ & & $\$ 0$ & $1,090,600$ \\
\hline PERSONNEL PROTECTION & & & & & & & & \\
\hline 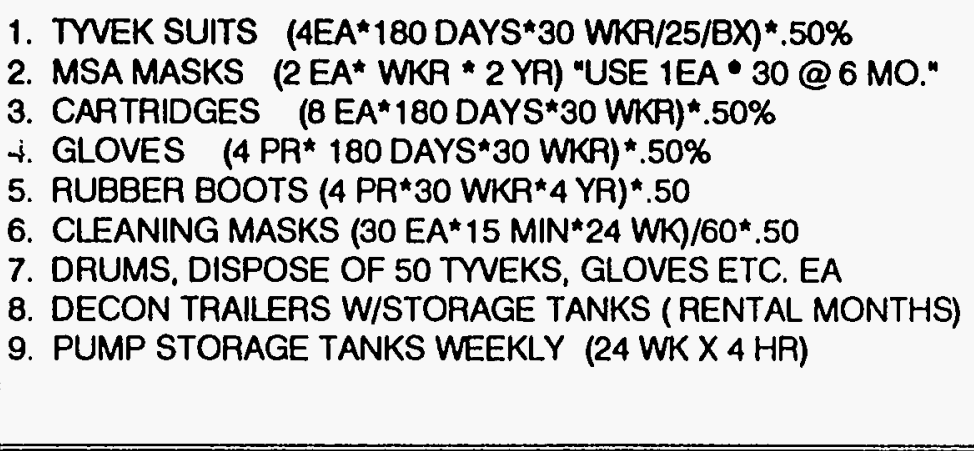 & & $\begin{array}{l}0.00 \\
4.00\end{array}$ & $\begin{array}{l}0 \\
0 \\
0 \\
0 \\
0 \\
0 \\
0 \\
0 \\
0 \\
0 \\
0 \\
\end{array}$ & & $\begin{array}{l}0 \\
0 \\
0 \\
0 \\
0 \\
0 \\
0 \\
0 \\
0 \\
0 \\
0\end{array}$ & $\begin{array}{r}85.00 \\
25.00 \\
1.00 \\
4.65 \\
20.00 \\
35.00 \\
35.00 \\
2.500 .00 \\
0.00\end{array}$ & $\begin{array}{l}0 \\
0 \\
0 \\
0 \\
0 \\
0 \\
0 \\
0 \\
0 \\
0 \\
0\end{array}$ & $\begin{array}{l}0 \\
0 \\
0 \\
0 \\
0 \\
0 \\
0 \\
0 \\
0 \\
0 \\
0\end{array}$ \\
\hline SUBTOTAL - PERSONNEL PROTECTION & & & (0) & & (0) & & - & 0 \\
\hline TOTAL CERCLA \& MISC COSTS & & & 34,479 & & $1,090,600$ & & 0 & $1,090,600$ \\
\hline
\end{tabular}




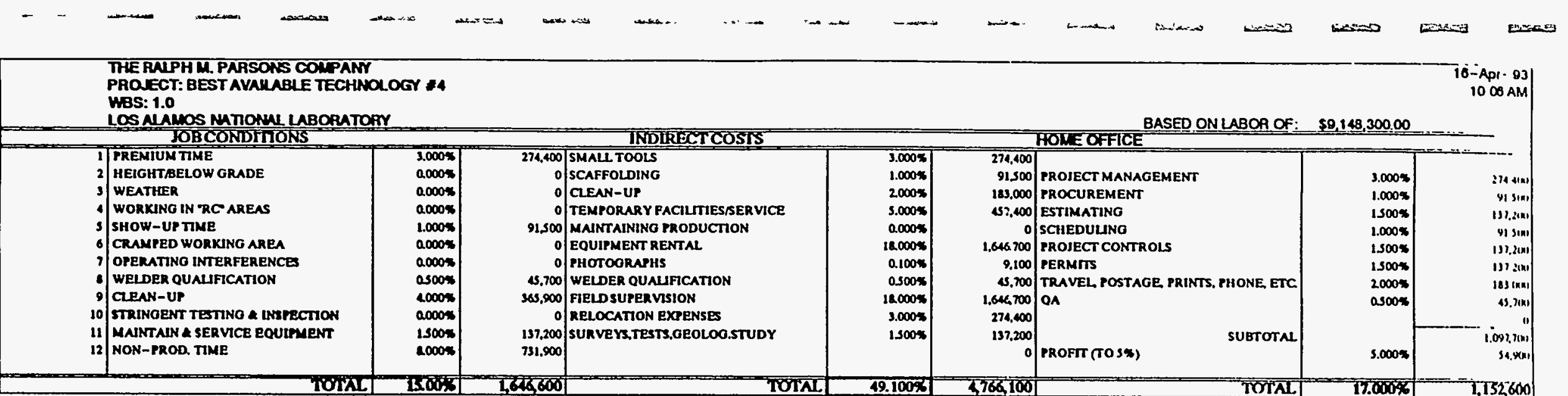




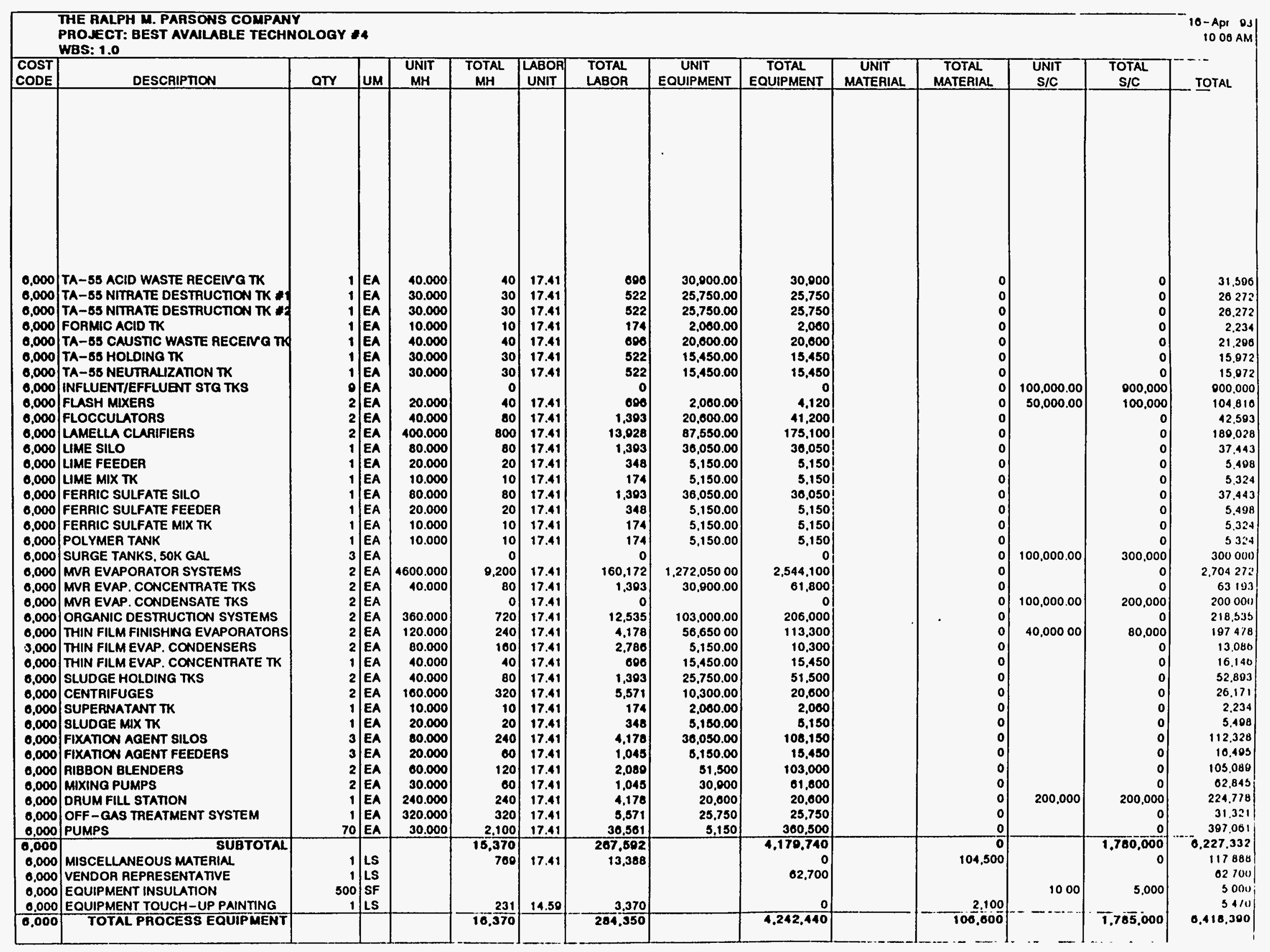




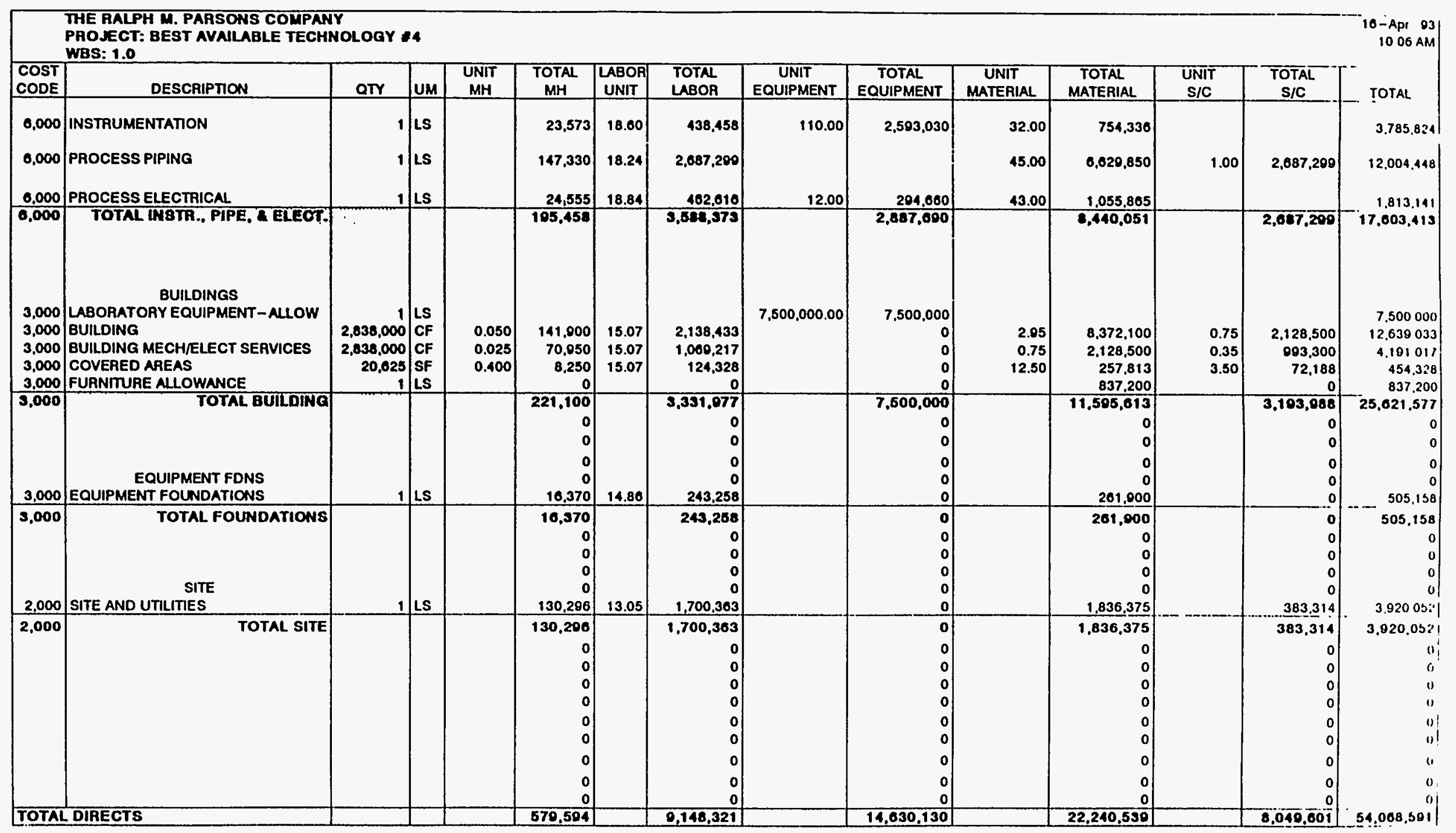


APPENDIX H

PROCESS CALCULATIONS 
These calculations are made to support a process design for a new Radioactive Liquid Waste Treatment Facility (RLWTF) to replace the current operations at TA-50. Design is based on treating the same volumes of waste and waste composition as is presently being processed at TA-50. But the design must be flexible enough to accommodate the many changes anticipated over the life of the plant. The first requirement is flexibility.

The present RLWP at TA-50 has been successfully meeting the required discharge limits during its many years of operation, but is having trouble meeting the increasingly more stringent release criteria; and this is expected to become even more stringent in the future. A second requirement is a high and predictable removal efficiency for any and all of the controlled pollutants.

The design capacity of the present plant is $250 \mathrm{gpm}$, but over the years the actual loading on the plant has decreased to $22 \times 10^{6} \mathrm{~L} / \mathrm{yr}$, and this has been worked off mostly in "batches"; that is, the plant has been run only 3 to 4 hours per day, 5 days per week, year round to support site operations, and operated successfully in this mode. In wastewater treatment, some of the more "high-tec" unit operations are not amenable to these kinds of operations. These unit operations include cross-flow filtration, reverse osmosis, electrodialysis, and ion exchange. They are not easily put on "standby" due to membrane fouling or ion migration; and if put on full recirculation, pumps must be kept running, back pulsers pulsing, anti-fouling chemicals continued to be added, etc. A weekly shutdown (as in five days per week operation) requires draining, flushing, and refilling with standby fluids. Two shifts per week may be lost for shutdown and start up. And even these, round the clock operations appear inconsistent with other Los Alamos operations.

The choice between a small (say $18 \mathrm{gpm}$ ), "high tec," round the clock operation and a larger (say $100 \mathrm{gpm}$ ), batch-continuous, 4hour per day operation is an important consideration, and often difficult to make or to justify. In the present case the choice was easier:

1. Large batch-continuous operations are typically much more flexible than closely-designed "high tec" operations.

2. Redundancy for reliable operations is easier to provide with the large batch-continuous type of operation.

3. $\quad \mathrm{R} / \mathrm{O}$ and $\mathrm{E} / \mathrm{O}$ cannot operate with high silica levels so a flocculation/sedimentation operation would be required in any event - so why not make this the primary operation?

4. The batch-continuous operation is much more compatible with the perceived "culture" established at TA-50 from past operations.

This includes type of operators, type of equipment, schedule of operations, control analyses, etc. Changes in these cost times and money for retraining and reduced performance on a new learning curve, so it is better not to change things unless really necessary.

Information available on current plant operations consists of a series of five annual reports (1987 through 1991), several standard operating procedures and safety analysis reports, five detailed analyses of the liquid waste influents representing different mixtures over the period March 25, 1991 through April 23, 1991, some sludge analyses, chemical use rates, and some bench scale test results. Since the five annual reports provide most of the information on what it is to be processed, these reports were extensively analyzed; but it must be recognized that this is "production" data, collected and reported for other purposes. This leaves much to be desired when attempting to use such information as a design basis. The five samples collected for detailed analysis represent a very short period of time. On an annual basis, the TA-55 flow treated through Room 60 , and released to mix into the main TA-50 inflow, represents only about $120,000 \mathrm{~L} / \mathrm{yr} \div 20.5 \times 10^{6} \mathrm{~L} / \mathrm{yr}=0.6$ percent of the total influent. But during its periodic additions it has a large impact on TDS because of its very high concentration of dissolved solids ( -20 percent or more). Unless the percentage of Room 60 effluent contained in a sample is closely known, it is difficult to extrapolate such data to annual loads. The results of the five analyses do indicate that instantaneous values of TA-50 influent vary from about $900 \mathrm{ppm}$ to $6,200 \mathrm{ppm}$ TDS depending on the concentration of TDS in the "neat" TA-50 inflow and how much Room 60 effluent is being blended into it. 
This means that for the small continuous designs with a fixed DF (e.g, R/O) either large surge volumes for influent and effluent must be provided to smooth out the variations, or considerable over design, based on peak values, is required.

It appears that most of the TDS will have to be removed from the wastewater to meet the $\mathrm{NO}_{3}$ release criteria, and most probably the soluble radioactivity. This TDS will comprise a large secondary waste; 1990 releases were $53,000 \mathrm{Kg}$, still contaminated with some residual radioactivity. Disposal costs will be significant. It is, therefore, important to carefully estimate the expected size of this waste stream. The values abstracted from the annual reports are shown at the top of the next page, sheet No. 3 .

There is an obvious reversal in the 1990-1991 values as compared to what went before, almost as if the inlet and outlet values were switched. Examination of a further breakdown to individual components, as shown on sheet No. 4, provided an explanation. The reported sampling problem arose because of a change in operations due to equipment problems. Neutralization caustic $(\mathrm{NaOH})$ bad to be dumped in by hand overdosing the influent in most cases. Then because the $\mathrm{NaOH}$ was not well mixed into the stream by the time it reached the sampling permit, the sample was "rich" in $\mathrm{NaOH}$. Note the 30,800 and $42,000 \mathrm{Kg} / \mathrm{yr} \mathrm{Na}^{+}$reported in 1990 and 1991 . Then since there is no recognized means of removal of $\mathrm{Na}^{1}$ ions between influent and effluent (see $\mathrm{Na}^{+}$values for 1990 and 1991 effluent), there is an obvious overly high value of $\mathrm{Na}^{+}$and $\mathrm{M}$ and $\mathrm{P}$ alkalinity which measures $\mathrm{OH}^{-}$ions.

This removes this particular anomaly, and since the influent is a yearly average of a BLENDED stream it is concluded that an "upside" average value of TDS in the blended stream is $2,500 \mathrm{mg} / \mathrm{L}(\mathrm{ppm})$, not the $6,200 \mathrm{mg} / \mathrm{L}$ suggested elsewhere. The range of this value is probably $600 / 6,000$ for the blended stream.

The higher effluent values for 1987, 1988, and 1989 were most influenced by the increases in $\mathrm{Na}^{+}$and $\mathrm{NO}_{3}{ }^{-}$ between influent and effluent. The reduction in these increases (between in and out) in 1990 and 1991 is assumed to reflect a change in operation.

The $\mathrm{Cl}^{-}, \mathrm{F}^{-}$, and $\mathrm{K}^{+}$are all constituents associated with the waste coming in from TA-55. There is acceptable agreement between inlet and outlet in the context of known operations; and a gradual decrease from 1987 to 1991, reflecting a reduction in the volume of TA-55 flow. This confirms quality of the data. The $\mathrm{Ca}^{++}$and $\mathrm{SO}_{4}{ }^{*}$ reflect the additions of $\mathrm{Ca}(\mathrm{OH})_{2}$ and $\mathrm{Fe}_{2}\left(\mathrm{SO}_{4}\right)_{3} 3 \mathrm{H}_{2} \mathrm{O}$ used in the flocculation/clarification operations. The $\mathrm{Ca}^{++}$in the influent must reflect $\mathrm{Ca}(\mathrm{OH})$ used in the Room 60 operations; but since saturated values of $\mathrm{Ca}^{++}$ are low, and dilution is high, the values look reasonable.

The $\mathrm{Mg}^{++}$and $\mathrm{PO}_{4}{ }^{-}$represent species which should be reduced by the known plant operations and the data correctly reflects this. All of those results engender confidence in the reported results for current operations on a BLENDED stream.

There is less information available on the solid waste streams. Volumes are reported but there is little information on composition or the dose rates of the various chemicals used to form the sludges. Reported chemical usage does not seem to agree with the waste produced; material balances would be meaningless. Even the D.F across the main TA-50 process has an uncertainty of 2.4 in it.

The TA-50 alpha removal process is quite efficient, reducing the influent from $1.43 \mathrm{Ci} / \mathrm{yr}$ of gross $\propto$ (per 1990 annual report) to an effluent of $0.00367 \mathrm{Ci} / \mathrm{yr}$, a 99.74 percent removal and a $\mathrm{DF}=390$. Based on the gross $\propto$ reported in the filter cake, however, (this is where the removed $\propto$ has to go) of $3.47 \mathrm{Ci} / \mathrm{yr}$, the performance is even better, 99.89 percent removal for a DF $=946.946 / 390=2.4 \mathrm{x}$ difference.

The difference between the $1.43 \mathrm{Ci} / \mathrm{yr}$ reported in the influent and the $3.47 \mathrm{Ci} / \mathrm{yr}$ reported discharged in the filter cake is unexplained. A transfer from Room 60 was suspected, but would be included in the influent measurements. Measurement problems are now suspected. This points out a problem of representative numbers to use as a basis of design. 


\begin{tabular}{||c|l|l|l||}
\hline & \multicolumn{1}{|c|}{$\begin{array}{c}\text { Total Cation } \\
\text { meq/L } \\
\text { Low/High-Average }\end{array}$} & $\begin{array}{r}\text { T.D.S. } \\
\text { mg/L } \\
\text { Low/High - Average }\end{array}$ & \multicolumn{1}{c||}{$\begin{array}{c}\text { T.S.S. } \\
\text { mg/L } \\
\text { Low/High - Average }\end{array}$} \\
\hline $\begin{array}{c}\text { 1987 Influent: } \\
\text { Effluent: }\end{array}$ & $\begin{array}{l}15.6 / 38.3-26.5 \\
29.4 / 163-56.8\end{array}$ & $\begin{array}{l}956 / 3230-2140 \\
2080 / 13,000-4150\end{array}$ & $\begin{array}{l}20 / 167-59 \\
0\end{array}$ \\
\hline $\begin{array}{c}1988 \text { Influent: } \\
\text { Effluent: }\end{array}$ & $\begin{array}{l}9 / 27-17 \\
23 / 87-44\end{array}$ & $\begin{array}{l}802 / 2330-1480 \\
1700 / 15,140-3120\end{array}$ & $0 / 95-33$ \\
\hline 1989 Influent: & $14 / 37-25$ & $1230 / 3120-2040$ & 0 \\
\hline Effluent: & $25 / 103-53$ & $2010 / 7960-4070$ & $11 / 99-34$ \\
\hline 1990 Influent: & $9.1 / 146-43.3$ & $986 / 11,100-3680^{*}$ & $0 / 206-59$ \\
Effluent: & $14.6 / 47.2-33.4$ & $1330 / 3570-2550$ & 0 \\
\hline 1991 Influent: & $5.6 / 118-52.6$ & $646 / 10,000-3570^{*}$ & $22 / 163-70$ \\
Effluent: & $17.5 / 32.9-26.5$ & $1080 / 2370-1810$ & \\
\hline 1991 Samples ${ }^{+}$ & $10.5 / 26-$ & $916 / 6200-$ & Filtered \\
\hline \hline
\end{tabular}

+5 samples analyzed for W. Midkiff 3/22/91 - 4/15/91

${ }^{*}$ Known sampling problems 
Reported Annual Releases in Kilograms

Based on Volume-Weighted Averages

(Based on Daily Sampling)

\begin{tabular}{|c|c|c|c|c|c|c|c|c|}
\hline Year & \multicolumn{2}{|c|}{ M-ALK } & \multicolumn{2}{|c|}{ P-ALK } & \multicolumn{2}{|c|}{$\mathrm{Na}^{+}$} & \multicolumn{2}{|c|}{$\mathrm{NasNO}_{3}^{-}$} \\
\hline 1 & IN & OUT & IN & OUT & IN & OUT & IN & OUT \\
\hline 1987 & 3320 & 16,000 & 1990 & 0 & 8420 & 21,800 & 5580 & 13,400 \\
\hline 1988 & 806 & 12,500 & 64 & 0 & 7240 & 24,200 & 6050 & 12,200 \\
\hline 1989 & 1150 & 11,500 & 275 & 63 & 8080 & 22,000 & 5900 & 11,400 \\
\hline 1990 & 31,900 & 11,200 & 30,100 & 0 & 30,800 & 12,300 & 5740 & 6210 \\
\hline 1991 & 44,000 & 11,400 & 41,700 & 29 & 42,000 & 8630 & 2420 & 3570 \\
\hline Year & \multicolumn{2}{|c|}{$\mathrm{Cl}^{-}$} & \multicolumn{2}{|c|}{$\mathrm{F}^{-}$} & \multicolumn{2}{|c|}{$\mathrm{K}^{+}$} & \multicolumn{2}{|c|}{$\mathrm{Mg}^{++}$} \\
\hline$\downarrow$ & IN & OUT & IN & OUT & IN & OUT & IN & OUT \\
\hline 1987 & 2140 & 3250 & 188 & 187 & 3560 & 4430 & 84.2 & 27.1 \\
\hline 1988 & 2400 & 3920 & 313 & 306 & 5550 & 8360 & 101 & 12 \\
\hline 1989 & 2360 & 4090 & 293 & 234 & 3360 & 3670 & 92.3 & 18.4 \\
\hline 1990 & 1860 & 2040 & 282 & 237 & 2770 & 3050 & 95.3 & 0.5 \\
\hline 1991 & 1480 & 1820 & 76 & 72 & 689 & 1100 & 92.4 & 4.3 \\
\hline Year & \multicolumn{2}{|c|}{$\mathrm{Ca}^{++}$} & \multicolumn{2}{|c|}{$\mathrm{SO}_{4}{ }^{=}$} & \multicolumn{2}{|c|}{ TDS } & \multicolumn{2}{|c|}{$\mathrm{PO}_{4}{ }^{=}$} \\
\hline 1 & IN & OUT & IN & OUT & IN & OUT & IN & OUT \\
\hline 1987 & 509 & 4200 & 1120 & 6410 & 48,500 & 108,000 & 298 & 40 \\
\hline 1988 & 510 & 6700 & 15 & 5730 & 42,900 & 106,000 & 72 & 8 \\
\hline 1989 & 508 & 4660 & 537 & 4620 & 41,000 & 95,000 & 116 & 6.7 \\
\hline 1990 & 633 & 5160 & 750 & 4090 & 68,700 & 53,300 & 83.6 & 4.7 \\
\hline 1991 & 492 & 6350 & 612 & 2830 & 71,600 & 39,500 & 111 & 17 \\
\hline
\end{tabular}


The efficiency of the TA-50 flocculation/clarification/sedimentation operations is high as compared to other reported operations of a similar kind. This improved efficiency can be explained in several ways.

1. The burden may be different, larger, easy to remove particles.

2. A long retention time allows more of the near-colloidal material to settle out.

3. There is an enhanced "gettering" action from long contact with a high concentration of recirculated solids.

The third choice seems most likely, and is assumed for this process design. Unfortunately, there is insufficient information to define "LONG" or "HIGH."

Bench scale tests have been suggested to help define these parameters, but results cannot be expected in the time frame of this study. It becomes quite apparent that any proposed design will suffer from large "holes," areas where data does not exist and must be bridged by assumptions. Under these circumstances the design reflects an approach which attempts to minimize the impact of these "holes."

All of the TA-50 influent analyses reflect some added fraction of Room 60 effluent, always a "blended" case. To develop a TA-50 "neat" influent, the Room 60 effluent must be "subtracted" out of the average "blend." But this gives no feel of the expected range of a "neat" influent for design. And all this mathematics has to be based on a SINGLE Room 60 effluent analysis which may or may not be representative. We must assume that it is.

Based on a ratio of flows of $120,000 \mathrm{~L} / \mathrm{yr}$ to $20.5 \times 10^{6} \mathrm{~L} / \mathrm{yr}$, assuming that $96,000 \mathrm{ltr}$ of the 120,000 (from previous ratios) contains $3.5 \mathrm{~N} \mathrm{HNO}_{3}$, and calculating the required dilution based on the $\mathrm{NO}_{3}{ }^{-}$found in the Room 60 effluent sample (this calculation is shown elsewhere, sheet No. 10), the Room 60 effluent contributes about $1,540 \mathrm{mg} / \mathrm{L}$ of T.D.S. to the TA-50 influent $(0.168 / 20.5)(188,000)=1,540$. Assuming $2,500 \mathrm{mg} / \mathrm{L}$ average for the blended TA-50 influent, minus $1,540 \mathrm{mg} / \mathrm{L}$ from TA-55 flow leaves $967 \mathrm{mg} / \mathrm{L}$ for the AVERAGE neat flow with a range of (see sheet No. 7) about 500 low to 3,000 high about the 967 average.

This is our basis and we now proceed to the calculations.

Examination of the previously developed calculations showed a certain "looseness" and variance in the calculated numbers appearing at different locations in these calculations. These occurred because there was no "established" values to use as a design basis; most of the values used were extracted from annual reports and these values were not developed specifically for use as a design basis.

As an example the 1990 report was used as a "typical case." But the 1990 report shows an influent to TA-50 of $19.1 \times 10^{6} \mathrm{~L} / \mathrm{yr}$, not the $20.5 \times 10^{6} \mathrm{~L} / \mathrm{yr}$ used for design. The effluent is $21.1 \times 10^{6} \mathrm{~L} / \mathrm{yr}$, not the $22 \times 10^{6} \mathrm{~L} / \mathrm{yr}$ used for design. When concentrations are used with these numbers to calculate mass values and then these masses are redivided by a different number to get back to concentrations, variations of 4 to 7 percent appear. Conclusions are not affected, but the variations appear "messy," so corrections were necessary for appearances.

The assumption is made that TA-55 effluent equals the current Room 60 effluent at $120,000 \mathrm{~L} / \mathrm{yr}$ as a design basis.

But there is a volume increase (calculated as a 1.4 factor) in going from Room 60 influent to Room 60 effluent, and since the ONLY analysis of this flow is based on a sample of the effluent, ignoring this factor will have a significant impact on the calculation of what the TA-50 influent would be if this stream was not mixed in, a TA-50 "neat" influent.

We use as a design bases $120,000 \mathrm{~L} / \mathrm{yr}$ from TA-55.

1. 80 percent contains $3.5 \mathrm{~N} \mathrm{HNO}_{3}$ plus other dissolved material. 
2. 20 percent contains $0.5 \underline{\mathrm{N}} \mathrm{HNO}_{3}$ plus other dissolved material.

Based on the calculated dilution factor and an analysis showing $188,000 \mathrm{mg} / \mathrm{L}$ TDS (numbers in this summary are calculated elsewhere),

$120.000 \mathrm{~L} / \mathrm{yr} \times 1.4=168,000 \mathrm{~L} / \mathrm{yr}$

$168,000 \mathrm{~L} / \mathrm{yr} \times 188,000 \mathrm{mg} / \mathrm{L}=31,584 \mathrm{Kg} / \mathrm{yr}$,

added to the TA-50 influent by Room 60 effluent.

If TA-50 total influent is $20.5 \times 10^{6} \mathrm{~L} / \mathrm{yr}$, at assumed $2,500 \mathrm{mg} / \mathrm{L}, \mathrm{TA}-50$ "neat" influent $=20.5 \times 10^{6} \mathrm{~L} / \mathrm{yr}$ $168,000 \mathrm{~L} / \mathrm{yr}=\underline{20,332} \mathrm{~L} / \mathrm{yr} \times 10^{6}$.

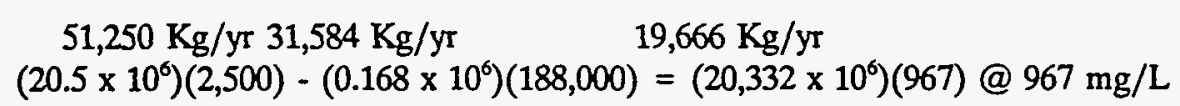

This number is important for a design based on treating a "neat" TA-50 stream.

$96,000 \mathrm{~L} / \mathrm{yr} @ 3.5 \mathrm{~N}$ gives $217 \mathrm{~g} / \mathrm{L} \mathrm{NO}_{3}{ }^{-}$and $0 \mathrm{NaOH}$

$24,000 \mathrm{~L} / \mathrm{yr} @ 0 \quad 0 \mathrm{~g} / \mathrm{L} \mathrm{NO}_{3}{ }^{-}$and $20 \mathrm{~g} / \mathrm{L} \mathrm{NaOH}$

The $\mathrm{OH}^{-}$is only 3.6 percent of required to neutralize the $\mathrm{H}^{+}$

Add $\mathrm{NaOH}$ to:

$\sum 120,000 \mathrm{~L} / \mathrm{yr}$ contains $173.6 \mathrm{~g} / \mathrm{L} \mathrm{NO}_{3}{ }^{-}$and $64.4 \mathrm{~g} / \mathrm{L} \mathrm{Na}^{+}$

TOTAL SALTS $=188 \times 1.4=263 ;-(173.6+64.4)=263 \mathrm{~g} / \mathrm{L}-238 \mathrm{~g} / \mathrm{L}=25 \mathrm{~g} / \mathrm{L}$

if the $\mathrm{Na}^{+}$and $\mathrm{NO}_{3}^{-}$were not in mixture

or at $168,000 \mathrm{~L} / \mathrm{yr}$, reduce conc. of $\mathrm{Na}^{+}$and $\mathrm{NO}_{3}{ }^{-}$by 1.4 factor at $168,000 \mathrm{~L} / \mathrm{yr}$ contains $124.0 \mathrm{~g} / \mathrm{L} \mathrm{NO}_{3}{ }^{-}$and $46 \mathrm{~g} / \mathrm{L} \mathrm{Na}^{+}$.

By analysis $-188,000 \mathrm{mg} / \mathrm{L}$ TDS; $188 \mathrm{~g} / \mathrm{L}-124-46=17.9 \mathrm{~g} / \mathrm{L}$ if no $\mathrm{NaNO}_{3}$ add "back" the 3.6 percent $\mathrm{HNO}_{3}$ to neutralize the $\mathrm{NaOH}$.

$(168,000 \mathrm{~L} / \mathrm{yr})=4.46 \mathrm{~g} / \mathrm{L} \mathrm{NO}_{3}{ }^{-}$and $1.66 \mathrm{~g} / \mathrm{L} \mathrm{Na}^{+} ; 17.9+4.1=24 .^{+} \mathrm{g} / \mathrm{L}$ which is close to the $25,000 \mathrm{mg} / \mathrm{L}$ TDS assumed for the Room 60 effluent after partial nitrate destruction. Thereafter $\mathrm{NO}_{3}{ }^{-}$partial destruction, the blended stream is:

$$
\begin{aligned}
& \left(20,332 \times 10^{6}\right) \mathrm{L} / \mathrm{yr}(967 \mathrm{mg} / \mathrm{L})+\left(0.168 \times 10^{6}\right) \mathrm{L} / \mathrm{yr}(25,000 \mathrm{mg} / \mathrm{L})=23,866 \mathrm{Kg} / \mathrm{yr} \\
& 19,666 \mathrm{Kg} / \mathrm{yr} \quad 4,200 \mathrm{Kg} / \mathrm{yr} \\
& \underline{23.866 \mathrm{Kg} / \mathrm{yr}} \doteq 1,164 \mathrm{mg} / \mathrm{L} \text { design value for blended stream } \\
& 20.5 \times 10^{6} \mathrm{~L} / \mathrm{yr} \quad \text { AFTER Partial } \mathrm{NO}_{3}{ }^{-} \text {DESTRUCTION }
\end{aligned}
$$

As shown elsewhere, based on historic data, the number used for "present" operations is (blended).

$$
20.5 \times 10^{6} \mathrm{~L} / \mathrm{yr} @ 2,500 \mathrm{mg} / \mathrm{L} \text { TDS influent }
$$

In passing through the plant, the volume is increased to $22.0 \times 10^{6} \mathrm{~L} / \mathrm{yr}$ from various operational additions. Changes in concentration will be caused by:

$\begin{array}{ll}\text { Dilution } & \text { decrease } \\ \text { Precipitation } & \text { decrease } \\ \text { Partial } \mathrm{COD} \text { remove } & \text { decrease } \\ \text { Add } \mathrm{SO}_{4}{ }^{-} & \text {increase }\end{array}$


There is insufficient information to quantify these changes, or to complete a material balance; and indeed, the numbers appear to be changing mostly in recent years. A reasonable assumption is the same $2,500 \mathrm{mg} / \mathrm{L}$ TDS used for the influent, assuming all these changes will just about cancel out, assuming a 2,500 $\mathrm{mg} / \mathrm{L}$ EFFLUENT of $2.2 \times 10^{6} \mathrm{~L} / \mathrm{yr}$ does not affect the design, but does influence the calculations of secondary waste.

Comparing the $2,500 \mathrm{mg} / \mathrm{L}$ TDS concentration to the influent and effluent values on sheet No. 3, show the $2,500 \mathrm{mg} / \mathrm{L}$ is high for the influent if the two "known to be bad" values of 1990 and 1991 are excluded. The $2,500 \mathrm{mg} / \mathrm{L}$ is $\underline{\text { low }}$ for the effluent vs. a $5-\mathrm{yr}$ average of $3,140 \mathrm{mg} / \mathrm{L}$, but close to the 1990 values assumed to be typical of CURRENT operations and what can reasonably be expected for future operations (particularly elimination of the recarbonation step).

Five years of historic data was analyzed and averaged to develop information on past decontamination factors (DF) and to determine further DFs required to meet the various release criteria. Several aspects of this analyses should be noted. First, all the data represents performance on a blended stream of Room 60 effluents and other (neat) TA-50 influents. Second, there is a high probability that there was a significant improvement in certain DFs as a result of the change in operation from parallel to series on the present flocculators.

Third, on average, no further DF was required to meet the "OLD" NPDES. The plant was in compliance, on an average basis. It is known that some releases exceeded the NPDES limits, or the DOE 5400.5 limits on rare occasions. This points out the value of having "equalization" tankage and recycle capability to smooth out peak values causing non-compliance.

In present operations, the TA-55 effluent is processed twice; once in Room 60, then in the main TA-50 plant. In the proposed processing scheme, the TA-55 effluent will be processed only once in an operation similar to present Room 60 practice. It was, therefore, necessary to look carefully at the contributions of Room 60 effluent to TA-50 influent to try and detect any adverse effect on the predicted DF of the single treatment. Based on the single 1991 analysis of Room 60 effluent, the following table is developed. (See sheet 8)

Except for Silver, Ag, the major contributions of Room 60 are all apparently soluble constituents not expected to be effected by precipitation. Considering the expected DF from improved operations (more like the $1991 \mathrm{DFs}$ rather than the 5-yr averages), the design looks acceptable.

The nitrate wastes from TA-55 is recognized as adding a significant fraction of the total TDS in the TA-50 effluent presently being released to the environment and the major fraction of the nitrates. Since the nitrates in TA-50 effluent must be reduced, various approaches are considered.

Using as a basis $120,000 \mathrm{~L} / \mathrm{yr}$ from TA-55 with 80 percent nitrate wastes at $3.5 \mathrm{~N} \mathrm{HNO}_{3}$ and 20 percent "caustic" wastes at $0.5 \underline{\mathrm{N}} \mathrm{NaOH}$ (plus $\mathrm{CL}^{-}, \mathrm{F}^{-}, \mathrm{K}$, etc.)

$$
\begin{aligned}
-3.5 \mathrm{~N} \mathrm{HNO}_{3} \times 96,000 \mathrm{~L} / \mathrm{yr}=336,000 \mathrm{~g} \text {-mole of } \mathrm{HNO}_{3} / \mathrm{yr} \\
\begin{aligned}
(\mathrm{mole} / \mathrm{ltr}) \quad \text { x } 63 \mathrm{~g} / \mathrm{g} \text {-mole }=21,168,000 \mathrm{~g} / \mathrm{yr} & =21,168 \mathrm{Kg} / \mathrm{yr} \\
& =46,667 \mathrm{lb} / \mathrm{yr}
\end{aligned}
\end{aligned}
$$

or if considering only the $\mathrm{NO}_{3}{ }^{-}$ion, in $22 \times 10^{6} \mathrm{~L} / \mathrm{yr}$;

$$
\frac{21,168 \mathrm{Kg} / \mathrm{yr} \times 62 / 63}{22 \times 10^{6} \mathrm{ltr} / \mathrm{yr}}=947 \mathrm{ppm} \text { or } \mathrm{mg} / \mathrm{L}
$$

Even considering evaporation as the method of removal if this $\mathrm{HNO}_{3}$ is neutralized with $\mathrm{NaOH}$, the resulting

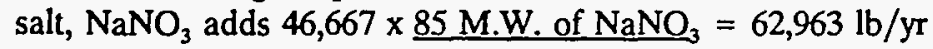

$63 \mathrm{M} . \mathrm{W}$. of $\mathrm{HNO}_{3}$

to the evaporator bottoms, a low leyel radiological waste, and a significant disposal cost. 


\begin{tabular}{||c|c|c|c|c|c||}
\hline & \multicolumn{2}{c}{$\begin{array}{c}\text { 1991 Analysis } \\
\text { Room 60 Effluent }\end{array}$} & \multicolumn{2}{c|}{$\begin{array}{c}\text { 1991 Average } \\
\text { TA-50 Influent } \\
\mathrm{mg} / \mathrm{L} \times 168,000 \mathrm{~L} / \mathrm{yr}=\mathrm{g} / \mathrm{yr}\end{array}$} & $\begin{array}{c}\text { \%g/L } \times 20.5 \times 10^{6} \mathrm{~L} / \mathrm{yr}=\mathrm{g} / \mathrm{yr} \\
\text { from } \\
\text { Room } 60\end{array}$ \\
\hline $\mathrm{As}$ & 0.77 & $129.4^{*}$ & $3.4 \mathrm{E}-3$ & 69.7 & 100. \\
\hline $\mathrm{Ba}$ & 0.21 & 35.3 & 0.1 & 2050 & 1.7 \\
\hline $\mathrm{Cd}$ & 0 & 0 & 0.01 & 205 & 0 \\
\hline $\mathrm{Cr}$ & 0.44 & 73.9 & 0.1 & 2050 & 3.6 \\
\hline $\mathrm{Cu}$ & $<0.003$ & $<0.5$ & 1.2 & 24,600 & 0 \\
\hline $\mathrm{CN}-$ & 6.15 & 1033 & 0.2 & 4100 & 25 \\
\hline $\mathrm{Pb}$ & 0.33 & 55.4 & 0.3 & 6150 & 0.9 \\
\hline $\mathrm{Hg}$ & 0.004 & 0.67 & 0.01 & 205 & neg. \\
\hline $\mathrm{Ni}$ & 0.02 & 3.4 & 0.3 & 6150 & 0.5 \\
\hline $\mathrm{NH}$ & 4.6 & 773 & 6.3 & 129,150 & 0.6 \\
\hline $\mathrm{Se}$ & 1.6 & $269^{* *}$ & $3.7 \mathrm{E}-3$ & 76 & ALL ? \\
\hline $\mathrm{Ag}$ & 0.44 & 73.9 & 0.02 & 410 & 18 \\
\hline $\mathrm{Zn}$ & 0 & 0 & 0.50 & 10,250 & 0 \\
\hline
\end{tabular}

*Questionable data

** Very questionable data! 


\begin{tabular}{||c|c|c|c|c||}
\hline Year & $\begin{array}{c}\text { Process Caustic } \\
\text { Liters/Yr }\end{array}$ & $\begin{array}{c}\text { Process Acid } \\
\text { Liters/Yr }\end{array}$ & $\begin{array}{c}\text { Total } \\
\text { Liters/Yr }\end{array}$ & $\begin{array}{c}\text { Cement Paste } \\
\text { BBL/Yr }\end{array}$ \\
\hline 1991 & $\begin{array}{c}9236 \\
16 \%\end{array}$ & $\begin{array}{c}49,458 \\
84 \%\end{array}$ & 58,694 & 0 \\
\hline 1990 & $\begin{array}{c}24,077 \\
20 \%\end{array}$ & $\begin{array}{c}97,474 \\
80 \%\end{array}$ & 121,551 & 24 \\
\hline 1989 & 27,176 & $\begin{array}{c}139,661 \\
84 \%\end{array}$ & 166,857 & 32 \\
\hline 1988 & $16 \%$ & $\begin{array}{c}138,910 \\
82 \%\end{array}$ & 170,365 & 21 \\
\hline 1987 & $18 \%$ & 148,166 & 185,240 & 47 \\
\hline
\end{tabular}

Assume $120,000 \mathrm{Ltr} / \mathrm{Yr} ; 80 \%$ Acid $(96,000 \mathrm{Ltr} / \mathrm{Yr})$ and $20 \%$ Caustic $(24,000 \mathrm{Ltr} / \mathrm{Yr})$

Average 30 BBL Cement Paste/Yr 
This nitrate should be removed at the source, or, barring that, either removed and recycled or destroyed. The $\mathrm{HNO}_{3}$ can be removed by "acid retardation," a form of ion exchange using standard resins or the more chemical resistant "durasils," or by electrodialysis, E/D. Either method removes, selectively, the $\mathrm{HNO}_{3}$ from the main process stream and recovers it as a concentrate ready for recycle. This is probably the best choice if it could be implemented. But in view of the many unknowns to such a recycle scheme, chemical destruction of the nitrate is shown on the PFD.

Chemical denitrification is a well-established unit process, particularly in the nuclear industry.* Organics such as formic acid, formaldehyde and even sucrose (sugar) are reacted with $\mathrm{HNO}_{3}{ }^{-}$and nitrates at prescribed times and temperatures to break the $\mathrm{NO}_{3}$ down into $\mathrm{N}_{2}, \mathrm{~N}_{2} \mathrm{O}, \mathrm{NO}$, and $\mathrm{NO}_{2}$, with the organics going to $\mathrm{CO}_{2}$ and $\mathrm{H}_{2} \mathrm{O}$. Some bench scale work may be required to define the exact conditions required, but we are dealing with a relatively small stream, and, as shown in the following, complete destruction is not required.

The $\mathrm{NO}_{3}-$ ion is present at $(3.5 \underline{\mathrm{N}})(62 \mathrm{~g} / \mathrm{N})=217 \mathrm{~g} / \mathrm{ltr}$ in the acid flow, and $217 \mathrm{~g} / \mathrm{L} \times \frac{96,000 \mathrm{~L}}{120,000 \mathrm{~L}}=173.6 \mathrm{~g} / \mathrm{ltr}$ $120,000 \mathrm{~L}$

in the mixed flow. If as shown by analysis of the Room 60 effluent, $\mathrm{N}$ as $\mathrm{NO}_{3}{ }^{-}=28,000 \mathrm{ppm}$, $\mathrm{NO}_{3}=28,000 \times \underline{\mathrm{NO}}_{3}=\underline{62}=124,000 \mathrm{ppm}$

$$
\mathrm{N} \quad 14 \quad \text { (densities not considered in this approximation) }
$$

there has been a dilution of from $173.6 \mathrm{~g} / \mathrm{L}$ to $124 \mathrm{~g} / \mathrm{L}$

$\underline{173.6}=1.40$, a 40 percent dilution, or $48,000 \mathrm{~L} / \mathrm{yr}$

124

added to a flow of $120,000 \mathrm{~L} / \mathrm{yr}$.

It is known that $\sim 660 \mathrm{gal}$ of sludge (22 gal/BBL $\times 30 \mathrm{BBL} / \mathrm{yr}$ ) was "washed" at $-3: 1$ volume ratio three times $\left(\sim 70: 1\right.$ dilution of salt content and $\left.4^{3}=64\right)$ which used -1980 gal per washing.

$1980 \mathrm{gal} /$ wash $\times 3$ washes $\times 3.785 \mathrm{~L} / \mathrm{gal}=\underline{22.483} \mathrm{~L}$, plus water produced by reaction:

$$
\begin{aligned}
& \mathrm{NaOH}+\mathrm{HNO}_{3} \rightarrow \mathrm{HOH}+\mathrm{NaNO}^{3} \\
& 3.5 \underline{\mathrm{N}} \quad 3.5 \mathrm{~N} \\
& \text { Water formed: } \\
& 3.5 \underline{\mathrm{N}} \text { or mole } / \mathrm{ltr} \times 18 \mathrm{~g} / \mathrm{mole}=63 \mathrm{~g} / \mathrm{L} \\
& 63 \mathrm{~g} / \mathrm{L} \times 0.8=5 \text { percent }
\end{aligned}
$$

So, considering water formed from chemical reactions, back flushes, washings, water in chemical solutions added, etc., this is considered an explained degree of dilution. Using the Room 60 effluent analyses, if all concentrations (not affected by operations in Room 60) are multiplied by 1.4, we can estimate the concentration (mg/L) of Room 60 influents.

Since the analysis of the effluent from Room 60 shows $188,000 \mathrm{mg} / \mathrm{L}$ TDS, the influent (or before dilution) is about

$$
188,000 \mathrm{mg} / \mathrm{L} \times 1.4 \text { factor }=263,000 \mathrm{mg} / \mathrm{L} \text { TDS }
$$

If the $\mathrm{HNO}_{3}$ is removed by destruction, and no $\mathrm{NaOH}$ used to neutralize it, $173.6 \mathrm{~g} \mathrm{NO}_{3}{ }^{-} / \mathrm{L}$ are removed and

$$
173.6 \times \frac{\mathrm{Na}^{+}=23}{\mathrm{NO}_{3}^{-}=62}=64.4 \mathrm{~g} \mathrm{Na}^{+} / \mathrm{L} \text { are not added, }
$$

a total difference of $173.6+64.4=238$ gram $/ \mathrm{ltr}$. The mixed TA-55 effluent is then reduced from $263,000 \mathrm{mg} / \mathrm{L}$ to $\left(263,000-238.000=25,000 \mathrm{mg} / \mathrm{L}=25 \mathrm{~g} / \mathrm{L}\right.$ (based on $120,000 \mathrm{~L} / \mathrm{yr}$ ) of mostly $\mathrm{Cl}^{-}, \mathrm{F}^{-}, \mathrm{K}^{+}, \mathrm{Na}^{+}$, etc. While this is not as low a concentration as the TA-50 "neat," it can still be volume reduced by a factor of 6 , to a 15 percent TDS or more typical of the bottoms from an evaporator, and on this basis is shown mixed into the feed to the evaporator. 
There is now enough information to make an estimate of the TDS in the TA-50 "neat" stream before the Room 60 effluent is mixed in:

"Nuclear Chemical Engineering," Benedict, et.al,. p 592, 595, McGraw-Hill, N.Y., (1981) Second Addition

$$
\begin{gathered}
{\left[\left(20.5 \times 10^{6} \mathrm{~L} / \mathrm{yr}\right) \times 2,500 \mathrm{mg} / \mathrm{L}\right]-[(120,000 \times 1.4 \mathrm{~L} / \mathrm{yr}) \times 188,000 \mathrm{mg} / \mathrm{L}]} \\
51.25 \times 10^{6} \mathrm{~g} / \mathrm{yr} \quad 31.584 \times 10^{6} \mathrm{~g} / \mathrm{yr} \\
=19.667 \times 10^{6} \mathrm{~g} / \mathrm{yr}=19,667 \mathrm{Kg} / \mathrm{yr} \\
19,667 \mathrm{Kg} / \mathrm{yr}+\left(20.5 \times 10^{6}-.120 \times 10^{6} \times 1.4\right) \mathrm{ltr} / \mathrm{yr}=967 \mathrm{mg} / \mathrm{L} \\
.168 \times 10^{6}
\end{gathered}
$$

which, as stated, is much lower than the $25,000 \mathrm{mg} / \mathrm{L}$ coming in after $\mathrm{NO}_{3}$ - removal.

If leave enough $\mathrm{HNO}_{3}$ in the acid waste to react with the $-0.5 \mathrm{~N} \mathrm{NaOH}$ in the caustic waste

$24,000 \mathrm{~L} / \mathrm{yr} @ 0.5 \mathrm{~N}=12,000$ gram equivalent

M.W. $\mathrm{NaOH}=40 ; x 40$ g/g.eq. $=480,000$ grams $\mathrm{NaOH}$

need 12,000 g.eq. of $\mathrm{HNO}_{3}$ to react.

$$
12,000 \text { g.eq. } x 63 \text { g/g.eq. of } \mathrm{HNO}_{3}=756 \mathrm{Kg} \mathrm{HNO}
$$

So from $21,168 \mathrm{Kg} \mathrm{HNO}$, leave $756 \mathrm{Kg} \mathrm{HNO}_{3}$ and remove $(21,168$ - 756 =) 20,412 Kg; a 96.4 percent removal of nitrate.

This is the design requirement for nitrate removal or destruction. A lower removal efficiency will be reflected in an increase in salt quantity in the evaporator bottoms. The combined feed of TDS into the process is:

TA-50 influent, "neat" $=20.332 \times 10^{6} \mathrm{~L} / \mathrm{yr}$ at $967 \mathrm{mg} / \mathrm{L}=19,667 \mathrm{Kg} / \mathrm{yr}$

Room 60 effluent $=168,000 \mathrm{~L} / \mathrm{yr} @ 25,000 \mathrm{mg} / \mathrm{L}=4,200 \mathrm{Kg} / \mathrm{yr}$

(16 percent of Total)

$120,000 \mathrm{~L} / \mathrm{yr} \times(25 \mathrm{~g} / \mathrm{L}+[238 \times .03 \mathrm{~L}]) \approx 168,000 \times 25 \mathrm{~g} / \mathrm{L}=23,866 \mathrm{Kg} / \mathrm{yr}$ (Mixed Feed)

There is thus a reduction in TDS of from:

to:

$$
19,667 \mathrm{Kg} / \mathrm{yr} \text { from TA-50 }+31,584 \mathrm{Kg} / \mathrm{yr} \text { from Room } 60=51,251 \mathrm{Kg} / \mathrm{yr}
$$

$$
19,667 \mathrm{Kg} / \mathrm{yr} \text { from TA-50 }+4,000 \mathrm{Kg} / \mathrm{yr} \text { from Room } 60=23,867 \mathrm{Kg} / \mathrm{yr}
$$

This $50^{+}$percent reduction in a large volume of secondary radioactive waste is the justification for the nitrate removal or destruction step in the process. Recycle vs. destruction would save the cost of $0.964 \times 46,667=$ $45,000 \mathrm{lbs} / \mathrm{yr} \mathrm{HNO}_{3}$ and have much less impact on the environment.

An alternative considered was use of the $\mathrm{CaCO}_{3}$, produced by carbonation of the clarified overflow to neutralize the $\mathrm{HNO}_{3}$. The overflow from the clarifiers contains dissolved (excess) $\mathrm{Ca}(\mathrm{OH})_{2}$. In the present operations this is reacted with gaseous $\mathrm{CO}_{2}$ and the $\mathrm{CaCO}_{3}$ formed $\left(\mathrm{Ca}(\mathrm{OH})_{2}+\mathrm{CO}_{2}-\mathrm{CaCO}_{3} \downarrow+\mathrm{H}_{2} \mathrm{O}\right)$ is removed by filtration. Upon backwashing the filters, this $\mathrm{CaCO}_{3}$ could become available for reaction with $\mathrm{HNO}_{3}$ per

$$
\mathrm{CaCO}_{3}+2 \mathrm{HNO}_{3} \rightarrow \mathrm{Ca}^{++}+2 \mathrm{NO}_{3}^{-}+\mathrm{H}_{2} \mathrm{O}+\mathrm{CO}_{2}^{\top}
$$

replacing the $\mathrm{NaOH}$ presently being used for this purpose. A sludge waste volume would be eliminated, $\mathrm{CaCO}_{3}$, but a salt waste volume $\mathrm{Ca}\left(\mathrm{NO}_{3}\right)_{2}$ would be produced. The savings is in not having to use about $28,000 \mathrm{lb} / \mathrm{yr}$ of $\mathrm{NaOH}$ to neutralize this stream.

The problem with this alternative is an anticipated "common ion" effect from the soluble $\mathrm{Ca}^{++}$salt. There would be a major reduction in the solubility of the $\mathrm{Ca}(\mathrm{OH})_{2}$ in such a high $\mathrm{Ca}^{++}$solution, reducing the concentration 
of $\mathrm{OH}^{-}$and $\mathrm{pH}$. The $\mathrm{OH}^{-}$concentration and $\mathrm{pH}$ are critical parameters in the flocculation and precipitation steps using $\mathrm{Fe}_{2}\left(\mathrm{SO}_{4}\right)_{3}$ and lime $\left(\mathrm{Ca}(\mathrm{OH})_{2}\right)$.

$$
\begin{array}{ll}
\mathrm{Fe}_{2}\left(\mathrm{SO}_{4}\right)_{3}+3 \mathrm{Ca}(\mathrm{OH})_{2} \rightarrow 2 \mathrm{Fe}(\mathrm{OH})_{3} l+3 \mathrm{Ca}^{++}+3 \mathrm{SO}_{4}{ }^{2} \\
\text { and } \mathrm{Pu} \text { or } \mathrm{Am}+4(\mathrm{OH})^{-} \rightarrow \mathrm{Pu}(\mathrm{OH})_{4} l \text { or } \mathrm{Am}(\mathrm{OH})_{4} ! \\
\text { plus Heavy metals }+\mathrm{OH} \rightarrow \text { Heavy metal hydroxides } \downarrow
\end{array}
$$

This idea can be pursued further in future developmental work. There is no basis to make comparisons at this time. Use of an alternate flocculent such as shown may have some promise. Numbers showing the reduction in solubility are shown as part of the sludge calculations.

With most of the nitrate removed, an approximate composition of the TA-55 effluent (Room 60 influent) looks like this:

$\begin{array}{llll}\mathrm{Cl}^{-} & 6,310 \times 1.4= & 8,834 \mathrm{mg} / \mathrm{L} \\ \mathrm{F}^{-} & 250 \times 1.4 & = & 350 \mathrm{mg} / \mathrm{L} \\ \mathrm{K}^{+} & 6,500 \times 1.4 & = & 9,100 \mathrm{mg} / \mathrm{L} \\ \mathrm{Alk}(\mathrm{OH} ?) & 2,150 \times 1.4 & = & 3,010 \mathrm{mg} / \mathrm{L} \\ \mathrm{Na}^{+} & 1,666 \times 1.4 & = & 2,332 \mathrm{mg} / \mathrm{L} \\ \mathrm{NO}_{3}^{-} & 4,491 \times 1.4 & = & 6,288 \mathrm{mg} / \mathrm{L} \\ \mathrm{COD} & 303 \times 1.4 & = & 424 \mathrm{mg} / \mathrm{L} \\ \mathrm{Ca}^{++} & \text {?? Minor } & = & \left(300^{+}\right) \mathrm{mg} / \mathrm{L} \\ \mathrm{SO}_{4} & 35 & & \\ \mathrm{Al}^{+++} & \text {?? Minor } & & \\ \mathrm{PO}_{4} & \text { ?? Minor } & & \\ \mathrm{CO}_{3}{ }^{2} & \text { ?? Minor } & & \\ \mathrm{SiO}_{2} & \text { ?? Minor } & & \\ \mathrm{Fe} & \text { ?? Minor } & \\ \mathrm{Cr} & \text { ?? Minor } & \\ \mathrm{Ni} & \text { ?? Minor } & \\ \text { etc. } & & \\ \left(\mathrm{Ca}^{++} \text {should be saturated at pH }=12^{+}\right) & \end{array}$

accounting for 90 percent of a calculated TDS $=33,570 \mathrm{mg} / \mathrm{L}$ (DILUTED by 1.4 factor, TDS $=24,000 \mathrm{mg} / \mathrm{L}$ ) which is felt to be good agreement considering only a single analysis of Room 60 effluent as a basis.

Design is based on the numbers reported in the five annual reports, but it has been recognized from the start that these are significant "holes" in this data and a better waste characterization is needed before proceeding into a CDR. Further evidence of this need turned up after preparation of the summary sheet showing the 5-yr averages and DF requirements.

When using the given information on Room 60 influent of -80 percent of $120,000 \mathrm{~L} / \mathrm{yr}$ is $3.5 \mathrm{~N} \mathrm{HNO}_{3}$ as only information available dilution of $96,000 \mathrm{~L} / \mathrm{yr}$ at $3.5 \underline{\mathrm{N}}$ into $22 \times 10^{6} \mathrm{~L} / \mathrm{yr}$ reduces the concentration to $3.5 \underline{\mathrm{N}} \mathrm{x}$ $26,000=0.0153 \underline{\mathrm{N}}=\mathrm{mole} / \mathrm{ltr}$ $22 \times 10^{6}$

$$
0.0153 \underline{\mathrm{N}} \times \frac{62 \mathrm{~g}-\mathrm{NO}_{3}-}{\mathrm{g}-\mathrm{mole}}=0.947 \mathrm{~g} \mathrm{NO}_{3}-/ \mathrm{L}
$$

The 5-yr average value of $\mathrm{N}_{\text {as }} \mathrm{NO}_{3}{ }^{-}$is $362 \mathrm{mg} / \mathrm{L}$, then (5-yr average) $\mathrm{NO}_{3}^{-}=392 \times 62 / 14=1,603 \mathrm{mg} / \mathrm{L}$ (TA50 effluent). So only $947 / 1,603 \times 100=59$ percent of the $\mathrm{NO}_{3}{ }^{-}$comes through Room 60 . Destruction of 96.4 percent (sheet No. 11) of the TA-55 $\mathrm{NO}_{3}{ }^{-}$still leaves $1,603-(947 \times .964)=690 \mathrm{mg} / \mathrm{L} \mathrm{NO}_{3}{ }^{-}$in the TA-50 effluent after $\mathrm{NO}_{3}{ }^{-}$destruction. Since the $\mathrm{NO}_{3}{ }^{-}$is a significant species in terms of required DF to meet the L.S. $\&$ W.L. release criteria, these numbers are really not good enough for a solid design. 
It is further noted that the sum of the 5-yr average values is just about equal to the $3,140 \mathrm{mg} / \mathrm{L}$ TDS without including a 20 to 25 percent contribution from "Alk-MO."

Process design is predicated on removing most of the alpha $(\alpha)$ contamination in a precipitation/flocculation clarification process much like present practice in Room 60 and the TA-50 clarifiers; residual $\alpha$ 's and soluble radioactivity ( $\mathrm{Sr}, \mathrm{Cs}$, etc.), heavy metals, nitrates, etc. will be removed by evaporation. This is expected to result in a relatively small volume of TRU wastes (high disposal costs) and a larger volume of low level radioactive waste that has lower disposal costs.

The concept proposed for this duty is shown on the following page. Based on processing $20.5 \times 10^{6} \mathrm{ltr} / \mathrm{yr}$, an operating feed rate of $100 \mathrm{gpm}$ is chosen, $6,000 \mathrm{gph}$ or $22,710 \mathrm{ltr} / \mathrm{hr}$, requiring less than 1,000 operating hours per year $(4 \mathrm{hr} /$ day $\times 5$ day/wk $\times 50 \mathrm{wk} / \mathrm{yr})$.

There is little information available as to the efficiency of the contact process vs. the precipitation step even from the present operation; it is known that the efficiency of the combination is very good. For this reason the design is kept flexible. As an example, if most of the $\alpha$ 's were taken out in the contactor, the sludge would be internally recycled and a high $\propto$ sludge withdrawn for disposal. This could reduce the volume of TRU sludge for disposal. Bench scale tests can answer such questions as the design proceeds.

It is desired to bave about 1 percent sludge (wt), on average, in the contactor. This is a judgement value based on a review of the literature and considering known characteristics of a $\mathrm{Fe}_{2}\left(\mathrm{SO}_{4}\right)_{3}-\mathrm{Ca}(\mathrm{OH})_{2}$ sludge. Consulting with a knowledgeable supplier of Lamella Separators indicated a 1-hr residence time and use of a thickener would produce a 5 wt percentage sludge for recycle.

$$
\text { Then: } \frac{25 \mathrm{gpm} \times 5 \%+100 \mathrm{gpm} \times 0 \%}{125 \mathrm{gpm}}=1 \% \text { average }
$$

so the recycle is set at $25 \mathrm{gpm}$.

At $125 \mathrm{gpm}$ total flow to the contactor, a 1 -hr average residence time requires $125 \mathrm{gpm} \times 60 \mathrm{~m} / \mathrm{hr} \times 1 \mathrm{hr}=$ 7,500 gal working volume. Due to internal recirculation, the contactor will not produce as high a solids content sludge at the bottom draw off. Assuming about 3 percent, the flows from the contactor to the Lamella is about

$$
\begin{aligned}
& (\mathrm{x})(.03)+(125-\mathrm{x})(0.0)=(125)(.01) ; \mathrm{x}=42 \mathrm{gpm} \\
& \text { clear liquid }=125-42=83 \mathrm{gpm}
\end{aligned}
$$

The sludge is recycled to the "Flocculator" so as to not make a lot of fine material in passing through the high shear Flash Mixer. This may or may not be an actual requirement, and if not would simplify control of the flow from contactor to clarifier. Note that feed to the contactor and recycled sludge are shown going to a high solids mixing zone to optimize contact. The design is based on the principles used in "Slurry Recirculation Clarifiers."

The chemical feed to form the sludge adds little volume to the flow, so for 1-hr residence time the total volume of clarifier and thickener is 7,500 gal. Parkson Corporation's model No. 1120/55 LGST with a shortened sludge holding tank is typical $19 \mathrm{ft}$ high $\times 17 \mathrm{ft}$ deep $\times 10 \mathrm{ft}$ wide. Chemical additions are now calculated. Note that, in operation, the fresh feed to the contactor dissolves some of the excess $\mathrm{Ca}(\mathrm{OH})_{2}$ in the recycled sludge and becomes saturated, so the excess $\mathrm{Ca}(\mathrm{OH})_{2}$ added in the clarifier merely passes through the recycle. The 3 percent and 5 percent solids in the sludge are nominal values.

The following information (see Sheet 14 ) is abstracted from the 5 annual reports: (There is unfortunately no information on $\mathrm{Fe}^{+++}$.)

For a relatively constant through-past, this reduction over the years represents a gradual reduction in "dosages." The data was smoothed by plotting and drawing a straight line through the five points $\mathrm{BBL} / \mathrm{yr}$ vs. $\mathrm{Kg} \mathrm{SO}{ }_{4}{ }^{-}$ ADDED/yr, giving an excellent fit. Reading off the point on the line $3,500 \mathrm{Kg} / \mathrm{yr}$ (some scatter due to year to year carryover of unfiltered sludge) $\mathrm{SO}_{4}{ }^{2}-300 \mathrm{BBL}$ FILTER CAKE/yr, gives a requirement of: 


$$
\text { Reported } \mathrm{SO}_{4}=\text { Values }
$$

\begin{tabular}{||c|c|r|c|r||}
\hline & $\begin{array}{c}\text { TA-50 Inlet mg/L } \\
\text { Average/High/Low }\end{array}$ & $\mathrm{Kg} / \mathrm{Yr}$ & $\begin{array}{c}\text { TA-50 Outlet mg/L } \\
\text { Average/High/Low }\end{array}$ & $\mathrm{Kg} / \mathrm{Yr}$ \\
\hline 1991 & $32.3 / 101 / 7.7$ & 612 & $128 / 288 / 61.5$ & 2830 \\
\hline 1990 & $39.9 / 121 / 0$ & 750 & $194 / 501 / 99.5$ & 4090 \\
\hline 1989 & $27 / 37 / 9.9$ & 537 & $194 / 1180 / 61$ & 4620 \\
\hline 1988 & $23 / 34 / 15$ & 671 & $169 / 675 / 20$ & 5730 \\
\hline 1987 & $49.6 / 246 / 22$ & 1120 & $240 / 490 / 17$ & 6410 \\
\hline
\end{tabular}

\begin{tabular}{|c|c|c|c||}
\hline & $\Delta \mathrm{Kg} / \mathrm{Yr}=\mathrm{SO}_{4}{ }^{-}$ & $\begin{array}{c}\text { To Clarifier } \\
\text { Influent }\end{array}$ & BBL's of Filter Cake \\
\hline 1991 & 2218 & $20.52 \times 10^{7} \mathrm{~L} / \mathrm{Yr}$ & $259 \mathrm{BBL} / \mathrm{Yr}$ \\
\hline 1990 & 3340 & $19.11 \times 10^{7} \mathrm{~L} / \mathrm{Yr}$ & $294 \mathrm{BBL} / \mathrm{Yr}$ \\
\hline 1989 & 4083 & $20.08 \times 10^{7} \mathrm{~L} / \mathrm{Yr}$ & $323 \mathrm{BBL} / \mathrm{Yr}$ \\
\hline 1988 & 5059 & $25.07 \times 10^{7} \mathrm{~L} / \mathrm{Yr}$ & $404 \mathrm{BBL} / \mathrm{Yr}$ \\
\hline 1987 & 5290 & $22.59 \times 10^{7} \mathrm{~L} / \mathrm{Yr}$ & $391 \mathrm{BBL} / \mathrm{Yr}$ \\
\hline
\end{tabular}


supplied as

$$
\mathrm{Fe}_{2}\left(\mathrm{SO}_{4}\right)_{3}, 160 \mathrm{mg} / \mathrm{L} \times \frac{\mathrm{Fe}_{2}-\left(\mathrm{SO}_{4}\right)_{3}}{3 \mathrm{SO}_{4}{ }^{-}=288.0 \mathrm{M} . \mathrm{W} .}=222 \mathrm{mg} / \mathrm{L}
$$

$3 \mathrm{CaO}+3 \mathrm{H}_{2} \mathrm{O} \rightarrow 3 \mathrm{Ca}(\mathrm{OH})_{2}$

$3(56) \quad 3(18) \quad 3(74)$

$$
\begin{array}{rrr}
\mathrm{Fe}_{2}\left(\mathrm{SO}_{4}\right)_{3} & +\underset{399.7}{3 \mathrm{Ca}(\mathrm{OH})_{2}} \rightarrow \underset{3(74)}{2 \mathrm{Fe}(\mathrm{OH})_{3}}+\underset{2(106.85)}{3 \mathrm{SO}_{4}{ }^{2}}+3(96) & +3 \mathrm{Ca}^{++} \\
3(40)
\end{array}
$$

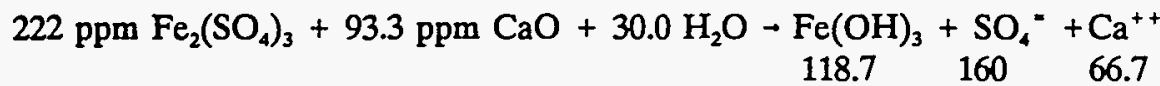

Therefore $222 \mathrm{Fe}_{2}\left(\mathrm{SO}_{4}\right)_{3}$ requires $93.3 \mathrm{CaO}$ or $123.3 \mathrm{Ca}(\mathrm{OH})_{2}$ for reaction.

Saturation with $\mathrm{Ca}(\mathrm{OH})_{2}$ requires $-700 \mathrm{ppm} \mathrm{Ca}(\mathrm{OH})_{2}$ or $-530 \mathrm{ppm} \mathrm{CaO}$.

Total use of $\mathrm{CaO}=93.3+530=623.3 \mathrm{ppm}$

We round these numbers to a total of $1000 \mathrm{ppm}\left(250 \mathrm{mg} / \mathrm{L} \mathrm{Fe}\left(\mathrm{SO}_{4}\right)_{3}+750 \mathrm{mg} / \mathrm{L} \mathrm{CaO}\right)$ as being a "TYPICAL" treatment level using an $\mathrm{Fe}_{2}\left(\mathrm{SO}_{4}\right)_{3}$ based treatment system. For comparison, Rocky Flats uses $1,500 \mathrm{ppm}$ in the first stage and $500 \mathrm{ppm}$ in the next two stages of a non-recycle system.

$$
\begin{aligned}
& 250 \mathrm{ppm} \mathrm{Fe}{ }_{2}\left(\mathrm{SO}_{4}\right)_{3} \quad 134 \mathrm{ppm} \mathrm{Fe}(\mathrm{OH})_{3} \\
& 750 \mathrm{ppm} \mathrm{CaO} \text { using } 105 \mathrm{ppm} \mathrm{CaO} \text { for reaction } \\
& 700 \mathrm{ppm} \mathrm{Ca}(\mathrm{OH})_{2} \text { in } \quad 530 \mathrm{ppm} \mathrm{CaO} \text { for saturation } \\
& \text { leaving } 115 \mathrm{ppm} \mathrm{CaO} \text { to form solution } \\
& 152 \mathrm{ppm} \mathrm{Ca}(\mathrm{OH}) \text { in suspension. }
\end{aligned}
$$

The required chemical rates are then assumed to be:

$250 \mathrm{ppm} \mathrm{Fe}{ }_{2}\left(\mathrm{SO}_{4}\right)_{3} \times 100 \mathrm{gpm} \times 60 \mathrm{~min} / \mathrm{hr} \times 8.33 \mathrm{lb} / \mathrm{gal}=12.5 \mathrm{lb} / \mathrm{hr}$ $750 \mathrm{ppm} \mathrm{CaO} \times(50,000)=37.5 \mathrm{lb} / \mathrm{hr}$ about $10 \mathrm{ppm}$ POLYMER for flocculation

After dissolution and reaction:

$134 \mathrm{ppm} \mathrm{Fe}(\mathrm{OH})_{3}$ are formed

$152 \mathrm{ppm} \mathrm{Ca}(\mathrm{OH})_{2}$ remains in suspension

$-90 \mathrm{ppm}$ ppt formed or T.S.S. removed

$$
\begin{aligned}
& =6.7 \mathrm{lb} / \mathrm{hr} \\
& =7.6 \mathrm{lb} / \mathrm{hr} \\
& =4.0 \mathrm{lb} / \mathrm{hr}
\end{aligned}
$$

\section{IN SLUDGE FORMED}

$$
=18.3 \mathrm{lb} / \mathrm{hr}
$$

(90 ppm comes from $70 \mathrm{ppm}$ T.S.S. $+3.5 \mathrm{ppm}$ metals $+5.7 \mathrm{ppm} \mathrm{PO}_{4}{ }^{*}+4.8 \mathrm{ppm} \mathrm{Mg}^{++}$.) The overflow from the clarifier contains $-700 \mathrm{ppm} \mathrm{Ca}(\mathrm{OH})_{2}$ in solution (assumed saturated, $\mathrm{K}_{\mathrm{sp}}=5.5 \times 10^{-6}$, Lange's). In the present operation some of this is precipitated out by reaction with $\mathrm{CO}_{2}(\mathrm{~g})$ and then removed by filtration. Backwashing the filters ultimately returns this $\mathrm{CaCO}_{3}$ to the sludge, and then to the filter cake. Of the $700 \mathrm{ppm}$ $\mathrm{Ca}(\mathrm{OH})_{2}, 378 \mathrm{ppm}$ is $\mathrm{Ca}^{++} ; \times 22 \times 10^{6} \mathrm{~L} / \mathrm{yr}=8,316 \mathrm{Kg} / \mathrm{yr}$. 


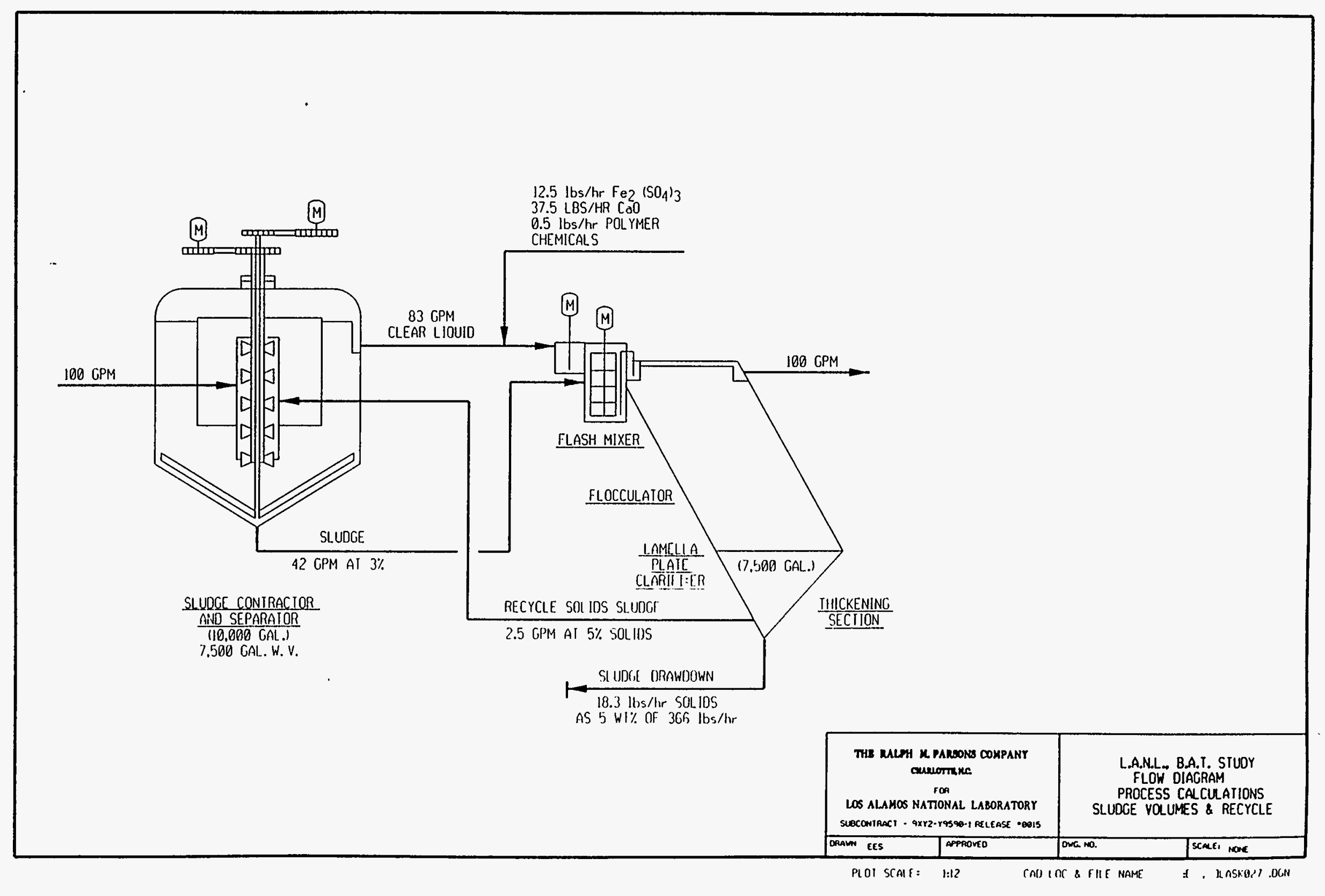


Presently, about $4,500 \mathrm{Kg} / \mathrm{yr}$ of this $\mathrm{Ca}^{++}$is added to the effluent. From sheet No. 4, for 1990 , $5,160 \mathrm{Kg} \mathrm{Ca}{ }^{++}$(out) $-633 \mathrm{Kg}$ (in) $=4,527 \mathrm{Kg} \mathrm{Ca}^{++}$added. $8,316 \mathrm{Kg} \mathrm{Ca}^{++} / \mathrm{yr}-4,527 \mathrm{Kg} \mathrm{Ca}^{++} / \mathrm{yr}$ to effluent $=3,789 \mathrm{Kg} \mathrm{Ca}^{++} /$yr to form $\mathrm{CaCO}_{3}$.

$$
3,789 \mathrm{Kg} \times \frac{\mathrm{CaCO}_{2}=100}{\mathrm{Ca}^{++}=40}-9,472 \mathrm{Kg} \mathrm{CaCO} \text { to sludge. }
$$

The reported release of filter cake from TA-50 for 1990 was 294 barrels, $61,134 \mathrm{ltr}, 62,241 \mathrm{Kg}=137,231 \mathrm{lb}$, while the use of filter aid was $21,000 \mathrm{lb}$, processing $21.15 \times 10^{6} \mathrm{~L}$.

$21.15 \times 10^{6} \mathrm{~L} / \mathrm{yr} @ 100 \mathrm{gpm}$ requires $931 \mathrm{hr}$ of operation.

$931 \mathrm{hr} \times 18.3 \mathrm{lb} / \mathrm{hr}=17,043 \mathrm{lb}$ sludge other than $\mathrm{CaCO}_{3}$

$9,472 \mathrm{KgCaCO}_{3}=20,882 \mathrm{lb} \mathrm{CaCO}_{3}$ (based on assumed usage, and 1990 release

$21,000 \mathrm{lb}$ of filter-aid used

$58,925 \mathrm{lb}$ solids in filter cake

compared to: $\quad 137,231 \mathrm{lb}$ Total filter cake

$78,306 \mathrm{lb}$ water in filter cake $=57$ percent

This 57 percent moisture compares to a reported 65-70 percent moisture (basis unknown).

The above is not intended as a material balance; there are too many unknowns, including the assumed dosage rates, and an "average" composition of the filter cake is an unknown. What is shown is "numbers in the same ballpark" useful for a conceptual design as the basis for a BAT study.

The annual reported chemical usage was (for 1990)

$\begin{array}{llll}\text { Lime } & 12,870 \mathrm{lb} & = & 4,170 \mathrm{KgCa}^{++} \text {if } \mathrm{CaO} \\ \text { Ferrie sulfate } & 4,332 \mathrm{lb} & = & 1,416 \mathrm{KgSO}_{4}=\text { if } \mathrm{Fe}_{2}\left(\mathrm{SO}_{4}\right)_{3} \\ \text { Perlite } & \underline{21,050 \mathrm{lb}} & & \end{array}$

TOTAL $\quad 38,252 \mathrm{lb}$

Considering weight losses due to $\mathrm{Fe}_{2}\left(\mathrm{SO}_{4}\right)_{3} \rightarrow 2 \mathrm{Fe}(\mathrm{OH})_{3}$ and solubility losses of $\mathrm{Ca}^{++}$which did not go to $\mathrm{CaCO}_{3}$, there is no way to account for even 30 percent of $137,000 \mathrm{lb}$ (of filter cake). Actually the gain in $\mathrm{Ca}^{++}$and $\mathrm{SO}_{4}{ }^{*}$ between influent and effluent shown on sheet No. 4 are larger than the $\mathrm{Ca}^{++}$and $\mathrm{SO}_{4}{ }^{*}$ in the reported usage, leaving none to add to cake. This is why a more detailed material balance could not be made.

The processing as described in this section is principally aimed at treating the $20.5 \times 10^{6} \mathrm{ltr} / \mathrm{yr}$ of TA-50 influent without the TA-55 effluent (or current Room 60 effluent) being added into it. As such, it duplicates much of the present TA-50 operation, in smaller sized, enclosed equipment for ALARA considerations. Removal efficiencies for the $\alpha$ 's and the heavy metals is expected to be comparable to present operations with improved contacting compensating for shorter residence time.

But a major factor in the choice of this equipment was a need for something to process the small TA-55 flow which contains most of the $\propto$ contamination. This flow at $120,000 \mathrm{ltr} / \mathrm{yr}$ is only $1 / 170$ of the $20.5 \times 10^{6} \mathrm{ltr} / \mathrm{yr}$ of the TA-50 (neat) flow, and it was felt to be unrealistic to have to provide a large batch system to process only this flow; a small continuous operation at $1 / 2 \mathrm{gpm}$ was even more unrealistic. What is believed to be a good compromise has been worked out, but to understand it, one must understand the planned operations which is described in the following. First it is necessary to document a few numbers derived from recent annual reports (1990).

The influent to Room 60 , TA-55 effluent contained a reported $148.627 \mathrm{Ci}$ of $\propto$ radioactivity. $124.5 \mathrm{Ci}$ of $\propto$ radioactivity was released in the cement paste. The difference is measurement errors and possibly carryover of some sludge volume to the next year. Sampling and analysis methods suggest that the $124.5 \mathrm{Ci}$ was actually 
contained in the 24 barrels of sludge produced. Room 60 effluent had a reported average $50 \mathrm{nCi} / \mathrm{L}$ in the $\left(121,500 \mathrm{ltr}\right.$ received) estimated $198,000 \mathrm{ltr}$ released to mix into TA-50 influent. This $\left(50 \times 10^{\rightarrow} \mathrm{Ci} / \mathrm{L} \times 198,000\right.$ $\mathrm{L} \Rightarrow) 9.9 \mathrm{mCi}$ is a small part of the reported $1.426 \mathrm{Ci}$ in the TA-50 influent. The reported release of $\propto$ activity was $3.47 \mathrm{Ci}$ in $62,247 \mathrm{Kg}$ of filter cake and $0.003674 \mathrm{Ci}$ in $22 \times 10^{6}$ ltr of effluent, a release of $0.167 \mathrm{nCi} / \mathrm{L}$. The difference between the $1.43 \mathrm{Ci}$ in and $3.47 \mathrm{Ci}$ out is unexplained, but here, because of sampling and measurement methods, the influent is felt to be the more reliable number. One problem arises with the use of these numbers (and data from the other years show the same problem), $3.47 \mathrm{Ci} / 62,247 \mathrm{Kg}=56 \mathrm{nCi} / \mathrm{gram}$ vs. a limit of 100 $\mathrm{nCi}$ /gram above which the wastes become TRU wastes. If the cake wt in this year was reduced from the contents of 294 barrels to the wt in $(.56 \times 294=) 164$ barrels, all of this waste would become TRU waste = VERY HIGH DISPOSAL COST!! The $1.43 \mathrm{Ci}$ value would allow a much larger volume reduction before reaching the $100 \mathrm{nCi} /$ gram limit.

Averaging the data for the past several years predicts 20 barrels of cement paste from $120,000 \mathrm{~L}$ of TA-55 wastes.

At 22 gal of sludge per barrel and a sp.g. $=1.11$

$20 \mathrm{BBL} \times 55 \mathrm{gal} / \mathrm{BBL} \times \underline{22 \mathrm{gal}} \times(8.33 \times 1.11) \mathrm{lb} / \mathrm{gal}=4,068 \mathrm{lb}$ "sludge"

$55 \mathrm{gal}$

at $18 \mathrm{wt} \%$ solids, $4,068 \times 0.18=732.3 \mathrm{lb}$ solids

$732.3 \mathrm{lb}+\frac{120,000 \mathrm{~L}}{3.785 \mathrm{~L} / \mathrm{gal}}=0.0231 \mathrm{lb}$ solids/gal processed $3.785 \mathrm{~L} / \mathrm{gal}$

$137,231 \mathrm{lb}$ Wet filter cake

For TA-50 $\frac{62,247 \mathrm{Kg} \times 2.205 \mathrm{lb} / \mathrm{Kg}}{2.05 \times 10^{7} \mathrm{~L}+3.785 \mathrm{~L} / \mathrm{gal}}=\frac{0.0253 \mathrm{lb} \text { "cake" }}{\text { gal processed }}$

$5.416 \times 10^{6} \mathrm{gal}$

considering all the water and filter aid in the filter cake, it is apparent there is much lower chemical usage in the TA-50 operation. Rationing the data for 24 barrels of "cement paste" from Room 60 in 1990, the 20 barrels of cement paste containing the $732.3 \mathrm{lb}$ of sludge solids would contain $(4,992 \times 20 / 24=) 4,160 \mathrm{ltr}$ and weigh $(6,731 \times 20 / 24=) 5,609 \mathrm{Kg}$. For both of these waste streams it is seen that most of the secondary wastes are "packaging" required for disposal of a much smaller mass of chemical precipitates used to clean up the wastewaters.

It appears generally accepted that to meet the more stringent release requirements most of the TDS presently being released in the TA-50 effluent will have to be removed. This is shown in the flow diagram of the Value Engineering presentation Executive Summary, P.I. 10411, April 1992, Section 9 and 10, and what is the present practice at SRS-ETF and Rocky Flats. There is no practical way of removing only the offending soluble species presently available. If the current TA-50 effluent is treated by evaporation, the current 2,500 ppm of TDS in $22 \times 10^{6} \mathrm{ltr} / \mathrm{yr}$ results in

$$
2,500 \mathrm{ppm} \times 22 \times 10^{6} \mathrm{~L}(=\mathrm{Kg}) / \mathrm{yr}=55,000 \mathrm{Kg} / \mathrm{yr}=121,250 \mathrm{lb} / \mathrm{yr}
$$

that will have to be disposed of as a low level waste. The form for disposal has not been positively identified, but the reference cited above shows disposal in "Portland Cement." This indeed is the current practice at SRS and Rocky Flats to produce a material called "Salt Stone."

An overall block diagram can thus be drawn to show the current operations plus evaporation which would meet expected requirements, using SALT STONE criteria (sheet No. C-20). 


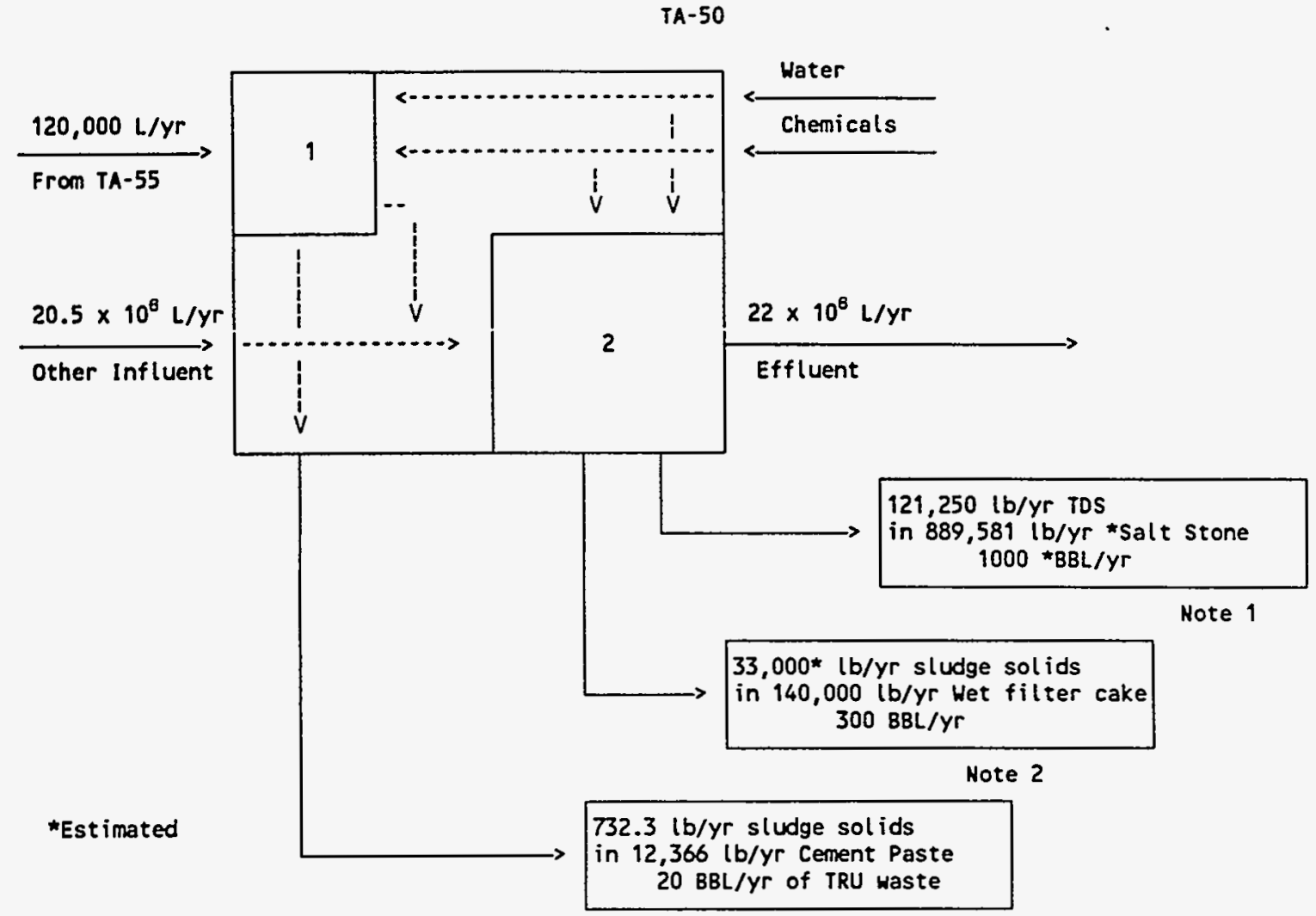

Note 1

Nitrate destruction in Block 1 reduces the 121,250 lb/yr TOS to about $(121,250-(62,963 \times$ .964 )(Sheet Nos. 7 and 11$) \Rightarrow 60,558$ (b/yr TOS with a proportionate decrease in volume of Salt Stone to $500 \mathrm{BBL} / \mathrm{Yr}$

Note 2

If "fixation" of this waste is required for disposal, there will be a major increase in this volume.

The present operation in TA-50 plus a projected volume produced by salt removal gives a total of $20+300+$ 1,000 barrels of secondary waste per year, $275,000 \mathrm{gal} / \mathrm{yr}$. This is 1.25 percent of the throughput and is comparable to the experience at other sites such as SRS and Rocky Flats. The lower numbers of past years has been the result of sending the TDS loading to the out fall and wet filter cake to burial, but this cannot continue.

Much of the above was derived from considerations of what can be done to upgrade the existing plant, since it is necessary to get a handle on existing practices and how efficient it is, but these numbers can serve as a basis for comparison of proposed alternatives. As an example, the simplest approach is to simply evaporate everything using redundant, low fouling, easy to clean evaporators. This appears feasible but would, of course, have to be demonstrated. The problem with this approach is that $150 \mathrm{Ci} / \mathrm{yr}$ into $1,000 \mathrm{BBL} / \mathrm{yr}$ of Salt Stone evaporator bottoms is $150 \mathrm{Ci} / \mathrm{yr}+900,000 \mathrm{lb} / \mathrm{yr}=367 \times 10^{-9} \mathrm{Ci} / \mathrm{gram}=1000 \mathrm{BBL}$ per year of TRU waste! This is NOT considered acceptable.

For the recommended design (from sheet No. 15), the treatment of the TA-50 "neat" part of the throughput results in $18.3 \mathrm{lb} / \mathrm{hr}$ of solids in the sludge formed from a $100 \mathrm{gpm}$ feed rate. $20.5 \times 10^{6} 1 \mathrm{lt}$ would result in

$$
18.3 \mathrm{lb} / \mathrm{hr} \times \frac{20.5 \times 10^{6} \mathrm{ltr} / \mathrm{yr}}{100 \mathrm{gpm} \times 60 \mathrm{~min} / \mathrm{hr} \times 3.785 \mathrm{~L} / \mathrm{gal}}=16,519 \mathrm{lb} / \mathrm{yr} \text { solids }
$$

coming off the Lamella Separator as a 5 wt percentage sludge. 
Except for the $\mathrm{CaCO}_{3}$ formed in present operations, by recarbonation of the feed to the mixed media filters, and added into the sludge (see sheet No. 16), the generation of sludge solids, $16,519 \mathrm{lb} / \mathrm{yr}$, is comparable to present operations. If the present practice of disposing of wet filter cake can continue, these solids can be easily handled by a R.V.P.F. (as present) resulting in a wet cake of:

$$
\begin{array}{ll} 
& 16,519 \mathrm{lb} \text { solids } \\
\text { containing } & 12,000 \mathrm{lb} \text { filter aid } \\
& 40,000 \mathrm{lb} \text { water } \\
68,519 \mathrm{lb} / \mathrm{yr} \text { wet filter cake }=\underline{150} \mathrm{BBL} / \mathrm{yr}
\end{array}
$$

Based on an INLET $\mathrm{Ci}$ of $\sim 1.5 \mathrm{Ci} / \mathrm{yr}$,

$$
\frac{1.5 \mathrm{Ci} / \mathrm{yr}}{68,519 \mathrm{lb} / \mathrm{yr} \times 454 \mathrm{~g} / \mathrm{lb}}=\frac{1.5 \mathrm{Ci}}{31.080 \times 10^{6} \mathrm{~g}}=48 \mathrm{nCi} / \mathrm{g}
$$

if the inlet $\mathrm{Ci}$ is over $3.0 \mathrm{Ci} / \mathrm{yr}$, this waste becomes TRU!

Alternatively, if this -5 wt percentage sludge is dried or dewatered (centrifuge ??) to about 30 wt percentage solids,

$$
16,519 \mathrm{lb} / \mathrm{yr} \text { solids }+0.3=55,063 \mathrm{lb} / \mathrm{yr} \text { sludge }
$$

and incorporated into "Salt Stone" at 47 wt percentage,

$$
55,063 \mathrm{lb} / \mathrm{yr} \text { sludge }+0.47=117,150 \mathrm{lb} / \mathrm{yr} \text { S.S. and at }-900 \mathrm{lb} / \mathrm{BBL}=130 \mathrm{BBL} / \mathrm{yr} \text {. }
$$

The denser Salt Stone could accommodate a possible $3.47 \mathrm{Ci} / \mathrm{yr}$ without becoming TRU waste.

$$
\frac{3.47 \mathrm{Ci} / \mathrm{yr}}{117,150 \mathrm{lb} / \mathrm{yr} \times 454 \mathrm{~g} / \mathrm{lb}}=\frac{3.47 \mathrm{Ci}}{53.140 \times 10^{6} \mathrm{~g}}=65 \mathrm{nCi} / \mathrm{g}
$$

This is the recommended route in view of the uncertainty of continuing to bury the wet cake.

\section{COMMON ION EFFECTS - TA 55 EFFLUENT}

If use $\mathrm{CaCO}_{3}$ formed from $\mathrm{Ca}(\mathrm{OH})_{2}+\mathrm{CO}_{2}(\mathrm{~g})$ to react with the $\mathrm{HNO}_{3}$ in TA-55 acid waste,

$$
\mathrm{CaCO}_{3}+2 \mathrm{HNO}_{3} \rightarrow \mathrm{Ca}^{++}+2 \mathrm{NO}_{3}^{-}+\mathrm{CO}_{2} \mathrm{I}+\mathrm{H}_{2} \mathrm{O}
$$

the $3.5 \mathrm{~N} \mathrm{HNO}_{3}$ results in $1.75 \mathrm{~g}$-moles of $\mathrm{Ca}^{++}$ions in solution in this volume. Diluting $96,000 \mathrm{ltr}$ of acid waste into $120,000 \mathrm{ltr}$ of total volume gives a $\mathrm{Ca}^{++}$concentration of 1.75 mole $/ \mathrm{ltr} \times \underline{96,000}=1.40 \mathrm{~mole} / \mathrm{ltr}$

From Lange's Handbook on Chemistry, $1985 \mathrm{Ed}$.

$$
\mathrm{K}_{\mathrm{Sp}} \mathrm{Ca}(\mathrm{OH})_{2}=5.5 \times 10^{-6}\left(20^{\circ} \mathrm{C}\right)
$$

$$
\mathrm{Ca}(\mathrm{OH})_{2}=\mathrm{Ca}^{++}+2 \mathrm{OH}^{-}
$$

No common ions: $\quad[\mathrm{Ca}][\mathrm{OH}]^{2}=5.5 \times 10^{-6} \quad[\mathrm{Ca}]=\mathrm{x}[\mathrm{OH}]=2 \mathrm{xmole} / \mathrm{ltr}$

$$
(x)(2 x)^{2}=5.5 \times 10^{-6}
$$

$$
4 \mathrm{x}^{3}=5.5 \times 10^{-6} ; \mathrm{x}=0.01112 \mathrm{~mole} / \mathrm{ltr}
$$

$[\mathrm{Ca}]=0.01112 \mathrm{~mole} / \mathrm{ltr}=0.4448 \mathrm{~g} / \mathrm{ltr}=445 \mathrm{ppm} \backslash 823 \mathrm{ppm}$

$[\mathrm{OH}]=0.02224 \mathrm{~mole} / \mathrm{ltr}=0.3781 \mathrm{~g} / \mathrm{ltr}=378 \mathrm{ppm} / 0.823 \mathrm{~g} / \mathrm{ltr}$

$$
\mathrm{pH}=12.347
$$

With common ions: $\quad(1.40+x)(2 x)^{2}=5.5 \times 10^{-6}$

$$
\begin{array}{cc} 
& x\left(x^{3}+1.4 x^{2}\right)=5.5 \times 10^{-6} \\
x<<1.4 & 1.4 x^{2}-1.375 \times 10^{-6}
\end{array}
$$


Information on Salt Stone

Abstracted from D.P.S.T.-88-372, March 15, 1988

Revised May 19, 1988

Table 4

Acceptable Formulation Range for Slag Salt Stone

\begin{tabular}{||l|c|c|c|}
\hline \multicolumn{1}{|c|}{ Material } & Minimum & Reference & Maximum \\
\hline Grade 120 Slag & $10 \mathrm{wt} \%$ & $25 \mathrm{wt} \%$ & $40 \mathrm{wt} \%$ \\
\hline Class F Fly Ash & $10 \mathrm{wt} \%$ & $25 \mathrm{wt} \%$ & $40 \mathrm{wt} \%$ \\
\hline Cement or Ca(OH) $)_{2}$ & $0 \mathrm{wt} \%$ & $3 \mathrm{wt} \%$ & $10 \mathrm{wt} \%$ \\
\hline Salt Solution & $40 \mathrm{wt} \%$ & $47 \mathrm{wt} \%$ & $55 \mathrm{wt} \%$ \\
\hline$\frac{\text { Water } \quad \text { Ratio }^{* *}}{\text { Total Solids }}$ & $\begin{array}{c}0.4 \\
\mathrm{pH} \geq 12.0\end{array}$ & $\begin{array}{c}0.5 \\
10^{\circ} \mathrm{C}-35^{\circ} \mathrm{C}\end{array}$ & 0.64 \\
\hline
\end{tabular}

*The salt solution used contained $29 \mathrm{wt} \%$ dissolved salts.

${ }^{* *}$ Total solids include slag, fly ash, cement or $\mathrm{Ca}(\mathrm{OH})_{2}$, and dissolved salts.

$$
\begin{aligned}
& 29 \% \text { of } 47 \%=13.63 \mathrm{wt} \% \text { salts } \quad 55 \mathrm{gal}=962 \mathrm{lb} \\
& 33.37 \mathrm{wt} \% \text { water say } 900 \mathrm{lb} / \mathrm{BBL} \\
& 3.0 \text { wt \% cement or } \mathrm{Ca}(\mathrm{OH})_{2} \text { or } 410 \mathrm{Kg} / \mathrm{BBL} \\
& 25.0 \text { wt \% fly ash } \quad 100 \mathrm{nCi} / \mathrm{g} \times 410,000 \mathrm{~g} / \mathrm{BBL} \\
& 25.0 \text { wt \% slag TRU LIMIT }=0.041 \mathrm{Ci} / \mathrm{BBL} \\
& 100.0 \text { wt \% } \bar{p}-2.1 \mathrm{~g} / \mathrm{cc} \quad 0.1363 \times 900^{+} \mathrm{lb}=\underline{123 \mathrm{lb} \text { salt }}
\end{aligned}
$$

\begin{tabular}{|c|c|c|c|}
\hline & Added & & \\
\hline Portland Cement & \multirow{4}{*}{$\begin{array}{c}7-1 / 2-12 \mathrm{lb} \text { to } \\
\rightarrow\end{array}$} & \multirow{4}{*}{$\begin{array}{c}1.0 \mathrm{Gal} \\
\text { of } \\
\text { Liquid } \\
\text { Waste }\end{array}$} & \multirow{4}{*}{$\begin{array}{c}\text { Swells to } \\
1.3 \text { to } 1.6 \\
\text { Gallon Volume }\end{array}$} \\
\hline Fly Ash & & & \\
\hline Potters' Clay & & & \\
\hline Attapulgas Clay & & & \\
\hline
\end{tabular}

Hanford "Transportable Grout Equipment"

Typical grout materials

Information on grout mixtures used to design equipment

Additive Mixture

$\mathrm{pH}=7-14$ Viscosity 10-500 c.p., Non-Newtonian

Liquid wastes are "dilute" to $30 \%$ salts

Mixtures will range from 11 to $18 \mathrm{lb} / \mathrm{gal}$

1.32 to 2.16 sp.g.

Liquid may contain: $\quad \mathrm{F}^{-}, \mathrm{NH}_{3}, \mathrm{Al}_{2} \mathrm{O}_{3}, \mathrm{NO}_{2}^{-}, \mathrm{Na}^{+}, \mathrm{K}^{+}, \mathrm{Al}^{+++}, \mathrm{Ca}^{++}, \mathrm{PO}_{4}^{-}, \mathrm{CO}_{3}{ }^{-}, \mathrm{NO}_{3}^{-}, \mathrm{SO}_{4}^{-}, \mathrm{Fe}$, $\mathrm{Ni}, \mathrm{Cr}$, Little $\mathrm{Cl}^{-}$ 


$$
\mathrm{x}^{3}<<1.4 \mathrm{x}^{2} \quad \mathrm{x}=1 \times 10^{-3} \quad \mathrm{pH}=11.0
$$

The common ion effect was not as pronounced, in terms of $\mathrm{pH}$ reduction, as first thought. But recycle of $\mathrm{CaCO}_{3}$ would require a recarbonation step and a filtration step to recover the $\mathrm{CaCO}_{3}$ for recycle. If the evaporator bottoms are made into Salt Stone (S.S.) for final disposal, it is beneficial to leave the $\mathrm{Ca}(\mathrm{OH})_{2}$ dissolved in the feed to the evaporator. The evaporator is designed to handle scaling solutions (has to handle $\mathrm{SiO}_{2}$ ) and the high $\mathrm{pH}$ is protective of the materials of construction and beneficial to the formation of S.S. As a partial replacement for cement (see sheet No. 20), it could actually reduce the final volume of waste. Two unit operations, recarbonization and filtration are eliminated. Chemical destruction of the $\mathrm{NO}_{3}{ }^{-}$in the TA-55 waste stream is the basis of this design.

Starting with a clean, empty system, the required sludge volume is built up by adding chemicals while on a complete recycle of clean water. A "working" volume of sludge must be available to contact the wastewaters before starting the wastewater flow.

Requirements of sludge solids are $1 \mathrm{wt}$ percentage (average) in the 7,500 gal (working volume) contactor, 625 pounds, and 2 wt percentage (average) in the 7,500 gal Lamella Separator and Sludge Thickener, 1,250 lb. Then a day's batch of wastewater can be put through.

$100 \mathrm{gpm} \times 60 \mathrm{~min} / \mathrm{hr} \times 4 \mathrm{hr} / \mathrm{batch}=24,000 \mathrm{gal}$

$20.5 \times 10^{6} \mathrm{ltr} / \mathrm{yr}$ will require 226 days/yr

The equipment can then be shut down and the solids allowed to settle. No problems are anticipated in resuspending the solids to start the next run. At a calculated (sheet No. 15) $18.3 \mathrm{lb} / \mathrm{hr}$ production rate, $73.2 \mathrm{lb}$ of sludge solids per 4-hr run are drawn off as a 5 wt percentage slurry and stored for further processing.

It is desirable to put the TA-55 effluent through this same equipment, on an "as needed" basis. After nitrate destruction on this stream, the TDS concentration in this stream is low enough to be beneficially fed into the evaporator, so no change is required. The only difference is that for the same feed rate more bottoms are produced from the evaporator to go to the finishing evaporator.

There is an increase in the volume of TRU level sludge. If one starts with a working volume of $1,875 \mathrm{lb}$ of solids in the sludge held up in the two units, puts through a batch of $24,000 \mathrm{gal}(90,000 \mathrm{~L})$ of TA-55 effluent to produce $73.2 \mathrm{lb}$ of additional solids, and then drains and flushes the equipment to remove all high $\mathrm{Ci}$ sludge, the result is $1,953 \mathrm{lb}$ of potentially TRU solids. This is larger than the present $732.3 \mathrm{lb}$ solids/yr (sheet No. 17) in $20 \mathrm{BBL}$ of cement paste, so a way was sought to reduce this volume.

Any design for a plant of this type must incorporate redundancy! This can typically be provided by the use of 3-50 percent units (or 2-100 percent units). If 3-50 percent units are used, the TA-55 stream can be run through one of the units in use resulting in

$$
\frac{1953}{2}+73.2=1,049.7 \mathrm{lbs} \text { solids } / 24,000 \mathrm{gal}
$$

or

$$
1,049.7 \times \frac{120,000 \mathrm{~L} / \mathrm{yr}}{90,000 \mathrm{~L} / \text { Batch }}=1,400 \mathrm{lb} \text { solids } / \mathrm{yr}
$$

at 30 percent solids in the sludge:

$$
1,400 \mathrm{lb} \text { solids } \div 0.3=4,667 \mathrm{lb} \text { sludge }
$$

incorporated into Salt Stone at 47 wt percentage

$$
4,667 \mathrm{lb} \text { sludge }+0.47=10,000 \mathrm{lb} \text { Salt Stone } / \mathrm{yr}
$$


and at $900 \mathrm{lb} / \mathrm{BBL}=11 \mathrm{BBL} / \mathrm{yr}$ containing about $124.5 \mathrm{Ci}$ of $\propto$ 's. This is 50 percent reduction in number of barrels from present operations. If this reduction in the number of barrels results in potentially too high a concentration of Pu or Am per barrel. The TRU sludge can always be diluted with non-TRU sludge as a diluent. The total number of barrels TRU and non-TRU remains the same.

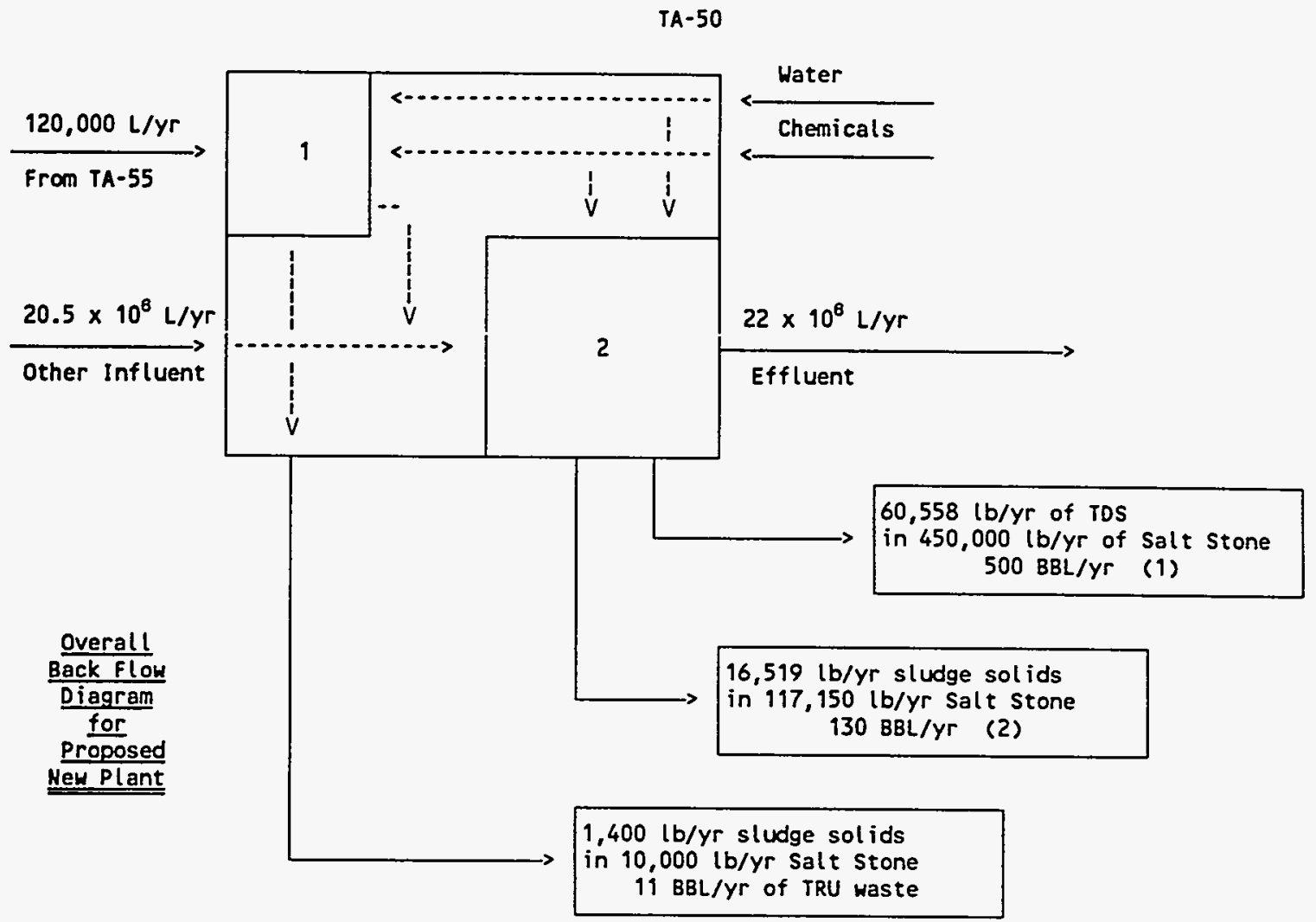

Note 1

Nitrate destruction in Block 1 reduces the $121,250 \mathrm{lb} / \mathrm{yr}$ TDS to about $(121,250-(62,963 \times$ $.964)$ (Sheet NoS. 7 and 11$) \Rightarrow 60,558$ (b/yr TDS with a proportionate decrease in volume of Salt Stone to $500 \mathrm{BBL} / \mathrm{Yr}$

Note 2

It is assumed that this non-TRU sludge must be "fixed" for acceptable final disposal.

With this volume for the expected amount of TRU waste, and the calculated volumes or the new operations from sheet Nos. 18 and 19, an overall block flow diagram for the proposed process can be developed as shown above. Comparison to the existing operation, sheet No. 19, (plus the waste from evaporation) projects a major reduction in the volume of solid waste produced. We now look at the details of treating the overflow from the clarifier by evaporation.

For a volatile constituent, the usual assumption is a DF of 1.0; output = input. The steam-stripping action will volatilize all but very high boiling components. For non-volatile components, the DF is determined by carryover from the evaporator. If there was no carryover, the DF would be infinite, but all evaporators will have some carryover, sub-micron droplets carried up with the vapors flowing out of the evaporator. The concentration in the condensate depends on the carryover and the bottoms concentration, but not on the feed.

DF is defined across an evaporator as:

Concentration in Feed $\equiv \mathrm{DF}$ Concentration in Condensate 
and can be measured; but really depends on the bottoms concentration selected (sort of an independent variable constrained by waste treatment considerations), and mechanical design of the equipment.

This can be illustrated using the design parameters of the SRS ETF Evaporators:

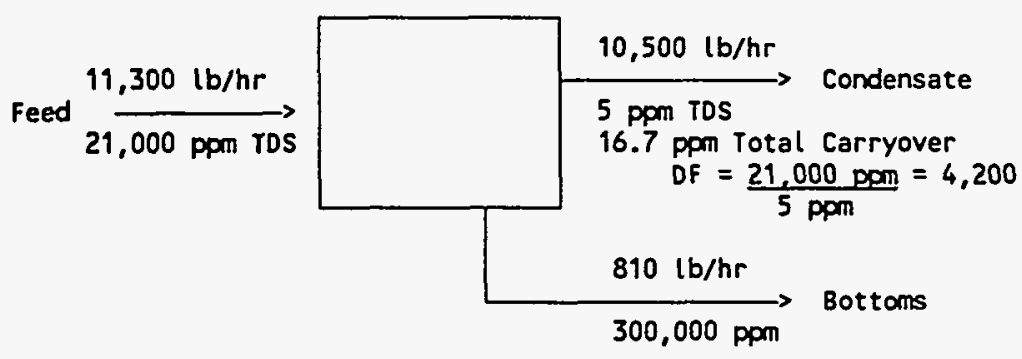

The required DF is 4,200 (4,600 was specified) to meet the required $5 \mathrm{ppm}$ in the condensate, and with a bottoms concentration (the "working volume" is the same) of $300,000 \mathrm{ppm}$, carryover must be limited to:

$$
\text { (0.3) }(\text { Carryover })=5 \mathrm{ppm} \quad \text { c.o. }=\frac{5 \mathrm{ppm}}{.30}=16.7 \mathrm{ppm}
$$

This is a "tight" carryover limitation, and is typically met by limiting the vapor velocity within the evaporator body. This entails the cost of a larger unit and becomes very significant if expensive materials of construction are required for corrosion protection. In the SRS ETF case this appears to be a gross over design (5 ppm in the condensate) because the condensate is recycled and does not have to meet release criteria. It was probably an initial intent to release this stream and then, when this was changed to recycle, the evaporator requirements were not reexamined.

As shown on the table of required DFs, the required DF to meet the L.S. \& W.L. criteria for $\mathrm{NO}_{3}{ }^{-}$of $\leq 10 \mathrm{mg} / \mathrm{L}$ is 160 without $\mathrm{NO}_{3}{ }^{-}$destruction on TA-55 effluent or (from sheet No. 12) 69 with $\mathrm{NO}_{3}$ - destruction. These are the maximum required DF's to meet this criterion.

If the limit in the condensate is $10 \mathrm{ppm} \mathrm{NO}_{3}{ }^{-}$and the $\mathrm{NO}_{3}{ }^{-}$is $1,603 \mathrm{ppm}$ of $2,500 \mathrm{ppm}$ TDS, $1,603 / 2,500=0.64$, and there can be $10 \mathrm{ppm}\left(\mathrm{NO}_{3}-1\right) / 0.64 \mathrm{NO}_{3}{ }^{-} / \mathrm{TDS}=15 \mathrm{ppm}$ TDS. If the operating fluid in the evaporator is about 20 percent TDS, the total carryover can be as large as $15 \mathrm{ppm}$ TDS $/ 0.20$ TDS/liquid $=75 \mathrm{ppm}$ total carryover which is easy to design to; $50 \mathrm{ppm}$ is a good design value. At 15 percent TDS in the bottoms or working fluid, the carryover can be as large as $100 \mathrm{ppm}$ of the circulating fluid. Summarizing: 


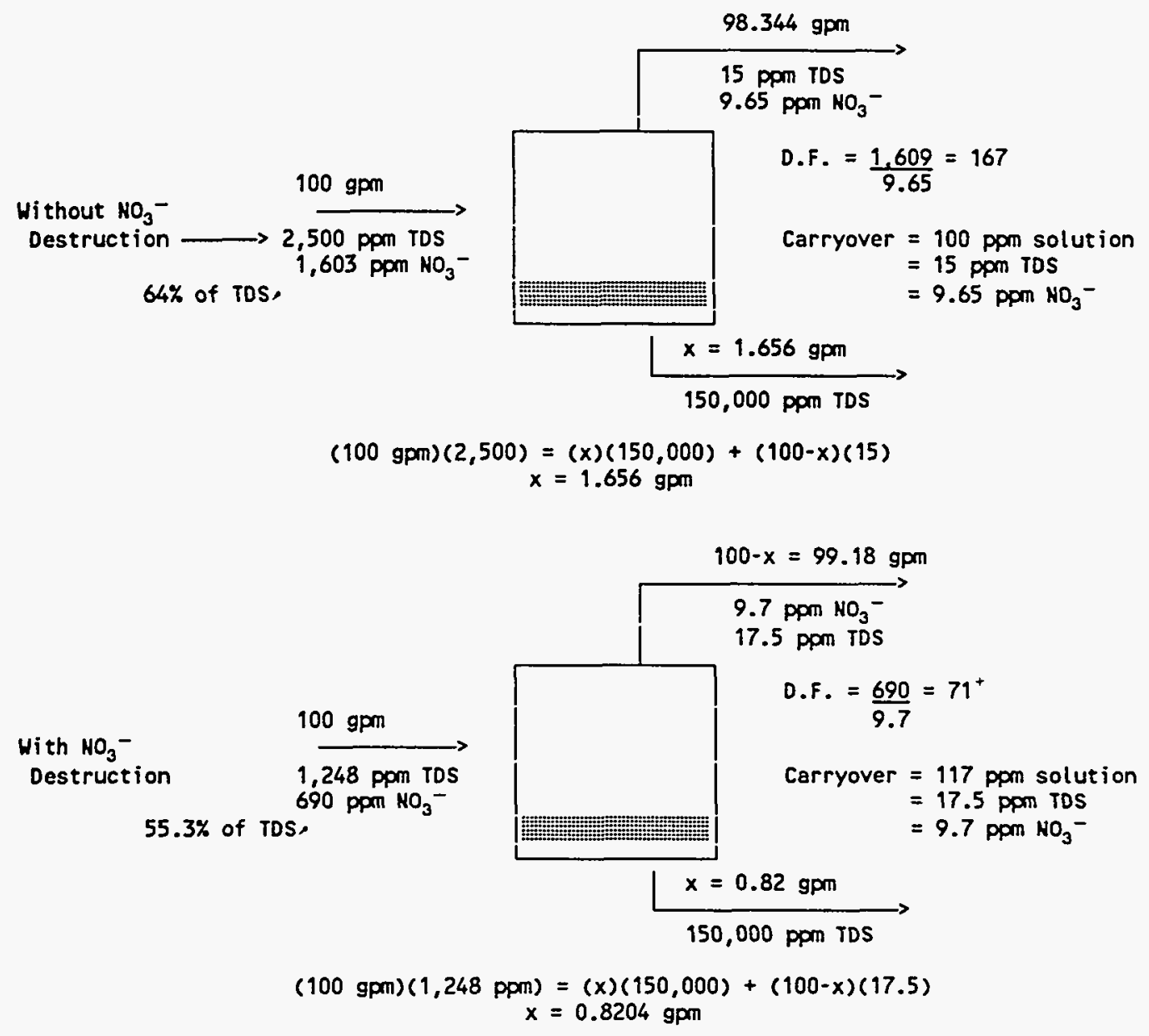

Use 15 percent TDS bottoms as design basis as lower TDS gives a lower boiling point elevation (b.p.e.) which is a better operation for a vapor recompression evaporator (V.R.C.E.).

Note the large difference in calculated DFs despite very small changes in operating parameters. This is, as stated, because the importance of the various variables, in terms of their influence on the required $s 10 \mathrm{ppm}$ $\mathrm{NO}_{3}{ }^{-}$, is

1. Carryover

2. Concentration of $\mathrm{NO}_{3}-/ \mathrm{TDS}$ (which changes little between cases $64 \% \mathrm{NO}_{3}--117 \mathrm{ppm} \mathrm{C.O}$

3. TDS in bottoms

$55.3 \% \mathrm{NO}_{3}-\frac{\mathrm{ppm} \mathrm{C.O}}{110 .}$

the DF is a purely calculated number.

Also nitrate destruction is not necessary to meet the $\mathrm{NO}_{3}{ }^{-}$release criteria of $s 10 \mathrm{ppm} \mathrm{NO}_{3}{ }^{-}$, but is used as a method to reduce the volume of salt stone wastes.

Since the required DFs, on all the other species, to meet the L.S. \& W.L. criteria are all less than 70 (actually $1 / 10$ of 70 or less), the proposed chemical precipitation and evaporation will meet all the requirements for case 6 - and also 4 and 5. A finishing evaporator as a thin-film wiped surface type is used to reduce the volume of the evaporator bottoms to the 30 percent TDS recommended for incorporation in Salt Stone. This is a relatively easy duty for this type of equipment. Depending on the final choice of a method of waste disposal (Salt Stone vs. other options), the duty on the main evaporator and finishing evaporator can be easily adjusted. A 10 percent 
TDS bottoms reduces the carryover by one third (say $9.7 \mathrm{ppm} \mathrm{NO}{ }_{3}^{-}$to $6.4 \mathrm{ppm}$ ) and increases the load on the finishing evaporator from $0.82 \mathrm{gpm}$ to $1.23 \mathrm{gpm}$. This study addresses feasibility, not optimization.

Examination of the required DFs to meet the more restrictive release criteria shows that only a few of the required DFs exceed ten:

\begin{tabular}{|c|c|c|}
\hline \multirow[b]{2}{*}{ for } & $\begin{array}{c}\text { Required DF } \\
\end{array}$ & \multirow[b]{2}{*}{$\begin{array}{l}\text { To Meet } \\
\text { September 11, } 1992 \text { NPDES }\end{array}$} \\
\hline & $\begin{array}{l}\text { To Meet } \\
\text { N.M.E.D.-051 (wildlife) }\end{array}$ & \\
\hline $\mathrm{Cu}$ & 850 & 9.6 \\
\hline $\mathrm{Cn}^{-}$ & 30 & 47 \\
\hline $\mathrm{Hg}$ & 12.2 & 30.5 \\
\hline $\mathrm{NH}_{3}$ & 115 & 384 \\
\hline $\mathrm{NO}_{3}-$ & 160 & \\
\hline $\mathrm{Ag}$ & 42 & 35 \\
\hline $\mathrm{Zn}$ & 25 & \\
\hline
\end{tabular}

The $\mathrm{NO}_{3}{ }^{-}$is taken care of by evaporation, and even the lower evaporator DF associated with reduced $\mathrm{NO}_{3}{ }^{-}$, of 70 is more than sufficient for everything but one of the copper, $\mathrm{Cu}$, numbers and the ammonia, $\mathrm{NH}_{3}$. The $\mathrm{NH}_{3}$ is easily reduced by conversion to an ammonium, $\left(\mathrm{NH}_{4}{ }^{+}\right)$ion by reaction with a mineral acid and removal as a non-volatile salt. This leaves only the copper. The required DF for copper, 850 , is so high because of an apparently unrealistic requirement of 0.2 parts per BILLION in the effluent, which is judged beyond the present limits of technology to either measure or treat.

The "worst" case of evaporation for removal of $\mathrm{Cu}$ is the $\mathrm{NO}_{3}{ }^{-}$destruction case shown on sheet No. 24, because the removal of some $\mathrm{NO}_{3}{ }^{-}$increases the relative concentration of $\mathrm{Cu}$ in the bottoms:

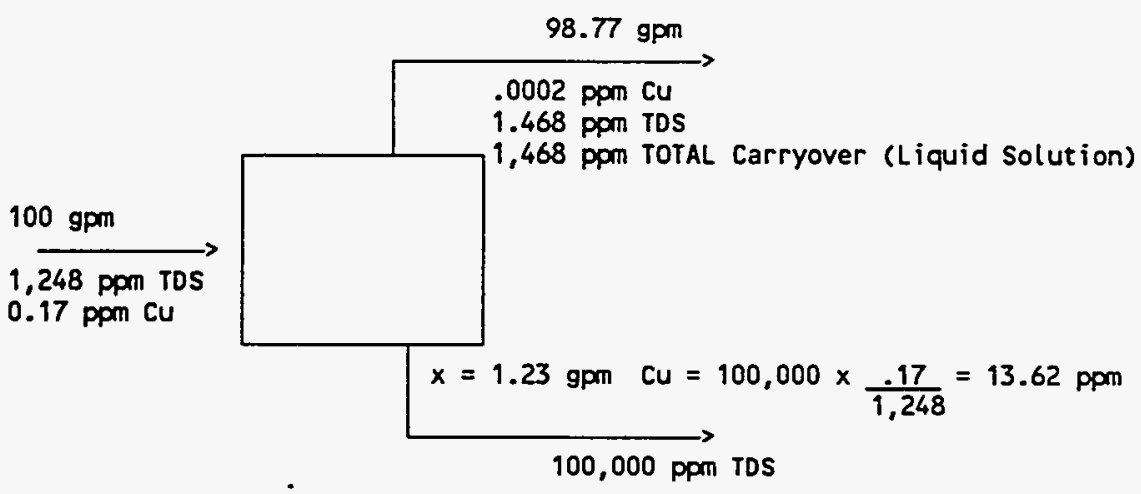

This is POSSIBLE, but is not considered a desirable design. Its right at the ragged edge. The Pritchess evaporator used as a model produces $<10 \mathrm{ppm}$ TDS condensate which is considered a good water quality and used for boiler feed makeup water. $10 \mathrm{ppm}$ TDS is three times larger than Cu removal requirements, even allowing for bottoms concentration differences. Even if designed, every time there was minor process upset or variation there is a high probability of having to recycle large quantities of condensate wastewater.

The high required DF for $\mathrm{Cu}$ is at least partially the result of the "high" average effluent value, $0.17 \mathrm{mg} / \mathrm{L}$. This is by far the highest concentration, in the effluent, of the various metals which require removal, and suggest a low removal efficiency in the precipitation/flocculation process. It is known that $\mathrm{NH}_{3}, \mathrm{CN}^{-}$, and EDTA, as well as other organics can complex copper and interfere with its removal. The $0.17 \mathrm{mg} / \mathrm{L} \mathrm{Cu}$ is quite a bit above the 0.02 to 0.07 expected achievable by hydroxide precipitation, so complexing is suspected. 
This is an area that shows the necessity for better waste characterization and process development work. If it was known where the copper was coming from, such as corrosion in certain equipment, it would be possible to reduce this source. If most of the copper is coming from one waste generator, it may be possible to treat it at or near the source, improving removal and reducing quantity in the effluent. Finally, if it could be shown that upstream removal of $\mathrm{NH}_{3}, \mathrm{CN}^{-}$, or EDTA would put the residual copper into the 0.02-0.07 range expected for precipitation, reducing the effluent to say $.0045 \mathrm{ppm}$, a DF of only 225 would be required to meet even the $0.0002 \mathrm{ppm}$ limit. Then evaporation could be all the post-treatment required. Upstream destruction of EDTA, other complexing agents and surfactants, is already being considered to enhance removal of transiaranics; but design requires data as a basis.

COD destruction is shown on the evaporator condensate, before final $\mathrm{pH}$ adjustment and release, using U.V. and ozone $\left(\mathrm{O}_{3}\right)$ as the oxidizer. This is precautionary, since the DF table does not indicate this requirement. But the composition of the COD is unknown and there may be certain constituents which must be destroyed and it may be useful to control $\mathrm{CN}^{-}$and $\mathrm{Cl}_{2}$ in the effluent. Using U.V. $+\mathrm{O}_{3}$, the logical location is on the clean condensate stream, where the U.V. tubes are not apt to foul or scale.

An alternate is the use of chemical destruction with strong oxidizers such as persulfates, permanganates, perchlorates, $\mathrm{Cl}_{2}$, and chromic acid. If this can be shown to be feasible, then COD destruction should be moved upstream (fouling or scaling of the U.V. tubes is no longer a consideration) with the advantage that destruction of chlorates and surfactants would result in improved operation.

Whether upstream reduction of the copper loading, or enhanced removal of copper can be achieved, there should still be an effort to have the permitted release of copper changed to something more reasonable. The 0.0002 $\mathrm{mg} / \mathrm{L}$ limit is $1 / 250$ of the World Health Organization's most restrictive Drinking Water Standards*, and less than 1/2,500 of the Livestock Watering and Wild Life standards, the released water's most restrictive likely use. The source of this value is completely indeterminate and it would be poor engineering judgement to attempt compliance. U.S. Public Health Drinking Water Standards, 1962 is $1.0 \mathrm{mg} / \mathrm{L}$ copper. Essential trace element, not very toxic.

If non-engineering considerations prevail, it is recommended that the problem be eliminated rather than solved. Design to zero release (of water) by maximizing evaporator performance to produce a high quality distilled water and then dispose of it by use of solar evaporation ponds. Previous study has shown that solar evaporation is a completely (technical) feasible operation for the LANL climatic conditions.

\section{A. NEW PLANT TO COMPLY WTTH TASK 015 (W.L. \& L.S.) RELEASE LIMITS}

1. A combined process is proposed using two stages of precipitation/flocculation, evaporation, and a finishing evaporator which can easily meet the requirements of processing the wastewater and discharging an effluent in compliance with the "Task 015" release criteria (also called the "L.W. \& W.L." criteria). $\mathrm{COD}$ destruction and nitrate, $\mathrm{NO}_{3}^{-}$, destruction are provided to remove volatile organics and reduce the secondary waste volume, but are not crucial to operation of the process. This addresses case 6, a new plant to treat the blended stream and meet the Task 015 release criteria.

2. Case 4, a new plant to treat the TA-50 "neat" influent is essentially the same design with a little less operating time required and a little less waste produced. The TA-50 "neat" stream is all the present influent to TA-50 less the present Room 60 effluent which is "blended" in.

3. Case 5, a new plant to treat only the TA-55 effluent to Task 015 release criteria would use the same process steps but on a much reduced scale. Cases 4 and 5 make little sense since the one plant for Case 6 can efficiently handle both streams (also see Case 2).

-The Nalco Water Handbook, page 35.3, Second Edition, 1988 
There is insufficient waste characterization data and process data information to attempt a material or energy balance on the existing processes. Fortunately, for the type of processes involved and the approach taken in this B.A.T. study, this lack of information did not prove an insurmountable problem. It should not be inferred that this lack of information dictated the type of processing chosen. The approach taken was that the feed, over the years, was subject to great variability and the plant had to be designed to handle just about anything that might come down the sewer line. It also had to be designed so that future required capabilities could be easily handled. The existing plant has many of these same characteristics.

The design basis is discussed in Section $A$ of these calcs, but it can be pointed out that the recommendation for a B.A.T. looks a lot like present operations at Rocky Flats - in fact it is highly likely that the Rocky Flats plant could successfully process the LANL wastes. On the other hand, one hell of a lot of additional information (waste characterization) would be required to make the same assessment for the ETF at SRS or the PCTF at Hanford.

In terms of functional requirements, the approach is quite simple:

1. Selectively remove the $\alpha$ 's to minimize the volume of TRU waste produced. Some heavy metals and TSS are co-removed.

2. Remove the TDS to remove residual heavy metals, radioactivity, $\mathrm{NO}_{3}{ }^{-}$. Residual TSS and other innocuous TDS is removed at the same time. (No known process has the selectivity to remove only the chemical species that must be removed for permitting.

3. Volatile contaminants remaining in the purified condensate (because of their volatility) are removed or destroyed. This is called the (volatile) COD fraction.

4. All materials removed from the wastewater by non-destructive means must be packaged for final disposal.

These four functional requirements are ALL that is required for a functional system, and could be implemented in a number of different ways. We have chosen:

1. Precipitation/Flocculation/Sedimentation

2. Evaporation

3. Oxidation using U.V. and Ozone

4. Fixation in Salt Stone

to meet these requirements. A supporting operation, of nitrate destruction, is used to reduce the volume of secondary waste produced but is not essential to functionality.

If alternate processing schemes are chosen, it will be necessary to more completely characterize the influent wastewaters in terms of expected variations in flow rates and in composition. For the present design, a number of deficiencies were noted and had to be worked around. These are listed to indicate the type of information that may be required to support alternate design approaches.

a. There is no information on the TA-55 effluent.

b. There is only one (1) compositional analysis of Room 60 effluent.

c. There is no information on several NPDES species.

d. We have no information on Room 60 chemical dosages or

e. on required TA-50 flocculator chemical dosages.

f. Consequently, the composition of the Room 60 sludge is unknown

g. as in the composition of the TA-50 filter cake. 
h. Use of $\mathrm{Ca}(\mathrm{OH})_{2}$ and $\mathrm{Fe}_{2}\left(\mathrm{SO}_{4}\right)_{3} \times \mathrm{H}_{2} \mathrm{O}$ is assumed as the flocculent; other chemical systems may be more effective and/or result in less secondary waste. Dosages are engineering judgement.

i. The 5-yr average TDS in TA-50 influent is $3,140 \mathrm{mg} / \mathrm{L}$ vs. $2,500 \mathrm{mg} / \mathrm{L}$ (1990 value and) assumed design basis. An arbitrary reduction of each specie by 25 percent is questionable and probably will not be representative.

j. COD, what is it and where does it presently go?

k. U.V. and $\mathrm{O}_{3}$ vs. chemical destruction of $\mathrm{COD}$ is speculative; there is no information on chemical dosages or efficiencies on which to base a choice.

1. Chemical destruction of $\mathrm{NO}_{3}{ }^{-}$is feasible, but the details of dosage, efficiency and off-gases are inadequate for material balance purposes.

m. Fixation requirements have not been defined.

n. TRU limits of max Pu content are speculative.

o. DF averages are based on numbers that are seen to be changing over the 1987-1991 period so there is a question as to which is more representative, averages or recent values?

p. The same holds true for quantities of individual species.

q. There are no detailed material balances shown in these calculations; the quality of the available data does not justify that depth of analysis. A typical example is the $1,603 \mathrm{mg} / \mathrm{L} \mathrm{NO}_{3}{ }^{-}$in $3,140 \mathrm{mg} / \mathrm{L}$ TDS (TA-50 average influent). $1,603 \mathrm{mg} / \mathrm{L} \mathrm{NO}_{3}{ }^{-}$will be reduced to $690 \mathrm{mg} / \mathrm{L}$ by nitrate destruction on the TA-55 effluent; but $690 \mathrm{mg} / \mathrm{L}$ in ??? $\mathrm{mg} / \mathrm{L}$ TDS ?? Assuming we start with $2,500 \mathrm{mg} / \mathrm{L}$ TDS, $2,500 \mathrm{mg} / \mathrm{L}-\mathrm{NaNO}_{3}=1,248 \mathrm{mg} / \mathrm{L}$ TDS. This is the number used even though it is not derived from the 3,140 value. $690 \mathrm{mg} / \mathrm{L} \mathrm{NO}_{3}{ }^{-}$in $1,248 \mathrm{mg} / \mathrm{L}$ TDS comes from two different sources. 